






\title{
GERMAN-ENGLISH DICTIONARY
}

for

\section{ELECTRONICS ENGINEERS}

and

PHYSICISTS

With a Patent-Practice Vocabulary

\author{
By BERNARD R. REGEN \\ Researcher and Translator \\ AND RICHARD R. REGEN \\ Physicist and Field Engineer \\ Magnetic Analysis Corporation
}

First Edition

1946

J. W. EDWARDS $0 s$ ANN ARBOR, MICHIGAN 


\section{Copyright 1946 by \\ Bernard R. Regen and Richard R. Regen}

This book or any part thereof must

not be reproduced without the permission of the publisher

Lithoprinted by Edwards Brothers, Inc. Ann Arbor, Michigan, U.S.A. 


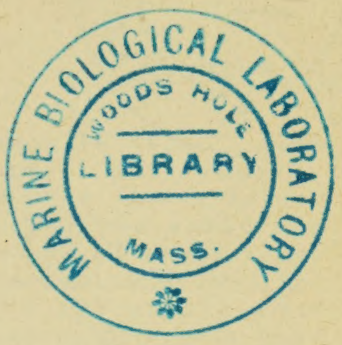

To

$\mathrm{Nel}$ son M. Cooke and

John Markus,

Authors of

Electronics Dictionary 



\section{PREFACE}

\section{"L'avenir de électricité, c'est dans}

future of electricity lies in the vacuum.) This remark, made before the Académie a generation ago by a famous French physicist, has proved to be prophetic. For the world has witnessed amazing developments in that branch of electricity which is based upon the employment of a vacuum: ELECTRONICS. Yet in spite of these great developments (or possibly because of them), and in spite of an increasingly urgent need for foreign-language dictionaries, students of electronics and allied problems have been forced to proceed without a lexicographical tool which brings together the terminologies of the two languages most vital to their work, German and English.

The authors believe that this volume will be welcomed by all electronics engineers and all physicists, notwithstanding certain limitations in size and scope which have been imposed by the war. It contains nearly 21,000 entries, representing most of the terminology current in 1939 and an appreciable number of terms introduced during the war years.

Owing to a lack of earlier German-English dictionaries in the general field of physics, the task of compilation has proved unusually difficult. The only outstanding reference books hitherto available for consultation have been Sattelberg's Wörterbuch der elektrischen Nachrichtentechnik (1926) covering the signal art in general, and Austin M. Patterson's famous German-English Dictionary for Chemists (1935). The bulk of the material in the present volume has been drawn from magazines, scientific journals, books, and patent literature. The definitions gleaned from other books have in most instances been amplified and re-worded.

A somewhat unorthodox policy has been followed by frequently adding explanatory annotations to the English language expressions. This has been done in order to supply related terminology for the benefit of the reader who may be unfamiliar with a given subject. Ample cross-references have been inserted throughout. And for those who desire fuller information, source and reference books--including a number of outstanding works of British origin--have been listed in a bibliography. The authors recommend purchase of some of the items included in this list, particularly the Electronics Dictionary by Nelson M. Cooke and John Markus. 
Indeed, the authors are deeply indebted to these authors and their book for much valuable information.

We also wish to express our gratitude to all those who have aided us in one way or another, especially to E. R. Weinmann, Arthur Worischeck, Walter Brunberg and Dr. G. Ornsen who assisted in the proofreading. We shall be equally grateful to those who bring to our attention such errors, imperfections, and omissions as are inevitable in the first printing of a work which is a pioneer in its field. A revised and amplified edition is being planned for a future date, and correspondence with the authors is solicited from those wishing to co-operate in the compilation of a revised text.

The Twelve Acres,

Stone Ridge, N.Y.

Bernard R. Regen and

55 W. 42 nd Street, New York, 18, N.Y.

August, 1946 


\section{BI BL IOGRAPHY}

Andretto, P. C. Principles of Aeronautical Radio Engineering. 1942 .

Chamber's Technical Dictionary. 1944.

Cooke, Nelson M. \& Markus, John. Electronics Dictionary. 1945.

Drake's Cyclopedia of Radio and Electronics. 1943.

Gherardi, A. A. Modern Radio Servicing. 1936.

Jacobs, Donald H. Fundamentals of Optical Engineering. 1936.

Keen, R. Wireless Direction-finding. 1938.

Manly, Harold P. Radio and Electronics Dictionary. 1931.

Olson, Harry F. Elements of Acoustical Engineering. 1940.

Patterson, Austin M. A German-English Dictionary for Chemists 1935.

Roget's Dictionary of Electrical Terms. 1943.

Zworykin, V. K., and Mortion, G. A. Television. 1940.

Ardenne, Manfred von Handbuch der Funktechnik. 3 vols. $1935-1938$.

Kohl rausch, F. Praktische Physik. 1944.

Sattelberg, Otto. Wörterbuch der elektrischen Nachrichtentechnik. 1926.

Schröter, F. Fernsehen. 1937.

Magazines covering radio, sound-film, television and related physical sciences and technological arts; foreignliterature reviews regularly appearing in some of them. Abstract journals such as Science Abstracts (A \& B), Physikalische Berichte, etc. Patents.

\section{ABBREVIATIONS}

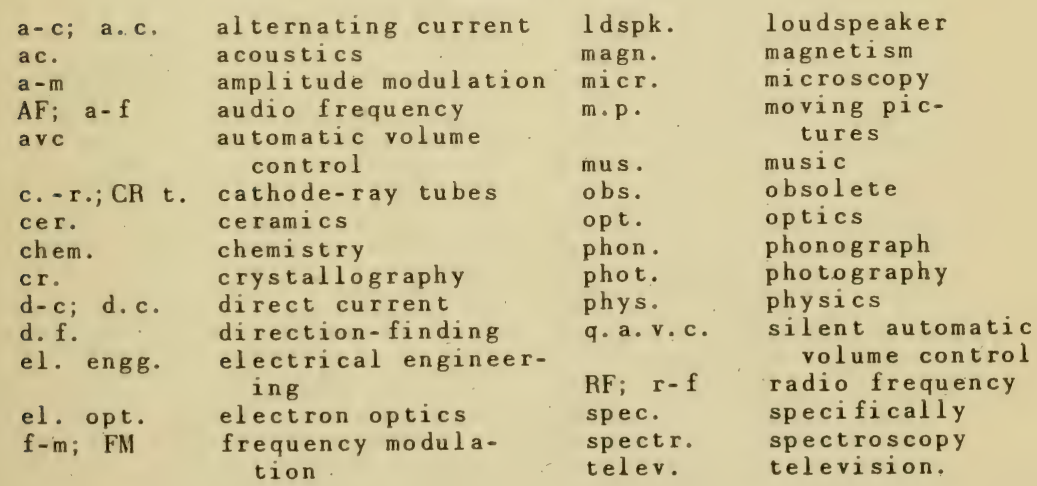



a.a.0. in the place cited, $10 c 0 \mathrm{ci-}$ tato, quoted reference.

Abänderung change, alteration, modification, amendment.

Abänderungspatent re-issue patent.

abarten vary, degenerate.

Abbau decomposition, disintegration, degradation, attrition. Frequenzfrequency division, f.submultiplication. -mittel disintegrant. -verhinderndes Mittel anti-disintegrant, stabilizer.

abbiegen bend off or away, bend angularly; turn off:

abbilden image, focus.

abbildende Ringelektrode focusing - ring (el.opt.).

Abbildung imaging, imagery, image formation; figure(Fig.), illustration, drawing, sketch, cut.

Abbildung, geteilte split focus (Walton method of scanning). -, komatische comatic image. -, konforme conformable representation. -, punktförmige point image. -, punktuelle point focal vision or imagery.

Abbildungs-fehler defect of image, aberration(opt.). - -gegenstand imaging object. -optik imaging "optic", focusing 0 . -vermögen resolving power (opt., micr.).

abblättern scale (off), shell, chip or peel off, descale, defoliate, exfoliate.

Abblendekapotte dimming cap.

abblenden (cf.abdecken) mask off, stop down (with masking diaphragm), occult, diaphragm, gate, blank, dowse (a beam), wipe, dissolve, fade or black out.

Abblender, Strahl- means to mask, stop, dowse, occult or eclipse rays or a beam, blanking or blanketing means.

Abblendung, Iris- irising out. abbreiten stretch out, flatten. abbremsen decelerate, retard, brake (electrons, etc.).

Abdeck-aufzeichnung masking recording, matting. -blende shutter, mask, shutter m. -doppelzackenspur duplex variable -area track - einfachzackenspur unilateral variable-area track.

abdecken (cf,abblenden) diaphragm, mask, eclipse (beam or pencil, by shutter, etc.), occult (a lens); uncover; cover; provide a resist coat (in metal etching).

Abdeck-fluigel masking or shutter blade or vane. - scheibe shutter disk with spiral slot, stop disk with spiral slot cyclically cooperating with quadruple-spiral scanning disk apertures (telev.). - seite masked side (m.p.). -streifen squeeze unit, barrier (m.p. film).

Abdeckung, Einfachzackenschrift mit unilateral variable-area track made with single-vane shutter.

Abdeckverfahren shutter mask method.

abdichten seal, pack, provide glands

Abdichtung tight seal, packing, hermetic plumbing.

Abdrift drift (off course, of air plane, etc.). -visier drift meter.

abdrosseln throttle, choke.

Abdruck copy, impression, print, cast, counterpart, replica.

Abdruck, Kontakt- contact print (phot.). Probe- proof, p.print, p. sheet. 
abdrucken

abdrucken print, reproduce or copy

(by printing or impression); stamp.

abdunkeln darken, cut off or dim (light), occult, eclipse, obscure, blanking or gating of spot (in telev.).

Aberkennung abrogation, abjudication, deprivation.

Aberration, Flächen- zonal aberration.

aberregen de-energize, de-activate. Aberregung, Relais- de-energization of a relay.

abfachen classify; partition, form compartments.

Abfall drop, fall, droop, decline, descent; waste, scrap, refuse, discard.

abfallen fall or drop down or off, slope down, droop, release (a relay).

Abfallprodukt by-product, waste p. abfangen capture, catch, intercept; pull (an airplane) out of a dive and level off.

abfasen chamfer, face.

abfasern lose fibers, fuzz.

abfassen frame, draft, prepare

(text), write, draw up (a specification, etc.).

abfiltern (cf.Filter, ausfiltern)

filter or strain off (by sieve, etc.).

Abfindungssumme indemnity, money paid in settlement (of claims, etc.).

abflachen flatten, smooth down, broaden, make oblate.

Abflachung bevel(ing), rounding off (a curve), leveling, flattening, smoothing, oblateness (at poles).

Abflachungsdrossel smoothing coil, s.choke.

Abfliegen outward course, flight away from airport.

abfliessen leak off, flow or drain off or away.

abfluchten align, line out.

Abflug, Ziel- flight away from object.
Abflugszeit outward flying time (away from airport, in ZZ landing). Abfluss leak path (for grid current). -hahn discharge cock, drain c.

-ventil discharge valve, drain v. abfressen corrode, eat away or up. Abfühlnadel pecker, selecting needle.

Abfuhr outlet (e.g., in water-cooled tube).

Abführung, Wärme- heat dissipation, h.loss, h."abduction", effect of carrying or conducting away heat. abfuttern case, line.

Abgabe import duty, tax, fee, royalty; emission (of radiations, electrons, etc.).

Abgabefläche, El ektronen- electron emitting area.

Abgangswinkel angle of departure. abgeflachte Spannung flat-topped potential (wave).

abgeglichen balanced, equalized, aligned; flush.

abgehender Strom outgoing current. abgehende Wirbelbahnen trailing vortices(airfoil).

abgekantet chamfered, beveled, canted.

abgeklungene Emanation emanation with diminished or decaying radioactivity.

abgelagert aged, seasoned.

abgeleitete Grösse derived or subsidiary quantity or magnitude. abgeschirmte Stelle dead spot, radio shadow.

abgeschlossen, in sich selbst selfcontained.

abgeschmolzene Röhre sealed off tube. abgeschrägt beveled, chamfered, canted.

abgesetzt offset.

abgestimmt, nicht- untuned, nonresonant, aperiodic, not in syntony or resonated.

abgestumpft truncated, blunt-ended, with frustum.

abgewickelte Linie evolute.

abgezweigte Anmeldung divisional application (portion divided of $f$ 
from original a.).

abgleichen equalize, compensate, balance, trim, align, level.

abgleichend, selbst- self-balancing.

Abgleich-fehler unbalance, balancing or alignment error, misalignment. -kondensator trimming condenser or capacitor, aligning $c$. -platte tuning spade, tuning plate, t. wand.

Abgleichung, Fehl-misalignment, faulty alignment.

Abglelchvorrichtung, Phasen- phase balancer, p. shifter, p. changer, p. compensator network.

Abgreifen der Entfernung measuring map range with dividers, etc.

abgrenzen mark of $f$, bound, demarcate, define (the limits of), delimit, circumscribe.

Abgrenzung und Einteilung, Wellenfrequency and wave-band designation: very low frequency (vif) 10-30 kc; low f. (If) 30-300 kc; medium f. (mf) 300-3000 kc; high f. (hf) 3-30 mc; very high f. (vhf) 30-300 mc; ultra-high f. (uhf) 300-3000 mc; super high f. (shf) 3000-30000 mc.

Abgriff tap.

abhängen depend on; lift off (receiver).

abhängig, druck- oder temperaturbe a function of pressure or temperature, pressure-or temperaturedependent or responsive.

Abhängigkeit, Frequenz- frequency dependence, having $f$. effect, being affected by, or being a function of, frequency.

Abheber plow (to peel film from teeth).

Abhebestange damper rod (piano).

Abhilfe remedy, redress, relief, cure, obviating a difficulty.

Abhörbox monitoring box.

Abhorchen (von Flugzeugen) auditory locating, spotting, detecting, or fixing position (of aircraft, etc.) by sound locator devices.

Abhorchgerät monitor, check or pick- up microphonic device; standby

circuit means.

abhören intercept, listen in; monitor.

Abhören, Maschine zum - von Ton-

filmstreifen machine for editing and rehearsing sound film.

Abhören der Negative direct sound reproduction from negatives.

Abhör-gerät (Flugzeug, etc.) airplane, etc., sound locator or detector; radio locator, Radar (using uhf waves and echo principle). - lautsprecher monitoring loudspeaker. -raum mixing room, monitoring booth, "box". -tisch

(und Schneidetisch) sound film editing machine.

Abhör-, Klebe- und Schneidetisch movieola.

Abisolierer wire skinner.

abkanten leyel, take off edge, chamfer, cant.

abkappen lop off (peaks or crests), clip, limit amplitude.

Abkapper amplitude or output limter.

abkehren turn away or aside, divert; sweep or brush off.

abkl appen swing or hinge down, out or away, let down.

abkl ingender Kurvenzug decay train. Abkling-kurve decay curve. -zeit decay period, die-out p.; quench p. (in super-regeneration). -zeitmesser(Fluoreszenz) fluorometer.

abkneifen pinch off, nip off. abknicken bend or deviate (a ray, at sharp angle).

Abkommen sight graticule or reticle (opt.), point of aim; agreement, convention, contract, arrangement; deviation.

$\mathrm{Abl}$ agerung deposit, sediment.

Ablagerungen, inselartige "island" deposits.

Ablass, Wasser- draining or drainage of water.

Ablauf expiry, expiration or lapse (of a period of time); rotation 
or flight (of electrons); film feed (from magazine spool). -bahn runway, landing ramp (of airplanes).

ablaufen run down (of a spring), return (of dial switch); run off, drain; expire (rights, contracts, etc.).

ablaufen 1 assen release, let go or return (dial switch).

ablaufend(e) Kante trailing edge. -(er) Kurs course or direction away from airport (in $\mathrm{ZZ}$ landing), outward course.

Ablaufzeit return time (of a dial switch).

ablegen(Zeugnis) bear testimony or witness.

ablehnen decline, reject, refuse, challenge, dismiss.

ableiten derive, deduce; turn aside; draw off, let escape, lead or carry off, dissipate or abduct (heat, etc.), shunt, drain to earth.

Ableiter arrester, means to let a charge leak or drain away.

Ableiter, Kathodenfall- valve arrester.

Ableitung leakance (line constant), shunt conductance; derivation (with respect to), differential coefficient (math.); downlead, lead-in (of an antenna).

Ableitung, dielektrische dielectric leakance, conductance. dritte, der Charakteristik third differential of characteristic. Antenne mit - shunt-excited antenna.

Ableitung, Oberflaechen- surface leakage. Wärme- heat dissipation, h. evacuation, loss or abduction, the carrying away or conducting away of thermal energy. Wasserdraining or drainage of water.

Able itungs-dämpfung leakance loss. -glied shunt element. -strom leakage or stray current. -widerstand leak resistance, resistance leak (of a tube).

ablenken deflect, deviate, inflect, diffract; turn of $f$ or aside.
Ablenk-gerät deflection or deflector means, time-base. -kammer deflection chamber, d. space (in electron microscope). -platte deflection plate, deflector $p$. -spannungen, symmetrische equal and opposite or symmetric sweep voltages or deflection potentials. -spule deflection coil, deflector yoke.

Ablenkung, herizontale horizontal or line scan, sweep or deflection.

Ablenkung, Bild-frame or picture scan or sweep. Bord-aircraft error, distortion of bearings (due to ship's field, etc.). Horizontal- horizontal or line scan, sweep or deflection.

Strahl- ray deviation, r. deflection; r. refraction. Zeilenline scan, line sweep. Zeittime base.

Ablenkungs-empfindllchkeit defloction sensitivity (reciprocal of d. factor). -generator deflection generator, sawtooth g., time-base g., sweep oscillator. -spannung sweep voltage. -spule deflecting yoke, sweeping y., s. coil. -wechsel, Vertikal- vertical deflection cycle, v. or frame d. or scan. - we ite sweep amplitude. -winkel angle of deviation, a. of refraction.

Ablese-fernrohr reading telescope. - l upe reading lens. -marke index line, i. mark.

ablesen (Karte) read (off from) map or chart.

Ablesescheibe (Peilen) dial of direction-finder.

ableugnen deny, abnegate.

abliegen be spaced apart or away, differ from.

Ablösung, Phasen- phase switching (in polyphase operation of ignitron).

Ablösungs-arbeit, -energie des Elektrons total internal work function (amount of energy re- 
Abmachung

quired by electron to break away from incandesced or other metal surface), excitation energy.

Abmachung agreement, arrangement, convention, stipulation, contract.

Abmass tolerance.

Abnahme-elektrode output electrode, collector e. -magnet pickup magnet. -stelle, Ton- sound gate.

abnehmen tap, transfer (potential, etc.), take off, pick up.

Abnehmer-sprechkopf sound pickup head, reproducing $h$. -stelle, Ton- sound gate, s. pickup.

Abnutzung abrasion, wear and tear, attrition.

abplatten flatten, oblate. abprallen rebound, be reflected. Abprallwinkel angle of ricochet. abrechnen make an accounting, settle an account.

Abrede, in - stellen deny. Abreiss-effekt (Schwingungen) discontinuity effect (oscillations). -kontakt arcing contact (of a switch). -methode adhesion method (used in surface-tension testing). -spannung stopping potential.

abrichten fit, adjust, true, trim, dress.

Abriss abridgment, abstract, synopsis.

abrollen (über) ride (on), roll on or off, roll in contact; unroll. abrunden round out, $r$. of $f$.

Abrundungsradius contour radius. Abrutschen, seitliches side slip (of an airplane).

Absatz wear and tear (of film); offset, reduction, recess, shoulder; subsection (in text).

absatzweise intermittently, discontinuously, progressively, gradually, by steps, stages or degrees, with intervals, stepwise.

absaugen (treat with a) squeegee. Absaug-feld positive field, suction f., f. designed to draw away, drive or concentrate electrons. -ring ring-shaped or annular collector.

\section{Absenkung}

abschaltbar disconnectible, severable, disengageable; adapted to be disconnected, switched off or cut of $f$.

Abschaltleistung (eines Schalters) rupturing, circuit-opening or circuit-breaking capacity (of a switch).

abschattieren shade of $f$.

Abschattierungsstörsignal spurious signal, shading $\mathrm{s}$. (in iconoscope operation, known also as "black spot", "tilt and bend", "shading", etc.).

abschichten separate into layers, stratify.

abschiessen (des Gettera) set off, cause vaporization (o' a getter).

Abschirm-becher shield(ing) can. -koeffizient absorption coefficient (screening function).

Abschleifmesser grinding blade or knife.

Abschlusswand (Kondensator) end plate.

abschmirgeln abrade (with emory), grind off.

Abschneiden (von Amplitudenspitzen) clipping or lopping off of amplitude peaks or crests (in a limiter). - (von Silben und Worten, in Telephonie) clipping, obliteration or mutilation of speech. abschrägen bevel, skew, scarf, slope, slant, taper, cant.

Abschrift, beglaubigte certified or legalized copy.

abschuppen scale or flake of $f$. Abschützung shielding, screening. abschwächen reduce (contrast, phot.). Abschwächer attenuator.

Abschwächungslösung reducer solution.

Abschwörung denial under oath, abjuration.

absedimentieren sediment, out.

absehen (von) dispense with, disregard, let alone.

Absehen sight graticule.

Absenkung in den Höhen de-accentuation or attenuation of high fre- 


\section{absetzen}

quencies, using treble de-emphasizer means.

absetzen lay off or plot (on a map or chart), shape course for; deposit, put down; interrupt.

absitzen lassen allow to settle, deposit or clarify.

Absorptionskante, $K-K$ edge. Unkehr- reverse absorption edge or limit.

Absorptionskonstante absorption coefficient. -küvette absorption cell. -modulation absorption or Heising modulation method. -rohr absorption column or tube.

-sprung absorption discontinuity. -streifen absorption bands, a. spectrum.

abspálten cleave (cryst.).

abspannen relieve of tension or strain, relax, release; stay, strain back, dead-end or terminate (a wire).

Abspann-isolator terminal or strain insulator. -seil guy or span rope or cable. -stange stay terminal, strain pole or mast.

absperren (cf sperren) shut off, cut off, confine, stop, stopper, seal off.

Absperrklappe, Drossel- throttle clack valve.

Abspieldose electric phonograph soundbox.

abspielen, direktes direct playback.

Abspielnadel phonograph needle.

Abspulen (cf. Ablauf) feeding film from magazine or feed spool.

Abstand nehmen (von etwas) refrain or desist from, dispense with.

Abstand, Augen- interocular distance. Betrachtungs- viewing distance. Frequenz- interference guard band, tolerance frequency. Geräusch- signal: noise ratio. Hauptebenen- distance between principal planes or points. Haut(focal) skin distance. Knoteninter-nodal distance. Netzebenen-
Abstimmung, rohe

grating constant (spectrometer), interlattice plane distance.

Pfeifpunkt- singing, whistling or stability marǵin. Wellen- interference guard band, tolerance frequency. Zeilen- line separation, inter-line distance, pitch (telev.).

Abstand-halter spacer. -i sol ator stand-off insulator.

Abstands-messer, Augen- interpupillary distance gage. -taste spacer key, blank k.

absteifen strut, prop, stiffen, reinforce, support, shore, truss, stay.

Absteller automatic disconnector or cut-off device (for soldering irons, etc.)

Abstieg, Ballon- descent of a balloon.

Abstimm-änderung tuning drift (due to temperature effects). - anzeigeröhre tuning indicator tube, maǵic eye. -bereichüberstreichung scanning a tuning range. -einrichtung tuning device or means, tuning control, selector.

abstimmen tune, syntonize (circuits), shade off (phot.), harmonize.

abstimmen, auf Resonanz resonate, tune to resonance.

Abstimm-glimmröhre tuning indicator glow-tube, flashograph. -kette tuning arrangement comprising a plurality of oscillation circuits. -kreis tuned or tuning circuit, tank c. -melder tuning indicator (e.g., of c-r. tube or magic eye type). -platte tuning spade or plate, t. wand. -schärfe sharpness of tuning or of resonance condition, selectivity.

Abstimmung, feine sharp or fine tuning. -, fühlbare lazy tuning (condenser sensibly detained on passing carrier wave). -, leise silent tuning, quiet (avc) tuning. -, rohe broad tuning, coarse tun- 
ing, flat tuning (with little selectivity). -, scharfe sharp, fine or precise tuning. -, schlechte mistuning. - , ungenaue off-resonance tuning. -, unscharfe broad or flat tuning.

Abstimmung, Falsch- mistuning. Primär-pre-selector means.

Abstimmungs-aggregat tuning set, tuning unit ( $\mathrm{L}$ and $\mathrm{C}$ ). - $\mathrm{kreuz}$ tuning indicator (magic eye, etc.). -stromkreis, Still-silent (q.a. v.c) tuning circuit.

Abstoppen von Zeitdauern stopwatch measurement of time intervals. abstossend, wasser- water-repellant, non-hygroscopic.

Abstossung, Coulomb- Coulombian repulsion. Eigen-, der El ektronenwolken repelling action of electron charges, negative space charge formation.

Abstossungs-kraft repelling force, repulsion. -potential repulsion potential.

Abstrahl reflected ray.

abstrahlen blast (with shot or sand); radiate, reflect.

Abstrahler radiator (ac., antenna). Abstrahlfläche radiating, radiation or radiant surface (loudsp.); reflecting surface or area.

Abstrahlung, Schall- sound radiation or projection.

Abstrakte tracker, prolonge (organ). Abstrebekraft centrifugal force. abstreichen scrape or strike of $f$, skin, strickle, lute (clay in brick mold).

abstreifen strip, wipe (off).

Abstrich downstroke or trailing edge (of an impulse).

abstufen grade (phot.), graduate, step.

Abstufung, empfindungsgerechte natural or subjective grading or spacing (in chromaticity or chromatic scale, etc.).

Abstufung, Licht- gradation of light. Schwärzungs- density graduation. Ton-grading of tanes, graduation of intensity.

absturipfen neutralize, saturate

(acids); truncate, blunt; dull, deaden.

Abstumpfungsfläche truncating face (cryst.).

absuchen search, sweep, scan, explore.

Abtast-band scanning belt. -blende

(Farnsworth, etc.) scanning aperture or hole (in Farnsworth dissector, Nipkow disk, etc.). - dose electric or pickup soundbox.

abtasten scan, explore, sweep, pick up, probe, search.

Abtaster, El extronen- electronic scanner, beam sweep, spot or lever electron brush or pencil.

Fernseh- televisor, video scanner. Film- film scanner (in intermediate film system). Kathodenstrahl- electron beam scanner. Lichtstrahl- spotlight scanner, scanning brush or pencil, sweep, flying or exploring spot scanner. Linsenkranz- lens drum scanner. Oberflächen- surface analyzer (to record irregularities).

Personen- (mit wanderndem Licht) spotlight scanner for persons. Abtast-frequenz scan frequency (= lines $x$ frame $f$.$) . -geschwindig-$ keit pickup velocity, pickup rate (phon.). -scheibe (cf. Spirallochscheibe) Nipkow scanning or exploring disk, spiral disk. - spalte, Ton- sound scanning slit. -stelle, Bild- picture or film gate. -strahl, El ektronen- electron spot, e. beam, e. brush (iconoscope).

Abtastung scanning, scansion, exploration, sweeping out (of picture area).

Abtastung nacheinanderfol gender Bildpunkte sequential or progressive scanning of elementary "areas. -, quadratische squarelaw scanning. -, sprungweise 


\section{Abtastung}

interlaced scanning. -mit unsichtbaren ultraroten Strahlen noctovisor scan (using infra-red rays). Abtastung, Fernseh-, von Kinofilm telecine scan of moving-picture film. Film- film scanning. Geschwindigkeits- variable-speed scanning. Schwingspiegel- vibrating mirror scanning (in Mihaly Telehor). Strahl- electron beam scan, spotlight sweep or scan. Tell-partial or fractional scan, coarse scan. Ton- pickup of sound. Vielfach-multiple scan.

Abtastzeile scanning line, s. strip. Abteilung, Berufungs- appeal division or department. Beschwerdeappeal dept., board of appeals. Nichtigkeits- annulment dept. (of G.P. Office).

abtonen, abtönen shade of $f$, tint, tone down.

abtragen plot or lay of $f$.

Abtragung disintegration, attrition, degradation.

Abtreibschmelze refining melt.

Abtrenner, Impuls- synchronizing separator, amplitude s., clipper.

Abtrennung severance, separation; division (of a patent application).

abtreten assign, cede, convey, transfer.

Abtretender assignor.

Abtretungserklärung declaration of assignment.

Abtrift drift (of ships or airplanes). -messer drift meter.

Abtropf-schale jar, dish, drip, drainer. -ständer draining stand.

Abwägeschaltung comparator circuit orǵanization.

abwalzen (cf. abrollen))ride on, roll.

abwandeln vary, change, alter, modify.

Abwärtstransformator step-down transf ormer.

abwassern (Flugzeug) rise or take off from water (airplane).
Abzïge

abwegig reden argue beside the point, tergiversate, dodge the issue.

Abwehr, Lärm-, Schall- noise abatement, $n$. suppression, n. attenuation, combating of $n$.

abweichen differ, vary, deviate, diverge, depart from; soak off. Abweichung departure, difference, disparity, divergence, deviation, variation, declination, anomaly, swing, drift. -, seitliche sphärische transverse spherical aberration.

Abweichung, Farben- chromatic aberration. Flächen- zonal aberration. Frequenz- frequency departure, swing, deviation or drift, lilt (slow fluctuation). Kursуаw, deviation from course. Linsen- lens aberration. Nullpunktzero error. Rest- residual aberration. Seiten- lateral deviation.

Abweichungskorrektur achromatization (opt.).

abweisen (eine $\mathrm{Klage}$ ) dismiss (a suit), non-suit.

Abweisungsmaterial anticipatory material or references, anticipa-. tions (in prior art).

abwenden avert, avoid. abwerfen release (a keeper). Abwerg waste tow or oakum. Abwesender absentee. Abwickelachse feed spindle. abwickelbar developable. abwickeln wind of $f$, unwind, reel off; rectify (math.).

Abwickelspule feed spool, f. reel. Abwickler, Film- magazine drum, feed reel.

Abwind down current. abzapfen draw off, tap. abzlehbares Papier transfer paper. abziehen print, copy; trim or true (a wheel); draw off or tap. Abzug print, copy.

Abzug, Korrektur- proof sheet. Abzüge machen take prints, print, 
copy, multigraph.

Abzugsrohr vent stack (of arc-lamp); waste or drain pipe or tube, vent p., outlet.

abzweigen (von Strahlen) split off or deviate (rays).

Abzweigpunkt tap, distributing point, branch-off point.

Abzweigung tap, branch-off, tie line, ramification; division (of a patent application).

Abzweigwiderstand leak resistance.

Achromasie achromatism, achromatization, degree of achromatic correction.

Achse, ausser extra-axial, abaxial. -, grosse major axis. -, kleine minor axis.

Achse, Abwickel- feed spindle. Dreh-axis of rotation, center of r., pivot, fulcrum, hinge. Drillrotator (quantum theory). Flugzeuglängs - fore-aft line, major axis of airplane. Gegen- antiaxis. Halb- semi-axis. Hauptprime or major axis, vertical axis (of rhombic crystal). Kristall- crystal axis, crystallographic a. Lande- correct bearing or line for approach or landing. Längs- longitudinal axis, fore-aft line (of air and water craft). Linsen- optical axis (of a lens). Makro- macro-axis (cryst.). Neben- lateral axis (of rhombic crystal), secondary axis. Säulen-prismatic axis. Spulen-bob or core of a spool (film).

Achsenankerrelais axial-armature relay.

achsenentfernt abaxial, of $f$ axis.

Achsenkreuz coordinate system.

Achsenrichtung, Kristall- crystallographic axis orientation. achsensymmetrisch axially symmetric, in axial symmetry. Achsenwinkel axial angle. achsig, ein- uniaxial. ein-undein- or thorhombic (cryst.). zwei- biaxial.

achsparallel axis-parallel, paraxial (e.g., rays beside, or parallel to, axis).

Achs-schenkel axle journal. -stand wheel base.

achteck Ig octagonal, eight-cornered. Achtelkreis octant.

achtelkreisförmige Komponenten octantal error components (df). achteraus, Backbord- port aft. recht- right astern. bord- starboard aft.

Steuer-

Achterbündel eight-wire core, quadruple twin.

achterliche Kurslinie astern course, direct line away from transmitter, "come-from" indication.

Achter-schraube octet ring. -spule figure "8" coil.

Acht-flach octahedron. -polröhre octode, eight-electrode type of tube or valve.

adaptiertes Auge, dunkel- dark adapted eye.

Adaption, Dunkel- dark adaptation, scotopia.

Adaptionszustand, Augen- (state of) retinal adaptation.

Adatom adsorption atom.

Adcocksystem (U-System) (spec.) vertical aerial or Adcock system (Usystem); also spaced aerial df system.

additive Modulation upward modulation, negative $\mathrm{m}$.

Ader, Zähl-pilot wire, marked w., meter $w$.

AEF (Ausschuss für Einheiten und Formeln) Committee on Units and Formulae, Standards or Standardization Committee.

"Aeo" Aufzeichnungslampe Aeo light, alkaline-earth oxide lamp, Aeo lamp.

Aeol s-glocke, $\mathrm{Gl}$ as- glass Aeolian bell. -harfe Aeolian harp.

affinieren refine.

After-kristall pseudomorph. -kugel spheroid. 
Aggregat, Lampen- bank or pillar of lamps, battens (used on stages).

Molekül-molecular cluster.

Aggregatzustand state of aggregation.

Agraffe stud (piano).

Ah. (Amperestunde) ampere hour.

Ähnlichkeits-gesetz, -theorie similarity principle, similitude theory.

Akkomodations-entspannung relaxing of adaptation. -fähigkeit power of accommodation.

akkomodationsloses Auge unaccommodated or fixed eye.

Akkordion-balg accordion bellows. -griff keyboard.

Akkordzitter auto harp.

Akkumulator, kochender agitated or gassing accumulator (just disconnected from charger).

Akten, Anmeldungs- file wrapper, application papers.

Aktendal arm reel-end signal.

Akten-zeichen serial number, filen., docket $n$., reference n. -zeichnung drawing incorporated in docket or case records.

aktinisch undurchlässig. adiactinic. Aktiniumemanation actinon.

aktive Masse active paste.

Aktivator, Schwermetall- phosphorogen (e.g., lin, promoting phosphorescence).

Aktivierung, Neu- reactivation, rejuvenation (of a thoriated cathode, etc.).

Akustik s,a. Schall, Ton, Klang, Geräusch.

Akustik, Bau-architectural acoustics, enclosure, space or room a. akustisch(e) Blendung aural dazzle. -(e) Empfindung acoustic perception. - (e) Gegenwirkung acoustic reactance. - (er) Kurzschluss acoustic feedback or short-circuit. -(e) Ortung sound location, acoustic orientation. - (e) Rückkopplung acoustic feedback. - (er) Widerstand acoustic resistance; $a$. screen.

Al arm, Aktend- reel-end signal. Luft- air-raid alarm. - ende, Luftall-clear signal.

Albedo albedo (reflection factor of diffusely reflecting surfaces, say, a planet).

d'Al embert'sche Wellengleichung $D^{\prime}$ Alembertian wave equation.

Alhidade alidade, sight rule.

Alkal imetall, Erd-alkaline earth metal.

Alkalizelle alkali metal photo-emissive cell.

Allein-berechtigung sole or exclusive right. -inhaber sole owner or holder.

allitieren coat with aluminum, aluminize.

Allstromempfangsgerät universal receiver set.

Alphabet, Fünfer- oder Fünfströmefive-unit code.

Alt alto, counter tenor.

Alt, tiefer contralto.

Altersrank priority.

alterssichtig presbyopic.

Altposaune alto trombone.

Al uminium-knetlegierung malleable aluminum alloy. - töne aluminum reeds (accordion).

Ametall non-metal.

Ampere-stunde(Ah) ampere hour. -windungszahl number of ampereturns (ats).

Ampl itude, Bewegungs-, Geschwindigkeits- velocity amplitude. Membranschwing- diaphragm excursion amplitude. Stör- noise level.

Amplituden-abschneider peak limiter, clipper, lopper. - anzeiger glowtube amplitude, tuning or resonance indicator, flashograph t.i. -begrenzer peak limiter, clipper, lopper.

amplitudengetreu of equal amplitude, with a. fidelity.

Amplituden-gl immröhre glow-tube amplitude, tuning or resonance indicator, flashograph t.i. -schrift 
variable-area (v,-width) recording or sound track. -selektion, -sieb amplitude filter, a. separator or selector, a. discrimination selector. -spitzen, Abschneiden der clipping or lopping off of amplitude peaks or crests, by limiter means.

Amt, Eich- gage or calibration of fice, standards bureau. Nottemporary of emergency exchange or office.

amtlicher Bescheid Patent Office action, decision, of ficial notice or notification.

Amtsblatt official gazette or journal.

Amtverkabelung central wiring, office wiring.

Analysator, Suchton- heterodyne analyzer.

anarbeiten attach, join or work onto.

anätzen etch or cauterize superficially or slightly.

anbacken bake on, fix by firing, bake superficially.

anberaumen, Termin zur Verhandlung einer Sache set or fix date for hearing a case.

Anblaston Aeolian tone.

anbohren tap, bore, drill.

Anbruch fracture.

Änderungsgeschwindigkeit remaining velocity (ballistics).

Androhung warning, caution, giving notification (of a threatened action).

Andruck-fenster pressure plate, aperture p. (m.p.). -rolle, Gummi- rubber-tired pressure or pad roll (m.p.). - schiene pressure pad, p. guide.

aneinandergesprengte Prismen broken contact (uncemented) prisms (closely fitted together).

anerkennen acknowledge, admit, recognize.

Anerkennungsurteil consent judgment.

\section{Anfressung}

Anfachraum generator or working space (beam tube).

Anfachung continuous excitation of waves (or point where oscillating begins); fanning, kindling.

Schwingungs- wave excitation, w. generation.

Anfachzeit building-up period (time required for oscillating to start).

Anfäll igkeit, Stör- susceptibility to trouble, interference or noise.

Anfangs-drift initial drift (of photo-cell). -geschwindigkeit initial velocity; muzzle v. (ballistics). -konzentration (cf. Vorsammellinse und Vorkonzentration) pre-focusing. -strom starting, initial, incipient or original current. -wert initial or early value. -zustand initial state or condition.

anfärben stain (superficially). anfechten, Gültigkeit eines Patentes challenge validity of a patent, argue non-validity.

Anfechtungsprozess contested action. anfeuchten wet, moisten or dampen (superficially).

Anflug approach (flight), coming $f$. Anflug, Ziel-flight towards object or target.

Anflug-grundlinie approach of center of sector. -kegel sector of approach. -manoever approach procedure. -richtung direction of approach, homing direction (of an airplane). -richtungsfunkbake runway localizing beacon (marker). -schneise approach sector, corridor of approach. - sektor sector of approach. -zeit time for flight to airdrome.

Anflussgeschwindigkeit velocity of approach, afflux v. (of fluids). anfressen corrode (superficially or slightly), eat away or eat at, erode, pit, attack, stain.

Anfressung, Rost- corrosion, honeycombing, pitting, tubercular or 


\section{anführen}

channel-shaped corrosion.

anfihren quote, cite, mention.

Angeber, Ton, chromatischer chromatic pitch pipe.

Angeklagter defendant, respondent. angelagerte Elektronen trapped electrons.

Angelegenheit case, matter, affair, in re:

angepasste Leitung matched line. -, nicht- mismatched line.

angeregte Gruppe exciton (in dielectric breakdown).

angeschärften Enden, Verbindung mit scarfed joint.

angezapfte Wicklung tapped winding.

-, in der Mitte center-tapped

winding, with midpoint tap.

angiessen cast on, pour on; color (by coat of clay).

Angleich, Farb-color matching, $c$. comparison.

angleichen equate, correct, compensate.

Angleichung adjustment, adaptation, (approximate) matching (of brightness, color, etc.).

angrenzend border on, adjacent, adjoining, contiguous.

Angrenzung contiguity, adjacency, adjoining.

Angriffspunkt point of engagement, attachment, action, application or attack (say, of a force).

Anguss lug (cast on); feedhead.

anhaftend adherent, inherent, be attached to.

Anhallen building-up of acoustic energy.

Anhaltspunkt clue, criterion, index; stopping point, fulcrum.

anharmonische Terme anharmonic terms or constants.

Anhäufung aggregation, accumulation, collection, damming up, concentration.

Anhäufung, Korn- grain aggregation.

Anhäufungszone zone of accumulation (in ultrasonic waves)

anheben (von Tönen, Bändern, etc.)

\section{Anl agerungsverbindung}

emphasize, accentuate, underscore or favor (certain sounds or frequency bands, by tone-control or tonalizer means).

Anhebung, Bass- bass boosting, b. compensation, tone-control means

to accentuate low-pitch or b. range, b. emphasis.

anheizen heating up, initial h. (of a tube); kindle.

Anheizzeit cathode heating time (till stable temperature is reached), thermal time constant ( of a cathode), warming-up period. anisotropische Verzeichnung anisotropic distortion, shear d., d. of orientation.

Anker armature, keeper (of permanent magnet); anchor, stay, guy; escapement.

Anker, Doppel-T- shuttle armature, H-type a. Dreh- pivoted armature. Eichkatzen- squirrel-cage armature. Schwing- oscillating armature.

Anker-gesperre anchor escapement. -relais, Schneide- knife-edge relay. -ruhestellung, Relais mit mittlerer neutraler neutral relay, relay with differentially moving armature. -umschlag armature travel, a. transit, armature excursion.

Ankl ang concord, accord, sympathy, tuned state.

Anklang finden find acceptance, become popular.

Anklingen gradual rise or waxing (of vibrations, etc.), initial sounding, intonation.

Anklingzeit starting or onset time (of a tone).

ankohlen char or carbonize (superficially, slightly or partially). ankommender strom incoming current. anlagern combine, become added to, adsorbed or attached.

Anlagerungs-reaktion addition reaction. -verbindung addition compound. 
Anl ass-anode starting anode (in arc rectifier). -beständigkeit tempering resistance.

anlassen start, turn on, let in; temper, anneal (glass, metal, etc).

Anl asstemperatur tempering temperature, annealing t., drawing $t$.

Anl auf pickup, start, acceleration. - beständigkeit resistance to tarnish, t.-proofness. - charakteristik transfer characteristic.

anl aufen become coated with oxide or moisture, tarnish, become dull or dim; start; buck against (a potential).

anlaufendes $\mathrm{Flugzeug}$ approaching airplane.

Anlauf-farbe temper color. -stromgebiet range or region of incipient current flow (say, at grid). -winkel angle of approach.

Anlege-kassette clamp-on dark slide. -punkt aiming point.

anlenken pivot, fulcrum, hinge.

Anlenkungspunkt pivotal point, fulcrum, hinge.

annetzen moisten, dampen (superficially).

Anmassung, Patent- usurpation of patent rights.

anmelden ( $\mathrm{ein}$ Patent) apply for letters patent, file an application.

Anmelder applicant.

Anmeldung, abgezweigte divisional application (part divided off from the original case or docket).

Anmel dungsakten file wrapper, application papers.

Anmerkung annotation, memorandum, remark, comment, (foot, marginal) note.

Anmerkung, Rand- marginal note or annotation, marginalia. Streitannotations of litigation.

Annahme acceptance, acceptation, adoption; assumption, supposition, presumption, conjecture, surmise.

Anode, Anlass- starting anode (to strike initial arc in $\mathrm{Hg}$ rectifier). Auffang- collecting or gathering anode (of 'multiplier); catcher (of klystron). Beschleunigungs- gun anode. Di aphragmaaperture anode, anode stop. Fangcollecting anode, gathering a., (Farnsworth telev., multiplier). Lochscheiben- apertured disk anode, ring a. Nachbeschleunigungs- second gun anode. Netz- plate fed from power pack. Prall-target anode, impactor a, reflecting electrode or dynode (of electron multiplier). Sammel- collector anode, gathering a. Voll- unsplit anode (in magnetron). Vor-first anode (c-r. tube); screen grid connected with cathode $g$. and acting as "fore-anode" in power pentode (Pierce circuit).

Anoden-absinkkurve anode or plate current curve. - ansatzpunkt spot on anode (of an arc). -aussortierung anode sorting. -blende (anode) diaphragm or partition (in cathode-ray tube), first anode, defining aperture (of telev. tube). -fleck anode spot. -gleichrichtung plate-current rectification or detection, anode bend $r$. - loch (cf. Anodenblende) gun (in cathode-ray tube). -rïckwirkung anode or plate feedback, plate reaction. -schale anode segment (of magnetron). - schutznetz screen grid, plate shield. - spannungsussteuerung plate voltage excursion. -strahlen anode rays, canal r., positive $r$. -stromaussteuerung plate current excursion. - unruhe fluctuation of plate or anode feed current. -verlustleistung anode dissipation.

Anomalie, Nullpunkt- origin distortion.

an ort und Stelle in situ, in the site, on the spot.

anpassen match, adapt; accommodate, adjust, fit, suit.

Anpassung, Augen- adaptation (to change in distance or focus), accommodation (to change in light intensity). Dunkel- dark adapta- 
tion, scotopia. Falsch- Fehl- mismatching; defective focus. Helllight adaptation, photopia. iiberovermatching. Unter- under-matching.

Anpassungs-dämpfung matching attenuation, non-reflection a. -fähigkeit adaptability, accommodation powers (of eye). -netz matching network. - schaltung circuit means designed to form or shape synchronizing signals. -transformator matching transformer.

anpeilen take bearings from or on (a radio station).

Anregung excitation, stimulation, stimilus, response, sounding (as of a Kundt glass tube).

Anregung, Kern- nuclear excitation. Anregungs-energie excitation or stimulation energy (e.g., potential). - faktor excitation factor. - spannung exciting or stimulating potential, pre-ionization p., p. to raise an electron to a higher level out of its atomic bond. -welle exciton (strictly:an electron in a crystal moving in the field of a positive hole).

anreichern enrich, strengthen, concentrate.

Anreicherungsfaktor concentration factor.

anreihen arrange in, or attach to, a series or row, align.

anreissen pluck (a string or chord). Anriss cracking, critical break. ansässig domiciled, residing at. Ansatz side arm or appendage (of a bulb or tube); adapter (phot.); loss, extension, tail, ear, lug, nipple, shoulder; deposit, sediment.

Ansatz einer Gleichung laying down a formula or equation.

Ansatz, Lichtton- sound-film head. Nadelton- sound-on-disk attachment or head.

Ansatzfläche des 0bjektıvgewindes screw shoulder, screw collar or seat of objective amount.
Ansatz-punkt contact point (airplane landing); spot (of arc, etc., on cathode or anode); point of attachment or insertion. -stuick extension piece, adapter piece. -tubus extension tube.

anschichten pile in layers, stratify.

anschiessen shoot into crystals, crystallize.

Anschlag limitation or buffer, stop, dog.

Anschlag, Auslösungs- trip dog, trigger means. Begrenzungs- back stop, limiting means. Fingerfinger stop (dial switch).

Anschl agbund stop collar.

anschlagen touch (piano), strike (a string, in percussion instruments), sound or tap (a tuning fork).

Anschlag-linie striking line (piano). -stelle striking point.

Anschliff ground and polished surface (under microscopic examination).

Anschluss, Kopfhör- telephone jacks. Sanmel- p.b.x. line. Vollnetz(Radioempfänger) all-electric set (with mains supply).

Anschluss-berufung cross appeal. -teil terminal part, connector. -teilnehmer, Sammel-subscriber with several lines.

anschmelzen melt, fuse or join onto, join by fusion; begin to melt.

Anschmiegung, enge intimate or snug adhesion, adherence, engagement or fit, close contact, hug.

anschmoren scorch.

anschneiden intersect; determine a fix, obtain a bearing.

Anschnitt gating (foundry work).

Anschuss, Kristall- crop of crystals, crystallographic growth, crystallization.

Anschwingzeit build-up period (time required for oscillations to start).

Anschraubring lens flange. ansetzen crystallize, effloresce; make up (a solution), prepare, 
mix; start or strike (an arc).

an sich per se, basically or fundamentally speaking, in or by itself.

Ansicht, Gesamt- total view, general v., assembly v. Hinter- rear elevation, posterior view. Seiten- side or lateral elevation. Vorder- front elevation, anterior view.

Ansichtskopie release print.

anspannen strain, stretch, bend, tension, tense.

anspielen auf make allusion to, hint at.

Ansprache response; inception, onset.

ansprechen in freier Luft response in free space or air (of a microphone).

Ansprech-konstante sensitivity constant (microphone), m. responsiveness. -wahrscheinlichkeit

(eines Zählrohres) efficiency of counter tube (likelihood to respond or register).

Anspruch (eines Patentes) claim (of letters patent).

Anspruch, Uniter- sub-claim.

Anspruch geltend machen advance, prefer, raise or plead a claim or point.

Anstalt, Forschungs- research institution.

anstechen pierce, tap, open.

Anstellwinkel angle of attack; a. of pitch, blade a.

ansteuern take bearings from, keep on course, head for.

Ansteuerungsfeuer line of approach beacon; directional beacon.

Anstoss nehmen take exception to. anstossen butt against, collide with, knock against, impulse (a circuit).

Anstrebekraft centripetal force. Anstreichen stroking (a string), (initial) bowing (of a violin).

Anstrengung, Augen- eye strain. Anströmrichtung direction of air flow.
Anströmungssgeschwindigkeit afflux velocity, approach $\mathrm{v}$.

Anteil constituent, portion, share, component.

anteilig proportionate, in proportion, pro rata.

Anteilziffer. percentage share. Antenne Siehe auch "Luftdraht"

Antenne, abgeschirmte shielded (downlead) antenna, anti-static antenna, screened a. - mit Ablei'tung shunt-excited antenna. -, ausgekurbelte reeled out, lowered or paid out antenna or aerial (through fairlead of airplane). -, eingebaute built-in antenna. -, eingefahrene retracted or drawn-in antenna. - einziehen reel in, haul in or retract antenna wire. -, künstliche artificial, mute or phantom antenna. -, vom Sender entkuppelte balanced antenna. mit seitlichem Minimum directive antenna. -, ungerichtete non-directional antenna.

Antenne, Baken- beam antenna, beacon a., radio range a. Band- tape antenna. Beggerow-Zeppel in Zeppelin antenna. Behelfs- temporary or makeshift antenna. Boden- ground or earth antenna; underground or buried antenna. Dach- gable roof antenna. Doppelkegel-, Doppelkonuscage antenna, double-cone a., sausage a. Doppel-V- double-V antenna, doublet a. Einfach-plain antenna. Erd- ground or earth antenna; underground or buried a. Ersatz- artificial antenna, mute a., phantom a. Fächer-fan antenna, harp a. Fi schbauch- fishbelly antenna (mast antenna in which maximum cross-section and minimum characteristic impedance are in middle). Flächen- sheet antenna. Flossen- skid-f in antenna (on airplane wing). Frei- outdoor antenna. Gegengewicht- screened aerial. Gemeinschafts- block antenna, communal or party a., centralized or shared a. Gitter-parasol-type 
antenna. Harfen- harp antenna, fan a. Hoch- open outdoor antenna (installed at, or reaching, a point high above ground). Innenindoor antenna. Käfig- cage antenna, sausage a., hoop antenna. Kreuzrahmen- crossed-coil antenna. Kurbel- reel antenna, trailingwire a. Lichtnetz- light-socket or -circuit antenna. Linearplain or straight one-wire antenna. Mikrophon- microphone boom or outrigger. Nahschwund- lowangle or short-range fading antenna. Netz- light- or powercircuit antenna, lamp-socket a.; a. adapter, a. eliminator. Peildirectional antenna. Rahmenframe antenna, loop a., coil a. Rauten- rhombic antenna. Reusencage antenna, sausage a., hoop a. Richt-directional or directive antenna, unilateral a. Richtungsgeber-director. Rundstrahlpolydirectional antenna, omni-directional a., omni aerial.

Schlepp-trailing-wire antenna. Schwundverminderungs- anti-fading antenna. Spiral- (flat) spiral aerial, extensible spiral or coiled antenna, s. loop. Stabrod antenna, whip antenna, upright antenna stick. Tannenbaumchristmas tree antenna. Unterwagen- under-car antenna. Weihnachtsbaum- christmas tree antenna. Wellen- wave antenna, Beverage a.

Antennen-ableitung antenna lead (in), downlead. -effekt antenna effect (arror in df). -ei, -endgewicht fish (of trailing antenna). -gebilde antenna array. -nahfeld vicinity or short-range field of antenna. -ringleitung multiple-receiver connection to antenna. -schwingung swaying or swinging of antenna. -system, $\mathrm{Peil}$ - directional antenna system. - verkuirzungskondensator antenna (shortening) condenser, aerial series cond., padding condenser. -verlängerungsspule serial-capacity or loading coil. -zuleitung antenna lead, downlead, feeder line.

Antikathode target, anti-cathode (of X-ray tube).

Antragsteller applicant; petitioner.

antreten, Beweis offer evidence. "Zum Tanz"- phase focusing, formation of groups of electrons in beam tube, bunching.

Antrieb, schlupffreier (cf. zwangsläufig) non-slip drive, geared d., positive d. Druckluft- pneumatic drive.

Antriebs-element motor element (of loudspeaker). -zahntrommel welle drive sprocket shaft.

antworten answer, respond, plead, reply.

Antwort-geber revertive signal means, check-back or monitoring position indicator (of measuring instruments). -schrift answer.

A.N. Verfahren A.N. method (of instrument landing).

anvisieren sight, aim at.

Anwaltsgebihren counsel fee. anwassern "land", alight or descend on, or make contact with, the water (airplane).

Anweisung, Fehl- incorrect indication or reading, misreading.

Anwuchs growth, increase, increment; accrescence, accretion. anzapfen tap.

Anzapf-transformer (center) tapped or split transformer. -turbine bleeder turbine.

Anzapfung, Mittel- center tapping, mid-tap.

Anzeige reading, indication, notice, notification, advertisement.

Anzeige, gedämpfte deadbeat, nonballistic or aperiodic reading (measuring instruments). -, ungedämpfte ballistic reading or 
indication (measuring instruments).

Anzeige, Richtungs-, eindeutige unidirectional direction-finding (with sense finding). Standdeadbeat indication or reading (measuring instruments). Zuckballistic reading, flash or kick r. (of instruments).

Anzeigemarke, schwarze black dot pointer or mark (on dials) to observe or read bearings.

Anzol gor s.a. MELDER

Anzeiger, Amplituden- flashograph (and similar neon types of indicators of resonance and volume). Aussteverungs- volume indicator (in sound recording). Fern- telemetric device (e.g., metameter of GE Co. using impulse carriers). Fernblitz- Keraunophone. Gl immlicht-flashograph, Tune-a-lite, neon tube, etc., tuning indicators. Höchstwert- crest indicator, peak i. Mindest-, Minimumminimum (voltage) indicator. Resonanz- resonance indicator, tuning i. Richtungs- directionfinder, df indicator. Schattenshadow-type tuning indicator, shadowgraph. Seitenabrutsch-side slip indicator. Spannungsspitzencrest or peak voltage indicator, crest voltmeter. Stand- bearing indicator, direction-finder dial. Verhältnis- exponent (math.). Wanderwellen- surge indicator, klydonograph. Well en- cymoscope. Windrichtungs- wind-direction indicator (say, of oscillatory-vane type). Anzeige-röhre, Abstimm- tuning indicator tube (e.g., magic eye, etc.). -vorrichtung indicator device, visual reading instrument

anziehen der Rückkopplung tighten feedback, make regenerative coupling closer.

anziehend, wasser - attracting water or moisture, hygroscopic.

Anziehung, Flächen- surface attraction, adhesion. Haarrohr-capil- lary attraction. Massen- gravitation.

Anziehungskraft attraction, attractive power, adhesive force, gravitation.

Anziehungskraft, Frd- gravitational force.

Anzielen des Zielpunktes sighting of target.

Anzugsdrehmoment starting torque. anzünden ignite, kindle, light, start, strike (an arc).

anzweifeln call in question, doubt. Aeol s-glocke, Glas- glass Aeolian bell. -harfe Aeolian harp.

Apertur, Zwitter- split focus (Walton method of scanning).

Aperturblende aperture stop or diaphragm.

Apparate-gamma apparatus gamma. -zuleitung instrument lead, apparatus 1 .

Apparat-geräusch system noise (sound recorder). -patent apparatus patent.

Apparaturgamma apparatus gamma.

Aquipotentialfläche isopotential or equi-p. surface or plane.

Arbeit, Ablöse- total internal work function (energy acquired by electron to break away from incandesced surface). Reibungs-frictional work, magnetic hysteresis.

Arbeits-eichkreis working reference system. -fähigkeit capacity for doing work, energy. -gang working, operating or procedural step or stage, run, course of manufacture, working operation or schedule. -kennlinie operating curve, working c. or characteristic, performance characteristic. -kontakt marking contact, make or operating c. (closure occurs upon energization of relay). -kopie studio copy, s. print. -leistung work, task, performance, efficiency, output. -linie und -punkt load line and operating point. -punkt, Ruhe- $Q$ point, quiescent $p_{\text {. }}$ (of a valve). -raum (Triftröhre) gener- 


\section{Arbeitsstellung}

ating space, working $\mathrm{s}$. (of drift or beam tube). -stellung operating position, working p. -strom watt current, working c. -strombetrieb open-circuit operation.

Architektur, Film- settings and composition.

arglistig fraudulent.

argumentierbar arguable. $\operatorname{arm}(z . b$. verlust-armes Material) low-(e.g., low-loss material). Arm, Kontakt-wiper arm. Lampenlamp bracket. Längs- line or series arm (network). Quer-cross arm, shunt arm (network). Tastwiper $(\mathrm{arm})$. Verhäitnis- ratio arm (Wheatstone bridge).

Armatur, Mast- pole hardware or fittings, accessories.

armiert, stahlband- steel tape armored (cable).

Art (Besselfunktion) kind (of a Bessel function; e.g., $J_{n}$ denotes Bessel function of first kind and order $\underline{n}$ ).

Atelier studio, stage, teletorium, telestudio. Synchronisier- scoring stage. Ton- sound stage, studio.

Atelier-direktor studio manager. -kamera studio camera. -szenen stage scenery, s. sets.

Atlas, Farb- color chart, c. scale (e.g., Munsell).

atmen breath, respire, throb, inhale, exhale.

Atmen des Films in and out of focus effect. -des Kohlemikrophons breathin' of microphone (slow changes in resistance and response of carbon m.). -des Störgeräusches fluctuations, or waxing and waning of noise.

atmende Membran flexible or non-reflecting diaphragm.

atmendes Mikrophon breathing microphone.

Atmosphärendynamo (Stewart) atmospheric dynamo.

Atmosphärilien atmospheric agencies, the elements. atmosphärische Störungen atmospherics, statics, strays, "X's".

Aton, Rückstoss- recoil atom.

atombindende $\mathrm{Kraft}$ atomic combining power, valence.

Atom-formfaktor atom form factor, atomic scattering or structure factor. -gewicht atomic weight. -gramm gram atom. -huille atom shell. -modell (Rutherford-Bohr) $\mathrm{R}-\mathrm{B}$. atom model or conception. -strahlmethode molecular or atomic ray method. -verrückung atom displacement. -zahl atomic number, nuclear charge number. -zerfall disintegration of atom, atomic d. -zertrümmerung atom splitting, nuclear transmutation, a. fission.

Atü (Atmosphärenüberdruck) atmospheric excess pressure.

ätzen corrode, etch, cauterize, treat with mordants.

ätzen, tief intaglio etching (whole metal coated with resist, pattern cut out with scriber).

Ätz-figur etch pattern, etching figure. -grund resist, protective coating, "ground". -schliff ground section prepared for etching of metals.

Audienz hearing, reception.

Audiogramm audiogram (gives hearing loss vs. frequency).

Audion, rückgekoppeltes regenerative grid-current detector, ultraudion. -, selbstschwingendes autodyne.

Audion, Bremsfeld- electron oscillation detector or rectifier (negative anode connected with leak resistance and condenser). Gegentakt- push-pull grid detector. Kraft- power (grid-current) detector. Schwing- oscillating detector, autodyne.

Aufbau construction, structure, assembly, assemblage, mount, mounting, superstructure; synthesis or building up (line by line, of a telev. picture).

Aufbau, Ionisierungsspiel- Townsend structure or build-up. Mikroskop- 
microscope mounting.

Aufbauten superstructural parts; set (filmwork).

Aufbauzeit, Funkenentladungs- formation time of spark discharge.

aufbereiten prepare, work up, dress (ore), separate.

aufblähen swell (up), bulge out, inflate, belly.

aufblättern exfoliate.

aufblenden dissolve or fade in (filmprojection).

Aufblendung, Fächer- fan fade-in. Iris- irising-in. Kulissen- sidecurtain fade-in. Vorhang- curtain fade-in. Winkel-angle fade-in. aufblitzen flash (up).

aufdämpfen deposit by evaporation, smoke or vaporize upon, distil (a coat or film) onto.

aufdrücken impress (a potential upon).

aufdrucken imprint, stamp upon.

Aufenthal tsort residence, domicile. auffallendes Licht incident light. Auffanganode (cf. Auffänger) collecting or gathering anode (of Farnsworth device), target (in a multiplier); catcher (of Klystron).

auffangen pick up (a signal by antenna), collect, intercept, catch or capture (electrons).

Auffänger collector electrode (in Farnsworth dissector), target (of cyclotron), catcher (in beam tube or Klystron).

Auffänger-dicke target thickness. -käfig collector electrode (in Farnsworth dissector). -platte target plate, impactor p. (of multiplier), collector (electrode). - pl attenverlustleistung collector dissipation (of multiplier).

auffasern separate into fibers, unravel.

Auffassung, indeterministische indeterministic conception or interpretation.

aufflackern flare or flash up.

aufflammen flame up, blaze, deflagrate.
Auffrischen re-processing, renewing, reviving, restoring, regeneration. Auffrischer replenisher (film development).

Aufführung, Privat- preview, private show.

aufgeben abandon (an application or a patent), forfeit, discontinue, relinquish, renounce, waive, surrender.

aufgelöste Linienbilder resolved line patterns.

aufgeschrumpft shrunk-fitted.

aufgeteilte Abbildung split focus (scanning method).

aufgewundener Exponentialtrichter twisted or coiled exponential loudspeaker.

Aufgussverfahren infusion process.

Aufhängebügel loop hanger.

Aufhängung, Mikroskop- microscope mounting. Schneiden-knife-edge suspension. Spitzen- pivot suspension, point $\mathrm{s}$.

aufheben lift, raise; preserve, keep; abolish, discontinue, terminate, revoke, abate, cancel, quash, reverse (a judgment), compensate; reduce (a fraction) to its lowest terms.

Aufhebung abrogation, abolition, suspension.

aufhellen spotlight.

Aufheller Klieg light.

Aufhellung schwarzer Stellen blooming, excessive brightness, loss of contrast (in picture).

Aufhellungsorgan intensity or brilliance modulation or control electrode (of c-r. tube).

aufkeilen key on.

aufklappbar capable of being swung open or raised (on hinges).

aufklappen open up on hinges, lift up, drop (a leaf).

Aufkohlung carburization.

Auflager abutment, seat, bearing surface, support.

Auflaufen des Films take-up (by spool) of film strip. - der Gleichlauffehler compounding or integration of differences of synchronism. 
Auflaufrolle take-up reel.

Aufleuchtdauer illuminated or light period.

aufleuchten light up, flash up, shine, glow.

Auflicht azimuthal or top illumination, light from vertical sources. auflockern loosen (up), relax, slacken, make less rigid or less compact.

auflösen resolve (into), solve, analyze, clear out; release, open (connections).

auflösen (eine Gleichung nach $\underline{n}$ ) solve an equation with respect of n.

auflösende Kraft resolving power (opt.).

Auflösung, Bild-picture resolution, p. definition; scan.

Auflösungsvermögen resolving power (micr.); solvent power. Minimumminimum separabile (of eye).

auflöten solder on; unsolder.

Aufmachung scenery, settings, composition.

Aufnahme reception, detection; plotting (of graphs), pickup (telev. and film work), recording (sound), shooting or taking (of pictures), uptake or absorption (say, of a gas), sorption.

Aufnahme, weitwinklige magnascopic picture.

Aufnahme, Augenblicks- instantaneous photograph, snapshot. Aussenoutdoor shot, exterior shot or take, outdoor scene; phosphor screen pattern photographed with outside camera (in oscillograph work). Ball- bulb exposure. Bild-, Kofferapparat für box equipment, trunk unit. Blindstrom drawing or taking wattless, idle or reactive current. Doppelduplex shot. Fahr- running, follow or traveling shot. Farbenfilm- color photograph. Farbraster-color screen photograph. Fern- telephotograph, telephotographic work; long shot (m.p.).
Flieger-, Flugzeug- aerial or airplane picture or photograph.

Freilicht- outdoor or exterior shooting or shot. Funkbeschikkungs- calibration of directionfinder. Gas- occlusion of gas, absorption of gas (in metal's, etc.). Gitterstrom- drawing or taking of current by grid. Gleichtakt- in-phase recording (on film). Gross- (big) close-up (m.p.). Hoch-vertical or upright picture. Innen- indoor or studio shot; oscillogram directly recorded on photographic emulsion inside vacuum space (in oscillograph work). Kombinations- combination exposure, composite shot (made by mask method). Kurbel- hand-driven shot. Lichtton- photographic sound-film recording. Luftbildair or aerial photography. Nachre-take. Panorama- panning shot, panoramic picture or view, pam or pan shot (studio slang). Probetest picture, trial shot. Querhorizontal picture. Raff- timelapse photography (low-speed take, high-speed projection). Roentgenbeugungs- X-ray diffraction exposure. Roentgen (strahl)- Roentgenography, X-ray picture, skiagraph. Rundblick- panoramic photograph, p. view, panning shot, "pam" or "pan" (studio slang). Schall- sound pickup, s. recording (in form of a track). Schallbandmagnetic steel tape recording. Sprach-Sprech- speech, voice, vocal or dialog recording. Stahlband- magnetic steel tape recording. Stahldraht- magnetic steel wire recording. Strom- current input, the taking of current (say, by a grid), current absorption. Taucher- submar ine shooting. Ton-, Kofferapparatur für box equipment, trunk unit. Verfolge- running shooting. Wieder- re-take (m.p.); re-opening (of law case). Zeitlupen-, Kamera für camera for 
slow-motion or retarded-action picture projection work. Zeitrafftime-lapse photography (low-speed shooting, high-s. projection).

Aufnahmefähigkeit pickup performance (of antenna); absorbability, absorptiveness, absorptivity.

Aufnahme-fähigkeit, magnetische magnetic susceptibility. - gelände location ( $\mathrm{film}$ work). -kamera, Zeitlupen- camera for slow-motion or retarded-action picture projection work. -kamera, Zeitrafftime-lapse camera (low-speed shooting, high-speed projection). - I ampen studio lights. -leiter chief camera man, first c. -magnet recording or pickup magnet (in steel band or wire sound recording). -platte platter (16" electric transcription record). -raum studio (of broadcast station), teletorium, telestudio. -stelle location (film shooting); receiving or translating place (telegraphy). -techniker camera technician. -verfahren pickup method. -verstärker recording amplifier. -wagen, Fernseh- television or electron camera truck, video bus, pickup camera truck, televising car. -winkel shooting angle.

aufnehmen trace or plot (graphs); take (current); collect or pickup (waves, vibrations); record (sound); take or shoot (pictures); copy (messages); receive, accommodate, lodge.

Aufnehmer, Schall-, Ton- sound collector (microphone), pickup; recorder.

Aufprall (cf. Prall) impact, bombardment, impingement, striking (of electrons, etc.); bound, rebound.

Aufpunkt "space" point. aufquellen swell (up), well up. aufrauhen granulate, roughen or grind superficially, knurl, rag, produce a nap.

aufrecht upright, erect.

\section{Auf spaltung}

aufrechterhalten maintain, keep alive (an application or patent), uphold.

aufreiben ream, broach.

Aufrichtung, Bild-erection of an image, rectification of i., inversion (when left and right, top and bottom are simultaneously interchanged).

Aufrichtungs-blende erector stop. -prisma erecting prism.

Aufriss elevation, vertical section, sketch.

aufrührbar stirrable, agitable.

Aufsatz any part put or set on, attachment, head-piece, neck, top, dome.

Aufsatz, Geschütz- optical instruments for artillery. Peil-bearing plate (in direction-finder).

Photometer- photometer head.

Aufschaukeln resonant rise, buildup or increase of amplification (of oscillations, in proper phase).

Aufschaukelzeit time-constant of resonant amplification.

aufschichten pile up, stack up, stratify, layer.

Aufschiebung postponement, adjournment, suspension, arrest (of judgment).

Aufschlemmung solution, suspension.

Aufschl emmverfahren floatation method.

Aufschlussraum part of prospected volume subject to current or displacement lines.

Aufschrumpfung shrunk-fit.

Aufschub adjournment, time-extension, grace of time, stay, postponement, prolongation, deferment, respite, suspension, delay, moratorium.

Aufsetzpunkt (Flugzeug) landing point, ground-contact point.

Aufsicht plan or top view.

Aufsichts-betrachtung direct or frontal viewing (of television pictures). -sucher view finder.

Aufspaltung separation, split-up, cleavage, division, dissociation. 
Aufspaltung, Dubletten- doublet separation. Spinbahn- spin crbit splitting.

Aufspaltungs-bilder splitting patterns (opt.). -faktor splitting factor.

aufspannen stretch or spread out, unfold.

aufstapeln pile up, stack up, store. aufstäuben (einen úberzug) spray, dust or atorize (a coat, film, etc.) upon.

Aufsteck-glas slip-on lens segment. - glas, Okular- eyepiece correcting lens. -kappe slip-on cap. -spule plug-in coil.

Aufsteller, Bühnen- set dresser. Aufstellpunkt location.

Aufstellung, Gitter-, Rowl andsche (Rowland's) mounting for concave grating.

Aufstieg, Ballon- balloon ascent. aufstreichen spread on, brush on, stain (paper).

Aufstrich leading edge or upstroke (of an impulse).

Aufsuchen, Ziel-detection of target or object (by reflection of radar or sonar pulses).

Aufteil ungs-filter crossover network (sound production). -verhältnis potentiometer ratio.

auftragen apply, charge (furnace), plot (curves), protract (geometry).

Auftrag-geber mandator, principal, client, customer. -rollen application rollers, inking $r$.

Auftreff-geschwindigkeit striking velocity. -winkel angile of impact.

auftreiben, das Loch enlarge, expand, broach, ream (a hole).

Auftreiber reamer.

auftrennen sever, rip up or open.

Auftrieb upward thrust, up-thrust, lift, buoyancy, ascendency.

Auftrieb, Luft- air buoyancy.

Auftriebmethode float method, buoyant-force $\mathrm{m}$. (hydrometer). auf "Vordermann stehen" be or lie in the shadow of.

Aufwand, beträchtlicher, von Mitteln elaborate means or circuit organization, appreciable outlay.

Aufwand, Leistungs- power input, p. expenditure, energy dissipation. Mehr- additional or extra expenditure, outlay or means (used for circuits, apparatus, etc.), complement (of tubes).

Aufwärtstransformator step-up transformer.

Aufweiterung, Gitter-increase or expansion of lattice spacing.

Aufweitung expansion, bulge-out. aufwickeln wind up, wrap up or upon, take-up (film); unwind, unwrap. Aufwickelspule take-up reel. Aufwind anabatic wind, upslope w. aufzählen enumerate, list, itemize. Aufzehrung, Gas- gettering, gas absorption, outgasing.

aufzeichnen (Stromlinien, etc.) map or plot (tubes or lines of force, etc., of ten in a tank).

Aufzeichner, Schall- sound recorder. Schallwellen- phonodeik (recording on film). Ton-, magnetischer magnetic sound recorder (magnetophone, Blattnerphone, Poulsen telegraphone). Schwingungs- vibrograph (to record mechanical vibrations).

Aufzeichnung, Abdeck- masking recording, matting. Bild- picture recreation, p. delineation, $p$. tracing, p. synthesis. Vielkanalmulti-channel recording.

Aufzeichnungs-kammer recording chamber (in electron microscope). -kontrolle monitoring (of sound recording or sound tracks). -l ampe, "Aeo"- Aeo light, alkaline-earth oxide lamp, Aeo lamp (variable-density sound picture recording work).- -objektiv recording objective. -schlitz, -spalt, recording slit or aperture. -träger record or track support (sound on film, disk, etc.); record sheet. -umfang re- 
cording range.

aufziehen mount (a photograph); wind (a dial switch).

Aufziehhebel lever ratchet.

Augapfel eyeball, bulbus.

Auge, akkomodationsloses unaccommodated eye, fixed eye. -, alterssichtiges presbyopic eye. -, berichtigtes corrected eye. -, bewaffnetes aided eye. -, dunkel adaptiertes dark adapted eye. -, entspanntes relaxed eye. -, fehlsichtiges defectively sighted (amietropic) eye. -, fernakkomodiertes relaxed eye. -, fernsichtiges far-sighted eye, hyperopic eye. -, kurzsichtiges near - or short-sighted eye, myopic eye. -, nachtblindes night-blind eye, nyctalopic eye. -, nahsichtiges near-sighted eye, myopic eye. rechtsichtiges normal-sighted eye, emmetropic eye. - unbewaffnetes unaided eye, naked eye. -, weit. sichtiges far-or long-sighted eye, hyperopic eye.

Augen-abstand interocular distance. - abstandsmesser inter-pupillary distance gage. -adaptationszustand state of retinal adaptation. - anpassung eye adaptation (to luminosity or wave-length of light), eye accommodation (to changes of distance or focus). - anstrengung eye strain. -blicksaufnahme instantaneous photograph, snapshot. - brechungs-messer skiascope, retinoscope. -eigenlicht self-light, intrinsic light of eye or retina, eye's own 1. (associated with photopsia). -empfindlichkeit intensity discrimination, contrast sensitivity (embodied in Weber-Fechner law). -farbenempfindlichkeit color or spectral sensitivity of eye, spectral response of eye. -höhle socket of eye, orbit, orbital cavity. -kreis exit pupil. - lehre ophthalmology. - lichtempfindlichkeit luminosity response of eye. -linse eye lens. -lin- senfaserung fibrillation of lens. -messer ophthalmometer, optometer. -muschel eye cup. -punkt exit pupil, eyepit. -spiegel ophthalmoscope, skiascope. -stäbchen cones (of nerve endings). -täuschung optical illusion. -zäpfchen rods (of nerve endings). -zerstreuungsbilder blur circles of eye.

ausätzen cauterize, destroy by caustic.

Ausbau demounting, taking apart or out, dismantling.

ausbauen disassemble, dismantle, demount.

ausbauchen bulge, swell, hollow out, belly.

ausbeulen take out dents (in a metal part); swell out, round out.

Ausbeute yield, crop, output, gain, returns, efficiency.

Ausbeute, photoelektrische photoelectric emissivity or yield. Quanten- quantum yield or efficiency.

ausbiegen bend out, turn out.

ausbilden fashion, form; develop; educate, train.

Ausbildung, Fach- technical education, schooling or training.

ausbleien provide with a lead lining or coating.

ausblenden mask with apertured diaphragm or stop, limit the field (of beam, pencil, etc.), diaphragm out, cut down aperture of a stop, use a field stop, iris out, circle out.

Ausblend-mittel limiting aperture, screening, masking or stopping means (for rays and radiations). -steverung obturator or cut-off modulation.

Ausblickstutzen objective lens socket (opt.').

ausbohren bore or drill out. ausbreiten spread (out), extend, expand, diffuse, propagate (waves).

Ausbreitprobe hammering test (specimen).

Ausbreitung, Licht- distribution of 
exposure (on film). Schall-, vertikale vertical spread or diffusion of sound (should be angle subtended by audience at loudspeaker).

Ausbreitungs-geschwindigkeit velocity of propagation (of waves, etc.). -strom dispersion current. -vorgang propagation phenomenon or action. -widerstand diffusion resistance.

ausbringen yield, produce, bring out, take away.

Ausbruch, Gas- eruption, (violent) liberation or escape of gas. Sonnen- solar eruption.

ausdehnbar expansible, extensible, ductile.

Ausdehnung dimension, extent; extension, expansion; latitude, scope, range.

Ausdehnung, Längen- linear expansion.

Ausdehnungsmesser dilatometer, extensometer.

Ausdeutung interpretation, evaluation, explanation.

Ausdruck expression, term (of an equation), denotation (of a factor).

Ausdruck, Fach-technical term, t. expression.

ausdünnen thin (out).

auseinander-gehende Werte divergent values, discrepant v. -ziehen spread apart, draw a. or asunder. Ausent-wicklung full development. ausexponiert fully exposed.

Ausfall, elektrischer eleciric shock. Intensitäts- fading, fadeout (of radio signals). Strahlenemergence of rays.

ausfällen precipitate, deposit. ausfall ender Lichtstrahl emergent light ray.

Ausfäll apparat precipitator, precipitron (electronic).

Ausfallwinkel angle of emergence. Ausfertigung office copy, official c.

ausfiltern filter out, exclude. ausfindig machen ascertain, trace, discover.

ausfliessen flow out, issue, discharge, emanate.

Ausflockung (de)flocculation, coagulation.

Ausfluss outflow, efflux, discharge, emanation; mouth, outlet, drain.

Ausfluss-einschnitt contractio venae. -röhre outflow tube, discharge pipe. -ton jet tone, slit tone.

ausfransen fray.

ausfräsen notch or recess (in milling machine).

ausfrieren freeze out (e.g., in liquid-air trap).

Ausfrier-gerät freeze-out device. - tasche liquid-air trap (vacuum pump).

Ausführbarkeit practicability, feasibility.

Ausführung lead-in (wire).

Ausführungs-beispiel exemplified embodiment, form of construction (cited by way of example in patent specifications). -zwang compulsory working (of a patent).

ausfuttern (ausfiuttern) line; case, bush, pad, upholster.

Ausgängen, Schalter mit 5 five-point switch.

Ausgangs-funktion trial function. - gleichung starting or initial equation. -leitwert output admittance, output conductance. -material starting, raw, initial or parent material. -pupille exit pupil. -schlitz exit slit (spectrograph). -stellung home position (of dial switch).

ausgefahrene Meterzahl paid-out or reeled-out yardage (of airplane antenna).

ausgefressene Kontakte pitted or worn contacts.

ausgeglichener Verstärker balanced amplifier.

ausgehen go out, become extinguished; originate, start, emanate, issue from. 
ausgepeilte Richtung bearing. ausgeprägt distinguished, peculiar, characteristic, singular, particular, marked, decided, significant, excellent; salient (re: poles).

ausgespannte Saite stretched or tensioned chord or string.

ausgesteuerter Tonstreifen, schwach low-modulation sound-track.

ausgetuchtes Loch bushed hole.

ausgezackte Linie, ausgezahnte Linie jagged line, serrated $1 .$, notched 1., dented 1.. dentated 1 , ausgezeichnet distinguished, marked, excellent, singular, significant, particular.

ausgezeichnet(en) Lichtweg, Satz vom law of extreme path. - (e) Punkte cardinal points (opt.).

ausgezogene Linie solid line, full line, unbroken line (in graphs, drawings, etc.).

Ausgiebigkeit productiveness, fertility, abundance, yield(iness). ausglätten (Kurven) smooth, flatten (graphs).

Ausgleich, Farb-color balancing (in color film). Ladungs- charge equalization. Pegel- level equalization, equalizing 1 . Schrumpfungs- shrinkage compensation. Schwund- fading compensation, volume control.

ausgleichen balance, compensate, equalize, align, bias out (by counterforce), counterbalance.

Ausgleichen (von Schallwellen) neutralization of sound waves (prevented by baffles).

Ausgleichentzerrung complementary recording.

Ausgleicher, Dämpfungs- equalizing network, compensating $n$.

Ausgleich-fläche (der Erdkruste) isostatic surface. -getriebe differential gear. -schwungscheibe rotary stabilizer (m.p. projector), impedance wheel. -spannung transient voltage; compensating v. -strom compensating current; balancing current (of a bridge). -transformator hybrid transformer, balanced t., differential t., 3-winding $t$.

Ausgleichung, Ladungs- charge equalization. Schallwellen- neutralization of sound waves (prevented by baffles).

Ausgleich-vorgang transient. -widerstand compensating resistance, balancing resistance, ballasting $r$., building-out $r$.

Ausguss lip, spout, outlet, drain, sink; delivery, effusion. -schnauze pouring lip, nozzle or mouth.

aushärten harden, set, indurate (thoroughly).

ausheizen heat, anneal, subject to (thorough) thermal treatment. auskehlen channel, flute, groove. auskeimen germinate; cease germinating.

auskellen ladle (out). ausklagen (einer Geldstrafe) sue for a penalty.

ausklappbar capable of being swung out or dropped (on hinges).

auskleiden line, clothe.

ausklinken release, trip, trigger, disengage, throw out (of gear).

Ausknickung buckling, bending (at a sharp angle).

Auskopierpapier printing-out paper. Auskoppelfeld output field, absorbing $f .$, uncoupling $f .$, delivery f. (in Hollmann's inverted cyclotron or electron turbine).

auskoppeln tune or balance out, neutralize, decouple (to suppress or lessen feedback).

auskurbeln (Antenne) reel out, lower, pay out the aerial (on airplane, through fairlead).

ausladend projecting, outrigging. Ausladung projection, overhang, reach, length of action, working radius.

auslagern adsorb (at points of attachment).

Ausl assventil escape valve, delivery v. 
Auslauf von der Rolle point where film strip leaves drum.

Ausläufer tail, extension, streamers, off-shoot, satellite line.

Auslauf-kurve coasting curve, deceleration c. -spitze discharge tip.

ausleeren empty out, evacuate, drain auslegen (für jedermann zur Einsicht) lay open (say, a patent application for public inspection); design, plan, lay out; interpret, construe.

Auslegung (eines Gesetzes) interpretation or construction (of an act or a law). -, falsche misinterpretation, erroneous interpretation or construction.

Auslenkhärte deflection hardness (force required to deflect phonograph needle point $100 \mu$ ).

Auslesefähigkeit selectivity.

ausleuchten illuminate. flash (an image).

Ausleuchtung, gleichmässige even illumination.

Ausleuchtung und Tilgung extinction or quenching (of fluorescence), evanescence.

Ausleuchtung, Spalt- slit illumination.

auslöschen erase, extinguish, wipe out, quench, blot, obliterate, cancel, put out, (cause to) evanesce.

Auslöschmagnet obliteratjus magnet, obliterating pole piece (to wipe out sound record, on wire or tape, for re-use).

Auslöschung, Fluoreszenz- fluorescence quenching, evanescence (say, by poison).

Auslöschzone dead spot, region of silence.

Auslöse-arbeit (cf. Ablösearbeit) total internal work function, excitation energy. -daumen releasing cam, resetting cam. -hebel trip lever, detent 1 .

auslösen emit, give off (electrons, etc.); trip, trigger, disengage, render operative, release.
Auslöser, Ball- ball release.

Auslöserknopf jack or escapement button, set-off button (piario).

Auslösespule trip coil.

Auslösung, Selbst- automatic release means, a. tripping action.

Auslösungs-anschlag trip dog. -feder escapement spring, release or trip spring. -knopf jack, escapement or set-off button or knob.

Ausmass amplitude, dimension, size, extent, quantity.

Ausmessung, Flächen-planimetering. ausmultiplizieren eliminate by multiplication (math.).

Ausmündung orifice, outlet.

ausnehmen von except, exempt from.

Ausnehmung recess.

Ausnitzungskoeffizient utilization coefficient.

auspressen press or squeeze out, express.

auspumpen pump out, exhaust, evacuate.

ausquetschen squeeze, crush or ring out, squeegee.

Ausrede plea, evasion, excuse, pretext.

Ausregelzeit decline period of control potential.

ausrichten orient, orientate, straighten, adjust, set (right).

Ausrichtung, fal sche misalignment (of image or track).

ausrïcken disengage, ungear, unmesh, throw out (of gear).

ausrunden round of $f$.

Ausrundung fillet.

Aussage affidavit, allegation, declaration, assertion, affirmation, deposition, testimony, evidence, averment.

Aussagender declarant, deponent, af fiant, witness.

Aussal zungskoeffizient salting-out coefficient.

ausscheiden (Teile einer Anmeldung) divide (an application for letters patent).

Ausscheidung precipitation, separation, elimination, deposit, ef f'lorescence. 
Ausscheidungs-effekt filtering, separating or excluding action or ef fect. -härtung precipitation hardening. -punkt point of separating out.

Ausichlag throw, excursion, deflection, deviation, kick, travel (re: a coil, diaphragm, instrument needle, beam, etc.); exudation, efflorescence, scum.

Ausschlag, Schall-displacement (of a particle) (ac.).

ausschleudern centrifuge, spin out. ausschliessen exclude, preclude, bar.

Ausschliesslichkeit uniqueness.

Ausschliessungs-grïnde reasons for exclusion. -prinzip Pauli's equivalence principle (obs.), exclusion p.

ausschmelzen melt or fuse out, render, liquate, purify by melting.

ausschmieden forge, hammer out.

Ausschnitt cutout, notch, hole, opening, cutaway portion, louver, aperture, recording window. Bildframe (of a camera); image area (on scanning disk). Blenden- aperture, slit (of diaphragm or stop).

Ausschuss für Einheiten und Formeln (AEF) Committee on Units and Formulae, Standards or Standardization C.

Ausschwingstrom decay current, decaying c. (of transients).

Ausschwingung decay, dying out (of an oscillation).

Ausschwingungs-verzerrung decay (transient) non-linear distortion, facsimile transient $d$. (in form of tailing, or as overthrow or underthrow distortion). -verzug hangover, tailing, excessive prolongation of decay of wave tail, in facsimile.

aussedimentieren sediment out

Aussehen, ver schwommenes foggy, bleary or blurred appearance (of an image).

Aussenaufnahme outdoor or exterior picture or shot, o. scene; (c-r.) screen pattern photographed with outside camera (oscillography). aussenden transmit, send (out), emit, give off, issue, dispatch, disseminate (of standard frequencies).

Aussen-gewinde male screw-thread, outside ścrew thread. -leiter "outer" (conductor or wire). -linie contour (line). -mass overall dimension, outside $d$.

aussenmittig eccentric, off center. Aussen-schenkel outer leg, o. limb. -taster outside calipers. -welt external world, ambient.

ausser Achse extra-axial, abaxial. ausserachsiale Strahlen extra-axial rays.

ausserachtlassen disregard, neglect, leave out of consideration.

Ausserfokusbild out-of-focus picture. ausser-gerichtlich extra-judicial, out of court. -irdische störungen extra-terrestrial or inter-stellar space noise or disturbances. -ordentliche Komponente extraordinary component (of rays). - Phase dephased, out of p. -Tritt fallen fall out of step or synchronism.

Äusserung, Kraft-manifestation or effect of force.

aussetzende Belastung intermittent load.

Aussetzer failure in ignition, slips (ignitron).

Aussetzung adjournment, discontinuance, deferment, stay (of proceedings), arrest (of judgment).

Aussichtsdichte reflection or specular density.

aussickern trickle out, ooze out, percolate.

aussieben filter out, exclude (by filter action); select, sift.

Aussiebung, Impuls- synchronizing separator (for sync and video signal separation), amplitude separator, clipper (in telev.).

Aussortierung, Anoden- anode sorting. Phasen- phase focusing, bunching (in klystron and other beam tubes). 
aussparen recess, remove material from; set aside (say, marginal portions for sound track on film). ausspringend salient, protruding, projecting, jutting.

ausspritzen squirt (out), wash by squirting, inject.

Aussprungwinkel angle of reflection.

Ausstattung scenery, settings, set, composition.

ausstellen (einer Urkunde, Vollmacht, etc.) execute (a document, power of attorneý, etc.).

Aussteuerung, prozentuale percentage modulation.

Aussteverung, Gitter- grid swing, g. sweep, g. excitation. Lautsprecher-operating condition in which input suffices to fully utilize loudspeaker power and performance; excursion of diaphragm. Licht strahl-, maximale clash point of light valve (in sound recording). Röhren- operating condition when signal (a.c.) voltage impressed on grid suffices in amplitude to swing plate current from zero to saturation; grid swing, g. excitation, amplitude of signal (a.c.) voltage. Schirm-full utilization of screen (up to its very border, in cathoderay tube operation), sweeping out of screen.

Aussteuerungs-anzeiger volume indicator (sound recording). - bereich drive range (of a grid in an amplifier tube), grid swing, Erid base, range below point where overload begins, that is, in straight portion of characteristic (maximum limit is 100 per cent modulation). - grad modulation percentage, depth of $\mathrm{m}_{\text {. }}$, percentage $\mathrm{m}$.; carrier amplitude (in telemetric or teletransmission work). -intervall control range, drive range. -kontrolle mit Neonröhre neon tube volume indicator. -kontrollgerät modulation meter, load indicator. Ausstrahlung radiation, irradiancy, emission of rays, radiations, os- cillations, vibrations and waves (in general, including heat and sound).

ausstreichen cancel, strike out, cross out, delete.

ausströıen emanate, emit, flow out, stream out, effuse.

Ausströmerscheinungen effusion phenomena.

austasten cut of $f$ or gate (pencil or beam, on flyback), blackout (by blanking signal); key off (a carrier).

Austastimpuls blanking pulse. austauschen exchange, interchange. Austausch-integral exchange integral. -kräfte exchange forces, e. energies. -operator, Ladungscharge exchange operator. -reaktion substitute reaction, exchange $r$. -werkstoff substitute material.

austragen deliver, distribute, carry out, discharge.

austreiben expel, drive out, outEas. (gas or vapor), cleanup, getter:

Austreibung, Gas- gas cleanup, outgasing, degasing, gettering.

austretendes Licht emergent light. Austritts-arbeit (der Elektronen) work function (of electrons). -blende exit slit. -geschwindigkeit exit velocity, muzzle v., (re: Euns); emergence v. -pupille exit pupil.

Austuchung bushing.

Ausübuny (eines Gedankens, etc.) carrying into practice or effect, execute (an idea, invention, etc.).

Auswahl-prinzip, -regel selection principle, selection rule (in electron transition).

Auswandern shift of beam or beacon course.

auswechselbar exchangeable, interchangeable, replaceable, capable of substitution.

Auswechslung, 0ptik- optical system or assembly (comprising interchangeable intermediate lens barrel). 


\section{Auswertung}

Auswertung evaluation, valuation, assay, analysis, appraisal, computation, interpretation; plotting (map).

Auswertung, punktweise point by point evaluation.

auszacken indent, notch, jag, serrate, dentate.

Auszeichnen, scharfes, einer fläche durch objektiv sharp focusing by lens of image in angle of field. ausziehbar telescoping, extensible.

Ausziehtubus drawtube (re: microscope).

Auszug abridgment, extract, excerpt, synopsis, abstract.

Auszug, Balg- bellows extension. $\mathrm{Bl}$ au- blue record. Farb-color record. Kamera- camera extension. Posaunen- telescoping or extension means (for tuning Lecher-wire or ultra-h-f systems, by short-circuiting bridge sliding along wires).

Auszugfilter, Farben- selective filter, s. screen.

Auto-elextronenemission auto-elec- tronic emission, field or cold emission of electrons. -frettage cold drawing, auto-frettage. - matenlegierung machining alloy stock. -matik automatic (sharp) tuning means; any automatically operable mechanism. -matik, Fading-, Schwund- automatic volume control means (avc). automatisch(e) Gittervorspannung automatic or self-biasing of grid. -(e) Kurssteuerung automatic or mechanical piloting. - (er) Verschluss automatic shutter.

A-Verstärker class A amplifier.

A W Zahl (Amperewindungszahl) number of ampere-turns (ats).

Ayrton'scher Nebenschluss Ayrton shunt, universal shunt (box). Azetil-film acetate film stock. -zellulose cellulose acetate. Azid azide.

Azımuth, Funk-, missweisender magnetic bearing. Funk-, rechtweisender true bearing.

Azimuthwinkel azimuth angle. Azoverbindung azo compound. 
Backbord achteraus port aft. -voraus port bow. -kurve port (left-hand) curve (in airplane manoeuvring and landing procedure).

Backe jaw, bit, die.

Backe, Einspann- clamping or gripping jaw, chuck jaw, grip (of a tester). Schweiss- welding die.

backen, zusammen- cake together, clinker.

Bahn path, orbit, shell (of an electron), trajectory, way, track; ecliptic (apparent path of sun); web (of paper, cloth, etc.).

Bahn, Ablauf- runway, landing ramp. Blitz-, verschlungene tortuous path of lightning flash. Elektronen- electron path, e. orbit, e. trajectory, flight of electrons. Erd- earth's orbit, terrestrial o. Fahr-track, runway (airfield), travel beam. Film-film track, f. chanrel. Flächenstrom- current sheet. Gleit- chute (film), slide (way), shoot. Herz-cardioid-shaped path (of electrons). Kreiscircular path, orbit. Landerunway (for landing of airplanes). Leit- transit path. Papier-paper web. Roll-cycloidal path (of electrons); roller type conveyor; runway (airport). Rosetten- (der Elektronen) rosetteshaped path (of electrons). Rückstoss- recoil track. Schwungsound take-off drum (m.p.). Spinspin orbit. Start- runway. Tauch-(der äussersten Na-Elektronen) dip orbit (of peripheral $\mathrm{Na}$ electrons). Wirbel-, abgehende trailing vortices (airfoil)
Bahn-aufspaltung, Spin- spin orbit splitting. -durchmesser diameter of orbit. -elektronen orbital electrons.

bahinen beat, smooth, clear (a way or path)

Bahn-ende, Mesonen- mesotron track end. -impuls orbital moment. -krels, Roll-cycloidal path of motion (of electrons). -schling orbital loop. -spur track. -verlauf ray path, r.tracing, trajectory.

Bajonettverschluss bayonet joint, b.union (as used in bayonet-type lamp holders).

Eake, Gleitweg- glide path beacon. Bakenantenne beam antenna, beacon a. , radio range aerial.

Balancier beam.

Balg(en) bellows.

Balgenauszug bellows extension.

Balgfalten bellows folds.

Ballast-röhre absorber tube or valve (operative during spacing periods). -widerstand ballast resistance, b.resistor.

Ballaufnahne bulb exposure. -auslöser ball release.

ballen form into balls, conglomerate, cake.

ballig crowned, cambered.

Ballon carboy, balloon (flask). Ballon, Säure- acid carboy.

Ballon-abfüller pump-equipped balloon flask. -abstieg balloon descent. -aufstieg balloon ascent. -variometer balloon statoscope.

Ball-sender re-broadcast station, repeating station, $\mathrm{s}$. belonging to a network.

Ballungsfähigkeit coalescing, bal- 
ling or caking property, conglomerableness, spheroidizing property (cryst.).

Bal sam, Kanada- Canada balsam.

Bananenstecker split (banana-shaped) plug, b.plug.

Band ribbon, tape, band, strip, tie, strap, bond, belt, hoop.

Band, besetztes full band. am laufenden-fabrizieren conveyorbelt manufacture, large-scale production.

Band, Führungs- control strip (of a printer). Lautschrift- sound recording band, tape or strip (magnetic sound recording). Lochscanning belt (telev.). Übertragungs- signal band.

Bandabschattierung, nach rot oder violett degradation of bands to red or to violet.

bandagieren fit with tires, tire; bandage, wrap or tie with ribbon or tape.

Band-antenne tape antenna. -aufnahme, Schall-, -aufzeichnung, Schallmagnetic steel tape recording of sound. -breiteregler band-width control.

Bändchen-galvanometer twisted strip or band galvanometer. -mikrophon ribbon microphone, velocity $\mathrm{m}$.

Band-einengung squeezing or compression of band. -eisen hoop iron, strip i., band $i$.

Banden, Ober- overtone absorption bands. Schwanz-tail bands (in afterglow)

Banden-besetzungsg rad degree of filling of bands. -kopf bandhead, band edge (spectral analysis). -zug band progression. -zweig branch part of series of lines forming a band.

Bandfilter band selecsor (circuit), preselector.

Band-filter von grosser Lochweite broad band filter. -förderer band or belt conveyor -führung,
Film- aperture guide. -haken bridle wire, tie hook. -kante band edge, b.head. -mikrophon ribbon microphone, band $\mathrm{m}$.

-mitte mid band. -öse bridge guide. -sperre band-exclusion filter. -umkehrung speech band inversion (secret telephony). Bank, optische optical bench. Bank, Prüf- (lens) test bench (opt.) Bär ram (of a press).

Barkhausensprung Barkhausen effect or jump (abrupt changes in magnetization).

Barometer, registrierendes barograph. Barometer, Dosen-. (Feder-) aneroid barometer. Gefäss- cistern barometer. Gefässheber- combination cistern and syphon barometer. Heber - syphon barometer.

Barometer-korrektur barometric check (df). -säule barometric column.

barometrische Reglerdose control aneroid.

Baryumazid barium azide.

Basenbildner base former, basifier, basifying agent.

Basisentfernungsmesser base rangefinder.

Bass-anhebung bass boosting, b.compensation, tone control means to accentuate, emphasize or underscore b. or low-pitch notes, b. emphasis. - balken bass bar (string instrument). -kasten bass box (musical instruments).

Bassin tank, cistern, reservoir, basin, bowl.

Bastler amateur, fan, "ham".

Batterie-glas battery jar. -klinke battery jack.

Bauakustik architectural acoustics, theater and auditorium acoustics.

Bauch (einer Schwingung) loop or antinode, internode (of an oscillation).

bauchig bellied, bulgy,

Baufehler, Kristall- crystal defects. 
Baukunst, Film- setting and composition art.

baumähnl ich tree-like, arborescent, dendriform.

bauschen swell out, bag, bulge, puff up; refine ( $t$ in).

Bauteil construction part, structural part or element.

Bauten, Auf- superstructural parts. beabsichtigen intend, plan, contemplate.

Beamter, Funk- radio operator, station official.

Beanspruchender claimant.

Beanspruchung stress, strain, load; claim.

Beanspruchung, Prioritäts- priority claim. Schlag- shock load or stress, blow 1., impact 1.

beanstanden object, reject, criticize adversely.

beantragen apply (for), petition, make a motion.

Beantwortung, Klage- reply, answer, defence (plea).

bearbeitbar workable, machinable, processable, treatable.

Bearbeitbarkeit, spanabhebende free cutting machinability.

bearbeiten work (on), tool, finish, dress, machine.

bearbeiten, kalt cold-work. -, warm hot-work.

Beaufschlagung electric stress (placed on an insulator link or unit); acoustic action or sound pressure (brought on microphone); impacting (by electrons).

Beauftragter attorney, agent, delegate, commissioner, mandatory.

Bebenmesser seismometer, seismograph (device to measure and record tremors).

Becher, Abschirm- shielding can, can, shield. Zink- zinc case or cylinder (of dry cell).

Becher-block encased fixed tubular paper or mica wound or wrapped condenser, solid-dielectric fixed capacitor. $-g l$ as beaker.
- glaskolben Erlenmeyer flask.

Becken cymbals. Tamborin- tambourine jingles.

Bedämp fung (introduction of) resistance, damping, attenuation.

Bedämpfung, Eingangs- input resistance, damping.

Bedarf need, demand, requirement.

Bedarf, Leistungs- power absorption, p.dissipation, p.requirements.

Bedarfpegel, Leistungs- power reference level.

bedeckt mit Cäsium caesiated.

Bedeckung surface coverage. -des Netzes coverage factor.

Bedeckung, Wandstoff- (cf Bekleidung) wall draping (ac.).

Bedeckungsfaktor, Pol- pole arc, p. pitch percentage.

Bedeutung import, importance, meaning, sense, significance.

bedienbar, leicht easily manipulable or operable.

Bedienbarkeit manipulability, manoeuvrability.

Bedienungsvorschrift service or working instruction, directions for use.

bedingen stipulate, contract for, condition.

Bedingungsgleichung equation of condition.

Bedrahtungsplan wiring diagram.

Beeidigung confirmation by oath.

Beeinflussung influence, control action, modulation, interference. -,gegenseitige mutual action, interaction. Gitter- grid.control, grid modulation. Sprachvoice control, modulation by voice.

Beeinflussungsröhre modulating tube, modulator $t$.

beeinträchtigen impair, injure, prejudice, affect adversely, encroach upon, infringe, detract. from.

Befehl, Einhalts- injunction, interdict. Vollziehungs- writ of execution, warrant. 
Befehls-rohr (cf Folgerohr) pilot or master thyratron (fires first, in a trigger circuit). -stelle control unit (airport). -übertragung zwischen Flugzeugen inter-aircraft voice or command communication.

befestigen secure, fasten, attach, fix; fortify, strengthen.

Befestigungsring ring fastener, securing ring.

befeuchten moisten, dampen, wet, water.

Befeuerung, Flughafen- airport beacon service. Mast- mast beacon. Nachtstrecken- route beacon for night flying service.

beflecken spot, stain, soil, blur. befördern forward, further, promote, convey, aid, transport. begehen inspect or patrol (lines, on foot).

begiessen coat (film), wet, moisten, water, irrigate.

beglaubigte Abschrift certified, legalized, authentic or attested copy.

Begleit- concomitant, accompanying, attendant.

Begleiten zum Fernsehbild sound or audio action accompanying television or video program.

Begleiter associate, satellite, congener (if similar).

Begleiter, Eisen- elements accompanying iron, iron associates, congeners of iron (if simílar).

Begleiterscheinung attendant, accompanying, or secondary phenomenon or action, satellite effect.

Begleit-körper accompanying substance or body, congener (if similar); impurity, foreign substance or admixture. -linien satellite lines. -saiten accompaniment strings. -stoff accompanying body or substance, congener (if similar); impurity, foreign substance.
Begrenzer, Amplituden- peak limiter, p.clipper, p.lopper. Verstärkungsoutput suppressor or limiter, gain spoiler.

Begrenzungsanschlag back stop, limiting means.

Begriff concept, conception, idea, notion, precept.

Begriff, ober- preamble, introductory portion of German patent claim setting forth prior art.

begründen establish, found, constitute, substantiate, create.

Begründung (eines Einspruches) argumentation (in support of an opposition).

Begründung, Berufungs- arguments or grounds for appeal.

Begründungsfrist period for giving grounds or to argue a case.

Begussmasse slip, engobe (cer.). behaftete Oberfläche, gas- gascontaminated surface (gas adhering to or adsorbed by surface).

Behagl ichkeitswert comfort value, c. factor.

behandelbar manipulable, tractable, workable, processable, treatable.

Behandl ung treatment, processing, manipulation.

Beharrungs-moment moment of inertia, rotational i. -punkt center of inertia, c. of mass, centroid. -vermögen inertia. -zustand steady-state condition, perman. ence, persistence, freedom from transients, resistance (of a machine).

behaupten contend, allege, state, hold, affirm, assent, maintain.

Behelfsantenne makeshift, temporary or emergency antenna or aerial.

beherrschen master, control, override, rule, overcome, dominate.

behinderte Rotation hindered or inhibited rotation (of molecules).

Behinderung hindrance, hindering, inhibition, restraint, impediment, obstacle. 
Behinderung, räumliche steric hindrance. Fliess- inhibition of plastic deformation.

Behmilot fathometer, echo depth sounder; Behm sound ranging altimeter, sonic a.

beidäugig binocular.

beidrehen auf Anfluggrundlinie turn to or steer for center of approach sector.

Beikreis epicycle.

Beilage annex, schedule, supplement, enclosure.

Beilauf, Jute-cable filler, jute f. or compound.

beimengen, beimischen admix, add, intermix, intermingle.

Beintaste bone key.

Beisitzer aid, aide, assessor.

Beispiel, Ausführungs- exemplified embodiment (re:patent specifications).

beispiel sweise by way of example, exemplified.

beistimmen accede, assent or agree to, acquiesce in.

Beiwert constant, coefficient, parameter, factor, co-ordinate.

Beiwert, Schruck- absorptivity.

Beiwerte (Peilung) correction values (to compensate bearing errors).

beizen corrode, mordant, cauterize, etch, píckle.

Beiz-brichigkeit acid brittleness. -mittel mordant, corrosive, caustic. - tonung mordant toning.

bejahenden sinnes in the affirmative (sense).

Bekämpfung, Geräusch-, Lärm- noise abatement, n.suppression, combating of $n$.

Bekanntmachung über Erteilung eines Patentes announcement of grant or allowance of a patent.

Bekanntmachungsgebiihr lay-out fee, announcement $f$.

Beklagter defendant, respondent. Berufungs- appellee. Widercross- or counter-claim defendant. bekleiden clothe, cover, coat, line, face, box.

Bekleidung, Wand-, schall schluckende baffle blanket, sound-absorbent wall draping or lining, tormentor, gobo (consisting of portable wall covered with absorbent material, used in m.p. studios).

Bekräftigung, Wahrheits- affirmation (of testimony).

beladen, eine Oberfläche mit Wasserstoff, etc, activate, sensitize or charge a surface with $\mathrm{H}$, form an $\mathrm{H}$ skin.

Beladung, Vor- pre-charge, preliminary c. (of sorptive in sorbent).

Belag, Prioritäts- priority proof. Spiegel- mirror coating, reflecting film, silvering. Strom- current coverage or distribution (amperes per centimeter of periphery).

Belagverlust surface leakage loss (dielectric).

bel angen, gerichtl ich bring legal action against.

Bel astbarkeit, Hoch- high load or current-carrying capacity.

bel asten load, charge, weight, burden.

belasten, punktförmig lump-load.

belastet, stark heavily or highly loaded.

Belastung, aussetzende intermittent load. Querspulen- leak load.

Belastungsspitze peak load, crest 1., maximum 1 .

beledert leather covered, leatherlined.

Belegung coat (of condenser), plating, deposit, film, covering, density (of electrons in beam).

Belegung, magnetische magnetic induction, m.charge, m.field, seat of m.flux. Kondensator-, bewegliche rotor plate.

Beleuchter light electrician; scaffold or top light controller.

Beleuchtung illumination, illuminance, irradiance (in Iux or 
meter-candle units).

Beleuchtung, gerade direct illumination. -, senkrechte scaffold lighting, overhead 1., top 1. -, spaltfreie apertureless illumination.

Beleuchtủng, Dunkèlfeld- dark ground illumination. Effekteffect light, spot or fancy 1. Gegen- background illumination. Hellfeld- bright ground illumination. Hinter-Rembrandt illumination, half-back i. Rampenfootlights. Zweizweck- dualpurpose illumination.

Beleuchtungs-brücke traveling light crane. - linse condenser lens, illuminating 1. , bull's eye 1. -messer illumination meter, i.photometer, illuminometer. -mittel illuminant. -stärke illumination (ratio luminous flux to area of element of surface, in lux units, etc.). -stärkemesser illuminometer, illumination photometer. -trommel exposure drum, recording $d$. (on which film is exposed to modulated light.beam).

Belichtung, Logarithmus der log. exposure. Mehrfach- superimposing, multiple exposure. Nachpost-exposure. Ruhe- average lighting, steady 1., unmodulated or no-sound 1. (in sound picture recording). Vor-priming illumination (of a cell), preexposure, preliminary exposure, or 1.

Belichtungs-bereich, Unter- toe gradient of characteristic H. \& D. (Hurter \& Driffield) curve, toe range of underexposure. -fenster aperture (in a.plate), gate, photocell window. -kanal film track, f. channel (camera). -l ampe exciter lamp. -mass exposure level (of Decoutes). -messer exposure meter (e.g. with barrier-layer cell, used in sound recording, photography, etc.), cinophot (m.p. film pocket photometer); brightness meter, turbidity $m$. -rolle printing drum (in p.machine), sound recording drum; scanning point, translation p. (in sound film reproduction). -schieber exposure lid, e. shutter. - spielraum latitude or range of exposure, e.range. -stelle sound gate. -tabelle exposure time table. -teil, Unter- toe region of underexposure (film). -trommel exposure drum, recording d.. (sound film). -zahntrommel printing sprocket, main s.

Bel ieben discretion, (at) random, selection, at will, ad libitum. bel iebig haphazard, (at) random, arbitrary, any....at all, any... whatever, optional.

Bemessung dimensioning, proportioning, choosing; size, dimensions or proportions.

Benachteiligter aggrieved party. Benachteiligung der hohen Frequenzen de-accentuation, de-emphasizing, slighting or partial suppression of high frequencies, attenuation of treble or highpitched frequencies.

benetzbar wettable, capable of being wetted or moistened.

Benetzungswärme heat of wetting. Benutzung eines Patentes working or reduction to practice of a patent.

Beobachtungs-fehler error of observation. -fenster bezel, observation window, peep-hole. -rohr observation tube (in electron microscope).

Beratung advice, consultation, counsel.

berechenbar calculable, computable, appraisable.

Berechnung, Bildkraft-. halbklassische semi-classical image- 
forve calculation.

Beregnungsversuch rain test, wet $t$. (of insulator).

Bereich, Einfluss- sphere of influence, radius of action.

Entzerrungs- frequency range of equalization. Klangintensitätsdynamic range. Lautstärke-, der menschlichen Stimme human voice intensity range. Mitnahmerange of forced oscillation, entrainment range, coherence $r$. , pull-in-step range. Schwärzungsdensity range $(Y$ ) (say, from .3 to 3$)$. Sperr- suppression range. attenuation $r$., exclusion band.

Bereich-filter, Frequenz- bandpass filter. -melder, Wellenwave-band indicator. -schalter, Wellen- wave-band switch.

Bereinigung des Minimums (cf

Enttrübungsrahmen) zero clearing (df).

Berg, Energie- energy hill, e. barrier. Potential- potential barrier. Wellen-peak, crest or hump of a wave.

Berg-fett mountain tallow, ozocerite. fleisch mountain flesh (asbestos). -fluss colored quartz. -glimmer margarite. -harz mineral pitch. -kristall rock crystal. -öl petroleum. - pech mineral pitch, asphalt. -wachs ozocerite, mineral wax.

Bericht, zusammenfassender survey report, review, summarizing article.

berichtigen correct, amend, adjust, rectify, redress.

berichtigendes $\mathrm{Gl}$ as correcting lens.

berichtigtes Auge corrected eye.

Berichtigungs-gerät compensation means (cam, etc., in df). -walze adjustment drum (range finder). - wert correction value, c.factor.

beriesein cause to flow or trickle over, water, irrigate, douche, spray, scrub, wash, sprinkle.
Berieselungsapparat sprinkler.

Bernstein amber.

Berstdruck breaking limit, bursting 1., explosion or cracking 1 .

Berufshandkamera professional hand camera.

Berufung appeal, brief for appeal. -einlegen gegen eine Entscheidung bei höherer Instanz take an appeal from a decision to a higher court.

Berufungs-abteilung appeal division, a. department. - begründung arguments and grounds for appeal, confirmation of appeal. -beklagter appellee, defendant in appeals court, respondent in an appealed cause. - instanz, zuständig sein als to have appellate jurisdiction. -klage appeal. -kläger appellor, appealer, appellant. beruhen auf based on, due to, derived from, conditioned by, predicated upon.

beruhigen quiet, steady, stabilize, smooth, calm, kill (melt).

Beruhigung stabilizing (weight). Beruhigungs-flügel intermediate blade, anti-flicker b. -frequenz fusion frequency, critical noflicker frequency (m.p.). -kapazität smoothing capacitor -spule., Elsenkern- smoothing choke, filter c. -vorrichtung arc-silencer (suppresses hum and frying noise). -widerstand steadying resistance, ballasting $r$.

berïhrend, doppelt- bitangent. Berihrende tangent.

Berïhrungs-ebene tangential plane. - gefahr risk or hazard of electric shock. - linie tangent. -punkt contact point, touching p. p. of tangency or osculation. -schutz protection against electric shock hazard.

berihrungsicherer Stecker shockproof plug.

Beriuhrungs-spannungsschutz protection against electric shock 
hazard. -stelle place of contact, osculation point (between two curves).

besäumen hem, edge, border, shear, trim, square (sheets).

Beschallung radiate or cause sound or ultra-sound waves to act or impinge upon, acoustic irradiation.

Beschaltung wiring.

Bescheid, abschlägiger adverse action or decision, refusal, disallowing action of a Fatent office Examiner.

bescheinigen certify, attest.

beschichten coat (a film with emulsion), form a layer or a film, apply emulsion.

beschichteter Film, doppeltdouble-coated film, sandwich $f$.

beschicken load (phot.), charge, feed.

Beschicker, Funk-, automatischer automatic radio quandrantalerror compensator or corrector means, cam compensator.

Beschickung correction or compensation (in df work), radio compass calibration.

Beschickung auf gleiche Temperatur reduction to equal temperature.

Beschickungsaufnahme, Funk- calibration of radio directionfinder.

beschiessen bombard (with electrons, etc.).

Beschlag, 0xyd- coating or film of oxide.

Beschläge fittings (of metal), fixtures.

Beschlagnahme seizure, attachment, arrest, distraint, distress.

beschleunigen accelerate, expedite, hasten, speed up.

Beschleuniger, Resonanz- magnetic resonance accelerator, cyclotron, induction electron a., betatron, rheotron. Vielfach- cyclotron (type of) tube with two dees and spiral beam accelerating elec- trons or ions in stages.

Beschleunigung, Erd-, Fall- gravitational acceleration, acceleration due to gravity. Nach- postacceleration (by second lens) (in c. r. tube).

Beschleunigungs-anode gun anode. -einrichtung high-potential transformer and vacuum tube (for nuclear disintegration). -el ektrode accelerator electrode. - elektrode, Nach- second or additional gun anode or accelerator electrode, post-accelerator, af ter-accelerator (c. -r. t.). - gitter accelerator grid (c.-r. tube); screen-grid (of tetrode) -linse aus zwei Lochelektroden double aparture accelerator lens (c. -r. tube). -messer accelerometer. -spannung gun potential, accelerator p., beam voltage. -stufe target stage, accelerator s. (of multipler). -system gun system (c.-r. tube).

Beschluss action (of Fatent Office), decision, decree, order (of a court of law).

Beschneidegl as trimming glass, print trimmer.

Beschneidung clipping (loss of initial or final speech sounds).

Beschreibung specification (of a patent), description, outline, sketch, characterization.

-, weitgehende broad specification. -von Bildern auf Schirm picture tracing, re-creation or delineation on screen.

Beschriften making a record or sound track upon, impressing a film or blank with acoustic actions.

Beschriftung recording (on film). Beschussprobe shooting or bombardment test.

Beschwerde-abteilung appeals department, Board of Appeal. -einrede rejoinder (in an appeal). -gericht court of appeals. 
-schrift appellatory plaint, appeal papers. -verfahren appeal action or procedure.

beschweren load, weight, burden; complain.

Beschwerung impedance.

beschwören take an oath, sign an affidavit, declare under oath.

Beseitigung, Geräusch- (an Klebestellen) blooping elimination.

besetzen populate.

besetztes Band full band.

Besetzung cast ( $m$.p.)

Besetzungs-grad der Banden degree of filling of bands. -verhältnisse size of audience in a playhouse. zahl (der Elektronen in einem Niveau) extent to which level is populated by electrons. -zahl, Höchst- maximum number of electrons in shell.

Besitzurkunde title deed.

Besonnung insolation.

Bespannung baffle cloth.

besponnen covered, braided.

Besprechung mit Eisendrossel modulation by voice action with magnetic modulator.

Besprechungs-anlage sound pickup outfit. -mikrophon sound pickup microphone, s. collector. -punkt point where sound action is impressed. -raum sound studio.

besprochener Modulator voice-impressed or voice-actuated modulator.

Bespulung provide with winding, coil or loading means.

Besselfunktion erster Art Bessel function of the first kind.

beständig stable, constant, durable, permanent, steady, fast (colors), resistant, proof; continuous, invariable.

beständig, feuer - resistant to fire or heat, fireproof, refractory. fl ammen- flame-proof, flameresistant. frost- freeze-proof, resistant to frost. hitzestable or resistant to heat, heat-proof, thermostable, refractory. koch- resistant, stable or fast to boiling (dyes, etc.). lichtbogen- arc-proof, arcresistant. säure- stable to acid action, fast to a. wasserstable in or towards action of water.

Beständigkeit, Anlauf- tarnishproofness, resistance to $t$. Raum- volume stability. Temperatur- temperature stability, unaffected by temperature (changes).

Bestandteil constituent, ingredient, part, component. Fremd- foreign or extraneous matter or substance. Bestätigungsurteil confirmatory decision.

Besteck dead reckoning, ship's position; set of instruments or utensils. Funk- radio fix.

Besteckungskosten trimming expense (re : arc-lamps).

Bestimming determination, definition, clause, provision, stipulation, ascertainment.

Bestimmung, Mengen- quantitative determination, q. analysis.

Bestimmungs-gleichung defining equation. -grösse determinant. -stïck factor, parameter, operating datum.

Bestrahlung, Schall-, hahe high radiation (distribution) efficiency. Sonnen- insolation, irradiation by solar rays.

bestreichen wipe (over), sweep out, scan, explore (say, the picture area or screen with beam, pencil or spot); cover (a range), contact, stroke (a magnet).

bestreitbar disputable, contestable. bestreuen strew or sprinkle (say, powder) over.

Bestwert optimum value, optimal v., most favorable v.

Betätigungsspule working coil, tripping c., differential c.

Beteiligter party concerned, interested or involved. 
Betonung accentuation, emphasis, action or stimulus (brought upon).

Betrachtung, un.nittelbare direct viewing. Aufsichts-, Direktdirect or frontal viewing (of television pictures).

Betrachtungs-abstand viewing distance. -apparat viewing apparatus, kinetoscope. -richtung sight-iine, line or direction of regard. -tisch film viewing machine. zeichen reference letter, r. numeral, symbol.

betreiben run, work, operate, exploit.

betreiben mit Arbeitsstrom operate on open circuit. -mit Ruhestrom operate on closed circuit.

Betrieb, Schwer- heavy-duty operation or service.

Betrieblautsprecheranlage works or plant public address system (loudspeakers distributed throughout a factory, etc.).

betrieblich operational, operative, functional, working, concerning actual use in service.

Betriebsbuch station log.

betriebsfähig operable, workable, in (good) working order.

Betroffener aggrieved party. betupfen dab, dip, tip, spot, stipple.

Beugungs-aufnahme, Roentgen- Xray diffraction exposure or pattern. -bild, -figur diffraction pattern, picture or image. - fransen diffraction fringes. -gitter diffraction grating. -scheibchen diffraction disk. -winkel diffraction angle.

beurkunden authenticate, legalize, record.

Beutel-filter bag filter. -sieb bolting sieve, bolter.

Bevollmächtigter attorney, agent, proxy, assignee, mandatory, mandatary.

bevorzugte Kristallachsenrich- tung preferred crystallographic axis orientation. -orientierung non-random orientation, privileged 0 .

bevorzugte, nicht- Orientierung randomly oriented, with nonpreferential or non-privileged orientation.

Bevorzugung (von NF oder HF) accentuation or emphasizing (of $\mathrm{AF}$ or of $\mathrm{KF}^{\mathrm{H}}$ and de-accentuation or de-emphasizing of the other).

Bevorzugung, energetische energy preference.

bewaffnetes Auge aided eye.

bewähren prove useful or good, give satisfactory results, prove (itself).

bewegl iches, entfesseltes Mikrophon following microphone.

Bewegl ichkeit mobility, fluidity (being the reciprocal of dynamic viscosity).

Bewegung motion, movement, stir, travel, action (in film projection).

Bewegung, fortschreitende progressive motion. -, hin- und hergehende reciprocating movement, to-andfro m., rocking m., oscillating m., shuttling motion.-, rein sinusförmige plain harmonic or sine movement. Brown'sche Brownian movement. Kreis-, Kreisbahn- circular motion, gyration, movement in an orbit, orbital m. Leitbahn- transit path motion. Rollbahnkreis- cycloidal path motion. Rotations- rotational motion, rotary $m$.

Bewegungs-amplitude velocity amplitude. -eindruck sensation of motion, illusion of $\mathrm{m}$. -elektrizitätslehre electrokinetics. - empfänger velocity microphone; pickup or detector of motion. -energie kinetic energy, motional e. -gleichung equation of motion. -grösse kinetic or motional quantity or magnitude, impulse, 
momentum. -impedanz motional

impedance. -kraft motive force, m. power. -lehre mechanics (re: machines), kinetics (re: motion of bodies and forces acting. thereon), kinematics (re: motion in the abstract), dynamics (re: force action on bodies). -mikrophon velocity microphone, pressure-gradient $m$. -rohr mobility tube. -stufe stage of motion, phase of progress. -ibergang motional transient. -vorgang motional action, cinematographic action.

bewehrt, stahlband- steel-tape armored.

Bewehrung re-inforcement, armoring, fittings (hardware, of an insulator), sheathing.

Beweis, der Gültigkeit eines $\mathrm{Pa}$ tentes Abbruch tut evidence impeaching, or prejudicial to validity of, a patent.

Beweis, Neben- secondary proof, collateral evidence, circumstantial e.

Beweis-beschluss direction or order for evidence. -kraft conclusiveness, evidential value.

Beweislast onus of proof, burden of $p$.

Beweis-material (documentary) evidence, evidential material, testimony, proofs. als- vorlegen tender in evidence. -recht law of evidence. -stück exhibit, document, proof. -wert evidential value.

bewerten weight.

Bewertungsfilter weighting network. Bewetterung air conditioning.

Bewilligung grant, allowance, permission.

Bezeichnung marking, denotation, notation, sign, symbol, designation labeling (for identification), color coding (of conductors).

bezichtigen accuse of, charge with.
Bezieher, Piano- piano stringer. Beziehung, Wechsel- interrelation, correlation, reciprocal or mutual relationship.

beziehungsweise and/or; ... or ... or both; and; or; as the case may be, respectively, or rather.

Bezirksgerichtshof district court. bezogene Farben related colors.

Bezugsdämpfung volume loss, volume equivalent. Rückhör- side-tone reference equivalent.

Bezugs-ebene datum level, d. plane, reference 1., fiducial 1. -punkt reference point, fiducial p., datum p. -stromkreis reference circuit. -system reference system, standard or norm (used for comparison). -wert relative value. zeichen reference symbol (numeral or letter).

bezw. s. beziehungsweise

bezweifeln call in question, doubt. Bibl iothekar librarian.

Bidipentode duodiode tetrode with suppressor grid; duodiode pentode (with bifilar filament).

Biegekristall "bender" crystal.

biegen bend, flex, curve, warp; diffract; refract.

Biegemoment flexural moment, bending m., flexural torque.

Biege-probe, Schlag- shock, blow or impact bending test piece or specimen. -versuch, Dauerbending fatigue test. -wechselfestigkeit alternating bending strength.

biegsam flexible, pliant, pliable, bendable, ductile, supple. -machen ductilize (as a tungsten wire).

Biegungsschwingung flexural vibration.

Bienen-harz bee glue, propolis. -korblampe beehive neon lamp.

Bifilardraht bifilar wire, twisted pair. -wicklung bifilar winding, Ayrton-Ferry winding.

Bild, aufgerichtetes, aufrechtes 
erected image, erect i., rectified i. -, detailreiches contrasty picture (rich in details and contrasts). - flaches very soft picture, flat picture (lacking contrast). - , fl aues non-contrasty picture or image (presenting a limy condition or fuzziness, contrast of ten impaired by white tint permeating whole picture). -, hartes contrasty, crisp or harsh picture or image. -, hochzeiliges high-definition (television) image or picture.

-, l ichtschwaches low-luminosity picture, p. with low brightness. -, reelles, umgekehrtes real reversed image. -, ruhendes still, still picture, unanimated p., ordinary photograph. -, scharf eingestelltes sharp picture, sharply focused image. -, tönungsreiches picture with proper shading values and contrast.

-, umgekehrtes reversed or inverted image. -, unbewegtes still picture, unanimated p. -, unscharfes fuzzy picture, ghosty p. -, unverzeichnetes, -, unverzerrtes orthoscopic image, undistorted picture. -, weiches (zwi schen $\mathrm{fl}$ au und normal) weak or soft picture (between flat and normal), uncontrasty $p$.

Bild, Stehen des-(es) steadiness of image. -, mangelhaftes stehen jumping or unsteadiness of picture or image.

Bild, Aufspaltungs- splitting pattern (opt.). Ausserfokus- outof-focus picture. Beugungsdiffraction pattern. Durchsichts(cf Durchprojektion) transparent picture, glass transparency, diapositive, lantern slide; translux p. Durchstrahler- transmission image. Eigenstrahler - image obtained by self-emissive method (electron microscope). Einzelunit frame, individual picture (in pictorial sequence). Empfangsrecorded copy (facisimile).

Ersatz- equivalent circuit diagram. Feld- field pattern, f. configuration or map (made in electrolytic tank). Fenster-window transparency. Fern-television picture, televised p.; telephoto. Flugbahntrajectory diagram. Formelstructural formula. Funk- photoradio (transmission), radio picture, wired p., photo-radiogram, photo-telegram. Gegen- counterpart, antitype. Geister- ghost image. Halbton-half-tone or mezzo-tinto picture (in black and white, with grey shading values). Interferenz- interference figure. KI ang- sound pattern, acoustic p. Klatschenbeat picture. Komik- funny picture, "funnies," cartoon. Komacomatic image. Kontroll-monitoring picture (telev.). Koordinaten- pattern of co-ordination. Ladungs- charge pattern or image (re : iconoscope). Lauf-animated, moving or motion picture (in a sequence or series of pictures or pictorial actions). Licht- photoimage, photograph. Linien-, unaufgelöstes unresolved line pattern. Luft- aerial image (formed in space). Mehrfachmultiple image, double i., ghost i. Moment- instantaneous picture, snapshot. Nach- after-image; copy, imitation, replica. Neben- ghost image, multiple or double $i$. (telev.). Netzhaut- retinal image. Offnungs- aperture image. Panorama- panoramic view or picture, panning shot, "pan" or "pam" (studio slang). Raff- time-lapse picture, fast-motion p. Raumspace diagram; stereoscopic picture, plastic p. Rechen- nomogram. Reihen- sequence of pictorial actions or pictures, motion p. Schatten- silhouette. 
Schau- diagram, graph. Schwarzweiss- black and white (facsimile) picture or phototelegraphic transmission. Sende- subject or outgoing copy (in facsimile). Sinn- symbol. Spalt-, optisches slit image, optical slit. Spiegelmirror or speculum image, flare ghosts or spots (camera), double or reflected (ghost) i. Stand-; Steh- still (film) picture, nonanimated p., still. Stimmungskey picture, sentiment $p$. -, dunkies low-key picture. -,überhelles high-key picture. Sucherseeker or monitoring picture. Teil- (Farbenauszug) compound picture (color record). Tontonal pattern or spectrum. Tricktrick shot, t. picture. Ursprungsoriginal subject copy. Weitwinkel- wide-angle picture, "widescope" p. Zeitlupen-slow-motion picture. Zerr-distorted picture (made with anamorphotic lens). Zerstreuungs- image formed by divergent lens. Zerstreuungs-, des Auges blur circles of eye.

Bild-ablenkung frame or picture scan. -abtaster televisor, television scanning device. -abtasterröhre, doppelseitige two-sided mosaic pickup tube with separate image anode, iconoscope with twosided mosaic screen. -abtasterRöhre mit mechanischer $\mathrm{Blende}$ Farnsworth dissector of electron camera, Dieckmann magnetic scanner. -abtaststelle picture or film gate (m.p.). -abtastung frame scan, picture s. -anode barrier grid mosaic. -aufbau picture synthesis, build-up of picture (line by line). -auffänger, elektronischer electronic scanning device. -auflösung resolution, picture definition, scan. -aufnahme, Kofferapparat für box equipment, trunk unit. -aufnahme, Luft- (cf Luftbild) aerial photography, airplane picture. -aufnahmevorbereitung lining up for shooting pictures.

bildaufrichtendes Mikroskop imageerecting microscope.

Bildaufrichtung erection of image, rectification of $i$., inversion of i. (reversing in both axes simultaneously, right and left, top and bottom interchanged at the same time). -aufzeichnung picture re-creation, tracing or delineation. -ausleuchtung "flashing" of image. -ausschnitt frame (of camera); image area (on scanning disk). -beschreibung (auf Schirm) picture tracing, re-creation or delineation (on screen). -bühne aperture or film trap. - bühneneinstellung framing, racking (by framing device). - charakter key of a picture or image. -drehung image rotation. - einstell ampe framing lamp. -einstellung centering control, height c., horizontal c., framing (in telev., of ten with rack and pinion for focusing). -empfänger picture receiver, p. reconstructor, video receiver, telev. receiver, picture viewing tube, p. reproducing device. - empfangsstelle picture or facsimile (telegraphic) receiving station or office, recording point. -entwerfung formation of an image, imagery, imaging. -entzerrung, Licht- rectification of aerial photos.

Bilder-kasten-, Laternen- lantern slide box. -masken, Lanternenlantern masks.

Bild-erzeugung formation of an image, imagery, picture ( $\mathrm{re}-$ ) creation, p. tracing. - fänger video pickup camera, television c. -fangröhre, Sender-pickup tube, iconoscope, dissector tube (of Farnsworth) - fehler picture distortion, image defects. -feinheit detail, (degree 
of) definition of $p$.

Bildfeld frame, picture area, image field. -ebnung flattening of image field, elimination of curvature (opt.). -krümmung,-wöl bung curvature of image field. -zerleger picture scanner, p. dissector (Farnsworth), p. exploring means (telev.). -zerlegung image (field) definition.

Bild-fenster film gate, aperture or

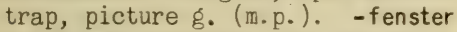
mit 2 Ausschnitten film gate with two frames. - fläche image area, picture field, image plane (opt.). - flachheit flat, very soft or noncontrasty condition or quality of picture. - flauheit flat, soft or non-contrasty quality of a picture (contrast impaired by white tint permeating $p_{.}$), limy condition, fuzziness. - flieger aerial photographer. -folge sequence or series of optic or pictorial actions (m.p.). -format picture format, frame size or format. - frequenz picture frequency, frame (repetition) f., repetition rate (telev.); video f., visual f. - frequenzverstärker amplifier for frame sawtooth time-base. -funk photo-radio, wired picture, facsimile transmission. -geber pickup camera. -gerät, Spaltphotographic soundhead (m.p.). - geräusch frame noise (m.p.). -greifer in-and-out-claw (of threading mechanism). -grösse size of picture, format of $p$. -grösse und Brennweite, Linse für veränderliche zooming lens for variable magnification and variable focus, "vario-objective". - güte (figure of) merit or quality of picture. -helligkeit, Mittel- average or background illumination, mean picture brightness. -helligkeitssignale video signals, shading-value signals (telev.). -hintergrund picture background. -höhe picture or image height (opt.), frame height (of a camera). - inhalt picture content, p. subjectmatter, "information". -kadre, Normal - standard picture frame. -kantenschärfe resolution on border. -kippeinsatz incipient frame flyback or beam return (telev.). -kipper frame timebase. -kippschwingung frame or picture time-base oscillation or impulse. -kraft (zwischen Metal) und Elektron) image force, image potential (between metal and electron). -kraftberechnung, halbklassi sche semi-classical image-force calculation. -linie focal line, image line.

bildlos amorphous, non-crystalline. Bild-meister director of photography, picture chief or director. -nachleuchten afterglow, phosphorescence of picture. -nachstellung framing, phasing, racking (m.p.; telev.), centering control (telev.). -nachstellung, $f$ al sche misframing, out-of-frame condition.

Bildner, Basen-base former, basifier, basifying agent. Korrosions- corrosive, corrodent, corroding or rusting agent. Schleifen- loop setter.

Bildnis portrait, picture, photograph.

Bildplastik plastic effect, relief e., stereoscopic e.; distortion of telev. image in form of multiple contours of diminishing intensity, plastic effect.

Bildpunkt image point, picture p., picture unit or elementary area, point image (telev.). -, dunkelster shadow. -, hellster highlight. -, nachl euchtender phosphorescent picture point. -signal video signal, electrical impulse resulting from elementary area. -verlagerung spot shift (causing plastic effect, in telev.). -verteiler picture scanner (at 
receiving end).

Bild-raster (cf Raster) scanning field, picture raster (telev.). -raum image space. -reihe sequence of pictorial actions, frames or pictures (m.p.); strip mosaic (aerial phot.). -röhre, El ektronen- electron image tube.

bildsames Gleiten plastic deformation.

Bildsamkeit plasticity, capability of being kneaded, fashioned, formed or molded (by pressure and/or heat application), formability, moldability, fictility.

Bild-schale image shell. -schallplatte sound and picture on disk. -schaltung feed or movement of frame (m.p.); frame ratcheting, frame timebase (telev.). - schärfe picture definition. - scherung shearing of an image. -schicht, Film- film emulsion or coat. -schirm projection screen, picture s. -schreibröhre televisor tube, viewing tube, picture reproducing tube. - schreibröhre, Projektions- projector-type television receiver. - schritt frame gage. - schwingungen picture or video impulses or signals. - seite image side (of a lens). - seitenverhältnis picture aspect ratio. - sender video transmitter; facsimile t. -signal video or picture signal. - spannung, Untergehen der swamping of video or picture signal. -speicherröhre image storing tube, charge or signal storage tube, normal iconoscope. - spei cherrobre, Bildwandl er- super-iconoscope. - sprung picture repetition frequency, picture cycle; break or shift of vision. -spurzeit Watkins factor, development $f$. -stelle picture scanner.

Bildstrich-einstellung phasing or framing of picture. -einstellung, $\mathrm{fal}$ sche misframing; out-of-frame condition. -geräusch frame line noise.

Bildstrom video current, picture c. -stürzung toppled condition of pictures (turned an angle in relation to one another).

Bildsynchronisierungs-impuls frame synchronizing impulse, f. sync. pulse, low sync i. -impulsverstärker frame synchronizing impulse amplifier, $f$. sync pulse a. - lïcke synchronizing gap (between end of field or frame traversal and beginning of next known also as underlap or interstitial period during which syne signal is introduced).

Bild-tanzen unsteadiness or jumping of picture. -teile verschiedener Gliederung picture portions of dissimilar nature, composition or make-up. -telegraphie picture telegraphy, wired or radio phototelegraphy, radio or wire transmission of pictures, facsimile, wire-photo. -transportrolle picture feed roller. -ibertragung (elektromechanische und el ektrochem.) picture, photo or facsimile transmission by electromechanical or electrochemical means, writing or copying telegraphy, by telautograph, telectrograph, Bartlane device, etc. -umkehrung image reversion, image inversion (when in two axes, i.e., interchange of left and right, and top and bottom of image); solarization (reversal of gradation sequence due to overexposure to light).

Bildungs-energie energy of formation. - wärme heat of formation, enthalpy.

Bild-unschärfe lack of picture definition. -verbreiterung image spread. -verfahren, Steh- lantern slide projection method. -verschiebung slow drift or hunting of image. -, seitl iche lateral shift of image. -verstärkung image intensification. -verstellungsknopf framing knob. -verstellungswelle framing shaft. 
-verwackel ung frame unsteadiness. - verzerrung image or picture distortion, jiggers (in facsimile). -vorführung, Raum- stereoscopic picture projection. -vorschubbewegung frame sweep scansion. -walze picture cylinder (in Bakewell app.). -wand projection screen. - wand, Ton- transoral screen (m.p). -wandler imąge converter, picture transformer, transducer (changes optic into electron image). - wandlerBildspeicherröhre super-iconoscope. - wandleuchtdichte brightness or luminous density of scræn. Bildwechsel picture cycle.

-, stetiger continuous, steady or non-intermittent feed or motion of film. - frequenz, -zahl frame or picture frequency, repetition frequency (telev.), number of frames per second. -zeit feeding time, picture cycle, moving period (m.p.).

bildweise Filmschaltung intermittent film feed, discontinuous film movement.

Bild-weite distance between screen and lens, image intercept.

- well enspannung video signal wave potential. -werfer picture projector. -wiederaufbau reconstruction (e. $\varepsilon_{\text {. }}$, of a facsimile picture). -winkel angle of image, a. of view. -wirkung, plastische (cf Bildplastik) illusion of depth (of picture), stereoscopic effect. -wölbung curvature of image, c. of field. -wurf picture projection. -wurfelektrode target electrode. - zahl frame or picture frequency. - zähler frame counter, $f$. indicator. -zeile picture line, p. strip (telev.). -zeil endurchlauf line traversal. -zerdehnung radial image distortion. -zerdrehung rotational, tangential or orientational distortion, twist of image. -zerleger picture scanner or explorer, dissector (of Farnsworth). -ziehen photographic travel ghost. -zittern unsteadiness of picture, jumping of $p$. -zusammensetzvorichtung picture reproduction means, p. recreator or delineator, p. scanner.

Billigkeitsverfahren equity suit.

Billigung approval, approbation, consent, assent, acquiescence.

Bimetall streifen bimetallic strip (consisting of two strips of dissimilar metals with different expansion coefficients), bimetallic trip or tripping gear (of a circuit breaker).

Binantenschachtel box with a twosegment electrometer system.

bindend, wasser - hydrophylic, waterabsorbent.

Bindeton ball clay.

Bindung binding, bond, union, association, affinity.

Bindung, Dreifach- triple bonding. Gas- gettering. Neben- secondary union or bond, second-order linkage.

Bindungs-energie (ElektronenKonzentration) binding or bond energy. -kraft (chemische) chemical linkage force. -stärke bonding strength.

Binode diode-triode (rectifier and $A F$ or $R F$ amplifier in one bulb, w1th joint cathode).

Binokularspule binocular (form of closed-field) coil.

Bitter'sche Streifen Bitter (magnetic powder) patterns, Bitter bands.

Bkw reactive power, $r$. voltampere, var.

Blähungsgrad expanding or swelling property, degree of inflation or imbibition.

Bl ank, Zahlen- figure blank, figure space,

Bl ankfilm film base. -,gel atinebeschichteter gelatine-coated film base. - unbeschichteter plain, uncoated film base.

$\mathrm{Bl}$ ank-seite cellulose face or side 
(of film). -verdrahtung bare wiring.

Blasdiuse blasting nozzle, gun, discharge $n$.

$\mathrm{Bl}$ ase, Dampf- steam-heated still; bubble of vapor or steam. Gl as(generally) bubble in glass, seed (when small), air bell (when of irregular shape). Guss- flaw or defect in a casting. Schweissarc crater.

blasen blast, blow, smelt (blast furnace), inject (steam).

$\mathrm{Bl}$ asen, Stahlkugel- steel shot blast.

$\mathrm{Bl}$ asenbildung blister formation, blistering (metal, phot., etc.); cavitation, occlusion of gases.

81 äser, $\mathrm{Fl}$ amm- blowing magnet.

$B I$ asinstrument wind instrument. $\mathrm{Blech}$ - brass instrument.

Blatt leaf, foil, sheet; page, folio; blade (of knife switch), lamination (of a magnetic core).

Blättchen lamina, lamella, small leaf, flake.

blättchenartig lamelliform, laminiform.

$\mathrm{Bl}$ attelektrometer leaf electroscope.

Blätter-bürste laminated brush. -kondensator foil condenser, tubular capacitor.

Blatt-feder leaf, plate or flat spring; reed, blade. -grïn chlorophyll. -kurve folium.

81 auauszug blue record.

bl auempfindlich blue sensitive.

81 aufilter blue filter, viewing filter (phot.).

Blech plate, sheet, foil, lamination of metal.

$\mathrm{Blechblasinstrument}$ brass wind instrument.

blecherner $\mathrm{Kl}$ ang tinny sound.

Blech-gestell chassis (of receiver set). -instrument brass instrument (mus.).

Bleicherde Fuller's earth.

Bleiglätte litharge.

Blende light-stop, diaphragm, aper- ture (stop), slit; shield (with scanning aperture, in Farnsworth dissector); shutter (e.g., iris diaphragm, focal-plane blind, disk with cut-out sector for exposing film or plate, in m.p. work).

Blende, Abdeck- mask, shutter, shutter mask. Abtast-(Farnsworth) scanning aperture, hole (of dissector tube of Farnsworth).

Anoden- anode diaphragm or partition (re: cathode-ray tube). Apertur- aperture stop, 2. diaphragm. Aufhell- reflecting screen. Aufrichtungs- erector stop. Austritts- exit slit. Blickfeld-field stop. Einhängeinset diaphragm. Einsatz- interchangeable diaphragm. Einschnïrungs- crossover aperture. Eintritts- input diaphragm or capacity disk (of klystron). Fächerfan fading shutter. Feld-field stop. Fenster-apertural stop, a. diaphragm. Gesichtsfeld-field stop. Halb- half stop, scale. $\mathrm{KI}$ ang- tone-shading means, tone control, tone switch, tonalizer. Kombinations- Goerz effect shutter. Kreis- iris stop or diaphragm. Licht- light stop, diaphragm, light shield (in sensitometer). Loch-stopping aperture, apertured partition, diaphragm, shield (in cathode-ray tube). Noiselessbias or noise-reduction stop, shutter vane or gate. 0kularm eyepiece diaphragm, eyepiece stop. Raster- scan hole, scan aperture (Farnsworth dissector), raster screen aperture. Reinton- bias or noise-reduction stop, shutter vane or gate. Schlitz- slit stop. Schluss- final diaphraóm.

Schnürspur- mask for making squeeze track. Sehfeld-field diaphragm, field stop. Sonnen- sunshade, black screen. Spalt- aperture stop, slit. Sperr- (blocking) light stop. Steuer- modulating 
electrode, modulation shield or grid (cathode-ray tube). Tontone-control means, tonalizer; fader. Trommel-drum with scan apertures or scan holes, scanning $d$. Vorhang- curtain fading shutter. Hechsel-stop disk with spiral slot cyclically cooperating with quadruple scanning disk holes, auxiliary rotary shutter disk with spiral slot. Zacken- triangular aperture (in film recording), vane with serrated or triangular edge. Zerleger-dissector aperture. Zonen- stop to diaphragm out a certain zone or area. Zusammensetz- picture receiver or $\mathrm{p}$. reproducer scanning hole.

Bl enden-ausschnitt aperture, slit. - ebene diaphragm plane. -l inse, Loch- aperture disk lens. -l och anode aperture (cathode-ray tube). -nachstellung phasing of shutter. -öffnung aperture of diaphragm or stop. -rohr screening tube. -scheibe horizontal edge (film recording); auxiliary rotary shutter disk with spiral slot (for quadruple scanning). - schirm gobo (for sound absorption, in studio). - träger diaphragm support. -trommel scanning drum.

$B l$ end-gl as moderating glass. - scheibe stop. - schirme gobos (for sound absorbing, m.p.).

Blendung glare, blinding, dazzle (of eye). -, akustische aural dazzling.

Bl endverfahren diaphragm method (for pencil modulation).

Blickfeld field of vision, range of v., field of view (opt.). -beidäugiges binocular field of view.

$31 \mathrm{ick}-\mathrm{fel}$ dblende field stop.

-linie line of vision, 1. of sight. -punkt point of view. -richtung sight-line, direction of sight, d. of regard. -winkel angle of view.
Bl ind-buchse dummy socket or jack. -komp onente wattless, idle, quadrature, reactive, or reactance component. -1 andeachse blind or instrument landing line or path. -1 andedienst beacon service (to assist in blind or instrument landing). -leistung reactive power, $r$. voltampere, var. -leitwert susceptance. -schwanz arrangement to make resistance alternately zero and infinite, in dipole feeder operation, stub. -stecker dummy plug. -stromaufnahme drawing or taking of wattless, idle, or reactive current. -verbrauchszähl er reactive voltamp. -hour meter, sine m., wattless component m., var-hour m. -vol tampere var (unit of reactive power). -widerstand, induktiver inductive reactance, positive $r$., inductance. -widerstand, $k$ ap azitiver capacitive reactance, negative $r$., condensance.

Blinker, Zeit- chronographic camera, time recording $c$.

Bl ink-l ampe flash lamp. - schaltung blinking arrangement, ratchet circuit scheme (in relaxation or sawtooth wave generator with $\mathrm{K}-\mathrm{C}$ time-base and neon lamp).

Blitz (Filni) statics (film).

Blitz, rückl aufender return lightning stroke.

Blitz, Kapsel- flashlight capsule. Kugel- flashbag; globular lightning, ball 1. Licht-pulse or flash of light, scintillation.

Blitz-anzeiger, Fern-keraunophone. -bahn, verschlungene tortuous path of lightning flash. -1 ampe flashlight, flashlamp. -licht flashlight; flash bulb, magnesium light.

Block, Gitter- grid condenser; staircase-like structure of echelon grating. Kopplungsfixed coupling capacitor.

Block-empfang party reception,

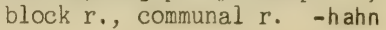


stopcock.

Blockierung, Gitter- negative biasing of grid, g. cut-off.

Block-kondensator fixed capacitor, blocking c., stopping c., insulating c. -kondensator, Wi ckeltubular capacitor, paper c.; Mansbridge condenser (of fixed value). -zeichnung schematic or diagrammatic illustration, block diagram.

Blut-farbemesser plethysmograph (monitors color changes, etc.), electroarteriograph. -körperchenzähler haemacytometer, blood counter.

Bock, Lager-pedestal of a bearing. Boden, gewölbter arched back (mus.). boden, Resonanz- sounding board.

Schrift- recording base.

Boden-antenne ground antenna, earth

a.; underground or buried antenna. - flugleiter ground control operator. -funkstelle ground directionfinding station; aeronautical $g$. radio s. -funkstellen-Rufzeichen ground station call sign or signal. -geschwindigkeit ground speed (of an airplane). -peilapparat ground station directionfinder. -peilstelle ground direction-finder station. -welle ground wave, direct $w$. (which hugs earth's curvature).

Bogen bow, arc, curve, curvature, bend, arch, clip.

Bogen, $\mathrm{Fl}$ ammen- flame arc, electric arc. Grad-graduated arc, bow, protractor. Hochstrom- heavycurrent arc, large-current arc. Kreis- arc of circle, circular arch. Pol- pole arc. Schwingungs- amplitude of oscillations. Voll-semi-circle rail (re: microscope).

Bogenabstand pitch or distance along an arc (of holes in Nipknow disk).

bogenbildend, nicht- non-arcing (metal).

Bogen-frosch bow-frog (string instrument). -gitter, Kreis- arcuate grid. -lampe mit breitwinklig gestellten Kohlen scissors-type arc-lamp. -l ampe, Freifallgravity-feed arc-lamp. -l ampe, Wolfram- tungsten arc-lamp, pointolite. -l ampenabzugsrohr vent stack of arc-lamp. -linie arc line, arcuate 1., curved 1. -mass radian measure. -rückschlag arc-back. -spektrum arc spectrum. Bohreinsatz, -eisen bit.

Bohrer, Zwick- twist drill. Bolzen, Knebel- toggle bolt. Öseneyebolt. Schlissel- clutch key.

Bolzen-mutter nut. -scheibe washer.

Bomben-sauerstoff tank oxygen. - flugbahn bomb trajectory. -richtgerät, -visier bombsight. Bord-ablenkung distortion of bearing on site (by re-radiation, etc., or by ship's field), aircraft error. -effekt board effect, ship field error (in df work), quadrantal error (due to structural parts, metal, etc.). bördeln bead over, edge, flange (an edge).

Bördelrand flanged or beaded edge. Bordsprechgerät, - telephon intercommunication telephone, interphone.

Bottich vat, vessel, tub, tun, tank. Bottich, Entwickler- developing tank.

Box, Abhör-monitoring box.

Bramme, Roh-slab, ingot.

Brand-messer pyrometer. -stein brick.

Bratpfannengeräusch frying noise. Bratsche viola, tenor violin. brauchbar usable, useful, serviceable, suitable.

Brause rose, douche.

brechbar frangible, fragile, breakable, brittle, refrangible, refractable.

brechend, doppelt- birefringent, birefractive. einfach- unirefringent.

brechende Brennfläche diacaustic (opt.). 
Brechkraft refractive power (opt.).

Brechung, Strahl-refraction or splitting up of rays.

Brechungs-brennfl äche diacaustic. - einheit diopter, dioptry (unit of focal length). -exponent refractive index, refractivity. -gesetz, Snellius'sches Snell's law of refraction. -indexbestimmung refractometry. -messer refractometer. -messer, Augendiascope, rétinoscope. -vermögen, Licht- optical refractive power, refrangibility (of light). -winkel angle of refraction, refracting a.

Brech-wert, Hauptpunkt- principal point refraction. -winkel angle of refraction, refracting a.

Breitbandempfänger broad-band receiver, wide-band $r$.

Breite width, breadth, latitude. -, nördl iche northern latitude.

Breite, Doppler- Doppler broadening.

Breiten-effekt latitude effect (cosmic rays). -höhe meridian altitude. -minute one minute of latitude. -wert azimuth value (of vision).

breitwinkliger Stellung der Kohlen, Bogenlampe mit scissors-type arclamp.

Bremsdynamometer Prony brake, brake dynamometer.

Bremse, Freilauf- free-wheeling brake, coaster b.

Bremselektrode reflecting electrode, retarding-field e. (in positivegrid tube).

bremsen brake, retard, check (motion), decelerate.

Bremsfeld reflecting field, retarding $\mathrm{f}$. (in electron-oscillation tube). -audion positivegrid detector, electron-oscillation detector or rectifier (negative anode associated with leak resistance and condenser).

- el ektrode reflecting electrode, retarding-field e. (in positive- grid tube). -generator negative transconductance generator or oscillator, positive-grid or retarding-field o. -röhre retardation valve, Barkhausen-Kurz retarding-field tube. - schaltung retarding-field or positive-grid circuit scheme (operating with grid maintained at a higher potential than plate), BarkhausenKurz circuit organization, oscillating-electron or electronoscillation scheme with reflecting electrode.

Brems-gitter suppressor or cathode grid (in a pentode). -kreis negative-anode or negative-plate circuit. -moment (Zähler) brake or retarding torque (of a meter, etc.). - spannung negative-anode potential (in electron-oscillation tube). - strahlen rays caused by particle retardation.

Bremsung der Elektronen reflection (in Barkhausen-Kurz tube); deflection (in magnetron); deceleration or retardation of electrons.

Brems-vermögen stopping power (of photographic emulsion); braking or retarding power. -wirkung der negativen Rauml adung repelling action of electron charge, negative space charge. -zylinder, Relais mit dashpot relay.

Brennebene, hintere back focal plane. -, vordere front focal plane.

brennen, dicht vitrify (cer.). Brenner, Fischschwanz- fishtail burner, batswing b. Schlitzbatswing burner, slit b. Schneidcutting torch. Zirkon- zirconium burner, z. filament.

Brennfläche caustic surface (opt.). -, brechende diacaustic. -, reflektierende, -, rückstrahl ende catacaustic.

Brenn-fleck (focused) spot. -glas burning glass, convex lens. - I inie focal line, image 1. 
-punkt focus, focal point. -punkt, vorderer first focal point. - schwindung shrinkage in firing (cer.). - spannung constant \$low potential (is slightly lower than firing or striking p.); normal running p. (of glow tube, etc.). -spiegel reflector sheet. -strahlhärtung flame hardening. -weite focal distance, $f$. length, convergence $d$. - weite, hintere back focal length. -weite und Bildgrösse, Linse für veränderliche zooming lens for variable focus and variable masnification, "vario-objective", zoom lens.

Brennweitenmesser focometer. brennweitige Linse, kurz- shortfocus lens. I ang- long-focus lens. Brett, Misch-mixing panel, monitoring p. objektiv-lens panel, objective board. Schall- baffle (board); sounding b. Verstärkeramplifier rack, a. panel.

Brillanz (akustische Wiedergabe) "bounce" (in sound reproduction).

Brille, Zweistärken- bifocal spectacles.

Brillengläser, durchbogene meniscus spectacle glasses. -, punktuell abbildende point-focal glasses.

Brisanz shattering power.

Bristolkarton Bristol board.

bröckelig brittle, friable, crumbly, fragile.

brodeln boiling noise.

8rom-öldruckpapier bromoil printing paper. -silberpositiv positive in silver bromide. $-z$ ahl bromine number.

Brown'sche Bewegung Brownian movement.

Brown Zungentelephon Brown reedtype telephone receiver.

Bruch break, breakage, fracture, rupture, failure, fraction, fragment; scrap, waste.

Bruch, Längen- longitudinal fracture. Vertrauens- breach of confidence.
Bruch-dehnung total extension or elongation (to fracture), breaking tension, stretch (of paper). -festigkeit ultimate strength.

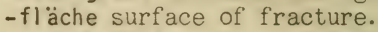
brüchıg brittle, short, friable, fragile, cracky.

Brüchigkeit, Beiz- acid brittleness. Bruch-methode, Ketten- method of infinite continued fractions (math.). -platten bursting disks. -punkt brittle point (of glass). - spannung ultimate stress, breaking s. -stücke fission products, fragments, fracture or rupture pieces. -teil fraction, fractional part, aliquot part. -versuch, Rot- hot breaking test.

Brücke bridge, platform; rider (piano).

Brücke, Beleuchtungs- traveling light crane. Kapazitäts- capacitance bridge, Wien b. Querleitungstransconductance bridge. Thermokreuz- thermo-couple or thermojunction, thermal cross. Verhältnisarme der ratio arms of bridge. Widerstands- resistance bridge, Wheatstone $b$.

Brücken-ausgleichstrom bridge balancing current. -gleichgewicht bridge balance. -H-Schaltung, Entzerrungskette mit $\mathrm{H}$-type attenuator network (balanced). -Scheitel bridge apex. -TSchaltung, Entzerrungskette mit T-type attenuation network (unbalanced). -übertrager differential transformer. -verhältnisarme ratio arms of bridge. Brumm-kasten bass keys. - spannung hum potential, ripple potential. -spur, -streifen buzz track; hum (broad bands on screen of c.-r. tube). -tastenfeder spring for bass keys (accordion). - ton, Netz- motor boating (of a.c.), a.c. pickup.

Brust-fernsprecher head and chest set. -mikrophon breastplate microphone. 
Buch, Dreh- shooting script.

Bücherei Iibrary.

Buchgold leaf gold.

Buchse sleeve, bush, socket.

Buchse, Blind- dummy socket, dummy jack.

Büchse, Fernrohr- telescope tube, barrel. Kompass- compass bowl, c. kettle. Lager- bearing bush.

Buchse, Rad- wheel bushing. Schaltjack.

Büchse, Widerstands- resistance box.

Bucht, Verstärker- amplifier rack, repeater rack or bay. Wählerbay of selectors, selector rack, switch frame.

Büffelleder buffalo skin.

Bug bow.

Bïgel clamp, clip, strap, staple, bow; yoke (of a magnet); loop (of a magnetron); harness (of headphones); saddle bracket (microscope); bout (string instrument).

Bügel, Aufhänge- loop hanger. Kopfhörer- harness, headband or strap (of headphone or headset). Stirn- head rest (of magnifier).

Bügel haken, $\mathrm{Fl}$ aschenzug- block tackle.

Bugwelle (eines Geschosses) nose wave (of a projectile).

Bühne, Bild- apenture or film trap Bühnen-aufsteller set dresser. -einstellung, Bild-framing.
Bund, Anschlag- stop collar.

Buindel beam, pencil, bunch, cone (of rays); wave packets.

Bündel, Achter-eight-wire core, quadruple twins. Strahlenbundle, bunch, beam or pencil of rays or radiations.

Bündel ling bunching (of electrons in Klystron), focusing, beaming, concentration, insuring beam or directive antenna effect.

bündig succinct, brief, concise, summarized, short, valid; flush, snug.

Bunker (für Verstärker) bay or rack (of amplifiers).

Bunsenphotometer Bunsen photometer, grease-spot p.

bunte Farben hue colors, chromatic c.

Gürde burden, load.

Bürste, Blätter- laminated brush. Kontakt- contact-brush, wiper.

Bürsten-entl adung brush discharge (between glow discharge and sparking). -maschine, Zwi schenmetadyne, metadynamo.

Büschel tuft, bunch, cluster, brush (discharge).

Bussole, Sinus- sine galvanometer.

Bütte tub, vat, tank, chest.

Büttenrandpapier deckle edged paper. Butzenscheibenlinse bull's eye lens. B Verstärker class B amplifier.

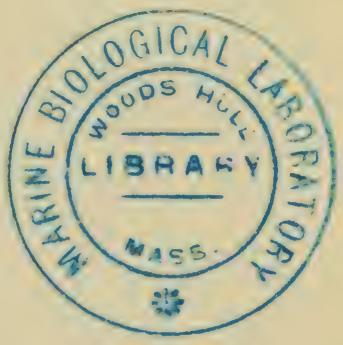


c

C Verstärker class C amplifier.

CW Schaltung capacity-resistance circuit scheme.

Callierfaktor (cf Kontraststeigerung) Callier factor (of print contrast).

cartesische Fläche Cartesian surface.

caesiumbedeckt caesiated.

Centradian centraradian (onehundredth radian).

Charakter, Bild-key of a picture.

Charakteristik s.a. Diagramm \& Kurve.

Charakteristik, fallende drooping characteristic, falling $c$. -, quadratische square-law characteristic. Anlauf- transfer characteristic. Herz-cardioid characteristic or space pattern. Maschinen- speed-load characteristic. Nachglüh- persistence characteristic. Richtungs- directive or directional characteristic or diagram, space pattern.

Chargengang heat run.

chargieren charge, feed, load.

Chiffrierung encipherment, coding. Chor assembly of strings (mus.). chörig, drei- trichord.

chromatischer Fehler chromatic aberration. - Schaum chromatic soft focus or bleeding.

Chromatisierung, Ortho- or thochromatic processing. conphas in-phase (condition), in phase coincidence.

$\operatorname{cotg} \alpha \cot \alpha$, cotangent $\alpha$.

Coulombabstossung Coulombian repulsion.

C Verstärker class C amplifier. Cycloidhöhe (Magnetron) cycloidal height.

Cyclotron (cf Resonanzbeschleuniger) cyclotron, magnetic resonance accelerator using two dees and a spiral beam. 
D Wert quadrantal error due to airplane fuselage, etc.

Dach-kapazität top (loading) capacity. -kohle upper coal.

d'Al embert'sche Well lengleichung d'Alembertian wave equation.

Damm margin or narrow strip of material between adjacent tracks or grooves of phonograph dislis, barrier, land.

Dammarharz Dammar resin or gum. dämmen stop up, dam.up, curb.

Dämmerungs-effekt night effect. -schalter twilight switch.

Dämmung, Schall- sound reduction or attenuation, $s$. deadening or absorption, silencing (of noise).

Dämmzahl, Schall- sound transmission characteristic.

Dampf, direkter live steam.

-, indirekter latent steam.

Dampf-auflösung volatilization. -bl ase bubble of vapor or steam; steam-heated still.

dampfdicht steam tight, vapor tight. Dampf-dichtemesser dasymeter.

- dichtung steam packing. -druckmesser manometer, steam gage, tensimeter. -druckzünder vaporpressure igniter.

dampfecht fast to steam.

dämpfen attenuate, damp (out), dissipate, absorb (sound in air, etc.); muffle, deaden, silence, quench, die away, decay, mute (mus. instnument).

dämpfend, schall- sound absorbing, s. attenuating, s. damping or deadening.

Dåmpfer mute (in string instrument), damper (of piano). -, rotierender rotary stabilizer (film feed).

Licht- silk, light softener (m.p.).
Schall- silencer, muffler, sound absorber, gobo (sound absorbing sheet) (m.p.).

Dämpferwicklung amortisseur winding, damping $w$.

Dampf-hülle steam jacket; vaporous envelope or sheath, vapor shroud. -hunger steam avidity. -kolben steam piston.

Dampflampe, Quecksilber-,mit Heizkathode phanotron, tungar tube (with argon filling). -, Quecksilber, mit steverelektrode mercury-vapor tube with control grid (of thyratron, ignitron, etc., type).

Dampf-messer manometer, steam gage, tensimeter. -schale evaporating dish or basin.

Dämpfung attenuation, damping, loss (of energy), dissipation, drop; impedance. -, kilometrische attenuation per kilometer of line. -, mechanische mechanical resistance, m. impedance.

Dämpfung, Ableitungs- leakance loss. Bezugs- volume loss, v. equivalent. Fehler- balance attenuation, return loss. Flïssigkeit's- liquid or fluid damping or cushioning, dashpot action. Kenn- iterative attenuation constant. Klirrcorrection of non-linear harmonic distortion. Leitungs- line loss, 1. dissipation, 1. attenuation. Nebensprech- crosstalk transmission equivalent, c. attenuation. Nutz- damping resulting in useful radiation or signal power, effective transmission equivalent. ชl- oil damping means (re:sound recording vibrators); oil dashpot. Pseudo- loss of selectivity by 
parallel internal resistance.

Rückfluss- structural return loss. Rückhörbezugs- side-tone reference equivalent. Schall- sound absorption, s. attenuation, s. deadening. Schwingungs- damping of oscillations or vibrations; shock absorption. Sperr- stop band attenuation. Strahlungsradiation damping, $r$. resistance. übersprech- crosstalk transmission equivalent. Verlust- damping resulting in outright loss or dissipation of energy (by ohmic resistance). Vierpol- image attenuation constant. Wirk- (cf Wirkwiderstand) transducer loss. Dämp fungs-ausgleicher equalizing network, compensating network. - dekrement, scheinbares logarithmisches equivalent logarithmic decrement. - entzerrer equalizing network, compensating n. -Frequenzkurve attenuation-f requency curve. - gang frequency response of attenuation, attenuationfrequepcy characteristic. -glied attenuation network or mesh. -klotz damping plate. -konstante, Wellen- wave attenuation constant (real part of transfer c). -mass unit of attenuation (in decibels or in nepers); attenuation standard (denotes also real component of image transfer constant). -messer transmission (efficiency) measuring device, decremeter. -mittel, Schall- sound absorbing, attenuating, insulating, damping or deadening means or material. - periode quench period. -pol damping pole (point of infinite attenuation or suppression); point of attenuation. -reduktion regeneration, gain, de-attenuation. - spule damping coil, amortisseur c. -verminderung regeneration, gain, de-attenuation. -verzerrung frequency distortion; tone d. -vorrichtung damper, dashpot. -wert decrement, value of damping or of attenuation. -wicklung damping or amortisseur winding. -widerstand non-reactive, effective, dissipative or ohmic resistance; attenuator. -widerstand, Kenn- iterative impedance, characteristic i. (for uniform line). -winkel phase angle difference, loss a. -wirkung quench action. Dampfwassertopf steam trap.

Darbietung entertainment (radio, acoustic or visual), proǵram (material), offering.

Darmsaite gut string.

darren kiln, dry, kiln-dry; liquate (copper).

darstellen represent, prepare, produce, make, manufacture, exhibit, display, describe.

Darsteller actor, character player.

Darstellung, vektorielle vectorial representation. Spektral- representation by spectra.

Darstell ungsweise method of preparation; manner of representation, style.

darunterliegend subjacent, underlying.

Dauer, Leucht- illuminated period, light p., time of luminescence or fluorescence.

Dauer-biegeversuch bending fatigue test. -bruch fatigue fracture.

Dauerfestigkeit fatigue strengtl. Kerb- notch fatigue strength. Korrosions- corrosion fatigue strength.

dauerhaft durable, lasting, permanent, stable, fast.

Dauer-haftigkeit durability, keeping quality, stability, permanence, endurance. -hal tbarkeit fatigue endurance. -hal tbarkeit, Verdrehungs- endurance under torsion stress. -prozess d-process (in phosphorescence decay). -registrierung long-period recording. -riss fatigue crack. -schl agprobe continuous or fatigue 
shock or impact test (specimen). -schlagwerk continuous or fatigue impact test machine. -standfestigkeit (long-time) creep strength, creep resistance, static fatigue stress, creep limit. - standfestigkeitspruffung (longtime) creep test. - standversuch creep test, fatigue test. -strich long dash, permanent note ( $N$ and A interlocking, in df). -strom persistent current. -ton permanent note, tone, sound or signal. -zustand steady state.

Daumen mechanical operating part resemblung a thumb, as a cam.

Daumen, Hebe- cam, tappet, finger, disk with lobe. Steuer- sequence switch cam.

Daumen-mutter thumb nut. - rad sprocket (wheel). - scheibe cam (wheel), disk with lobes.

Däuml ing cam knob.

dazwi schentreten intervene.

Debye-Scherrer-Ring D.-S. ring or circle, powder pattern, Hull $r$. Decke belly (of string instrument). deckeln provide with lid or cover. Deckenprojektor ceiling projector (to determine cloud height).

Deck-fähigkeitsmesser (Farbe) sryptometer (measures concealing power of paint). - $\mathrm{gl}$ as einer Linse anterior surface of a lens. - gläschen cover glass (of microscope). -kraft density (phot.), opacity, hiding, concealing or covering power (of paints).

-kraftmesser cryptometer. -mittel covering material, resist, protective coat (in etching work), "ground". -peilung alignment bearing. -plättchen cover slides.

Deckung registry, cover, covering, coincidence, congruence (geom.); intensity or coverage (of a negative, opt.), stereoscopic contact (in range finder).

deformierte Fläche deformed surface, figured s. dehnbar machen ductilize (wire). Dehnbarkeitsmesser ductilimeter, dilatometer, extensometer.

Dehner expander (in volume-range control).

Dehnung, Bruch- total extension or elongation, breaking tension, stretch (paper). Rück-damping capacity (re:metals). Zeithigh-speed camera shooting; time scale increase.

Dehnungs-di agramm, Spannungsstress-strain diagram, stressdeformation d. -fuge expansion joint. -grenze yield point. -messer dilatometer, extensometer, ductilimeter. -modulus modulus of extension, (often) Young's modulus of elasticity. - schlitz slip joint (Martin furnace). -welle dilational wave. -zahl coefficient of expansion or extension.

dekadi sche Logarithmen common logarithms.

dekonzentriert de-focused, debunched, dispersed, out of focus. Dekoration set (m.p.).

Dekrement, Dämp fungs-, scheinbares logarithmi sches equivalent logarithmic decrement.

Dekrementmesser decremeter.

Delta-Verfahren "toe" negative method (phot.).

Demodulation demodulation, detection, rectification.

demontieren demount, disassemble, dismantle, take apart, knock down.

Densitometer, Kugel-, integrierendes integrating spherical densitometer.

Densogramm, Mikro- microdensitometer record.

derb solid, compact, dense, firm, rough, strong.

Derbheit ruggedness, robustness, solidity, strength.

Desakkomodation, zeitl iche magnetic ageing (irreversible decrease of permeability with time). 
detailreiches Bild picture rich in detail and contrasts, contrasty $p$.

Detektor, linearer linear detector or rectifier. quadratischer square-law detector or rectifier. quantitativ arbeitender integrating detector. Fest- contact detector, fixed crystal d. (with non-adjustable cat whisker).

hebel- contact detector or crystal d. with cat whisker adjustable by lever action. Pinsel- contact or crystal detector with cat whisker. wellen-cymoscope, wave or oscillation detector, coherer.

Determinante, Gleichungs-, für Leitungen determinant for line equations.

detonierender Verstärker heterodyne amplifier.

Deutl ichkeit, Konsonanten- consonant articulation. Sprach-articulation, intelligibility of voice, clarity of speech audition. Yokal- vowel articulation.

Deuton deuteron.

Deutsche Industrie-hormen (DIN)

German industrial standards.

Deutung explanation, interpretation, evaluation.

Dewargefäss Dewar vessel.

Dexel adze (cutting tool).

Dezentrierung decentration, decentering, eccentricity.

Dezimalstelle decimal place.

Dezimeterwelle micro wave (for $w$. below $20 \mathrm{~cm}$ ), decimeter wave.

Diagonalbeziehungen diagonal relationships (in periodic system).

Diagramm see al so Charakteristik, Kurve.

Diagramm, Doppelkreis- figure "g" diagram or space pattern. Kreiscircle diagram, circular loci. Orts- locus or circle diagram. Pulver- powder pattern. Rückstrahl- back reflection photogram. Strahlungs- radiation diagram or characteristic, space or $r$. pattern. Zeiger- vector diagram. Zustands- phase diagram.
Dialogpegel dialog level.

Diaphonie (cf libersprechen) crosstalk; babble (when from a great number of disturbing channels).

Di aphragma diaphragm, stop, baffle, mask, membrane, shutter. -anode apertured anode, a. stop.

Diapositiv, Strich- line diapositive, line transparency.

Diapositiv-projektion lantern slide projection. - sender film transmitter.

Diaprojektion lantern slide projection.

dichroitischer Schleier dichroic or dichromatic fog, silver or red fog.

dicht dense, close, tight, firm, compact, thick, impervious, sealed, hermetic.

dicht, zu overdense.

dicht brennen vitrify (ceramics).

dicht, dampf- steam or vapor tight. licht- opaque, (sealed) lighttight or light-proof, not transmitting 1. luft-hermetic(ally sealed), airtight, excluding air. vakuum- vacuum-tight, sealed to insure vacuum.

Dichte, Einsatz- number of stations (satisfactorily operable) in a given band and their closeness. Leucht- luminous brightness, intrinsic brilliance, intensity or brightness of illumination or light in terms of stilb (sb) or apostilb (asb) units. Lichtstromlumirous flux density (in lumens). Massen- mass density.

Dichte-gitter density grid, (charge) d. control or regulating $g$. -messer, Flïssigkeits- liquid density meter, densimeter, hydrometer, areometer. -messer, Gasdasymeter. -messung, photogr. densitometry, photodensitometry, photographic density measurement. -modulation density modulation, charge-density $m$.

dichten make tight, lute, pack, caulk; condense, compact, densify. 
dichtend, selbst- self-sealing, tightening or packing.

Dichte-reguliergitter density grid, (charge) density regulator or control g. -schrift variabledensity sound track. -skala density scale. -steuerung, Ladungs- charge-density modulation.

dichtgepackt close-packed (cryst.).

Dichtung, Labyrinth-, Turbinenturbine labyrinth seal, t. gland. Vakuum- vacuum plumbing, sealing or packing.

Dichtungs-ring gasket ring. -stoff luting, calking, packing, plumbing material (vacuum work).

Dicken-messer thickness gage, calipers, gage c. -schwingungen thickness vibrations (cryst.). -taster, Faden- calipers with jaws to measure thread diameter capacitively.

Dickte thickness.

dielektrische Verschiebung dielectric displacemént.

Dielektrizitätskonstante specific inductive capacity, dieletric constant, permittivity.

Dienstvorschriften service or working rules or regulations.

Dietrich skeleton key, picklock.

Differenzfrequenz difference frequency (re:beat, heterodyning).

Differenzialrelais differential or discriminating relay.

differenzierbar differentiable.

Differenzton difference tone, differential $t$.

diffundieren diffuse.

Diffusionswärme diffusion heat (gas).

Diktiermaschine dictograph.

D I N (Deutsche Industrie Normalien) German industrial standards.

Dinaston Dinas clay.

Ding object. -punkt object point. dingseitig on the object side. Diodengleichrichter, Zwe ifachduodiode rectifier.
Diopter alidade, diopter.

Dioptrik dioptrics (now covering refraction branch of optics).

Dipole dipoles, doublets.

Cipolzeile dipole array.

direktanzeigender Peiler directreading direction-finder.

direkt(es) Abspielen direct playback. - (er) Dampf live steam.

-(er) Gegenbeweis rébutting testimony or evidence. -(e) Kopplung direct coupling (by resistance, inductance or capacity).

Direktionskraft directing force, directive f., versorial $f$.

Direktor, Atelier- studio manager.

Direktverstärker straight-ahead amplifier.

Diskant treble. -posaune soprano trombone.

Diskontinuität, El ektronenflussshot effect.

Dispersion, Drehungs- rotatory dispersion.

dissoziierbar dissociable.

Distanz, Kern- inter-nuclear distance.

Distanz-halter spacer, separator, spacing means. - isol ator spacing insulator, stand-off $i$.

-relais distance relay. -stück spacer, separator piece.

DK (dielektrische Konstante) dielectric constant.

Dochtkohle cored carbon.

Döckchen stud.

docken wind (yarn, etc.).

Dockenfeder poppet spring.

Dolle nick.

Dompfaffenorgel bird organ.

Donnereffekt peculiar effect in

sound recording when white and ultra-violet light is used; (nonlinear) photographic distortion in variable-area recording known as "donner" or thunder effect.

Doppelaufnahme duplex shot. doppelbeschichteter Film doublecoated film stock, sandwich $f$. Doppel-blickzielfernrohr double 
direct sighting telescope.

-brechung double refraction, birefringence. -diode duodiode, double diode.

doppeleindeutig one to one.

Doppel-ferngl as field glass, bino-

culars. -fernrohr binocular

telescope. -gängeraufnahme dual-

role work. -gestaltung dimorphism.

- gitterröhre bigrid valve, twin-

grid tube, four-electrode tube

(tetrode, pliodynatron, etc.).

- gleichrichter full-wave recti-

fier.

doppelhörig binaural.

Doppel-impul sverfahren double-

impulse method. -kegel antenne

cage antenna, double-cone antenna.

-klang echoed sound, hearing

sound twice (by electric and by

air transmission). -knoten

binode. -konusantenne cage

antenna, double-cone atenna.

-kreisdiagram figure-8 diagram,

tangent circle pattern. - I inse

double lens, doublet. - lötungen

(Dewar-Gefäss) double seals

(Dewar vessel).

doppeln duplicate, copy.

Doppeln, Film- film duplication.

Doppel-pedalharfe double-action

harp. -platten twin plates or crystals, composite crystal, biquartz. -plattenkristall bimorph crystal, divided-plate crystal, biquartz. -quarzkeil double quartz wedge. ringoberflächenspannungsmesser twinring type surface tensiometer. -röhre twin-tube, two-system $t$., dual amplification t., reflex tube. -röhrenschaltung tandem tube (push-pull) circuit organization.

doppel sägeförmige Spannung double sawtooth potential.

Doppel-schichtfilm sandwich film, double-coated f. -schichtl inse double-layer lens. - schnürspur, Gleichtaktsprossen- variable- density double-squeeze sound track.

doppelseitige Bildabtaströhre twosided mosaic pickup tube (with separate image anode). -Mosaikröhre image multiplier iconoscope. doppelsinnig ambiguous, equivocal, uncertain, double meaning, "double entendre".

Doppelsperrkl inke ' (cf Klinke) double dog, d. pawls, d. detent.

doppelspuriger Tonstreifen doubleedged variable-width sound track or record.

Doppelsteverröhre power pentode in which cathode grid is connected with control g. rather than with cathode.

Doppel-T-Anker shuttle armature, H-type $a$.

doppelt-berührend bitangent. - brechend birefringent, birefractive. -hochrund convexoconvex. -hohl concavo-concave.

Doppel-umkehrprisma inversion prism (reverses or éxchanges in both axes of image). - V-Antenne double-V-anterna, doublet. -weggleichrichter full-wave rectifier. - wegschaltung double or full-wave rectifier circuit organization. -wendel system biplane filament system. -zackenschrift mit Abdeckung bilateral track recording with double-vane shutter; double-edged variablewidth track, duplex v.-area $t$. - zackenschrift, Viel-multiple double-edged variable-width track. -zackenspur, Abdeck- duplex variable-arca track. -zünder time and percussion fuse, combination f. -zungenpfeife double-tongued flute. -zweipol röhre duodiode. -zweipol-Ereipol röhre duodiodetriode tube. -zweipol-Vierpolröhre duodiode-tetrode tube. Dopplerbreite Doppler broadening (causing D. width). Dorn mandrel, spine, pin, tongue. 
Dorn, Lehr- inner cylinder or plug gage.

Dose, Abspiel-electric phonograph soundbox. Abtast-electric pickup or soundbox. Wiedergabesound pickup head.

Dosen-barometer aneroid barometer. - fernhörer watchcase telephone receiver.

dosiert in measured quantities, quantitatively regulated, dosed (pharmacology, etc.).

Dosierung, Dosis dosage (re:rays, etc.).

Dosismeter, Roentgen- X-ray dosimeter.

Doubeln duplicating.

Dptr. diopter, dioptry.

Drachen, Karton- box kite.

Draht, durchgehender through-wire. -, schwingender filament swing (magnetron, etc.). -, spannungsführender live wire, charged w., "hot" w. -, stromführender currentcarrying wire, live $w$.

Draht-,Durchgangs- through-wire. Fasson- profile wire, shaped or fashioned w. Gitterwickel- grid coil wire. Haar- Wollaston wire, capillary w. Klingel-bell wire, annunciator w. Litzen- stranded wire, Litz wire.

Draht-gaze wire gauze, w. mesh, w. cloth. -gebilde, Luft- aerial or radiating array. -gewebe wire gauze, w. mesh, w. cloth. -kreuzung wire crossing, transposition of (line) wires, crossover. -netz, Erd- ground mat. -schirm wire screen. -schleife, Funkbeschickungs- QE (quadrantal error) correcting, clearing or compensatirg loop. -wal zwerk wire rod mill. -wellentelegraphie wired radio, w. wave telegraphy.

Drall twist, spiral thread, torsional force, moment of momentum (phys.), spin (of electrons), rifling (gun).

drallfrei non-kinking, non-twisting; unrifled.

Drallwinkel angle of twist (rifling). Dreh-achse axis of rotation, center of r., pivot, fulcrum, hinge.

- anker pivoted armature (re:loudspeaker, etc.). -ausgleich torque compensation.

drehbar gelagerter Hebel pivoted lever; fulcrumed 1. (when with two arms).

Dreh-buch shooting script, screen play. -dose crank box.

drehen, plan- face planing. übersuper-speed operation (m.p.). unter- low-speed operation.

Dreh-federwage torsion balance. -fehler, Nord- northerly turning error. -feld rotating field, rotary $f$. - feldschwingungen rotating field oscillations. - funkfeuer rotating radio beacon or radio range, omnidirectional b, - impuls, Spin- spin angular momentum, spin moment, moment of momentum of electron. -knopf rotary (tuning) knob. -lichtsignal revolving beacon. -ling crank.

Drehmoment torque, moment of rotation, twisting or torsional moment, angular momentum (re: atomics). Anzugs- starting torque. -ausgleich torque compensation.

Dreh-platte turntable (re:phonograph). -polarisation rotatory polarization, optical rotation. -punkt fulcrum, pivot, center of rotation, c. of motion. -resonanz torsional oscillation resonance. -scharnier swivel hinge. -scheibe dial (re:automatic tuning, etc.); turntable (part of m.p. studio equipment). -schwingver such oscillation or alternating torsion test. -späne turnings, borings, drillings. -spiegelung rotatory reflection, combined rotation and reflection (cryst.). -spule, mit einem Lager versehene unipivotal 
moving coil. -stabfeder torsion bar spring. -tisch rotating stage, rotator (opt.).

Drehung rotation, revolution, turn, angular motion, gyration, twist, torsion. -, magneto-optische magneto-optical rotation, magnetic r., Faraday effect.

-,optische optical rotation, rotatory polarization. -,optischen, Umkehrung oder Wechsel der mutarotation.

Drehung, Eild-image rotation.

Links- levogyration, left-handed polarization, counter-clockwise rotation. Phasen- phase rotation, p. shift. Rechts-dextrorotation, right-handed polarization, clockwise rotation.

Drehungsdispersion rotatory dispersion.

drehungsfrei irrotational, rotationfree.

Drehungs-kristal। "twister" crystal. -schwingung torsional vibration.

- vermögen rotatory power.

-vermögens, Jimkehrung oder Wechsel des mutarotation. -winkel angle of rotation; a. of deflection.

Dreh-wage torsion balance. -wähler, Heb- vertical and rotary selector. -zapfen pivot, trunnion, journal. (of an axle):

Dreibein tripod.

dreichöriges Piano trichord piano, three-stringed $p$.

Dreidimensional itat tridimensionality, three-dimensionality; stereoscopic property (opt.).

dreieckig triangular, threecornered.

Dreieckrechner triangulator (for triǵonometric calculations).

Dreiecks-keil triangular wedge. -lehre trigonometry. -rechnung, wind-air-drift triangulation. oreiecksternschalter delta-star switch.

Oreier-alphabet three-unit code. -stösse triple collisons, threefold $c$.

Dreifachbindung triple bond(ing). dreifach(e) Oberharmonische triple harmonic. - (er) streifenfilter tricolor banded filter.

dreifachrechtwinkl ig trirectangular. Dreifach-röhre three-unit or threepurpose tube (three independent valves in one bulb). -stecker three-pin plug, triplug.

Dreifarben-l euchtdichten tricolor KD (reflection density) values. -trennung three-color separation. -verfatiren tricolor method. dreifarbig trichromatic, threecolored.

Dreifingerregel right-hand rule, thumb rule, Fleming's $r$.

dreiflächig three-faced, trihedral. Dreifussring tripod ring.

dreigliedrig trinomial (math.). -(e) Farbgleichung three-color equation, three-stimulus e.

Drei-kreisempfänger three-circuit receiver. -punktschaltung Hartley circuit scheme (terminals including center tap of coil connected with three electrodes of tube), potentiometer circuit scheme. -schenkeldrossel threelegged reactor or choke coil. drei-schenkelig three-legged, threelimbed. - seitig three-sided, trilateral, triangular. -stellig three-place.

Dreistoffsysten ternary system. dreiteilig three-part, tripartite. -(er) Stecker three-point plug, three-way $p$.

Drei-teilung trisection, triequipartition. -wegehahn threeway tap or cock. -wegeschalter three-way switch. -wegverbindung three-way connection. -wicklungstransformator hybrid transformer. dreizählig trigonal (cryst.); threefold, triple, ternary.

Drillachse rotator (quantum theory). drillen drill, turn, trist. 
Drillung torsion couple, twisting c.

Drillungs-kristall "twister" crystal. -modulus torsion modulus, shear m., rigidity m. -schwingungen torsional vibrations.

dritter Ordnung, Kurve cubic curve. Drittwicklung tertiary winding (of a transformer).

Drossel choke coil, choke, inductor, reactance coil, impedance $c$. kicking c., tickler c. (regeneration).

Drossel, eisenfreie air-core coil, air-c. choke. Abflachungssmoothing coil, s. choke. Dreischenkel - three-legged reactor coil or choke coil. Eisen- ironcored choke coil. Entzerrungsanti-resonant coil. Kipp- cutoff choke (regulating battery charging). Klang- tone-contról choke. Hetz-hum-bucking coil, hum eliminator c. Stever- magnetic modulator, modulator choke, Heising modulator. Tast-, Telephonie- magnetic modulator coil. Tonbeseitigungs- hum eliminator choke, h. bucking coil.

Drossel-absperrklappe throttle clack valve. -kette low-pass filter. -satz, Niederfrequenzlow-pass filter. -spule, Funkair-gap reactor. -wirkung attenuation (filter, etc.), damping, choking or throttling action.

Druck pressure, head; compression; impression, print(ing).

Druck, Gegen- counter-pressure, back-pressure, reaction. Hochhigh pressure; relief print. Licht- photo-mechanical printing, photographic p.; radiometer vane effect. Lichtfarben- photochemical color printing. Rückreaction pressure. Schallsound or acoustic pressure. Schweiss- welding upset. Strahlungs- radiation pressure. Tief- low pressure; intaglio printing process. Um- reprint. Vorfirst impression, proof.

druckabhängig pressure-responsive, pressure dependent, being a function of $p$.

Druck-amplitude pressure amplitude. -eigenspannung residual compressive stress. -empfang typescript reception, printing $r$. -empfänger (cf Bewegungsempfänger) pressure (-responsive) microphone. -entlastung pressure relief.

Drucker, Fern- teleprinter, teletype, stock ticker.

Druck-erhöher intensifier, pressure step-up means. -feder compression spring. -fenster pressure plate or gate (m.p.). - filter pressure filter. -gasschalter gas blast switch, (cross) airblast s., autopneumatic circuit breaker.

- gebiet compressed or pressure region or area. - und Gefrierpunktsmesser manocryometer. -gleichgewicht, osmotisches isosmotic equilibrium. -kanmerlautsprecher pressure-chamber loudspeaker, pneumatic 1. (without diaphragm). -knopfempfänger push-button or press-button receiver set, receiver with automatic tuning means. -kolben pressure flask, p. piston, piston ram plunger; digestion flask. -körper indentor (rubber). -kufe pressure pau (m.p.). -lack, Gummi-gum printing varnish. -luftantrieb pneumatic drive. -luftschalter (cross) airblast switch, autopneumatic circuit breaker.

Druckmesser pressure gage, piezometer, liquid manometer, fluid m. -, osmoti ischer osmometer. Dampfsteam gage, manometer, tensimeter. -\& Gefrierounktsmesser manocryometer.

Druck-minderer pressure reducing or relief valve. -platte platen (of a printing press). -probe, Kugel- 
ball pressure test, Brinell ball impression or indentation $t$. -rahmen film trap (projection work). -raster printer's screen. -regler pressure regulator, barostat, piezostat. -rolle presser or pad roll (film feed). - schiene pressure shoes (film proj.). -steg pressure or harmonic bar (piano). -verbreiterung pressure broadening (re: gas spectrum). -verschiebung pressure shift (re: spectral lines). -wage piston manometer. -windkessel compressed airtank, blast pressure tank. -Zug-Verstärker push-pull amplifier. -zünder pressure igniter.

D-Spannung Dee voltage (cyclotron). Duanten duants (cyclotron), dees. dubbeln, dubben duplicate.

Dublette doublet.

Dubletten-Aufspaltung doublet

separation (spectrum). duff dead, dull.

Dulling dulling (suppression of AF by dubbing or scoring, m.p.). dumpf all bottom, tubby, boomi; dull dunkel, Modulierung auf modulation to dark condition.

Dunkel-adaptation, - anpassung dark adaptation, scotopia. -entladung dark discharge, dark current. -feldbeleuchtung dark ground illumination. -kaniner dark room, camera obscura. -methode observation in darkness. -pause dark period, cut-off or obscuring period (m.p.). -raum, Aston'scher Aston dark space, primary d.s. -raum, Faraday'scher Faraday dark space. -, KathodenCrookes dark space, cathode d.s. -stösse background counts (in Ceiger-Müller tube). -strahlung obscure radiation, heat $r$. (from a light source), invisible (actinic) $r$. beyond the violet. -strom dark current (of photoelectric cell flowing in absence of illumination). -wertsteuerung adjustment to value of darkness. -widerstand dark resistance.

-zeit dark interval or period. dunkles Stimnungsbild low-key picture.

duinn thin, tenuous, slender, dilute, feeble, rare, weak.

diinnwandig thin-walled. Dunst, Fernen- distant fog. Dunst-abzug hood (for fumes).

-abzugsrohr vent pipe. -kreis atmosphere. -messer atmometer. -rohr ventilating pipe.

Dup-emulsion dupe emulsion. -negativ duplicating negative. -positivfilm duplicating positive stock, master $\mathrm{p}$.

Dur (Tonart) major (musical pitch or mode).

durchbelichten fully expose or ir radiate.

Durchbiegung deflection, flexure, bending, coflexure, sag, dip (of line wire).

Durchblickvorrichtung dioptric device.

durchbohren perforate, bore, drill, punch, pierce, penetrate. durchbohrt perforated, punctured, punched, bored, apertured. durchbrochen apertured, perforated. Durchbruch burst (discharge); fracture, break. -stoss burst pulse. durchdringen pervade, permeate, penetrate.

durchdringend (inter) penetrating (e.g., two crystal lattices), penetrant.

Durchdringungs-frequenz penetration frequency, critical f. -vermögen penetrating power, penetrativeness. Durcheinander confusion, mixup, bedlam.

durchfallen fall or drop through (a potential).

durchfallendes Licht transmitted light, transcident 1. Durch-flussmesser flow meter. -flutung circulation (magnetic 
potential); flow (of RF, etc.).

Durchführung wall entrance, bushing, duct, lead-in. Innenraum- indoor bushing.

Durchführungs-i sol ator, Wand- wall lead-in or bushing insulator. -stromwandler bushing-type current transformer.

Durchgangs-draht through-wire. - frequenz penetration frequency. -richtung forward or low-resistance direction (rectifier).

-widerstand volume resistance (exclusive of surface $r_{.}$), insulation $r$.

durchgeben filter, strain.

durchgehend(er) Draht through-wire. -(es) Licht transmitted light, transcident 1.

Durchgriff grid controllance, $g$. penetration factor, grid transparency, transgrid action, gain reciprocal $1: \mu$

Durchgrifflinse (cf Lochblendenlinse) aperture lens (through which field or anode may act or draw electrons).

Durchhang sag, slack. -verfahren "toe" method of recording (underexposure of film, Tobis method using tœe region of Hurter \& Driffield curve).

durchkopieren print through.

Durchlass clearance, lumen. -breite band-width, pass band. durchlassen filter, strain, transmit (light, etc.), let through or pass.

Durchlass-frequenz pass wave. -grad (von Wasser) transmission factor (of water), radiation through water.

durchlässig, halb- semi-permeable, semi-transparent.

Durchlässigkeit transmittancy, transmissivity, transmitting property or power (for rays, electrons, etc.), transparency, perviousness, penetrability, permeability (magnetic).
Durchlässigkeit für ultrarote Strahlen diathermancy, property of being diathermanous.

Durchlässigkeit, Filter- transmittance (ratio of filtered to unfiltered current). Kopietransmission of picture. Lichtlight transmittance, transmittancy (spec., of unit thickness). Luftair penetrability, a. perviousness. Ultrarot- infrared transmittance or transmittancy, diathermancy.

Durchlass-kurve selectivity curve (of a direction-finding receiver). -richtung forward direction, lowresistance d. (of a rectifier). -widerstand forward resistance (of rectifier).

Durchlauf running through or passage (of a film). Bildzeilenline traversal.

durchlaufen pass through, traverse, cover, sweep (a line).

Durchlaufkopiermaschine continuous film printer.

durchleuchtend transparent, diaphanous.

Durchleuchtung trans-illumination, transmission of light; radioscopy . -mit Roentgenstr. (diagnostic) radioscopy, radiography.

Durchleuchtungsappar at fluoroscope, cryptoscope.

durchlöchert punctured, perforated, pierced, punched, apertured, having holes or pits. - (er) Bügel beehive shelf (gas drying).

Durchmesserwicklung full-pitch winding.

Durchmesserwicklung des Senders operating transmitter at high modulation percentage.

durchmustern survey, review, catalog or count (stars).

durchnässen wet (thoroughly), soak, steep.

Durch-perlungselektrode bubblingtype electrode. -projektion back projection, diaprojection. 
-projektionswand translucent screen.

durchruhren stir or agitate (thoroughly, to the bottom).

Durchsatz throughput (furnace work). durchscheinend translucent, partially transparent, diaphanous, shining through.

Durchschlag breakdown, rupture, puncture (of insulation); punch, drift; filter, strainer; carbon copy. Wärme- breakdown due to thermal instability.

Durchschlagen (von Nachbarsender) interference by neighboring station in volume, neighboring stations "come through" or "break through" or swamp signals (by image frequency effect).

Durchschlagen der Gleichstromkomponente (über Synchronisierzeichen zum Schirm) d.c. component reaches screen by way of synchronizing pulses.

Durchschl ags-festigkeit, Stossimpulse-voltage breakdown strength. -geschwindigkeit propagation speed (of fluid in pipes); rate of insulation breakdown.

Durchschlagspannung breakdown, puncture, rupture, disruptive or flashover potential.

Durchschmelzung burnout, melting, fusion, blowout.

durchschneiden traverse, cut across, cross, intersect.

Durchschnittsverminderung reduction of area (of a test piece pulled apart, resulting in a "waist"). durchschwingen swing through; operating condition in which wave is not interrupted or cut of $f$. durchseihen strain, filter, percolate.

durchsetzen thread (a film); permeate, traverse, pervade, mix, intersperse, thread (turns by magnetic flux).

durchsichtig transparent, dia- phanous, translucent (when only partially transparent), clear, lucid, pellucid, limpid.

Durchsichts-betrachtung rear viewing (of pictures). -bild (cf Durchprojektion) diapositive, lantern slide, glass transparency (picture thrown from rear on frosted glass pane, etc.); translux picture. -dichte transparent density (m.p.). -schirm (Speicherprojektionsröhre) transparent screen (of a storage-type television tube). -sucher direct view finder, eye level finder). durchstarten eines Flugzeuges "open up" and repeat landing procedure (in 22 landing method). durchstechen pierce, stab, cut through.

Durcnsteuern operating inside straight portion of characteristic close to point where overload begins; grid excitation sufficient to swing plate current from zero to saturation point (re: amplifier). Load a tube to full capacity (re:transmitter).

Durchstossverfahren descent (or piercing) through clouds, penetration method (in airplane landing).

Durchstrahl ung transmission of light or of radiations, penetration by rays, irradiation.

Durchstrahl ungs-methode transmission method. -ibermiskroskop transmission-type electron or ultramicroscope. -verfahren transmission method (using thin foil, in ultra-microscopy work).

durchstreichen flow, pass, circulate or travel through; cross out, cancel.

durchstreichende Linie trajectory. Durchziehgl as slide.

Düse nozzle, tip (welding), tuyere, twyer.

Düse, $\mathrm{Bl}$ as- blasting or discharge nozzle, gun. Fang- mixing nozzle 
Düse, Schwe iss-

(steam jet). Schweiss- welding tip. Venturi-throat or constricted piece of Venturi meter, nozzle-like reducer.

Düsenkopf nozzle tip.

D-Wert quadrantal error due to airplane fuselage, etc.

Dynamik contrast or sound volume ratio between lowest and highest intensities of notes or passages (in music, etc.); practical volume compression and expansion; speech or signal energy volume range. Also: Eatio between undistorted maximum signal strength and noise, noise-signal ratio.

Dynamik-einebnung compression. -einregelung adjustment of contrast (by compandor action). -entzerrer dynamic expander.

- I inie volume-range or contrast characteristic. - presser compressor (in volume-range control). -regler volume- or dynamic-range control means, compandor (comprising compressor and expander means), contrast regulator. -steigerung (cf. Wuchtsteigerung) dynamic-range expansion. -verzerrer dynamic compressor.

dynami scher Lautsprecher, permanentdynamic loudspeaker with permanent magnet.

Dynamometer, Brems- brake dynamometer, Prony brake.

Dynatronsummer dynatron ( $A F$ ) OScillator. 
Ebentild image, likeness, similarity. Ebene optical flat (opt.); plane, plain.

Ebene, in einer - mit coplanar with, flush with.

ebene Welle plane wave.

Ebene, Bezugs- datum level, d. plane, reference 1., fiducial 1. Blenden- diaphragm plane. Brenn-, hintere back focal plane. Brenn-, vordere front focal plane. Einfalls- plane of incidence. Einstell- focal plane. Einstellungsfocusing plane. Flucht- vanishing plane. Halb- semi-plane, half-p., H-p. Haupt-principal plane, p. surface. Hyper-hyperplane. Kristall- crystal plane, c. face. Netz- lattice plane, plane-family of a crystal.

Ebene, Polarisations- plane of polarization. Drehuna derpolarization error.

Ebene, Reflexions- plane of reflection. Schmiegungs- osculating plane. Spalt- cleavage plane, c. face. Symmetrie- plane of symmetry, crystallographic p. Teilungs- plane of division.

Ebenenabstand, Haupt- principal plane distance.

Ebenheit planarity, flatness, smoothness, evenness.

Ebenmass symmetry.

Ebnung, Bildfeld-flattening of image field.

Ebnungslinse, Feld- field flattener. Echelettegitter echelette grating (opt.).

Echo, stufenförmiges echelon-formation of multiple echoes.

Echo, Hörer- listener echo. Mehrfach- multiple echo, reverbera- tion, flutter e. Schein- pseudo echo. Vielfach-multiple echo, flutter e. Wellen- radio (signal or wave) echo, wave reflection (involving one or more hops to ionosphere and back).

Echo-lot sonic altimeter, soundranging a., echo depth sounder, fathometer. -lotung echo sounding, echo or reflection altitude or distance measurement and ranging; radar or radio locator, sonar work (predicated upon echo principle). -paare paired echoes. - stromdämpfung echo current attenuation; active return loss. -unterdrïckung echo suppression, e. killing, damping of reverberations (by sound absorbing material). - weite echo area, effective scattering cross-section of radar target. -werk swell organ. -wirkung echo effect.

echt, dampf-fast to steam. kochfast to boiling. licht- fast to light, non-fading.

echtes, absolutes Gehör genuine absolute pitch.

echte Längenmessung direct measurement of length.

Eck, Seiten-lateral summit Würfelcorner of a cube, cubic summit.

Ecke corner, angle, edge (of a plane angle), summit (of a crystal), quoin.

Ecke, Mittel- lateral summit.

Eckfrequenz corner frequency, sharp cut-off frequency (filter). eckig angular, cornered, angled. edel noble, non-base, rare, precious, rich, vital, inert (gas). Edel-erde rare earth. -gas rare or noble gas, inert gas (in atmos- 


\section{Edelgasglüh}

67

phere). -gas-Glühkathodengleichrichter tungar (argon-tungsten) rectifier. -metall noble metal, precious m., rare m. -stahl refined or superior alloy steel. Effekt, kreismagnetischer gyromagnetic anomaly.

Effekt, Bord- board effect, ship field error (in df work), quadrantal error (due to structural parts, metal, etc.). Funkellow-frequency variation of local emission density. Richt- rectification effect; directional. $e$. Effektbeleuchtung effect lighting, spot or fancy 1.

Effektivwert effective value, rms (root-mean-square) value, virtual $v$.

Effekt-kohle salted carbon, carbon for flame arc. -lampe studio light (ordinary hand or table lamp, m.p.). -szenen scenics, light effects, etc.

egal isieren equalize, level, regulate (piano).

$\mathrm{Ei}$, Antennen- $\mathrm{fish}$ (of trailing antenna).

Eichant gage or calibration of fice, standards bureau.

Eichelröhre acorn tube.

eichen ǵage, measure, standardize, calibrate, test, stamp, adjust (weights), log.

Eich-katzenanker squirrel-cage armature. -kreis, Arbeits- working reference circuit. -mass gage, standardized measure. -reizkurven (typical) visibility curves.

eidesstattliche Erklärung affirmation in lieu of oath, statutory declaration.

eidliche Versicherung affidavit, sworn statement, testimony or declaration.

eigen inherent, self, "eigen", own, individual, characteristic, proper, specific, special; exact, pre-

\section{Eikonal}

cise, delicate.

Eigen-abstossung der Elektronenwolke repelling action of electron charge, negative space c. -dichte, Energie- proper energy density. -drehimpuls eigen or characteristic angular momentum. -energie "eigen" energy, characteristic e. -funktion eigen function, characteristic f. -geräusch inherent film noise. -geschwindigkeit indicated air speed (of an airplane). - gewicht specific gravity, own or dead weight, proper w. -heit singularity, particularity, peculiarity. -kapazität selfcapacitance. -licht des Auges eye's own light, self-l. or intrinsic 1. of retina (associated with photopsy). - peilung board aircraft direction-finding, taking bearings with homing device on board, on two or more stations.

eigenschwingungsfrei aperiodic, deadbeat.

Eigen-spannung residual stress, inherent or internal tension or strain, -spannung, Druck- residual compressive stress. -störung internal trouble. -strahlerUbbermikroskop self-emission electron microscope, s. -illuminating e.m. (based on electrons arising on object surface).

eigentümil ich peculiar, characteristic, inherent, specific.

Eigen-tumsrecht ownership, proprietary or property right, proprietorship. -verständigung inter-phone communication (airplane), inter-vehicular c. -wärme specific, body or animal heat. -wert "eigen" value, "eigenwert", characteristic, representative, typical or inherent value, characteristic number. -zeit proper time. -zustand "eigen" state.

Eikonal, Winkel-angle eiconal (opt). 
einachsig uni-axial.

einäugiges Sehen monocular vision, non-stereoscopic vision.

einäschern incinerate, calcine, burn to ash.

ein-atomar, -atomig monatomic.

Einausschalter of $\mathrm{f}-\mathrm{on}$ switch.

Einbandübertragung single-sideband transmission (with vestigial s. $-\mathrm{b} \cdot$ ).

Einbau inner player (piano).

einbetten imbed, place in matrix.

Einbettungsmaterial matrix or embedding material.

Einbeulungsprüfung bulging test.

Einbiegung curvature, inflection, flexure.

einblasen blow in or into, insufflate, inject.

Einbläser punctiform pits produced by atmospheric pressure in glass.

Einblenden der Mikrophone fade-in and mixing of microphones.

Einblick-linse eyepiece lens. -rohr eyepiece tube.

Einbrennzeit heating-up period.

Einbruch (Kurven, etc.) break, breaking in.

Einbruchsmelder burglar alarm.

Einbuchtung bay, bight, indentation, niche.

eindampfen thicken by evaporation, boil down, inspissate.

eindeutig unique, plain, clear, unequivocal, single-valued.

-, doppelt- one to one.

eindeutige Peilseite absolute direction. -Richtungsanzeige unidirectional direction-finding (with sense finding or sensing).

Eindeutigkeitsprinzip ( $P$ auli) exclusion or equivalence principle.

eindicken inspissate, thicken, concentrate, boil down, condense.

eindrehen recess, tap (a screwthread), indent.

Eindringungsfähigkeit penetrativeness.

Eindruck, Mitten- binaural balance (in sound locating).
Eindrucksgleichheit equality of sensation or impression.

Eindruckverfahren ball test, Brinell hardness t. method.

einebnen level, smooth, flatten (curve or wave).

Einebnung, Dynamik- compression.

Einergang-Kurbel und -welle singlepicture crank and shaft (m.p.).

Einfachantenne plain antenna.

einfach-brechend singly refracting, unirefringent. -frei univariant, monovariant, having one degree of freedom. -wirkend single-acting.

Einfachzacken-schrift mit Abdeckung unilateral variable-area track with single-vane shutter. -spur unilateral variable-area track. einfädeln thread (up) film. einfädig unifilar.

Einfall, schiefer oder streifender grazing, oblique or glancing incidence. Glanz- grazing incidence (at a glancing angle).

Einfallen incidence, snap-in motion; collapsing, implosion (reversed explosion).

einfallendes Licht incident light.

Einfallgeschwindigkeit bombardment velocity.

Einfalls-ebene plane of incidence. -winkel angle of incidence. -winkel, Haupt- angle of principal incidence.

einfaltige Gruppe one-dimensional group.

Einfang, Neutronen- neutron capture. einfangen capture, captivate, trap (electrons).

einfarbiges Licht monochrome, monochromatic light, light of one color.

Einfärbung dying, tinting, staining, inking, imbibition.

einfassen border, hem, surround something, enclose, case, bind, trap, trim, set.

Einfassleisten binding strips.

Einflug-richtung direction of approach (in airplane landing). 
-schneise approach track. -sektor approach sector. -sender boundary marker beacon.

Einflugzeichen boundary marker signal, come-in s. Haupt- main marker, inner marker or main entrance signal nearest airport boundary (in airplane landing). Vorouter marker (beacon) signal, fore $m$. beacon.

Einflussbereich sphere of influence, radius of action.

einförmig uniform, monotonous.

Einfräsung milled slot or recess.

einfrieren freeze up, congeal.

einfügen insert, interpose, intercalate.

einführen thread (up) film, introduce, insert.

Einführungs-isolator lead-in or feed-through insulator, bushing. -isolator mit Vergusskammer pothead insulator. -verlust insertion loss.

Eingabe amendment, petition (in Fatent Office practice).

eingängig single-thread (mech.).

Eingangs-bedämpfung input resistance, $i$. damping. -klemme input terminal. -leltwert input admittance. -pupille entrance pupil. -schlitz entrance slit (spectr.). -wirkleitwert input conductance, $i$. active admittance.

eingebaute Antenne built-in antenna.

eingefahrene Antenne retracted or drawn-in antenna, reeled-in a.

eingefroren latent, dormant.

eingehen (in eine Gleichung) enter into, occur, or appear in, an equation.

eingekittete Linse cemented lens.

eingeschwungener Zustand steadystate (of current, oscillation, etc.).

eingestrahl tes Licht incident light, exciting 1 .

eingestrichene Oktave one-stroked octave.

Eingiessung pouring-in, infusion, transfusion.

Eingreifen (der Greifer) in-and-out movement (of claws), engagement of $c$.

Eingrenzung von Fehlern tracking down seat or source of interference or trouble, localize trouble.

Eingriff engagement, mesh, gearing.

Einhal tsbefehl injunction, interdict.

Einhängeblende inset diaphragm.

einhängen des Hörers hang up or replace telephone receiver, clear the line.

Einheit (constructional or structural) unit, integral part; unity.

Einheit, Ladungs-, positive elek. unit positive charge. Massenunit of mass. Mehrheit von-en array of units, plurality or multiplicity of u. Oberflächenunit of area. Radiumemanationscurie unit of radon. Raumwinkelsteradian, spheradian. $X-$, (Roentgenstrahl-) wave-length unit of X-rays, etc., Siegbahn unit, $X u, X$ unit.

Einheiten und Formeln, Ausschuss für (A E F) Committee on Units and Formulae, Standards Committee.

Einheits-frequenz standard frequency. -funktion von Heaviside unit function of Heaviside. -gitter unit lattice. -ladung unit charge. -masse unit mass. -punkt unit point, principal point. -spin unit spin. -zelle unit cell, elementary c., unit crystal; lattice unit.

einhörig monaural.

einhüllen envelope, enwrap, imbed, shroud, sheath.

Einhülllende envelope, contour, outline.

Einkanal tastung single-channel pulsing (telev. synchronization).

Einkerbung notching, indentation, denting.

einkeilen (in) dent, nick, groove, notch.

einkernig mononuclear, uninuclear, 
single-cored.

Einkl ang syntony, consonance, unison, concord, accord, agreement. einklinken lock, engage, ratchet.

Einknopfkontrolle uniselector, unicontrol.

einkopieren over-print, double print.

Einkrei sempfänger single-circuit receiver, ultraaudion (ultraudion) receiver.

Einkristall faden single-crystal filament, mono-crystal $f$.

einlagern intercalate, embed, incorporate, store.

Einlagerung embedding, occlusion, inclusion, insertion, matrixing.

Einlagerungs-rate rate of incorporation (cryst.). -verbindung, komplexe complex intercalation compound.

einlaufen shrink, contract; flow in; run in (as a bearing, etc.). einläufig unicursal; single-barreled, s.-tracked, s.-channeled.

Einlauf-punkt auf die Rolle point where film is fed onto drum. -seite feed or threading end (m.p.).

einlegen, Berufung gegen eine Entscheidung bei höherer Instanz lodge or take an appeal from a decision to a higher court.

einlegen (Film) thread up the film. einleiten lead in, initiate, originate, induce, introduce.

einmalig single, solitary, unique. -(er) Vorgang non-recurrent, unique or singular action or phenomenon, single event.

Einordnungsstellung position of grating resulting in a one-order spectrum:

Einpegelung leveling.

einpeilen (Funksender mit Richtempfänger) taking bearings from, or tune in, a radio transmitter, with directional receiver.

einprägen impress, imprint, emboss (sound track on film). inpressen press-fit, press in.
Einpulsverfahren single-pulse or monopulse method.

Einpunktschreiber single-point recorder.

Einquell enempfänger solodyne receiver.

Einrasten locking by a detent, snap shutting.

Einrede plea, defense. Beschwerderejoinder (in an appeal).

Einregelung der Dynamik adjustment of volume range or contrast (by compandor action).

Einregelungszeit building up or waxing period (say, of a control potential).

einreichen file, petition, apply for.

Einrichtung arrangement, device, disposition, equipment, contrivance, outfit, organization, means.

Einriss rent, fissure, tear, flaw.

einrücken engage, throw in gear or mesh.

Einriuckhebel trip or engagement lever, starting lever.

Einsattelung crevass or dip (of a resonance or other curve).

Einsatz (acoustic) intonation, onset (of sound, discharge or other action), start, incipiency, initiation, insertion; charge (of a furnace); stake, application, use, what is put to work.

Einsatz der Schwingungen, harter hard start of oscillations. -, weicher gentle or smooth start of oscillations.

Einsatz, Bildkipp- incipience of frame flyback or beam return (telev.). Leuchtfaden- streamer onset. Zeilenkipp - incipient (line) flyback.

Einsatz-blende interchangeable diaphragm. -dichte number of stations (satisfactorily) operable inside a given wave-band. -härtung case hardening, surface cementation. -punkt, Gitterstromgrid-current point, point of incipient grid-current flow. -spannung (cf Zündspannung) spark or 
flashover potential, breakdown potential (in grid-glow tubes), starting potential.

Einsaugemittel absorbent, imbibent, adsorbent.

einsaugend absorbent, absorptive, imbibent.

einschichten imbed, (inter) stratify, interleave, arrange in layers.

Einschlaglupe folding magnifier.

einschliessen enclose, confine, include, seal in, lock, embed, matrix, occlude.

Einschlussröhre sealed tube.

einschmelzen seal-in (a wire or lead in a tube).

einschneiden engrave (sound track on film).

Einschnitt cut-off (spectrum); incision, cut, notch, dent.

Einschnürung squeezing, compression (re:film); bind-up, constriction, recess; waist, reduction in area (metal testing).

Einschnürungs-blende crossover aperture. - punkt crossover (in electron-optics).

Einschwing- und Ausschwing Verzerrung transient (non-linear) distortion, build-up and decay d. (in ac., facsimile, etc., associated with hangover, tailing, underthrow and overthrow).

Einschwingvorgang build-up transient oscillation, onset or initiation of impulse.

einseitig offen unilaterally open (-ended).

Einseitenbandübertragung singleside-band transmission (with vestigial s. -b.).

Einsenkung crevass, dip, depression. einsetzen case-harden (metal).

Einsetzen der Zündung initiation or incipience of striking or firing.

einsickern infiltrate, soak in, ooze in.

Einspannrand, gewell ter corrugated suspension means (of loudspeaker cone).
Einsprache acoustic inlet (of a microphone).

einspringen spring in, shrink (of fibers), re-enter (of an angle). einspringend re-entrant.

Einspruch plea, objection, protest, demurrer, opposition. -unterstützen argue in support of an opposition.

einspuriger Tonstreifen singleedged variable-width sound track or record.

Einsteckseiher plug-in inlet strainer.

einstellen set, adjust, position, focus. -, neutral adjust or set to neutral.

Einstell-fassung focusing mount fof lens). - fehler faulty focusing or spot control, decrease of spot with intensity increase, large diffuse speck on screen in gasfocused picture reproducing tube. -knopf focusing knob (of camera). -lampe, Bild- framing lamp. - l upe focusing magnifier. -marke reference mark, gage m., index dot (on dial), measuring m., wander $\mathrm{m}$. (of telescope). -mikroskop focusing microscope. -schraube set screw, adjusting s., leveling s. - tafel focusing board, test chart. -tubus micrometer adjustment (electron microscope), adjusting tube.

Einstellung, kritische critical adjustment, delicate a., setting or focusing. Bildbühnen- framing, racking (with framing device). Bild- centering control, height c., horizontal c., framing (in telev., with rack and pinion for focusing). Fein-vernier (dial) adjustment, tuning or setting. Fern- long-range focus. Wahshort-range focus. Nullpunktzero adjustment. Phasen- phasing, phase adjustment, shift or regulation. Scharf- automatic tuning; sharp focusing. Still- silent 
tuning, q.a.v.c. tuning. Unendliche-infinity adjustment (of range-finder).

Einstellungs-ebene focusing plane. - fläche surface of reference. -klage complaint or action for discontinuance, suspension or stay (of infringement, etc.), action for issuance of an injunction, i. suit.

Einstellweite focal range, focusing $r$.

Einstich puncture, perforation. einstimmig monophonic, one-voiced, unanimous.

Einstrahl-funkstelle beam station. - sender beam transmitter, unidirectional $t$.

Einstreuung stray effect.

einstufen grade (by quality).

einstweilige Verfügung temporary, provisional or interim injunction, provisional decreo.

e insumpfen digest with water, soak พอt.

eintasten key on, key in.

eintauchen (cf Tauch) dip, plunge, immerse, steep; telescope (a coil).

Eintauch-refraktometer immersion refractometer, dipping $r$. zeit immersion time.

Einteilchenmodell single-particle model.

einteilen divide, subdivide, distribute, separate, grade, sort, classify, graduate (in terms of degrees or other units), index.

einteilig uni-partite.

Einteilung, Nonius- vernier scale.

Eintrag charge, input.

Eintragende feed or charge end.

Eintragschnecke feed worm.

Eintragung entry, registration.

Eintragungs-bescheinlgung certificate of registry. -zwang compulsory registration.

Eintritts-blende input diaphragm or capacity disk (re:klystron, resonance cavity). -feld area of applicator at skin surface
(X-ray work). -geschwindigkeit approach or inlet velocity. -phase entrance phase (re:filter).

Ein-und Ausrïckhebel starting and stopping lever.

ein-und-einachsig orthorhombic (cryst.).

einverstanden sein agree to, acquiesce in.

Einwand demurrer, objection (for instance, of Fatent Office Examiner).

einwandfrei satisfactory, unobjectionable, acceptable, tolerable.

Einweggleichrichter half-wave rectifier.

einwegig single-channel, simplex, one-way.

einweichen steep, soak, macerate, digest.

Einwendung plea, defense, objection, demurrer.

einwertige Salze 1 :l salts.

Einzackenschrift single-edged variable-width sound track or record.

Einzahn-stift striking roller (m.p.). -scheibe drive wheel (m.p.).

einzel single, individual; various, unitary, separate.

Einzel-abschnitt-Filter single-section filter. -bild einer Bildrelhe individual picture or unit frame in a series or sequence. -darstellung single representation; separate treatise, monograph. -heiten details, particulars, minutiae. -klang (sounding of an) individual note. - linse unit lens (of focusing field). -lupe magnifier unit. - pellung taking bearings from one object. -streuung single scattering (of electrons). -vorgang single or separate process, happening, action, event or reaction.)

einziehbar retractile, retractable, withdrawable.

einziehen wind or reel in (an antenna), retract (airplane undercarriage); turn down, decrease (say, diameter of a shaft).

Einziehungsmittel absorbent.

Einzigkeitstheorem uniqueness 
theorem.

Einzugsgebiet cross-sectional area of undistorted portion of field (direction-finding frame).

eirund, halb- semi-oval.

eis-artig ice-like, icy, slacial. -blau ǵlacier blue.

Eisen, kadmiertes cadmiated iron. Band- hoop, strip or band iron.

Eisen-begleiter elements accompanying iron, $i$. companions or associates, congeners (if of similar sort). -bügel iron staple, yoke. -drossel iron-cored chokecoil. -drossel, Besprechung mit modulation by voice action with magnetir modulator.

eisenfreie Spule air-cored (choke) coil.

Eisen-goniometer iron-cored goniometer. -kern, geblätterter laminated iron core. -metalle, Nichtnon-ferrous metals. -metallurgie siderurgy. -panzer iron shield, $i$. screen, i. cladding. -pulverkern Ferroçart core, compressed iron dust c. -pulververfahren magnaflux inspection method. -rahmen iron-cored frame (d.f.). -schluss path closed, or passing, through iron (for magnetic flux), magnetic shunt, keeper (of permanent magnet). - späne iron turnings, borings, filings or chips. -venlust iron loss, core 1. -wasserstoffwiderstand ironhydrogen resistance. -weg iron path, magnetic or ferro-m. circuit.

Eiskalorimeter ice calorimeter.

elastisch gestreut elastically scattered.

elastische Nachwirkung plastic flow persistence, after-flow, elastic after-effect.

Elastizität elasticity, compliance (mechanical filter, ac.).

El astizitäts-messer elasmometer (for Young's modulus), torsometer. -modulus modulus of elasticity (of extension, torsion, compression or volume).

Elektrete electrets.

elektrisch, gleichnamig similarly electrified or charged. glühthermionic. ungleichnamig oppositely electrified or charged.

elektrisch symmetrisch homopolar.

elektrischer Ausfall electric shock.

Elektrizität, Druck- piezo-electricity. Glas- vitreous electricity, positive e. Harz- resinous electricity, negative e. Reibungsfrictional electricity, tribo- er

Elektrizitätslehre, Bewegungselectrokinematics, electrokinetics. Ruhe- electrostatics.

El ektrode, Abnahme- output electrode. Beschleunigungs- accelerator electrode, gun e. (c.-r. tube). Bildwurf- target electrode. Bremsfeld- retlecting electrode, retarding-field e. (in electronoscillation, Barkhausen-Kurz or positive-grid tube). Durchperlungs- bubbling-type electrode. Entnahme- output electrode, catcher e. (klystron). Gegencounter electrode, opposite e., co-operating e.; signal plate, metallic screen (forming foundation of mosaic, in telev. tube). Nachbeschleunigungs- second or additional gun or accelerator electrode, af ter-accelerator, postaccelerator. Quecksilbertropfdropping mercury electrode. Ring-, abbildende image focusing ring. Sammel- output electrode, collector e., gathering e., catcher (in klystron). Seiten- end or wing electrode (in magnetron). Spitzen- needle or point electrode. Steuer- control(ing) electrode, shield, Wehnelt cylinder or grid (in cathode-ray tube). Suchexploring, probe, search or collector electrode (vacuum tube). Zitter- vibrating electrode. zünd-ignitor (of ignitron). 
Elektroden-abstand electrode spacing, inter-electrode distance or gap. -fahrsäule electrode mast or support. -kapazität (inter-) electrode capacitance.

elektrodenlos electrodeless, devoid of, or without, electrodes.

Elektrodenschluss electrode shortcircuit.

Elektrometer, Faden- filament electrometer. Goldblatt-gold-leaf electroscope. Kipp-Wilson electrometer (casing tiltable giving gold leaf different positions).

Elektronen, angelagerte trapped electrons. -, innere inner electrons (on K.L.M... shells).

-,kernferne outer (level) electrons, conduction, valence, peripheral, orbital, or planetary electrons. -,kernnahe electrons adjacent nucleus, inner (fixed) electrons. - -,klebende captured electrons, electrons sticking to electro-negative gases, surfaces, etc. -,kreisende orbital electrons. -,positive positrons, positive electrons. -, schwere heavy electrons, barytrons, dynatrons, mesotrons, penetrons, $\mathrm{x}$ particles, etc.

Elektronen, Bahn- orbital electrons. Feld-field electrons (from cold or auto-electronic emission caused by an electric field). Gitter- lattice electrons. Hiillen- shell electrons. Kreiselspin electrons. Leitungs- conduction electrons (from outer levels). Ruickstoss- recoil electrons, Compton e. Superleitungssuper-conduction electrons.

Unterschale- sub-shell electrons. Zerfall- decay electrons, e. resulting from disintegration (say, mesotrons).

El ektronen-abgabe, glühel ektr ische thermionic emission, Edison or Richardson effect. -abgabefläche electron emission or emitting area. -abtaststrahl electron scanning spot or brush (iconoscope), e. pencil, beam or lever. - auffang electron capture. - austrittsarbeit work function of electrons. -bahn electron path, orbit or trajectory, flight of electrons. -belegung density of electrons (in beam). - beugung electron diffraction. -bildröhre electron image tube. -bremsung reflection (in Barkhause electronoscillation tube), deflection (in magnetron); retardation or deceleration of electrons. -drall electron spin. -einfang electron capture or trapping. -emission, Autoauto-electronic, cold or field emission of electrons. -energie electron energy, electron affinity. -entladung, Glith- thermionic discharge. - fleck electron spot, scanning s. -fluss- Diskontinuität shot effect. -gas, entartetes degenerate electron gas. -gruppierung phase focusing (in beam tubes). -hülle electron shell, electron cloud. -konzentration electron focusing. - l aufzeit transit time of electrons, orbit time of $e .-l$ awine avalanche of electrons. -lehre electronics. - I inse electron lens. (electromagnetic or electrostatic). -mikroskop electron microscope, immersion objective. -mikroskopSelbstleuchtverfahren selfluminous, self-emission or s.illuminating method (using electrons arising on object surface). -niveau electron level. -optik electron optics. -ort electron position, e. locus. -pendelung oscillating of electrons. -physik electronics. -plasma residual electrons, plasma (region without resultant charge). -quelle electron (emission) source, $e$. emitter. -raster-Mikroskop raster microscope, electron-scan $\mathrm{m}$. 
-relais electron relay, thermionic r. -rumpf core, kernel, stable electron group. -schalen, beinahe geschlossene nearly closed eleatron shells. - schleuder electron gun. -schwingungen electron oscillations. -spritze electron gun. -sprungspektrum electron transition (or jump) spectrum. -stauung electron cloud or accumulation (in a virtual cathode).

El ektronenstrahl, gaskonzentri erter gas-focused electron beam. -, geschwindi gkeits-gesteuerter velocity-modulated electron beam. -, weisser heterogentous beam.

Elektronen-strahlabtaster electron scanning pencil, beam, spot. brush or lever. -strahlengang electron ray path. strahlröhre (cf Kathodenstr.) cathode-ray tube; beam tube; thermionic t. (obs); c. $-r$. tuning indicator $t$. -strommodulation electron quantity modulation. - tanz oscillatory movements of electrons (due to magnetic or electric forces). -turbine cyclotron and inverted (or Hollmann) forms thereof. -technik electronic art or technology, electronics. -übergangswahrscheinlichkeit transition probability (of electron passing from one level to another level). -überhol ungsgebiet catchup or overtake region (in which electrons arrange themselves in groups for phase focusing in beam tubes). -überkreuzungsstelle crossover of electrons (where principal rays from first lens cross axis, equivalent to exit pupil of eye). -übermikroskop electronic super microscope, e. ultra microscope. - unterhülle sub-shell (of eleatrons). -welle electron wave, phase w., de Broglie w. -wolke cloud of electrons. --Ei genabstossung der repelling action of electron charge, negative space charge. -wucht collision force, bombardment $f$. (of electrons). -zustand, entarteter degenerate electron state.

Elektroniker electronician, electronics engineer.

elektro-opti scher Verschiluss electrooptic shutter.

Elektrophoresezelle electrophoresis cell.

elektrostatisch geschirmt electrostatically shielded, e. screened. -(er) Lautsprecher electrostatic loudspeaker, condenser 1., capacitor 1 .

Element, Lager- inert cell. -Rasterpicture unit, p. element, elementary area. Schalt- circuit element, c. means. Streckenlinear element (math.).

El ementar-ladung el ementary charge, electronic c. - well en Huygens wavelets. -zelle unit cell, elementary c., lattice unit.

Elementenmessung stoichiometry.

Emanation, abgeklungene emanation with diminished or decaying radioactivity. Aktinium- actinon. Radium- radon. Thorium- thoron.

Emanationseinheit, Radium- curie unit of radon or radium emanation $\left(10^{-10}\right.$ curie-liter $=$ eman. $)$.

Emission, glühelektrische thermionic emission. Autoelektronen- autoelectronic, cold or field emission of electrons.

Emissions-strom, Feld- field emission of electrons, cold e. -vermögen emissivity, emission power.

EMK (elektromotorische Kraft) emf (electromotive force).

EMK von rechteckiger Kurvenform square-shaped or rectangular emf, square-topped emf.

Empfang, Block- block reception, communal or party $r$. Druck- telescript reception, typed r., teleprinting $r$. Einquell- solodyne reception. Geradeaus- single circuit reception, non-heterodyne r., straightahead r. Hör- audible reception, auditory r., aural r., 
reception by ear, hearing or earphones. Musa- multiple-unit steerable antenna reception. Primär - single-circuit reception. Richt-directional reception. Rückkopplungs-, mit Hilfsfrequenz super-regenerative reception. Schreib- visual reception, recorder r. Such-, direkter singlecircuit or straight-ahead (nonheterodyne) reception. Superheterodyne-, mit selbsterregter Trägerwelle homodyne reception, zero-beat $r$. Tertiär- threecircuit reception. Ton- AF modulated or tone-modulated c.w. reception. Transponierungs-,

Uberlagerungs- heterodyne reception.

Empfänger receiver; collector, pickup (microphone). Empfänger, verdrahteter fully wired receiver set.

Empfänger, Allstrom- universal receiver set, $a-c / d-c r . s$.

Bewegangs- velocity microphone, v. pickup; pickup or detector of motion. Bild-(cf Bildempfangstelle) picture receiver, television or video r., picture reproducing device, p. viewing tube, image reconstructor.

Bildton- audio-video receiver combination. Breitband- broadband or wide-band receiver.

Dreikreis- three-circuit receiver. Druck- pressure (responsive) microphone. Druckknopf- pushbutton or press-button tuned receiver. Einkreis- ultra-audion, ultraudion or single-circuit reception. Einquell- solodyne receiver. Fernseh- television receiver apparatus, video-signal receiver, kinescope (Zworykin), oscillight (Farnsworth). bleichstrom- d.c. electric (radio) set. Heimfernseh- home television receiver. Kontroll-monitoring receiver. Peil-directionfinding receiver. Peilfunk- radio compass, directionfinder (avigation). Pendel- superregenerative receiver, Armstrong r. Primär- single-circuit receiver. Richt-, Einpeilen auf Funksender tuning directional receiver to radio stations (to take bearings). Schall- sound collection device, acoustic receiver, sound pickup or microphone. Schallschnelle- velocity microphone. Strahlungs- radiation responsive or sensitive record or receiving sheet or surface (on which radiations impinge to measure color temperature, etc., such as photographic plate, selenium cell, bolometer). Superregenerativ(Armstrong) super-regenerative receiver. - und Sender kombiniert transceiver radio set; microtelephone. Unterwasserschallsubaqueous or submarine microphone, pickup device or sound detector, hydrophone. Wechsel stroma.c. electric receiver set. Zielflug- homing receiver apparatus. Zweikreis- two-circuit receiver set. Zwischenfrequenz-. mit Kristallsteuerung crystal-monitored or c. stabilized supersonic or superheterodyne receiver, stenode radiostat.

Empfänger-blechgestell metal chassis - fläche radiation-sensitive surface; record sheet. -reichweite (distance) range of receiver, distance-getting ability of radio set. - Senderkombination microtelephone; transceiver radio set.

Empfangfeldstärke incoming signal level.

Empfängl ichkeit susceptibility, receptivity, responsiveness.

Empfangs-antenne, vom Sender entkoppelte balanced receiver aerial (uncoupled from transmitter). - bild recorded copy (in facsimile). - gebiet service area (in broadcasting). - spannung, ger ichtete directional incoming or signal 
potential. -verfahren, Mehrfachdiversity receiving method.

empfinden sense, perceive, feel, apprehend, experience, have consciousness. .. emp findl ich responsive, sensitive or reactive to (say, temperature), be a function of, be dependent on.

empfindlich, blau- blue sensitive. flecken- susceptible to staining or spotting. richtungs- with directional response.

empfindlich machen, licht- photosensitize.

Empfindlichkeit sensitivity, sensitiveness, sensibility, responsiveness; efficiency, yield, output.

Empfindl ichkeit, Ablenkungs- deflection sensitivity (reciprocal of deflection factor). Augenintensity discrimination, contrast sensitivity (embodied in NeberFechner law). Hand- hand or body capacitance or capacity effect. Hör- auditory sensitivity, hearing capability. Kerb- stress concentration index. Korrosionscorrodibility, susceptibility to corrosion. Licht- photo-sensitivity. Richtungs- directional response.

Emp findl ichkeits-kurve response characteristic. -messung sensitometric measurement (phot.). -verteilung sensitivity distribution, spectral response (of eye). -ziffer sensitivity factor.

Empfindl ichmachung sensitization, activation, sensibilization.

Empfindung sensation, feeling, perception, sentience.

Empfindung, akustische acoustic perception. Farb-color sensation, c. perception, chromatic s. Lautstärken-aural sensitivity. Ton-acoustical perception, sound sensation.

empfindungs-gemässe, -gerechte Farbstufe, subjective chromatic scale value. -gerechte Abstufung subjective or natural grading or spacing (in chromaticity or chromatic scale, etc.).

Empfindungs-grenze des Ohres loudness contour of ear, auditory sensation area (between limits of maximum tolerable and minimum perceivable intensities). -lautstärke response of ear to signal strength; sensible loudness of sound.

- schwelle, Schall- threshold of audibility or acoustic perception. -zunahme sensation increment.

Emulsions-seite face (of film) bearing emulsion. -steilheit steepness of gradation.

End-al arm, Akt- reel-end signal. -ausschlag full excursion, limiting deflection. -geschwindigkeit terminal velocity. - gewicht fish of a trailing antenna. -impedanz terminating or end impedance, load i. -kapazität top capacity, terminating or end c. -kondensator terminating condenser or capacitor.

endloser Filmstreifen endless film strip.

End-mass, Parallel- standard gage block, end-to-end s. bar. -punkt terminal, terminating point, terminus. -röhre power tube, final t. -röhre, Doppelsteverpower pentode in which cathode grid is connected with control g. rather than with cathode. -streifen trailer (of a film). -urteil final judgment. -verschluss cable box, terminating box. -verstärkerstufe final amplifier stage, power amplifier s. -wert final, resultant or ultimate value.

energetische Bevorzugung energy preference.

Energie, Ablösungs- excitation energy, work function (of an electron). Anregungs- excitation energy, stimulation energy (say, a potential). Bewegungs- kinetic 
energy, motional e. Bindungsbinding energy, bonding e. Eigeneigen energy, characteristic e. Elektronen- electron energy or affinity. Selbst- self-energy (of electrons).

Energie-berg energy hill, e. barrier. -eigendichte proper energy density. -erhaltung energy conservation, preservation of $e$. - impulstensor energy momentum tensor. -leitung (energy) feeder lead, feeder downlead, transmission line.

energielos (ohne Energieverbrauch) non-dissipative, wattless.

Energie-messer ergometer. -niveau energy level, quantum'state.

energiereich full of energy, energyrich, energetic.

Energie-rïckgewinnung durch Bremsen energy recuperation by (regenerative) braking. - schwelle threshold of energy. -stufe energy level, energy term, quantum state. - übertragung energy transfer. - umwandler transducer, sink. -verbrauch consumption, dissipation, loss or expenditure of energy. -verbrauch, ohne nondissipative, wattless. -zerstreuung scatter of energy.

enge Anschmiegung intimate or snug adhesion, adherence, fit, engagement or contact, "hugging."

engjähriges Holz close-grain wood. entaktivieren render inactive, deactivate, de-energize, desensitize.

entarteter El ektronenzustand degenerate electron state.

entartetes Elektronengas degenerate electron gas.

Entartung degeneracy, degeneration. Entbindung, Wärme- disengagement of heat, evolution of h., release of h.

Entbrummer hum eliminator, humbucker.

Entbündein de-bunching (in klystron), unbunching; defocusing.

Entdämpfung gain, regeneration, deattenuation. -Frequenzkurve gainfrequency curve.

enteignen expropriate, dispossess. entei sen de-ice.

entfallen lose (a chance), drop out, fall.

Entfärbung decoloration, bleaching. Entfärbungsmittel decolorant, bleaching agent.

Entfernung, Kern- inter-nuclear distance. Sprung- skip distance, skip zone.

Entfernungs-messer, Basis- base range finder. -messer, Halbbildsplit-field coincidence range finder. -schleier distance fog. -skal a distance scale.

entfesseltes, bewegliches Mikrophon following microphone.

Entfritter decoherer, tapper. entgasen extract gas from, outgas, cleanup, degas, getter.

Entgasung, Trocken- dry distillation.

entgegen-arbeiten counteract, buck, work against. -drehen, dem Wind head into the wind, crab. -gesetzte Pole opposite poles, unlike p.

Entgegenhaltung anticipation, anticipatory disclosure or reference (from prior or earlier art). entgegen-wirken oppose, counteract. -wirken, der Resonanz anti-resonant. entglasen devitrify.

entgraten debur.

enthärten soften, anneal.

entionisieren de-ionize, unionize.

Entisolierer wire skinner, insulation stripping tool.

Entknurrungswiderstand anti-growl resistance, resistor designed to suppress growling noise due to $\mathrm{RF}$ or tickler coil, in regenerative path.

Entkohlung decarburization, decarbonization.

entkoppel te Antenne (vom Sender) balanced antenna. 
Entkopplung decoupling, balancing, tuning out.

entkräftigen exhaust, debilitate, weaken, vitiate; invalidate. entladen discharge; unload (phot.). Entladeschaltung circuit means causing fast rise of sawtooth potential.

Entladung, aperiodische aperiodic, deadbeat, impulse or non-oscillatory discharge. -, kontrahierte contracted discharge. -, selbständige self-sustained, spontaneous or unassisted discharge. -, stille corona, silent discharge, efflove (in skin therapy). -, strahlartige needle-point, corona or streamer discharge, leader-stroke d. -, überspannte overvolted discharge. -, unselbständige assisted or non-selfsustained discharge.

Entladung, Büschel-brush discharge, corona (ranges between glow and spark discharge). Dunkel- dark discharge, dark current. Fackeltorch-form of discharge. Fadenstrahl- (cf Fadenstrahl) threadray discharge. Glühlelektronenthermionic discharge. Hochstrombogen-, nichtkondenslerte heavycurrent uncondensed discharge. $\mathrm{Kipp}$ - condenser discharge (in time-base). Neben- lateral, stray or secondary discharge. Schwing- oscillatory or oscillating discharge. Spitzen- point discharge, needle (-gap) d. Spritz-initial discharge caused by rapid surge of ions; needlegap discharge. Vor-pre-discharge. Entl adungs-aufbauzeit, Funkenspark-discharge formation time. -kanal, Vor-pre-discharge track. - leuchtstreifen luminous streamers. Entl adungsröhre, Gas- gas discharge tube or valve. KIpp- time-base discharge tube; kipp relay. Quecks I l berhochdruck- highpressure mercury discharge tube.
Entladungs-stoss burst, pulse, corona. -verzug discharge delay.

entlang streifen brush, skirt or wipe over or along, graze.

entlasteter magnetischer Lautsprecher balanced-armature magnetic loud'speaker.

Entlastung relieving something of weight, traction, stress, etc., say, by counterpoise and the like, unburdening, removal of load.

Spannungs- stress relief; antifatigue or anti-vibration means (for lines). Zug- traction relief.

entlüften evacuate, exhaust air, deaerate.

entmagnetisieren de-Gauss, demagnetize, de-ener bize.

entmischen demulsify, liquate out, unmix, separate, sort (ions in mass spectrometer).

Entmodulierung demodulation, detection, rectification.

Entnahme, Leistungs- energy or power output, delivery, absorption or pickup, tapping, taking or draining of power or energy. Probe- sample taking, sampling, taking or selecting at random.

Entnahmeelektrode output electrode, catcher (in beam tube).

entnehmen absorb, pick up, catch or abstract (energy from an electron beam); derive or infer from.

Entorientierung desorientation.

Entquellen (Gele, etc.) shrinkage, cyneres is.

entregen de-energize.

entriegeln unblock, unlatch, trigger, release, trip, unlock (a tube previously cut of $f$, by causing it to break down and discharge).

entrinden strip of $f$ bark, bark, decorticate.

Entschädigung indemnity, indemnification, compensation, consideration, damage payment. -festsetzen assess or fix damage or indemnity. 
-zuerkennen award damages.

Entscheidung decision, sentence, verdict, judgment. Berufung einlegen gegen eine- take or lodge an appeal from a decision.

Entscheidungskern crux of a decision. entschlickern dross.

Entschliusselung de-coding, ungarbling, deciphering, breaking a (secret) code, decryptographing.

Entspannen einer Feder relaxing, relieving or untensioning of a spring.

Entspannung relaxation, strain, stress or tension relief, untensioning or slackening, untensed state.

entsperren see öffnen

Entsprechung equivalent, denotation, analogy.

Entstehung origin, genesis, nascence. Entstehungs-ort origin, source or seat of generation or production. -potential appearance potential, ionization p. -zustand nascent state, status nascendi.

Entstörung interference elimination. entströmen flow, stream, escape or issue from.

Enttrübungsrahmen frame designed to make zero or minimum point sharp (free from night effects, etc.), zero cleaning or sharpening frame. Entwarnung all-clear signal.

Entweichungsventil escape, drain or outlet valve, vent valve.

entwerfen design, project, plan, trace, sketch, outline, focus or form (an image).

entwickel bar developable.

entwickeln (cf erzeugen) develop, evolve, disengage, generate, give off (gases).

entwickelnd, licht- emitting or producing light, photogenic.

Entwickelung, Rahmen- rack or tray development. Reihen-, Serienexpansion in a series, power series or seriation (math.). Umkehr- reversal processing or development.

Entwickel ungs-arbeit development (al) work. - faktor Watkins factor, development f., time of appearance. -rahmen developing rack. -steilheit slope (of an $\mathrm{H}$ \& D curve). -vorrichtungen, Film- film processing equipment. Entwicklerbottich developing tank. entzerren correct or eliminate distortion, equalize, compensate.

Entzerrer corrective network, attenuation compensator, attenuator (sound recording and reproduction); sound clarifier. -zum Anheben und Senken compensator to accentuate and deaccentuate frequencies or bands, accentuator and deaccentuator, emphasizing and deemphasizing means.

Entzerrer, Dämpfungs- equalizing or compensation network. Dynamikdynamic expander. Längs- seriestype attenuation compensator or equalizer. Quer- shunt-type attenuation compensator or equalizer. Entzerrung (Dynamik) expansion (by an expandor). Ausgleich-complementary recording (of film). Phasen- correction of phase, delay equalizer; echo weighting term.

Entzerrungs-anordnung distortion corrector device or compensator, anti-distortion device. -bereich frequency range of equalization. -drossel anti-resonant coil. - filter filter-type equalizer, network e. -gerät rectifying camera (aerial phot.). -grenze frequency limit of equalization. -kette mit Brücken-H-Schal tung H-type network attenuator (balanced). -kette mit Brïcken-T-Schaltung T-type network attenuator (unbalanced). -kreis (zum Hervorheben einiger Frequenzbereiche) balancing network (to accentuate or underscore certain bands). entziehen abstract, extract, take 
away, withdraw, deprive, rid.

Entziehung, Wasser- dehydration, removal or expulsion of water, drying.

entziffern decipher, decryptograph. entzünden ignite, kindle, inflame.

Entzündung, Selbst- spontaneous combustion, self-ignition.

Ep iskop reflecting projector, episcope.

erbreiten broaden.

erbringen produce, adduce.

Erd-alkal imetall alkaline-earth metal. - antenne ground or earth antenna, underground or buried antenna. -anziehung gravitation. - anziehungskraft gravitational force. -bahn earth's orbit, terrestrial o. -bebenmesser seismometer, seismograph (when recording). - beschleunigung acceleration due to gravity.

-drahtnetz ground mat. -drahtschleifenmessung loop test.

Erde, Edel- rare earth.

Erderwiderstand grounder resistance. Erd-kabel leader cable, buried cable. -kapazität, Teil-direct earth capacitance. -klemme ground clamp, g. terminal. -kruste, Ausgleichfläche der isostatic surface (of earth).

erdmagnetisches Feld geomagnetic field.

Erd-magnetismus terrestrial magnetism. - schelle ground clamp. -schleife earth or ground circuit.

Erdschluss earth connection, (arcing) ground. Flammenbogen- arcing ground. -fehler ground fault. -löschvorrichtung groundfault neutralizer.

Erdung, Lichtbogen-, Erdungslichtbogen arcing ground.

Ereignis occurrence, happening, action, event, phenomenon. -, einmal iges non-recurrent, unique or singular event or action.

erfahrungsgemäss according to, or in the light of, experience, empirical, usual.

Erfahrungstatsachen empirical, experimental or practical facts or data.

erfassbar, zahlenmässig numerically evaluable.

Erfindergeist inventive genius, creative conception, inventor's spirit.

Erfindungs-gedanke basic or underlying idea of an invention, idea on which an invention is predicated. -gegenstand object of invention, disclosure. -geltungsbereich scope of invention or patent, command of a p. -patent patent for invention, letters $p$. -schritt object of an invention. erfillen fulfill, perform, satisfy, comply with, measure up. to.

Ergänzung complement, supplement, completion, addendum (addenda, pl.).

Ergänzungs-farbe complementary color. -stoff vitamine. -winkel complementary angle.

erhaben raised in relief, olevated, embossed; high, grand, salient, outstanding.

erhaben, hohl- concavo-convex. rund- convex.

Erhaltung der Energie, Gesetz der law of conservation of energy. - der Masse conservation of the mass.

Erhaltungs-gesetz law of conservation: -satz, Impuls- theorem of conservation of momentum.

erheben (Anspruch, $\mathrm{Kl}$ age, etc.) make (a claim), bring or file (a suit or an action). -, in die $n$ te Potenz raise to the $n$th power.

Erhebung elevation, projection, prominence, salience, peak, point, pip (on c. $-r$. screen tracing).

Erhebungswinkel angle of elevation. erhitzen heat, warm, fire, incandesce, ignite, anneal.

Erhöhung elevation, prominence, projection, peak, jutting, lobe (as 
of a cam), pip (as on a c. $-r$. screen tracing).

Erhöhungszeichen sharp (mus.).

Erholung recovery, recuperation (from fatioue, stress, etc.).

Erichsen-prüfer Erichsen cupping machine. -prüfung Erichsen cupping test.

Erklärung, eidesstattliche affirmation in lieu of ath. Nichtigkeitsdeclaration of nullity.

erlauben allow, permit, authorize.

erlaubt(es) Energieniveau permitted level, p. quantum state, p. energy zone. $-(\mathrm{er})$ übergang permitted transition (according to selection principle).

Erlöschen eines Patentes expiry or expiration of a patent, annulment of a patent.

ermächtigen empower, authorize.

Ermangelung, in...von in default of, in the absence of, failing.

Ermittelung investigation, ascertainment, discovery.

Ermüdung fatigue, sluggishness, inertia, tiredness.

Ermüdungsgrenze fatigue limit.

Erniedrigungszeichen mol (mus.).

Erneuerungsschein certificate of renewal.

erörterbar discussable, arguable, disputable.

erproben test, try out, prove (ordinance).

errechnen calculate, compute, figure (out), reckon.

erregen excite, enerojize, impulse (by shock), stimulate, activate.

Erreger-lampe (cf Tonl ampe) exciter or exciting lamp (m.p.). -spule, Feld-field (exciting) coil (of electrodynamic loudspeaker).

Erregung excitation, energization, impulsing. Fremd- separate excitation. Selbst- self-oscillation, s. -sustained o., spontaneous (unassisted) o., spilling over (of a regenerative receiver, with fringe howl and whistling). Stoss- shock, impact or collision excitation. Summer- buzzer excitation.

Erregungslampe exciter lamp, exciting 1. (operating photo-electric cell).

errichten, eine Senkrechte erect a perpendicular.

Errichtungsprotokoll minutes of proceedings.

Ersatz-anspruch indemnity claim, claim for compensation. - antenne artificial antenna, mute a., phantom a. -bild equivalent circuit diagram. -elektron equivalent or replacing electron. -fähigkeit capability or susceptibility of being replaced, equivalency. -kapazität equivalent capacity. -schema equivalent circuit schema.

Erscheinung phenomenon, action, event, manifestation, appearance. Nicht- non-appearance, nonattendance, absence, default, contempt.

Erscheinungsform form of manifestation, phase, state, physical appearance.

erschüttern shake, jar, percuss, chatter, vibrate.

erschütterungsfrei resilient, shockabsorbent, non-vibratile.

erschweren make (more) onerous or difficult, impede, aggravate.

erstarren solidify, freeze, congeal, harden, set.

Erstarrungspunkt freezing, coagulation or solidification point.

Erstattungspflicht liability to make restitution.

Erst-strom primary current. -wicklung primary winding.

ersuchen um Gehör file application or petition to be heard.

Erteiler, Lizenz- licenser, grantor, issuer of a license.

Erteilung eines Patentes grant or allowance of letters patent.

Erteilungsakten file wrapper (of a patent application). 


\section{Erwärmungskraft}

Erwärmungs-kraft heating power, calorific p. -verlust loule loss, joulean heat, thermal or heat 1.

Erwartungswert expected or anticipated value.

erweichen soften, plasticize.

erweitern widen, expand, enlarge, dilate, spread.

erweiterte Pupille dilated pupil.

Erwerber acquirer, purchaser, transferee.

Erwerbungskosten acquisition cost, purchase cost or price.

Erwiderung answer, rejoinder, reply, replication (of plaintiff to defendant's answer).

Erz, selbstgehendes self-fluxing ore.

erzeugen (cf entwickeln) generate, produce, create, set up, cause, result in.

erzeugend generant. elektronenelectronogenic (electron-emission under influence of light, from moles). farben- color producing, chromogenic. fluoreszenz- fluorogenic, with activator properties. kälte- cryogenic, frigorific. leuchtenergie- fluorogenic, phosphorogenic, photogenic, having phosphor properties. licht- emitting or producing light, photogenic. phosphoreszenz- phosphorogenic, causing persistence of fluorescence. schwingungsvibromotive, oscillation-generative.

Erzeugnis, Neben- by-product.

erzwungen(e) Schwingungen forced or constrained vibrations or oscillations. (opposite of free o.). -(er) iibergang forced or nonspontaneous transition.

Esse stack, chimney.

Estrichgips Keene's cement, flooring plaster.

Etagen-kessel battery boiler. -ofen storey furnace. -rost step grate. -ventil multi-seat valve.

Etagere rack, stand, shelt', support. Etalonapparat reference standard, calibrated instrument.

eventuell optionally, contingently; if desired or necessary; eventually, ultimately.

Evolventenverzahnung involute tooth gear.

E.K. (Elektrizitätswerk) - rachrichtenübermittelung power line signaling or intelligence transmission.

Exolierung anodic treatment, anodizing.

Expansionsschalter hydroblast or expansion circuit breaker or switch.

explosiver Laut explosive sound.

Exponentialtrichter exponential horn, logarithmic $h$. (either of uniform or of multiple-flare type, for loudspeaker). -, aufgewundener twisted, coiled or curled exponential horn. -, gefalteter folded exponential horn.

Expositionszeit exposure time, exposure scale.

Extinktionskoeffizient totalreflection coefficient, extinction c.

extra matt rough matt.

extremkurze Wellen (cf Welleriabgrenzung) ultra-short waves.

Exzenter cam, disk. with lobes.

Exzenterbewegung, Herz- cam movement (of Livin). 
Fabrik-marke trade-mark. -zeichen anmelden register a trade-mark.

facettierte Linse bevel-edged lens. Facettspiegel, Scheinwerfer- segmented or facetted searchlight reflector.

Fach-arbeiter skilled worker, specialist. -ausbildung technical education, schooling or training. -ausdruck technical term or expression.

Fächer-antenne fan antenna, harp a. or aerial. -aufblendung fan fade-in. -blende fan fading shutter.

facherndes Prisma, stark highly dispersive prism.

Fach-genosse professional colleague, p. collaborator. - literatur technical literature or press. -mann professional man, expert. specialist, man skilled or trained in an art. -ordnung classification. -photograph professional photographer. -presse technical press or literature. -sprache technical language, professional terminology. -welt technical world.

Fackel-entladung torch form of discharge. - erscheinung flicker effect (of a cathode). -kohle cannel coal.

Faden, biegsamer ductilized filament; flexible thread or fiber. -,thorhaltiger thoriated filament. Faden, Einkristall- single-crystal filament, mono-c.f. Glas- glass fiber or thread; thread-like defect of glass. Kenn-colored tracer thread (for color coding). Leucht- streamer; \$low column (in a tube). Quarz- quartz thread, q. filament. Quecksilber-mer- cury column, m. thread. Stromstreamer (in discharge); current path, streamline, c. tube.

Faden-dicketaster calipers with jaws to measure thread gage capacitively. -einsatz, Leuchtstreamer onset. -elektrometer filament electrometer. -galvanometer string §alvanometer. - $g$ las spun glass, filigree -kabel (HF-Leitung) co-axial or concentric cable with silkthread-supported central conductor. -kreuz reticule, cross or hair lines, reticle, sraticule, cross-spider hairs. -kreuzlupe reticle magnifier, $r$. glass, $r$. lens. -lange, Gl imm- length of neon glow column (in tuning, etc., indicators). -strahl fuzzy hose-like pencil of non-uniform cross-section in gas-focussed c. $-r$. tube, consisting of positive ions and secondary electrons, thread. beam. -strahlen pencil rays (electrons). - strahlentl adung thread ray discharge. -strom filament current, cathode c. - umschnirrung serving of thread (on cables) -zähler thread counter, linen tester.

fädig, ein- unifilar.

Fading-automatik automatic volume control means (a.v.c.). - mischhexode a.v.c. mixer hexode.

Fagott bassoon. -wasserabguss bassoon syphon.

Fahne, Rauch- smoke steamer. Fahnenbildung signal inertia-drag. Fahr-arm hinged tracing lever (of planimeter). -aufnahme running, follow or traveling shot. -bahn track, taxiway (airfield), 
runway; travel beam.

fahrbares Mischpult dolly or cartype mixer, teawagon console mixer.

fahren run, move, ride, travel. Fahr-gestell, einziehbares, rückzlehbares retractable or retractile undercarriage. -säule, Elektroden- electrode mast or support. -stift tracing or tracking point (of planimeter). -strahl radius vector. -stuhl, Spulen-, (in Bandbreiteregler) band-width regulator comprising auxiliary coupling coil parallel to oscillation coil.

Fahrtwindgenerator wind-driven generator.

Fahr-widerstand (Flugzeug) drag. - zeug (power) craft (marine, land or air), power-propelled vehicle.

Faktor factor, coefficient, submultiple (of a number).

Faktor, Anregungs- excitation factor. Anreicherungs- concentration factor. Atomform- atom form factor, atomic structure factor, structure amplitude f., f-value; scattering factor (of X-rays or electrons by gases). Aufspaltungs- splitting factor. CallierCallier factor. EntwickelungsNatkins factor (time of appearance) Filter-filter factor, screen $f$. Form- shape factor, theoretical stress concentration f. Füllspace factor, activity $f$. or coefficient. Klirr-non-linear harmonic distortion factor, klirr, blur or rattling $f$. Kugel- coefficient of sphere (photometry) Leistungs- power factor. Polbedeckungs- pole arc, pole pitch percentage. Q-Q factor, magnification f. Spur-tracking error, t. distortion (phonograph). Streu- scattering factor, $S$ value (of x-rays). Trenn- separating factor. übertragungs- transfer factor, image t. constant. Verlust- power factor (e.g., of a cable, condenser, etc.), ratio of watts to volt-amps.; phase angle difference $(\tan \delta)$. Verstärkungsamplification factor, voltage $f$. Widerstand-Reaktanz- $Q$ factor, magnification $f$.

fakultativ optional, non-obligatory, non-compulsory, permissive.

fällbar precipitable.

Fall-bär ram, tup, rammer. -Beschleunigung "gravitational acceleration.

Falle, lonen- ion trap (in gun of c. $-r$. tube to attract negative ions). Quecksilber- mercury trap (in vacuum pump).

fällen, ein Lot let fall a perpendicular. -, ein Urtell render a judgment, decision, sentence, verdict or award, issue a decree.

fallen lassen abandon ( $a$ patent, an application).

fallende Charakteristik drooping characteristic, falling $c$.

Fall-klappenrelais drop indicator relay. -körper, Zähigkeitsmesser mit ball-drop type of viscosimeter. Fäl Imittel precipitant.

Fall-raum cathode,drop or fall space. - scheibe, - schieberverschluss drop shutter. -system$\mathrm{Pl}$ attenwechsel drop system of record changing. -werk vertical drop or blow-impact testing machine. -winkel angle of inclination.

falsch (e) Ausrichtung misalignment (of image or track). - (es) Licht light fog, leakage 1., stray 1. (in film printing). - phasig misphased, dephased, out of phase. - (e) strahlung stray radiation, s. rays.

Fal sch-abstimm ung mistuning, offresonance condition. - anpassung des Widerstandes mismatching of impedance, mismatched i.

Fal schanpassungsfaktor mismatching factor, reflection f., transition $f$. 
Fâl schung distortion, vitiation, falsification, adulteration, fraud, forgery, counterfeiting. Fal te pleat, fold, crease, wrinkle, lap.

Fal ten-filter plaited, folded or fluted filter. -horn folded, coiled, curled or twisted horn (of a loudspeaker).- - lautsprecher folded-horn loudspeaker. -schl auch pleated or corrugated hose or tube, accordion tube.

faltig having folds, pleats or creases, wrinkled, puckered, Faltung (Spektrallinien) faltung (of spectral lines).

Faltversuch folding, doubling or bend-over test.

Falz fold, groove, flute, notch. Falz-beständigkeit folding endurance (of film). -făhigkeit foldableness. -festigkeit folding strength. -membran non-rigid noncircular (breathing) cone with curved radiating surface. -widerstand folding resistance. -ziegel interlocking tile.

Familie, Gleichungs- equations grouped in a family.

Fang-anode (cf Auffanganode) collecting or gathering anode. -blech baffle sheet (in a masnetron). -duse mixing nozzle (steam jet). fangen catch, capture, captivate, collect, trap, secure.

Fănger, Bild- pickup camera. Schleifen-feed and take-up sprocket mechanism (film projection).

Fang-gitter suppressor grid, cathode g. (in power pentode), interceptor g. -mittel getter(ing substance) -platte target or impactor plate -rohre, Farnsworth' sche F. dissector tube -spiegel collecting mirror.

Faraday's(cher) Dunkel raum Faraday dark space. -(ches) Gefäss Faraday ice pail, F. cylinder. -(cher) Käfig Faraday screen, shield or collector.
Farb-angleichung color matching, c. comparison. -atlas color scale, c. chart (e.g., of Munsell). - ausgleich color balancing (of c. film). - auszug color record, chromatic selection. -auszug, negativer separation negative. -deckfähigkeit concealing, covering or hiding power (of paint); opacity.

Farbe color, dye, paint, pigment, stain, hue, tone, shade, tint. -, bezogene, related color. -, bunte hue color, chromatic c. -, unbezogene unrelated color. -, unbunte hueless color, achromatic c., color devoid of hue. Ergänzungs- complementary color. Grund-primary color; ground or priming c. Interferenz- interference color. Kern- nuclear stain. $\mathrm{Kl}$ ang- timbre, tone color, quality. Leucht- luminous paint. Stammprimary color. Ton- timbre. Umdruck- re-printing ink.

Färbekraft dyeing power, tinctorial p.

Farbemesser, Blut- plethysmograph (tests blood flow), electroarteriograph.

Farbempfindung color sensation, $c$. perception.

Farben-abweichung chromatic aberration. -ausgleich color balancing (in color film). -auszugfilter selective filter, s. screen.

farbenblind color blind.

Farbendruck, Licht- photo-chemical color printing.

farbenemp findliches Zäpfchen color-distinguishing cone (of eye).

Farben-empfindlichkeit des Auges, -empfindl ichkeitsverteilung color sensitivity or spectral response of the eye. -empfindung color sensation, chromatic s.

farbenerzeugend color producing, chromogenic.

Farben-fehler chromatic defect, color d., chromatic aberration. 
-fehl sichtigkeit color-vision deficiency. -filmaufnahme color photograph. korrektion achromatization. -lehre science of color, chromatics. -mass colormeter, colorimeter. - messer chromatometer, colorimeter; c. knife. -messung chromatometry, chromatometrics, colorimetry. -rad rainbow wheel. -reste residual chromatic aberration, uncorrected color.

farbenrichtig orthochromatic (phot.).

Farten-saum color fringe. -schichtfilm dye-coated film. - sehen color perception, color vision. - skal a chromaticity scale, color scale, color chart. -spektrum color spectrum, chromatic s. -stufe color gradation, shade, tint. -messer tintometer. -ton tint, hue, color tone. -tuchtigkeit color vision. -unterscheidung color discrimination. -veränderung change of color, discoloration. - vergleich color matching, c. comparing.

-wahrnehmung color vision, c. perception. -wiedergabe color reproduction. -zerstreuung chromatism, dispersion, chromatic aberration (opt.).

Farberegler, $\mathrm{Kl}$ ang- tone control, tonalizer.

Färbetabletten varitone tablets. Farbfehler chromatic aberration. farbfehlerfrei achromatic.

Farb-film, Natur- natural-color film. - glas colored glass, stained g., c. filter. -gleichung, dreigliedrige threecolor equation, tristimulus e.

farbig, flammen- flame or rainbow color. -, mehr- polychromatic, pleochroic (cryst.), multi-colored. -miss- discolored, inharmonious in color. -, regenbogen- rainbow colored, iridescent. -, voll- full or saturated in color.
Farb-kennzeichnung color coding (for identification and wire tracing). -komponente color or image component (in color reproduction). -kopie color print.

fart-korrigiert color-corrected, achromatized. - los colorless, hueless, achromatic.

Farb-lossung staining solution (microscopy). -massystem (IBK) chromaticity scale system, color chart, CIE diagram. -messung colorimetry. -muster color sample $-r a d$ inking wheel, i. roller. -raster color embossing. -rasteraufnahme color screen photography.

farbrichtig orthochromatic, isochromatic.

Farb-rolle inking roller, i. wheel. - schaum fringing (in film).

- schreiber inker, printer.

-schwellenwert liminal value or threshold of intensity of a color. -stufe color gradation, tint, shade. -stufe, empfindungsgemässe subjective chromaticity scale value. - temperatur color temperature. -toleranz color tolerance. - ton (warm, kalt) color hue, tone or tint (warm, cold).

farbton-gleiches, reines Gelb psychologically unique yellow. -richtig orthochromatic.

Farb-treue color fidelity (of color film). - umschlag discoloration, change of color.

Farbung (Schall) timbre, tone or sound color, quality.

Farbunterscheidungsvermögen color discrimination faculty.

Farb-verfahren, Linsenraster- lenticular color printing method. -wiedergabe color reproduction. -zentren F. centers.

Farnsworth'sche Bildfangröhre

F. image dissector tube.

- scher Vervielfacher F. multiplier tube.

faserformig fibrous, filiform. 
Faserung, Linsen- fibrillation of lens.

Fassondraht sectional, shaped, fashioned or profile wire.

Fassung socket, holder, mount (ing) fitting. -für Kristalle crystal (oscillator or resonator) mount or holder. -für objektiv mount or barrel of lens or objective.

Fassung, Einstell- focusing lens mount. Kristall- holder or mount of a crystal. Linsen-, objektiv-objective or lens barrel or mount. Röhren- tube socket, valve socket. Röhren-federnde cushion socket, anti-microphonic valve holder. Schneckenganghelical lens mount.

Faustregel rule of thumb, rough and ready $r$.

Fazettspiegel, Scheinwerfersegmented or facetted searchlight reflector.

Feder spring; pen, feather; (nech., corresponds to capacitance in electricity).

Feder anspannen tension, tighten or bend a spring. -entspannen relax, relieve or untension a spring.

Feder, Auslösungs- escapement spring, trip or release s. Blatt- leaf spring, flat or plate s., reed, blade. Dockenpoppet spring. Drehstab- torsion bar spring. Druck- compression spring. Gegen- counter (acting) spring, retractile s. Haupt- main spring, master s. $\mathrm{K}$ egel- volute spring. Nebenschluss- shunting spring, of fnormal s. Nut und - slot and feather (union), slot and key, groove and tongue joint. Pendel(des Zerhackers) vibrator blade, v. reed or spring (of chopper). Rückführ, Rückzugs- retractile spring, restoring s. 'Schlagimpact spring.
Schleif- wiper, slide spring, contact spring. Schnecken-coil (ed) spring. Schrauben-helical spring, coil s. Schreib- stylus, style, recorder pen, inker, scriber.

Feder, Schwing- (des Zerhackers) vibrator spring, reed s. (of chopper). Sperr-click spring. Stromstoss- impulse spring (of dial). Zentrierungs- spider (of loudspeaker coil). Zug-tension spring, traction s., retractile s.

Feder-barometer aneroid barometer. -keil feather key. -klinke spring catch. -kraft elasticity. -kraftantrieb spring motor, clockwork (motor) drive. -manometer, $\mathrm{Gl}$ asspoon-type glass manometer. -motor spring motor.

federnd springy, elastic, resilient, yielding, cushioned, flexible, supple.

federnd (er) Kontakt spring contact, flexible c., c. spring. - $-(e)$ Leitrolle spring-loaded idler. -(e) Röhrenfassung,-(er) Röhrenhalter cushioned tube nolder or socket, anti-microphonic valve holder.

Feder-raster spring catch, s. dentent. -ring lock washer. -satz spring bank, s. assembly. -spannung spring tension. -uhr, Präzisions- chronometer.

Federung, Federungswiderstand compliance, mechanical capacitance (reciprocal of stiffness).

Feder-wage spring balance, s. scale. -weg spring deflection, s. excursion or elongation. -werk spring mechanism. -zange spring pliers.

Fehl-abgleichung faulty alignment, misalignment. - anpàssung mismatching; defective focus. - anpassungsfaktor mismatching factor, reflection f., transition $f$. -anweisung incorrect indication or reading, misreading. 


\section{Fehl bauer scheinung}

-bauerscheinung mosaic structure (of metal crystals).

Fehler (cf Verzerrung) error, defect, fault, flaw, failure, distortion, aberration (in lenses), trouble (in circuits).

Fehler, chromatischer chromatic aberration. -, stroboskop ischer stroboscopic aberration. Abbildungs- defect of image, aberration. Abgleich- unbalance, balancing error, alignment error. Beobachtungs- error of observation, experimental e. Bild-picture distortion, image d. Einstell-faulty focusing, faul ty spot control, decrease of spot with intensity increase, large diffuse speck on screen, in gasfocused picture reproducing tube (telev.) Farb-, Farbenchromatic aberration, c. or color defect. Form- informality, formal defect. Geometriegөometric distortion. Geschwindigkeits- distortion due to synchronous speed differences. Glass- glass defects or flaws: striae, cords (when large), bubbles (seeds when small, airbells when of irregular shape), stones, crystallization bodies. Gleichgewichts- imbalance, unbalance. Gleichl auf- jitters (distortion caused by nonsynchronism in facsimile).

Instrumenten- variations of bearing due to instruments (df). Kugelgestalts- spot-size distortion. Leitungs- line failure or fault. Linsen- lens defects, 1. aberrations. Norddreh- northerly turning error. Offnungsapertural defect, a. effect or error. Orts- seometric location error. Peil- direction-finding error (e.g., due to night effects), distortion of bearing. Phasen- error of phase, phase distortion. Richtungs- deviation (of pencil); directional distortion (of bearing). Teilungsfaulty pitch (of an image), underlap (of lines). Tonnenbarrel distortion, positive distortion. Trapez- trapezoidal, keystone or trapezium distortion (due to dissymmetry to earth of deflection plates). Verschiebelack of synchronism, out-of-frame defect. Versuchs- experimental, error. Zerdehnungs- (eines Bildes) radial distortion of a picture or image (pincushion effect). Zerdrehungs- (eines Bildes) rotational or tangential distortion, twist (barrel effect). Zonen-, Zwischen- zonal aberration, zonal error.

Fehler-beseitigung curing or elimination of errors or of defects, "trouble shooting." -dämpfung balance attenuation, return loss. -dreieck triangle of error. -eingrenzung tracking down seat or source of interference or trouble, localizing t.

fehlerfrei free from flaws, faults or defects, faultless, unmarred, sound; aplanatic (free from spherical aberratibn), aberrationless (of a lens). -,farbachromatic. -, pol arisationsfree from polarization error (df).

Fehler-funktion,-gleichung (Gaussian) error equation. -grenze limit of error.

fehlerhaft faulty, defective, impaired, having flaws.

Fehlerscheibchen circle of confusion; apertural effect. -schutzeinrichtung fault clearing device. - sucher aniseikon (electronic crack detector), electromagnetic flaw d. (using flash magnetization), magnaflux inspection means, device used for flaw detection by $\mathrm{x}$-ray or materiology methods. 
-tiefenbestimmung stereoscopic radiography for locating defects; depth determination of flaws. -verteilungskurve error distribution curve.

Fehl-griff blunder, mistake, error of judgment; wrong manipulation. -ordnungerscheinung, thermische formation of holes (cryst.).

fenlsichtiges Auge defectively sighted eye, ametropic eye.

Fehl-sichtigkeit, Farben- color vision deficiency. -weisung, Funk- (cf Funk- Beschickung und Funktrübung) direction-finding or directional error, quadrantal error $(2 E)$, compass deviation, distortion of bearing. -winkel phase displacement angle, shift angle. -zündung failure to fire or ignite (rectifier, iǵnitron); misfiring.

Feilhalten (von Waren) delivery on sale (of goods), exposing or exhibiting for sale.

Feilhieb notch.

Feilicht, Feilsel filings, filing dust.

Fein-abstimmung sharp or fine tuning. - bewegung, Zahnrad- slowmotion gear. -blechstrasse sheet rolling mill. -einstellung vernier (dial) adjustment or tuning.

feinfühlig delicately sensitive.

Feingefüge fine structure, microstructure.

feingepulvert finely powdered, pulverized, comminuted or atomized.

Feinheit (des Bildes) detail or (degree of) definition of picture or image. Raster- raster detail, resolution or definition of scanning patterr.

Fein-neitskoeffizient fineness coefficient (of Fabry), coefficient de finesse. -höhenmesser (aneroid) barometer measuring atmospheric pressure at airdrome not reduced to sea level, sensitive altimeter. -korn fine sight; fine grain (phot.). fein-mahlen grind fine, triturate. -maschig fine-meshed.

Fein-messlehre micrometer caliper or gage. -messchraube micrometer gage screw. -rasterung highdefinition scan (of picture). -regulierung sharp tuning (of radio).

feinschuppige magnetische Struktur fine-scaled magnetic structure.

Fein-sicherung glow-tube type, etc., of lightning arrester. -stellschraube micrometer screw, vernier. -struktur fine structure, microstructure (cryst.).

feinverteilt finely divided.

Fein-verschiebung fine displacement. -wage precision balance, microbalance. -zerkleinern fine grinding or crushing, comminution, finely dividing.

feinzerteilt finely divided.

Feinzug finishing block, f. die.

Feld field, compartment, bay, section, panel, pane, frame (film pictures).

Feld, erdmagnetisches geomagnetic field. -,krümmungsfreies flattened field (by field flattening lens). -, totes dead zone (of ribbon microphone), plane of ribbon. - wöl bungsfreies flattened field.

Feld, Auskoppel- output field, coupling $f$., absorbing $f$., delivery $f$. (of a Hollmann electron turbine or inverted cyclotron), catcher $f$. (of klystron). Bild-printing plane (film printer); frame, picture or image field, picture area. Brems- reflecting field, retarding $f$. (of Barkhausen electron-oscillation tube). Eintritts- area of applicator (at skin surface, in X-ray work). Gleich- constant or steady (magnetic) field. Gravitations- gravitational field. Kenniinien-family of characteristics or curves. Lampen- bank or panel of lamps, battens (stage lighting). Längs- longitudinal 
field, paraxial f., f. parallel to axis. Lösch- magnetic obliterating field (in magnetic sound recording, telegraphone and magnetophone). Mittel-central field (of vision). Prüftest field; proving ground (ordinance). Quer- transverse or cross (magnetic) field. Rückmelde- revertive signal panel. Saug- suction or positive field, field which draws away. Schärfenfocal field. Spiegel- revertive signal panel, check-back position indicator p. (giving readings of remote instruments). Stossprüfpotential impact or impulse testing field. Stör - stray field, interference $f$. Tasten- keyboard. Teilchen- particle field. Veraleichs- matching field (in photometry). Versuchs- proving ground, field for making tests or experiments. Wellen- wave field.

Feld-ausschnitt, Bild- image area (on scanning disk). -beleuchtung, hell- bright ground illumination (micr.) -bild field pattern, configuration or map (made in electrolytic tank). -blende field stop. -durchgriff action of field through aperture lens (el. opt.). -ebenungslinse field flattener (opt.). -elektronen field electrons (resulting from emission caused by intense electric f.). -elektronenemission auto-electronic, cold or field emission (and current caused thereby). -erregerspule field (exciting) coil (in electrodynamic loudspeaker).

feldfrei field-free.

Feld-funkentelegraphie military service radio telegraphy, noncivilian wireless. -generator, Brems- negative transconductance generator or oscillator (using retarding field circuit), positive grid o. -krümmung, Bildcurvature of image field. -krümmungskorrektur field flattening. -krümmungsverzerrung distortion due to curvature or lack of flatness, curvilinear distortion. -linse field lens (anterior or front lens in telescope and in microscope). -messung plotting field map or configuration of electrostatic field (in electolytic tank); land surveying or measurement. -profil field patterr. -röhre, Zwei-drift tube norkino with two fields. -spule field coil, magnetizing c. -stärkenprofil field pattern. -stecher fieldglass magnifier. -strom field current (emission of electrons due to an electric field). -tiefe, Schärfen- depth of focus, d. of definition, d. of field. -trich-tertheorie field funnel theory. -verlauf field map, f. pattern, f. configuration. -welle field wave. -wölbung, Bild-curvature of image field, lack of flatness of image field. -zerleger, bild-picture scanner, dissector (of Farnsworth), p. exploring means.

Fell, Trommel-drumhead; tympanic membrane, tympanum, eardrum.

Fel söl petroleum.

Fenster projection aperture (film projection); window (in envelope of a cell, iconoscope, etc.); bezel (in casing or panel of radio apparatus).

Fenster, Andruck- pressure plate or gate (m.p.). Belichtungs- aperture (in aperture plate), photocell window. Beobachtungs- bezel, observation window, peephole. Bild- picture gate, aperture or trap; range-finder (m.p.). Bild-, mit zwei Ausschnitten film gate with two frames. Druck- pressure plate or gate (film). Film- film gate (of projector), film trap. Kopier-printing plane, p. station. Pendel- internal pressure plate. Projektions- projection aperture. Rollen- roller film gate. Ton- 
sound gate.

Fenster-bilder window transparencies. - blende apertural stop or diaphragm. -druck gate pressure, gate friction. führung aperture guide. -kopiermaschine step printer, intermittent p. -linse aperture lens.

fensterlose Roentgenröhre windowless $X-r a y$ machine or tube.

Fern-anzeiger telemeter (e.g., metameter of GE Co., using impulse carriers). -aufnahme telephotography, long shot. -besprechgerät remote-control unit. -bild television picture, televised p., telephoto. -bildgrossaufnahme (Ferns.) projection video picture (of large size). -blitzanzeiger keraunophone. -drucker teleprinter, teletyper, stock ticker.

Ferne, Sonnen- aphelion.

Fern-einstellung long-range focus. -glas, Doppel- field glass, binocular $§$.

Fernendunst distant fog.

Fern-hörer, Dosen-watchcase telephone receiver. Kopf- headphone, headset, "can". -kino telecine, telecast, intermediate-film system (of television). -linse telephoto lens. - meldeleitung communication or signal circuit or line. -mel dewesen, Flugzeug- aircraft communication service, avigation signal service. -messer telemeter. -messgerät telemeter (e.g., metameter of $\mathrm{GE} \mathrm{Co}$. using impulse carriers); range-finder. -messummengeber telemetric integrator. -objektiv telephoto lens. -punkt far point (opt.).

Fernrohr, Ablese- reading telescopic tube. Doppel- telescope of binocular type. Doppelblickzieldouble direct- sighting telescope. Rundblick- panorama sight. Zieltelescope sight, bombsight, rifle sight, etc.

Fern-rohrbrille telescopic spectacles. -rohrbïchse telescope tube, barrel. -rohrlinse telescopic magnifier. -schreiber telegraph, teletype, teletypewriter, telegraph printer; t. operator.

Fernseh-abtaster televisor, scanner (at sending end). -abtastung (von Kinofilm) telecine scan of (motionpicture) film. - aufnahmewagen television or electron (pickup) camera truck, video bus, televising car.

Fernsel-bild, plastisches stereocopic or three-dimensional (appearance of) video picture. -empfänger television receiver, picture reproducing device, teleview apparatus (e. ..., Zworykin's, Farnsworth's oscillight, etc.). -empfänger, heim- home television receiver.

fernsehen televise, teleview.

Fernsehen, direktes direct pickup (and viewing). Gegen- two-way television. Mehrkana! - multichannel television.

Fernseher (cf Kathodenstrahlröhre) television transmitter, video or telev. camera, televisor (e.g., emitron, iconoscope, Farnsworth dissector, etc.).

Fernseh-raster (cf Raster) television raster, scanning surface, unmodulated screen pattern.

-röhrenprüfer monoscope, monotron, phasmajector. - sender television transmitter (comprises strictly both video and aural signal transmitters). -signal video signal, picture s., telev. s. -sprecher, -telephon television telephone, video telephone. -wandlerorgane photo-cells and light-relays changing light into current and back (in telev. work), transducer means.

Fernsprech-formfaiktor telephone influence factor. -messtechnik telephonometry. -störfaktor telephone interference factor.

Fern-steuerung distance, telemetric or remote-control action (by 
Selsyn type motor). -thermometer water temperature gage. -übertragung, wettermessinstrument mit funkentelegraphischer radio telemeteorographic (or telemetric) instruments, r. sonde. -wirkung distance action, remote-control a., telemetric a. zeichnung perspective drawing.

Fertig-erzeugnis finished article, manufactured goods. -form finishing mold (glass bottle making).

Fertigung, Fliess- conveyor belt or assembly-line production, large-scale manufacture.

fest (cf beständig) solid, firm, unvarying, compact, strong, fast, fixed, stationary, tight, rigid, secure, proof, stable. -(e) Kopplung close coupling, strong c. festbacken cake together (by heat or pressure), clinker.

Festdetektor contact or crystal detector (with non-adjustable cat whisker).

fest-fressen seize, jam, freeze. -halten, photographisch record photographically (on record sheet).

Festigkeit strength, solidity, sturdiness, ruggedness, firmness, resistance.

Festigkeit, Dauer-fatigue strength. Falz-folding strength. Feuerfire-proofness, refractoriness. Gestalt- form stability, nondeformability. Kerbdauer- notch fatigue strength. Knitter- crumpling strength. Riss- crack strength, c. resistance. Spannungs- puncture potential, dielectric strength. Stossdurchschlags- impact breakdown strength. Streck- resistance to stretching or elongation. Trennungs- separating strength, static crack strength. Zerbrechungs- breaking strength, resistance to breaking strain. Zerdrückungs- crush strength, resistance to compression or crushing strain.

estkondensator fixed or non- variable condenser or capacitor. fest-machen consolidate, solidify, make concrete or firm; fasten, secure, attach. - setzen fix, settle, stipulate, determine, establish.

Festsetzung, Entschädigungs- assessing or fixing damage or indemnity.

Festsitz tight fit, snug $f$.

feststellbar determinable, ascertainable; lockable (in position), fixable, securable.

feststellen ascertain, determine, establish, state; lock (in position).

Feststellungs-klage declaratory action. -urteil declaratory judgment.

festweich semi-solid.

Fett, Vakuum- vacuum grease.

Fett-fleckphotoneter Bunsen photometer, grease-spot p. -schliff greased (ground-in) joint. -ton Fuller's earth.

Feuchtemesser hygrometer, psychrometer.

Feuchtigkeit moisture, moistness, wetness, dampness, humidity.

Feuchtigkeitsmesser hygrometer, psychrometer. -, registrierender hygrograph.

feuchtigkeitssicher damp-proof, moisture-proof.

Fever beacon, (radio) beam station, radio range.

Feuer, Ansteverungs- line of approach beacon, directional $b$. Drehfunk- rotating radio beacon, omni-directional b. Funk-, mehrstrahliges multi-beacon, triple-modulation b. Navigationsnavigation or maritime beacon, $b$. or beam station for aerial navigation or avigation.

feuerbeständig resistant to fire or heat, fire-proof, refractory.

Feuerbeständigkeit fire-proofness, ref ractoriness.

feverflüssig liquid at high temperature, molten, fused.

Fever-führung firing (schedule). 
-punkt focus (opt.); hearth (in mining). -schutztrommel safety magazine (of film projector). -stein flint. - -ton fireclay. -versilberung hot-dip silverplating. -verzinkung hot galvanization, pot g. -verzinnung hot-dip tinning.

Figur, stehende stationary pattern or figure.

Figur, Atz- etch pattern, e. figure. Beugungs- diffraction pattern, d. picture. Fliess- strain pattern. $\mathrm{Kl}$ ang- sound pattern, acoustic $\mathrm{p}$. or figure. Ring- Fresnel or Huygens zone, F. zone plate. Schlag- percussion figure. Staubdust figure, powder pattern (of Lichtenberg, Bitter, LebyeScherrer, Hull, etc.). Streckflow lines, 1. of stress, surface bands.

Film, doppelbeschichteter sandwich film, double-coated f. -,gegossener coated film. -,glasklarer pellucid film stock (of maximum transmittance). -, mehrfachbeschichteter double-coated film, sandwich f. -,plastischer stereoscopic film, plastic f. -, unverbrennbarer slow-burning film, safety f. -, verregneter rainy film.

Film, Atmen des...s in-and-out-offocus effects. Auflaufen des...s take-up (by spool) of film. Einlegen des...s threading of film.

Film, Blank- film base. Doppelschicht- double-coated film, sandwich $f$. Duppositiv- duplicating positive film, master positive. Farbenschicht- dye-coated film. Flach- non-stereoscopic film, non-plastic f., plain $f$. Gravar - film with directly engraved track. Hör- (Tonfilmrundfunk) video and audio film, sound telecine or telecasting film. Kino-, Fernsehabtastung von tele- cine scanning of motion-picture film, scanning of film for television. Klein- substandard film, narrow f. Kultur-, Lehr- educational film, cultural f., instruction or school film. Linsenrasterlenticular film, lenticulated $f$. Milieu- "milieu" film. Naturfarbnatural-color film. Präge-film with directly embossed track. Raster- lenticulated screen film. Reportage- newsreel, news film, topical f. Roh- blank film, raw stock. Rundfunkton- sound telecine, telecast (broadcasting of video and audio signals). Schmalsubstandard film, narrow $f$.

Spezial-, hoher steilheit highcontrast emulsion film. "stummsilent film. Trickton- sound cartoon. Umkehr- film resulting from developing by reversal. Vorführungs- display film. Zeichentrick- animated cartoon. Film-abheber plow (to peel film from teeth. -absatz packing, wear and tear. - abtastung film scanning. - abwickler magazine drum, feeding reel. - architektur setting and composition. -archiv film archives. - atmen in-and-out-of-focus effects. - aufl aufspule, -aufwickel spule takeup spool or reel. -bahn film track, f. channel. - bandführung aperture guide. -baukunst setting and composition. -belichtungsstelle film gate. - bewegung, bildweise intermittent film feed or movement. - bewegung, stete continuous film feed or movement. -bildschicht film emulsion, f. coat. -blitz statics (m.p. film). -doppeln f’ilm duplicating. - einfädelung, - einfuihrung threading of film. - einschnürung squeezing or compression of film (for making squeeze track). -entwickel ungsvorrichtung film processing equipment. -fenster filin gate, film trap. -fernsehabtastung telecine scan (of 
m.p. film). -fernsehsystem intermediate film television system. -fortschaltung film feed, f. motion, f. travel. -führung film feed, film guiding, f. gate. -geber film transmitter, film pickup, f. scanner, tele-cinematographic device. -justierung registration of film. -kameral aufwerk camera film feed mechanism. -kanal film track, film channel. -kern reel, spool. -kittlehre film splicing gage. -messer,-meterzähler footage counter. -methode, Zwi schenintermediate-film (pickup) method of television transmission.

-sotic, Ton- sound lens, s. optic (m.p.). -projektionsfenster aperture, gate. -registerhaltigkeit reçistration or stability of film. -reqler, film traction regulator (stabilizer). -rolle film take-up spool or reel. - rundfunk, Tonsound telecine, video and audio broadcast. - schaltung, bildweise intermittent film motion, discontinuous $f$. feed. -schaltung, stete continuous film feed or movement. -schleife film loop. -schneiden editing. - schwanz trailer run-out (piece of blank film at end). - sensitometer sensitometer, timer. - spannung film tension, f. traction. - spulenhalter film roll holder. - steilheit, hohe high contrast of film. -streifen, endloser endless film strip. -träger film base, f. support. - transport, absatzweiser intermittent film feed. -transport, steter continuous film feed. - transportrolle film (feed) sprocket. - tür film gate. -uhr footage counter. - verarbeitung film processing. -verblitzung dendriform exposure of film (due to static charges). -vertrieb release of film, distribution of $f$. - vorführung film projection; f. exposure (in gate). -vorlauf lead or precession of sound recording over pictures $=19$ frames $=361$ millimeters. -vorratsspule film magazine roll, magazine. - wagen, Ton- sound truck, location t., sound van. -wirkung merit or quality of picture. -wölbung film buckling. -zähler footage counter. -zug, Instetigkeit im flutter in film pull. -zusatzgerät, Ton- soundhead, sound film attachment.

Filter, tonrichtiger true color filter, pure c.f., orthochromatic f.

Filter, Aufteilungs- crossover network (sound production). Band-, von grosser Lochweite broad-band filter. Bewertungs- weighting network. Blau-blue filter, viewing $\mathrm{f}$. Durchlass- band-pass filter, b.-transmission f. Einzelabschnittsingle-section filter. Entzerrungsfilter-type equalizer, network e. Farbenauszug- selective filter, $s$. screen. Frequenzbereich- bandpass filter. Gitter- lattice filter (used in electric signaling). Elocken- bell jar filter. Kanaichannel filter. Kontrast- filter for selective contrasts, contrast screen filter. Kreuzglied- lattice section filter. Licht- light filter, color f. Mikro-filter for micrographic work. Nadelgeräusch- needle scratch filter. Streifen-, dreifacher tricolor banded filter. Tiefpass- lowpass filter. Trenn- dividing filter, separating f. Verlaufsky filter (of gradual action). Wellenstrom- ripple filter.

Filterabschl uss dch. 1/2 Längsglied mid-series filter termination. -dch. 1/2 Querglied mid-shunt filter termination.

Filter-durchlässigkeit, Glas-filter or glass transmittance or transmittancy. -faktor filter factor, screen $f$. -federungswiderstand compliance (of mechanical filter). -glied filter section, $f$. mesh, f. 
unit. - ochlage position of transmission range. -massenwiderstand inertance (of mechanical filter). -steilheit sharpness (of cut-off) of selecting network, slope.

Filz-kufe felt pad. unterlagscheibe felt washer.

Finger, Kontakt- contact finger, wiper.

Finger-anschlag finger stop (of dial switch). -bildner digitorium (for piano practicing). -hut(ionisierungs)kammer thimble ionizing chanber. -regel, Dreiright-hand rule, thumb rule, Fleming's $r$. -scheibe dial switch, finger disk or wheel.

Fisch-bauchantenne fish-belly antenna (mast antenna in which maximum cross-section and minimum characteristic impedance lie in the middle). -schwanzbrenner bat's wing burner, fishtail b.

Fis-Klappe F sharp key.

Fixier-flüssigkeit fixing liquid, f. liquor. -mittel fixative, fixing agent. -natron sodium hyposulfite, sodium thiosulfate, "hypo". -salz fixing salt.

fixierter Kathodenfleck des Quecksilberlichtbogens anchored spot of mercury arc.

flach flat, without contrast, wi th low gamma (picture); flat, flattopped (curve, e.g., in broad tuning).

flach werden level off, flatten out, slope, become less steep, be smoothed.

$\mathrm{Fl}$ achdruck lithoprinting.

Fläche surface, face, facet, plane, area, zone, plain, level, sheet, flatness.

Fläche, cartesische Cartesian surface. -, deformierte deformed or figured surface. - konstanten Potentials equipotential surface, isopotential $\mathrm{s}$.

$\mathrm{Fläche,} \mathrm{Abstrahl-} \mathrm{radiation} \mathrm{surface,}$ radiant s., reflecting s., pro- jector s. (loudsp.). Abstumpfungstruncated face (cr.). Ausgleich-, der Erdkruste isostatic surface (of earth). Aquipotential- isopotential or equi-p. surface or plane. Bild-image area, picture field; image plane (opt.). Brenncaustic surface (geometry). Empfänger - radiation sensitive surface; record sheet. Gleit- glide plane, slip p. (cryst.), sliding surface, slide. Grenz- interface, boundary layer, surface of contact. Grund-basal surface, base, area. Ketten- catenoid. Knotennodal surface, n. plane (opt.). Kristall- crystal face, c. plane. Leit- deflection vane (in ldspk. horn, to reduce directional property), labyrinth, baffle. Lichtverteilungs- isophote, isolux diagram, luminosity or isophotic surface or curve. Neben- secondary face. Niveau- level, surface, equipotential s., isopotential s. Potential-, konstante equipotential or isopotential surface. Prismen- prismatic surface, $p$. face. Rückstrahlbrenn- catacaustic (surface). Schärfen- surface of sharp or distinct vision. Schiebungs- slip plane (cryst.). Spalt-cleavage plane, c. face (cryst.). Stirn- end face, face, front surface. Strahl(ungs)emitting surface, emitter area. (electrons), radiator surface, radiant surface. Translationstranslation plane, slip p. Trennungs- cleavage plane, parting $p_{\text {. }}$, surface of separation. wellen-, Fresnel'sche Fresnel zone (of half-period elements).

Flächen-aberration-, -abweichung zonal aberration (opt.). - antenne sheet antenna. -anziehung surface attraction, adhesion, adsorption.

flächenartige Strombahn (cf flächenhaft) current sheet, laminar 
or areal path of current.

Flächen-ausmessung planimetering. -element (cf Bildpunkt) elementary area, unit a. -erhaltungsgesetz principle of conservation of area.

flächenförmig areal, laminar, of sheet-like nature or form.

Flächengl imml ampe glow-lamp or neon 1. with plate-shaped cathode, plate neon 1 .

flächenhaft areal, laminar, involving two-dimensionality, of sheet or plane form. - (e) Berührung surface or areal contact (as in osculation). -(e) Spule laminar or strip-like coil, non-filamentary $c$.

Flächen-helle, -helligkeit luminous brightness of a surface, intrinsic brilliance. -inhalt surface area. - integral area integral (of a surface). -kathode plateshaped cathode, larǵe-surface c. (as distinguished from cratershaped, punctiform orfilamentary c.). -l ampe, - leuchte bank or pillar of lamps or luminous sources, battens. -messer planimeter, integraph (for curves). -potential, Grenz- interface potential.

flächenreich polyhedral, with many faces or sides.

Flächen-satz theorem of conservation of areas. -stück area element, unit a., elementary a.

flächentreue Projektion equal-area projection (in mapping).

Flächenwinkel plane angle. -winkel, Grenz- interfacial angle (dihedral between two crystal faces).

-zeichnung, Spalt- cleavage plane marking, crystal m.

flächenzentriert face-centered (cryst.).

$\mathrm{fl}$ acher Frequenzgang flat frequency characteristic (free from peaks and dips).

Flach-film plain film, non-plastic f., non-stereoscopic f. -gewinde square (screw) thread. -heit (eines Bildes) flat or noncontrasty quality or condition of a picture or image.

flächig...f faced, areal, laminar.

flächig, drei- trihedral, three-

faced. viel- polyhedral, many-

faced. vier- tetrahedral, four-

faced. zwei-dihedral, two-

faced.

Flächner, halb-hemihedron. Vollholohedron.

Flach-spule flat coil, pancake $c$. -streicher flat squeegee, strickler.

Flackereffekt flicker effect.

flackern flicker, flare, flash.

$\mathrm{Fl}$ ackerrelais flashing relay.

$\mathrm{Flageolettöne} \mathrm{harmonics.}$

$\mathrm{Fl}$ ammbläser blasting magnet, blowing $\mathrm{m}$.

Flamme, leuchtende luminous flame. -, nichtleuchtende roaring flame. Lock- pilot flame. Lötrohrblowpipe flame.

flammenbeständig flame-proof, f. -resistant

Fl ammenbogen flame arc, electric a. -erdschluss arcing ground.

flammenfarbig flame-like, flamecolored.

Flammen-mikrophon flame microphone, f. transmitter. -spektrum flame spectrum.

flammicht watered (of fabrics), veined or grained (of wood).

Flammpunkt flashpoint, ignition p., kindling $p$.

Flanken-spiel backlash (of screw thread). -steilheit (cf Filtersteilheit) width of transition interval (between transmission and attenuation bands); steepness of sides or slopes of a curve, or of upstroke and downstroke of an impulse. -streuung side leakage, s. stray, end s.

$\mathrm{Fl}$ ansch, Kühl- radiator vane, cooling vane. 
Flasche, Kugel- balloon flask; spherical flask.

$\mathrm{Fl}$ aschenzugbügel haken block shackle.

Flattereffekt flutter offect (called พоพ ow when changes per second are less than 6, flutter 6-30, gargle $30-200$, and whiskers when over 200 ps.)

flattern flutter, flicker, wave.

Flatter-russ lampblack. -widerhall multiple reflection (between two parallel surfaces), flutter echo.

flau flat, without contrast (c. of ten diminished by white tint permeating whole picture); limy or blooming condition, fuzzy (of picture); flattened or flat (characteristic or response).

flau werden flatten out.

flechten plait, twist, braid, interweave.

Fleck, Kathoden-, fixierter anchored cathode spot (of an $\mathrm{Hg}$ arc). Lave- Laue spot. Licht- spot (in scanning); hot spot (of film). Reflexions- flare ghost, f. spot. flecken spot, stain, speckle, mottle, patch.

fleckenemp findlich susceptible to staining or spotting.

Fleckentheorie patch theory (of metals).

Fleck-schärfe (sharpness of) spot focus. - Verformung, -verzerrung spot distortion.

flicken repair, patch, mend.

Flickerpeilung Robinson flicker direction-finding method, make and break, loop reversing or switching method.

fliegen, gegen den wind fly up wind. -mit dem Wind fly with tail wind.

fliegende Kathode floating cathode.

Flieger-aufnahme aerial picture, airplane p. or photo. -horizont artificial horizon. -visier anti-aircraft sight.

Fliehkraft centrifugal force, flywheel $f$. -regler centrifugal governor.
Fliess-behinderung inhibition of plastic deformation. -bereich plastic range. -eigenschaften rheological properties.

Fliessen, plastisches viscous flow, streamline $f_{.}$, laminar $f_{\text {. }}$, steady f. -, Wi ssenschaft der Verformung unter rheology.

Fliess-feder fountain pen. -Fertigung production on conveyor belt or assembly line. -figur strain figure. -grenze flow limit, yield point. -körper fluid or liquid body or substance, non-solid. -verzug yield or flow distortion or deformation. -vorgang flow process.

Fl immer, Zwischenl inien-, Zeileninter-line flicker, shimmer, weave (telev.).

flimmerfrei flickerless. - (e) Verschlussblende non-flicker shutter. - (e) Wiedergabe flickerfree reproduction.

$\mathrm{Fl}$ immerfrequenz fusion frequency, no-flicker $f$, , critical flicker $f$.

fl immern flicker, glisten, glitter, sparkle, scintillate.

Fl immer-peil ung loop reversing or switch method, Robinson flicker d. f. method. -photometer flicker photometer.

flittern glisten, glitter, sparkle, scintillate.

flocken flake, form flakes or flocks, flocculate.

Flödel purfling (mus, instrument).

Flöte flute, flageolet. -mit Stimmzug flute with tuning slide.

Flöte, Harmonie- organ accordion. Liebes- flute d'amore. Papagenopandean pipe. Piccolo-, Pickelpiccolo flute. Stimm- tuning or pitch pipe. Stock- stick flute. Terz- third flute. Trommel- fife. Fluchtebene vanishing plane. fluchtgerecht truly aligned or flush (with).

Fluchtl inientafeln alignment charts. flüchtig volatile, fugitive, tleeting, 
transient, cursory, superficial, evanescent.

Flüchtigkeit volatility, fugacity, evanescence, fleetingness.

Fluchtpunkt vanishing point.

fluchtrecht flush, aligned, in true alignment.

Flug, Gehörziel-aural indication or reading of homing device, aural method. Schleifen- ("U") turn, loop. Sichtziel-visual homing flight.

Flug-bahn path of flight, trajectory -bahnbild trajectory diagram.

Flügel wing, vane, flap, lobe, blade.

Flügel, Abdeck- masking or shutter blade or wing. Beruhigungs- intermediate blade, anti-flicker blade, balancing blade. Radiometer- radiometer vane. Zwischenintermediate blade, anti-flicker b., balancing $b$.

Flügel-horn bugle, vocal horn. -messer vane-type fluid meter. - mutter winged nut, butterfly n., thumb $n$.

$f l$ ügeln wing.

Flügel-rad screw wheel, worm w., $\mathrm{s}$. propeller. -schraube wing or thumb screw or nut.

Flug-fernmeldewesen avigation or aerial navigation signal service. - funkdienst aeronautical radio service. - geschwindigkeit rate of flying speed, traveling velocity (electrons, etc.).

Flughafen airport, airdrome, landing field, flying $f$. Verkehrscommercial airport, civil a. -befeverung airport beacon service. -grenze airport boundary, a. border line.

Flug-leiter, Boden- ground control operator. -leiter, Peil- ground direction-finding operator. ort (flying) position (of craft). -probleme avigation, aeronatical or flying problems. -sicherung avigation or aerial navigation safety, air communication safety. -strecke airway, avigation route. -wesen aviation, aerial navigation, avigation, aeronautics.

Flugzeug, anlaufendes approaching airplane. -, schwanzloses tailless airplane. Nurflügel- tailless airplane.

Flugzeug-abhörapparat airplane sound locator or detector. - aufnahme aerial photograph, airplane $p$. - aufsatzpunkt ground contact point, landing point. - befehl sübertragung inter-aircraft voice or command communication. - führer pilot; navigator, avigator. -führung avigation or piloting of an airplane. - gleichgewicht balance or trim of an airplane. -längsachse fore-aft line of airplane, major axis. -leitung aircraft guiding or guidance, avigation, piloting. - navigation aircraft guidance, piloting of aircraft, avigation. - propeller, Versetz- variablepitch (feathered) airscrew or propeller. -schieben crabbing of an airplane. -stützpunkt airplane base, base airport. -versetzung drift, lateral displacement or shift (in course) of an airplane, deviation from course, yaw.

Flugziel flying course, airplane neading or head (towards destination), home.

Fluoreszenzauslöschung fluorescence quenching, evanescence, f. extinction (e. g., by poison).

fluoreszenzerzeugend fluorogenic.

Fluoreszenz-messer fluorometer; phosphoroscope (measures decay). -strich fluorescent screen tracing or line. -unterdrückung quenching of fluorescence, suppression or killing of $f$.

fluoreszieren fluoresce, scintillate (when excited by $\alpha$ rays), luminesce.

Fluoritlinsensystem semi-apochromatic lens system.

Fluss flow, flux, circulation, stream, 
current, drift (of electrons).

Fluss, Streu- leakage flux, stray flux. Windungs- turn flux, flux turns, magnetic linkage.

flüssig liquid, fluid, non-solid, aquiform. -machen liquefy. -werdend liquescent.

flüssig, feuer- liquid at high temperature, wolten, fused. streng-viscous, semi-fluid, difficultly fusible.

Flüssigkeits-dämpfung liquid or fluid damping or dasn-pot action. - dichtemesser hydrometer, liquid density meter, areometer. -l inse (oil or water) immersion objective. -prisma liquid prism.

Fluss -mittel fluxing material, flux. -richtung low-resistance or forward direction (of a dry or oxide rectifier, e. . $_{\text {. }}$-spat fluorspar, fluorite. -verkettung flux linkage.

Flutlicht floodlight.

Fokustiefe depth of focus, d. of field.

Folge sequence, series, succession, consecution, future, consequence, result, conclusion, sequel, compliance.

Folge, Bild-series or sequence of optical or pictorial actions. $\mathrm{KI}$ ang- sequence or series of acoustic actions. Linsen- lens combination, system of lenses. Reihen- sequence; consecution, succession, series, order. Tonseries or sequence of acoustic or sound actions.

Fol ge-kern, Nacil- product nucleus. -kontakt make-before-break contact. - produkt metabolons, products of successive disintegration of radio-active parent material. -punkt consequent points or poles (magn.) -reaktion consequent reaction, secondary $r$. -rohr piloted thyratron (fires second in a trigger circuit). -rung deduction, inference, conclusion, induction, corollary. - schal terkamm sequence switch cam Folie, Träger- supporting foil. Fol iendurchinesser record (disk) diameter.

Forcierkrankheit strain disease (metals).

Förderband, jandförderer band or belt conveyor, c. belt.

Fördermechanismus, Platten- plate feed mechanism (of electron microscope).

fördern convey, transport, feed, haul; boost, promote, favor, further, advance, nelp, hasten.

Form, Fertig- finishing mold (glass bottle making). Mutter-master mold (cer.), parental form. Press- pressure mold. VorEathering mold (glass manuf.). Zwischen- passage type.

Formänderung deformation, distortion, strain, warp.

Formänderungsfähigkeit (plastic) deformability, rheologic or flow property.

Formant narmonic determining timbre or tone color, formant. hallresonance formant. naupt- main or basic formant. Unter- sub-formant Format, bild-picture or frame size or format.

formbar vlastic, capable of being plastically shaped or fashioned, plastically deformable or formable, moldable, fictile.

Formbeständigkeit stability of shape, non-deformability.

Formelbild structural formula. Formeln, Ausschuss für Einheiten und (AEF), Committee on Units and Stardards, Standards Committee. Raum- spatial formulae.

formengleich conformal.

Form-faktor aspect ratio (picture). -faktor. Fernsprech- telephone influence factor. -fehler formal defect, inf ormality. 
-festigkeit stability of shape, non-def ormability. -gebung fashioning, shaping, molding, imparting shape by outside action (e. है., pressure). -gebung, spanabhebende (und spanlose) shaping or fashioning by machining with (and without) removal of material by cutting tools (in the form of chips, etc.)

form-gerecht, -getreu true to form or shape, undistorted, having fidelity, faithful (e.g., in amplitude or phase of signals). Formierung forming (of a valve or cell); activation, sensitization.

Formling molded article or blank. formlos amorphous, formless, noncrystalline.

Form-mangel abhelfen oder heilen remedy or cure formality defect. -maschine, Rüttel- jolt molding machine, jar ramming $\mathrm{m}$.

formschlüssig form-closed or -locking.

Form-stich shaping pass (in rolling). -stück, Ton-, mehrzïgiges multiple tile or clay conduit.

Formulierung, Strich-chemical formula using dashes or lines to represent bonds.

Formzlffer shape factor; theoretical stress concentration factor.

For schungs-anstalt research institution. -gebiet research field or domain. -geist inquisitiveness, inquiring or searching spirit.

Fortdiffundierung diffusing away.

Fortepedal forte pedal, loud $p$.

fortführen carry away, convey; continue, prosecute (a case).

for theben, sich cancel out or eliminate (a factor).

fortlaufend sequential, continuous, uninterrupted, non-intermittent. -(e) Reihe unbroken, uninterrupted or continuous sequence or series.

Fortpflanzungs-geschwindigkeit velocity of propagation. -konstante propagation constant, transfer c. -mass (hyperbolical) line angle.

Fort-rückung translation, advance, feed, shift, removal, moving away. -schaltrelais stepping or impulsing switch or relay. -schaltung, Film- film feed, travel or movement. -schal twerk stepping mechanism.

fortschreitende Welle progressive wave, advancing $w$.

Fortschreltungs-bewegung translational movement, stepping, propagational m. -geschwindigkeit speed of progression, s. of travel.

Fortschritt advance, progress, improvement, advancement (in an industrial art, etc.)

Foto... see Photo...

Fourier'sche Intergralzerlegung Fourier integral seriation.

Fourierreihe, doppelte double Fourier series.

Frage-bogen questionnaire, list of queries. -recht right to crossexamine. -stellung interrogatory.

Fransen fuzz, fringe. Beuqungsdiffraction fringes.

fräsen cut, mill, face.

frei, drehungs- irrotational, rotation-free. farbfehler-achromatic. funken- non-sparking, non-arcing, arcless. kohle-carbon-free, free from carbonaceous admixture. polarisationsfehler- free from polarization error (in df, etc.). ...frei -less, -free, non-, an-, free from, devoid of.

frei(e) El ektronen (Leitungselektronen) free electrons, conduction e. -(er) Platz nole (nuclear theory).-(e) Schwingung free oscillation. -(e) Weglänge, mittlere mean free (length of) path.

Frei-antenne outdoor antenna. - fallbogenl ampe gravity-feed arclamp. -gabe unblocking, release, disengagement, opening, clearing, liberation. 
Freiheitsgrad degree of freedom. -zahl variance (of a system).

Freilauf-bremse free-wheeling brake, coaster b. -kupplung freo-wheel clutch, slip coupling.

Frei-lichtaufnahne outdoor or exterior shooting or shot.

- luftdurchfuhrung open-air wallduct, leadin out-door bushing (insulator). -luftisolator outdoor insulator. -schwinger free radiator (loudspeaker).

freitragend self-supporting, s. - supported.

Fremdbestandteil foreign, extraneous or external matter, material or substance.

fremderregt (er) Magnet electromagnet, non-permanent $m$. -(er) Schwingröhre, - (er) Sender master-excited oscillator or transmitter.

Fremd-erregung separate excitation. - gas foreign gas.

fremdgesteuerte steverstufe crystal-stabilized master stage. -unselbständige Kippmethode distant-operated non-self-running time-base method.

Fremd-körper foreign or extraneous body or substance. -lichtLichtmodulator light relay of Kerr cell or Karolus c. type (to re-convert currents into brightness variations with constant, independent ray source). -peilung ground direction-finding (bearing or directional data supplied to airplane from ground stations). - schichtverfahren self-luminous or s.-emissive method (using electrons on surface of object, in electron microscope). -töne alien frequencies or tones (in sound reproduction).

Frequenz, mittlere medium frequency, mid frequency. -technische commercial frequency, industrial $\mathrm{f}$.

Frequenz, Abschneide- cut-off frequency, critical f. Abtastscan frequency (= lines $\mathrm{x}$ frame frequency per second).

Beruhigungs- no-flicker frequency, fusion or critical f. (m.p.). Bild-picture frequency, frame $f$., video f., visual f. Differenzdifference frequency. Durchdringungs- penetration frequency, critical f. Durchgangs- pass wave, p. frequency. Ecken- corner frequency, sharp cut-off frequency (of a filter, etc.). Einheits- standard frequency. Grenz- cut-off frequency, limiting $f$. Gruppen- group frequency (number of wave trains per second), sparkf. Hilfs-, Rückkopplung mit super-regeneration. Kreis- pulsatance, angular velocity, cyclic frequency, frequency in radians $(\omega)$. Leitkreistransit-time frequency (magnetron). Mittel- medium-wave frequency, mid freq. Mutter-master frequency. Null-frequency at which phase shift is zero. Pendel- electron oscillation frequency; quench or bias $f$. furnished from auxiliary oscillator circuit (in superregeneration). Raster-, Rasterwechsel- field frequency (in interlaced scanning). Resonanzresonance frequency, natural $\mathrm{f}$. Schwankungs- frequency flutter. Schwellen- threshold or critical frequency, photo-electric threshold. Seiten- side frequency (one of sum or difference frequencies). Spiegelimage frequency. Sprech- voice frequency, speech $f$. Steuerpilot or synchronizing frequency. Summen- summation frequency, sum $f$. Teil- component frequency. Trenn- 
cut-off frequency. Überhör- supersonic, ultrasonic or ultra- audio frequency. Umlauf- rotational frequency (electrons). Unterhörsubaudio or infrasonic frequency. Unterresonanz- submultiple resonance frequency, subsynchronous $f$. Verschmelzungs- critical flicker frequency, fusion $f$., no-flicker $f$. Wach- watch frequency, distress $f$., $\mathrm{f}$. of international automatic alarm signal. Wellenzug- group frequency, wave-train f., spark $f$. Well igkeits- ripple frequency. Wiederhol ungs- repetition frequency, $f$. of recurrence. Winkel- angular frequency, radian f., pulsatance. Zeichen- signal frequency. Zeilen (wechsel)- line frequency, strip f., horizontal scan $f$. Zwischenintermediate-beat or transfer frequency (in superheterodyning).

Frequenz-abbau frequency division, $f$. submultiplication. -abgrenzung s. Wel lenabgrenzung,- abhängigkeit frequency dependence, having a $f$. effect, condition of being affected by, or a function of, frequency. - abstand interference guard band, tolerance frequency. -atweichung frequency deviation, drift or swing, lilt (slow fluctuation). -amplitude amplitude proportional to frequency. - bereichfilter band-pass filter. - bereichüberstreichung durch

Kondensator frequency range or band swept, scanned or covered by a condenser or capacitor. -Entdämpfungskurve frequency-gain curve. Frequenzgang frequency characteristic or response curve, influence of frequency, $f$. effect. -, $\mathrm{fl}$ acher flat frequency characteristic (without peaks or dips). - der Amplitude, starker marked dependence of amplitude upon frequency. -normung standardization of frequency response curve.

Frequenzoemisch frequency spectrum. frequenzgerader Kondensator straight line frequency condenser.

Frequenzgerät, siliben- syllable vodas (Bell Co.).

frequenzgesteuerte Senderöhre frequency-stabilized transmitter tube.

Frequenz-hub frequency fluctuation, swing or variation (between two limits); f. deviation (above and below the assigned center or resting $f_{.}$, in $f$. modulation), amount by which instantaneous carrier $f$. differs from r.f. -kennlinie frequency-response characteristic. -kurve, Entdämpfungs gainfrequency curve.

frequenzmässig, hoch- for high frequency, so far as high frequency is concerned, relative to hf.

Frequenz-messer frequency meter, wave $\mathrm{m}$., ondometer, sonometer. -messer, Zungen vibrating reed frequency meter. -modulation frequency modulation. -reihe series of frequencies. -schnitt cross-over of frequencies (in sound film production); point where wave-bands handled by loudspeakers intersect. - schwankung lilt (slow fluctuations of frequency), drift of $f_{.}$, f. flutter, f. departure, swinging (momentary variation of received $f$.). - schwankungsschwächer discriminator (converts drift into direct potential difference). -selektion frequency selection. - sieblochbrei transmitted band, band-width of a filter, spacing between cut-off points. -skal a frequency spectrum, f. scale. -sperre, Spiegel- image frequency stopper. -steuerung (von Senderohrschwingungen) frequency stabilization (of transmitter tube oscillations).

Frequenz-teiler (cf Frequenzweiche)

frequency divider, f. submultiplier. - libersetzung fraquency transformation.

frequenzunathängig free from frequency 
effect, independent of $\mathrm{f} ., \mathrm{f}_{\text {.- }}$ independent.

Frequenz-unterdrückung, Spiegelimage frequency stopper, image frequency rejection. - unterscheidung frequency discrimination. -verdreifacher frequency tripler. -verschiebung frequency distortion, tone d.-verwerfung slow frequency drift (in transmitter or receiver apparatus occurring after tuning). -verzerrung attenuation-frequency distortion, amplitude-f.d., attenuation d.,f. distortion. -wandlung heterodyne or superhet, action; frequency change, $f$. transformation. -weiche dividing filter or network, separator (sync and video signals, in telev.) comprising limiter tube and network, -wiedergabe frequency response characteristic.

Fresnel's Mitführungskoeffizient Fresnel coefficient of drag. fressen seize, bind, freeze, attack, corrode:

freundlich, licht- photophilic, photophilous, favoring highlights over shadows.

Freyagerät German version of radar or radio locator.

Friscn-arbeit fining process (metals). -aufnahme new recording, re-recording or new pickup of film (sound track film). -wirkung oxidizing or purifying reaction.

Frist grace, limitation, respite, term, (fixed) time allowance or extension. moratorium.

fristgerecht in time, within time limit.

Frist-gesuch dilatory plea. -gewährung grant of respite. -verlängerung time extension, respite, grace.

fritten frit, sinter, concrete.

Frittporzellan soft porcelain, frit $p$.
Front, Wellen-, geneigte tilted . wave front or wave head.

frontale $\mathrm{H}$ al bkugell inse hemispheric front lens.

Frontwelle onde de choc, impact wave, bow wave (which precedes a projectile).

Frosch cam, dog, adjustable stop, arm; frog (of violin bow). - perspektive worm's eye view.

frostbeständig resistant to frost, freeze-proof.

Frostpunkt freeze point.

Frühzuindung premature ignition, pre-ignition.

$F$ S $P$ see Funkseitenpeilung

Fuge joint, junction, slit, groove, crack.

Fuge, Dehnungs- expansion joint. Schweiss- welding seam or line, weld, shut. Stoss- joint, junction.

fühlbare Abstimmung tuning condenser sensibly detained or braked on passing carrier, "lazy tuning".

Fühler-kondensator pickup condenser, scanning c. -lehre thickness gagé.

Fühlhebel tactile, feeler, contactor or probing lever, scanner, pickup means; indicator of micrometric caliper.

fühlig, fein-delicately sensitive. FühInadel selecting needle or pin, pecker. Kugelspitzen- scanning or pickup needle with spherical point (sound record).

Fühlratsche micrometer friction thimble.

führen lead, conduct, guide, pilot, steer, channel, carry (current), support, hold.

Führerstand (Flugzeug) pilot's cockpit, p. compartment (airplane).

Führung, seitliche lateral guidance (of airplane).

Führung, Fenster- aperture guide, gate. 
Film- film feed, f. guiding, $f$. gate. Nach- (des Rahmens) resetting or re-adjustment (of frame). Nieder-downlead. Rückreturn path, return circuit, $r$. wire, r. lead. Schmelz- smelting practice, conduct of heat, smelting schedule. Seiten-(von Film) lateral guidance, side guiding (of film). Wellen-wave guide (either conducting or dielectric). Fuhrungs-band control strip (of a printer). -kanal film track, $f$. channel. -loch (guide) perforation (for engagement of sprocket). -rille track or groove (of phonograph record). rolle sprocket wheel (of film feed). -schlitten slide (carriage).

Fülle, Klang- sound volume.

Fill-faktor space factor, activity coefficient. -flüsigkeit immersion liquid (microscope). -körper filling body, packing material. -kurve single-loop oscillogram. -mittel filler, filling, stuffing or loading material or compound. - perspektive plenary perspective -strich filling mark, gage m. or line.

fünf-dimensional in terms of fivedimensional world. -eckig pentagonal.

Fünferalphatet five-unit code.

Fünfflach pentahedron.

fünfgliedriger Mischer five channel mixer.

Flinf-pol röhre pentode (either power pentode or screen-grid $p_{\text {.) }}$

-strömealphabet five-unit code.

-stufenschwächer five-step weakener (phot.)

Funk, Bild- radio picture, wired picture, facsimile transmission.

Funkazimuth, rechtweisender oder geographischer true bearing. ,- missweisender oder magnetischer magnetic bearing.

Funkbake radio beacon, radio range (sometimes marker beacon).
Funkbake, Kurs- radio range or beacon. Landungs- landing beacon. Richtungs- (zum Anfliegen) runway localizing beacon, terminal marker beacon (used in approach procedure).

Funk-beamter radio station official, board radio operator. -beschicker, automatischer oder mechanischer automatic (radio) quadrantal error compensator, cam compensator. - beschickung (cf Funktrübung) goniometric error or directionfinding deviation and its correction, clearing or compensation; shift of minimum or zero point; (local) calibration of radio compass.

Funkbeschickungs-aufnahme calibration of direction-finder. - drahtschleife QE (quadrantal error) compensating or clearıng loop. -kurve error curve. -kurvenscheibe compensator cam, zero-clearing $c$.

Funk-besteck radio fix. -bild photo-radio, radio picture, wired p., radiophotogram. -drosselspule air-gap reactance coil.

Funkeleffekt. sparkling, scintillation, glitter; low-frequency effect of local emission density (of electrons).

funkeln sparkle, scintillate, glitter.

Funken, Gleit- slide spark, creepage s. Öffnungs- break spark, sparking at break. Schliessungs-make spark. Unterbrechungs- break spark, wipe s., circuit-opening s.

Funkenent 1 adungs-Aufbauze it formation time of spark discharge.

funkenfrei sparkless, non-sparking, non-arcing.

Funken-geber spark coil, Ruhmkorff c. -kanal spark track. -linien spark lines. - löscher spark quench, blowout or extinguisher, s. killer. -mikrometer micrometric spark-gap or spark-discharger, spintharometer: -sender, Ton- 
singing or musical (quenched) spark transmitter.

Funkenstrecke, Kugel- sphere (spark) gap, ball gap, Löscnquenched spark gap. Nadelneedle (spark) gap. Reihenmultiple spark gap. Schutzspill gap. Ton- quenched gap. Uberspannungs- surge arrester, s. gap, s. absorber.

Funkensystem, Takt- timed spark system (wireless transmission).

Funkentstörer static eliminator.

Funken-überschlag spark breakdown, flashover, sparkover. -verzögerung spark lag. -zähler spark counter.

Funk-fenlweisung radio af error, directional e., quandrantal e. ( $\left.2 E^{\prime}\right)$, compass deviation, distortion of bearing. -feuer radio beacon, radio range; marker beacon.

Funkfeuer, mehrstrahliges multi (-ray) beacon, multiple-or triplemodulation beacon. Dren- rotating radio beacon or range. Hauptmain route or airway radio beacon. Kreis- rotary beacon, omnidirectional beacon, circular navigational beacon (sending non-directional signals in all directions). Landungs- landing beacon (for instrument or blind landing). Richtdirectional beacon station or transmitter s. Strecken- (air) route beacon.

Funk-feuerkennung call signal or distinctive signal of a beacon station. -freund radio amateur, $r$, fan, ham, -geber radio transmitter; spark coil (obs.). -horchdienst radio intercept service. -mutuna radio prospecting, $r$. metal locating. -ortung radio position finding, obtaining $r$. fix.

Funkpeil-scheibe bearing plate of radio df. - schwund radio fadeout or fading. -sektor approach sector. - station, -stelle radio direction-finder station or post (on ground or on craft). -stationmittellinie center line of approach sector.

Funk-platte spark plate (condenser of automobile radio). -querverbindung intercommunication channel. -rufzeichen radio call, code signal, "signature" (of a station). -schatten radio shadow, dead spot, pocket. - schneise radio beacon course, equisignal track, corridor of approach. -schwund fading, fadeout (of radio signals).

Funkseitenpeilung, beschickte od. verbesserte true or corrected radio bearing. -, unverbesserte oder abgelesene uncorrected or read radio bearing.

Funksender, Einpeilen eines Richtempfängers auf einen tuning in a transmitter station with directional receiver, to take bearings. Leit- radio range, $r$. beam station, $r$, beacon.

Funkspektrum see Well enabgrenzung Funkspruch, geschlüsselter radio code message, coded $r . m$. - spruchweg radio channel.

Funkstelle, Boden- aeronautical ground radio station, g. direction-finder s. Einstrahl- beam station. Gross- high-power radio station, long-distance or long-range s. Land-ground radio station; shore or coast $\mathrm{s}$. Leitnet control station. Luft- aircraft radio signal station.

Funktechnik radio engineering, $r$. art; $r$. communication or signaling art. -techniker radio technician, r. engineer.

funktelegraphi sche Verbindung radio telegraphic connection, r. signal communication (channel).

Funktelephon radiophone.

Funktion, konjugierte conjugate function. Ausgangs- trial function. Bessel- erster art 
Bessel function of the first kind. Eigen- eigen function, characteristic f. Einheits- unit function (of Heaviside). Hyperbelhyperbolic function. Kraftpower function. Kugel- spherical function. orts- position function. minkel- trigonometric function, angular $f .$, function of angles. Funk-trübung uncleared zero, blurring or lack of precision of minimum or zero (in radio d.f. or bearing). -verkehrsbezirk radio controlled aerial navigation (or avigation) district or zone. -wechsel sprechen alternate. twoway radiophone communication. -wesen radio technology, $r$. signaling art.

Furche furrow, channel, wrinkle, ruling or line (of diffraction grating); groove or track (with undulations, of phonograph or gramophone disk).

Fuss pinch, press, squash, stem or foot (of a lamp, tube or valve). -,umgestuilpter re-entrant squash or press. Strahlerzeuger-gun press (of c. -r. tube).

Fusskreis root line, dedendum 1. fusslose Röhre loctal tube. Fussplatte base plate (camera). Fussounkt foot (math.), nadir (point opposite the zenith, in astronomy). -, Gegen- antipode.

Fusspunkt-kurve pedal (or podal) locus curve. -linie podal line. -widerstand terminal impedance or base-loading (of antenna).

Futter, Mantel- shell lining. Fütterungsstoff lining material. 
Gabe, Impuls-, Stromstoss- pulsing, impulsing, emission or transmission of impulses. Impuls-, rückwärtige revertive pulsing. gabelartig forked, furcated, bifurcate.

Gabel-kl ammer forked clamp. -umschalter hook switch. -zinken, Stimm- tuning fork prong or tine. Galgen, Mikrophon- microphone boom. Galle, Gl as- glass gall, sandiver. Galtonpfelfe Galton pipe.

galvanisch galvanic, conductive. galvanische Kopplung resistance coupling.

Galvanometer, kompensiertes rneograph (of Abraham) in which inertia and damping are compensated. Bändchen- twisted strip or band galvanometer. Fadenstring galvanometer, vibrating $g$. Saiten- Einthoven 8. Stoss- ballistic galvanometer.

Galvanostegie galvanoplastics, electroplating, electrodeposition. liamma, Kopie- negative gamms. liang motion, working state, gear; course, passage, pass, travel, stroke; trend, drift; heat run; thread or spire of screw; action (of camera); function, dependence, variation, response (characteristic).

Gang, toter lost motion, backlash, play. -zügiger intimate or positive threading of gear (without play or backlasn).

Gang, Chargen- heat run. Dämpfungsfrequency response of attenuation, attenuation-frequency characteristic. Frequenz- frequency characteristic, frequency-response curve, effect of frequency. Fre- quenz-, starker, der Amplitude, marked dependence of amplitude on frequency. Gefälls-gradient variation. Gleich- unison, synchronism. Min-forward motion or working stroke, travel, pass or journey, scansion (telev.) Hin- und Her- reciprocating motion, rocking movement. Ortslocal variation. Rück - return stroke, retrace or flyback (of pencil); decline. Selbstautomatic feed, operation or action. Strahlen-path of rays, ray tracing, geometric configuration of rays. Tages-, örtlicher local diurnal variation. Temperatur - temperature dependence. Vorwärts- direct action (of camera)

gangbar salable, marketable; practicable.

Ganghöhe, windungs- pitch of turns. gängi g.....thread(ed), pitched. gängi g, l inks- left-handed, counterclockwise. rechts- clockwise, right-handed. scharf- (screw cut) with triangular thread. Gangunterschied path or phase difference (of waves, rays, etc.) ganz whole, entire, total, complete. -(es) Vielfaches integral multiple. - (e) Zahl integer, whole or integral number.

Ganz-heit totality, whole, aggregation. -metallwand all-metal (projection) screen. -polkegel polehode cone, body cone. -ton whole tone.

ganzzahlige Ladung integral spin, charge.

Ganzzahligkeit integralness, property of being an integer. 
Gar-brand finishing burn (ceramics). -brenne fired to maturity (ceramics). Gare refined state, finished s. Garnierung bushing, lining. Garnitur fittings, trimmings, mounting. Gasabgang gas issue, withdrawal or offtake, g. delivery.

gasartig gasiform, gaseous.

Gasaufnahme occlusion or absorption of gas (in metals). -aufzehrung gettering, absorption of gases. -ausbruch gas eruption, (violent)

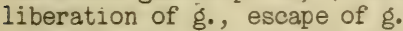
-austreibung. gas cleanup or expulsion, outgasing, degasing, gettering. gasbehaftete 0 berfläche gas contaminated surface, s. with adsorbed g. (skin).

Gas-bindung gettering. - bl asenbildung cavitation, occlusion of gases (in metals, etc.). -dichtemesser dasymeter. -entl adungsrohr gas discharge tube or valve. -entwicklung evolution, disengagement or giving off of gas.

gas-formi g gasiform, gaseous. - gefull te Röhre gas-content tube, gaseous t., gassy t.

Gas-konzentrierung bas focusing, ionic f. (in c. r. tube). - schalter, Druck- (cross) airblast switch. - schmelzschweissung gas torch autogenous welding. -strecke gaseous path or gap (in a tube). - verschluss gas seal, bell and hopper, cup and cone arrangement (furnace). -wage gas specific gravity balance. -zelle gas-filled photo-emission cell.

gattieren classify, sort.

Gattung kind, genus.

gaufrieren goffer, emboss.

Gaze, Draht- wire gauze, mesh or cloth. gebend s. erzeugend

Geber, Bild- pickup camera. Filmfilm transmitter, pickup or scanner, telecinematographic or film scanning device. Funk- radio transmitter; spark coil (obs.) Funken- spark coil, Ruhmkorff c. Impuls- impúlsing device, impulse transmitter; tonal generator, tonic transmitter. Kontakt- contact maker, timetapper or marker. Kristall taktcrystal monitor, c. stabilizer. Takt- impulsing means, impulser; time beater, t. tapper, metronome.

Gebiet, Druck- pressure or compression area or region. Einzugs- cross-sectional area of undistorted portion of field (re: df frame).

Empfangs- service area (broadcasting). Interferenzmush area (chain broadeasting). Orts- local area. Reiss- discontinuity or breakof $f$ region (in oscillations). Störmush area (in chain broadcasting), disturbed area or zone. Gebilde structure, system, organization, form, image.

Gebilde, Gewebe-, winzige ultramicrons, (of eye tissue). Leiter- conductor structure, c. system, array. Leuchtluminous pattern (lines, bands, or continuous spectra). Luftleiter-aerial extension, a. structure, a. network. Raumspace diagram; solid 3-dimensional structure. Siebselective system, filter means, network.

Gebläse, Knall gas- oxyhydrogen blowpipe.

geblattert laminated.

Gebrauch custom, usage, practice, use.

gebrauchsfertig ready for use, readied.

Gebrauchs-kop ie usable or working copy (for display). - l ast working load, service 1. -muster (German) petty patent, utilitymodel patent. -vorschrift directions for use.

Gebuhr fee, royalty, tax, dues, 
duty, payment. Bekanntmachungslay-out fee. Zeugen- witness fee. Zuschlags- extra fee, supplementary fee or dues, extra tax.

gebundene Ladung bound charge.

- Wárme latent heat.

gedacht imaginary, fictitious, conceived, assumed.

gedackte Pfeife stopped pipe.

gedămpft(e) Anzeige deadbeat, aperiodic or non-ballistic reading or indication. - (er) Raum, mittel- moderately live room. Gedanke, Erfindungs- basic idea underlying an invention. gedeckte Lichter covered lights. - Pfeifestopped pipe.

gedrăngt, gedrungen compact, compendious, of restricted dimensions.

Gefahr danger, peril, risk, hazard, chance, jeopardy, menace.

Gefäll, Grenz- limiting slope, 1. gradient. Potential- potential gradient, p. barrier, threshold of $p$.

Gefällsgang gradient variation. gefalteter Exponentialtrichter folded exponential horn (of a loudspeaker).

Gefass container, vessel, tank, jar, pot, box. -,Faraday'sches Faraday ice pail, F. cylinder.

Gefäss-barometer cistern barometer. -heberbarometer combination cistern and syphon barometer.

gefasste Linse mounted lens.

Gefăsswirkungsgrad bulb efficiency (rectifier).

Geflecht plaited or woven work, network, reticusation.

gefrierbar coagulable, freezable, congealable.

Gefrierpunkt-lehre cryoscopics. -messer cryometer. -messer und Druckmesser manocryometer.

Gefuge structure, texture, bed, stratum. Fein-fine structure, micro-s. Grob- macro-structure. Klein-fine structure, micro-s. Streifen- laminated structure, banded s.

Gefuhl, Raum- space feeling, spatial feeling (in vision).

gegebenenfalls if necessary, under certain circumstances, in an emergency, optionally.

gegen den Wind fliegen fly up wind. gegenarbeiten buck, oppose, counteract, work in opposition.

Gegen-beleuchtung back (ground) illumination. - beweis, direkter rebutting testimony or evidence. -bild counterpart, antitype, replica. -druck counter-pressure, back-p. reaction. -druck, Strahlungs- radiation reaction. gegene inander schalten connect in opposition, c. differentially.

Gegenelektrode counter-electrode, opposite e., cooperating e.; signal plate or metallic screen (forming foundation of mosaic in telev. tube).

gegenelektromotorische $\mathrm{Kraft}$ counter electromotive force (c emf), back emf.

Gegen-feder counter(-acting) spring, retractiles. -feldmethode retarding potential method. -fernsehen two-way television. -fusspunkt antipode. -gewicht counterpoise, counterweight, counterbalance. -gewichtantenne screened aerial. -keil counter-wedge. -klage cross-claim, counter-claim, -kontakt (pol) co-operating contact or terminal, opposite contact. -kopplung negative or stabilized feedback, reverse f., anti-regeneration, degeneration. -kraft reaction, counter (-acting) or opposing force. -kufe counter pad. - kurs course, head or flight away from port, outward c., opposite c. - lager abutment, any relatively immovable point or surface 
sustaining pressure.

gegenläufig oppositely directed or oriented, of contrary sense.

Gegen-läufigkeit opposition, 0 . of phases, etc. -modulation push-pull modulation, $m$. in opposition. -mutter lock, clamp, check, jam or binding nut. - parte i adversary, adverse party, "the other side" -phasigkeit phase opposition, anti-phase condition. -polung opposition of polarity, opposite p., reversal of p. -probe contrasting sample, c. test, check test. -satz,im... zu contradistinct from, in contradistinction to. -schaltung differential connection, connection in opposition. -sehen, Vorbeisehen beim not to look straight at each other (in twoway video telephony). -seher 2 -way video or television apparatus.

gegen-seitig mutual, reciprocal, one another (when more than two) each other (when two), contralateral (on the opposite side). --mischbar consolute. - sinnig in or of opposite or contrary sense, direction or orientation (e.g., of rotation); irrational, devoid of sense, non-sensical, illogical. Esinnig in Reihe series opposing.

Gegensonne parhelion, mock sun.

Gegenstand object, (sometimes:) image of cathode formed at crossover by first lens, in c.r. tubes, and acting as object for second lens; subject-matter.

Gegenstand, korperlicher solid or three-dimensional object.

Abbildungs- imaging object. Erfindungs- object of invention, disclosure. Ubertragungsobject to be televised or transmitted by video signals, teleview o., televised o.
Gegenstands-gl as object glass -weite distance from electron source (aperture or crossover) to lens, object d.

Gegen-stoff antisubstance, antibody, antidote. -strahl reflected ray, reflection. -strom counter-current, inverse c., reverse c. -stromrelais reverse-current relay. -stuck counterpart, match.

Gegentakt-arbeiten push-pull operation, work in phase opposition. -audion push-pull grid detector. -brensfeldaudion electron-oscillation detector or rectifier (negative anode associated with leak resistance and condenser) in push-pull circuit scheme. -kippe gerät push-pull time-base. -mikrophon push-pull microphone. -noiseless split-wave noi seless recording. -schrift push-pull track.

Gegen-teil opposite, contrary, antithesis, converse, contrast. -versuch control or check test or experiment: -werk equivalent, counter, value. -wind contrary wind, head w.

gegenwirkend counteracting, reacting, antagonistic.

Gegen-wirkung, akustische acoustic reactance. -zelle counter electromotive force (battery) cell.

gegliederte Bildteile, verschieden picture portions of different gradation, composition or organization.

Gegner adversary, objector, opposer, opponent, "the other side."

gegossener $\mathrm{Film}$ coated film.

gegrundet auf based upon, predicated on, grow out of, result from, conditioned by.

Geh-Steh-Apparat start-stop type of telegraph printer.

Gehalt, Kern- nuclear concentration, content of nuclei. 
Gehause case, box, shell, housing, cabinet, chamber (camera).

Hörer- receiver case, r. shell. Trieb- gear casing, g. box, g. housing.

Geheim-kamera detective camera, concealed c. -telephonie garbled, scrambled or secret telephony (based upon speech band inversion, use of scrambler circuits, speech inverters, etc.)

geheizte Röhre, indirekt heater type or indirectly heated tube. Gehör... auditory, acoustic, audio, aural, hearing.

Gehor, echtes, absolutes genuine absolute pitch. um-ersuchen file application or petition to be heard, petition for a hearing.

Gehor-empfindungsskal a auditory or aural sensation scale. -maskierung auditory making. - schädigung hearing impairment. - scharfe acruity of hearing. - schärfemessung audiometry. -zielflug aural homing.

Gehrung mitre, bevel.

Geige violin. Stroh- violin in which bridge vibrates a diaphragm attached to a horn.

Gei gen-harz rosin, colophony. -lack violin varnish.

Geist, Erfinder- inventive genius, inventor's spirit. -Forschungsresearching or inquiring spirit, inquisitiveness.

Geister, Gitter- spectral (grate) ghosts.

Geisterbild ghost image.

Gekräz- Metall- waste metal.

gelagert, drehbar- rotationally supported, journaled, fulcrumed, pivoted.

Gelănde, Aufnahme- location.

Gel ănde-arbeit field work (geology, etc.) -winkelmesser angle of site instrument, clinometer. gel at inebeschichteter $\mathrm{Bl}$ ankfilm gelatine-coated film base

Gelb-giesser brass founder. -glut yellow heat.

Gelbildung gel formation.

Geldstrafe, eine - ausklagen sue for a penalty.

Gelenk joint, link, articulation, hinge. Kardan- universal joint, cardan $j_{.}$, hall and socket $j$. Knie- knee joint, knuckle j., elbow j. Kreuz- universal joint, Hooke's j. Kugel-, Universalball and socket joint, universal j., cardan j.

gelenkig flexible, pliable, supple; articulate, jointed, linked.

Gelenk-kette link chain. -kondensator swing-out condenser. -kupplung Hooke's joint. -viereck linked or articulated quadrilateral or quadrangle.

gellend shrill, yelling, tingling. gelten be valid or in force, be alive, apply to, hold good for. geltend machen, einen Anspruch advance or make a claim.

Geltungsbereich einer Erfindung scope or command of an invention. Gemagerat German type of radar. gemasert speckled, streaked, veined, grained.

gemeinsam common and joint, shared. gemeinschaftlich mutual.

gemeinschaftlich und einzeln jointly and severally. gemeinschaftlich betrieben ganged (of condensers), geared or locked together, operated jointly or in interlocked relationship. Gemeinschafts-antenne party antenna, block a., communal a., centralized or shared a. -sender chain broadcast station.

Gemisch, KI ang- sound spectmum. Gemischkomponenten spectral components (sound, etc.)

gemischtpaariges Kabel composite cable. 
gemischte Stimme mutation stops (organ)

genau exact, correct, precise, close, falthful (of reproduction).

Genauigkeit, funfstellige fivefigure accuracy.

Genehmigung approval, approbation, permission, allowance, assent, consent, sanction, concession.

General-nenner common denominator. - unkosten overhead or general expense. -vollmacht general power of attorney.

Generator, Ablenkungs-deflection generator, sawtooth g., timebase generator. Bremsfeldnegative transconductance oscillator or generator, positivegrid or retarding-field 0 .

Fahrtwind- fan-driven generator, wind-driven g. (aimplane). Impuls- impulse generator, surge g., "lightning" g. Lochscheitenlight chopper. Pruf- signal generator, all-wave oscillator, test o. Quadratwel len- square or rectangular wave generator. Resotank- micro-wave generator (electron-oscillation tube confined in a cavity, e.g., c. resonator magnetron). Sägezahn sawtooth generator, ratchet g. Steuer- drive oscillator, master o. Ton- musical generator, tonal g. Wanderwellen- surge generator, impulse g., "lightning" g.

Geometriefehler geometric distortion.

Geometrisierung der Wellenmechanik geometric derivation of wave equation.

geordnet, regellos with random orientation.

geordneter Zustand ordered state or arrangement (of a structure), perfect configuration (of units in crystal lattice).

gepackt, dicht close-packed. gepanzerte, wenig streuende Spule iron-core deflecting yoke (w口th minimized soot and pattern distortion).

gepeilter Sender tuned-in beacon

or transmitter (to take bearings). gepufferte Lösung buffered solution.

Gerade straight-line characteristic or "curve," (Schottky)

line.

gerad(e) Beleuchtung direct illumination(micr.) -(er)

Kondensator, frequenz- straightline frequency condenser or capacitor. - (er) Kondensator, kapazitäts- straight-line capacity condenser. - (er) Kondensator, wellen- straight-line wave condenser. - (e) Oberharmon i sche even harmonic.

ger ad-linig (recti)linear, in direct proportion. -sichtiges

Prisma direct-vision prism. -wertig of even valence.

gerasterte Schicht, $-(r)$ Schirm mosaic screen, photosensitized m.

Gerăt implements, gear, apparatus, outfit, equipment, instrumentation, tools. Lichtton- sound film head or attachment.

Geräteglas apparatus glass (stock). Geräusch s.a. Akustik, Klang, Schall.

Geräusch (Radio, Film, etc.) und akustische Eigenarten: Noise (in radio, film, etc.) and acoustic peculiarities;

Apparatgerăusch system noise (in recording).

Bildgerăusch frame noise.

Bildstrichgeräusch frame line noise (form of motor boating).

blecherner $\mathrm{Kl}$ ang tinny sound.

Bratpfannengerausch frying noise, zoop.

Brodeln boiling. 
Brummton, Netz- mains hum, hum. dumpf all-bottom, boomy, dull, tubby.

Eigengerăusch inherent film noise.

gellend shrill, tingling, yelling.

GeschwindigkeitsschwankungsGerausch - flutter, wowows (when intensity pulsation or variation is up to 6 cycles a second); flutter (6-30); gargle (30-200), and whiskers (over 200).

gleichmässiges Gerausch smooth noise, uniform $n$.

aleitender Ton glissando, sliding note.

Grundgerăusch (back) ground noise.

hallend reverberant (multiple echoes).

He iserkeit pops (at splices), raucousness, hoarseness.

helle Klangfarbe strident or shrill timbre.

heulen howl.

lleulton warble tone, hf warble note, "multi-tones."

hoher Ton tweeter, treble, highpitched or high-frequency note ar sound.

hohler Ton boomy sound, dull s. (higher pitches cut off).

Kellerton tunnel or boom effect.

Klappern chatter, rattle.

Klebestellengeräusch blooping, splice bumps, dull sound due to blooping patches.

$\mathrm{kl}$ impernder $\mathrm{Kl}$ ang harsh or strident sound (with strong harmonics or high partials), shrill, tinkling, chinkling s.

$\mathrm{Kl}$ ingen sounding, ringing (of a tube), microphone noise.

klirren clink, clatter.

knacken click, crack, crackle, crepitate.

knallen detonate, explode, report,

crack, pop, pistol shot noise.

knarren bloop, jar, crack, rattle creak, squeak.

knattern crack.

knirschen grind, crunch.

knistern sizzle, grate, crackle, rustle.

knurren growl, snarl, rumble. kochen boil.

Kohlekorngerăusch carbon noise (microphone).

Kornrauschen film grain noise.

krachen crack, crash, crackle, rustle.

krächzender Ton all-top sound or voice.

kratzen scratch, scrape, rasp, bumps (film splices).

kreischend strident, all-top (of voice).

lautgetreu of acoustic fidelity, orthophonic.

Lautsprechergerausch rattle of loudspeaker.

lebendig live, realistic.

Mikrophon-geräusch microphonic noise, valve n. -schmoren mike stew.

moiréartiges Gerăusch moiré pattern of noise.

nachhallend reverberant, echoing, resonant.

Nadelgeräusch needle scratch $n$. chatter, record noise.

Mebengerăusch extraneous noise.

Netz (ton) brummen mains hum.

offen hollow (of sound).

Perforationsgeräusch sprocket hole modulation or noise.

pfeifen whistle, ring, squeal, howl, sing.

$\mathrm{Plattengerausch} \mathrm{record} \mathrm{noise,}$ surface n., needle scratch. -schlag disk wobble.

plötzliches Gerăusch impulse excitation noise.

prallen rattle.

prellen(am Kontakt) chirp, thump, clink, chatter (caused at 
contacts and keys).

quieken squea., squeak.

raspeln rasp.

rasseln rattle, clatter.

Rauhigkeit hissing, raucousness, hoarseness.

Raumgeräusch set noise.

Rauschen (von Röhren) tube or valve noise (thermal aøitation of electrons), hissing, rustle.

Rauschen, Schrammen- scratch noise. Widerstands-circuit noise (due to Brownian movement).

reiner Ton pure note or tone. sausen rush, whiz, whistle, hum. schallen sound, resound, ring. Schallolattengeräusch record noise, surface $n$.

schallhart acoustically rigid or hard, non-absorbent.

schallweich sound absorbent. scharf shrill, piercing, strident.

scharren scrape, scratch.

Schlag disk wobble (phonograph).

Schlacen(Kontakt.) key click, thump, or chatter.

Schmutzceräusch dirt noise.

Schmoren frying, mike stew.

schnarren, burr, buzz, jar.

Schrammenrauschen scratch noise.

Schrotgeräusch shot noise (due to Schottky effect).

schwirren whiz, whir, buzz, hum.

selbsttönen singing or squealing (of a tube).

Staubrauschen dust noise.

Strichger äusch frame-line noise (sort of motor boating).

summen hum, buzz.

surren motor boating.

tiefe Töne low AF or bass notes; bassy condition (in sound reproduction, low AF overemphasized).

trillern warble, trill, quaver. überschreien overmodulate. unreiner Ton impure tone, ragked tone or note. gerecht, flucht-

Wärmerauschen thermal noise (due to thermal motion of electrons).

Widerstandsrausch circuit noise (due to Brownian movement).

Wobbeln warble, wobble,

Zischen fuzzy noise (of film), hiss, sizz, sizzle, fizz, high harmonic alien tones, in film reproduction.

Zwitschern birdies, canaries. Geräusch, Blld-frame noise.

Eigen- inherent film noise.

Lautsprecher- rattle of loudspeaker. Mikrophon- microphone or valve noise. Neben- extraneous noise. Perfor ations - sprocket noise (m.p.). Raum- set noise. Wärme-thermal noise (sort of Brownian movement of electrons in input circuit).

Geräuschabstand sional-noise ratio. geräuscharm of low noise, noiseless, silent, silenced.

Geräusch-bekämpfuna noise abatement, suppression or attenuation. -beseitigung (an Tonklebestellen) blooping elimination. -beseitigung, automatische silent tuning, noise suppression, noise gate. -filter, Nadelneedle scratch filter. -kasten

(Erzeugung besonderer Geräusche) equivalent acoustics (for generation and imitation of noises and sound effects). -losigke it noiselessness, silence, quietness, calm(ness), absence of noise. - messer noise meter, sound-level meter (e.g., measuring in terms of decibels), noise reasuring set, acustimeter. -messer (in elektrischen Kreisen) psophometer. -muster noise pattern. -spiegel noise level. -unterdrückungsstromkreis noise reduction, squelching, suppression or silencing circuit. -verdeckung overriding of noise. gerecht, flucht- truly aligned or 
gerecht, form-

flush (with). form- true to form or shape.

gerechte Farbstufe, empfindungssubjective chromatic scale value. Gerechtiqkeit justice, equity,

equitableness.

qereckte Probe stretched specimen. gereifelt grooved, milled, knurled, fluted.

Gericht, Bezirks- district court. gerichtet, einseit/g- unidirectional (ized).

gerichtet(e) Empfangsspannung directional sínal or incoming potential. - (er) Lautsprecher directional loudspeaker. -(es)

Licht parallel light (rays).

gerichtlich belangen bring lekal or judiciary action or proceedings against.

Gerichts-barkeit erster Instanz original jurisdiction. - befehl writ or warrant. -beisitzer assessor, associate judge.

-bezirk court circuit, court district. -hof, zuständiger court of competent jurisdiction. -stand competency of a court, jurisdiction, venue. - verhandlung hear ins, trial.

gerieft, geriffelt grooved, milled, knurled, fluted.

geringhaltig low standard, worthless, of low content.

Gerippe, Kristall- crystal skeleton, skeletal c., crystallite.

gerissene Kurve dotted-line curve, broken-line c. or graph.

Gerïst structure, skeleton, frame, rack, scaffold. Trocken- dryinó frame or rack.

Gerüstkran gantry crane. gesamt total, entire, aggregate, complete, whole.

Gesamt-ansicht total view, general v., assembly v. -heit totality, entirety, assembly, senerality, whole. spin total spin.

-verhalten general behavior.

-wirkungsgrad overall efficiency, total e., commercial e.

Geschädigter aggrieved, injured party. sescheckt cross-hatched.

Geschehnis action, event, occurrence, happening, phenomenon. aeschichtet, zwischen- inter-stratified.

geschichtete Kathode coated (oxide) cathode, photo-c. with lightsensitive layer.

Geschicklichkeit skill, cleverness, art, dexterity, expertness. geschlagene Saite percussed string, p. chord.

qeschlitzter stöpsel split plug. qeschlossen, in sich closed upon itself, self-contained. geschlüsselter Funkspruch coded radio ressage.

geschmeidig pliable, flexible, supple, soft, ductile, malleable. Geschoss-bugwelle nose wave of a projectile. -geschwindigkeit projectile velocity.

Geschütz-aufsotz optical instruments for artillery. -zielender gunlayer

Geschwindigkeit (in Mechanik) velocity (being, in mechanics, the analog of current in electricity). Geschwindigkeit, ungeordnete velocity of agitation. Abtastpickup velœity, p. rate (phonograph). Anfluss-velocity of approach, afflux v. (of fluid). Apparat zur Bestimmung derveloity selector. Austrittsexit, efflux, muzzle or emeroence veloity. Boden- fround speed (of airplane). Durchschlagspropagation speed (of fluids in pipes); rate of insulation breakdown. Eigen-air speed (of airplane). Einfall-bombardment velocity. Eintritts-approach or inlet velocity. Fluq- rate of flying speed, traveling velocity (electrons, etc.). Fortpflanzungsspeed of propagation or transmission. Fortschreitungs- speed of progression or travel, forward speed. Grund- ground speed. 
Gruppen-envelope velocity, group v. Kriech-rate of creep (of meta 1). Masseteilchen-particle velocity. Mündungs- muzzle velocity. Phasen- phase speed, p. velocity, wave v. Rückkippflyback speed, time-base unlock s. Teilchen-particle velocity (ac.) Umfangs-circumferential speed, peripheral s. Wanderungs-rate of migration, crawl or creep, m. velocity.

Geschwindigkeits-abtastung variable-speed scan. -amplitude volume current (ac.). -fehler distortion due to synchronous speed differences. -feld velocity gradient, v. field pattern.

geschwindigke itsgesteverter

Elektronenstrahl velocity-modulated electron beam or pencil (klystron, etc.).

Geschwindigkeits-gitter accelerat or grid. -messer speedometer, tachometer, tachy graph, (when recording). -messer, wind- anemometer. - potential velœity potential.-raum momentum space, velocity s. - schwankungen w ow Ows (when intensity pulsation is up to 6 cycles per second); flutter (when 6-30 cps.); garnlle (30200 ) and whiskers (over 200). -steuerung velocity modulation. -verteilung velocity distribution, d. in energy. -wechselgetrlebe change-speed gear.

gesehnte Wicklung short-pitched winding, chord w.

Gesenk die, block forging d., swage. -schmiede drop for óe.

Gesetz, Inkrafttreten eines -(es) commencement of an act or law.

Gesetz, Ähnlichkeits- similarity principle. Brechungs-,

Snellius'sches Snell's law of refraction. Erhaltungs- law of conservation. Flächenerhaltungs- principle of conservation of areas. Impulserhaltungs- principle of conservation of momentum. -, Lambert'sches emission law, Lambert 1. Teilungs- law of partition. Zufalls- law of probability, 1. of chances.

Gesetz-blatt, Reichs-imperial law journal, i. gazette. -buch code, statute book.

Gesetzessuslegung law inter pretation.

gesetzlich lawful, leǵal, licit, legitimate, statutory.

qesetzliches HIndernis statutory bar.

gesetzmässig according to law or statutes, conf ormable to (natural) law, in a regular or legal way. Gesetzmässigkeit lawfulness, legality, legitimacy, procedure according to statutes.

gesicherte Mutter lock nut, jam $n$. Gesichts-eindruck visual impression. -feld field of view, $f$. of vision. -feldblende field stop. - sinn vision, faculty of seeing. -strahl visual ray. -wert face value, facial brightness (of a screen perf ormer). -winkel visual angle, optic a., facial a.; camera or viewing angle.

Gespensterstrom ghost current. Gesperr, Anker- anchor escapement. Malteserkreuz - cross wheel, (cam and) star w. of Maltese cross, Geneva stop or intermittent movement.

Gespinst spun fabric, yarn goods. Gesprächspausen intervals of no speech.

gespritzt squirted, extruded; sprayed, atomized. - (er) Glühfaden squirted filament. -(es) Metall die-cast metal. gestaffelt stagkered, echeloned, graded.

Gestalt, Kugel-spherical shape, bulbous or ball form.

Gestaltfestigkeit form stability, 
non-deformability.

gestaltlos amorphous, non-crystalline, formless.

Gestalts-fehler, Kugel- spot size distortion. -veränderung change of form, change of shape, deformation.

Gestaltung formation, configuration, form, shaping, fashioning, organization, state. Doppeldimorphism. Neu- re-organization, re-arrangement, modification, re-formation.

Gestell frame, stand, rack, bay, shelf, mount. Verstärkeramplifier rack, repeater bay. wăsserungs- washing rack (phot.). gesteuerter 0 szillator, leitungsline-controlled oscillator. gestochene Schärfe microscopic sharpness (phot.).

gestreckt straight, stretched, linear.

gestreut, el astisch und unelastisch elastically and unelastically scattered. kern- deflected by nucleus.

Gesuch application, petition. -steller applicant, petitioner. geteil ter Ring split ring, segmented getreu, amplituden - of equal amplitude, with a. fidelity.

form- true to form, undistorted, orthoscopic (opt.)

$\mathrm{kl}$ ang- orthophonic, of high

fidelity.

getreue Wiedergabe faithful reproduction, fidelity in $r$. , good definition of image (with fine detail formed by a lens, telev. or facsimile receiver or a c.$r$. oscilloscopé ).

Getriebe, Ausgleich- differential gear. Kegelräder- bevel gearing, mitre-wheel g. Pl anetenplanetary gearing, epicyclic gear train. Reduktions- reduction gear, stepdown gear.

Schraubenzahn- helical gear, spiral g., worm. Umlauf- planetary gear, sun and planet $g$.
Winkelverzahnungs- double helical gear.

Getriebelehre kinematics.

Getter-abschiessen set off or cause vaporization of getter. -pille getter tab, g. patch, g. pill. -spiegel getter patch, getter film.

gewährbar allowable, grantable. gewähren (ein Fatent) allow a patent, grant a p., issue a p. Gewebe tissue, texture, textile, fabric, web, cloth, gauze, netting. Draht- wire gauze, w. cloth, w. mesh. Hart- indurated fabric, impregnated $f$.

Gewebegebilde, winzige ultramicrons (of eye tissue).

gewell ter Einspannrand corrugated suspension means (of cone.)

gewerblich industrial, concerning trade or profession.

Gewicht, Atom- atomic weight. Gegen- counterpoise, counterweight, counterbalance. Molmolar or gram-molecule weight. gewichtsanalytisch gravimetrical. Gewichtsprozent percent by weight. -verhältnis proportion by weight. Gewinde thread, winding, coil. - schneiden tapping, thread cutting.

Gewinde, Aussen- male screw thread, outside s.t. Flach- square thread. Innen-, Mutterfemale or internal screw thread. Rechts- right-hand screw thread. Gewinde-lehre thread gage. -steigung pitch of a screw thread. -stift headless screw, grub s. -taster thread caliper.

gewöl bt arched, arcuate, domed, convex. - (e) Lamellen, kygelförmig dished laminae.

gezackte Linie jagged line, serrated 1., notched 1., dentated 1., dented 1 .

gezupfte Saite plucked string or chord.

Gier, Elektronen- electron affinity. 
Gierungsmesser

Gierungsmesser yaw meter.

giessen coat (a film).

Giesser, Gelb- brass founder.

Giesserei, Kunden- jobbing foundry.

Giessform casting mold.

Giltigkeit validity, lawfulness, legality, life (of a patent).

Gipfel, Wellen-peak, crest or hump of a wave.

Gipfel-höhe ceiling. -spannung peak voltage, crest $v$.

Gips plaster of Faris, gypsum, calcium sulfate.

Gisklappe G sharp key.

Gitter, automatisch vorgespanntes automatic- or self-biased grid. -,freies floating grid, free g. -weitmaschiges open-meshed grid, wide-meshed $g$.

Gitter, Anodenschutz- screen grid, plate shield. Beschleunigungsaccelerator grid (of c.r. tube); screen g. (of tetrode). Beugungsdiffraction grid. Brems-

suppressor grid, cathode g. (in pentode tube). Dichte (regulier)(charge) density grid, density control g. Echel ette- echelette grating (opt.). Einheits- unit lattice. Fang- suppressor grid, cathode g. (in power pentode), interceptor g. Geschwindigkeitsaccelerator grid. Grund- fundamental lattice. Haupt-parent lattice. Hohl- concave grating. Kathodenstrahlröhren- grid or shield, Wehnelt cylinder, of a cathode-ray tube. Konkav- concave grating. Kreisbogenarcuate grid. Kreuz- cross grating. Kristall- crystal grating, surface lattice. Maisch- stirrer, rake (brewing). Plan- plane grating. Raum- space-charge grid; space lattice, "raumgitter". Reflexions- diffraction grating with parallel reflecting surfaces. Saug- space-charge grid. Schichtstratified lattice. Schirm-, Schutzscreen grid; shield g. (in thyratron). Stau- suppressor grid, baf-

\section{Gittermittenabstand}

fle g. Steg- grid in which all wires are parallel to axis and surround cathode cage-fashion. strich- ruled grating. stromatomic lattice. Stufen- echelon grating. Zug- positive grid, space-charǵe $g$.

Gitter-ableitung grid leak (resistance). -absorption, Grundfundamental lattice absorption. - antenne parasol-type antenna. - aufstell ung, Rowl and 'sche Rowland mounting of concave grating. - aufweiterung expansion or increase in lattice spacing. - aussteuerung grid (voltage) swing, grid sweep. -beeinflussung, -besprechung grid control, grid modulation. -block grid condenser; staircase-like structure of echelon grating. -blockierung negative biasing of grid, g. cut of $f$. -durchgriff grid transparency, g. controllance, g. penetration factor. - ebenenabstand inter-lattice-plane distance. -einheit lattice unit, unit cell, elementary cell.

-einsatzpunkt grid current point, point of incipient grid current flow. -elektronen lattice electrons. -filter lattice filter. -furchen rulings or ruling grooves (of diffraction grating). - geister spectral grate ghosts. - gleichrichtung grid-current rectification or detection (in audion valve). -haltedraft grid stay wire, g. supporting $w$. -holm grid stay or supporting member. - interferometer grating interferometer. - ion, Zwi scheninterstitial ion. -kathodenstrecke grid-cathode path. -konstante grating constant, g. space; lattice c. -loch, lattice vacancy, 1. hole. -masche grid mesh. -mast lattice pole, girder p. -mikrophon grille-type microphone. -mittenabstand distance between centers of successive rulings (in diffraction grating); grating constant. 
-modulation grid modulation, 8 . control (Brit.) -nebenschluss grid leak (resistance). platz lattice place, 1. point. -punkte lattice points. -rohr, Doppelbigrid valve, twin-grid tube, four-electrode $t$. (pliodynatron, tetrode, etc.). saum grid skirt (in telev. tube). - schwingung (Kristall) lattice vibration (cryst.) -spektrograph, Vakuumvacuum grating spectrograph. -steg grid stay; solid portions forming g. meshes; grid strip used in lieu of wire. -steverung grid (voltage) sweep, g. swing, g. excursion, g. excitation; g. control (in ignitron, thyratron, etc.); g. modulation. -störungen lattice distortions, 1, dislocations (cryst.). -strebe grid stay, g. supporting means. -strich grating line, ruling or ruling groove of a grating.

Gitterstrom-aufnahme drawing or taking of current by grid. -aussteuerung grid current swing or sweep. -einsatzpunkt grid current point, point of incipient grid current flow or where g. begins to draw current. - gleichrichtung grid current detection, cumulative grid detection. -punkt grid current point, point of incipient current flow at $g$.

Gitter-verschlebung lattice distortion, 1 . dislocation. -vervielfältiger mesh multiplier. -vorspannung, automatische automatic or self-biasing of grid. -wickeldrähte grid coil wires.

Gl anz, Halbmetall - submetallic lustre.

Glanz-einfall grazing incidence. - garn glace or glażed cotton, lustrous or mercerized yarn. -messer gloss meter, glossimeter. -winkel glancing angle.

Glas s.a. Linse, Objektiv
Glas, berichtigenaes correcting lens. -,spannungsloses strainless glass, strain-free g. -, ungefasstes rimless lens.

$\mathrm{Gl}$ as, Aufsteck- slip-on lens segment. Brillen- spectacle glass.

Durchzieh- slide (micr.) Farbcolored glass, color filter, stained g. Hart- pyrex glass. Lese-, viereckiges oblong reading glass. Milch-frosted glass, opal $\xi_{\text {., }}$ light-differing $g$. Muschei- globular lens. Ob iektslide, mount (micr.). Rauchsmoked glass, tinted gl. Sammelconverging lens; preparation tube, specimen t. Sol in- crown glass. Spiegel- plate glass. Trübopal glass, frosted g., lightdiffusing $g$. überfang- flashed opal glass.

glas-ähnllch, -artig glasslike, glassy, vitreous.

Gl as-bildner vitrifier. $-b l$ ase glass bubble, seed (when small), air bell (when of irregular shape). -faden glass thread, thread-like defect of flass. -federmanometer spoon-type glass manometer. -fehler (cf Schlieren) glass defects or flaws; glass striae or chords (when heavy), bubbles (seed when small); airbell (when of irregular shape), stones; crystallization bodies, cloudiness, strain. -flächensplegel ung glare or reflection of glass surfaces, specular $r$. -galle glass gall, sandiver.

glasig glassy, vitreous.

glasklarer film pellucid film stock (having maximum transmittance).

Glas-kolben glass bulb.-plattenstaf-feln (Michelson) echelon spectroscope. -satz glass batch, composition or charge. - schaum glass gall, sandiver. - scheitel lens vertex. -schlieren schlieren, striae or chords (when large), reams (when broken by rolling into fine bands). -schliffuerbindung graund-glass joint. 
-schmutz glass gall. -schneider, Roll- wheel-tyne glass cutter. -schweiss glass gall. -spannuno strain or internal tension of glass. -stöpsel glass stopper. -trübung glass cloudiness.

gl astechnisch glass technological.

$\mathrm{Gl}$ as-ïberfangschicht glass lining. -ver guitung refining or treating of optical glass (e.g., by producing low-reflectance coating, dimming, etc., as by Bausch and Lomb magnesiumfluoride anti-reflection fjilm on glass).

Gl asur, halbmatte half-matt glaze. glätten smooth (ripples, fluctuations or pulsations of currents); make level, even or uniform, stabilize.

Gl at theitsprïfer smoothness meter or tester, glossimeter (measures ratio of reflection to total light), surface analyzer.

Glättung smoothing (ripples of current, potential, etc.). Spannungs- voltage stabilization, v. smoothing.

Glättungs-kondensator smoothing condenser or capacitor. -röhre glow-tube stabilizer.

Glauben, in gutem- in good faith, bona fide.

Gl aubwïrdigkeit credibility, trustworthiness, credit.

gleichachsig co-axial, equi-axial.

Gl ei chbeanspruchung static loading, constant 1 .

gleich-bedeutend equivalent, tantamount, synonymous. - bel astete Phasen balanced phases.

Gleichen, Kurs- loxodromes, rhumb lines. Misswei sungsisogenic lines, isogonals.

gleichentfernt equidistant, equally spaced (apart).

Gleichfeld constant or steady field

gleichförmig uniform, steady, smooth, even, homogeneous.
Gleichgang unison, synchronism. gleich-gerichtet equidirectional, unidirectional, equi-axial, of like orientation. - gestal tet isomorphic, isomorphous

Gleichgewicht balance, equilibrium, poise, trim (of airplane), stability. -, radioaktives radioactive equilibrium (being either transient or secular). Brïcken-bridge balance. Druck-, osmotisches isosmotic equilibrium. Gleichgewichts-fehler unbalance, imbalance, lack of balance or equilibrium. -lehre statics. - potential equilibrium potential (of mosaic). -verhältnis equilibrium ratio. -zustand state of equilibrium or balance, stable or stabilized state.

Gleichheit state of balance or equality, likeness, sameness, uniformity, constancy. Druck-, osmotische isosmotic equilibrium. Phasen- in-phase state, coincidence of phases. Spiegelmirror symmetry,

Gleichheits-photometer, Lummer Brodhun L. - B. contrast photometer, total-reflection $\mathrm{p}$. -zeichen equality sign.

Gleich-kl ang unison, consonance, resonance. -lauf, lokal geregelter local synchronizing (e.g., by a (uning fork).

gleichl aufende Kondensatoren gang(ed) condensers, synchronized capacitors.

Gleichlauf-fehler, Auflaufen der compounding or integration of errors or differences in synchronism. -impuls synchronizing impulse, corrective $i$. -signal, -zeichen synchronizing pulse, sync signal; tripping s.

Gleichluftstrom direct flow of air, d.c. air $f$.

gleich-machen equalize, balance, stabilize, make even or uniform, steady. -mässig uniform, steady, constant, smooth. -namig like, 
similar (of poles). -phasig co-phasal, in phase, (equi-) phased. -richten rectify, detect, demodulate.

Gleichrichter, linearer linear rectifier, detector or demodulator. -quadrati scher square-law rectifier or detector.

Gl eichrichter, Doppel (weg)- fullwave rectifier. Einweg-, $\mathrm{Hal}$ bweghalf-wave rectifier. Hochvakuumkenotron, electron-type rectifier. Kontakt-contact rectifier, crystal $r$., natural $r$. Pendel- vibrating rectifier, tuned-reed $r$. Schwingkontakt- vibrating-reed rectifier. Sperr- barrier-film rectifier, blocking-layer $r$., metal $r$. electronic r., cuprox r. Trockendry rectifier, metal $r_{\text {. , copper }}$. , cuprous-oxide r., selenium-oxide $r$. Verstärker- amplifying detector. Voll weg- full-wave rectifier. Zweif achdiodenduodiode rectifier.

Gleichrichter-pille oxide or metal rectifier of reduced size (e.g., Westector). -rückzündung backfiring, arcing-back (of Hg rectifier), backlash (imperfect rectification). -vol tmesser rectifying voltmeter. -zelle rectifier cell, barrier-layer c.

Gleichrichtung beider $\mathrm{Hal}$ bwellen full-wave rectification. -einer Halbwelle half-wave re tiffication -, unvoll ständige backlash

Gleichrichtung, Anoden- platecurrent rectification or detection. Gitter-grid-current rectification or detection.

Gleichrichtung, Volumen- volume rectification.

gleich-schenklig isosceles. -sinnig in Reihe series aiding.

Gleichstrom, Luft- current of breath (in sound recording).

Gleichstromempfänger d.c. electric radio set.

gleichstrom-vormagnetisierte Spule d.c. controlled saturable or three-legged reactor or coil. - iberlagert superposed on d.c., superposed with d.c.

Gleichtakt-aufnahme in-phase recording (on film). - sprossen-Doppel-. schnürspur variable-density double squeeze track. - sprossenspur single variable-density track. - sprossenschnür spur variabledensity squeeze track. -verstärker in-phase amplifier.

Gleichteilung equipartition.

gleich-temperiert isothermal, uniformly heated. -tönend unisonant, consonant.

Gleichung, eine - nach $n$ auflösen solve an equation with respect to n.

Gleichung, quadratische quadratic equation. Ausgangs- starting equation, initial $\theta$. Bedingungsequation of condition. Bestimmungsdefining equation. Farb-, dreigliedrige three-color equation, 3-stimulus e. Kontinuitätsequation of continuity. Summensummation equation. WiederhallEyring formula. Zustandsequation of state.

Gleichungs-ansatz laying down a formula. -determinante für Leitung determinant for line equations. -famille equations grouped in families.

Gleichverteilungssatz law or principle of (energy) equipartition (of Maxwell-Boltzmann).

gleich-viel fach equimultiple. -warm isothermal, uniformly heated.

Gleich-well enbetrieb chain or network broadcast (from a key station, on one wave), operation of stations on one w. -wertigkeit equivalence.

gleich-winklig isogonal, equiangular, equi-angled. zeitig simultaneous, synchronous, isochronous, contemporaneous, contemporary, co-incidental.

Gleisweg rail track. 
Gleit-bahn chute (of fllm), slide, slideway, shoot. -drahtbrïcke slide wire bridge. -element glide element.

Gleiten, bildsames plastic deformation.

gleitender Ton sliding note, glissando.

Gleit-fläche glide plane, slip p. (cryst.); sliding surface, slide. -flug, Seiten- side slip (airplane landing). -funken slide spark, creepage s. -Kontakt sliding contact (with selfcleaning or self-wiping action). -lager plain bearing, slide b. - lineal slide rule, s. bar. -linien slide or slip bands or lines. - modulus modulus of elasticity (in shear), torsion m.,

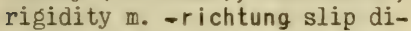
rection (cryst.). - schuh pressure pad, p. guide. -sitz, schlichtplain sliding fit.

Gleitung slip (cryst.). Zwillingstwin slipping.

Gleitungskoeffizient slip coefficient.

Gleitweg glide path, landing curve, 1. beam. -, äquipotentieller isopotential glide path.

Gleit-wegbake glide path beacon. -winkel glide angle.

Gl ied member, term (math.); limb, joint, link; section or mesh (filter), element.

Glied, $L, T, H$, und $\pi$ (oder II)$\mathrm{L}, \mathrm{T}, \mathrm{H}$, and pi type section or mesh (of a filter, attenuator or network). Ableitungs- shunt element. Brücken-lattice section. Dämp fungs- attenuation network or mesh. Kettenleiternetwork mesh. Kreuz- lattice section (of filter or network). Längs- line arm, series element. Mittel- intermediate member (of a series). Quer - cross arm, shunt element. Regel- regulating attenuator. Schwächungs- attenuator. Sperr- suppressor. Spin- spin term. übergangs- transition type, transition member. Verbindungs- connecting link. Wechselwirkungs- inter-action term. Zwischen- intermediate member, connecting $\mathrm{m}_{\text {., link, }}$ intermediat $\theta$.

gliedern arrange, organize, classify, divide methodically (say, contents of a book, etc.); articulate, joint. verschieden gegliederte Bildteile picture portions of different nature, make-up or organization.

Gl iederung organization, arrangement; jointing, articulation.

gliedrig, vier-four-membered, tetragonal, quadrinomial (math.) -(e) Farbgleichung, vier- fourcolor equation.

Gl imm-entl adung glow-discharge (spontaneous or unassisted, in glow-tube), brush d. (when $\propto$ curring at sharp points), corona. - fadenlänge length of (neon) glow column (in tuning indicator).

Gl immlampe gaseous-conduction tube, glow-tube (e.g., neon tube), flashing lamp (in sound recording). Flächen- neon tube with flat plate-shaped cathode, plate neon tube or Iamp. Polsuch-polarityfinder glow-tube.

Gl imml ampen-gerät, Universal- universal neon-tube tester. -kippschal tung neon lamp time base circuit scheme. - schall aufnahme glow-lamp sound recording (Aeolight).

Gl imml icht-anzeiger flashographtype indicator, Tune-A-Light tuning indicator. -gebiet, Vorsustaining voltage range, prephotoglow region (arises at a voltage slightly below striking voltage).

Gl immröhre, Abstimm- tuning indicator glow-tube (e.g. flashograph). Amplituden- glow-tube amplitude indicator, resonance or tuning indicator (e. g., flashograph). 
Resonanz- resonance indicator glow-tube (flashograph).

Gl imm-säule light column, glow c. (of tuning indicator). - spannung glow point or potential, breakdown p. (indicates incipient glowdischarge in grid-glow tube); anode p. (at which glow-discharge begins, in photocell), stopping p. (lies slightly below glow p., in photo-glow or grid-glow t., and is the value to which $p$. must be dropped to stop glow-d. once it has started). -( strecken ) spannungsteiler glow-tube potentiometer. -summer glow-tube type buzzer oscillator (produces relaxation waves), neon time base. -verlust corona loss. -zelle photo-emissive gas-filled cell foperating at a potential slightly below critical discharge potential), photo-glow tube.

Glocke globe, bell, jar, glass; receiver (of air pump).

Glocken-filter bell-jar filter. -klöppel beIl hammer, bell striker. -magnet bell-shaped magnet. -spiel carillon (set of bells), orchestra bells. -trichter bell funnel. glühen heat, incandesce, anneal, glow, fire, ignite.

glühelektrisch thermionic.

Glüh-el ektronenabgabe thermionic emission, Edison or Richardson effect. -elektronener. tiadung thermionic discharge.-faden, -biegsamer ductilized filament. -faden, gespritzter squirted filament. -fadenpyrometer disappearing-filament pyrometer, optical p., monochromatic p.

kathodengleichrichter mit Edelgas tungar (argon-tungsten) type rectifier. -körper incandescent body or mantle, Welsbach mantle. -rijckstand residue on ignition. -span mill iron, hammer scale. Glutmesser pyrometer.

gnomonische Karte gnomonic chart (straight-course chart).
Gold-bergkeil Goldberg wedge, neutral (grey) w. -blatt gold leaf, g. foil. -bl attel ektrometer gold-leaf electroscope or electrometer. -gl immer yellow mica. goldhaltig auriferous.

Goldquarz auriferous quartz. Goniometer, Eisen-iron-cored goniometer. Reflexions- reflecting goniometer.

Grabstichel graver, burin.

Grad, vom ersten linear.

Grad, Aussteuerungs- modulation percentage, percentage modulation; carrier amplitude (in tele-transmission). Blähungs- expanding property, swelling p., imbibition power. Durchlass- (von Wasser) transmission factor (of water), radiation through water. Winkeldegree of angle, radian (unit of circular measure of angle).

Gradation contrast.

Gradationskurve, Steilheit der gamma value or slope of response line.

Grad-bogen graduated arc, bow, protractor. -netz graticule, reticule, reticle; map grid.

Gramm, Atom- gramatom.

Grammophon, Licht- sound-track film player (without pictorial actions). Granalien shot, granulated metal. Graphitbel ag Aquadag coating. Grätzschaltung Grätz (full-wave) rectifier circuit organization (four electrolytic $r$. per phase in a bridge scheme).

Grau-keil neutral (grey) wedge, Goldberg w. -keilstufe intermediate shading value (of wedge). - schleier grey fog (phot.). - strahlung grey radiation. - stufe intermediate tonal or shading value (between white and black, of picture).

Gravarfilm engraved sound film, embossed groove recording.

Gravitationsfeld gravitational field. Greifer claw (of in-an-out movement or feeder mechanism); pull-down 
(of projection printer). Bildthreading mechanism, in-and-out claw. Justier-pilot claw (m.p.). Greifer-maschine step-type printer. -mechanismus pull-down claw mechanisi. - spitze dowel pin (film feed). -stift film feeder pin, pilot p., registration $p$. Greifloch finger hole.

Grenz-bedingung limiting condition, critical c., boundary c. -durchmesser limiting diarreter (of dielectric line).

Grenze limit, boundary, bound, end, limitation, confines, demarcation. Grenze, Empfindungs-, des Ohres loudness contour of ear, auditory sensation area. Entzerrungs- frequency limit of equalization. Ermüdungsfatigue limit. Loch- transmission range of band-pass between cut-off points. Schärfenfeldcamera lines. Schmerz- threshold of feeling.

Grenz-fall limiting case, critical case, -fläche interface, boundary layer, surface of contact. -flächenpotential interface potential. - flächensp annung interfacial tension. -flächenwinkel interfacial angle (dihedral between two crystal faces). - frequenz cut-off frequency, limiting $f$. -gefälle limiting slope, 1. gradient. -l astwechselzahl limiting number of load alternations. -linie boundary line, demarcation 1 . separation 1., limiting 1., junction 1. -schicht interface, boundary layer. -strahl border line ray, inf ra-Roentgen $r$., grenz $r$.

-wellenlänge threshold wave length. -wert limiting value, critical v., saturation v. -winkel critical angle, limiting a. (of refractometer).

Gries, Stör- low noise in picture background due to thermal effect,
Brownian movement, mechanical properties of tubes and lowfrequency fluctuations of local emission density, i.e., shot effect, etc.

Griff, Durch- grid transparency, penetration factor, transgrid action, gain reciprocal 1: $\mu$ Kordel-milled l-n $\urcorner$, knurled $k$. Rück- inverse grıu transparency.

Griffbrett finger board (of string instrument).

Griffel style, stylus, (slate) pencil. -stopfen stopper with thumb piece.

grobfädig coarse-threaded, c. filamentous.

Grob-gefüge macro-structure, coarse or gross s. -höhenmesser (cf. Fein-) barometer measuring actual atmospheric pressure reduced to sealevel.

grobkristallinisch coarsely crystalline.

Grob-sicherung arrester of sparkgap type. -struktur reduced (or reduction of) resolution. -strukturanal yse gross, coarse or macro-structure analysis.

Grossaufnahme (big) close-up (m.p.). Fernbild- large viewing screen projection (for public entertainment).

Grösse quantity, magnitude, entity, size, dimension, format (of pictures, etc.). -, abgeleitete subsidiary quantity, derived q. -,kon jugierte, komplexe conjugate, complex quantity. Wirkungsaction quantity, a. magnitude.

Grössenlehre mathematics; geometry. grössenmässig dimensional(1y), as regards size or quantity.

Grössenordnung order of magnitude. Gross- $\mathrm{fl}$ ächen lautsprecher power loudspeaker, (public) address l., announce, call or paging 1 . system. -funkstelle high-power radio station, long-distance signal station. -kreis (peilung) great-circle (used in bearing calculation). 
-kreisrichtung great-circle direction or course. -lautsprecher power loudspeaker, (public) address loudspeaker (system). -projektion large picture projection (with magnascope).

- schal libertragungsanl age (public) address loudspeaker system, announce, call or paging 1. system. -struktur coarse structure, gross s., tracro s.

Grösstwert maximum (value), maximal or crest $v$.

Grossumfangkople "hi-range" or wide-range print.

Gribchen-bildung, Gruben- pitting, formation of pits.

Grund, Atz- resist, protective coating. Ausschliessungs- reason for exclusion.

Grund-bande fundamental band. - farbe primary, ground or priming color. -fläche basal surface, base; area. -fluissigkeit suspending liquid. -form primary, basic or fundamental type or form.-geräusch (back) ground noise.- -stromkreis (back) ground noise reduction circuit, squelching, suppression or silencing circuit.-geschwindigkeit ground speed. -gitterabsorption fundamental lattice absorption. -gleichungen, Maxwell'sche Maxwell electromagnetic equations. -harmonische fundamental frequency. -helligkeit background, mean or average shading component, d.c. component. -körner fundamental substance, parent s. -kurs true course, head-on c. -linie, Anflugcenter line of approach sector. -masse ground mass. -material matrix, elementary material, base m., ground m.-nachbildung basic network.-niveau background level (or average illumination, telev.). - platte mounting plate, bed p., base, chassis. - punkt cardinal point (of a lens). -rlchtungswinkel base angle (gunnery). -riss ground plan, floor p. - sätzliches fundamentals, rudiments, basic or elementary facts. - scheitelbrechwert primary vertex refraction. -schicht primary or fundamental layer. -schleier inherent fog, ground $f$. (of emulsion resulting in background noise). -schwingung fundamental or first harmonic oscillation or vibration. -stimmen foundation stops (organ). -teilchen smallest particle, fundamental p., atom. -ton pitch, keynote, fundamental or primary tine or frequency; back-ground (noise). -zug leading or salient feature or characteristic, basic trait. -zu-stand ground state (of an atom). -zustand, Zuriuckfallen in

return or re-transition to lowermost enerǵy level.

Grünauszug green record.

grünempfindlich green sensitive.

Gruppe, angeregte exciton (in dielectric breakdown).

Gruppen-erscheinungen group phenomena (cryst.). - frequenz group frequency, number of wavetrains per second, (wave) train frequency, sparking frequency. - geschwindigkeit envelope velocity, group v.; group retardation. -schaltung series-parallel connection. -wähler group selector (aut. telephony).

Gruppierung, El ektronen- phasefocusing (in beam tubes), bunching.

Guckloch peephole, bezel (in radio set panel).

Guitarre guitar.

Guiltigkeit eines Patentes

anfechten challenge validity of a patent, plead or argue invalidity of a $p$.

Gummi, Hart- ebonite, hard rubber, vulcanite. 
Gummi-andrïckroll en rubber-tired presser or pad rollers, -chromverfahren bichromated gum process. - dichtung rubber gasket, $r$. packing. -druckl ack gum printing varnish, - harz gum resin. -propf rubber stopper. - puffer rubber grommet. -stopfen rubber stopper. - überzug(auf Roll enlaufoahn) rubber-tread (of pad roller). günstigst optimal, most favorable, most propitious.

Gurtband belt strap, b. band. Gürtel belt, girdle, band, zone. Kl ima- climatic belt.

Guss, hart- chilled casting. Spritz- (pressure) diecasting, feeder casting, die molded casting.
G. W.

Guss-al uminium cast aluminum. -blase flaw or defect in a casting. -haut casting scale, c. skin. -masse pourable compound, dope.

Gutachten expert opinion, judgment, decision, verdict.

Güte quality, merit, grade, "goodness"; legibility or definition (in telegraphy, television, etc.) Bild- (figure of) merit or quality of pict. übertragungstransmission performance (rating).

Gute-wert, -zahl figure of merit, quality (6) factor (of coil, etc.)

gutschliessend tight-fitting, tightly sealed.

G. W. (Gruppenwähler) group selector (in automatic telephony). 
H Brïckenschaltung, Entzerrungskette mit balanced H-type attenuator network.

$H$ Strahlen $\mathrm{H}$ rays (consisting of $\mathrm{H}$ particles, positive hydrogen $\mathrm{p}$. or protons).

H \& D Kurve Hurter \& Driffield curve (phot.).

haarartig hair-like, capillary. Haardraht capillary wire, follaston พ.

haar-faserig filamentous. -förmig capillary, hair-shaped. Haar-riss craze, micro flaw, (hairline) crack, capillary fissure. -rohr, Heber- capillary syphon, c. tube syringe. - rohranzlehung capillary attraction.

haarscharf very (or microscopically) sharp, precise or correct.

Haarzellen hair cells, capillary c. Habannröhre type of split-anode magnetron; dynatron oscillator (though the latter strictly is predicated on secondary emission). hachieren hatch.

Hacker chopper.

Hafenbefeuerung, Flug- airport beacon service.

naftbar liable, responsible, answerable.

haften adhere, stick, cling. Haft-spannung adhesive stress. -vermögen adhesive power, adhesion. Hahn (stop). cock, tap, faucet, cutoff (vacuum pump).

Hahn, Kreuz- four-way cock. Lichtelectromagnetic mirror vibrator (with vil damping, for variablearea recording). Schraubquetschscrew pinchcock. Tropf- dripping cock. Vakuum- vacuum tap. Vielwege- multi-way stop cock. Vierwege- four-way cock. Wechsel-
H

change cock.

Hahn-fett tap grease, joint g. , vacuum g. -kegel, -küken plug of a cock. -stopfen cock stopper.

Häkchen little hook, clasp.

Haken hook, catch, fastening.

Band-bridle wire, tie hook.

Sperr- pawl, catch, latch, trip, dog, click, detent, ratchet.

wider-barb.

Hakenharfe hook harp.

Halb-achse semi axis. -achsenver-

hältnis ellipticity coefficient.

-art sub-species. -bildent-

fernungsmesser split-field coincidence range finder. -blende half stop, scale. -dunkel twilight, semi-darkness.

halbdurchlässig semi-permeable, semi-transparent.

Halbebene semi-plane, half-p., Hplane.

halbeirund semi-oval.

Halb-erzeugnis, -fabrikat semifinished product, intermediate p. - flächner hemihedron.

halbgeschränkter Riemen quarterturned belt.

Halbheit imperfection, half-measure, half-way step, "half-cooked" condition.

halbieren halve, bisect, disect, cut in half, part.

Halbierende, Winkel- bisector (of angle).

Halblerungslinle bisector, bisectrix (line).

halbklassische Bildkraftberechnung semi-classical image-force calculation.

Halb-kokung semi-coking, partial carbonization. -kreis semi-circle, hemicycle. -kugel hemisphere. -kugellinse semi-circular lens, 
hemispherical 1. - I ängsglied, Abschluss eines Filters durch mid-series termination of a

filter. -leiter semi-conductor, poor c.

halbmatt half matt.

Halb-mattgl asur half-matt or semimatt glazing. -metallglanz submetallic lustre. -öffnungswinkel semi-apertural angle. - periode semi-period, half-period, halfcycle. $\rightarrow$ risma hemi-prism (cryst.) -querglied, Abschluss eines Filters durch mid-shunt termination of a filter. -raster, Ineinanderpassen der interlocking or meshing of fractional scans (in interlaced scanning). -raum half-space.

halbrund half-round, semi-circular, hemi-spherical.

Halbschatten half-tone, half-shade, partial shadow, penumbra.

- apparat half-shade apparatus, half-shadow analyzer. -grenze penumbra boundary. -kompensation penumbral compensation.

Halbton semi-tone, semi-note; sharp (mus.) -bild half-tone picture, mezzo tinto (in black and white, with grey intermediate shading values).

Halbtotale close-medium shot, medium shot.

halb-tragbar semi-portable. -versenkt semi-sunk, semi-recessed. -versilberter Spiegel beam splitter (densitometer).

$\mathrm{Halb}$-vokale liquid consonants, semi-vowels (liquidae). -weggleichrichter half-wave rectifier.

halbwegs fairly, substantially, half-way.

Halb-weitschicht half-value layer. -wellenpotential half-wave potential.

Halbwert-breite half-width,-w. at half maximum intensity (opt., spectral line and band). -dicke half-value thickness or layer. -druck half-value pressure. -punkt half-maximum point. -zeit half-value life, half-l. period.

Halbwinkel half angle.

halbzahliger Spin half-integral spin, half-odd s.

Halb-zähligkeit half integer. -zeit period of half life or change, semi-period, time-to-half value, half-life period. -zelle half cell.

Hal leffekt, Raum- liveness.

hallend reverberant, with multiple echoes.

Hall formanten resonance formants.

Halligkeit liveness (realistic acoustic condition).

Hall raum acoustically live room, reverberating chamber or enclosure. -zahl chamber coefficient (ac.).

Halo-bildung halation (television, etc.). - chemie chemistry of salts, halochemistry.

Hals, Schwanen- goose neck. Stöpselneck, ring, collar or sleeve of a plug. Trichter- throat of a loudspeaker horn, neck.

Hal s-bandkurve collar curve. - lager journal bearing.

hal tbar durable, strong, stable, fast, permanent (of colors).

Hal tbarkeit, Dauer- fatigue endurance.

hal tbarmachen stabilize, preserve. Haltedraht, Gitter- grid stay wire, g. supporting $w$.

halten hold, support, keep, retain, stop, restrain.

Hal tepunkt break.

Halter holder, support, handle, keeper. Distanz- spacing means, spacer, separator. Röhren-, federnder cushioned tube holder or socket, anti-microphonic valve holder. Wicklungs- winding form, coil f., skeleton f., former.

Hal terelais guard relay, holder r. 
Hal terung, Kristall- crystal holder, c. mounting.

Hal terungssystem holder, mounting or supporting system.

Hal te-spule (eines Relais) restraining coil, holding-on c. (of a relay). -strom retaining current, holding c. -vorrichtung chassis (of loudspeaker). -zeit critical range, c. interval, thermal retardation.

hal tlos unstable, unsteady unsupported, unattached, untenable.

Hammer, Pendel-Charpy's impact testing machine.

hämmerbar malleable. .

Hammerkopf (für Bass, Mittellage und Diskant) piano hammer (for bass, tenor and treble).

Hand, an - von by reference to, by the aid of. auf der - liegen (it is) obvious, evident or patent, goes without saying.

Handbuch handbook, manual, compendium.

handel sgängig commercial, marketable. Handel smarke trade-mark.

Hand-empfindlichkeit hand (or body) capacity offect. -feilenklobe hand vise. -griff handle, grasp, grip, knob, button. -kamera, Berufs- professional hand camera. -kapazitat body capacity effect, hand c.e. -koffer carrying case, suitcase.

Handlung plot, story, treatment, theme, scenario, shooting script (m.p.).

hängen bleiben "take hold" (in silent tuning).

hantieren work, handle, manipulate, manage, operate.

Harfe, Doppelpedal - double-action harp. Haken- hook harp. Klavierkeyed harp, piano $h$.

Harfenantenne harp antenna, fan a.

Harmonieflöte organ accordion. hart (es) Bild crisp or harsh picture, high-contrast p., contrasty p., hard p. - (e) Kom- ponente der Höhenstrahlung penetrating or hard component of cosmic rays. - (e) Röhre hard tube, h. valve, high-vacuum $t$. - (e) Teilchen primaries.

härtbar hardenable, temperable, indurable, capable of being hardened" or cemented.

Härte, Auslenk- deflection hardness (force required to deflect phonograph needle point $100 \mu$ ). Schallsound or acoustic stiffness, rigidity, ratio pressure amplitude: velocity amplitude as function of frequency.

Härte-bad, Fixier- fixing and hardening bath. -bildner salt causing hardness (in water). -messer, Strahlen- penetrometer (for X-rays), qualimeter.

härten harden, temper, indurate.

Härteprüfer hardness tester

(Brinell, Rockwell sclerometer and scleroscope).

hartgeschlossenes Rohr rigidly terminated tube (ac.).

Hart-gewebe indurated or impregnated fabric. -glas pyrex. -gummi hard rubber, ebonite, vulcanite. -guss chilled casting. - lagerhärtung induction hardening.

Härtling (hard) dross.

Hartlötung hard soldering, brazing. Härtung, Ausscheidungs- precipitation hardening. Brennstrahlflame hardening, Einsatz- case hardening. Oberflächen- case hardening, surface cementing.

Härtungs-biegeprobe bending test in tempered state. -kohle hardening carbon, cementing $c$.

Hartwerden (einer Roentgenröhre) hardening of an X-ray tube (due to clean-up effect and gain of vacuum).

Harz, Dammar- Dammar resin or gum. harzhaltig resinous, resinic, resiniferous.

Haspel reel, spool, windlass. Haufen cluster, aggregation, 
accumulation, piles, agglomeration, clump. Molekuil-molecular clusters.

Häufigkeit frequency, incidence, abundance.

Häufigkeitsverhäl tnis abundance ratio.

Haufwerk, Kristall- aggregation of crystallites.

Haupt-achse prime axis, major a., vertical axis (of a rhombic crystal). -azimuth principal azimuth. -ebene principal plane, p. surface. -ebenenabstand distance between principal planes or points. -einfallwinkel angle of principal incidence. -einflugzeichen inner marker signal, main entrance s. (nearest airport boundary, in airplane landing), main marker. -formanten main formants, basic f. -funkfeuer main route or airway radio beacon. - gitter parent lattice. - l inie principal line (spectr.). -nenner common denominator. -patent

parent patent, main or original patent. -peilstelle main control direction-finder station (of an air communication safety district comprising several ground df stations). -punktbrechwert principal point refraction. -punkte principal points, unit p. -quantenzahl principal quantum number. -regler main governor or regulator (of a turbine). -rohr main oscillation generator (in independent drive system). - rolle leading star. -rollendarsteller principal, star, star performer.

hauptsächlich main, chief, principal. Haupt-sammell inse second lens (in electron gun, c. $-r$. tube). -satz fundamental principle, folaw, $f$. theorem, axiom (math.). -schnitt, erster (1.) primary plane, tangential p., meridional p. (opt.). -schnitt, zweiter (11.) secondary plane, sagittal p. (opt.).
- sender main oscillator, m. transmitter. -signal inner marker signal, main entrance s., main marker (in airplane landing). -streckenfever main or trunk route or airway beacon. - taste master key. -uhr master clock. -valenzkette primary valence chain. -verhandlung main oral hearings, m. proceedings. -zahl cardinal number. -zipfel major lobe (of space pattern). zug main object (of an invention), outstanding or principal characteristic feature or trait.

haustelephon domestic telephone, private home telephone, house 'phone, telephone extension, intercommunication $t$.

Haut skin, hide, film, membrane. Guss- casting scale, c. skin. Licht- cathode glow. 0xyd- skin or film of oxide.

Haut-abstand (focal) skin distance. -widerstand dermal or skin resistance. -wirkung skin effect.

Heaviside'sche Einheitsfunktion $H$. unit function.

Hebdrehwähler vertical and rotary selector.

Hebedaumen cam, tappet, finger, disk with lobes.

Hebel, drehbar gel agerter pivoted lever, fulcrumed 1 . (when with two arms). - , hin- und hergehender rocking lever, oscillating 1 .

Hebel, Aufzieh- lever ratchet. Ausiöse- trip lever, detent lever. Einrück- starting lever, tripping lever, engaging 1. Einrück- und Ausrück- starting and stopping lever. Fühl-tactile lever, feeler, scanning 1., pickup 1., probing 1., indicator (of micrometric caliper). Schwung- balance beam, rocker, rocking lever. Sperr- pawl, latch, arresting lever, lock 1.

Hebel-arm, Strahl-pencil leverage, deflectibility of $p$. -detektor 
lever-adjusted cat whisker crystal detector. -schalter, Zweipoldouble-lever switch. -wage beam scale, lever s. -wirkung leverage, lever action, purchase.

Hebepunkt fulcrum.

Heber, Kontrast- (dynamic-range)

compandor (comprising compressor and expander, to improve signalnoise ratio).

Heber-barometer syphon barometer.

- haarrohr capillary syphon. -pumpe

syphon pump. -rohr syphon tube,

syphon. -schreiber syphon recorder.

Hebeschritt, Wähler- vertical step

of selector (automatic telephony).

Heck stern.

Heft handle, hilt; number or issue

of a magazine, stitched booklet.

heilgerät therapeutic apparatus

(e.g. using short waves, rays, etc.).

Heimfernsehempfänger home television receiver.

heimlauf backstroke, return s. or motion; retrace or flyback (of pencil or beam, in telev. tube, often gated by blanking pulse).

Heiserkeit raucousness, hoarseness. heissbrüchig hot-short.

Heissdampf superheated steam.

heissgrädig difficultly fusible.

Heiss-kühlung cooling by evapora-

tion. -leiter hot conductor

(with negative temperature coefficient), third class conductor. Heiz-kathoden-Roentgenröhre hotcathode X-ray tube, Coolidge tube. -korper heater (e.g., for indirectly heated cathode), heating element; radiator. -kraft heating power, calorific p. -lötstelle hot junction (of a thermocouple). -mantel heating jacket. -unruhe fluctuation in heating current, ripples. -wendel heater spiral, heater coil.

hell bright, brilliant, light, pale, clear, transparent, limpid; strid- ent, shrill (ac.).

Hell anpassung bright adaptation, light a., photopia.

Hell-Dunkel-Intervall light-dark range. - I inien light-dark-lines.

helle $\mathrm{Kl}$ angfarbe strident timbre, shrill t. - Stelle (im Film) clear portion (in film).

Helle, Flächen- brightness of a surface, intrinsic brilliance.

Hellfeldbeleuchtung bright ground illumination (micr.).

Helligkeit (als physikalische Grösse) photometric brightness, luminance, radiance (in candles per unit area). -(als psychologische Grösse) visual sensation. -eines objektivs speed of a lens or objective. rielligkeit, mittlere average background illumination (telov.).

Helligkeit, Fyächen- surface brightness, intrinsic brilliance.

Grund- background, mean or average shading component, d.c. component. objektiv- speed of a lens or objective. Raum- volume brightness. Ton-pitch (sound sensation).

helligkeits-angleichung brightness matching. - schwebung brightness flutter. -signal, Bild- video signal, picture shading s. -steuerung brilliance modulation, brightness control, intensity $\mathrm{m}$. -stufe intermediate brightness stage, i. grey value (between black and white), shading or tonal v. -umfang brightness range, contrast, key (of a picture). -verteilung brightness distribution (of highlights and of shadows). -wechsel je Sekunde frame frequency per second, number of illuminations of a picture point p.s. - werte brightness values. density $v_{.}$, shading $v$. Hell-rotglut bright red heat. -schreiber Hell-type recorder. - sektoren shutter openings. - steuerung modulation to light condition. 
Hemieder hemihedron, hemihedral form, h. crystal.

hemmen stop, check, brake, impede, obstruct, retard, inhibit, clog, lock.

Hemm-rad brake wheel, escapement w. -schuh brake shoe.

Hemmung escapement, retarding action, brake a., stopping a., check, hindrance, restraint, inhibition (in reactions).

Hemmungs-grund disability (law). -körper restraining substance, decelerator.

Henkel handle, lug, ear.

Herabsetzung stepping down (of potential, gearing, etc.), decrease (speed, by step-down gear); submultiplication.

heranlotsen guide to (airplanes, etc.).

herausarbeiten work out of (say, a solid piece, by cutting, punching, etc.), blank out.

Heraus-führung lead-in (wire or conductor), lead-out wire. -gabe restitution, return, delivery, surrender, issuance, edition (of a publication).

heraus-hören apperceive (say, a certain note in a spectrum). -pipettieren pipet out, remove with pipette. -spritzen spurt or spout out, gusher, gush. -treten protrude, emerge, step out of.

herrschende Verhältnisse prevailing circumstances.

herrühren originate, proceed or come from.

herstellen make, produce, manufacture, close (a circuit, a connection).

Hertz-Einheiten cycles per second, cps. -'sche Welle Hertzian wave, $H$. radiation, electric wave.

herunter-gehen let down (of an airplane). -klappen let down in hinges, hinge down, drop (as a table leaf). -regeln, -stevern regulate (a quantity) in a downward direction, regulate down. hervor-bringen produce, create, set up, develop. -heben make conspicuous or prominent, underscore, highlight; accentuate, emphasize, underscore (certain frequencies or bands). -ragen project, stand out, be salient. Hervorrufungszeit developing time. Herz-bahn cardioid-shaped path (of electrons). - charakteristik cardioid characteristic or space pattern. - exzenterbewegung cam movement of Livin. -spannungskurve electro-cardiogram. heulen howl.

Heul-signal siren: -ton sound with large number of modes of vibration, warble frequency tone, highfrequency warble tone ("multitones").

Hexodenkappe top cap of hexode tube. H-Glied H-type section, filter mesh or network $\mathrm{m}$.

hiebton Aeolian note.

Hilfs- auxiliary, subsidiary, subordinate, ancillary, accessory, standby.

Hilfsfrequenz pilot frequency auxiliary f.; local f. oscillation (in superheterodyne). -rückkopplung super-regeneration (working with quench voltage).

hilfs-funke pilot spark. - I andeplatz emergency or auxiliary landing place or field. -mittel auxiliaries, accessories, supplemental or expedient means, makeshift, adjuvants. -netz auxiliary (control) grid. -peilstelle auxiliary, associate or affiliated ground direction-finding station (subject to main control station). -phasenkreis starting-winding circuit. (of split-phase induction motor). -sender auxiliary transmitter, local oscillator (in heterodyne scheme). - stoff adjuvant (substance), auxiliary or 
accessory material or agent. -träger auxiliary carrier, subcarrier. -voramt sub-control station.

Himmelfernrohr astronomic telescope, astrographic t.

himmel s-gegend point of compass, quarter of the heavens. -mechanismus astronomical dynamics, celestial mechanism or mechanics.

Hinaufpendeln (gradual) rise in resonance (amplifier).

Hindernis bar, barrier, difficulty, obstacle; estoppel (law). , gesetzliches statutory bar, legal impediment estoppel. Luftfahrt- avigational (air navigation) obstacle.

Hinderungsgrund sein be a bar to (law).

hineinziehen draw in, drag in, incorporate, involve.

Hinführung und Rückführung lead and return (path or circuit wires); scansion and fly-back (telev.).

Hin-gang, -lauf forward move, f. working stroke, travel, pass or journey; scansion, scanning motion, (active-line) sweep (telev.).

Hinl auf-Rückl auf-Verhältnis sweepflyback ratio.

hinleiter outgoing lead, o. wire, o. conductor.

hinten in the rear, behind, posteriorly.

Hinter-ansicht rear elevation, r. view. -beleuchtung Rembrandt illumination, half-back i.

hinterdrehen back-off, relieve eccentrically.

hintere Brennweite back focal length.

hintereinander sequentially, consecutively, serially, one after the other.

Hintereinanderschaltung series connection, seriation, cascading, concatenation or tandem connection (of motors).

\section{Hochfrequenzkamer a}

Hinter-grundeffekt background effect. -grundprojektion back

(ground) projection.

hinterlegen deposit, file, record, register.

Hinterlinse back lens.

hinterschnitten undercut.

Hinter-setzer false front. -wandzelle photo-voltaic barrierlayer cell with posterior metallic layer.

hinüberreissen carry over (in a distillation).

Hin- und Herbewegung shuttle, rocking, oscillating or reciprocating motion.

Hinuntertasten carrier caused to drop to low value (zero) by synchronizing signal.

Hirschleder buckskin.

Hitzdraht-anemometer, -sonde hotwire anemometer. -strommesser thermal ammeter, hot-wire a.

hitze-beständig stable or resistant to heat, heat-proof, thermostable, refractory. -empfindlich sensitive to heat, susceptible to thermal action.

hitzen, über- superheat, overheat. Hitzmesser pyrometer.

Hoch-antenne open outdoor antenna (installed at, or reaching, a point high above ground). -ätzung relief etching, $r$. engraving (metal bears outline of pattern in resist). -aufnahme vertical or upright picture or shot. -bel astbarkeit high-load or heavy-load or current-carrying capacity.

hochblau bright blue, azure.

Hochdruck high pressure; relief print. -kondensator pressuretype capacitor (in nitrogen tank). -rohr, Quecksilber- high-pressure mercury discharge tube. Hoch-frequenzbereich treble range, treble band (mus.). -frequenzheilgerät short-wave therapeutic apparatus. -frequenzkamera high- 
speed camera.

hochfrequenzmässig for high frequency, so far as high frequency is concerned, relative to high frequency.

Hochfrequenztelephonie, leitungsgerichtete carrier-current or wire-guided radio telephony. hochgekohlt high-carbon; with high carbon content (of metal).

Hoch-glanz high polish, h. lustre, brilliance, -heim'sche Verspiegelung special mirror plating or "silvering" (of I.G. Farben). - intensitätskohle high-intensity carbon (arc).

hochkantig on edge, edgewise. Hoch-l autsprecher tweeter loudspeaker, treble 1. - leistungslautsprecher high-power loudspeaker, (public) address 1., announcer, call or paging 1. hoch-l ichtstarkes 0 bjektiv highspeed or ultra-speed objective or lens. - rund convex. -schmel zend high-melting, difficultly meltable.

Hoch-schule, technische techological institute, polytechnical university, technical college. - spannungskondensator highpotential (fixed) condenser or capacitor.

hochspannungssicher safe against high-potential breakdown, having high puncture strength, proof against high p. and shock.

Höchst-besetzungszahl maximum number of electrons in shell. - leistung maximum power, peak p., crest p.; extreme record performance.

Hochstrombogen heavy-current arc, large-c. arc. - entladung, nicht kondensierte heavy-current uncondensed discharge.

Höchstwertanzeiger crest indicator, peak i.

Hoch-tastung working impulse transmitted on voltage substantially above normal; transmitter for impulse-keying operated at voltage far above normal. -tonkonus, -tonl autsprecher tweeter or treble (cone) loudspeaker. Hochvakuum-gleichrichter kenotron, electron-type rectifier. -röhre high vacuum tube, hard $t$.

hochzeiliges Bild high-definition

(telev.) picture.

hochziehen pull out (of a dive, airplane).

Höcker hump (of resonance curve); bump, protruberance; pip (of $c .-r$. screen tracing).

Hof, Licht- halo, halation. hoffreie Platte, Iicht- nonhalation plate.

Höhe, Breiten- meridian altitude. objektiv- height of objective or lens. Sprung- pedestal height or level; amplitude of return or flyback (of beam or pencil, in telev.). Windungs- pitch of turns.

Höhen high (audio) frequencies, h. pitches. Absenkung in den- deaccentuation or attenuation of higher (audio) frequencies, using treble de-emphasizer means.

Höhen-einstellung height control (telev.). - lader supercharger (with high blower ratio). -lage height, altitude, position in vertical sense. -messer altitude meter, altimeter (for instance, echo or reflection type sound ranging altimeter), statoscope, terrain clearance indicator (of W.E. Co.). -messer, Fein(aneroid) barometer measuring atmospheric pressure at airdrome not reduced to sealevel, sensitive altimeter. -messer, Grobbarometer measuring actual atmospheric pressure reduced to sealevel. -schritt vertical step (in automatic telephone equipment). -strahlen cosmic rays. -strahlenschauer, -strahlenstösse bursts 
or showers of cosmic rays, c. ray track (traced by odoscope). -strahlung, harte Komponente der penetrating or hard component of cosmic rays.

höhensymmetrisch symmetric about vertical axis.

Höhen-verstellung adjustment in elevation. -wert altitude value

(in vision). -winkel angle of elevation, azimuthal a., angular height, vertical visual a.

Höhepunkt culminating point, summit, top, crest, maximum, critical point.

hoher Ton tweeter, treble, highpitched or h.-frequency note or sound.

höhere Instanz higher court, superior c., c. of appeals.

hohl all bottom, boomy, dull (of sound or voice); hollow, concave (of mirror).

hohl(er) Ton boomy sound, dull s. (high AF frequencies cut of $f$ ).(e) Wiedergabe reproduction with high acoustic frequencies cut of $f$ or de-accentuated. höhle, Augen- socket of eye, orbit, orbital cavity. Ohr- ear cavity. hohlerhaben concavo-convex.Hohl-gitter concave grating. -heit cavity, emptiness.

höhl'g honeycombed, having cavities or pits.

Hohl-kathode concave cathode, hollow c. -kehlenhal bmesser fillet radius. -metall rohr, Well enausbreitung in wave propagation in wave guide or metal pipe (dielectric or conducting). -prisma hollow prism.

Hohl raum reverberation chamber (ac.); (blackbody) cavity. Resonanzresonance cavity, r. chamber, rhumbatron. -bildung cavitation (in supersonic waves). -kabel, Papier-airspace paper-core cable, dry-space c., dry-core c. -resonator cavity resonator, chamber r., rhumbatron. -strahlung black-body radiation, cavity $\mathrm{r}$.

Hohl-rohrleitung hollow pipe line, h. wave-guide (either dielectric or conducting); concentric line, co-axial 1., pipe 1. -schicht airspace. -schraube female screw. -sog cavitation. -splegel concave (mirror) ref lectar.

Höhlungsresonanz cavity resonance (of rhumbatron and klystron).

Holm, Flugzeug- spar of an airplane wing. Gitter- grid stay, g. supporting member. Tragflächenwing spar.

Holoeder holohedral form of crystal. Holoedrie holohedrism.

rolz, engjähriges close-grain wood. Knet- plastic wood. Kohlcharcoal (of wood).

Holzfaser wood fiber, ligneous fiber, woodpulp. -stoff lignin, cellulose, ligno-cellulose.

Hol z-mehl wood meal, w. powder, w. flour. -pech wood pitch. -p feife wood pipe (of organ). -schliff mechanical woodpulp. -schnitt wood stock, w. block, engraving, w. cut. -stoff woodpulp, cellulose. -wolle excelsior, woodwool.

holzzerstörend lignicidal. homochrom of uniform color, homochromatic, homochromous.

Honigwabenspule honeycomb coil. hörbar audible, aurally perceptible, auditory, capable of being heard or acoustically perceived or sensed. -machen render audible, audibilize.

Hörbarkeits-lautstärkeregler audibili-

ty network. - schwelle threshold of audibility or of auditory or acoustic perception (represented by audiogram).

Horch-dienst, Funk- radio intersept service. -gerät radio or sound locator, listening or detecting device (e.g., to spot airplanes 
by sound, based on echo principled range- and distance-determining device, intercept receiver, asdic (submarine detector). -ortung radio intercept service, sound location, s. ranging.

fiör-empfang auditory reception, aural r., r. by ear, hearing or earphones. -empfindlichkeit hearing ability, auditory sensitivity.

Hören, einohriges monaural listening or reception. -, zweiohriges binaural or plastic hearing or audition, two-channel listening. Richtungs- aural determination of direction (of airplanes), airplane spotting. Rück- side tone.

Hörer, Schau-aural and visual audience, video-audio a., persons receiving audible and visual impressions simultaneously. Sprechmicrotelephone, telephone handset, transceiver, walkie-talkie set.

hörer-echo listener echo. -gehäuse receiver case, r. shell.

Hörfilm (Tonfilm zur Rundfunkïbertragung) sound film broadcast, s. telecine or telecasting.

Hörfrequenz audio, audible, tonal or acoustic frequency, A.F. uber- supersonic, super-audible or ultra-audio frequency. Untersub-audio frequency.

hörig, doppel-binaural. einmonaural.

Horizont, kinstlicher Flieger- artificial horizon.

Horizontal-ablenkung horizontal or line scan, sweep or deflection. -wechsel horizontal or line synchronizing pulse or cycle. Hör-kopf sound (pickup) head. -muschel earpiece, earcup.

Horn, Exponential-, aufgewundenes twisted or curled exponential or logarithmic horn (of loudspeaker). Exponential-, gefaltetes folded exponential iudspeaker horn. Horn, Ruf- signal horn. Signal- bugle. Wald-French horn, concert h.

hornig horny, horn-like, corneous, callous.

Hör-peilung auditory direction-finding. -samkeit acoustic properties, acoustics. -schärfe acuity of hearing. -schwelle audibility chreshold. -sorechschalter talk-listen switch (in inter-communication systems).

Hosenrohr syphon pipe, Y-pipe.

Hub stroke, throw, travel, excursion; percentage modulation. Frequenzfrequency swing or deviation (above and below the assigned resting frequency, in f.-modulation), amount by which instantaneous carrier $f$. differs from resting $f$. Rück- return stroke, back s. Hubmagnet vertical stepping magnet (automatic telephony).

Hügel, Potential- potential barrier. Huille cover (ing), case, casing, wrapper, envelope, sheath, cloak, shell, hull (ship), contour, shroud. Atom- atom shell. Dampf- steam jacket; vaporous envelope or sheath. Elektronen- electron shell, e. shroud. Leucht- luminous sheath or envelope, aureole, photosphere (of sun), 1. shroud. Schutz- protective cover, p. coat, p. sheath.

Hïllen-elektronen 'shell electrons. -kurve envelope. -rohr encasing tube or pipe, jacket.

Hülse bush, sleeve, socket, case, collar, barrel.

Hülsenschliff female part of ground joint.

Hupe reed horn.

Hüpfer, Hüpferschal ter contactor (in multiple-unit control system).

Hütchenkondensator ceramic hoodshaped fixed condenser or capacitor.

Hüttenarbelt smelting, founding or foundry work.

Huygens'sche El ementarwell en Huygens wavelets.

H W Z (Halbwertzeit) half-value life, 


\section{hydrophob}

138

half-life period. hydrophob non-hygroscopic, non-

absorbent, water- or moisture repellent.

Hyperebene hyperplane.

hyperfeine Struktur hyperfine structure.
Hyperfunktion hyperbolic function. Hysteresemesser hysteresis meter. -, schreibender hysteresigraph. HZ (Hertz) cycle per second, Hertz unit (of frequency). 
IBK-System C I E (Commission Internationale d'Eclairage) chromaticity scale.

I conometer iconometer, wire-f rame view-f inder.

I gnitron-Stromtor-Kombination mutator.

im allgemeinen as a general rule, generally speaking, by and large.

Im Grunde genommen basically or fundamentally speaking, as a matter of principle.

Im übrigen as to the rest.

Immersionslinse immersion lens, i. objective (with oil or water, of microscope); double aperture lens.

Impedanz s. a. Scheinwlderstand. Impedanz, Abschluss- terminating, end or load impedance or impedor. Bewegungs- motional impedance. Bl Indkomponente der reactive impedance component. End- terminating impedance, end i., load i. streu- leakage impedance. Stromkrels mit-impedor. Wirkkomponente der - active, dissipative, effective or resistance component of impedance.

Impedanzglied, Reihen- series impedance element or impedor.

Impfen inoculate, vaccinate, inject.

Imorägnlermittel impregnating agent, impregnant.

Impuls momentum (in dynamics); impulse, pulse (telev.), surgé (when of unidirectional polarity).

Impul s, Austast- blanking pulse (to gate retrace of pencil, in telev. tube). Bahn- orbital moment. Bildsynchronisier- frame synchronizing impulse, low sync. i.
Dreh-(spin) angular momentum (spin). Gleichlauf- synchronizing impulse; correcting i. Kipptripping impulse. öffungsbreak impulse.

Impuls, Takt- synchronizing impulse, sync signal. Zellensynchronisierline synchronizing impulse, high sync. $i$. Zellenfolge- line sequence impulse, line set impulse, partial scan irpulse (in interlaced scanning).

Impuls-abtrenner, Synchronisiersynchronizing separator, amplitude s., clipper. -erhaltungssatz theorem of conservation of momentum. -gabe impulsing, pulsing, i. transmission or emission. - geber impulse generator, surge g., impulsing device, i. transmitter; tonal generator, tonic transmitter. -generator (cf-geber) impulse generator, surge g., lightning g. -messer peak or crest (voltage) meter.

I mpul smodul lerte Wellen pulsemodulated waves (as in radar). Impuls-öl schalter oil-blast circuit breaker. - peilverfahren impulse direction-finding method. -platte signal plate (iconoscope). -prüfung flash or impulse test (of insulation). -quantenzahl spin quantum number. - summe sum of momenta. - tensor, Energleenergy momentum tensor. -transporttheorie momentum transportation theory (of turbulent flow). - verfahren, Doppel- double impulse method.

Inaktivlerung inactivation, deactivation; racemization (opt.). - ,optische racemization (of a quartz crystal). 
Inanspruchnahme claim, right of taking advantage of.

Inbetriebsetzen setting in motion, rendering operative, starting, initiating.

Indeterministische Auffassung indeterministic conception or interpretation.

Indexstrich index mark, i. line, gage m.

indirekt geheizte Röhre heater type tube, indirectly heated tube.

-(er) Dampf latent steam.

Induktion, wechsel-mutual inductance.

induktionsfrei(es) Kabel screened (conductor) cable. - (er) widerstand non-inductive resistance, effective r., ohmic $r$.

Induktionsspule, variable, variometer.

Induktive Kopplung inductive coupling, transformer $c$.

Induktivität, punktförmige lumped inductance, concentrated i. (or electrical inertia). -, verteilte distributed inductance, continuous loading. streu- stray inductance, leakage $i$.

Industriegeräte commercial apparatus, industrial a. (as distinguished from precision and laboratory equipment).

ineinandergreifen interlock, interengage, intermesh (as partial scans).

inertmachen de-activate, devitalize, de-energize.

Influenz electrostatic induction.

Inhalt content (s), area, volume, substance, capacity. Bild-picture area, p. content or subjectmatter, "information". Flächen(surface) area.

Inhaltsangabe table of contents, index.

Inhibierung stay of proceedings, estoppel.

I nhomogen ität non-homogeneousness, lack of homogeneity, heterogene- ousness.

Inkl inationswinkel magnetic inclination, m. dip.

Inkrafttreten commencement (of an act or law), coming into force.

Innen-antenne indoor antenna or aerial. -aufnahme indoor shot, studio s.; oscillogram directly recorded on photographic emulsion inside vacuous space. -gewinde internal thread, female t. -grenzlehre plug gage. -photographieoszillograph internal photography oscillograph. -raumdurchführung indoor bush(ing). -taster inside calipers.

innere Quantenzahl inner quantum number. -Reibung solid viscosity, internal friction.

innermolekulare kette inter-molecular chain.

innewohnend inherent, intrinsic, innate.

Insel island.

Inselartige Ablagerung, Inselbildung "island" formation.

Insgesamt altogether, substantially, on the whole.

Instand-haltung servicing, maintenance work. - setzung repairing, reconditioning, restoration, rehabilitation.

Instanz, Gericht erster court below, lower court. Gericht höchster- supreme court. Gericht zweiter oder höherer - court above, higher c., c. of appeal. Gerichtsbarkeit erster - original jurisdiction.

Instrument, Blech- brass instrument (mus.). Dreheisen- moving-iron instrument (of plunger, vane or repulsion type). Mess-, gedämpftes deadbeat measuring instrument, aperiodic m.i. Mess-, ungedämpftes ballistic measuring instrument. Schreib- graphic or recording instrument.

Instrumentausrïstung instrumentation. 
Instrumentenfehler variations of bearing d'se to instruments (df), measuring instrument error.

Integral, Austausch-exchange integral. Raum- space integral.

Integralrechnung integral calculus.

Integrierbar integrable.

Intergrieren über integrate over.

Intensitäts-ausfall fading.

-messgerät level recorder.

-steuerung intensity, brightness or brillance modulation (of c. $-r$. tube), -verfahren variabledensity method (of sound recording).

Interferenz-bild interference figure. - Tarbe interference color. -gebiet mush area (in chain broadcasting).

interferenzialrefraktor interferometer.

Interferenz-streifen interference fringes (opt.) -ton beat tone, interference note.

Interferometer, Gitter-grating interferometer.

Interkombinationen inter-system combinations.

Intermediär intermediary, intermediat $\theta$.

Intermittenzschwingung relaxation oscillation, sawtooth o., ratchet o., timebase o.

Intervall-faktor interval factor. -regel interval rulo (of Landré). intervenieren intervene, interpose, interfere.

Intonation voicing.

intonieren tune (a piano), intone.

in Tritt kommen fall in step, synchronize, phase-focusing (in beam tubes).

Inverter inverter (thyratron).

Invertzeitrelais' inverse-time relay.

Ionen, Locker- defective ions, "Locker" i. 'Sprüh- spray

ions. Zwischengitter - interstitial ions. Zwitter-dual. hybrid or amphoteric ions.
Ionen-beweglichkeit ionic mobility. - falle ion trap (in electron gun of c. $-r$. tube). -komponente, Wasserstoff- $\mathrm{pH}$ value, hydrogen ion concentration. -rüickkopplung ion feedback. -rumpf ion care. -rundlauf circulation of ions. -spaltung ionic cleavage, ionization. -stärkemesser ionometer. -steverrohr thyratron. -windvoltmesser ionic wind voltmeter. -wolke ionic atmosphere.

Ionisationswärme heat of ionization, h. of dissociation.

Ion is ierungs-k ammer, . Finger hutthimble ionization chamber. -spannung ionization potential, ionizing p., p. sufficient to detach electron from its atomic bond. -spielaufbau Townsend structure or build-up. -stufe stage of ionization.

Iris-auf- und-Abblendung irising in and out. -blende iris diaphragm.

irisieren irisate, iridize, iridesce.

irreführen confuse, mislead, misdirect.

Irrung correction.

I sokl inen isoclinics (lines of equal darkness, in photoelasticity).

I solations-messer insulation tester, megger. -prüfung, Stoss- impact, impulse or flash test. -widerstand insulation resistance, insulance (reciprocal of leakance).

Isol ator, unvollkommener imperfect dielectric, leaky insulation. Abspann- terminal insulator, strain i. Abstand-, Distanzstand-off insulator, spacing i. Durchführungs-, Einführungsfeed-through insulator, lead-in i., wall duct, bushing.

Freiluft- outdoor insulator. Kreuzungs- transposition insulator. Pilz- mushroom, petticost or umbrella insulator (long leakage path). 
Stütz- pin insulator, rod i. wanddurchlass- wall lead-in or bushing insulator.

I solier-masse insulating compound,

i. paste, moldable or fictile i. substance, "dope." -masse, gepresste molded insulation. -röhre buffer tube, isolating valve.

I sotopieverschiebung isotope of fect, $i$. shift.
Jacarandahol z rosewood.

Jahresgebühr annual fee or tax. Jahreszeitliche Änderungen seasonal variations. Jalousiebretter louvres. Japan-1 ack Japan lacquer, J. varnish. - wachs dapan wax. jeweils under given or prevailing conditions; at the time, in each case.
J

Jodieren iodizing (of a negative). jungfräuliche Kurve virgin curve. Justier-greifer pilot claw (m.p.) -stift pilot pin, registration p. (film printing.) Justierung, Film-registration of film.

Jutebeilauf jute filler (cable making. ) 
$K$ Absorptionskante, $K$. Wert $\mathrm{K}$ edge, $\mathrm{K}$ absorption limit.

$K$ wert octantal component of quadrantal error (GE) due to fuselage.

$K$ and $L$ Schale-Abschirmungskoeffizient $K$ and $L$ shell absorption coefficient (screening function.)

Kabel, gemischtpaariges composite cable. - , induktionsfreies screened (conductor) cable. -,konzentrisches co-axial line, concentric 1. or cable, pipe 1. -,mehradriges multi-wire cable. Erd- leader cable, pilot cable, buried c. (for airplane landing.) Faden- co-axial or concentric cable with silk-thread-supported conductor. Leit- leader cable, pilot cable (for airplane landing.) Papierhohlraum- airspace paper-core cable, dry-core c. Photozellen-low-capacity shielded cable between photo-cell and amplifier, video c., concentric or co-axial c.

Kabel-schuh cable eye, c. socket, connector lug, sweated thimble. -verlegung cable laying, burrying of a c. -wachs cable wax.

Kabine, Kino-, Vorführungs- projection room, p. box; p. booth (in back projection.)

kadmiertes Eisen cadmiated iron. Käfig, Auffänger - collector electrode (in Farnsworth dissector.). Faraday'scher Faraday shield, F. screen, F. wire cylinder. Roll en- roller cage.

Käfig-antenne cage antenna. -spule canned coill, shield c., cage c. -transformer shielded transformer.

Kal iber bore, pass, caliber, diameter; groove. -dorn, Toleranz- tolerance plug gage.

kalkartig calcareous, limy.

Kalk-schleier chalk fog. -spat calcite.

Kalorimeter, Eis- ice calorimeter. Kondensations- steam calorimeter.

Kalotte cup-shaped, cap-shaped or hemispherical part, calotte.

$\mathrm{Kal}$ ottenmembran hemispheric or capshaped (metallic loudspeaker) diaphragm.

kalt(er) Farbton cold color hue.

kalt(e) Kathode cold cathode (as in photo-tube and $\mathrm{BH}$ type gaseous rectifier).

$\mathrm{Kal}$ taushärten cold tempering.

kalt-bläsig refractory (of metal.) -brüchig cold short-brittle.

Käl teeinheit frigorie.

$k a ̈ l$ teerzeugend frigorific, cryogenic.

Käl te-punkt solidifying point (of oil). -regler cryostat.

$\mathrm{K}$ al t-lack cold varnish. -schlag cold heading die. - sprödigkeit cold brittleness. - stauchmatrize cold upsetting die. -verformung cold working, c. shaping.

Kamera, Atel ier-studio camera. Berufshand- professional hand camera. Geheim- detective camera, concealed c. Hochfrequenz- highspeed camera. Laufboden- baseboard extensible camera. Lochpin hole camera. Mikrospektralmicrospectroscopic camera. Mebelcloud chamber. Rundblickpanoramic camera. Spreiz(en)strut camera, extension c. Tonsound recording camera. Tricktrick camera. Wil son-fog, cloud 
or expansion chamber (of Wilson). Kamera-auszug camera extension. -balgen camera bellows. -fahrgestell camera truck, dolly.

-laufwerk feed mechanism. -mann, Atelier-studio cameraman. -mann, Ton- recordist.

$\mathrm{Kamm}$, Folgeschalter - sequence switch cam.

Kämmen der Turbinendichtungen rubbing of labyrinth seals.

Kammer chamber, room, space, camera, compartment. Aufzeichnungsrecording chamber (of electron microscope). Fingerhut(ionisierungs) - thimble ionizing chamber. Klein-small (air) chamber for ionizing work. Leistungsenergy chamber (of klystron). Luft- air pressure space (of a loudspeaker.) Nachhall - reverberation room, $r$. space or enclosure. wilson-Wilson fog, expansion or cloud chamber.

Kammer-gericht Supreme Court (of Frussia.) - l autsprecher, Druckpressure chamber loudspeaker, pneumatic 1. (without diaphragm.) -ton "A" of tuning fork.

$\mathrm{Kamm}-\mathrm{l}$ ager (concealed) collar thrust bearing. - rad cog wheel. - zwecke tack.

$K$ amp f stoffe (chemical) warfare substances.

Kanadabal sam Canada balsam. Kanal, Film-, Führungs- film track, f. channel (in film camera.) Funken- spark track. Sendetransmission channel, signaling $c$. Teil- sub-channel. Ubertragungstransmission channel. Vorentladungs pre-discharge track.

$\mathrm{K}$ analfilter channel filter.

kanalig, mehr- multi-channel.

$\mathrm{Kanal}$-strahl canal ray, positive $r$., diacathode r., anode r. -tastung, Ein- single-channel pulsing (telev. synchronization.)

Kanonenbronze gun metal, g. bronze. Kanonier, Richt- gunlayer.
$K$ ante, abl aufende trailing edge. Leit- leading edge, 1. tip (of airplane wing). Schmal- narrow edge, n. side.

$\mathrm{K}$ anten-schärfe resolution on border (of a picture.) -schema Deslandres diagram (of spectral band systems.) -system zero-line system, edge s.

kantenwei se edgewise, on edge. $k$ antenwirkung edge effect, fringe $\theta$. Kapazität s. a. Kondensator

Kapazität, akusti sche acoustic capacitance, a. compliance (reciprocal of a. stiffness.) -, mechanische compliance. -, punktförmige concentrated capacitance, lumped c. -, verteilte distributed capacitance, self-c:

Kapazität, Beruhigungs--smoothing capacity. Dach- top (loading) capacity. Eigen- self-capacitance. Elektroden- (inter-) electrode capacitance. Ersatz- equivalent capacity. Kipp- time-base condenser. Röhren- (inter-)electróde capacitance. Sättigungssaturation capacity. Schaltcircuit capacitance. Selbstself-capacitance, distributed capacity (of a circuit, etc., due to proximity effects.). Streustray capacitance, distributed c., spurious capacity. Teilerddirect earth capacitance. Verdunkelungs- shading condenser. Verlust- imperfect capacity, leaky c.

kapazitätsarm of low capacity or capacitance.

Kapazitätsbrücke capacitance bridge, farad b., Wien b.

kapazitätsgerader Kondensator straight-line capacity condenser.

$K$ apazitäts-messer faradmeter. -probe capacity test. - reaktanz capacitive reactance, negative $r$. $\mathrm{k}$ apazitiver $\mathrm{Bl}$ indwiderstand capacitive reactance, negative reactance, condensance.

Kapotte, Abblende- dimming cap. 
Kappe cap, hood, top, dome, crown, can. Aufsteck-slip-on cap. Hexoden- top cap of hexode tube. Lichtschutz- light-hood (phot.) Ohren- ear muff.

Kapsel capsule, case, box (printing), cap, chill, mold (foundry), sagger (cer.). Mikrophonmicrophone button (housing), capsule. Sprech- condenser microphone.

Kapsel-blitz flashlight capsule. -guss casting in chills; chilled work. -ton sagger clay, capsule c.

karburiert, öl- oil carburetted.

Kardan-gelenk, -kupplung universal joint.

Kardinalpunkte cardinal points (opt.)

Kardioide, Wechsel- switched cardioid (space pattern).

Kardioidenbildung cardioid formation (of space pattern or directional diagram.)

kariert cross-hatched.

Karneol carnelian.

Karolus-Kerr Mehrfachol attenzelle Karolus multi-plate Kerr cell.

Karte, gnomonische gnomonic chart (straight-course chart.)

Merkator- Mercator chart. Strecken- route (airway) map.

Kartei card index, c. catalog.

Kartenaufnahme, photographische photographic mapping.

Karten-kurs map course. -netz graticule. -projektion,

Merkator- Mercator or cylindrical projection (orthomorphic p.)

-winkelmesser map protractor.

kartesische Fläche Cartesian surface.

Karton, Bristol- bristol board.

Kartothek card index, c. catalog.

kaschieren place on a support, base or other surface; coat with paper.

Kaskaden-schal tung cascade connection, cascading; concatenation or tandem connection (of motors.)
- verstärker cascade amplifier, repeating a.

Kassette spool box, darkslide (phot.); cassette. (X-ray work), sagger, coffer, casket. -, leise silenced magazine. Anlegeclamp-on darkslide. 'Tageslichtdaylight loading magazine (m.p.).

Kassetten-rahmen darkslide carrier. -schieber shutter of darkslide.

Kastagnetten castanets.

Kastanienholz chestnut wood.

Kasten, Geräusch- equivalent acoustics (for special sound effects and noise imitations.) Potentialpotential well, p. hole. -drachen box kite.

Katheten perpendicular and base adjoining hypothenuse (in a rightangled triangle.)

Kathode cathode, filamient, emitter (of electrons.) -, direkt geheizte direct-heated cathode. -, geschichtete (oxide-) coated cathode; photo-c. with light-sensitive layer. - , indirekt geheizte indirectly heated cathode (in heater-type tube), unipotential c., iso-p. c. -,kalte cold cathode (as in photo-tube and in BH type gaseous rectifier). -,körperliche physical cathode, material $c$.

Kathode, Anti-target, anti-cathode (of X ray tube). Flächenplate-shaped cathode (of neon tube as distinguished from point or crater.) Hohl- concave cathode, hollow c. Oxyd- oxide-coated filament, dull emitter. Paste- oxidecoated (pasted) cathode. Photo-, zusammenhängende mirror type or continuous photo-cathode, photoelectric c. Punkt- point-source cathode, crater c. Quecksilbermercury-pool cathode. Raster (photo)-mosaic, rastered photocathode screen. Rein- bright emitter (often thoriated c.) Schicht- cathode bearing a layer 
or coat (of oxide, photo-sensitive substance, etc.). Siedeklihlungvapor-cooled cathode.

Kathoden-anheizzeit cathode heating time (till stable temperature is reached), thermal ticie constant. - ansatzpunkt spot (of arc) formed on cathode. -dunkelrauri, Crookes cathode dark space, Crookes' dark s., Hittorf's dark s. - fackelerscheinung cathode flicker effect. -fallableiter valve arrester. -fleck des Hg-Lichtbogens, fixierter anchored cathode spot of mercury arc. -licht(haut) cathode glow. - I inie cathode line. -neuaktivierung cathode re-activation, c. rejuvenation. - siedekuhlung vapor cooling for cathode. -spratzen flicker or spitting of cathode. -sprung cathode jump of potential.

Kathodenstrahl-abtaster electronbeam scanner. -bundel cathode-ray beam or pencil; gun. -intensitätskontrolle catnode-ray beam intensity modulation (by shield, grid or Wehnelt cylinder). -röhre thermionic tubes (obs.); beam tube; c. $-r$. tube, oscilloscope, iconoscope, emittron, and other tubes operating with beam and screen; Lenard t. -röhre, gasgefüllte gas-filled c. $-r$. tube, soft $t$. working with a c.-r. (for ionic or gas focusing).

Kathoden-verstärker cathode follower. -zerstäutung cathode disintegration, c. spatter, splutter or sputter.

Kathodo-lumineszenzl ampe cathodeglow tube. -phon cathodophone, diaphragmless microphone, glowdischarge m.

Kation cation (positive ion moving toward cathode; negative ion called anion).

Katzenauge bull's eye (small thick lens). kaubar masticable.
Kauf nehmen, in accept something (at the expense of), be tolerable or tolerated.

käuflich commercial (grade), merchantable, purchasable.

Kaustik caustic (opt.).

Kaution stellen give bail, bond or security.

Kavitation cavitation.

Kegel, Anflug- sector of approach. Ganzpol- polehode cone, body cone. Licht- cone of light, luminous c. Rastpol-herpolnode, space cone. Reitungs- pole of friction. Sehangle of vision, cone of $v$. (camera work.) Strahlen-cone of rays. Stumpf-truncated cone, frustum.

Kegel-antenne, Doppel-double-cone antenna, cage a. -druckprobe cone findentation) test. -feder volute spring. -lehre taper gase. -mantel cone-shaped shell. -projektion conical projection (of a map). - $r$ ad bevel wheel, cone w., mitre w. -radgetriebe bevel gearing, mitrewheel ह. - schnitt conic section. -stumpf truncated cone, f'rusto c., frustum. - ventil conical valve, ball v.

Kehle throat, larynx, channel, flute.

kehlen groove, slot, flute, channel, key.

Kehl-kopfmikrophon 1 aryngophone, throat microphone. -nahtschweissung fillet weld.

Kehr-doppelwendel reversed double loop. -wendel return spiral, reversed s., helix, coil or loop. -wert reciprocal value, inverse $\mathrm{v}$. -wert, Zähigkeits- fluidity (reciprocal of viscosity).

Keil wedge, key, cotter, jib, liner, shim. Dreiecks- triangular wedge. Goldberg- Goldberg wedge, neutral (grey) w. Streifen- photometric wedge. Stufen- step tablet, stepped (photometric) absorption wedge (X-ray, etc., work). 
Keil-flach spheroid. -nute keyway. -schwärzungsmesser circular wedge densitometer.

Keim nucleus or seed (cryst.), germ. Kristall- crystal nucleus, seed $c$. Reif- digestion nucleus.

Keim-kristall crystal nucleus, nucleus crystal. -ling nucleus (cryst.), germ, embryo. -wirkung nuclear action, action of nuclei (cryst.) -zenter grain center.

Keller-armatur boiler fittings. -ton tunnel effect, boom e.

Kenndämpfung iterative attenuation constant.

Kenndämp fungswiderstand iterative impedance; characteristic $i$. (for uniform line).

Kennedy-Heavi side Schicht KennedyHeaviside layer.

Kenn-faden (colored) tracer-thread (for c.coding). -grösse characteristic quantity, c. magnitude. -grösse, Übertragungs- transfer characteristic. -l eitwert indicial admittance.

Kennlinie, Arbeits- operating or working characteristic or curve, performance c. Dynamik- volume (range) characteristic. Frequenzfrequency-response characteristic. Richt- directional diagram or space pattern (df); rectification characteristic. Schwing- oscillating characteristic, resonance c., c. of an oscillatory system. Touren-Belastungs- speed-load cha:acteristic. Ubernahmetransf er characteristic.

Kennlinien-feld family of characteristics or curves. -kippung characteristic straightening. -knick, oberer upper bend or knee of characteristic. -kick, unterer lower bend or knee of characteristic.

Kennsignal tuning note (characteristic audio signal for tuning), c. sign or signal (of Morse code or other identification), signature of a station, theme. kenntlich machen characterize, identify, make conspicuous, identifiable or distinguisable.

Kennung, Funkfeuer- call or distinctive signal (of a beacon station. )

Kennungssignal distinctive, identifying or code signal, signature.

Kenn-widerstand characteristic impedance, image i., indicial $i$. -winkelmass iterative phase constant. -zahl characteristic factor, office code. -zeichen charact. or distinguishing mark or feature, sign, identification, symptom; object (of invention or patent).

kennzelchnen characterize, distinguish, code.

Kenn-zelchnung, Farb- color coding. -zlffer characteristic.

kerbe, scharfe sharp notch, nick or groove; slot, slit. Rund- round notch, charpy notch (with root radius.)

Kerb-dauerfestigkeit notch fatigue strength. - empfindlichkeit stress concentration index. -nute keyway. - -schlagzähigkeit notch impact tenacity.

kerbverzahnt splined (externally or internally.)

Kerb-wirkungszahl fatigue stress concentration factor. -zähigkeit notch tenacity.

Kern nucleus, core, grain, kernel, pip, stone, pith, heart, crux (of a matter.) -, geblätterter laminated core. Ei senpulver-, gepresster compressed iron-dust core, Ferrocart c. Film- reel, spool.

Kristall (isations)- center or nucleus of cristallization. Lamell en- laminated core. Luftair core. Masse- compressed ironpowder or dust core, molded c., Ferrocart c. Nachfol ge- product nucleus. Spulen-bob or core of a spool (m.p.) Staub- duct or molded core, Ferrocart c. Verunreinigungs- impurity nucleus. 
Wasserstoff- $H$ particle, hydrogen particle or nucleus, proton, deuteron (when of heavy hydrogen or deuterium.) Zwischen- compound core, "intermediate".

Kern-abstand inter-nuclear distance. -anregung nuclear excitation. -bildung germination, nucleation. -distanz internuclear distance. -durchdringung (Potentialschwelle) nuclear penetration function. - farbe nuclear stain.

kernferne El ektronen planetary, peripheral, orbital or outer (-level) electrons (farthest from nucleus), valence e., conduction e.

Kerngehalt nuclear concentration, content of nuclei.

kerngestreut deflected by nucleus. Kernguss cored work (foundry).

kernig, ein- uninuclear, mononuclear. mehr-, viel- multinuclear, polynuclear, having a plurality of nuclei or cores, polycyclic.

Kern-körper(chen) nucleolus. - ladungszahl charge on nucleus, nuclear charge number, atomic number. -magneton (K.M.) nuclear magneton. -moment, magnetisches nuclear (magnetic) moment.

kernnahe Elektronen inner(fixed) electrons, e. adjacent nucleus.

Kern-physik nuclear physics. - potential schwelle, Durchdringung der nuclear penetration function. -schatten umbra. -schliff male part of ground-in joint. -spin nuclear spin. -spule, Masse- dust-core coil, compressed-iron-core coil. -teilung nuclear division.

-theorie nuclear theory. -umänderungsprozess nuclear disintegration process. -umwandl ung nuclear transmutation. -verlust core loss, iron 1. -wechsel wi rkung nuclear inter-action.-zerplatzen nuclear fission or disintegration.
Kerr Mehrpl attenzelle, KarolusKarolus multi-plate Kerr cell.

Kerze, römi sche Roman candle, magnesium torch, smoke pot.

Kerzenstärkencharakteristik candle-power characteristic.

Kessel boiler, vessel, tank, pot. Druckwind- compressed-air tank, blast pressure tank. Etagenbattery boiler.

Kessel steinbildung boiler scale formation, incrustation.

Kette filter, network; channel, chain, train, series, bondage. -, el ektroakusti sche channel (in sound recording or reproducing), electro-acoustic transducer. -, innermol ekul are intra-uolecular chain. Abstimm- tuning arrangement comprising a plurality of oscillation or tuning circuits. Drossel- low-pass filter. Entzerrer - corrective network, equalizing $n$. Gelenk- link chain. Neben- side chain, subordinate $c$. sieb- ladder-type filter, bandpass f. or network. Sperr- lowpass filter. Sternglied-T network. Transoort- conveyor chain. Verstärker-amplifier cascade. Verzögerer- delay network. widerstands- voltage divider, potentiometer.

Ketten-brichmethode method of infinite continued fractions. -durchhang slack or sag of a chain. -fläche catenoid. -flaschenzug chain tackle block. -glied network or filter mesh or section.

Kettenleiter iterative network, recurrent structure. -erster art II $(\pi)$ network. -, mehrgliedriger multi-mesh network. -, mit $1 / 2$ Längsgl ied endender mid-series terminated network. -, mit 1/2 Querglied endender mid-shunt terminated network. -, vielgliedriger multi-mesh network, multi-section n. -, zweigl iedriger two-section network. 
Ketten-leitermasche mesh, section of a network. -linie catenary. -molekil chain molecule. -rad sprocket wheel. - rollen scheibe chain sheave. - schal tung relay chain circuit. -widerstand iterative impedance.

$\mathrm{K}-\mathrm{H}$ Schicht Kennedy-Heaviside layer. Kiefernhol z pinewood.

Kiesel zinkerz willemite.

Kimm (sea) horizon. -tiefe dip of horizon.

Kino motion-picture theater, film playhouse, movie.

Kino, Fern-telecine, telecast, intermediate-film television system. Koffer- portable projector. Spielzeug- home movie.

Kino-film-Fernsehabtastung telecine scan of film, pickup of pictures from moving-picture f. -kabine projection booth. - positiv moving picture positive. -skal a station name projection scale.

kipp... time-base, relaxation, "kipp," trip, triggering.

Kipp-drossel cut-off choke (regulates battery charging). -einsatz, Bildincipient frame flyback. -einsatz, Zeilen- incipient line fly-back. - elektrometer Wilson electrometer (gold leaf given different positions by tilting case).

kippen retrace, reversal or return of pencil (in scanning); trip over, trigger; tip, dump, tilt.

Kippentl adung condenser discharge (in time-base).

Kippentladungsröhre time-base discharge tube.

Kipper, Bild-frame time-base. Zeilen-line time-base.

Kipp-erscheinung jump phenomenon, swing p. (in arts other than television). - frequenz relaxation frequency, time-base $f$. -gerät time-base, deflector means, relaxation oscillator, trigger device. -gerät, Gegentakt- push-pull tire-base. -hebel schal ter toggle switch (with projecting lever). -impuls tripping impulse, -kapazität time-base condenser. -kreis timebase circuit, trigger c., relaxation c. -moment maximum torque (mech.) -oszillator, Sperr-blocking oscillator. -periode time-base period. - schal tung, Gl imml ampenneon-lamp time-base circuit scheme. - schwinger time-base oscillator.

Kippschwingmethode, fremdgesteuerte, unsel bständige distant-operated (non-self-running) time-base method. -, sel bständige, mi tgenommene self-running controlled timebase method.

Kippschwingung relaxation, sawtooth or ratchet oscillation, time-base o., kipp o. - sel bständíge self-running time-base. Bildframe or picture time-base oscillation or impulse. Zeilen- line time-base impulse or oscillation.

Kipp-schwingungsdauer relaxation period. - sender, Sperr-blocking oscillator. - spannungsrïckl auf flyback of sweep potential, return of time-base p. to zero. -spiegel oscillating mirror, vibrating $\mathrm{m}$.

Kippung, Kennlinien- characteristic straightening.

kippvorgang time-base action, ratchet a.

Kirsche, Schmelz- igniting pellet.

Kirschrotglut cherry-red heat, cherry redness.

Kissen, Luft- air cushion, dashpot. kissenförmige Verzeichnung pincushion distortion, pillow d. (telev.).

Kittlehre, Film-film splicing gage. $k l$ agbar werden bring action or suit (against).

$\mathrm{Kl}$ age, Einstellungs- action for discontinuance (of an infringement, etc.) Feststellungs- declaratory action. Nichtigkeits- action or suit for cancellation, annulment or invalidation. Patent- patent suit, patent action, p. litigation. Patentverletzungs- action or suit for infringement of patent rights. 
Schadenersatz- action for damages. Unterschiebungs- suit for passing off goods as though of another person.

Kl age-abwei sung dismissal of claim, non-suit. -beantwortung reply, response, answer, defense (plea). -grund cause of action.

klagen bring action, sue, file a suit.

$\mathrm{Kl}$ agepatent patent in issue, in suit, at bar.

Kläger plaintiff, complainant. Berufungs- appellor. widercross or counter-claim plaintiff.

Kl ageschrift plaint, bill of charges, statement of claim, bill of particulars, plaintiff's declaration.

$\mathrm{KI}$ ammer clamp, clasp, clip; braçket (round or square, used in mathematical formulae, etc.). (Round brackets are strictly known as parentheses in typography). Gabel- forked clamp. Verbindungsbrace, clip connector.

$\mathrm{Kl}$ ang s.a.. Jon, Akustilk, Geräusch und Schall.

$K l$ ang, blecherner tinny sound. -,zusammengesetzter complex (musical) sound. Doppel- echoed sound (hearing sound twice, i.e., by electric transmission and by transmission through air). Einzel- (sounding of an) individual note or tone. Gleichunison, consonance, resonance. $\mathrm{Kl}$ ang-bild sound pattern, acoustic p. -blende tone-shading means, tone switch, t. control, tonalizel. -drossel tone-control choke (coil). -einsatz acoustic intonation, onset of a sound. -farbe timbre, tone color, quality.-- helle strident timbre, shrill sound. -färbemlttel tone-shading means, tone or timbre control m.; tonalizer. - farberegler tone control, tonalizer. - flgur sound pattern, acoustic figure. - fullle sound volume. -gemisch sound spectrum. $\mathrm{kl}$ anggetreu of high fidelity, orthophonic (of reproduction of sound).

$\mathrm{Kl}$ ang-intensitätsbereich dynamic range of sound. -reiniger acoustic clarifier (on ldpsk. baffle).

Kl appe (clack) valve, flap, trap, lid, damper, stop, key (of wind instrument), pallet (of harmonium).

kl appen strike, clapper; clap, flap, fold, move in various directions, of ten about pivots or hinges, to open or close, as the lid of a chest or the cover of a box. auf-, hoch-fold, raise or lift to open or erect, on hinges, as the cover of a talking machine. wegswing or fold out of the way. zudrop, move, swing or let down (for shutting) on hinges.

Kl appen-horn key horn. -mechani smus key work, k. action.

kl appern chatter, rattle.

Kl appensignal see Klatsche.

Kl app-kamera folding camera, collapsible c. -kassette hinged darkslide. -sucher, uml egbarer reversible folding finder. -variometer hinged-coil variometer.

klar clear, distinct, pellucid, lucid; intelligible, articulate. - bestimmt uniquely determined, definitely or clearly defined.

klär-bad clearing bath. -bassin, -bottich settling or clearing tank, tub, vat or reservoir.

$\mathrm{Kl}$ arinette clarinet, clarionet.

kl armachen standby, make ready, alert.

Klärmittel, Klärungsmittel clarifying or clearing agent, clarifier, fining substance.

$\mathrm{Kl}$ artonverfahren noiseless $\mathrm{film}$ method.

$\mathrm{Kl}$ atsche beat or clappers (to mark sound and picture tracks for synchronization).

Kl atschenbild beat picture.

Klaue claw, paw, pawl, catch, clutch, grasp.

Kl auen-kupplung claw clutch, c. coupling. 
Klavi atur keyboard, bank of keys.

Kl avierharfe keyed harp, piano harp.

Kl ebäther collodion.

$\mathrm{Kl}$ ebband friction tape, adhesive or sticky t.

$\mathrm{Kl}$ ebemittel adhesive, agglutinant, cement.

Kleben sticking (of a keeper), freezing, adhering, clinging; cementing, gumming, glueing.--der El ektronen capturing or sticking of electrons (to electro-negative gases, surfaces, etc.) Filmblooping, patching or splicing (of film).

Kl ebe-presse blooper, splicer (for making blooping patches in film). -stelle, Ton- bloop, blooping patch or splice (on sound film). -stell engeräusch splice bump, dull sound due to blooping patch, wOwOws, blooping. -tisch splicing table, s. bench.

klebrig sticky, adhesive, pasty, viscid, glutinous.

Kl ein-bildphotographie microphotography, photomicrography. -film narrow film, sub-standard $f$. - gefüge, micro-structure, fine structure. -kammer small (air) chamber, thimble (for ionizing wark). -l ampe miniature lamp. -lader trickle charger. -lebewesen micro-organism. -l l chtbll dkunst photomicrography, microphotographic art.

kleinlückig of fine porosity, closegrained.

Kleinröhre miniature tube (of acorn, midget, etc. type).

kleinsten Quadrate, Methode der method of least squares.

Kleinstmaschine miniature machine.

Kl einumfangkopien "lo-range" prints.

$\mathrm{K} l$ emme terminal, binding post, connector, clamp.

$K 1$ emmeffekt pinch of fect (in needle reproduction of sound).

$K$ Klerme, Elngangs input terminal. trdground clamp. Verbindungs- connector means, binding post, terminal.
$\mathrm{K} l$ emmenwiderstand terminal impedance, $t$. resistance.

$\mathrm{Kl}$ emmschraube clamp, lock or thumb screw; binding post, terminal.

Klick, Tast- key thump, k. click.

Kl ima-gürtel climatic belt. -technik air conditioning.

Kl imati sierung air conditioning.

klimpernd harsh or strident (of a tone or sound containing strong harmonics and high partials), grating, shrill, tingling.

$\mathrm{Klingeldraht}$ bell wire, annunciator w.

Klingen sounding, ring (of a tube).

Kl ingfähigkeit ringing noise, microphonic n. (of a tube).

Klinke, Batterie- battery jack. Doppel sperr- double dogs, pawls or detents. Rast- detent, latch, pawl. Sperr- pawl, catch, latch, click, detent, ratchet.

Kl inken-scheibe notched disk, ratchet disk. -stecker jack, plug switch.

klinkwerk trip gear, release g.

Kl irrdämpfung correction of nonlinear harmonic distortion.

klirren scratch (of a loudspeaker), clink, clatter.

Klirrfaktor non-linear harmonic distortion factor, klirr f., blur or rattle $f$.

klopfen beat, knock, tap.

Klopffeind anti-knock agent (engine).

kloppel, Glocken- bell hammer, b. striker. -stange clapper rod, c. stick.

kl ump i g clotted, clotty, lumpy.

Klystron klystron (ultra-highfrequency oscillator with two rhumbatrons acting as buncher and catcher).

K.M. (Kernmagneton) nuclear magneton.

knacken crack, crackle, click, crepitate.

Knagge cam, catch, peg, stop, lug, bracket.

Knall detonation, explosion, report, crack, pop, pistol shot noise.

Knall-gas, Wasserstoff- detonating 
gas mixture (of $\mathrm{H}$ and 0 ), oxyhydrogen gas. -gasgebläse oxyhydrogen blowpipe. -pyrometer explosion pyrometer. -satz detonating composition.

-schutzgerät pistol shot silencer, crack s., noise killer (radio). -welle shock wave (ac.)

knapp close, tight, narrow; scanty, meager, brief, concise.

Knarre ratchet.

knarren crackle, rattle, crack (of loudspeaker), creak, squeak.

Knebel-bolzen toggle bolt. -griffschraube tommy screw. kneifen nip, squeeze, pinch. kneten knead, pug (cer.)

Knet-holz plastic wood. -legierung plaștic alloy, wrought a., malleable a.

Knick break (in curves); crack, flaw, kink; bend or knee (e.g., upper and lower, of tube characteristic).

knicken crack, break, buckle, collapse.

Knick-festigkeit flexibility (of a film), breaking strength, buckling strength (of metal). -punkt, -stelle kink or knee, break (of a curve). widerstandsfähi gkeit folding endurance (of film), breaking or buckling strength. $\mathrm{knle}$ knee, bend (of a curve); elbow, knuckle.

knieförmig knee-shaped, geniculate.

Kniegelenk knee joint, elbow j.

Kniff (cf. Trick) artifice, trick, device; crease, pinch.

knistern crackle, crepitate, rustle (silk).

Knitterfestigkeit crumpling strength. Knöll chen nodule, little knob or node.

Knolle lump, knob, node, nodule, knot.

knollig knobby, knotty, bulbous. Knopf button, knob. Druck- push button, press button. Einstellfocusing knob (camera). Triebmilled head, pinion h. (micr.).
Knopf-empfänger, Druck- pushbuttontuned receiver, receiver with automatic tuning. -karte instruction chart. - röhre shoebutton tube, acorn $t$.

Knoten node, nodule, knot, knob; plot. - abstand internodal. distance. - ebene, - fläche nodal plane, n. surface (opt.). -linie neutral line, common 1., nodal 1. -punkt, - stelle nodal point (opt.); junction p. -strahl beam or pencil of non-uniform section, in gas-focused c. $-r$. tubes, due to poisons and secondary electrons. -verblindung transfer joint, temporary bridge.

Knüppel i sol ator club-type insulator, pin $i$.

knurren growl, snarl, rumble. Kochbecher beaker.

koch-beständig resistant, stable or fast to boiling (dyes, etc.) echt fast to boiling (dyes).

kochend(er) Akkumulator agitated or gassing accumulater (just disconnected from charger). - (es) Geräusch boiling noise.

Kochounktmesser ebullioscope. Koeffizient s.a. Faktor und Beiwert.

Koeffizlent, Ausnutzungs- utilization coefficient. Extinktionstotal-absorption coefficient, extinction c. Gleltungs- slip coefficient. MitflihrungsFresnel coefficient of drag. Schwächungs- absorption coefficient. Vereinigungs- combination coefficient.

Koerzitivkraft coercive force, coercivity (sometimes: retentivitv). -messer coercimeter.

Koffer, Spei sungs- current feed box. - apparat box equipment, trunk unit (for sound and picture pickup). -kino portable projector equipment.

Kohärenzlänge der Well lenzüge coherence length of wave-trains.

Kohäsionsfest i gkeit cohesion strength. 
Kohle coal, charcoal, carbon, carbonaceous material. -,sal zgetränkte mineralized carbon, impregnated c. Docht- cored carbon. Effekt- salted carbon, c. for flame-arc. Härtungshardening carbon, cementing $c$. Hochinten si täts- high-intensity carbon (for arcs). Rein-solid carbon.

kohlefrei carbon-free, non-carbonaceous.

Kohlen-korn carbon granule, c. grain. -korngeräusch carbon noise (of nicrophone). -mikrophon, atmendes breathing of microphone (slow changes of resistance and response of carbon m.)

kohlen char, carbonize, carburize. Kohl ennachschub carbon feed.

Kohl epuppe (Trockenel ement) carbon rod (of dry cell).

Kohlholz charcoal.

kohl schwarz coalblack, jet-black. Kokosfaser coco fiber, coir (fiber from husk of coconut).

Kolben piston, plunger, ram, bulb, iron or bit (for soldering). Mess- graduated flask, measuring f., gage $f$. Röhren- bulb or vessel of a tube (radio). Schliffground-in glass stopper. -membran piston diaphragm. - stange piston rod.

Kollektiv des Okulars field lens of eyepiece.

kolf ektor commutator, revolving contact-maker, distributer, rotary switch.

Kollimationslinie collimating mark (opt.).

Kollisionsverfahren interference action or procedure (law).

Koma-abbildung,-blld comatic image. -reste hoherer ordnung independent cona of higher order.

Kombinations-aufnahme combination exposure, composite shot, mask method of shooting. - blende Goerz offect shutter. - register combination stop (organ). -stimme mutation stop (organ). - töne combination (difference or summation) tones.

Komikbilder film cartoons, funnies. Kommando-gerät data computer, ballistic director (gunnery). - stelle order point, order place, o. station (bridge); impulsing or control-action transmittor (remote control). - übertragung zwiwchen Flugzeugen inter-aircraft command or voice communication or signalling.

kommutieren reverse (polarity, connections, etc.)

kompakt compact, solid, non-apertured.

Komp ar ator, selbstrechnender computing recording comparator.

Komparserieraum extras room .

Kompass, Kreisel- gyro-compass. Ortungs-,Peil- navigation compass. Tochter- auxiliary compass, repeater c. Trocken- dry compass.

Kompass-büchse compass bowl, c. kettle. -kurs compass course, steered c. - rose compass card, c. scale. strich point of compass.

Kompendium Goerz effect set (to obtain different effects, fading, etc. in motion picture work).

Kompl ementwinkel complementary angle. komplexe konjugierte Grösse conjugate complex quantity.

Komponente, ausserordentliche extraordinary component (of rays). -, ordentliche ordinary component (of rays). Blind-wattless, idle, quadrature, reactive or reactance component (of impedance). Wirkactive, wattful, energy or inphase component.

Komprimieren der Laufzeit transittime compression.

Kondensanz capacitive reactance, condensance.

Kondensationskal orimeter steam calorimeter.

Kondensator s. a. Kapazität

Kondensator, frequenzger ader straight-line frequency condenser or capacitor. (Note: Capacitor nowadays is preferable). -, gleichl aufender ganged condense. 
Kondensator, kapazitätsgerader

154

synchronized condenser. $\quad-, \mathrm{kapa-}$ zitätsgerader straight-lińe capacity condenser. -, quadrat 1scher square-law condenser. -, unvollkommener leaky condenser. -, well engerader straight-line wave (length) condenser. Abgleichaligning condenser, trimming $c$. Blätter-foil capacitor, tubular C., Mansbridge (paper) c. Blockblocking condenser, stopping c., insulating $c$. , fixed $c$. Endterminating condenser. Festcapacitor, fixed or invariable condenser. Fïhler-pickup or scanning condenser (used in sound reproduction). Gelenk- swing-out condenser, book c. Glättungssmoothing condenser. Hochdruckpressure-type capacitor (in nitrogen high-p. tank). Hütchenceramic hood-shaped capacitor. Mehrfach-, Mehrgang- multiple condenser, ganged c., synchronized c., tandem c. Mittellinienlogarithmic condenser (relation between capacity and angle obeys 1. law). Nachstimmungs- aligning condenser, trimming c.; vernier c. Quer- shunt condenser, by-pass c., bridging c. Quetsch- compression type condenser. Schluss- terninating condenser, short-circuiting c. Sperr - stopping cóndenser, insulating c., blocking c. Trockendry electrolytic condenser.

überbrïckungs- bridgir.s condenser, by-pass c. Verkürzungs- shortening condenser, padding $c$. Ver$\checkmark$ ielfäl ti gungs- condenser-type d.c. multiplier. wickelblocktubular condenser, paper capacitor, mica c. (made of metallized or foil-coated insulation strip).

Zeitkreis- time-base condenser. Kondensator-abschlusswände end shields (forming "tub" of ganged condenser). - bel agverlust surface leakage loss of a condenser. -klemme condenser type bush or terminal. -leltung high-pass filter. $-m i k r o p h o n$ condenser micro-
Konstante, Verschiebungs-

phone, electrostic m. -rïckstand residual charge of condenser. - sirene condenser siren (produces pulsating current). -wanne tublike container with end plates or shields of dielectric or sheetmetal to accommodate a condenser; ganged c., multiple c.

kondensierte, nicht-, Hochstromentl adung uncondensed heavy-current discharge.

Kondensorspule condenser coil (of eleotron microscope).

Konduktanz, Röhren- transconductance (of a tube).

Kondukte wind trunk (of an organ). konforme Abbil dung conformable representation.

Königsholz king's wood.

Konizität conicity, conicalness, coning, taper.

konjugierte Funktionen conjugate functions. -komplexe Grösse conjugate complex quantity. - Schnittweite conjugate intercept. Konkavgltter concave grating. konphas in-phase, in p. coincidence, co-phasal.

Konsistenzmesser viscosimeter. konsoli dieren consolidate, set, concrete, solidify.

Konsonanten, stimmhafte sonant, voiced or vocal consonants. -, stimmlose non-vocal, surd or breathed consonants. - deutlichkeit consonant articulation.

konstant constant, invariable, nonvarying, fixed, stable, steady, stabilized.

Konstante constant, parameter. Ansprech- sensitivity or response (of microphone). Dämp fungsattenuation constant (real part of transfer or propagation c.) Dielektrizitäts- dielectric constant, specific inductive capacity, permittivity. Fortpflanzungspropagation constant, transfer $c$. Gitter- grating constant, g. space; lattice c. Kraft- force constant. Richt- rectification constant. Verlust- attenuation constant. Verschiebungs- displacement constant 
specific inductive capacity (in vacuo). Well endämo fungswave attenuation constant.

Zerfalls- disinteoration constant. transformation c. , radio-active constant, decay coefficient.

Konst anthal tung stabilization. konstruktiv constructional, practical, concrete, constructive. Kontakt, ausgefressener pitted contact, worn c. -,federnder spring contact, flexible c., c. spring.

Kontakt, Abreiss- arcing contact (of a switch). Arbeits- marking contact, make or operating contact (closure occurring upon energization of relay). Folgemake-before-break contact; dummy $c$. Gleit- sliding contact (selfwiping and s.-cleaning). Leerl aufspare or vacant terminal or contact. Ruhe- spacing contact, rest c., non-operating c. (closure $\propto-$ curs upon de-energization of relay): contact of rear part of key lever. Schiebe- adjustable slide, slide contact, cursor. Schl epDmake-bef ore-break contact, trailing c. Wackel- variable contact, loose or defective c. Wechselmake-and-break contact. Wi schself-cleaning or -wiping contact.

Kontakt-abdruck contact print

(phot.) -arm wiper. -bürste contact brush, wiper. -finger contact finger, wiper. - geber contact maker, time tapper, time marker.-glei chrichter contact detector, crystal d. or rectifier. natural $d$. hebel contact lever. $-k l$ eben sticking of contacts. -mittel catalyst, contact agent, c. substance. - prell en contact chatter, c. click, c. thump, c. chirp. -satz bank of contacts. -schieber adjusting slider, slide contact, cursor. -schiene contact bar. -schmoren scorching, freezing or melting together of contacts. - substanz contact substance, catalyzer, catalyst. -wi rkung catalysis, contact effect. -zunge spring contact, reed, blade, ditton. -zu sammen schmoren scorching, freezing or melting together of contacts. Kontingentierung quota system. Kontinuitätsgleichung equation of continuity.

Kontinuum continuous spectrum.

Kontra-Alt contralto. -Bass -contra-bass (brass wind instr.) -Fagott contra bassoon.

kontrahierte Entl adung contracted discharge.

Kontraktionskoeffizient Foisson's ratio (lateral contraction: longitudinal extension), rho ratio.

kontraktlich contractual, by contract or agreement.

Kontrast contrast, gamma $(\gamma)$ -empfindl i chkeit,-empfindung contrast sensibility or sensitivity, intensity discrimination. -filter filter for selective contrasts, contrast screen, c. filter. -hebung compandor action, dynamicrange action means (comprising compressor and expander) to improve signal-noise ratio. - photometer mit iürfel cube-type contrast photometer, contrast $\mathrm{p}$. with cubical cavity.

kontrastreiches Bild contrast picture, high-gamma $p$.

Kontraststeigerung Callier effect, print contrast.

Kontrollbild monitoring picture (telev.).

Kontrolle control, check-up, supervision, monitoring, regulation, governins.

Kontrolle, Aussteuerungs-, mit Neonröhre neon tube volume indicator. Einknopf- uni-selector, unicontrol operation. Modul ationsgradnodulation meter.

Kontroll-empfänger monitoring receiver. - lehre master gage. - sender monitor transmitter, check-up t.; service test oscillator. 
Konturenversteilerung improvement in resolution and black-white contrast, insuring higher definition (telev.); expansion. Konus, weitgeöffneter wide-open cone or pencil. Hochtontweeter or treble cone loudspeaker. Riffel-corrugated cone. Tiefen-low-frequency cone loudspeaker, woofer. - antenne, Doppel- cage antenna. -membran diffusion cone, conical diaphragm; woofer.

Konventional strafe (monetary) penalty, contractual fine.

Konvergenz, winkel- half convergency error.

Konvergenzwinkel convergence angle. - unterschled binocular parallax difference.

Konzentration, Anfangs- pre-focus. El ektronenoptik - focussing by electron lens. Gas- gas focusing (in c.r. tube), ionic f. $\mathrm{Nach}-$ re-focusing. Selbst- gas focusing, self-focusing. Vor- (cf Vorsammellinse) preliminary focusing, pre-focus, first focus action (in c.r. tube).

Konzentrieren focusing, concentration, fasciculation.

konzentrisches Kabel co-axial cable or line, concentric c., pipe 1. Konzert-flügel concert grand piano. -piano upright grand piano.

Koordinaten-bild pattern of coordination. -papier crosssection paper, ruled p., coordinate p. - system, Pol arpolar co-ordinates. - system, rechtwinkliges rectangular coordinate system.

Koordinationszahl co-ordination number.

Kopf, verlorener deadhead, crop end, wastehead. Banden- band head, b. edge (spectr.) Düsen- nozzle tio. Einspann- fixing head, clamping h. Hör- sound (pickup) head. Lawinenhead of surge. Magnetisierungsmagnetic recording head (for sound, on wire or tape). Revolver- lens turret (camera). Ring- annular recording head. Schicht- striation head. Schreib-recorder head. Teil- index head. Verschlusscable terminal.

Kopf-fernhörer headphones, headset, "can". -hör anschluss telephone jacks. -hörerbijqel harness, head band, strap. - welle onde de choc, head or front wave, bow w., impact w., shell w., ballistic w. Kopie, Ansichts- release print. Arbeits- studio copy, s. print. Farb-color print. Gebrauchsworking copy, usable copy (for display). Grossumfang- highrange ("hi-range") or wide-range copy or print. Kleinumfang"lo-range" print. Lavenderlavender print. Mehrfach- multiple orint. Montage- first answer print. Muster - master copy, $\mathbb{m}$. print. Photo- photostatic cooy, photostat, photographic c., photoprint. Schnitt- edited print. Theater- release print. Tonsound film used for reproduction. Verleih- release print, distributing p. Zwi schen- lavender print, 1. copy.

Kopieranstal t orinting shop.

Kopieren, Durchlauf- continuous film printing. Verkleinerungs- reduction printing.

Kopier-fenster printing plane, $p$. station. - gamma negative gamma $(\gamma)$.

Kopiermaschine printer. -, automatische automatic printer. -,optische pro,iection printer, $p$. printing machine. Durchlaufcontinuous film printer. Fensterintermittent step printer. Schlitzslit printer, contact printer.

Kopler-schwärzung printing density. -stelle printing drum. -werk printing studio, p. shop or plant. Koppel, Oktav- octave coupler (organ).

Koppelnavigation navigation by dead 
reckoning. -ort fix, dead-reckoning position (avigation).

Kopplung, direkte direct coupling (by a resistance, capacitance or inductance common to both circuits), impedance coupling, conductive c. -,feste close coupling, tight c., strong c. -, gal vani sche resistance coupling, óalvanic c. -, induktive inductive coupling, transf ormer c.,$- k a b$ azi tive capacitive coupling, electric c., electrostatic c. -, lose loose coupling, slack c., weak c. -, veränderliche vario-coupler -, wilde stray, spurious or undesired coupling.

Kopplung, Gegen-negative or stabilized feedback, reverse f., anti-regenerative coupling, degeneration. Mit- positive regeneration, p. feedback; spurious or undesired coupling or feedback. Rück- regeneration, reactive coupling, feedback c., reaction. Sperrkreis- parallel resonance coupling. Spinimpul sspin orbit coupling. Streustray coupling, capricious c., spurious $c$.

Kopplungs-block invariable or fixed coupling capacitor. -faktor coupling factor, c. coefficient. -röhre coupling tube. - spule, veränderliche vario-coupler. -verlauf coupling coefficient, $c$. factor. -widerstand transfer impedance, coupling resistance.

Korb, Lautsorecher- loudspeaker chassis frame. Schutz- arcing ring, guard $r$. (of insulator). Korb-bodenspule spiderweb coil, basket c. - $f l$ asche carboy, demijohn. -wicklung basket winding, low-capacitance w.

Kordel-griff milled knob, knurled $k$. -mutter milled nut, knurled $n$. kordieren mill or knurl (an edge). Korn front sight, muzzle s.; grain, bead, granule.
Korn. Raster- silver @lobule (of iconoscope mosaic), silver plug (in barrier-grid m.) Schleierfog grain (of film). Sucherbacksight (of view-finder).

Korn-anhäufungen grain aggregations. -bildung granulation, crystallization.

Körner center point, punch mark, gage D. -rauschen carbon granule noise (microphone).

Kornet cornet.

Korngrenzen grain boundaries (cryst.) Körnigkeit graininess, granularity, granulation.

Körnigkei tsmesser granulometer.

Korn-rauschen film-grain noise. -zerfall grain disintegration.

Körper, optisch inaktiver racemate, racemized material (quartz), racemic substance.

Körper, Fliess- fluid or liquid body, non-solid (substance). Fremd- foreign, external or extraneous matter or substance, undesired admixture, impurity, contamination. Füll-filling body, packing b. Gl üh-incandescent body, incandescing $b_{\text {. , incandescent }}$ mantle (Welsbach).

Körper(chen), Kern- nucleolus.

Körper, Lager- bearing box, housing or body. Rotations- body or solid of rotation or revolution. Schicht- stratified structure, s. body. Stamm- parent substance, D. body.

Körperbeschaffenheit constitution. Körperchen corpuscle, particle. Körperlich solid, three-dimensional. bodily, corporeal, physical, material (e.g., of a cathode), molar.

körperlose Soule structure in which whole mass of vibrating system is contained in oil.

Körper-schaft body corporate, corporation. - schalli sound conducted through solids. -strahlung, Schwarz- black-body radiation, 
cavity $r$ - teilchen particle, corpuscle.

Korouskeln, Licht- photons. Korrektlonsglied correction factor, corrective term.

Korrektur-abzug proof sheet. - scheibe compensating cam (df). Korresoondenzorinzip (Bohr's) correspondence orinciple.

korrigiert, farb- color corrected, achromatized.

Korrosions-bildner corrosive, corrodent, corroding agent, rusting agent. - dauerfestigkeit corrosion fatigue strength.

- empfindlichkeit corrodibility, susceptibility to corrosion. -verhinderung corrosion inhibition.

kosmi sche Ultrastrahlen cosmic rays.

kostenpflichtig abweisen dismiss (a suit or legal action) with costs.

krachen crack, crash, crackle, rustle.

Krachtöter (automatic) silent tuning; noise suppressor, noise gate (in film work, etc.)

krächzender Ton all-top sound or voice.

Kraft force, power, enerky (force in mechanics $=$ voltage or emf in electricity). - , auflösende resolving power. -, atombindende atomic combining power, valence. -, lebendige-kinetic force, vis viva, momentum.

kraft meines Amtes by virtue of (power vested in) my office. $\mathrm{Kraft}$, rücktreibende repelling force, deflecting $f$. (acting on electron pencil). -, schwingungserzeugende vibromotive force. ,- zwi schenmol ekul are intermolecular force.

Kraft, Abstossungs- repulsion, repulsive force, repellency. Abstrebe- centrifugal force. Anstrebe- centripetal force. Antriebs- driving power, propel- ling force or p., propulsion. Anzlehungs- attraction, attractive force or power, adhesive $f$. Bewegungs- motive or motor force or Dower, motional f., kinetic $f$. Bild- image potential, i. force. Brech- refractive power, refractivity. Direktions- directive force, directing $f$. , versorial $f$. Erwärmungs- heatine power, calorific p. Färbe- tinctorial power, dyeing $p$. Feder- elasticity. Flieh- centrifugal force. Gegenreaction, counter-acting force, opposing f., reactive $f_{.}$, antagonistic f. Massen- inertance (of mechanical filter). Prallresiliency, elasticity; ricocheting force. Rand-force acting at an edge, marginal force. Richtdirective force, directing $f$, , versorial f. Rückstell resiliency, restorin£ force, retractile f., elastic f., restitution force or pressure. Schiebthrust, thrusting power, shear. Schnell-elasticity. Schwimmbuoyancy, floating capability. Schwing- centrifugal force; vibratory power, vibrating $p$. Soann- tension, extensibility, expandibility, elasticity.

Spring- sprinǵiness, power of recoil. Stoss- percussive power, motive $p$. Teil- partial force, componental $\mathrm{f}$. Thermo- thermoelectromotive force or power. Seebeck effect. Umfangs- circumferential force, tangential $f$., peripheral $f$. Verbindungscombining power, bonding strength, affinity. Wurf- projectile force. Zieh- drawing power, tractive power, traction, attraction, pulling, dragging or hauling power or force. Zug- traction, tractive force, tension, attraction.

Kraft-audion power grid-current detector. -äusserung manifestation or effect of force. -berechnung, Bild-, halbkl assi sche semi-classical 
image force calculation.

- fahrzeug motor vehicle, power craft. - funktion power function. -konstante force constant. - linle line of force, tube of force. - Iinlenschräglage slope of force lines. -loserklärung declaration of invalidity or cancellation. -mass unit of force. -messer dynamometer. -messer, Koerzitivcoercimeter.

kraftschlïssig (cf zwangsläufig) tensionally or positively connected (by positively acting means), force-locking.

Kraft-schwingröhre power oscillator. -stossmesser ballistic pendulum. - stufe power stage, final s. (of an amplifier). -wechsel energy exchange.

Krähl rechen rake, rabble. $\mathrm{Kran}$, Gerïst- gantry crane. Kranz, Rad- wheel rim, tire.

Spiegel- row, array, rim or set of mirrors, mirror drum (telev. scanning). Zahn- gear rim, tooth rim.

Kranz-abtaster. Linsen- lens drum scanner. - brenner ring burner, crown b.

krarupieren krarupize, load (a line) continuously.

kratzen scrape, scratch, rasp, grate. Kratzer, sichtbare visible scratches. kräuseln curl, crisp, crimp, mill (coins).

Kräuselung rippling (of a d.c.). Kreis (s.8. Stromkreis). circuit, circle, orbit, circular path.

Kreis, nichtschwingungsfähiger aporiodic or non-osoillatory circuit. -. wi derstandloser resistanceless circuit. -, unschreibender circumscribing circle. Achtel- octant. Arbeitseich- working reference circuit. Bei- epicycle. Bremsnegative anode circuit. Dunstatmosphere. Fuss- root line, dedendum 1. Halb-hemicycle, semicircle. Kipp- trıgger circuit, time-base c., relaxation c.

Krümmungs- curvature circle (lens). Kurs- direction or bearing circle. Licht- circle of light-halo. Luftatmosphere. Mittags- meridian. Nutz- active circuit, useful c., signal c. Parall el resonanzparallel resonance circuit, rejector c. Roll(bahn)- cycloidal path (of electrons). Saug- acceptor circuit, impedance or absorption wave trap. Schwungradparallel resonance circuit, flywheel c. Sechstel- sextant. Selektiv- selective circuit, selector c. Serienresonanz- series resonance circuit, acceptor $c$. Sieb- selective circuit, filter c. So annungs- potential circuit (of ammeter). Sperr-rejector circuit, wave trap. Stoss- impulse circuit, impulsing c. Teil-pitch circle, p. line, divided c., graduated c., dial, g. circular plate. Úbertragerintermediate circuit, transformer $c$. Um- periphery, circumference; surroundings, environs, ambiency. Ureich- master reference system. Viertel- quadrant. Zerrungs- annulating network, suppressing $n$. Zerstreuungs- circle of confusion, blur circle.

Kreis-bahn circular path, orbit. - bahnbewegung motion in a circle or orbit, orbital movement, circular m. - bewegung circular motion, gyration. - bewegung, Rollbahn- cycloidal path motion. -blende iris diaphragm. -bogen arc of a circle; circular arch. -bogengitter arcuate grid.

kreischende Stimme all-top voice (or sound).

Krei sdi agramm circle diagram, circular loci. Doppel- tangent circle pattern, figure-8 diagram or (space) pattern (df).

Kreisel top, gyro gyroscope, gyrostat.

Kreiselektron spinning electron. 
Krei sel-kompass gyro-compass. -molekuil top molecule, spinning m. -neigungsmesser bank-and-turn indicator. -pumpe centrifugal pump, turbine p. -verdichter rotary compressor, centrifugal c.

kreisend circulating, rotary, gyratory, eddying, vortical (i.e., of vortex motion).

Kreis-frequenz pulsatance $(\omega)$, angular velocity, cyclic frequency, $f$. in radians. -funkfeuer rotating or omnidirectional beacon, circular navigational beacon (sending non-directional signals in all directions). -funktion trigonometrical function, angular f. -grad degree of a circle or arc, d. of angular measure, radian. -keil schwär zungsmesser circular wedge densitometer. - lauf cycle, circulation (of fluid), circular course, circuit. - lochscheibe film scanning disk with apertures circularly rather than spirally arranged.

kreismagnetischer Effekt gyromagnetic anomaly.

Kreis-messung cyclometry. -parameter circular parameter. -prozess cyclic process, cycle. -scheibe mit veränderlichen sektoröffnungen sector disk (shutter) with variable apertures. - spannung "circle" voltage. -spule tuning coil. -ströme circular currents, c. flowing in a circular path. -welle circular electric wave.

Kreuz sharp (mus.).

Kreuz, Abstimmungs- tuning indicator (magic eye, etc.). Achsen-coordinate system. Faden- reticule, reticle, graticule, cross line, hair 1., cross spider hairs. Leucht- reticle image (reflector sight). Nalteser - cam and star wheel of Maltese cross, Geneva intermittent movement, crosswheel. strich-cross lines, c. ruling, reticle. kreuzen cross, intersect, traverse, transpose.

kreuzförmig cross-shaped, cruciform. Kreuz-gelenk universal joint, Hooke's j. -gelenkkupplung cross joint. - gesperre, Malteser-Geneva stop. - gitter cross grating, surface lattice. -glied lattice section (of network or filter). -hahn four-way cock. - libelle cross level, -modulation cross modulation. -peilung fix, bearirg taken from two or more ground directionfinders, cross bearing. -rahmenantenne crossed coil antenna.

kreuzsaitig cross strung, overstrung. Kreuz-schaltung s.H- \& T-8rückenschaltung. -schlitten compound slides. -teilungsmast cantilever type of tower (with double-tapered mast).

Kreuzungs-isolator transposition insulator. -punkt, Uber-crossover (of electrons in c.-r. tube).

«reuzverhör cross examination, cross questioning.

kribbeln swarming (of film).

kriechen crawl, sneal, creep (of materials); leak (of current).

Kriech-calvanometer quantometer, fluxmeter. -geschwindigkeit rate of creep (in metal). -strom creep current, sneak c., surface leakage c.

Kristall, einachsiges uniaxial crystal. -, langstrahliges needle-like elongated crystal. -, linksdrehendes levogyrate crystal. -,optisch inaktives Quarz- racemic quartz crystal, racemized c., racemate. -, zweiachsiges biaxial crystal.

Kristall, After-pseudomorph. Biegungs- "bender" crystal. Doppelplatten- biquartz crystal, bimorph c., bimorph cell. Drehungs, Drillings- "twister" crystal. Einmonocrystal, single c. Holoederholohedral form of crystal. Keimcrystal nucleus, nucleus c. Mehrpolycrystal. Misch-mix crystal, mixed c., crystalline solid solution. 
Quarz-, fertiges piezoid, finished blank. Quarz-, optisch inaktives racemic quartz crystal, racemized c., racemate. Schwing- crystal oscillator. SeignettesalzRochelle (salt) crystal.

Spaltungs- cleavage crystal. Sphäro- spherical crystal, sphero c. Tannenbaum- dendrite.

Zweielement- bimorph crystal (either of bender or twister type). Zwillings- (cf Doppelolatten-) twin crystal.

Kristall-achse crystal axis, crystallographic a. ( $\mathrm{x}=$ electric a.; $\mathrm{y}=$ mechanical a.; $\mathrm{z}=$ optical axis). -achsenmesser conoscope (determines optical axis). -achsenrichtung crystallographic axis orientation. -anschuss crop of crystals, crystallographic growth, crystallization, shoot into crystals.

kristallartig crystal-like, crystalline.

Kristall-ausscheidung separation of crystals. - bau crystal structure, crystalline form. -baufehler crystal (structural) defects. - beschaver inspectoscope. -blegungsschwingung crystal flexural vibration. -bildung crystal formation, crystallization, granulation (of sugar). -dickenschwlngung thickness vibration of a crystal. - drehungsschwingung, - drillungsschwingung torsional vibration. - ebene crystal plane, c. face. - ecke crystal angle. -faden, Einsingle-crystal filament, monocrystal f. -fassung (piezoelectric) crystal holder or mount. - fläche crystal face, c. plane. - gerippe crystal skeleton, skeletal c., crystallite. -gitter crystal grating (used for diffraction); crystal lattice. - gitterschwingung crystal lattice vibration. -hal terung crystal holder, c. mount. -haufwerk crystallite aggregation. - Inaktivierung, optische racemization.

Kristallisations-kerne centers of crystallization, nuclei of $c$. -wachstum crystal growth, germination.

Kristall-kanten crystal edges. -keim crystal nucleus, seed c. -kern nucleus of crystallization, center of c. -kernbildung crystallization, nucleation, germination (a gern being required in induced crystallization). -kyrperchen, rundes globulite, spherulite. -längsschwingung longitudinal vibration of a crystal. - lautsprecher piezo-electric loudspeaker. -lehre crystallography. - ilnse crystalline lens. -mikrophon piezo-electric microphone, crystal m. - quer schwingungen transverse vibrations (including flexural v.), contour vibrations or oscillations. - schnitt crystal cut ( $x$ cut or Curie cut, also known as the zero angle cut, faceperpendicular cut and normal cut; y cut, 30-degree cut or faceparallel cut; $T$ cut $=35$ degrees to $\mathrm{z}$ axis).-schwingung crystal vibration. - splitter crystal grain or chip. - steuerung crystal stabilizing, crystal control. -taktgeber (beim Sender) crystal monitor, $c$. control means, c. stabilizer. -winkelmessung crystal goniometry. kritisch critical, delicate. Kröpfung crank.

Krücke rake, rabble, crowbar.

Krümmer, Rohr-pipe bend, elbow or knee. -radius radius of curvature, $r$. of gyration.

Krümmung, Bildfeld-curvature of image field.

krümmungsfreies Feld flattened field (by flattening lens).

Krümmungskorrektur. field flattening.

Krummwerden warping, buckling, crooking, bending.

Kübel vat, tub, pail. 
Kubieren raise to third power, involution process (math.)

Kubikwurzelziehen extraction of cube root, process of evolution.

kublsch-raumzentrierte Metalle body-centered cubic metals, cubic body-centered $\mathbf{m}$. (cryst.)

Kubizlerung calculate volume of; raising to third power, involution process (math.)

Kufe pressure pad, p. guide (m.p.) Filz-felt pad. Gegen- counter pad.

Kugel ball, sphere, globe, bullet, shot, bulb, bead.

Kugel, After-spheroid. Halbhemisphere. Ulbricht'sche

Ulbricht sphere.

kugelähnl ich spheroidal, ball-like. Kugel-blasen, Stahl- steel shot blast. -biltz flashbag; globular lightning, ball 1 .

Kügelchen small bulb, globule, spherule, globulite, pellet, small ball or shot.

Kugel-densitometer, integrierendes integrating spherical densitometer. -druckprobe ball pressure test, indentation or Brinell ball impression $t$. - einsprache spherical mouthpiece. - faktor coefficient of sphere (photometry). -flasche balloon flask, spterical $f$.

kugelförmig spherical, globular, ball-shaped, orbioular. nichtaspherical, non-s. -qewöl bte Lamellen dished laminae.

Kugel-funkenstrecke ball-gap, sphere gap, spark-gap with ball electrodes. -funktion spherical function. -gelenk ball-andsocket joint, universal $j$. -gestalt spherical form, ball shape, bulbous s. -gestaltsfehler spot size distortion. -körperchen globulite, spherulite (cryst.) -kupplung ball-andsocket joint, universal j. - I inse spherical lens. -mikrometer spherical micrometer. -mikroskop ball stage microscope. - photometer sphere or globe photometer (of Ulbricht). - rücksprung ball rebound, ricocheting. - schale partial sphere, cup-shaped, or cap-shaped or hemispherical part, spherical shell, calotte. - schliff ground-in balland socket joint. -spiegel spherical mirror, s. reflector.

-spitzenfühlnadel scanning or pickup needle with spherical point (sound engraved film).

kugel symmetrisch spherically symmetric, sphero-s.

Kugel-tisch ball stage (micr.). -ventil ball valve. -welle spherical wave.

Kinhler cooler, refrigerator, condenser; radiator (automobile). Rückfluss- reflux condenser, return-flow c. -mantel cooling jacket, condensing $j$.

Kühl-mantel cooling jacket, condensing $j$. -rippe cooling or radiating, fin, vane or flange. - schl ange cooling coil, spiral condenser pipe or tube.

Kühlung, Heiss- cooling by evaporation.

Kühlvorrichtung cooling, refrigeration or chilling device.

kijken plug of a cock.

külbchen ball, piece, lump, parison (of glass).

Kulissenaufblendung side-curtain fade-in.

Kulturfilm cultural film, educationa. $f$.

Kümme $r$ form degenerate form.

Kundengiesserei jobbing foundry.

Kündigung (giving of) notice, denunciation (of a treaty), termination.

Kundschaft clientele, custorers, clients, following, goodwill.

Kunst-ausdruck technical term, art term. -griff artifice, trick. -hol z artificial wood, imitation w. - leder leatherette, art leather, artificial 1., 1. imitation. -leitung artificial line, cable or 
künstl ich

circuit, balancing or equalizing network.

küntllich artificial, synthetic. -(e)Antenne artificial antenna, mute a., dummy a., phantom a. -(e) Radioaktivität induced or artificial radio-activity. -(er) Zug forced draft.

Kunst-produkt, -stoff synthetic product, artificial p., synthetic, plastic. -trick, Schlelf- optical grinding artifice.

Küpe vat, boiler, copper.

Kup fermantelrelais copper-jacketed relay.

Kuppe cusp, crest, peak, meniscus (of a liquid colum).

kuppein couple, clutch, interlock, gang (e.g., condensers), join, unite.

Kupplung, Frell auf- free-wheel clutch, slip coupling. GelenkHooke's joint, universal j. Kardan- universal joint. $\mathrm{Kl}$ auen- claw coupling, c. clutch. Kreuzgelenk- cross joint. Kugelball-and-socket joint, universal j. Leerlauf- slip coupling, freewheoling clutch. Rutsch-,

Schl upf- slip clutch, s. coupling.

Kurbel und Welle, Einergang- singlepicture crank and shaft (m.p.)

Kurbel, Tret- foot pedal, treadle.

Kurbel-antenne reel antenna, trailing-wire a. - aufnahme hand-drive shot or shooting. -kröpfung crank (of a shaft). -schelbe crank disk, cam. -schlelfe crank guide. -zentrum crank center.

Kurbler crank turner.

Kurs, abl aufender course or direction away from airport, outward course. -, mi sswei sender magnetic heading, $m$. course, unc orrected bearing. -, rechtvoraus head-on course (fore-aft axis of craft directed to objective). -, rechtwelsender true (north) course. -mit Vorhal tewinkel course heading directly into wind, crabbing.
Kurve, ger issene

Kurs, Grund- true course, head-on course. Karten- map course.

Kompass- compass course, steөred c. Soll- prescribed course, charted c. stever- steered course, compass c. Vorhal tewinkel - course heading directly into the wind, crabbing. Wind- course headed directly to objective, corrected c. heading in wind (same as map c. in absence of wind). Wind-, rechtwel sender true heading. Ziel-, ablaufender course away from transmitter or beacon. Zlel-, anlaufender target course towards beacon. Ziel-, $\mathrm{mi} s$ swei sender magnetic course to steer. Zwischen- compound course. Kurs-absetzen laying off or plotting position lines or bearings, shaping course for. - abweichung (temporary) deviation from course, yaw. -fehler course error. - funkbake radio range, $r$. beacon. -gleiche loxodrome, rhumb line. -kreisel directional gyro. -linie, achterliche astern course, direct line away from transmitter. -linie, vorliche ahead course (towards beacon), "go-to" indication. - peil ung course bearing (taking bearing by directional signals, to keep craft on course). -richtung head, course bearing. - schel be bearing plate. -stativ class microscope.

-steuerung, automati sche automatic or mechanical piloting. -welser radio range, $r$. beacon. -weisung, rechtwel sende true course to steer, radio guidance or pilotage (from ground). -wei sung, $\mathrm{m} / \mathrm{sswe}$ l sende magnetic course to steer by radio guidance or pilotage (from ground). -winkel azimuth of target (in gunnery); magnetic a.

Kurve(3.a. Kennlinle, Charakteristik) curve, graph, diagram, characteristic. -drltter Ordnung cubic curve. -,flache flat curve, flattopped curve(e. g., in broad tuning), flat characteristic.,$- f l$ ave flattened curve (not rising steopiy), flat characteristic. -gerissene dotted-line or broken-line 
curve or graph. -, jungfräuliche virgin curve. -, zweispitzige double-peaked curve, doublehump c. Abkling- decay curve. Auslauf- coasting curve, deceleration c. Begrenzungsenvelope, contour line. Blattfolium. Durchlass- selectivity curve (of df receiver, etc.) Eichreiz- (typical) visibility curve. Empfindlichkeits- response curve, $r$. characteristic. Füll- single-loop oscillogram. Funkbeschickungs- error curve. H \& 0 - Hurter \& Driffield curve. $\mathrm{Hal}$ sband- collar curve. Hüllenvelope. Längungs- elongation curve, stretch c. Leit- directrix, pitch or contour of cam compensator (df). Ohren-auditory sensation curve. Peil-directional diagram, space pattern. Resonanz-, mit zwei Höckern double-hump resonance characteristic or curve. Schlangenserpentine. Schwärzungs- characteristic film or $H$ \& D (Hurter \& Driffield) curve. Sinus- sine curve, curve of sines. übertragungs- transmission (frequency) characteristic. Umrandungsenvelope. Verfestigungs- plastic stress-strain curve. Wachstumgrowth curve. Wende- turn in landing (by ZZ method), U turn. Zapfen- spectral response of eye cones. Zerfall- decay curve.

Kurven-einebnung flattening of a curve. -form, rechteckige rectangular curve shape (say, of an emf). -lichtmethode variablearea recording method (of sound).
- schaar family, group or system of curves or graphs. -scheibe, Funkbeschickungs- compensator or zero cleaning cam. -schreiber oscillograph, oscilloscope (when no graphic record is wade), curve tracer. - schwanzstück toe region of film characteristic. -zeichnen mapping a curve, plotting a graph (say, with stylus). -zug, abklingender decay train.

kurz-brennweitlges ob jektiv shortfous lens or objective. -brüchig short-brittle.

Kurzflammlampe hard light.

kurz-gefasst brief, concise, abbreviated, compendious, of reduced length or style. halsig short-necked. -lebiges Produkt short-life, short-lived, periodic or transient product.

Kurzschluss short-circuit, "short," bridge, by-pass, short-circuit path. -, akustischer acoustic feedback or short-circuit. -, vollständiger dead shortcircuit.

kurzsichtig near-sighted, short-s., myopic.

Kurzschluss-brïcke, -bügel shortcircuiting bridge. -1 äufer squirrel-cage rotor (of induction motor).

Kurz-wellenwandler short-wave converter. zeichen symbol.

Kïvette trough, vessel; bulb. Absorptions- absorption cell.

$k$ Wert octantal component of quadrantal error (or QE) due to fuselage. 
L Glied L-type section, filter s., mesh of network.

L Regler L-section attenuator. Labialpfeife flue pipe (of organ). labil unstable, non-stable, labile. Labium lip (mus.).

Labyrinthdichtung labyrinth seal, 1. gland (of turbine).

Lack, Tauch- dipping varnish.

Lackmuspapier litmus paper.

La Cour'sches Rad phonic wheel.

Lade, wind-wind chest, sound box (of organ). -pumpe charging pump, compression $p$.

Lader, Klein- trickle charger.

Ladeschaltıng circuit means causing slow $r$ ise of sawtooth voltage.

Ladestrom charging current (of dielectric).

Ladung, freie free charge. -, ganzzahlige integral spin, i. charge. -, gebundene bound charge, induced

c. -,punktförmige lumped loading. -, ungebundene free charge.

Einneits- unit charge.

Ladungs-ausgleich charge equalization. - austauschoderator charge exchange operator. -bild charge pattern, c. image (of iconoscope). -dichte charge density. -dichtesteuerung charge density modulation. -einheiten, positive elektrische unit positive charges. -sinn nature of a charge, sign. -speicher-Bildabtaster, einseitiger singlesided mosaic-type of storage iconoscope. -speicherung charge storage, c. accumulation.-träger charge carrier. -trägertrennung sorting or separation of ions and electrons (in a mass spectrometer). -vermögen, elektrisches electrostatic capacity. -zahl, Kern- nuclear charge number. -zusammenballungen bunching of charges, b. of electrons.

Lage layer, stratum, bed, coat, coating; state, situation, position, location, site; bearing (df). Lägel barrel, keǵ.

lagenrichtig positionally or geometrically correct.

Lagensicherung locking in position.

Lager bed, layer, stratum; bearing, bush. Mit einem einzigen - versehene Drehspule unipivot moving coil. -mit Weissmetallfutter babbitted bearing.

Lager, Gegen- abutment. Gleit- slide bearing, plain b. Hals- journal bearing. Kamm- (concealed) collar thrust bearing. Spur- thrust bearing. Spurzapfen- end thrust bearing. Steh- pillow block bearing, pedestal b. Stein- jeweled bearing. Weissmetallfutter-babbitted bearing.

Lager-bock pedestal of a bearing. -büchse, -buchse bearing bush, bushing of $a$ b. -element inert cell. -härtung, Hart- induction hardening. -körper bearing box, b. house. -prozess ageing, storage process. Lagerung, Schneiden- knife edge supporting or suspension. Spitzenpivot jewel, jewel bearing, point suspension or support. Stift-

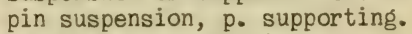

Lagerzapfen journal pin.

Lambertgesetz cosine emission law, Lambert $]$.

Lamelle, Pumpen- vane (in Gaede vacuum pump).

Lammellen, kugelförmig gewölbte 
dished laminae.

Lamellen-kern laminated core. -magnet compound magnet, laminated m. -spiegel strip-like mirror (in screw scanner).

I aminieren laminate; draw (in spinning).

Lammfell lambskin.

Lampe, punktförmige lamp with pointshaped or crater light source (e.g., tungsten arclamp, Fointolite). Aufnahme- studio light. Bienenkorb- beehive neon lamp. Bildeinstell- framing lamp. Blink- flash lamp. Blitz- flash (light) lamp. Bogen-, sprechende speaking arc, Simon or Duddell. Effekt- studio light. Einstellframing lamp. Erreger-exciter lamp, recorder or reproducer 1. Flächen-bank of lamps, pillar of 1. (studio, etc.), battens (stage). Flächengl imm- flat-plate neon tube. Gl imm- glow tube (e.g., neon tube or lamp), gaseous-conduction t., flashing lamp (for sound recording). Kathodolumineszenz- cathode glow-tube. Kleinminiature lamp. Kurzflammenhard light. Langflammen- soft light. Leuchtstoff- fluorescent, luminescent or luminous lamp or light, tube using phosphor substance. Mehrfaden- multiplefilament lamp. Melde- pilot lamp, telltalo 1., alarm 1., indicator 1. Mikronernst- Nernst microscope lamp, Pol suchgl immpolarity finder glow-tube. Punktpoint-type neon tube (e.g., tungsten arc lamp, Pointolite). Pustblow lamp. Quecksilberdampf-, mit Steuerelektrode mercury-vapor tube of thyratron or ignitron type with control grid. Reduktoradapter-transformer lamp. Reservespare lamp. Säulen-pillar (lamp), battens. Schlitz-slit lamp (micr.). Signal- signal lamp, telltale 1 . Sofitten- (overhead) scoop lamp, scaffold 1. Spiegelbogen- reflected (or reflector) arc- lamp. Ton- exciter lamp (operating photocell). Wolframmbogen- pointolite. Lampen-aggregat bank of lamps, pillar of 1. -arbeit blast-lamp work (glass making). -arm lamp bracket. - feld bank of lamps, panel of 1 . -fuss lamp squash, 1. press. -gehäuse lamp chamber, 1. housing. -stempel larp squash, 1. press. - iberlastung overrunning of a lamp (operated under overvolted condition).

Landé Interval Iregel Landé interval rule.

Landeachse correct bearing or line for approach (and landing, of an airplane). Blind- blind or instrument landing line or path.

Lande-bahn landing runway. -dienst, Blind- beacon service (to assist in blind or instrument landing of airplanes). -funkfeuer landing radio beacon. $-p l$ atz landing field, 1. strip, airport, airfield. -platz, hilfs- emergency or auxiliary landing field or airport, landing strip.

Landesgericht national court.

Landfunkstelle ground radio station, shore or coast $\mathrm{s}$.

Landgericht district court or county c., general court of justice.

Landung, Hebel- blind landing, instrument 1.

L andungsfunk-bake, - feuer landing beacon (for instrument landing).

l angbrennweitige Linse long-focus lens or objective.

Länge, 0st oder West east or west longitude.

I angeiförmig of extended oval shape. Längen-ausdehnung linear expansion. -bruch longitudinal fracture. - durchschnitt longitudinal section. -mass linear measure. -messung, echte und unechte direct and indirect measurement of length.

längentreu isometric. 
Langflammenlampe soft light.

I ang-gestreckt extended, elongated, obllong. - -gl iedrig long-linked, long-membered. -halsig longnecked. -lebig long-lived. -leblges Produkt long-life product. längl lchrund elliptical, oval, lenticular.

Langloch oblong hole, slot. -hebel slotted lever.

Längsachse major axis, longitudinal a., fore-aft line (ships, airplane, etc.).

langsam abfallendes Relais slowrelease relay. - ansprechendes Relais slow-response relay, slowoperating $r$. lösend slowly releasing or disengaging; $s$. dissolving.

Langsambinder slow-setting cement. Längsarm line-arm, series a. (of a. network).

Langschl iff long-fibered (mechanical) pulp.

Längs-durchschnitt longitudinal section. -entzerrer series-type attenuation equalizer or compensator. -feld longitudinal field, f. parallel to axis, paraxial f. - glled(einer Kette) series element, s. mesh (of network). - glled, in 1/2 - endendes fllter mid-series terminated filter. -pellung taking bearings from d.f. station situated more or less in direction of travel or course. - schärfe definition in line direction.

längsechlffs fore and aft.

Längsschwingung longitudinal vibration.

langstrahliges $\mathrm{Krl}$ stall elongated, needle-like crystal.

Langstreckend lenst long-range or long-distance avigation or navigation service.

Leangsstreifen longitudinal stripes, 1. striae, 1. streaks.

Längs- und Querverschlebung axial and cross adjustment (electron microscope).

Längs-widerstand (Filter) line resistance, series $r$. (of a filter). -zweig line arm, series arm.

Längung extension, elongation.

Längungskurve elongation curve, stretching $c$.

lang-wellig long-wave, of long wavelength. -wierig protracted, wearisome, tedious, time-consuming.

Lappen flap, tab, lobe, flange, lug; rag. - schraube thumb screw.

lappig lobed, lobate; flabby, flaccid. Lärm-abwehr, -bekämpfung combating of noise, noise abatement.

Lasche bond, cover plate, fish plate, splice strip (rail track).

Last, Gebrauchs- working load, service 1.

Lastwechsel cyclic stress, s. application cycle. -zahl alternating stress number, $n$. of load alternations.

Lasurstein lapis lazuli.

Laternen-bilderkasten lantern slide box. -bildermasken lantern masks. - elnfasslel sten lantern slide binding strips.

lau tepid, lukewarm, mild, balmy. Laveflecke oder -punkte Laue spots. Lauf course, path, run, race, current; barrel (gun). Heim- return or back stroke or motion, flyback (of pencil or cathode spot). HInforward move, f. working stroke, travel, pass or journey, activeline scansion (of spot or pencil, in telev.). Krels- cycle, circulation (of a fluid), circular course, circuit. Leer- no-load operation, idling. Rück- return or retrace, flyback (of pencil or cathode spot).

Lauf-bahn einer Rolle mit Gummiüberzug rubber tread of pad roller. -bild moving picture, animated p., one of a sequence. or series of pictures or pictorial actions. -bodenkamer base-board extensible 
camera. -einschaltung throwing in (of a gear), starting to run (of a mechanism, etc.).

laufend(en) Band, am-fabrizieren conveyor-belt manufacture, assembly line or large-scale $\mathrm{m}$.

- (es) Maass linear measure.

- (e) Nummer serial number, consecutive n. - veränderl/ch running variable.

Läufer rotor; cursor contact, sliding $c$.

Läufer, Kurzschluss- squirrel-cage rotor (of induction motor).

Läufermühle edge mill.

Lauf-fläche tread, bearing surface, journal. -gewicht sliding weight. -glasur flow glaze.

läufig, l inks- moving to left, m. counter $-c l \infty \mathrm{kw}$ ise.

Lauf-richtungskoeffizient directional effect (in film processing).

-ring race, raceway, ball-race

(of a bearing). -schaufel runner

blade, bucket (of turbine).

-umschaltung reversal of motion.

-werk feed mechanism, movement, working gear. -werk, Ton- soundfilm feed mechanism. -winkel transit angle. -zahl variable number, running variable. -zapfen journal, neck (of rolling mill). -zeit transmission time, transit t., propagation $t$. zeit, Elektronen- electron transit time, orbit time of electrons. -zeiteinfluss (electron) transit time effect. -zeitensteuerung velocity modulation.

Laufzeit-geräte (cf. Lautzeitröhre) devices predicated on transit time wherein electrons are bunched in proper phase to result in spacecharge concentrations and formation of groups. -glieder means involving transit time effects or phase delay. -komprimierung transit time compression. -korrektur echo or delay weighting term (telephony), delay equalizing. -röhre drift tube, beam t., $t$. involving transit time or phase of fects. -schwingungen electron oscillations. - störungen delay distortions. -verzerrungen transit-time distortion, phase d. Laugenflïssigkeit liquor.

launisch capricious, erratic, fickle. lauschen listen in.

Louschmikrophon pickup microphone, p. transmitter detectophone (connected to head-phones).

Laut (articulated) sound. -, explosiver explosive sound. Selbst- vowel. Zi sch-sibilant, friccative.

Laute lute.

lauter pure, clear, genuine, unalloyed, sheer.

läutern purge, refine, purify, clear, clean, cleanse, clarify, rectify, wash.

lautgetreu of high fidelity, orthophonic.

Lautheit loudness.

lautlos silent, quiet, noiseless.

Lautschriftband sound recording steel tape.

Lautsprecher, elektrostati scher capacitor or condenser loudspeaker. -, fremderregter dynamischer electrodynamic or moving-coil loudspeaker. -,gerichteter directional loudspeaker. -, induktordynami scher moving-iron loudspeaker. - , permanent-dynami scher permanentmagnet $(\mathrm{pm})$ or moving-coil dynamic loudspeaker. -,trichterloser direct-radiator loudspeaker (without horn).

Lautsprecher, Abhör-monicoring loudspeaker. Druckkammer- pneumatic loudspeaker (without diaphragm), pressure-chamber 1. Exponentialtrichter- exponential or logarithmic loudspeaker (with uniform or multiple flare in horn). Faltenfolded, curled, coiled or twisted horn loudspeaker. Freischwingerdirect-radiator loudspeaker, freer. 1. Gross- power loudspeaker. 
Grossflächen-, Hochle istungspower louds peaker, (public) address 1. , announce $1_{\text {. }}$, paging 1. , call 1. Hoch(ton)- tweeter loudspeaker, treble 1. Konusmembrancone loudspeaker. Kristallpiezo-electric loudspeaker. Luftkammer - air-chamber loudspeaker (c. coupling diaphragm and horn). Pilz- omni-directional (3600) exponential horn loudspeaker (mostly mounted on pole). Pressluftpneumatic loudspeaker, compressedair 1. (without diaphragm).

Riffel-Riffel loudspeaker (with corrugatiins and substantially horizontal radiation). Riffelfalten-folded and corrugated horn louds peaker. Tieftonkonus- lowfrequency cone loudspeaker, woofer. Vierpol- balanced-armature loudspeaker (with permanent magnet). Lautsprecher-abstrahlfläche projector surface, radiating s. (of a loudspeaker). - anlage, Betriebsworks address or public a. loudspeaker system (in factories, etc.). -antrieb motor element. -haltevorrichtung chassis of a loudspeaker. -horn, auf gewundenes twisted loudspeaker horn, coiled h. - horn, gefaltetes folded loudspeaker horn.

Lautsorecher-korb chassis frame of loudspeaker. -leitfläche deflecting vane, labyrinth. -leitwand deflecting vane; baffle (to prevent acoustic feedback). -luftkammer air space, a. chamber. - spinne spider of loudspeaker (inside or outside voice coill, to keep coil centered). -tonführung labyrinth of loudspeaker.

Lautsprechertrichter, aufgewundener twisted loudspeaker horn, coiled h. -, gefal teter folded loudspeaker horn. -hals throat of loudspeaker horn. -mundöffnung mouth opening of horn, largest flare. -mundstück thrcat (narrow inlet) of horn.

Lautsprecher-vorhof air chamber of louds peaker (between horn and cone). -wagen sound truck, loudspeaker $t$. Loutstärke, Empfindungs- response of ear to signal stimulus, sensible loudness of sound. Stör- noise level.

Lautstärkemesser, Neonröhre- neon tube volume indicator.

Lautstärken-bereich der stimme human voice intensity or dynamic range. -emofindung aural sensitivity. -stufe loudness unit, sensation unit.

Lautstärke-regler, logarithmi scher volume control with resistance varying according to a logarithmic law. -schwankungen fadings. -umfang volume range, dynamic $r$. Lavenderkopie lavender copy, 1. print.

Lawine, Elektronen- electron avalanche.

Lawinenkopf head of a surge.

lebender Kalk quicklime.

lebendige Kraft kinetic force, vis viva, momentum.

Lebendigkeit liveness, realistic properties (in architectural acoustics').

Lebensdauer life period, (working or useful) life, life time (say, of a quantum).

Lebewesen, Klein-micro-organism.

lebhaft lively, brisk, active, vivacious; bright, vivid, gay (of colors).

lebig, lang- long-lived.

Lecherleitung Lecher-wire line.

Leckstrom leakage current, leakance c.

Lederharz (India) rubber. -stulpen leather cuffs.

leer empty, blank, void, vacant, hollow.

Leere vacuum, emptiness, vacancy. Leergewicht tare, weight allowance for container, weight empty. Leerl auf no-load (operation), idling. 
- impedanz open-circuit impedance, no-load i. -kontakt spare or vacant terminal or contact, dumb or dummy c. -kupplung slip coupling, free-wheeling clutch. -nocke delayed pulse trip cam.

-widerstand open-circuit impedance. Leer-scheibe idler (wheel). -stelle hole, vacancy (in lattice).

-stell enmechanismus vacancy mechanism (metals). -versuch blank experiment, no-load test.

Legierung, sparstoffarme alloy low in critical or scarce metals. Automaten- machining alloy stock, alloy readily workable in automatic machine tools. Knet-plastic alloy, malleable a., wrought a. Lehm-guss loam casting. -stein unburnt brick, adobe.

Lehrdorn inner cylinder or plug gage. Lehre, Augen- ophthalmology. Bewegungs - mechanics (re:machines); kinetics (re:motion of bodies and forces acting thereon); kinematics (re:motion in the abstract); dynamics (force action on bodies).

Bewegungsel ektrizitäts - electrokinematics, electrokinetics. Dreiecks - trigonometry. Elektronen-electronics. Farbenscience of color, chromatics. Filmkitt- film splicing gage. Fühler- thickness gage. Getriebekinematics. Gefrierpunkts- cryoscopics. Gewinde- thread gage. Gleichgewichts - statics. Grössenmathematics, geometry. Innengrenzplug gage. Kegel- taper gage. Kontroll- master gage. Kristallcrystallography. Licht- optics, photology (or physiological o.). Loch- internal caliper, plug gage. Natur- natural science, n. philosophy. Normal - normal gage, masterg. Phrsen- solution theory, doctrine of phases. Rachen- gap gage. Ruheelektrizitäts- electrostatics. Schraub- micrometric gage, micrometer. Schub- sliding gage, s. caliper. Seh-optics. Uhrschraubdial micrometer. Wärmekr ofttherrodynamics. Wasserkrafthydrodynamics. Wurf- ballistics. Zahlen- arithmology, numerology. lehrenhaltig true to gage.

Lehr-film educational film, instruction f., school f. -satz abstract theorem, a.doctrine. - schau educational exhibition.

leicht-flüchtig readily volatile. - fliissig easily fusible, e. meltable, $\theta$. liquef iable; mobile. - lösslich easily soluble.

Leichtmetall light metal (of low $\mathrm{sp} g \mathrm{r}$ ).

leicht-schmelzbar easily fusible, e. meltable. -siedend low-boiling, of low ebullition (point).

Le im glue, size.

leimartig gluey, gelatinous, glutinous.

Leine line, chord, cord.

leise Kassette silenced magazine.

Leiseabstimmung silent tuning.

Leiste ledge, border, ridge, molding, strip. Einfass- binding strip.

Leistung power, work, performance, energy. Abschalt- rupturing capacity, circuit-breaking c. , circuit-opening c. (of a switch). $B l$ ind-reactive power, $r$. voltampere, var. Schall- response (of an acoustic system). Scheinapparent power. Spitzen-record (achievement, performance or accomplishment). Verlust- (power) dissipation, power loss. Verzerrungs- distortion power (in $\mathrm{d}$. volt-amps.). Watt- wattage, power in terms of watts. Wirk- (cf Bl ind-) active power, actual p., true p. (in watts).

Leistungs-aufwond power input, $p$. expenditure, energy dissipation. - bedarf power absorption; p. dissipation, p. requirements. - bedarfspegel power reference level. -entnahme energy output, power 0. , 
delivery, absorption or pickup of energy or power; taking, tapping or draining of power or energy. -fähigkeit performance, efficiency, serviceability, power, ability; resolving power (of a microscope). -faktor power factor. -faktorzeiger power factor indicator or meter. -kammer energy chamber (of a klystron).

leistungslos non-dissipalive, wattless.

Leistungs-messer power meter, ergometer. -reserve margin of power, standby p. - umsatz energy exchanǵ, transduction. -verstärker power amplifier.

Leitbahn transit path. -bewegung transit path motion. -welle

(Magnetron) transit-time or electron path oscillation (of magnetron).

leiten conduct, guide, lead, govern, steer, pilot, route (a message).

Leiter, Aufnahme- first cameraman, chief c. Aussen- "outer", outside conductor or wire. Halbsemi conductor, poor c. Heissthird-class conductor, hot c. (with negative resistance). Hinoutgoing lead, o. wire, o. conducior. Ketten-iterative network, recurrent structure. Nullneutral wire. Ruck- return wire, $r$. lead, r. conductor. Tonmusical scale (natural, diatonic or just scale, and tempered $\mathrm{s}_{\text {.). }}$. Voll-compact or unstranded conductor.

Leiter-gebilde conductor structure, c. system. -gebilde, Luft- aerial extension, a. structure, a. network, array of antennae. -stuck element of conductor, conductor element. - system conductor system, c. structure.

Leitfähigkeit, dielektrische leakance, dielectric conductance. -, elektrische conductance. -, magnetische magnetic conduc- tance, permeability, permeance. -, spezifische conductivity. Super- supra conductance, super $c$. Temperatur - temperature diffusivity, thermal conductivity. Ubersupra conductivity, super $c$.

Leit-fähigkeitsgefäss conductivity cell. -fläche deflecting vane (mounted in horn to reduce directional properties of a loudspeaker), labyrinth, baffle. -funksender radio range, $r$. beacon. -funkstelle net control station. -kabel leader cable, pilot c. (d.f.). -kante leading edge, 1. tip (of airplane wing). -kreisfrequenz transit-time frequency (of a magnetron). -kurve directrix; pitch or contour (of a cam compensator). -linie beacon course, beam, equi-signal line, e.-s. course or zone; directrix. -richtung course on beam or equisignal line. -rolle guide roller, idler, pad roller (without sprockets). -rolle, federnde springloaded idler. -satz rule, guiding principle, basic p. -stelle ground direction-finder station (sending directional or position data to craft). -strahl guideray, localizer beam, radio beacon course, landing beam; radius vector (math.). -strahl, streckenlong-distance or route navigation (or avigation) beam. -strahlsender beacon, radio range or beam station, aerophare. -strahlwinkel angle of beam. -strom conduction current; pilot c. (in wired radio). -system, Lichtcondenser relay system (in film printing).

Leitung conduction, conductance, transmission; electric circuit, wire, (supply) line, cable, lead conduit, guide, piping, mains. -, angepasste matched line. -,konzentrische co-axial line or cable, concentric c., pipe 1. 
-,kurzgeschlossene shorl-circuited line. -, offene (am Ende) openended line. -,verkabelte cable line, stranded-wire 1.. -,zusammengesetzte compound circuit.

Leitung, Antennenring- multiple receiver connection to an antenna. Ausgleichs- balancing network, b. line. Energie- energy f'eeder lead, e. leader, downlead, transmission line. Fern-long-distance line, loll circuit. Hohlrohrconductive (hollow or pipe) line, wave-guide. Kondensator-highpass filter. Kunst-artificial line, a. cable, a. circuil, balancing or equalizing network. Lecher-Lecher-wire circuil or line. Quer- shun line, cross 1.; leakage conductance, leakance. Regler-pilot wire. Rohr-pipe line, co-axial cable, concentric 1. Ruickwärts-high-resistance direction (of rectifier). Simultan-earthed phantom, composite circuit. Soeise-(aerial) feeder lead, down-lead, energy feeder cable or wire. Umweg- by-pass lead. Vorrats- spare circuic, standby c. Weg-directions for tracing or tracking trouble or for "trouble shooting". Wellen- ultra high-frequency feeder line; wave guide (either dielectric or conduc (ing).

Le itungs-dämpfung line loss, 1. alienuation. -elektronen conduction electrons (from outer levels of atoms). -fehler line failure, line faulc.

leitungs-gerichtete Hochfrequenztelephonie wired or carrier current RF telephony, t. along wires by RF. -gesteverter 0szillator line-controlled oscillacor.

Leitungs-induktivität lead induclance, line $i$. -messer conduclometer. -nachbildung balancing network, artificial b. line. -rundfunk wire broadcast, AF rediffusion. -schema diagram of connections, circuit d., hook-up. -schleife loop, metallic circuit. -strom-Modulation conductioncurrent modulation. -stück strelch of line, portion of 1. , line element. -taubheit (im äusseren $0 \mathrm{hr}$ ) conduction loss or impairment of hearing, conduction deafness (in ouler ear). -übertragung wired radio, w. wireless, carrier lelephony or telegraphy, wire broadcast. -verstärker telephone (circuil) repeater, communication line $r$. -wasser tap wacer, city mains w. -wechselstrom conduction $\mathrm{a}-\mathrm{c}$.

Leitvermögen, richtungsabhängiges asymmetric conductance. -, spezifisches specific conductance, conductivity.

Leitwand baffle, deflecting vane (of loudspeaker).

Leitwert admittance, conductance, susceptance (being, respectively, the reciprocals of impedance, resistance and reactance, the latter either inductive or capacitive). -, spezifischer conductivity. Blind- susceptance. Eingangs- input admittance. Eingangswirk- input active admittance, input conductance. Kenn- indicial admittance. Rückwirkungs- reactive admiltance, susceptance (either inductive or capacitive). Scheinadmittance. Ubergangs- transconductance, transfer characteristic. Wirk- conductance.

Leit-zahl, Wärme- thermal conductivity. -zone equi-signal or glide zone or path.

Lenardröhre c.-r. tube of Lenard (used in radiology and early experiments on phosphors).

Lese-band (ore) picking belt conveyor. -glas, viereckiges oblong reading glass.

Lettern-gut, -metall type metal.

lettig clayey, clayish.

Leuchtdauer illuminated period, light p.; period or duration of 
glow, luminescence or luminosity; phosphorescence $p$.

Leuchtdichte brightness (in terms of stilb, sb, or apostilb, asb, or other units), intrinsic brilliance (of a luminous surface). Bildwand- screen brightness, s. luminous density. Dreifarbentricolor RD (reflection density) value. Schwarz- brightness of black.

Leuchte lamp equipment (m.p.). Flächen- bank or pillar of lamps, battens. Natrium- sodium lamp.

Leuchtelektronen emitting electrons, optical e.

leuchten emit light, shine, glow, luminesce.

Leuchten, Nach- afterglow, phosphorescence, persistence of light or of vision (of an image, on retina).

leuchtende $\mathrm{Fl}$ amme luminous flame.

Leuchtenergie erzeugend photogenic, fluorogenic, phosphorogenic (as by manganese, in zinc sulfide).

Leuchter, Selbst- self-luminous substance, fluorescent s., phosphor.

Leuchtfaden luminous or glow column; streamer. -einsatz streamer onset.

Leucht-farbe luminous paint. - fleckdurchmesser spot diameter. - gebilde luminous pattern (lines, bands, or continuous spectra). -hïlle luminous sheath, 1. envelope; photosphere (of sun).

Leuchtkraft illuminating power, luminosity. -, gemessene luminance, photometric brightness. -bestimmung photometry. -verminderer, Nach- poison, killer (of phosphorescence). -verstärker phosphorogen:

Leucht-kreuz reticle image (reflector sight). -masse, -material phosphor, luminescent or phosphorescent substance or material. -mittel illuminant. -punkt (luminous) spot. -rakete flare. -resonator glow-tube resonance indicator. -röhre luminous discharge tube; fluorescent $t$. -schirm fluorescent screen, luminescent s., phosphor s.; target. -schirmsubstanz phosphor, fluorescent substance (for c. rr. tube screens). - spur tracer trajectory. -strahl quelle, einfarbige monochromatic illuminator, monochromator. -stofflampe fluorescent lamp, luminous or luminescent 1. or tube, t. containing phosphor. -streifen (bei Entladung) luminous streamer (in discharge). -verfahren, Selbst- self-luminous or s. -emissive method (using electrons on object surface). -zeiger dial light resonance indicator. -zifferblatt luminous dial.

L Glied L-type section, filter mesh or network $\mathrm{m}$.

Libelle level, spirits or water 1. Kreuz- cross level.

licht(e) light, pale, bright, luminous, clear, inside (of diameter). -(e) Weite inside diameter, $i$. width, lumen, clear aperture (of lens), useful or effective d.

Licht, auffallendes incident light. -, ausfallendes emergent light, transmitted 1. -,direktes specular light. -, durchfall endes transcident light, transmitted 1. -, einfallendes incident light. -,einfarbiges monochromatic light, homogeneous $1 . \quad-$, eingestrahltes incident light, exciting $1 . \quad-$, fal sches light fog, leakage 1., stray light (film printing). -,gerichtetes parallel light rays. -, spektral zerlegtes spectroscopically dispersed or separated light.

-, unzerlegtes undispersed light.

Licht, Blitz-flashlight, flash bulb, magnesium light, lime 1. Flutfloodlight. Gegen- backlight, counter light (m.p.). Kathodencathode glow. Neben- light-shot (film). Nord-aurora borealis. ober- overhead scoop light, scaffold 
1., skylight. Ruhe- unmodulated light, no-modulation 1., average or steady illumination, no-sound lighting (in sound recording).

See- marine phosphorescence. Sofitten- overhead scoop, scaffold light. Spitzen- highlights (of a picture). Streu- stray light. Sucher- searchlight. Südaurora australis. Ton- exciter lamp (operating a cell). Wechselbrightness variations (striking photo-cell), light intensity v.

Licht-abstufung gradation of light. -anzeiger, Glimm- flashograph, Tune-A-Light tuning device. -ausbreitung auf Film distribution of exposure on film.

Lichtbild photo-image, photograph. -, unvergängl iches imperishable picture or photograph. -entzerrung rectification of aerial photographs. -kunst, Klein-microphotography, photomicrography.

Licht-blende light stop, diaphragm, apertured partition; light shield (in sensitometer). -blitz pulse of light, flash of 1., scintillation. -bogen, Erdungs- arcing ground.

lichtbogenbeständig arc-proof, a. resistant.

Lichtbogen-erdung arcing ground. -löschkammer arc quench chamber. lichtbrechend optically refracting, light r., refractive or refringent. Licht-brechungsvermögen (optical) refractive power, refractivity. -bündelvignettierung vignetting of cone of light. -chaos chaotic light (of eye or retina), photopsy.

lichtchemisch photo-chemical, actinic. Lichtdämpfer silk, light sof tener (m.p. studio).

lichtdicht (sealed) light-tight or 1.-proof.

Lichtdruck photo-mechanical printing; photographic p.; radiometer-vane effect.

lichtdurchlässig light transmissive or transmitting, diaphanous, pervious or permeable to light rays, transparent, translucent.

Lichtdurchlässigkeit light transmittance, (specific) light transmittancy.

licht-echt fast to light, non-fading. -elektrisch (cf photo-) photoelectric; electronogenic. -el ektrische, -emofindliche Zelle photo (-electric) cell, light microphone. -empfindlich machen photo-sensitize.

Lichtemofindlich-keit photosensitivity; actinism. -keitskurve des Auges response curve of eye.

lichtentwickelnd emitting light, producing 1., photogenic, with luminophor properties.

Lichter highlights (in pictures), lights, transparent spots or areas (in photography, printing, etc.). -, gedeckte covered lights.

lichterzeugend emitting light, producing light, photogenic, with luminophor powers.

Licht-farbendruck photo-chemical color printing. -filter light filter, color f. -fleck hot spot (on film); spot (of light). -fleckverzerrung spot distortion.

lichtfreundich favoring high lights over shadows, photophilic, photophilous.

Lichtfreundlichkeit light transmittancy, l. transmissivity.

lichtgebend giving or emissive of light, luminous, photogenic, with luminophor properties.

Licht-gewinn light increment, 1. gain. -grammophon sound-track film player (without pictorial accompaniment). - hahn electromagnetic mirror vibrator (of ten) with oil damping (for film recording by variable-area method). -haut cathode glow. - hof halation, halo. -hof, Reflexions- halation by reflection.

lichthoffreie Platte non-halation plate, plate free from halo. Lich thof-schutzmittel anti-halo 
means, anti-halation substance. -störung halo disturbance.

Licht-kegel cone of light, luminous cone. -korpuskeln photons. -kreis circle of light, halo. -l ampe, Ton- exciter lamp. -lehre optics, photology (or physiological o.). -leitsystem condenser relay system (film printing). -loch pupil (of eye), opening for light. -messung photometry, optometry (re:eye). -mikroskop optical microscope, light m., light-optical m.

Lichtmodulator light relay, any device adapted to reconvert current into light or brightness variations, e.g., television receiver lamps, Kerr cells, modulated neon lamps, c.-r. tubes, etc. -, selbstleuchtender light relay of the glow-tube, neon arc lamp, sodium lamp, etc., type.

Fremdlicht- light relay of the Kerr cell, Karolus c., etc. types adapted to reconvert currents into brightness variations by the use of a constant independent ray source.

Licht-mühle radiometer. -netzantenne light-socket antenna, 1.-circuit a. -optik light optics, physical o., geometrical o. of light rays. - pause photographic tracing, blue print. -phonograph sound-track film player (without pictorial accompaniment). -quant light quantum, photon. -quelle source of light, illuminant. -quelle,-punktförmige punctiform light source, pointolite, tungsten-arc lamp. -quellenbild light source image. -rakete flare. -reflexionsvermögen light reflectance, 1. reflextivity. -regler light relay, 1. modulator (Kerr cell, etc.); light control means. -reiz luminous stimulus.

Lichtrelais light relay (Kerr cell, Karolus cell, modulator, recorder lamp, etc.); device converting current into light variations. -,elektrooptisches electro-optical cell, Faraday c., light-relay depending on F. effect. Uberschallsupersonic light-relay.

Licht-säule light column (of glow tube). - schacht light tunnel (of sensitometer). - scheue photophobia. -schleuse, Vierband- fourband or f.-ribbon light valve.

lichtschluckend light absorbing, optically absorptive, optically absorbing.

Licht-schranke light barrier. -schutzkappe light hood (phot.).

lichtschwaches Bild low-luminosity picture, p. of low brightness.

Licht-schwächung light absorption, 1. loss, 1. diffusion, 1. dimming, 1. intensity drop, cutting down or reduction of 1 . -sinnprüfer visual photometer. -sirene light chopper, c. wheel. -spalt light slit, 1. aperture, 1. opening. -soielhaus moving picture theater, film playhouse, movie. -spritze (Ewest'sche) recorder lamp with constricted neon arc (system Ewest). - spur light track, 1. pattern, 1. tracing.

Lichtstärke luminous intensity, light i.; power, speed, $F$ number (of a lens). -,gemessene luminance, photometric brightness. objektivspeed or power (of a lens, etc.), F number.

Lichtstärkemesser photometer.

lichtstarkes 0bjektiv high-speed or high-power objective, fast 0. objektiv, hoch-ultra-rapid lens, ultra-high-speed 1:

Licht-stärkeverteilung light intensity distribution (on photo-cathode). - staub luminous dust (of dark visual field). -steuerung light modulation; 1 . scanning. -steuerungseinrichtung light valve, light relay. -steuerzelle, Debye-Sears supersonic light valve, D.-S. 1 . modulator.

Lichtstrahl, wandernder flying spot (for spotlight scanning). -(es), maximaler Ausschlag des clash 
point of light valve (in sound recording). -abtaster spotilight scanner, scanning brush, s. pencil. Licht-streuung light scatter, diffusion. -strom luminous flux, light f. (in lumen units); lighting or lamp current. -stromdichte luminous flux density (in lumens). -strommesser lumen meter (of Blondel, etc.), photometer, lumeter. -stufe der Kopiermaschine printer step. -summe total light emitted, 1. sum.

Lichtton photographic sound recording. - ansatz photographic soundfilm head. -aufnahme photographic sound film recording. -gerät sound-film head or attachment. -punkte elementary shading values, e. density v. -spalt recording slit. -streifen veränderlicher Breite squeeze track. -zusatz sound-film head or attachment.

Lichtumfang range of light oscillations.

lichtundurchlässig light-tight, opaque to 1. impervious to 1 ., non-diaphanous.

Licht-ventil light valve, 1. relay (e.g., selenophone, Kerr cell, neon lamp, etc.). -verteiler beam splitter (m.p.). - verteilungsfläche luminosity curve, isophotic c., isophote, isolux diagram. -wagen light-truck. -wechsel variation of exposure. -weg, Satz vom ausgezeichneten law of extrere path. - werbung light advertising, propaganda using illumination or light effects. - wi ssenschaft optics, photology (or physiological o.). zähler counter tube. -zelle photo- (electric) cell. -zerhacker light (beam) chopper, episcotister. -zerlegung dispersion of light, decomposition of light (into primary colors).

lichtzerstreuend light diffusive, dispersive or scattering.

Liderung packing.

Liebesflöte flute d'amore.
Ligand attached atom or group.

Lindenholz lime.

lindern alleviate, soften, relieve, mitigate.

Lineal straight edge, rule.

Linearantenna plain or straight (onewire) antenna or aerial.

linear(er) Gleichrichter oder Detektor linear rectifier or detector.

-(e) Vergrösserung magnification in diameter.

Linearisierung linearizing action, correction of non-linear distortions (in an amplifier), say, by reverse feedback.

Linearskala slide-rule dial.

Linie, abgewickelte evolute. -, ausgehackte jagged line, serrated 1., notched $1 .$, dented 1. -, ausgezogene solid line (in graphs, etc.). - - durchstreichende trajectory. -, fal sche grating ghost. -, gezackte jagged line, serrated 1., notched 1., dented 1. -,in eine-bringen align, rectilinearize, straighten out in a line. -in gerader - mit in alignment, or flush, with. -, strichpunktierte dash-dot line. -, verbotene forbidden line. -, zusammengesetzte multiplet (in complex spectrum).

Linie, Anflug-center of approach sector. Anschlag- striking line (piano). Aussen- contour line, boundary 1. Begleit- satellite line. Bild-focal line, inage 1. Blick- line of sight, 1 . of vision. Brenn- focal line, image 1. Gleitslide or slip band or line. Grenzboundary line, demarcation 1 . Grund- center line of approach sector. Halbierungs- bisector (line), bisectrix. Haupt- primary line. Kenn- characteristic curve or graph, indicial diagram. Knoten- neutral line, common 1. , nodal 1. Kollimations- collimating mark. Kraft-tube of force, line of $f$. Mantel-directrix, line along a shell or cylinder. Mittags- meridian. Mittel- median 
line, center 1., axis, equator. Neben- secondary line, branch l.; satellite (spectr.). Nordlichtgreen auroral line. Rad-cycloid (stroboscopic aberration), epicycloid. Richt- guide line, guiding 1. Richtkenn- rectification characteristic; directional diagram, space pattern. Schaugraph, curve. Scheide- boundary line, separation 1. Schmelzfusion curve. Schrauben- helix, helical line. Seh- line of sight, 1. of vision, 1. of collimation. sinus- sinuous line, sinusoidal 1., sinuoid. Spektral- spectrum line, spectral 1. Stand- position line. Strom- stream line, flow 1., force 1. Translations- slip band. Trennungs- dividing line, boundary 1., partition 1., line of separation (of a solid moving through liquid). Umgrenzungscontour, line of demarcation or separation, boundary line, peripherical 1., circumferential 1., circumscription 1. Verschiebungsline of displacement. Visierline of sight. Wellen- wavy line, undulatory 1 ., sinuous 1 . winkeldiagonal. Hurf- line of projection, curve of p., trajectory. Zeuge- generating line, generatrix. Zug- tractrix.

Linien-bilder, aufgel öste resolved line patterns. -breite breadth or width of line; spot diameter. gleicher Spannung isoclines (indicating stress lines in glass and other materials). -komplex multiplet. -raster ruled plate, line screen or grating; raster.

linienreich rich in lines, with abundance of 1 .

Linien-skala variable-density sound track. - spektrum line spectrum. -steuerung velocity modulation. -summe line integral. -umkehr line reversal. -verbreiterung broadening or spread of line or strip (telev.). -zug line, trace (of a graph).

linig, krumm- curvilinear.

linksdrehend levo-gyrate, levo-rotatory, counter-clockwise rotating.

Links-drehung levorotation, lefthanded polarization. -händigkeit left-handedness. -kurve left-hand (or port) curve or loop (in airplane landing, etc.).

links-l aufende well en sinistropropagating traveling waves or surges. -läufig moving to the left, counterclockwise.

Links-quarz left-handed quartz, levogyrate crystal. - schraube lefthanded screw.

Linse (s.a. Objektiv, Lupe, Glas) lens, objective glass; bob (of a pendulur:).

Linse, eingekittete cemented lens. -, facettierte bevel-edge lens. -,fehlerfreie perfect, aberrationless lens. -, gefasste mounted lens. -,gekittete cemented lens. - ,krümmungsreduzierende fieldflattening lens. -,kurzbrennweitige short-focus lens.,- 1 angbrennweitige long-focus lens. -, lichtstarke high-power lens, h.-speed 1. -, magnetische, mit Ei senpanzer ironclad magnetic lens, iron-core deflector unit (of c.-r. tube). -, scharfzeichnende achromatic lens. -, ungefasste rimless lens. -, ungekittete uncemented lens, broken-contact 1.- veränderli cher Brennweite und v. Bildgrösse zooming lens for variable focus and variable magnification, varioobjective, -, verkittete cemented lens. -, weichzeichnende sof tfocus lens. -, weitwinklige wideangle lens. -, zus ammengesp rengte uncemented lens (lens units closely fitted together, as in Cornu device), broken-contact 1 .

Linse, Augen- eye lens. Bel euchtungscondensor lens, illuminating 1 ., bull's eye 1. Beschleunigungs-, mit 2 Lochelektroden doubleaperture (electron optic) 
accelerator lens. Brenn- burning glass, convex lens. Butzenscheiben- bull's eye lens. Doppeldouble lens, doublet. Doppelschicht-double-layer lens. Durchgriff-(cf. Lochblenden-) aperture lens (through which field or anode may act or draw electrons). Einblick- eyepiece lens. Einzel- unit lens. Elektronen- electron lens (either electromagnetic or electrostatic). Feld-anterior lens (in telescope and microscope), field 1 . Feldebnungs- field flattener.

Fenster- aperture lens. Ferntelephoto lens. Flüssigkeits(oil or water) immersion lens. Halbkugel- semi-circular lens. Haupt- second lens (in electron gun). Hinter- back lens.

Immersions- (oil or water) immersion lens; double aperture lens (electron-opt.). Kugelspherical lens. Lochbl enden(cf. Durchgriffs-) simple aperture lens (electronic). Lochscheiben- aperture lens (in scanning disk). Muschel- globular lens. Punktal - toric lens. Sammel- condensor lens, convergent 1., positive 1. Schauviewing lens. Scheitel-zenithal lens, vertical 1. Stufen- echelon lens. Trocken- dry lens, d. objective. Verzögerungs- cutoff lens, stopping 1. (el.opt.). Yorder-front lens, field 1. Vor sammel - cathode lens, first focusing 1. (like Wehnelt cylinder, aperture disk, etc., in c. $r$. tubes). Vorsatz- auxiliary lens, supplementary 1., 1. attached in front, magnascope (to enlarge projector image). Zerr-anamorphic lens, anamorphosing 1., distorting 1. Zerstreuungs- dispersive lens, divergent 1., negative 1. Zusatz- supplementary lens. Zwiebel - biconvex lens. Zylinder- cylindrical lens.
Linsen-abweichung aberration of a lens. -achse optical axis of a lens.

linsenähnlich lenticular, lenticulated, lens-shaped.

Linsen-deckglas anterior surface of a lens. - einschiebebrett slidable lens panel. - faserung, Augenfibrillation of lens (of eye). -fassung lens barrel, 1. mount. - fassung, Vignettierwirkung der vignetting effect or trimming of light by lens mount. - fehler lens defects, 1. aberrations. -folge lens combination, system of lenses. - grundpunkt cardinal point (of a lens). -kranzabtaster lens drum scanner. - prüfer lensometer.

-rasterfarbverfahren lenticular or lenticulated lens color printing method. - rasterfilm lenticular film, lenticulated f. -satz lens combination, system or assembly of lenses. -scheibe lens disk (scanner). -scheinwerfer spotlight. -scheitel vertex of lens, apex of 1. -system, Fluorit- semi-apochromatic lens system, fluorite 1.s. -trommel lens drum (scanner). -trübung clouding of a lens, opacity of a 1 . - vergutung s. Gl asvergutung. -weite, 1 ichte clear aperture of a lens (useful or effective diameter). -zusammenstellung lens combination, lens assembly.

Lippenpfeife flue pipe, flute p.

Lissajousfiguren Lissajous figures, L. curves. - apparat kaleidophone (of Wheatstone).

Literatur, Fach-technical literature, t. press.

Litze strand, stranded wire, braid, lace, cord, string.

Litzen-draht stranded wire, litzwire. - spule litzwire coil.

Lizenz, Zwangs- compulsory license.

Lizenz-erteiler licenser, grantor, issuer of a license. -gebihhr royalty, license fee. -nehmer licensee, grantee, concessioner. In natural, Naperian or hyperbolic 
logarithm $=\log _{\varepsilon}$ or $\log _{e}$.

Loch eye, pore, pocket, opening, aperture, perforation, puncture; hole (of nucleus). -auftreiben enlarge, expand, broach or ream a hole. -, ausgetuchtes bushed hole. Loch, Blenden - anode aperture (cathode-ray tube). Führungs- guide perforation (for sprocket engagement). Gitter-vacancy or hole in lattice. Greif-finger hole (in dial switch). Licht-opening for light admission, pupil (eye). schau-peep hole; inspection hole, bezel.

Loch-blende apertured partition, anode aperture, diaphragm or shield (of $c_{0}-r$. tube), stopping aperture. -blendenlinse (cf. Durchgrifflinse) apertured disk lens; simple (electron-optic) aperture lens. -breite (eines Frequenzsiebes) transmitted band, transmission range, bandwidth, band pass (of a filter), spacing between cutoff points. -breite, Bandfilter von grosser broad-band filter. -elektroden, Beschleunigungslinse aus zwei double-aperture accelerator lens (in c. $r$. tube).

löcherig punctured, perforated, apertured, porous, pitted.

Löchertheorie hole theory (re: nucleus).

lochfrassähnliche Zerstörung pitting, destruction in form of pits, honeycombing.

Loch-grenze transmission range of band pass (range between cutoff points), channel width or limit, cut-off frequency. -kamera pinhole camera. -lage (eines Filters) position of transmission range or of band pass (of filter). -lehre internal caliper gage, plug g. -niete hollow rivet, tubular $r$. -probe drift test.

Lochscheibe, konzentrierende focusing aperture lens or apertured disk (mounted anteriorly of cathode, in c. $-r$. tubes). Kreis- film scanner (with holes arranged circularly rather than spirally). Mehrfach-multi-spiral scanning disk. Spiral-Nipkow disk, spiral d. (for scanning). Vierfachquadruple spiral scanning disk. Lochscheiben-anode apertured disk anode, ring a. -generator light chopper. -linse aperture disk lens.

Loch-spirale scanning (hole) spiral; spiral row of apertures $\left(e_{0} g_{0}\right.$, in Nipkow disk). -trommel scanning drum (of Jenkins).

Lochung perforation, punched holes (in film), punching.

Lochweite band-width, band-pass, band between cutoff points; inside diameter. Bandfilter grosserbroad band filter. Well enbergpeak separation, inter-peak distance, inter-crest $d$.

Locker-ionen defective ions, "Locker" ions. -stellen loose spots, 1. places (cryst.).

Lockflamme pilot flame.

Löffelbohrer spoon bit.

Logarithmen, dekadische common logarithms.

ogarithmus der Belichtung log exposure.

lösbar detachable; soluble, dissoluble, dissolvable.

losblättern detach in leaves or scales, exfoliate, defoliate.

löschen (cf auslöschen) turn of $f$ (repeater); extinguish, quench, slake (lime), obliterate, clear, cancel, blot, erase, wash out (a magnetic record).

Löscher, Funken- spark quench, blowout or extinguisher, s. killer. Röhren- tube quench.

Lösch-feld, magnetisches obliterating field, erasing $f$. (of magnetophone, etc.). - funkenstrecke quenched spark gap. -kalk quicklime, slaked 1. -kammer, Lichtbogen- arc quench chamber. kohlie 
quenched charcoal. - magnet obliterating magnet (in magnetic sound recording equipment), washout I. - spannung stopping potential (at which glow discharge is stopped; s.p. is lower than glow p., in photo-cells and g.tubes), extinction p., critical p. (at which tube goes out), cutoff $p$. (of thyratron). -taste cancel key, clearing k., release k. -wirkung quench effect. -zündsp annungsdifferenz extinction-striking potential difference.

lösen solve, dissolve; loosen, untio, detach, disengage, disconnect.

Löser solving agent, solubilizer.

Löslichkeits-druck solubility pressure. -produkt solubility product.

löslich machen solubilize.

los-löten unsolder. -trennen separate, sever, tear apart, detach.

Lösung solution; discharge (of a gun); separation, detachment, opening (of a connection), severance. -, ungepufferte unbuffered solution. Abschwächungs- reducer solution. Zehntel (normal)tenth normal solution, decinormal $s$.

Lösungs-druck solution pressure. -magnet releasing magnet, trip $m$. Lot fällen let fall a perpendicular. Lot, Behm- fathometer, echo depth sounder; sonic altimeter. Messingschlag- brass solder. Mittelmedian perpendicular.

Löt-kolben soldering bit. -rohr blowpipe. -rohrflamme blowpipe flane.

lotsen, heran- guide to, pilot (a craft by radio signals).

Lötstelle soldered joint, shut; junction of a thermo-couple. Heiz- hot junction of a thermocouple.

Lötstreifen tag strip, terminal s., connection s.

Lotung sounding (determination of depth or altitude), probing (for depth).

Lötung soldering, brazing (by hard solder); agglutination; adhesion. Doppel- double seals (in. Dewar vessel, etc.).

Lotung, Echo- echo sounding, reflection s. ( $\mathrm{f} r \mathrm{r}$ depth and altitude measurement).

Lötung, Hart- hard soldering, brazing. Selbst- autogenous soldering.

Loxodrome oblique line, loxodromic line or course, great-circle bearing.

L-Regler L-section attenuator.

Lücke gap, void, space; deficiency. auf - stehen positioned so as to fill gaps (e.g., in a parallel row), staggering. -,positiv gel adene positive hole (cryst.).

Lücke, Bildsynchronisierungs- synchronizing gap (between traversal of frame and beginning of next), underlap or interstice period in which syne signal is introduced. Mischungs- miscibility gap.

Lücken-bildung unsaturated linkage. -satz vacancy principle. - -synchronisierungsverfahren gap, under lap, interstice or interval synchronizing method (sync signal introduced between end of line traversal and beginning of next line).

lickig porous, honeycombed, pitted. Luft-abschluss exclusion of air, $a b-$ sence of a., hermetic seal, airtight s. - al arm air raid alarm. - aufnahme air absorption, a. œclusion; aerial photograph, airplane picture. -auftrieb air buoyancy. -bild aerial image, i. formed in space by optical system. -bildaufnahme aerial photooraph, airplane picture. - bildkarte aerial mosaic; photographic map.

luftdicht air-tight, hermetical.

Luftaraht (cf Antenne) aerial (wire), antenna. -gebilde aerial structure, radiating syster, array of antennae. 
- verkürzungskondensator shortening condenser. -verlängerungsspule antenna load coil, aerial loading inductance.

Luft-druck air pressure, atmospheric p., pneumatic pressure. -durchlässigkeit air permeability, a. perviousness, -einschluss air cavity, a. bubble, a. occlusion.

luftelektrische Störungen atmospherics, statics.

lïften ventilate, air, aerate; raise or lift (clear of something).

luftentzündlich inflammable in contact with air, pyrophoric.

Lüfter ventilator, fan.

Luftfahrt, Verkehrs- commercial aviation, c. avigation. -hindernis air navigation obstacle, avigational o. -navigation avigation, aerial navigation.

Luft-funkstelle aircraft radio signal station. -gebilde aerial structure, radiating system. -gleichstrom current of breath (in sound recording). -gütemesser eudiometer. -hahn air cock. -kammer air pressure space (loudspeaker). - kammerl autsp recher airchamber loudspeaker (c. coupling diaphragm with horn). - kern air core. - kissen air cushion, dashpot.-kreis atmosphere.

luftleerer Raum vacuum, vacuous space, exhausted $s$.

Luft-leerspannungssicherung vacuum arrester. -leitergebilde aerial extension, a. structure. -mast ("Schnorchel") breathing mast, underwater air intake (supplying air, for Diesel engines, to submerged submarines). -meer atmosphere. - plattenspektroskop FabryFérot interferometer, étalon (when not variable). -rahmen air-cored frame (d.f.). -reibungsverlust windage loss. -sauger aspirator. -säure carbonic acid. -schall sound conducted or transmitted through air. - schleier air fogging (chemical action of air). -schraube, versetz- variable-pitch (feathered) propeller or airscrew. -schraubensteigungsmesser propeller pitch indicator. -schutzleiter air warden. -spule air-core coil, loop or frame (d.f.). -strahl air jet. -strom, Gleich-direct flow of air, d.c. air-flow. -strompendelung air jet pendulation. -strömungsmesser anemometer. -verdichter air condenser, a. compressor. -verdünnung air rarefaction, vacuum.

-verkehrskontrolle airways traffic control, avigation regulation.

-welle sky wave, space w., indirect w. -wichte specific gravity of air. -widerstand aerodynamic drag (on airplane). -winkel angle of the wind. zutritt access of air, admission of $a$.

Lumièreplatte autochrome plate.

Lumineszenzl ampe, Kathoden- cathodeglow tube.

Luminophore phosphors, luminophors, luminescent substances.

Lummer-Brodhun'sches Photometer oder wïrfel L.-B. contrast (or totalreflection) photometer, cube $\mathrm{p}$. (with cubical cavity).

Lunkerung shrinkage, contraction, piping, blowhole formation, cavitation (in metals).

Lupe, stark vergrössernde high-power magnifier. Ablese- reading lens. Einstell- focusing magnifier. (lens). Einzel-magnifier unit, individual $\mathrm{m}$. Fadenkreuz- reticle magnifying lens. Feldstecher-field glass magnifier. Fernseh- telescopic magnifier. Präparier- dissecting magnifier. Stativ- stand magnifier.

Lupen-aufnahme, Kamera für Zeitcamera for slow-motion picture projection. -ständer lens stand. -verfahren, Zeit- high-speed shooting, low-speed projection method. Luvwinkel drift angle, a. of lead. 1.W. (lichte weite) inside diameter, i. width, lumen.

Lyra lyre. 
Maass s. Mass

Mach'sche Streifen Mach bands. Mächtlgkelt size, thickness, magnitude, power, strength.

Madenschraube headless screw. Magazinbalg reservoir bellows. magern make lean or poor, shorten, reduce plasticity (of ceramics).

Magneslagl immer magnesia nica (biotite).

Magnesiuml icht magnesium light. Magnet, fremderregter non-permanent magnet, electro- m. Abnahmepickùp magnet. Aufnahme- recording magnet (for steel-band or wire recording of sound), pickup magnet. Auslösch- obliterating magnet, wash-out m. (to wipe away sound track or recording on wire or tape, for re-use). El inrïcktrigger magnet, trip $m$., starting m. Glocken- bell-shaped magnet. Hub- vertical maønet, stepping m., lifting magnet. Lamellen- laminated magnet, compound $m$. Löschobliterating magnet, wash-out $m$. (sound recorded on wire or tape). Lösungs- release magnet, trip $m$. Topf- ironclad magnet, potshaped m. Wähler-bank of (stationary) contacts, selector magnet (aut. telephony).

Magnetfeld, Quer- transverse magnetic field.

Magnetfeldröhre magnetron (or permatron) working with a constant magnetic field (strictly, a m. operates with a variable m.f.) magnetisch(e)Belegung nagnetic induction, $m$. charge, seat. of $m$. induction or m. field.-(er)Effekt, kreis - gyromagnetic anomaly. -(e)Leitfähigkeit permeance, magnetic conductance, permeability.
-(er)Schirm magnetic shield, $m$. screen, can (of a coil).

-(e) Sp annuno nagnetic potential (line integral of $\mathrm{m}$. intensity). -(er) Tonaufzeichner magnetic sound recorder, magnetophone, Foulsen telegraphone, Blattnerphone (modern form of F.t.)

Magnetisierungskopf magnetic sound recording head (on wire or tape). magneto-optische Drehung magnetooptic rotation, magnetic $r$., Faraday effect (rotation of plane of polarization).

Magneton, Kern- (K.M.) nuclear magneton.

Magnetorotationsspektrum magnetic rotation spectrum.

Magnetron, Zweischlltz- two-split magnetron, two-segment $\mathrm{m}$.

Magnetron-cycloidhöhe cycloidal height of a magnetron. -leitbahnwelle transit-time or electron path oscillation. -leitkrelsfrequenz transit-time frequency of a magnetron. -schwingungen magnetron oscillations ( 3 kinds: electronic, negative- resistance and rotating field o.)

Magnet-schale magnetic shell. -schenkel magnet limb, m. leg. -spannschraube magnetic chuck. -spule solenoid, field coill. -summer magnetic interrupter, buzzer type of audio-frequency oscillator. -tonverfahren magnetic sound recording method, telegraphone s.r.m.

Mahl-felnheit fineness of grind, $f$. of grain, $f$. of comminution. -gut material to be ground or milled.

Mai schgitter stirrer, rake. Makroachse macro-axis (of crystal). Mal mark, spot, sign, token; stigma. 
mal times $(x)$.

Malerfirnis painter's varnish.

Malteserkreuz Maltese cross, cross wheel, (cam and) star wheel in Geneva intermittent movement. - gesperre Geneva stop.

Mängel (in einer Anmeldung) defects (in a patent application).

mangels in default of, in the $a b-$ sence of, because of lack of. manipulieren manipulate, handle. Manko shortage, deficiency, deficit.

Mannigfaltigkeit manifoldness, multiplicity, variety, diversity.

Mann-loch manhole. -schaft men, force, crew, gang.

Manometer, Gl asfeder-spoon-type glass manometer. MicLeod- NicLeod (vacuum) gage.

Manschette flap, collar, cuff.

Mantel casing, jacket, case, shell, sheath, surface (math.), nappe, sheet, envelope, cover. Heizheating jackel. Kegel- coneshaped shell. Wasser- water jacket.

Mantel-fläche generated surface, shell s. -futter shell lining. -gefäss, Vakuum- thermos or Dewar vessel exhaust jackel. - I inie directrix. -rohr tubular shell, -transformator ironclad uransformer, shell t. -welle wave on outer surface of coaxial cable, "shell" wave.

Mantisse mantissa (fractional part of a logarithm).

Manual keyboard, manual.

Manuskript scenario script (m.p.).

Marien-bad water bath. -glas selenite, mica.

Mark marrow, pith, pulp, core. Marke, Einstell-reference work, bench m., gage m., measuring m., wander $\mathrm{m}$. (of telescope, to establish "stereoscopic con(act"). Fabrik- trade-mark. Zeit- time scale, t. marking. Ziel-sigh reticle or gralicule.
Markenplatte index plate (of telescopic sight).

Markieren mark, define, outline.

Markierung(Szenenwechsel) nothing on film edge. Interscheidungsdistinctive marking, telltale $\mathrm{m}$.

Markierungs-sender marker beacon, marker. -vorrichtung marker, puncher, notcher (m.p.).

Masche, Gitter- grid mesh. Kettenleiter-mesh or section of a network.

maschig meshed, netied, reticulated. grob-, weit-wide-meshed, coarsem. (e.g., of a grid).

Maschinensender alternator transmilter.

Maser speckle, spot, mark. -holz burwood.

maserig speckled, streaked, grained, veined.

Maske mask, mat (in film recording). Laternenbilder- lantern mask. Vignettier - vignet ting mask.

maskieren mask, blank, blanket, conceal, camouflage, cover, dissimulace, eclipse.

Maskierung, Gehör - auditory masking (loss of sensilivity of ear).

Mass measure, scale, dimension, proportion, degree, extent. auf-drehen turn true (to template or gage). auf genaues - bringen finish or true up (to template or gage). -, laufendes linear measure.

Mass, Aussen-over-all dimension, outside d. Belichtungs- exposure level (of Decoutes). Dämpfungsunit of altenuation, image attenuation constant. Eben- symmetry. Eich-standardized sage, s, measure, calibration unit. Endstandardized gage block, end-toend standard bar. Farbencolorimeter. Kraft- unic of force. Längen- linear measure. Parallelend-standardized gage block, end-to-end standard bar. Phasenphase constant, wave-length 
c. Rlcht- standard, gage.

Strlch- line standard. Vierpolwinkel- image phase constant. Winkel- phase constant, wavelength c. (denotes phase shift between input and output potential, and is the imaginary component of image transfer constant): set square. Zeit- time scale, t. morking.

Mass-abweichung (cf. Toleranz) of $f-s i z \theta$ condition, dimensional discrepancy or difference. - analyse volumetric analysis.

Masse mass (in mechanics; analogou to inductance in electricity and inertance in acoustics).

Masse, aktive active paste.

Einhelts- unit mass. Grundground mass. Isolier-, gepresste molded insulation. Leuchtphosphor, luminescent or fluorescent substance. Ruhe- (der

Teilchen) rest mass (of particles). Massekern dust core, molded $c$. -spule dust-core coil, compressed iron core c. Ferrocart c. Massen-absorptionskoeffizlent mass absorption coefficient. - anziehung gravitation. - dichte mass density. -einheit unif of mass. -gesteine unstratified rocks. -güter bulk goods; large-scale manufactured goods. -kopie release print; quantity production of prints. -kräfte mass, inertia (in mechanics, mechanical filters, etc.; analogous to inertance in acoustics and inductanco in electricity). -moment moment of inertia, rotationsl i. -platte pasted plate (of battery). -plet electric mass. -punkt quantum, point-mass (in quantum theory), rotator; center of mass, c. of inertia, centroid: -schwankungskoeffizient mass absorption coeficient. -spektrograph mass spectrograph, m. spectrometer. -spektrum mass

spectrum. -strahler masa radiator. -widerstand inertance (ac.). -zahlen mass numbers. -zentrum center of mass, c. of inertia, centroid. Masseteilchen-Geschwindigkeit particle velocity.

Mass-faktor dimensional constant, $d$. factor. -gabe, nach in accordance with, in proportion w.

massgebend determinative, determinant, decisive, authoritative, competent; conclusive.

Massgefäss measuring vessel, gracuated v. masshaltig true to size, measure or dimension (inside tolerance range, found by limiting gage). -mässig suffix signifying "in the manner of"; "ธ० far as.... is concerned"; according to, in terms of. For instance, hochfrequenzmässig: so far as RF is concerned, or, for $R R$.

massig massive, massy, molar.

mässig, gesetz- in accordance with law or statute, conformable to (natural) law, in a regular or legal way. -,grössen- dimensionally, quantitatively, numerically; pertaining to, or in relation to, quanta. -hochfrequenzfor $R E$, so far as $R F^{3}$ is concerned, relative to RF. -, quanten- pertaining or in relation to quanta. -, zahlen- numerically.

mässigen moderate, temper, mitıgate. massiv solid, strong; molar.

Mass-kunde metrology. - nahme mode of action, (precautionary) step, expedient, technique. -nahme, Schutz- preventive, protective, safety, or precautionary step, measure or means, safeguard. -regel cf. -nahme.-röhre measuring tube, graduated t., burette. Masstab scale, size, measure, yardstick, proportion, gage, standard. Parallelend- end-to-end measuring bar, standard gage block. Vergleichs-standard of comparison or reference, comparative "yardstick". Zeit- time scale.

Mass-stabtransformator gage (group) transformer. -stock gage, 
yardstick, linear measure. -system, Farb- (CIE) chromaticity scale system, CIE diagram. -zahl measure or numerical criterion.

Mast, Gitter- lattice pole, girder $D$. Kreuzteilungs- cantilever type of tower, double tapered mast.

Luft- ("Schnorchel ") breathing mast, underwater air intake (supplying Diesel engine air when submarine is submerged).

Mast-armatur pole hardware, fittings. -befeverung mast beacon.

Material, entgegenstehendes anticipatory references (found in prior art and literature). -, zertrümmerbares fissionable or disintegrable substance. - prüfer, el ektronischer aniseikon. -prüfung testing of materials. -wanderung flow of material, ureep of $\mathrm{m}$.

Materie-welle matter wave, de Broglie (elementary particle, electron, proton) wave, electron w., phase พ. -zer trümmerbarkeit disintegrability or fissionability of matter.

Matrize metal master, master negative, mother (in phonograph record manufacture), matrix die. Kaltstauchcold upsetting die. Press- negative stamper. Stanz- cutting dieplate, punching die.

matt dead, faint, feeble, weak, dull, matt (photographic paper).

mattgeschliffen ground, frosted, delustered, matted.

Mattglas ground glass, frosted g. mattieren tarnish, deaden, dull, deluster, matt, frost, impart a matt surface or look to, grind (gloss).

Mattierungsmessung film polish measurement, glossimetric m.

Mattl ack matt varnish. Mattolein matoleine (retouching varnish).

mattrot dull red.

Mattscheibe ground or frosted glass (pane or plate). Visierfocusing screen.

Maul-trommel Jew's harp. -weite interpolar distance, air-gap (of horseshoe magnet).

m. a.W. in other words, that is to say. Maxima, Neben- secondary or lateral radiations, s. lobes or ears (in space pattern).

maximale Aussteuerung des Lichtstrahles clash point of light valve (in sound recording).

Maximalpeilung maximum signal (strength) method (df).

Maximumwertzeiger peak indicator, crest i.

Maxwell'sche Grundgleichungen Maxwell electromagnetic equations.

Maxwellverteilung Maxwell (statistical) distribution.

mC milli-curie.

McLeod Manometer McLeod vacuum gage.

mechanischer Filter, Federungswiderstand compliance of a mechanical filter. -Filter, Massenwiderstand inertance of a mechanical filter.

Medien media, mediums.

Meerfarbe sea green.

mehlartig like meal or flour, farinaceous.

Mehlgips earthy gypsum. mehradriges Kabel multi-wire cable. Mehraufwand extra or additional expenditure or means (in equipment, etc.) Röhren-tube complement, extra outlay for tubes,

Mehr-deutigkeit ambiguity, equivocation. -drehung multirotation.

mehrfach manifold, multiple, reposted.

Mehrfachbelichtung superimposing, multiple exposure.

mehrfachbeschichteter Film sandwich film.

Mehrfach-bild multiple image (in telev.) -echo multiple echo (reverberation), flutter e. -empfangsverfahren diversity roception method (to minimize fading). -kondensator multiple condenser, gang c., tandem c., synchronized c., gang capacitor. -kopien multiple prints. 
Mehrfachnachrichten

-nachrichtensystem multiplex signal system. -platten KarolusKerrzelle Karolus type of multiple-plate Kerr cell. -röhre multi-valve, dual v., multipurpose tube, multi-unit $t$. -rückkoplung multiple, regeneration. -spirallochscheibe multi-spiral scanning disk. -strahler bank or pillar of lamps, battens. -telegraph multiplex telegraph. -telephonie multiple telephony. -verstärker multistage amplifier. -zackenschrift multilateral sound track. -zeichen multiple signal, echo s.

mehr-fädige Lampe multiple-filament lamp. - farbig polychromatic, pleochroic, multi-colored. Mehr-gangkondensator ganged condenser or capacitor, synchronized c., multiple c., gang capacitor. -gitterröhre multi-grid tube (tetrode, pentode, etc.) mehrgliedrig multi-mesh, m. section (e.g., of a network). Mehrheit plurality, multiplicity, majority. -von Einheiten array of units, Iplurality or multiplicity of $u$.

Mehrkanalfernsehen multi-channel television.

mehrkanal ig multi-channel.

Mehrkantprisma polyhedral prism. mehr-kernig with more tran one nucleus or core, polynuclear.

-köpfige Besatzung multiman crew. -köpfiges Stationspersonal multi-manned station.

Mehr-kristall polycrystal. -lagenspule multi-layer coil, banked c., pile c., honeycomb c. with banked winding. -platten Karolus-Kerrzelle Karolus multiple-plate Kerr cell. - punktschreiber multi-point recorder.

mehr-stimmig polyphonic (of an organ). -strahliges Funkfeuer
Membrane, Nawi-

multi(-ray) beacon, multipleor triple - modulation b. -teilig multipartite. -wegig having multi-channel or $\mathrm{m}$. path properties. -wegige übertragung multi-path transmission.

Mehr-welligkeit multi-wave property. -zahl plurality, multiplicity; majority.

mehrzigiges Tonformstück multipletile or clay conduit.

Meilerkohle charcol.

Meissel chissel, pledget, stylus or style (for sound recording), engraver tool.

Meister, Bild-director of photography, picture chief, p. director. Tor- monitoring operator (working at mixer desk), recordist, sound engineer, director of $\mathbf{s}$.

Meister-negativ metal master (in making phonograph records), master negative. $\rightarrow$ ositiv mother record, master positive.

Melde-feld, Rïck- revertive signal panel. - l ampe alarm lamp, pilot 1.. telltale 1.

Melder (s.a. Anzeiger) indicator Mel der, Abst immungs- tuning indicator. Einbruchs- burglar alarm. Rijck - revertive signal means, position-repeat-

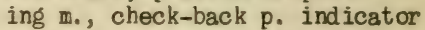
(in telemetric arrangements). stations- station indicator, tuning $i$. Wellenbereichwave-band indicator.

Membrane, al s Ganzes schwingende piston diaphragm, diaphragm moving like a p. or en blo. -, atmende flexible, non-reflecting diaphragm. Falz- non-rigid non-circular breathing cone (with curved radiating surfaces). $\mathrm{Kal}$ otten- hemispherical diaphragm, cap-shaped d. Kolbenpiston diaphragm. Konusconical diaphragm, cone d., diffusion cono. Hawi- cone 
diaphragm with curve profele, curvilinear c., para-curve. Riffel- pleated diaphragm (of loudspeaker), d. with pleated corrugations. Schirm- screened diaphragm (of microphone).

Sektor- (non-rigid) sector diaphragm, sectorial cone.

Membraneinspannung suspension of diaphragm.

membranloses Mikrophon diaphragmless microphone, cathodophone.

Membranschwingamplitude excursion amplitude of diaphragm.

Mengeeinheit unit of quantity.

Mengen-bestimmung quantitative determination, q. analysis. - verhältnis quantitative relationship, proportion (of ingredients), q. composition.

Meniskuskante meniscus edge. menschl ichte Stimme, Lautstärkeverhältnis human voice intensity range.

Mensur measure (string instruments), scale (organ); measuring vessel; measuring, mensuration.

Merkatorkarte Mercator chart.

Merkatorkarten projektion Mercator projection, cylindrical p., or thomor phic $p$.

merkbar perceptible, noticeable, appreciable.

Merk-mal characteristic feature, c. object (of an invention), c. mark, sign, indicator, index, criterion. -punkt index point, fiducial p., reference $\mathrm{p}$., point de repère (in Baudot apparatus), bench mark.

Mesonen-bahnende mesotron track end. -zerfall meson decay.

Mesotrontheorie meson theory, $t$. of mesotrons (also known as heavy electrons, barytrons, dynatrons, penetrons, \& particles, etc.)

Messbereich measuring range, measurement $r$. , indication or reading $r$.

Messer (s.a. Anzeiger, Melder, Messgerät, Messung, Meter, Probe, Prüfung, Zähler) measurer, meter, measuring instrument or device; knife, cutter, blade. Abklingzeitfluorometer. Abschleif- grinding blade, g. knife. Abtrift-drift meter. Augen- ophthalmometer, optometer. Augenabstand- interpupillary distance gage. Augenbrechungs- skiascope, retinoscope. Ausdehnungs- dilatometer, extensometer. Basisentfernungsbase range finder. Bebenseismometer, seismograph. Bel euchtungs(stärke)- illumination meter, 1 . photometer, illuminometer. Belichtungsexposure meter (in sound recording, etc.), brightness m., turbidity $m$. Beschleunigungsaccelerometer. Blutfarbeplethysmograph (tests blood flow), electroarteriograph. Brandpyrometer. Brechungs- refractometer. Brennweiten- focometer. Dampfdichte- dasymeter. Dampf(druck)- steam gage, manometer, tensimeter. Dampfdruck-, osmotischer osmometer. Dämp fungsloss or transwission (efficiency) measuring device, decremeter (measures logarithmic decrement or damping of circuits). Deckkraftcryptometer (measures concealing power of paint). Dehnbarkeits-, Dehnungs- ductilimeter, dilatometer, extensometer. Dichte-, photographischer (photo-) densitometer. Dicken- thickness gage, calipers. Dosis- dosimeter, dosage m., intensimeter. Druckpiezometer, liquid manometer, fluid m., pressure gage. Druck-, osmotischer osmometer. Druck- und Gefrierpunkt- manocryometer. Dunst- atmometer. Durchlässigkeitstransmissometer (for semi-transparent media). El astizitätselasmometer (measuring Young's modulus), torsometer. Energieergometer. Entfernungs- range finder, distance meter. Erdbebenseismometer, seismograph (when 
recording). Fallkörperzähigkeitsball-drop viscosimeter. Farbenchromatometer, colorimeter. Farbenstufen- tint ometer. Feinhöhen- (aneroid) barometer measuring atmospheric pressure at airdrome (not reduced to sea level), sensitive altimeter. Fern- telemeter. Fern-, mit Summenangeber telemetric integrator. Feuchte- hygrometer, psychrometer. Feuchtigkeits, registrierender hygrograph. Filmfilm footage meter or counter. Flächen(aus)- planimeter; integraph (for curves). Flügelvane-type fluid-flow meter. Fluoreszenz- fluorometer. Flussfluxmeter (for magnetic measurements). Flissigkeitsdichteliquid density meter, areometer, hydrometer (measuring sp.g.) Frequenz-(cf Wellenmesser) frequency meter, onometer, sonometer. Gasdichte- dasymeter. Gefrierpunkt- cryometer. Gehör schärfeaudiometer. Geländewinkelangle-of-site instrument, clinometer. Geräusch- sound level meter, noise m., n. measuring set, psophometer (f or telephone noises), acustimeter. Geschwindigkeits- speedoreter, tachometer. Gierungs- yawmeter. Glanz- gloss meter Glätte- glossimeter; surface analyzer. Gleichrichtervolt- rectifying voltmeter. Glutpyrometer. Grobhöhen- (aneroid) barometer or altimeter measuring actual atmospheric pressure reduced to sea level. Halbbildentfernungs - split-field coincidence range-finder. Herzschlag - cardiac tachometer. Hitz- pyrometer.

Hitzdraht- hot-wire anemometer (and other measuring instruments using a hot w.) Höhen- altimeter, altitude meter ( of echo or reflection type), sound ranging altimeter, statoscope. Hörschärfe- audiometer. Hysterese- hysteresigraph. Impuls- peak meter, crest m. (for potentials). lonenstärke- ionometer. lonenwindvolt- ionic wind voltmeter. I solations- insulation tester, megger. Kapazitätsfaradmeter. Kartenwinkelmap protractor. Kochounktebullioscope. Koerzitivkraftcoercimeter. Konsistenzviscosimeter. Körnigkeitsgranulometer. Kraft-dynamometer, force meter. Kraftstoss- ballistic pendulum. Kreiselneigungs- bankand-turn indicator (aviation). Kreiskeilschwärzungs- circular wedge densitometer. Kristallachsenconoscope (determines optical axis of crystals). Lautstärke- volume indicator. Leistungs- power meter, ergometer. Leitungsconductometer. Leuchtkraftphotometer. Lichtstärke- photometer, lumen meter, lumeter. Lichtstrom- lumen meter (of Blondel), photometer, lumeter; wattmeter for lighting circuits. Luftdruck - barometer, manometer. Luftdruck-, registrierender barograph. Luftfeuchtigkeitshygrometer, psychrometer. Luftgüte- eudiometer. Luftstromanemometer. Mattierungs- film polish meter, glossimeter. Neigungs. (winkel)- clinometer. Neonröhrenlautstärke- neon tube volume indicator. Oberflächenspannungssurface tensioneter. öffnungswinkel-apertometer. Pegeltransmission measuring set. Permeabilitäts- permeameter. Pol itur- glossimeter. Pul sschlagcardiotachometer. Pupill enweitekorometron. Quotienten- quotient meter, ratio m. Röhrenspannungs-, Röhrenvolt- vacuum tube voltmeter. Roentgendosis- X-ray dosimeter. Roentgenstrahlenhärte- penetrometer, qualimeter. RoentgenstrahlenRontgen meter, iontoguantimeter (Duane's), ionometer. RückstrahIungs- reflectometer. Sauerstoff- 
eudiometer. Saug- vacuometer. Schalenwindstärke- cup-type anemometer. Schallstärke- phonometer, sound level meter. Scheltelbrechwert- apex refractometer. Schlïpfungs- slip meter. Schnittbildentfernungs- splitfield range-finder. Schwärzungsdensitometer. Schwere- barometer, gradiometer (measures gradient of earth's gravity field). Schwere-, registrierender barograph.

schwingaudionwellen- autodyne wave or frequency meter. Sicht (barkeits) - visibility meter (of Lukiesh-Taylor, etc.) Sonnenstrahlungswärme- solarimeter, pyroheliometer, actinometer ( $\mathrm{f}$ ar solar flux density measurements). Splegelneigungs- Abney level (to measure vertical angles).

Steigleistungs- rate of climb meter ("variometer" measuring both rise and descent). Steilheitsderivator, tangent meter (for curves). Strahlen- radiometer (for heat rays), actinometer (for actinic r.) strahlenbrechungsrefractometer. Strahlenhärtepenetrometer (for $x$-rays). Strom-, registrierender recording ammeter. Summerwellen- buzzer wave meter. Temperatur- bolometer (of high precision). Temperatur-, thermoelektrischer thermel. Tiefen-fathometer (e.g., supersonic and sonic types), depth finder. Tontonvariator, pitchpipe; frequency record. Tonschwankungs- flutter meter. Trägerfrequenzferntelemeter, metameter (of GE Co., using carrier waves). Tropfenstactometer, stalagmometer. Trübungs- turbidimeter, opacimeter, nephelometer. übersteuerungsoverload indicator. Umfangsgeschwindigkeits- tachometer (e.g., stroboscopic using strobotron). Vakuum- vacuum gage, ionization g., vacuometer. Verbrauchs- supply meter. Handerwellen- klydonograph, surge indicator. Wärme- calorimeter, thermometer. Wärme-, thermoelektrischer thermel. Wassersiede- hypsometer. Wellen(s.a. Frequenzmesser) wave meter, frequency meter, Fleming's cymometer, ondometer. Widerstandsohmmeter, megger. Windgeschwindigkeits- anemometer, wind meter. Winkel-protractor, goniometer, angle gage ( $f$ or eye), angleometer ( $f$ or measuring external angles), theodolite (for surveying). Wirkverbrauchs- active power meter. Zähigkelts- viscosimeter, viscometer. Zeit-chronometer, time piece, clok, watch. Zeit-, registrierender chronoscope, chronograph. Zungenfrequenzreed frequency meter.

Messer-schalter knife (blade) switch. zeiger knife-edge pointer.

Mess-filmeinrichtung time recorder on film. - form parison (bottle making). -gefäss measuring vessel, graduated v., measure.

Messgerät, Fern- telemeter, metameter (of GE Co., using carrier impulses). Intensitäts- level recorder. Mikrozeit- microchronograph. Wetter-,mlt funkentelegraphischer Fernübertragung radio telemeteorographic instrument. Zeit- chronographoscope.

Messing-blasinstrument brass wind instrument. - schlaglot brass solder. - späne brass shavings, b. turnings.

Messinstrument, gedämpftes aperiodic or deadbeat measuring instrument. -, ungedämpftes ballistic measuring instrument. Wetter-, mlt funkentelegraphischer Fernübertragung radio-telemeteorographic instrument, $r$. sonde.

Mess-kolben graduated flask, 
measuring $f .$, gage $f$. -kunde metrology. -lehre, Feinmicrometer caliper, m. gage. -methodik measuring methodology. - okular micrometric eyepiece. - pegel measuring level, expected 1. - schallplatte frequency record, phonograph $r$. bearing sliding or constant notes, parlophone record (producing warble note). -technik, Fernsprechtelephonometry. -trommel graduated drum. -umformer measuring transducer.

Messung, quantitative quantitative measurement, $q$. analysis. -, stationäre deadbeat measurement. Abnahme- acceptance test, works test. Elementen- stoichiometric measurement, stoichiometry. Entdampfungs-Frequenz- gainfrequency measurement or test. Farb- colorimetry, chromatometrics, chromatometry. Feldland surveying, 1. measuring; mapping or plotting of field configuration or field pattern (in an electrolytic tank). Gehörschärfe- audiometry. Kreiscyclometry. Kristall- crystallometry. Kristallwinkelcrystal goniometry. Längen-, echte direct measurement of length.-, unechte indirect measurement of length. Leuchtkraft-, Licht- photometry, optometry (re: eye). Mattierungs- film polishing measurement, glossimetric m. strecken- end-to-end measurement, straight-away $m$.

Mess-wandler measuring transformer, instrument $t$. -zahl numerical value, measured v., measuring v. -zwelge measuring arms.

Metall, gespritztes die-cast metal. Edel- noble metal, precious m., rare m. Erdalkali- alkalineearth metal. Misch- mix metal, alloy m., "Misch" m. Spiegelspecular metal.

Metall-azid metallic azide. -beschreibung metallography. -blatt (thin) sheet of metal, $m$. foil, lamina, lamination. -gekrätz waste metal. $-g l$ as enamel. -kassette metal darkslide. - kunde metallography. - pickel bildung (corrosion) pitting. - probe assay, test for metal; metal testing. -schllff polished section, micro-section; metal filings. -späne metal shavings, turnings, borings or chips. -spritzmethode metal spray method (with Schoop gun). -tuch wire cloth. -versetzung alloying, alloy. -vorrat metal reservoir, pool of $\mathrm{Hg}$ (in a mercuryvapor arc rectifier, etc.)

Metastellung meta position.

Meter, Spektral photo- spectrophotometer. Vakuum- vacuometer, vacuum gage.

Meter-zahl, ausgefahrene reeled-out yardage, paid-out y. (airplane antenna). -zähler (Film) film footage counter.

Methodik methodics, methodology. Mess- measuring methodology.

Mikro-densogramm microdensitometer record. -filter filter for micrographic work.

Mikrometer, Funken- micrometric spark-discharge gap. Kugelspherical micrometer. Objektstage micrometer. Okularnetzmicrometer with reticle eyepiece. über- ultra-micrometer.

Mikrometerschraube micrometric screw, micrometer.

Mikro-nernstlampe Nernst lamp of a microscope. -nutsche microfunnel, m. suction filter.

Mikrophon, bewegl iches, entfesseltes following microphone. -, l ichtempfindliches light-sensitized cell, 1. microphone. -, membranloses diaphragmless microphone, cathodophone, glow-d ischarge m. -, nichtrichtungsemp findl I ches astatic microphone. Atmen des - s breathing (slow changes in resistance and response) of a 
carbon microphone. Band-, Bändchen- ribbon microphone, band m., veloity m. Besprechungssound pickup microphone.

Bewegungs- veloity microphone, pressure- gradient m. Brustbreast-plate microphone or transmitter. Doppelkohle- push-pull carbon microphone. Einblendungsfade-in and mixing microphone. $\mathrm{Fl}$ ammen- flame microphone, $f$. transmitter. Gegenkontaktpush-pull microphone. Gittergrille-type microphone. Kehlkopflaryngophone, throat microphone. Kohle-carbon microphone. Kondensator- condenser microphone, electrostatic m. Kristallpiezo-electric microphone, crystal m. Lausch- pickup microphone, p. transmitter, detectophone. Postsolid-back transmitter, carbonback microphane. Raum- non-directional (polydirectional) microphone. Richt- directional microphone (unidirectional, bidirectional, etc.), non-astatic $m$. Schallacoustic microphone. Schüttelgranule microphone. Stab- carbonstick microphone. Tauchspulenmoving-coil microphone.

Mikrophon-ansprechkonstante sensitivity constant of microphone. - antenne microphone boom, $\mathrm{m}$. outrigger. - atmen breathing of microphone (due to slow changes in resistance and response, in carbon m.) -einblendung fade-in and mixing of microphones. -einsprache acoustic inlet of microphone. -gal gen microphone boom. -geräusch microphonic noise (carbon noise), valve noise. -schmoren mike stew (noise). -verstärker microphone amplifier, m. pre-a.

mikrophotographisch photomicrographic.

Mikro-photozellen globules of mosaic. screen (in iconoscope). -raffer camera for stop-motion or time-lapse motion micrography.

Mikroskop, bildaufrichtendes imageerecting microscope. Durchstrahl ungsüber- transmission-type ultramicroscope. Einstell-focusing microscope. Elektronen- electron microscope. El ektronenrasterelectron scan microscope, raster m. Hornhaut- corneal microscope. Kugel - ball-stage microscope. Licht- optical microscope, light m., light-optical m. Rasterelectron-scan microscope, raster or screen-scan m. Schattenshadow microscope. über- electron ultra-microscope, e. supermicroscope.

Mikroskop-aufbau, -aufhängung microscope mounting. -bügel saddle of microscope, bracket of $\mathrm{m}$. Mikroskopie microscopic optics. Mikroskopiker microscopist.

Mikroskop-revolver revolving nosepiece. - schleuse air lock (chamber) of electron microscope. -spektralkamera microspectroscopic camera. -träger microscope stage. -verschiebung, Längs - und Queraxial and cross adjustment (in electron microscope).

Mikro-telephon microtelephone transmitter, transceiver, combination handset. -wage micro-balance. -wellen micro or hyperfrequency waves (I to $100 \mathrm{~cm}$ ). -zeitmessgerät microchronograph.

Milchgl as opal glass, frosted g., light-diff using $g$.

mild mild, soft, gentle.

Milderunsgründe extenuating circumstances, mitigating c.

Milieufilm"milieu" film.

Milopam German trade-name for polyvenyl chloride.

Minderer, Druck- pressure reducer, relief valve.

Mindest-vol tgeschwindigkeit appearance (or minimum) potential. -wertanzeiger minimum (voltage) indicator. 
Minimalrelais no-load relay.

Minimum-aufl 18 sungsvermögen

minimum separabile (of eye).

-bereinigung zero clearing (df).

- peilung minimum (or zero) sig-

nal direction-f inding method.

-schärfen zero cleaning, $z$.

sharpening (df). - verfahren

method based on minimum deviation (opt.) -wertzeiger minimum (voltage) indicator.

Minutenzeiger minutes hand (of timepiece).

mischbar miscible, mixable. -, gegenseitig consolute (miscible in all proportions).

Misch-barkeit, Wicht- non-miscibility, immiscibility. -brett mixing panel, monitoring $p$. , supervising $p$.

mischen mix, mingle, blend, adulterate, alloy, combine, unite.

Mischer mixer, fader, blender, (ac., etc.), mixing booth, "aquarium" (m.p. studio slang) -, fünfgliedriger five-channel mixer. Tontone fader, $t$. mixer.

Mischerschaltung keying or mixer tube scheme (for adding sync and video sionals).

Misch-gerät mixer bus. -hexode, Fading- a.v.c. mixer hexode. kristall crystalline solid solution, mixed crystal. -metall mixed metal, alloy, "Misch-metall." -molekiil mixed molecule (loosely associated molecular union). -peilung taking bearings on board, without directional receiver, from directive beacon. $\rightarrow$ pult mixing desk, monitoring d., supervising d. -pult, fahrbares dolly or cart-type mixer, teawagon console m. -regler mixing control. -röhre mixer tube. - röhre, selbstschwingende self-heterodyning mixer tube, autodyne. -signal signal spectrum (containing video and sync impulses, telev.) -stufe mixer stage, m.-first detector s. -tafel mixing panel, monitoring p., supervising $p$. -tisch mixing desk, monitoring $d$. Mischungs-lïcke miscibility gap. - rechnung alligation (math.)

Mischwalzwerk set of mixing rollers. miss-farbig discolored, inharmonious in colors. lungener Teil rejected také (m.p.)

Miss-ton distorted sound, of $f-p i t c h$ note. -verhältnis disproportion, asymmetry, disparity.

missweisend(er)Kurs uncorrected bearing, magnetic heading, $m$. course.

- Nord uncorrected or magnetic north.

Missweisung, Orts- local declination.

Misswe isungsglelchen isogonic lines, is ogons.

Miszelle micelle.

Mit-arbeiter fellow worker, collaborator, co-worker, contributor, assistant. -bewegung relative motion (of atomic nucleus), comovement, associate motion. - führungskoeffizient (Fresnel) coefficient of drag.

mitgenommene, selbständige Kippmethode self-running controlled time-base method (telev.)

Mithör-einrichtung side-tone circuit. -schalter, -schliussel listening key.

Mitkoppeln determining of course or fix, by dead reckoning (navigation).

Mitkopplung positive regeneration, p. feed-back; spurious or undesired $f$.

mitlaufende Spannung follower potential.

Mitlauter consonant.

Mitnahme synchronization, pull-instep, loking-in; locking of circuit. -berelch range of forced oscillations, "entrainment" range, coherence r., pull-instep $r$. 
Mitnehmen (bei Entladung) drift (in discharge).

Mitnehmer part carrying, dragging or driving along another part: driver, carrier, gripper, catch, dog, nose, clutch, engaging piece, etc., entrainment means.

mit-relssen entrain, carry, drag or pull along. -schwingen covibrate, oscillate in resonance, sympathy or unison, be in sympathetic vibration. - schwingend(er) Leiter equi-frequent conductor, resonant or co-vibrant c. -schwingend(e) Saite sympathetic string or chard.

Mitschwingung co-vibration, sympathetic $\mathrm{v}$.

Mittags-kreis,-linie meridian.

Mitte center (point), middle, midpoint (of tapping).

Mittel middle, medium, expedient, means, remedy, agency; mean (math), averago.

Mlttel, Fül-filler, filling, stuffing, loading material or compound. Schalt-' circuit element, c. means.

Mittel-anzapfung center tap, mid (point) t. -bildhelligkeit average illumination, background i., mean brightness value of picture. -blende middle diaphragm. -ecke lateral summit (cryst.) -feld central field (of vision). -frequenz(cf. Wellenabgrenzung) medium frequency $\mathrm{m}$. wave.

mittelgedämpfter Raum moderately live room.

Mittel-glied intermediate member (of a series). -lage tenor (of voice). -lichter half tones, average shading values. -linle der Funkpeilstation center line of approach sector. -linlenkondensator logarithmic capacitor (in which relation capacity: angle obeys 1. law). -lot median perpendicular.

mitteln average, ascertain mean value.
Mittelpunkt center, c. point, midpoint (of tapping). Krümmungscenter of curvature. -tastschaltung center-tap key modulator scheme.

Mittel-schenkel center leg, c. limb. - senkrechte median perpendicular. - wert, quadrati scher virtual or root-mean-square (rms) value.

Mitten-abstand distance between successive rulings (in diffraction grating, indicated by grating constant). -eindruck binaural balance (in sound locating).

mittenrichtig centered, co-centric. Mittenschärfe central definition, sharpness of center.

mitten symmetr i sch centro-symmetrical. mittig co-axial, concentric. -, ausseneccentric, off-center.

Mittigkeit centrality, centricity.

mittler mean, average, medium, central, mid...-(e) freie Weglänge mean free length of path. - (e) Helligkeit average (background) illumination (telev.)

Mittungsstift centering pin.

mitziehen (s.a.ziehvorgang und ziehen) draw, drag or pull along, pull in step, tug; warp.

Mn (Motorgeräusch im Norden) noise of motor north signal.

Modell, Atom- (R-B) atom model or conception. Einteilchen- single particle model. Mutter- master pattern.

Modellierton modeling clay.

Modell schreiner wood pattern maker. modeln modulate by pulse-time modulation ( $t-m)$, amplitude $m$. $(a-m)$, frequency $m .(f-m)$ or phase m. $(p-m)$; model, mold. Modul, piezo-elektrisches piezo-electric modulus.

Modulation, additive upward modulation. -, subktraktive downward modulation.

Modulation auf Dunkel modulation to dark condition, negative $m$. - auf Hell modulation to light condition, positive m. -der stimme 
inflection of voice.

Modulation, Absorptlons- absorp-

tion modulation, Heising $m$.

Dichte- (charge) density modula-

tion. Elektronenstrom- electron

quantity modulation, $m$. of

bunched $\theta$. stream. Frequenz-

frequency modulation Gegen-

opposite modulation. Ge-

schwindigkeits- velocity modula-

tion. Gitter- grid modulation, g.

control (Brit.). Intensitäts-

intensity, brightness or bril-

liance modulation. Kreuz-

cross modulation. Leitungsstrom-

conduction current modulation.

Nullphasen-null phase modulation.

Parallelröhren- Heising modula-

tion, choke m., plate m., con-

stant-current m. Phasen-phase

modulation. Puls- pulse modula-

tion (used in radar). Quer-

cross modulation. Relhenröhren-

constant-potential modulation.

Spannungs- velocity modulation.

Zelt- velocity (variable-speed)

modulation; variable-speed scan.

Modul ationsfrequenz modulation

frequency, signal $f$.

modul at lonsgesteuerter Sender

transmitter in which amplitude

varies with $A F$ amplitude, $A F$

modulated transmitter.

Modulations-grad percentage modu-

lation, $m$. percentage, degree of

m., depth of $m_{\text {. }}$-gradkontrolle

modulation meter. - röhre mixer

tube, modulator t. -tiefe of.

H. - Brad.

Modulator, besprochener voice-im-

pressed or voice-actuated modulator.

Modul ator, Llcht, - light relay, any device adapted to reconvert current into light or brightness variations, e.g., television receiver lamps, Kerr cells, modulated neon lamps, c. $-r$. tubes etc. Llcht-, selbstleuchtender light relay of the glcw-tube, neon arc lamp, sodium lamp, etc. type. Licht-, (Fremdl lcht)

light relay of the Kerr cell, Karolus c., etc., types adapted to reconvert currents into brightness variations by the use of a constant independent ray source.

Modul ierbarkelt modulability, modulation capability.

Modulus, Drlllings-, Schlebungstorsion modulus, shear $\mathbf{m}$., rigidity $m$.

modulieren $s$. modeln.

Mohr, Platinum- platinum black. Quecksilber-black mercuric sulfide.

moiréartlges Geräusch moire pattern of noise.

Moiréeffekt moiré or watered-silk pattern (in photography).

moirieren water; cloud (fabrics). Molekiil, unstarres non-rigid moleoule. Ketten- chain molecule. Kreisel- top molecule, spinning $\mathrm{m}$. Misch-mixed molecule (in a loosely associated or 1. bound molecular union).

Molekiil aggregate molecular clusters. Molekul ar-gewicht (Mol.-Gew.) molar weight, gram-molecular w. -strahlen molecular rays, m. beam.

Molekuil-haufen molecular clusters. - sieb micro-filter. -spektrum molecular spectrum.

Molenverhältnis molar ratio, mole $r$. Molisierung re-combination (of ions in gas).

molletieren mill, knurl (coins, etc.)

Mol-norm molal. -verhältnis molar ratio, mole $r$. -volumen molar volume. -wärme molar heat.

Moment, Brems- brake torque, retarding $t$. (in a meter). Drehtorque, moment of rotation. Kernnuclear (magnetic) moment.

Massen- moment of inertia, rotational i. Relbungs- friction torque.

Moment-bild instantaneous photograph, snapshot. -prozess 
m-process, evanescence p. (in decay of phosphorescence).

-transport momentum transfer. monaurales Hören monaural recep-

tion, $m$. listening.

Möndchen lune, meniscus.

mondförmig crescent-shaped, lunate, méniscal.

monoatomar monatomic.

Mono-chord monochord, sonometer

(with one string) -chromasie

monochromatism, monochromasia,

(condition of electrons being

of like wave-length and like

speed).

monochromati sierend, selbst-

auto-monochromatic (e.g., when

an instrument becomes its own

monochromator).

monomolekul arer FIIm monolayer, monomolecular layer (adsorbed film).

Monopackverfahren mono-pack method. Montage setting-up, mounting, erection, installation or assembly (work), fitting.

Montagekople first answer print. Morsepunkte, brelte lengthened dots. -spitze clipped dots.

Mosaik-block mosaic block (cr.). - platte target, mosaic plate (telev.). -röhre, doppel seitlge image multiplier iconoscope. -schirm mosaic (of iconoscope). -schirm, Uberfahren durch Strahl sweep out mosaic with beam.

Motor-geräusch im Norden(Mn) noise of motor North signal. -unterbrecher motor-drive make-and-break. $\mu \mathrm{F}$ microfarad (mfd, mf).

$\mu \mu \mathrm{F}$ picofarad, micromicrof.

Muffe muff, sleeve, bush, socket joint, clamp, coupling box.

Röhren- pipe socket.

Muffenverbindung socket joint.

Mühle, Licht- radiometer (of Crookes).

Mulde, Potentlal- potential well, p. hole.

Multipletten multiplets.

Mund mouth, opening, muzzle, vent, or if ice.
Mundharmonika mouth harmonica, m. organ.

mündliche Verhandlung oral hearing. Mund-öffnung, Trichter- mouth opening of horn, largest flare. -stück, Trichter-throat of horn. -stuicköffnung chink between mouthpiece and reed (of clarinet).

Muindung mouth, aperture, orifice. Mündungs-geschwindigkelt muzzle veloity. -wucht kinetic energy at the muzzle.

mürbe mellow, tender, soft, brittle, friable, short (of metal).

Musa-Empfang multiple-unit steerable antenna reception, musa $r$. Muschel, Augen- eye cup. Hör-, Ohren- ear cap, earpiece. Okul areyepiece cup.

Muschelgl as globular lens. muschelig shelly, conchoidal. Musikwerk musical automaton, mechanical music instrument, "jute box" Muster, Geräusch- noise pattern. Stör- spurious or false pattern (in iconoscope picture).

muster-gültig serving as a model, standard or ideal, classic. -haft standard, typical, $\theta x-$ emplary,

Musterkopie master copy, m. print. Mutter mother disk, master matrix. (in phonograph record making).

Mutter, gesicherte lock nut, jam $n$. Daumen- thumb nut. Flügelwinged nut, butterfly $n$. Gegenlok, clamp, check, jam or binding nut. Kordel- milled nut, knurled n. Schrift- type mold, matrix. Überwurf-castle nut.

Mutter-form master mold (cer.); parent (al) form. - frequenz master frequency. -gewinde female screw thread. -model I master pattern. -schlïssel (nut) wrench, spanner. -schraube bolt and nut. -sender key station, master s. (in chain broadcasting). -substanz parent substance. -uhr master clock. -verschluss nut lock.

Mutung, Funk- radio prospecting, $r$. metal locating. 
Nabe nave, hub, boss.

Nabelpunkt umbilic.

nachahmen imitate, copy, mimic; counterfeit, adulterate.

Nach-anmel dung supplementary application.

nacharbeiten finish, dress, remill, re-trim, re-true (up or off).

Nach-aufnahme re-take. -baratome, nächstnähere next nearest neighbor atoms. -belichtung post-exposure. -beschleunigung after-acceleration, post-a., acceleration by second gun. anode. -beschleunigungselektrode second or additional sun anode or accelerator electrode, after-accelerator. -bild after-image; copy, imitation, replica.

nachbilden copy, reproduce, imitate; balance, simulate. -, neu rebalance.

Hachbildung, Grund- basic network. Leitungs- bal ancing network, artificial b. line Neu- rebalancing. Nachbildwirkung persistence of vision (in form of after-image). nacheilen lag, trail (behind). Nacheilwinkel angle of lag. nacheinander one after another, successively, sequentially, consecutively, seriatim. -färben dye again, counterstain.

Nach-fliessen after-flow (of metals), persistence of plastic f., elastic after-effect. -fol gekern product nucleus. -forschung research, search, investigation. -frist time extension, respite. - fuhrung des Rahmens re-setting or re-adjustment of frame or coil (d.f.)

nach-geben give way, yield, concede. - geschal tete stufe higher stage, end s., power s. -giebig yield- ing, flexible, pliable.

Nach-giebigkeit compliance, yieldingness. -gluhen after-glow, phosphorescence. -gluhchar akter ist ik persistence characteristic. -hall echo, reverberation; resonance. -halldaver, ubertriebene excessive reverberation period.

nachhallen dying down of sound (in an acoustically live room).

Nachhall-kammer, -raum reverberation room, enclosure or space. -zeit reverberation period.

nachhaltig lasting, enduring, persevering.

Nach-konzentration re-focusing, action of second lens. - ladeerscheinungen phenomena associated with absorption current and residual charge (of condenser).

nachlassen temper, anneal (glass, metals, etc.); slacken, relax, abate.

$\mathrm{Nachl}$ auf-einstellung automatic resetting of direction-finder to zero. -peiler direction-finder in wnich readjustment to zero is effected automatically. -profil wake profile. -stufe (cathade) follower stage.

Nachleuchten after-slow, phosphorescence, persistence of light or of vision (on retina). Verminderer des Nachleuchtens killer, poison.

nachmessen check dimensions, check-up of measurements, test figures, etc. Nach-prufung re-examination, recheck, verification, review. -reifung maturing (of film).

Nachricht communication, intelligence, information, signals; news. -Ubermittel ung signalling, intelligence transmission.

nachrichten re-align, re-straighten, 
readjust, re-set.

Nachrichten-system, Mehrfachmultiplex signal system. -technik communication or signal art or technics, signal work.

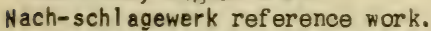
-schlUssel master key, pass key. -schrift picture re-creation; postscript. -schub, Kohlencarbon feed (arc lamp).

nachstellbar regulable, re-adjustable, resettable.

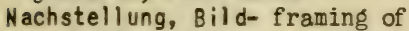
picture, racking of $p_{\text {. }}\left(\mathbb{m} . p_{.}\right)$, centering control (telev.)

Bild-,falsche misframing, outof -frame condition. Blendenphasing of shutter (film work). Nachstimmung; Rahmen- re-setting or re-check of frame or coil tuning (in automatic radio corpass).

Nachst immungskondensator alioning condenser, trimming capacitor; vernier $c$.

nachstnähere Nachbaratome next nearest neighbor atoms. nachsuchen apply (for a patent), file (a petition).

Nachsynchronisieren post-scoring (a silent film), dubbing, scoring (first picture, then sound), re-recording.

Nachsynchronisierung (von Trickfilm mit synchronisch springendem Ball) bouncing ball synchronization.

nachtblindes Auge night-blind eye, nyctalopic $\theta$.

$\mathrm{Nacht-bl}$ indhe it hemeralopia, nyctalopia. -effekt night of fect, polarization error, directional or quadrantal errors (in general).

nachteffektfrei night-effect-free, free from $n . \theta$. or polarization errors.

Mach-teil disadvantage, injury, prejudice, loss, detriment, shortcoming, demerit, inconvenience, difficulty. -trans- port take-up sproket action. $\mathrm{Nachtstreckenbefeverung} \mathrm{route} \mathrm{beacon}$ for night flying service.

Nach-verstärker amplifier in stage above input stage, (mostly) power amplifier. -vertonung dubbing, re-recarding of sound track.

nachwe isen detect, discover, prove, demonstrate, identify; point out, refer to

Nachwickel-rolle take-up reel or spindle, hold-back sprocket. -trommel take-up sprocket.

Nachwickler (lower) take-up sprocket. Nachwirkung after-offect, secondary

e., accompanying $\theta_{\text {. }}$; hysteresis, persistence, decay (period).

-, elektrische electric aftereffect (persistence of strain). -, magnetische magnetic fatigue, m. after-effect, residual loss (of matals). -,plastische plastic flow persistence, afterflow, elastic after-effect. Nachwirkungs-strom absorption current, c. of residual charge (of a condenser). -verlust residual loss.

Nachwirkzeit decay period.

$\mathrm{Nadel}$, Abflihl- selecting needle or pin, pecker. Abspiel- needle, stylus (riding in groove).

nadelformig needle-shaped, acicular. $\mathrm{Nadel-funkenstrecke} \mathrm{needle-point}$ spark-gap, needle g. -geräuschfilter needle scratch filter (phon.) -reibung needle friction (causing stylus drag). -schalter vibrating reed rectifier.

$N$ adelton stylus or needle-recorded and reproduced sound. -ansatz sound on disk attachment or head. -verfahren sound on disk method. -verstärker phonograph amplifier. -zusatz sound on disk attachment.

Wähe nearness, vicinity, neighborhood, proximity, propinquity, closeness, adjacency, contiguity. sonnen- perihelion.

Naheinstellung short-range focus. 
nabellegen be or lie near, border (on), be adjacent or contiguous; be patent, obvious, evident or self-suggesting.

Wäherung apprcach, approximation, proximation.

Waherungsformel approximate formula, a, equation.

nahestehend intimately related or connected, affiliated.

Nah-feld vicinity or shart-range field (of antenna). -pellung close-range direction-finding. -punkt near point.

$N$ ährboden nutritive substratum, nutrient medium.

Nahschwund radio fadeout or fading 60-100 miles around transmitter site. -antenne low-angle or short-range fading antenna.

Nah-signalmittel short-distance or s. range signal means. -sţrungen nearby interference (from sources close to receiver). nahtlos seamless, jointless. Naht schwelssung seam welding. Nahverkehrsbezlrk local control zone, approach c.z.

namhaft famous, well known, renowned, reputable, considerable, appreciable.

Napf bowl, basin, pan, cup. Narbe scar, seam, grain (of leather and paper), pit, defect, flaw, pock. Rost- rust pit. Schneld- cutting scar.

Narbung pocking.

Hase catch, lug, nose, beak, cam, tappet; projection, spout, nozzle. Klink-detent, latch.

Nasenkell jib, key.

Nassverfahren met process.

Natr l uml euchte sodium lamp.

naturähn Ilch realistic, true or similar to nature.

Natur-erscheinung natural phenomenon, $n$. process, $n$. action. - farbflim natural color film. -forscher investigator of nature or in field of natural sciences, n. philosopher, naturalist. naturharter stahl self-hardening steel.

Naturlehre natural science, $n$. philosophy.

naturliche Zahl natural number. NatUrllchkelt der Wiedergabe faithfullness, fidelity or realism of reproduction.

Naturtreue fidelity, faithfulness, realistic properties.

Navigation, Flugzeug- avigation, aircraft navigation, aircraft piloting. Koppel- navigation by dead rockoning. Richtungs-, Seltendirectional navigation. Streckenlong-distance (air) navigation or avigation. Vertlkal- vertical guidance, v. avigation.

Navigationsfeuer navigational or maritime radio beacon.

Nawimembrane cone diaphragm of curved profile, curvilinear cone, para-curve.

Nebel nebula (spectroscopy), mist, fog, veil (phot.). stor- chief interference zone, maximum noise z. -apparat atomizer. -kamera cloud chamber. - 1 andung blind landing, instrument 1 . -spurmethode Kilson cloud track or chamber method (streak of droplets produced by a particle).

Neben-achse lateral axis (of rhombic crystal); secondary a. -apparat accessory equipment, auxiliary apparatus. -bestandtell secondary ingredient. -betrieb by-operation, secondary process, side-line. -bewels secondary proof, additional evidence. -bild ghost image. -bindung secondary union, s. bond, linkage of the second order.

nebeneinander in parallel (electrical connection). -stellen juxtapose, place side by side or alongside.

Heben-entladung lateral discharge stray d., secondary d. -erzeugnis by-product. -fläche secondary face. -geräusch extraneous noise (m. p.); ambient $n$. (in sound locating). - Intervenient co-intervener. 
-kette side chain, subordinate c. $-k l$ age incidental action. -kläger co-plaintiff. -kopplungsschwingung spurious oscillation. - licht (auf Platte)light-shot (on film). -linie secondary line, branch line, satellite (spectr.) -maximum secondary radiation, lateral $r$., s. lobe or ear (in directional characteristic or space pattern). -patent sub-patent, collateral or subordinate patent. -peilstelle associate direction-finder ground station. -reaktion side reaction, secondary $r$. -rohr side or branch tube or pipe. Nebenschluss, Ayrton'scher universal shunt, Ayrton s: Gittergrid leak. -feder shunting spring, off-normal s. -kondensator by-pass condenser. - resonanz parallel resonance.

Neben-setzung juxtaposition. - sprechdämp fung cross-talk transmission equivalent, cross-talk attenuation. -stelle, Peilassociated direction-finder. - uhr secondary clock. -umstände collateral circumstances, c. factors. -weg by-path. -winkel adjacent angle, adjoining a.

-zipfel minor lobe, m. ear (of space pattern).

Negativ, Meister-,Originalmaster negative, metal master (of phonograph record). Rahmenrack negative.

Negativ-ebene negative plane, $n$. aperture (of film printer). - farbauszuge separation negatives. -gamma negative gamma $(\gamma)$. - lack transparent negative varnish. - schwärzung negative density. - umfang intensity range or latitude of a negative. -widerstandsröhre dynatron type of tube with negative resistance (e. g., kallirotron).

Hehmer, Lizenz- licensee, grantee. Neigekopf tilting head (tripod). Neigung inclination, tendency, slope, pitch, dip, trend. Schwingungs" tendency to oscillate or "spill-over"
Strahl- inclination of ray.

Neigungs-differenz difference of vergence. -ebene inclined plane. - messer clinometer (to measure angles of slope), Abney level (to measure vertical angles). -messer (zum Messen von Neigungen im Meeresboden) clinometer for measuring sea bottom slopes. -messer, Kreisel- bank-and-turn indicator. -winkel inclination (angle), a. of slope, a.of dip. -winkelmesser clinometer.

Nenner, General-, Haupt- common denominator.

Nenn-reichweite rated range, nominal r. - wert nominal, rated, assessed, normal, expected or face value. Neonflächengl imml ampe plate neon lamp (telev.)

Neonrőhren-aussteuerungskontrolle, - lautstärkemesser neon tube volume indicator.

Nernstl ampe, Mikro- Nernst microscope lamp.

Nerven-reiz nervous stimulus. - taubheit perception or nerve impairment of hearing, $n$. of perceptiun deafness.

Netz (of Gitter) net, netting, gauze, network, reticle, graticule, reticulation, reticulum, grate, lattice, grid.

Hetz, Anodenschutz- screen grid, plate shield. Anpassungs- matching network. Erddraht- ground mat. Grad-graticle, reticle, map grid. Hilfs- auxiliary (control) grid. Karten- graticle, map grid. Raumlade- spaco-charge grid. Schutz- shield or screen grid; protective network. Spannungsspace-charge grid. Streckenair routes. Verstärkeramplifier channel.

Hetz-anode plate fed from power unit or light socket. - anschlussgerät (strictly:) power pack, power unit; power converter set, electric s., mains-operated radio s. - antenne, Licht- light socket or lighting circuit antenna, lampsocket $a$. 
netzartig (s.a. Netz) netlike, reticular, reticulate, grate-, grid- or grating-like,

Netz-bedeckung coverage factor. -bildung reticulation, netting. -brummen mains hum, motor boating (of $a-c$ ), a-c pickup. -drossel hum eliminator coil, hum-bucking c. -ebene lattice plane, plane family of a crystal. -ebenenabstand grating constant (spectr.); inter-lattice plane distance, distance between successive planes indicative of lattice constant (cr.)

netzen wet, moisten, steep, soak. netzförmig net-shaped, reticular, reticulate.

Netzgerăt electric set, mainsoperated set, power-pack or powerunit s., power-unit electric radio $s$.

Netzhaut retina. -bild retinal image. -trägheit retinal persistence. -zentersehen foveal vision.

Netz-mikrometer, Okul ar-micrometer with reticle eyepiece. -stecker power supply plug, light-socket p. -struktur cellular network, lattice, reticular structure, reticulation. - ton mains hum, line h. -transformator power transformer, mains t. - unruhe mains or supply-line ripple or fluctuation. -verstärker, -vervielfacher mesh multiplier, m. amplifier (with secondary emission, seriation or cascading of electronpermeable fine-mesh targets). -werk network, reticulum. -werk, Trenn- dividing network.

Neu-aktivierung der Kathode reactivation or rejuvenation of a cathode. -gestaltung re-organization, re-arrangement, modification. -heit novelty, originality.

neu-he itsschädl ich anticipatory, rendering novel ty negative.
- nachbilden re-bal ance. neunwertig nonavalent. Neusilber argentan, German silver

neutral einstellen adjust to neutral, set to $n$.

neutraler Schirm neutral screen. Neutral isations-kondensator balancing or neutralizing condenser. - schal tung neutrodyne circuit organization.

Neutroneneinfang neutron capture. nichtabgestimmt untuned, aperiodic. $\mathrm{Nicht-anerkennung} \mathrm{non-acknowledg-}$ ment. - beobachtung non-observance. nicht-berechtigt disqualified, not entitled. - bevorzugte orientierung random orientation, nonpreferential o., non-privileged o. or direction. -dissoziiert undissociated, non-d.

Nicht-eisenmetalle non-ferrous metals, non-iron m. -erfullung non-performance, non-fulfillment, default. -erscheinen non-appearance, non-attendance, absence, default, contempt.

nichtgerichtetes Mikrophon astatic microphone, non-directional m. nichtig null, void, invalid, annuled.

Nichtigkeits-abteilung annulment department. - beschwerde plea of nullity. - erklärung declaration of nullity, annulment or cancellation. -klage actiun or suit for cancellation, annulment or invalidation.

nicht-ionisiert unionized, nonionized. -kommutative Algebra non-commutative (vector)algebra. -kondensierte Hochstromentl adung uncondensed heavy-curreńt discharge. -kristallisch amorphous, non-crystalline.

Nichteiter non-conductor.

$\mathrm{nicht-leuchtende} \mathrm{Flamme}$ roaring flame. -l ineare Verzerrung non-linear distortion. - 
- linearität lack of linearity, non-1. -metall nonmetal.

nichtmischbar immiscible, nonmiscible; unsoluble, insoluble, insolvable.

Nichtmi schbarkeit non-miscibility, immiscibility.

nicht-reproduzierbar non-reproducible, non-controllable. -resonierend aperiodic, non-resonant. -rostend rust-proof, stainless, rust-free. - sphärisch non-spherical, aspherical. -ständig nonpermanent, temporary, transient, transitory. -symmetrisch asymmetric, unilateral. -umwandelbar inconvertible. -vergasbar nongasifiable, non-volatile. -wässerig non-aqueous, anhydrous.

Nicht-zahlung default of payment, non-payment. -zerfallbarkeit non-disintegrability, non-fissionability.

nichtzerstörende Prüfung non-destructive test.

nieder-drücken press down, force d.; depress or touch (a key or button). -fallen fall down, drop, precipitate, settle.

Niederfrequenz-drossel satz low-pass filter. -röhre AF tube, low frequency t., "apple" (m.p. studio slang).

Nieder-führung downlead. -gang descent, decline, downward stroke or travel, lowering movement or stroke.

niederlegen lay down, put d., deposit.

Niederschlag precipitate, precipitation, (radio-active) deposit, sediment, settlement. -arbeit precipitation process, iron-reduction work. -gefäss precipitating vessel. -mittel precipitant.

Niederschlags-apparat precipitator (cottrell). -potential deposition potential.

nier enförmig reniform, kidney-shaped (for instance, condenser plate).
Niete, Loch- hollow rivet, tubular $r$. Niet-schaft rivet shank, r. stem. -teilung rivet spacing, $r$. pitch. -verbindung riveted joint.

Nipkowscheibe Nipkow scanning disk. Nitrierstahl nitriding steel, nitralloy $\mathrm{s}$.

Nitrierung nitrification, nitrogen, nitridation (steөl).

Nitrobenzol nitrobenzen $\theta$.

Niveau level.

Niveau, Energie- energy level, quantum state, term. Grund-background level (or average) illumination (telev.). stör- noise level.

Niveau-fläche level surface; equipotential or isop. surface. -röhre level tube, leveling t. -stufe term, energy level (of electrons).

nivellieren equalize, smooth, bring down to an average level, grade, even.

Nivellierer, Spannungsspitzen- voltage peak limiter.

Nocken cam, lifter, tappet, disk or wheel wi th lobes. -, zweistufiger double-lift cam. Leerlauf-delayed pulse trip cam. Schalt- trip cam, trigger c. Steuer- sequence switch cam.

Noiseless, Gegentakt- split-wave noiseless film recording. -blende biasing or noise-reducing stop or shutter vane or gate. -stromkreis back-ground noise reducing circuit, noise gating $c$.

Nomogramm straight-line or alignment chart, self-computing c.; monogram.

Nonius-einteilung, -skala vernier scale.

Nonne mold matrix.

Nord, misswei send uncorrected north, magnetic n. -, rechtwe i send corrected north.

Norddrehfehler northerly turning error.

nördliche, Breite northern latitude. Nordlicht aurora borealis. -linie green auroral line. 
Horm standard, norm, model.

Norm, Vor-preliminary standard, tentative s.

Hormal-bildkadre standard picture frame. -essig standard vinegar, proof v. -flüssigkeit normal or standard solution or lqquid.

normal gluhen normalize.

normal isieren standardize, normalize (brief heating slightly above $A_{3}$ point).

Normal-lehre normal gage, master g. - potential normal potential. -schliff standard (interchangeable) ground joint. -schrift standard track (sound recording). normalsichtig normal sighted, emmetropic.

Hormalweingeist proof spirit.

Mormenaufstellung formulation or laying down of standard rules or norms, standardization or normalization.

Normierung standardization, normalization, gaging.

Normung des Frequenzganges standardization of frequency response or characteristic.

Not-amt temporary exchange, emergency $e$. -anlage provisional or emergency plant or equipment. - behelf makeshift, expedient, last resort, temporary or emergency means, improvisation.

Note, Triller-trill note, warble n. Not-fall case of emergency, case of need. -mittel expedient, makeshift or emergency means. -ruf,signal, -zeichen S O S call, (radio-telegraphic) distress call or signal, mayday (radio telephone distress call).

Novokonstant $\mathrm{Cu}-\mathrm{Mn}$ alloy with $\mathrm{Al} \mathrm{Fe}$. Nuance shade, tint.

Null zero, nought, cipher, null. - frequenz frequency at which phase shift is zero. -leiter neutral wire. -linie zero line, missing 1., band center (spectr.) -l inienverlagerung zero setting, electrical biasing (in film recording). -methode zero method, null m., balance $\mathrm{m}$. -phasenmodulation zero phase or null phase modulation.

Nullpunkt zero point, null point, neutral p., origin (of a diagram). -abweichung zero error. -anomalie origin distortion. -einstellung zero adjustment. -empfindlichkeit zero-level sensitivity. -fehler origin distortion, ion cross effect. -schärfung zero point clearing, cleaning or sharpening (in df).

Nullstelle zero place; abscissa which gives zero value to Bessel function.

Nullung eines störers connecting casing of interfering device with neutral (in d.c. mains).

nullwertig avalent, non-valent.

Nullzweiglinien zero branch lines.

numerische objektivöffnung numerical aperture of objective.

Nummer; laufende serial number, consecutive $n$.

Nummernscheibe dial (switch), number plate, d. plate. Ablaufen der return of the dial. Aufziehen der - winding up the dial.

Nummernwahl impulse action or stepping, selection.

Nurflügelflugzeug tailless airplane. Nussbaum walnut.

Nute slot, notch, groove, recess, key-way, oilway. -und Feder slot and feather (union), slot and key, groove and tongue joint.

Nute, Schmier- oil groove or slot, worm.

nuten slot, groove, notch, flute, keyseat.

Nutenwellen slot ripples, tooth $r$.

Nutsche, Mikro- micro suction filter, m. funnel.

nutzbarer Linsendurchmesser useful or effective (clear) aperture of a lens.

Nutz-dämpfung damping resulting in useful radiation or signal power, effective transmission equivalent. -feldstärke signal strength or 
field intensity, "useful" field intensity. -kreis useful circuit, active c. -lautstärke signal level, sound s. volume. -lelstung useful power, signal p., useful work or effect; efficiency. $-n$ lesser usuffuctuary, beneficiar $y_{0}-$-signal intelligence signal, any s. with inf ormation.
- spannung useful potential, signal p. - strom useful current, signal c. -stromkrels signal circuit, utilization aircuit. -widerstand (cf WIrkwiderstand) useful or signal resistance. -wirkung of $\mathrm{f}$ ciency.

N.Z. (Neutral. Zahl) noutralizing number. 
Ober-banden overtone absorption bands. -begriff introductory part of German patent claims setting forth prior art, broad class and nature of invention and disclosure. - beleuchter chief light electrician; scaffold or top light controller (studio).

oberfläche, gasbehaftete gas-contaminated surface, s. with adsorbed gas. -, spiegelnde specular surface. -, vertonte sound-impressed surface, surface or area bearing sound track. oberflächen-ableitung surface leakage. - abtaster surface analyzer. -beladung (mit $\mathrm{Hg}$ ) activation, sensitization or charging of a surface (with Hg). -effekt surface effect (of photo-cathode). -einhelt unit of area.

oberflächengerichtet surface normal. oberflächen-härtung case-hardening

(metals), surface cerrenting.

-integral integral taken over a

surface. -normale surface normal. -phase surface phase. -schicht surface layer; s. barrier. -spannung surface tension.

-spannungsinesser od.

- spannungsprüfer, nach

Abreissmethode surface tensiometer of adhesion-balance type.

-taster surface analyzer (to measure irregularities). -widerstand surface resistance (of insulation); skin-effect resistance (in $a-c$ flow). -wirkung surface action; skin effect; surface work (cryst.)

oberflächlich superficial.

oberharmonische harmonic, higher $h$. -dreifache third harmohic, triple h. -, gerade even harmonic. -, ungerade odd harmonic. ober-leiter, künstlerischer art su- pervisor. -licht overhead scoop, scaffold light, skylight. -strich permanent maximum current or carrier, peak carrier amplitude, maximum or black-level picture signal (telev.) -taste sharp (mus.). -ton overtone (of ten a harmonic), upper fartial (NOTE: harmonics and overtones are partials of a frequency above fundamental, a partial being a pure-note component of a sound or complex note).

objekt, übertragungs- object to be televised or scanned. IIntersuchungstest specimen, $t$. sample, $t$. piece. -glas slide, mount (micr.)

objektiv (cf Linse, Lupe, Glas) objective, object glass, lens (receiving first light from object). -, höchstl ichtstarkes ultra-rapid or ultra-high-speed objective or lens. -, kurzbrennweitiges shortfocus lens. -, lichtstarkes highspeed objective. -, zusammengesprengtes broken-contact (unoemented) lens or objective. Aufzeichnungs- recording objective. Fern-telephoto lens. Immersionsimmersion objective (electron microscope).

Objektiv-brett lens or objective panel or board. -deckel lens cap. - fassung objective or lens mount or barrel. -gewindes, Ansatzfläche des screw shoulder or s. collar of objective mount. -halter lens holder. -høhe height of lens or objective. -öffnung, numerische numerical aperture of objective. -öffnungswinkel angular aperture of objective. -ring lens adapter. -satz set of lenses, lens combination. -spule objective coil. -wechsler revolving nosepiece (micr.) 
Objekt-kammer object chamber. -mlkrometer stage micrometer. -schle::se object airlock-sucher object finder (opt.) -tisch micrometer stage, object stage or stand, manipulator. -träger object slide, mount, stage or stand, o. support, o. carrier (plate), specimen holder (e.g., a celluloid film or skin). -trägerplättchen object support lamina or slide (film or skin). -umfang range of brightness (of subject).

ofen, Etagen- storey furnace. -sohle furnace bottom, bed, sole or floor.

ofentrocken kiln-or oven-dried, $d$. in kiln or oven.

offen hollow (of sound). -(er) Schwinger open oscillator. - (e) Sprache open language, uncoded 1. einseitig- unilaterally open (-ended).

offenbarer Unsinn manifest absurdity. offenbarung disclosure.

offenkundige oder offentliche Vorbenutzung public use, prior use. offentlichen Hand, Betrieb der public utility.

offnen, eine Röhre open, unlock, render conductive, allow discharge, cause breakdown of a tube, trigger a t. (say, a blocking oscillator). -, einen stromkreis break or open a circuit or a line.

öffnung aperture, orifice, opening, mouth, vent, leak, crack, slit, port; $F$ number (opt.)

-, numerische, des objektivs numerical aperture of objective. Blenden- aperture of diaphragm or stop. Mundstick-chink between mouthpiece and reed (of clarinet). Schall- louvre (of bell tower). Spalt-chink (of clarinet). Verschluss- shutter aperture, s. opening.

jffnungs-bild aperture image. -fehler apertural defect, error or effect. -verhältnis aperture ratio, relative a.; field of view (picture projection). weite flare, width of opening, (of horn, trumpet, etc.) -winkel, Halbsemi-apertural angle. -winkelmesser apertometer.

ohm'scher Widerstand ohm resistance, steady-current $r$.

Ohr ear, lug, eye, catch, loop. -, vertäubtes deafened ear.

öhr ear, handle, eye, catch, eyelet. ohrempfindungsgrenze loudness contour of ear, auditory sensation area.

ohren-kappe ear muff. -kurve auditory sensation curve. -muschel ear, cap, e. piece.

Ohrhöhle ear cavity.

okonomiekoeffizient efficiency of economy.

ökonomisch economic(al).

oktaeder œtahedron.

oktaedrisch octahedral.

Oktave, eingestrichene one-stroked octave.

oktavkoppel octave coupler (organ). okular, verzerrungsfreies or thoscopic eyepiece, distortion-free e. Augenlinsen- eyelens of eyepiece. Mess- micrometric eyepiece. sucher-fousing lens.

Okul ar-aufsteckglas eyepiece correcting lens. -blende eyepiece diameter, e. stop. -kollektiv field lens of eyepiece. -muschel өуepiece cup. -netzmikrometer micrometer with reticle eyepiece.

ölbildend oil forming, olefiant. -(es) Gas olef iant gas (ethylene).

ol dämp fung oil damping (in vibrators); o. dashpot. fänger oil catch, 0 . collector.

ölhaltig containing oil, oleiferous. ölharz oleoresin.

olig oily, oleaginous.

ölkarburiert oil-carburetted.

öl-nute oil groove, oil run, oilway. - pumpe oil pump; type of highvacuum pump, e.g., "Hyvac". - schalter, Impuls- oil-blast circuit-breaker. - schiefer oil shale. 
-schwungmasse rotary stabilizer

(in film feed). -tropfchenmethode oil drop method.

Opalglas opal glass, frosted g., light-diff using $g$. - schelbe, Vollpot opal diffuser disk (phot.)

operatorenrechnung operational calculus.

Optik optios (science), optic (optical means such as lenses, etc., placed in path of rays in physical apparatus, etc.)

optik, Elektronen- electron optics, $\theta$. lenses (and their control of beams). Licht- light optics, physical o., geometric o. of light rays. Spalt-microscopic aperture, imaging optic means, slit "optic". Ton (film)- soundhead lens, s. optic (m.p.)

Optik-auswechslung optical system or assembly comprising interchangeable intermediate lens barrels. -tubus optical barrel.

optisch, spannungs- photo-elastic. -(e) Bank optical bench. -(e) Drehung optical rotatory power, 0. rotation, rotatory polarization.(e) Inaktivierung elnes ouarzkristalles racemization.

ordentliche Komponente ardinary component (of rays, etc.)

ordnen order, arrange (methodically or systematically), regulate, settle, collect terms (math.)

ordnung arder, arrangement, arganized disposition, class; power (math.)

-, Kurve dritter cubic curve. -, nter of $n^{\text {th }}$ order.

ordnungsgemiss orderly, methodical. ordnungs-grad degree of arder. -stellung, Ein, -stellung, Zweiposition of grating resulting in 8 one-arder or a two-arder spectrum. ordnungswidrig irregular, disorderly, contrary to order, discipline or rule.

Ordnungszahl ardinal number; $n$. of a series, atomic n.

Orgel, Domp faffen-, Yogel- bird organ. -zungenpfeife reed organ pipe. orientiert, nicht bevorzugt

randomly oriented, non-preferentially 0 .

Orientierung orientation, direction; trend, inclination. -, bevorzugte non-random orientation, privileged orientation.

orientierungsabhängig orientationsensitive, o. - responsive, be a function of, or dependent upon, orientation, direction or angle.

ort place, locus, region, locality, site, position.

Original-negativ master negative. - positiv master positive.

Ort, geometrischer vector point, geometrical locus.

ort und stelle, an in situ, on the site, on the spot.

Ort, Elektronen- position or lous of an electron. Flug- position of flying craft, fix. Koppel-

fix, dead-reckoning position.

orthochromatisierung arthochromatic processing.

Orthodrome straight (or rhombic) line portion of a great circle. -ortig -place.

ortig,drei- three-place.

örtlicher Tagesgang local diurnal variation.

Orts-bestimmung position-finding. - diagramm locus diagram, circle d. - fehler geometric location errar. -funktion position function. -gang local variation. -gebiet local area. missweisung local declination.

ortsveränderlich non-stationary, movable, (trans)portable.

ortung position-finding, orientation, taking of bearings, navigation, avigation. -, akustische sound location, acoustic orientation. Funk- radio positionfinding, obtaining $r$. fix.

Ortungskompass navigation compass. öse lug, өуe, өar, hook, loop, өyelet. Löt-soldering tag, tab. ösen-bl att tong, lip, flange. -bolzen eyebolt. 
Oszillationsquantumszahl vibrational quantum number. Oszill ator, leitungsgesteuerter line-controlled oscillator. sperrkipp-blocking oscillator, squegging 0 .

oszillograph, Innenphotographieinternal photography oscillograph. Saiten- string oscillograph. Schlelfen- loop, string or vibrator oscillograph. oszillographenschlelfe oscillograph loop, 0. vibrator.

oxydations-flamme oxidizing flame. -mittel oxidizing agent. - stufe stage of oxidation, degree of 0 . oxyd-beschlag coating of oxide. -faden oxide-coated cathode, oxide filament.

oxydhaltig oxidic, containing oxide.

oxydhaut skin of oxide, film of 0 . oxydlerbar oxidizable.

oxydkathodenröhre tube with oxidecoated filament, dull emitter $t$. oxygenleren oxygenate, oxygenize. ozokerlt ozocerite.

ozonsaverstoff ozonized oxygen, activated oxygen in the form of ozone. 
P Zweig P branch (spectr.)

Paarbildung pairing; formation of pairs, drawing together of lines (due to hum, in interlaced scanning).

Paare,Echo- paired echoes.

Paarerzeugung pairing.

paarlg erschelnen(von Linien, im Bild) pairing of lines (telev.)

Paarigkeit pairing, formation of pairs (caused by hum, in inter laced scanning).

Pack pack, packet, bale, bundle.

Packung packing, serving, wrapping. störschutz- interference eliminator kit.

Packungserscheinung packing phenorenon.

Paket,Federn- bank, assembly or set of springs.

Paneel panel.

Panoramabild panoramic picture or view, panning shot.

fanzer shield, ironcladding, armor (of a cable).

Panzerhol z retal-plated plywood.

Panzerung shielding, screening, ironcladding.

Papageno-flöte,-pfeife Pandean pipe.

Papier, abziehbares transfer paper. -, selbsttonendes self-toning paper.

Papier, Koordinaten- ruled paper, coordinate paper, cross-section p.

Papier-bahn web of paper. -band tape of paper, web of $p$. -hohl raumkabel air-space papercore cable, dry-core c. -pergament parchnent paper. -strang web of paper. -streifen paper strip; paper web. -umhulliung wrapping of paper. -vorschub paper feed.

Pappbogen sheet of pasteboard or cardboard. -deckel pasteboard, paperboard, carton.

Pappe pasteboard, paperboard, cardboard, cart on, millboard.

Pappelholz poplar.
Pappenguss pressure-cast carton or cardboard (using paper pulp), papier mâché, milliboard.

Pappmasse papier-mâché.

parabolisch, rotations- full parabolic.

Parabol spiegel parabolic reflector.

parachsial paraxial.

Paraffinbad paraffin bath.

Parallax, Sucher- seeker or finder parallax.

parallel, achs- axis-parallel, paraxial.

Parallel-endmass standard gage block, end-to-end s. bar. -impedanz leak impedance.

$P$ aral lelogram, schiefwinkl iges rhomboid, oblique parallelogram.

Parallel-resonanzkreis parallelresonance circuit, rejector $c$. -röhrenmodulation Heising choke or plate modulation, constantcurrent $\mathrm{m}$.

parallelschalten (connect in) parallel, shunt (across).

Parallel-schaltung parallel connection, p. circuit organization, multiple connection, paralleling. -verschiebung translatory motion, t.shift. -versuch parallel experiment, check-up e., duplicate determination. -weg bypass, shunt, shunt path.

Para-lysator anti-catalyst. -stellung para position.

Pardune back stay, guy wire, g. cable.

Partei, Gegen- opponent, adversary, adverse party, "the other side" (in a suit).

Partialdruck partial pressure.

Paschen-Back Verwandl ung PaschenBack (magneto-optic) effect.

passend fit, suitable, convenient, appropriate.

Pass-fläche seat, surface where two 
Passierrohr

parts fit together or engage

(snugly). - sitz press-fit, snug

f., seat, lodgment.

Passierrohr indicating plug, inside gage.

Passivierung passivation.

Passung fit.

Pastekathode pasted (oxide-coated) cathode.

Pastille pastil, button, bead, lozenge.

Patent, angemeldetes filed p., pending p., p. applied for.

-, schwebendes pending patent.

$A$ bånderungs- re-issue patent.

Apparat- patent covering and disclosing apparatus or machine, a.p. Beweise, die Gulligkeit eines... es Abbruch tun evidence impeaching, or prejudicial to, validity of a patent. Erfindungs- patent of invention. Haupt- parent patent, main or original patent. Klagepatent in issue, in suit or at bar. stamm- parent patent. Stoffpatent covering a substance or material, product p. Verbesserungs. patent of improvement. Verfahrensprocess patent, method p. Zusatzpatent of addition (to parent p.)

Patent-anmassung usurpation of patent rights. - anspruch claim of a patent. -beschreibung patent specification. -dauer life of a patent, continuance of a p., term of a p. -erlbschung expiry or expiration of a patent; annulment of a. p. -erteilung allowance, grant or issue of letters patent. -gebuihr patent foe, p. tax. -gesetz patent law, p. code, p. statutes. -giltigkeit anfechten challenge validity of a patent.

patentiert im In- und AUsland covered by domestic and foreign patents.

$P$ atent-inhaber patente $\theta$, grante日, holder of letters patent. -klage patent suit, p. action. -kommissar stellvertretender deputy or acting patent commissioner. -streit patent action, p. litigation. -unteranspruch sub-claim of a

\section{Peiler}

patent. -verlängerung renewal of a patent. -vorwegnahme anticipation, anticipatory reference.

patentverletzendes Merkmal patentinfringing fact or feature.

Patentverletzungsklage action or suit for infringement of patent rights.

Patrize upper tup die, punch.

Patrone cartridge ( $f$ or specimen in electron microscope); pattern, stencil, mandrel.

Patronenreihe string of cartridges.

Pauke kettle drum.

Paukenstimmschlissel kettle drum wrench.

pauschen swell, refine (met.)

Pause copy, tracing; space, interval, intermission, pause. Dunkelobscuring period, cut-off p., dark p. Gespräch-interval of no speech. Licht-photo(graphic) tracing, blueprint. Telegraphierspace (in keying or telegraphing work).

pausen trace, calk.

Pedal, Forte- loud pedal. Pianosoft pedal. Tonhaltungs- tone sustaining pedal.

Pegel level, gain (in db units); water gage. -, mittlerer mean power level (as indicated in volume indicator). Dialog-dialogue level. Leitungsbedarf- power reference level. Rausch- noise level (due to Schottky effect and thermal noise). Schwarz- black level. Stör-, Untergehen der Bildspannung im swamping of picture or video signal in noise level.

Pegel-ausgleich equalizing level, $l$. equalizer. -messer transmission measuring set. -schreiber level recarder. -strom pilot current, p. carrier (in suppressed carrier system).

Pegelung level or volume (gain) regulation.

Peil O Gruppen $Q$ code groups.

Peiler, direkt anzeigender directreading direction-finder.

-, selbstanzeigender automatic 
direction-finder, direct-reading d.f.

Peiler, Bodenstation- ground station direction-finder. Nachlaufdirection-finder with automatic zero setting. Sicht- visual direction-f inder, direct-reading d.f.

Peil-abschnitt, geeichter calibrated sector. -antennensystem directional antenna system. -antrieb frame drive, loop d. (handle, wheel and spindle). -aufsatz bearing plate (of d.f.). -empfänger direction-finding receiver. -fehler direction-finding error, bearing $e$. (due to night effects, etc.), distortion of bearing. feld bearing field, -flugleiter ground directionfinding control operator. -funkempfänger radio compass, directionfinder. hauptstelle main control direction-finder station (of an air communication safety district). - haus direction-finder station building. -kompass navigator's compass. kurs, recht voraus head-on course (fore-aft axis or lubber line of craft directed toward objective). -kurve space pattern, directional diagram. - leitstelle main control direction-finder station (of an air communication safety district). nebenstelle associated d.f. ground station, sub-control station. -punktmarke, schwarze black dot, b. mark (on scale). -rahmen coil antenna, loop a., frame a. -richtung direct course or line to transmitter or beacon. -ring direction-finder loop or frame. -scheibe, Funk- bearing plate (of radio direction-finder). -seite, eindeutige absolute direction. -seitenschal ter directionfinding sense switch, sense-finding s. -spannung directional signal potential (picked up by directional antenna in df, as distinguished from auxiliary antenna potential). -stelle, Boden- ground df station, aeronautical g.s.-, Nebenassociate ground station df. -strahl, raumger adl iniger or thodrome. -strahl wegabl enkung distortion of bearing, deviation due to diurnal or seasonal factors, weather, terrain, local conditions, etc.; lateral deviation. -tabelle bearing chart.

peiltreve Bodenwelle ground wave resulting in undistorted bearing.

peilung taking bearings, directionfinding. -, fal sche false bearing, erroneous b. -, optische pilotage. -, wahre true bearing.

Peilung, Deck- alignment bearing. Eigen- board (aircraft) directionfinding, taking bearings with homing device on board. Einzelbearing taken from one object. $\mathrm{Fal}$ sch- erroneous bearing, false b. Fl immer - (cf Wechselkardioide) loop reversing switch method (d.f.) Fremd- ground direction-finding (directional data or radio bearing supplied on request to airplane from ground station).

Grosskreis- great circle bearing. Hör - auditary direction-finding. Kreuz- cross bearing (taken from two or more ground df stations). Längs- taking bearings from $d f$ stations roughly in direction of travel or course. Maximalmaximum-signal strength (df) method. Minimum- minimum or zero signal strength (df) method. Misch- taking bearings on board (without directional receiver) from directive beam. Nah- closerange direction-finding. Standl inienposition-line bearing, great circle bearing. Standort- obtaining a fix, position or location finding (by two or more direction-finders), position-line bearing, great-circle b. Ziel-,missweisende Richtung Flugzeug-Boden magnetic reciprocal course (from airplane to ground station). Ziel-, rechtwei sende true course to steer. 
Ziel-, rechtweisende Richtung vom Flugzeug zur Bodenpeilstelle true reciprocal course (from airplane to ground station).

peilungsbeiwert error compensation value.

Peil-verfahren, Impul s- impulse direction-finding method.

-verlagerung bearing shift, $b$. error, b. displacement. -vorsatz d.f. means added to normal receiver equipment. zeichen call sign, code signal (say, of a ground station). -zeiger pointer of df apparatus.

Pendel, Schwere- gravity pendulum. Pendel-bewegung shutter movement (m.p.) -feder (im Zerhacker) vibrator blade, reed or spring (of chopper). -fenster internal pressure plate. -frequenz quench or bias frequency furnished from ausiliary oscillator circuit (in super-regeneration); electron-oscillation frequency. - frequenzschaltung (Armstrong) super-regeneration circuit organization. -gewlcht pendulum weight, bob. - glelchrlchter vibratory rectifier, tuned-reed r. -hammer (Charpy's) impact testing machine. -kugellager self-aligning ball bearing. pendeln oscillate (of electrons), vibrate, swing (of phases), pendulate, undulate, pulsate, rock, hunt (around an average value).

Pendeln, Hinauf- (gradual) rise in resonance.

Pendel-rickkopplung super-regeneration. -rïckkopplungsspannung positive quench or biasing voltage (impressed on grid in superregeneration circuits). - schlagwerk (Charpy) Charpy impact testing machine. umformer vibrating rectifier.

Pendel ung, El ektronen- oscillating of electrons. Last- load fluctuation, hunting of load. Luftstrom- air-jet pendulation.

Pendel-verviel facher reciprocating and accelerating secondaryelectron multiplier. -wechselrichter vibratory inverter, inverted rectifier of vibrator type.

Peracidität superacidity, hyporacidity.

Perforationsgeräusch sproket hole modulation or noise (m.p.)

Periode cycle, period. Halbhalf cycle, h. period, semioscillation, alternation.

schwebungs- beat cycle. Viertel- quarter-period. Zugwechsel- field frequency in interlaced scanning ( $=2$ \& frame f., telev.)

Periodenzelt time of vibration, $t$. of oscillation.

perlen form bubbles, drops or beads; sparkle, glisten like pearls.

Perlenwand directive or buaded screen.

Perl-glimmer margarite. -mutterblech crystallized tinplate, moiré métallique. -mutterpapier nacreous paper. -rohr bead tube (filled with glass beads to break up a fluid). -schnur maryante (cryst.); string of beads or pearls, line of droplets. permanentdynami scher Lautsprecher dynamic or moving-coil loudspeaker using permanent magnet (to produce constant field), $\mathrm{p}-\mathrm{m}$ dynamic 1.

Permanentsatz permanence principle, sum rule.

Permeabil itätsmesser permeameter.

Perpendikel pendulum, perpendicular. -ende oder - Gewicht bob.

Personenabtaster mit wanderndem Licht spotlight scanner for persons.

Perspektive, Frosch- worm's eye view. FUll - plenary perspective. Vogel- bird's eye view.

Pesenantrieb belt drive. 


\section{Petent}

212

Petent petitioner, applicant, suppliant.

Petrolkoks petroleum coke, oil c. pfahl stake, pile, stick, pole, post.

pfeife, gedackte, gedeckte stopped pipe. Doppelzungen- doubletongued flute. Galton- Galton pipe. Labial-,Lippen- flue pipe, flute pipe. orgel-reed organ pipe. Quer-fife. Rohr- reed pipe. Signal-siren, whistle. stimm- tuning pipe, pitch p. Vogel- bird whistle. Zinkzinc pipe. Zinn- tin pipe. Zungenreed pipe, r. flute. Zwitscherbird warbler.

pfeifen whistle, sing, squeal, howl, self-oscillating (of a tube)

pfeifpunkt-abstand,-sicherheit singing, whistling or stability rargin (of radio tube).

pfeil arrow, dart; strain.

Pfeiler pillar, post, column, upright, standard.

Pfeil-höhe sag or dip (of a line wire), height (of an arch, reniscus, etc.), camber (of airplane wing). $-\mathrm{r}$ ad double helical or herring-bone gear wheel.

Pferdefleischholz Surinam wood.

$\mathrm{Pflanzenwachs} \mathrm{vegetable} \mathrm{wax.}$

$\mathrm{Pfl}$ ichtenblatt (contractual) specification.

$\mathrm{Pfl}$ ichtwert specification value, contract $v$.

pflock peg, pin, plug, stake.

pfosten pillar, post, upright, column, standard.

Pfropfen cork, stopple, stopper; graft.

$\mathrm{pH}$. Hert $\mathrm{pH}$ value (hydrogen-ion concentration).

Phase phase; stage, epoch, era.

Phase, ausser de-phased, out-of-phase

(condition). gleichbelastete - (n)

balanced phases. -, in in-phase, co-phasal, in step. -, ver schobene displaced phase, shifted p., dephased or out-of-phase condition.

Phase, Eintritts- entrance phase (of network or filter). Oberflächensurface phase. Verdunkelungsdark interval (m.p.)

\section{Phasenverdrehung}

Phasen-abgleichvorrichtung phase balance, p. changer, p. shifter. -ablösung phase switch (in polyphase operation of ignitron). -aussortierung phase focusing. -drehung phase rotation, p. shift. -einstellung phasing, phase adjustment, p. shift, p. regulation. -entzerrung phase correction, delay equalizer; delay or echo weighting term.

phasenfalsch misphased.

Phasen-fehler error of phase, p. distortion. fokus phase focusing. -geschwindigkeit phase speed, p. velocity, wave v. - gleichgewicht phase (rule) equilibrium. -gleichheit phase coincidence, in-phase state, co-phasal s. -hub phase variation or fluctuation. -kurve phase curve. -laufzeit time of phase transmission. - lehre solution theory, doctrine of phases. -mass phase constant, wavelength $c$.

phasenmässig in proper phase relation; looked at from phase viewpoint.

Phasenmodulation phase modulation. -nacheilung phase lagging. -raum phase space, extension-in-phase. -regel phase rule. -regelung phasing, phase adjustment, p. shift. -reinheit freedom from phase shift or angular differences. -resonanz phase resonance, velocity $r$.

phasenrichtig in proper phase relation.

Phasen-rückdreher phase lagger, p. shifter. -schwund phase fading. -spaltung phase splitting. -sprung phase angle shift.

phasenstarr in locked phase relation, p. -locked.

Phasen-teiler phase splitter. -transformator phase shifting transformer. -ibergang phase transition. -umkehrer phase inverter, p. reverter. -verdrehung phase shifting, $p$. rotation. 
phasenverkehrt wirken act in reverse polarity, act in opposite phases or in p. opposition.

Phasenverschiebung phase shift, $p$. displacement, p. advancing (if forward), dephased condition. -von $90 \mathrm{Grad}$ quadrature relation of phases. -von $180 \mathrm{Grad}$ phase opposition.

phasenverschoben dephased, out-of phase, in p. quadrature (when 90 degrees), in p. opposition (when 180 degrees).

Phasen-verzerrung phase distortion. -verzogerung phase lag, p. delay. -voreilung phase lead, p. advance. -vorschieber phase advancer. -welle phase wave, de Broglie w., electron w. -winkel impedance angle, phase,a., impedance factor, 2 factor, quality factor.

phasig,fal sch- misphased, dephased. gleich-co-phasal, equi-phased, in phase. richtig-true-phased, in correct phase (relationship).

Phonograph, Licht- sound track film player (without pictorial accompaniment).

Phonographen-drehplatte turntable. -platte phonograph disk, p. record. -plattenteller turntable.

Phoresezelle, Elektro- electrophoresis cell.

Phosphore phosphors, luminescent or fluorescent (screen or lamp) materials.

phosphoreszenzerzeugend phosphorogenic.

Phosphorogene phosphorogens, activating additions (promotive of phosphorescence).

photoelektrisch(e) Ausbeute,-(es)

Emissionsvermögen photo-electric emissivity, p. $\rightarrow$. yield. -(e) Schwellenfrequenz photoelectric threshold frequency, critical f. - (e) Wirkung photoelectric effect, Hertz $\theta .-(\theta)$ Zelle photo-electric cell, electric eye (either photo-conductive, $p$. -voltaic or p. -emissive, though the last-named should strictly be called a photo-tube, a light-sensitive tube or a photo-electric t.) Photogramm photograph, photographic record.

Photographie, Kleinbild- microphotography, photomicrography.

photographisch festhalten record photographically (on a record sheet).

photographisch(e) Dichtemessung (photo)densitometry, photographic density measurement. - (en) schicht, Bremsvermögen der stopping power of photographic emulsion. -(em) Zeichendruck, Telegraph mit photo-printing telegraph.

Photokathode photo-cathode, photoelectric c. - , zusammenhängende plain mirror or continuous (nonmasaic) photo-cathode (of Farnsworth dissector and superemitron). -,Lichtstärkevertellung auf light intensity distribution on photocathode.

Photokopie photostatic copy, photographic c., photoprint, photostat. Photometer, Bunsen-, Fettfleck-

Bunsen photometer, grease-spot p. with translucent central spot in screen. Flimmer- flicker photometer. Gleichheits- contrast photometer, total-reflection p. Kontrast-, mit WUrfel contrast photometer of cube-type with cubical cavity. Kugel- sphere photometer. UIbricht integrating sphere. Lummer-Brodhun- contrast photometer, Lummer-Brodhun $p$. (based on equality of illumination). Spektral-spectrophotometer. Topf-photometric integrator. Ulbricht- sphere or globe photometer of Ulbricht. WUrfelcube photometer, integrating $\mathrm{p}$. with cubical cavity.

Photometer-aufsatz photometer head. -schirm photometer screen. Photo-metrierung photometric 
evaluation or recording. -physik photo-physics. -strom photo-cell current. - volteffekt photovoltaic effect (as in a cuprousoxide cell).

Photozelle photo-electric cell (photo-conductive, photo-voltaic or photo-emissive), electric eye. Mikro- globule of mosaic screen. Photozellen-kabel low-capacity shielded cable between photoelectric cell and amplifier of the video cable, co-axial or concentric cable type. -verstärker electron multiplier phototube; photo-cell amplifier. -vorlicht priming or bias illumination. physikal isch-chemi sch physicalchemical, physico-c.

$\pi$ Glied (I1-Glied) pi $(\pi)$ type section, filter mesh or network $\mathrm{m}$.

Piccoloflöte piccolo flute.

Pickelbildung (corrosion) pitting (of metal).

piezo-elektrischer Modul piezoelectric modulus.

Piezo-kristall piezo-electric crystal. -quarzzelle supersonic light valve (predicated on Brillouin and DebyeSears effect).

Pikkelflöte piccolo flute.

Pilgerschrittverfahren Mannesmann, Perrins or reciprocating rolling process.

Pille,Getter- getter tab, g. pill, g. patch. Gleichrichter-oxide or metal rectifier of reduced size (say, $1 \mathrm{sq} . \mathrm{cm}$ ), Westector $\mathrm{r}$.

Pilz-isol ator mushroom, petticoat or umbrella insulator. - l autsprecher omnidirectional (360 degree) exponential horn loudspeaker or sound radiator (mostly mounted on a pole top).

Pinne pin, peg, tack; peen (of a hammer).

Pinseldetektor cat whisker detector.

Pinzette pincette, tweezers, for $\theta$ ceps, nippers, pincers.

Pistole, Spritz- spray gun, s. pistol. Pitotröhre Pitot tube (to determine fluid or liquid velocity of flow).

$\mathrm{Pl}$ an, Bedrahtungs- wiring diagram. - biegeversuch plane bending test.

$\mathrm{Pl}$ anck's Wirkungsquantum $\mathrm{Planck}$ 's constant (quantum of action).

$\mathrm{Pl}$ andrehen face planing.

Planetangetriebe planetary gear, epicyclic g. (train).

$\mathrm{Pl}$ angitter plane grating (for light dispersion).

planieren plane, planish, smooth, level, even.

plan-konkav plano-concave. -konvex plano-convex. - liegen in

Fokusebene flattened in focal plane. -mässig systematic, methodical, according to plan. -parallele Platte plane-parallel plate. - schleifen surfacegrind. - symmetrisch planisymmetric.

$\mathrm{Pl}$ asma, Rest- residual electrons, plasma (region without resultant charge).

Plastik plastic, stereoscopic or relief effect (pictures). Bildplastic or relief effect; form of telev. picture distortion (consisting of multiple contours of diminishing intensity giving pseudo or erroneous three-dimensional appearance).

plastisch(e)Bildwirkung illusion of depth (of a picture or telev. image). - (er) Film stereoscopic film. - (es) Fliessen viscous flow. - (e) Verformung plastic deformation, p. working.

Platine plate, sheet bar, p. slab; p. bar.

Platin-mohr platinum black. -papier platinotype paper.

Plationrohre amplifier tube with filament disposed between control grid and plate (with low internal resistance, one model with photoelectric g.)

Plätchen lamella, small plate, platelet, scale.

Platte plate, sheet, slab, lamina, lamination, leaf (of a table), 
Platte, lichthoffreie

flagstone. -, lichthoffreie non-halation plate, p. free from halo.

Platten, Ablenk- deflection plates, deflector p. Auffang- target, collector or impactor plates, dynodes (in multiplier). Doppel- twin plates, twin crystal, composite c., biquartz. Strichkreuz- cross wire plates, reticles, graticules (telescope and other optical instruments).

Plattenfordermechan ismus plate-feed mechanism (in electron microscope).

plattenformig laminar, laminated, plate-like, p. shaped, l amellar, lamell iform.

Platten-gerausch (phonograph) record noise, surface n. -halter plate holder. -kristall, Doppeldivided plate crystal, biquartz. - lampe flat-plate (neon) lamp; tube with center plate instead of grid. -schlag disk wobble (of phonograph record). -schnitt plate contour, plate section (of condenser, etc.). -spieler di sktype phonograph or gramophone. -staffeln, Gl as- (Michelson) echelon diffraction grating spectroscope. - teller turntable (of phonograph). -wechsler record changer.

Plattieren plating.

Plattine plate, mill bar, slab.

$\mathrm{Platz}$, freier hole (in nuclear theory). Gitter- lattice place, 1. point. Lande- landing field. airport.

Plätzchen little cake, lozenge, troche, tablet.

platzen burst, crack, break open, explode, implode (inward collapse or burst, as of a vacuum tube).

Platzwechsel transposition, crossing; exchange of places (of electrons, etc.)

plotzlich sudden, abrupt, precipitous. -(es) Schwingen spilling over (of an amplifier tube, attended with fringe howl).

Pochwerk steam stamp.

Pockholz lignum vitae, pockwood.

Pohl'sche Wippe Pohl commutator

(double-pole double-throw switch).

Polarisation, Dreh- rotatory polarization, optical rotation.

Polarisations-apparat polarizing apparatus, polariscope. -ebene plane of polarization. -ebene Drehung der polarization error. effekt polarization error.

polarisationsfehlerfrei free from polarization error (d. $f$.)

Polarisationskapazitat polarization capacitance.

polarisationsoptisch photo- $\theta$ lastic. Pol arisations-prisma polarizer. -winkel polarizing angle, Brewster a.

polarisiert, elliptisch elliptically polarized. -,geradlining planepolarized. Kreisbahn-,zirkular circularly polarized.

Polarlicht aurora (borealis or austral is).

Pol-bedeckung pole arc, circumferential length of pole face, p. pitch percentage. bildung pole formation, polarization. -bogen pole arc, p. pitch percentage. -eck summit (cryst.) -effekt pole effect.

Pole, ausgeprägte salient poles. -, entgegengesetzte opposite poles, unlike p. -,gleichnamige like poles, similar $p$.

-, ungleichnamige opposite poles, unlike p. Gegenkontakt- $\infty-$ operative contacts, opposite contacts.

polen, um- reverse polarity. polieren polish, burnish, shine. Polierschleifmaschine grinding and polishing machine.

Politur polish, gloss, shine, shining, lustre. -messer glossimeter, glossmeter. 
Polklemme pole terminal. -papier pole paper, p.-finding $\mathrm{p}$.

Polster,Luft- air cushion, a. pocket; dashpot.

pol-suchgl immlampe polarity-finder glow-tube, p. indicator. -teilung pole pitch.

Polung, Gegen- opposite polarity, opposition of $p_{\text {. , reversal of }} \mathrm{p}_{\text {. }}$

Pol-wechsel reversal of polarity, change of $p$. -zwischenraum pole clearance, inter-polar space or gap.

Porenraum pore space, cell s., cell cavity.

Porzellan-erde porcelain clay, China c., kaolin. -masse porcelain body. -schiffchen porcelain boat. -tiegel porcelain crucible. -ton porcelain clay, kaolin.

posaune trombone. -, durchschlagende trombone with free reed (organ). Alt- alto trombone. Diskant- soprano trombone. Zug- slide trombone. posaunenartig verschiebbar shiftable trombone-fashion or like a telescope, telescoping.

Posaunenauszug telescoping means, extension $\mathrm{m}$. (for tuning Lecher-wire system).

Positiv, Bromsilber- positive in silver bromide. Meister- mother record, master positive. Originalmaster positive.

positive elektrische Ladungseinheit unit positive charge.

positron positron, positive electron, antielectron.

Postmikrophon solid-back transmitter, carbon-back microphone or telephone instrument.

Potential, Abstossungs- repulsion potential. Entstehungs - appearance potential, ionization $p$.

Geschwindigkeits- velocity potential. Gleichgewichts- equilibrium potential (of mosaic). Grenzflächeninterface potential. Rückstossrecoil potential. Strömungsflow potential, streaming p.

potential-berg potential barrier.
- $f l$ ăche, konstante equipotential surface, isopotential s. -gëfalle, weltzeitlicher Anteil universal diurnal (variation) component of potential gradient. -hïgel potential barrier. -kasten, -mulde potential hole, p. well. -schwelle potential barrier.

Potenz, in die nte - erheben

raise to the nth power.

Potenz-gefäll potential gradient. -schwelle(Kerndurchdringung) nuclear penetration factor.

potenzieren raise to higher power (by process of involution, math.)

Potenzreihe exponential series, power s. (math.)

Präge-bär ram (of a press). -film (cf Gravarfilm) engraved sound film (track cut in $\mathrm{film}$ ).

prägen stamp, coin, imprint, impress, emboss.

prägepolieren roll polish, $r$. finish.

Prägepresse screw press.

Prägung, $R$ and- marginal embossing of $f$ ilm.

Präklusivfrist preclusion period.

Prall-elektrode electron mirror, dynode, reflecting electrode, impactor anode (of multiplier phototube or photo-electric electron-multiplier phototube), target electrode, rebound or reflecting impactor (of Weiss mesh multiplier ).

prallen(cf prellen) rebound, bound, bounce, be reflected.

Prall-kraft elasticity, resiliency. -winkel angle of reflection, a. of ricocheting.

Präparier-arbeit dissecting work (micr.). -lupe dissecting lens.

prasseln crackle, rustle, rattle.

Pratze claw.

Praxis, in die - überführen reduce to practice or to a practical form, work a patent or the invention disclosed therein.

Präzisionswage precision balance, p. scales. 
prellen (cf prallen) toss, make to rebound, thump or click (key), chatter, reflect.

prellfrel free from thumping or click (in key operation).

Prellkraft resiliency.

Press-bernstein ambroid. -bodenröhre lotal base tube. -druck ram pressure (in pressfits).

Presse,Fach- technical press, $t$. literature, professional men's press. Klebe- blooper, splicer (for film). Präge- screw press. Zleh- (cf Tlefzleh-) drop (forge) press (for drawing and shaping articles from sheet material).

pressen press, compress, stanp, mold (under pressure).

Presser compressor (in volume-range control).

Press-form pressure mold. - gasschal ter (cross) airblast switch. -körper,-ling pressed object or article, blank after pressure application or molding. -l uftl autsprecher compressed-air loudspeaker, pneumatic 1. (without diaphragm): -luftschalter (cross) airblast switch (similar to gas b. s.) -masse fictile substance, moldable material, plastic m., synthetic, (thermo-) plastic. -matrize, negative negative stamper (phonograph recard). -platten pressings (phonograph record disks). -prozess pressureshaping, molding or fashioning method. -sitz press-fit, force or driving f. -spahn glazed cardboard, presspan insulation material, pressboard. -stempel press ram. -stock stamper, die (built up from mother record, in phonograph record manufacture). -stoff plastic, moldable or fictile material,

Primär-abstimmung pre-selector means. -empfang single-circuit reception.

Prinzlp geringsten zwanges principle of least strain or resistance.

Prinzlp, Ausschllessungs- exclusion principle (formerly Fauli's equivalence p.) Auswahl- selection principle. KorrespondenzBohr's correspondence principle.

Prinzipal open diapason (organ stop).

Prinzipschaltung basic circuit diagram (showing underlying principle).

Prioritäts-beanspruchung priority claim. -belag priority proof. - jahr convention year.

Prlsma prism, wedge (when weak). -, aneinandergesprengtes brokencontact p., two quartz prisms closely fitted together without cementing. -, doppel umkehrendes inversion prism, inverting p. (reverses in both axial directions of image). -geradslchtiges direct-vision prism. -stark fächerndes highly dispersive prism.

Prísma, Ablenkungs- deviating prism. Aufrichtungs- erecting prism. Mehrkant- polyhedral prism. Polarisations- polarizer. Stufenechelon prism. Umkehr- (cf Blldumkehrung) inverting prism, reverting p. Vergleichs-compar1son prism. Wollaston- Wollaston prism, double-image p., Zwlllingsbiprism, double-image $p$.

prlsma-ähnllch prism-like, prismoidal. -formig prism-shaped, prismatic.

Prismen-fläche prismatic surface, p. face. -sucher prismatic finder, p. seeker.

Privatauffuhrung pre-view.

Probe (cf Prufer, Prufung, Messer, Messgerät, Messung) sample, specimen, test piece; trial, test. gereckte stretched specimen. -, verguitete quenched or tempered specimen or test piece. Beschussbombardment test, shooting $t$. Kapazitäts- capacity test. Kegeldruck- Brinell ball impression or indentation test, ball pressure t. Loch- drift test. Metall-assay, test for metal; m. testing. Ri sshärte- abrasion 
test, scratch t. schlag- impact test, blow t., percussion $t$. Tlefzleh- cupping test, cuppability t. (piece or specimen). Tonsound test; tonsil t. (in recording voice of a singer).

Zerreiss- tensile test, breaking t., rending test (specimen).

Probe-abdruck proof, proof print, p. sheet. -aufnahme test picture, trial shot. -entnahme sample taking, sampling (at random).

-machen testing, assaying.

-nehmen sampling.

Probenglas specimen glass, s. tube.

Probe-objekt specimen. -saal rehearsal hall. -stück specimen, sample, test piece. ziehen sampling.

Problergefäss testing vessel, assaying $v$.

Profil,Feld(styrken)- field pattern (often mapped in an electrolytic tank). -Tragfligel- aerofoil section.

Profll-begrenzung contour line, boundary of a section or profile. - prifer (electronic) profilometer. -stempel profile stamp (surface pressing).

Projektion, flächentreue equal-area projection (mapping) Dla- oder Dlapositiv- lantern slide projection. Durch- back projection. Gross- large-picture projection work (with magnascope, etc.) Hintergrund- background projection. Kegel-conical projection (of a map). Merkatorkarten- Mercator projection, cylindrical p., orthomorphic $\mathrm{p}$.

Projektions-anlage, RUck - back projection equipment; re-p. apparatus. -aussteuerungsanzelger projected volume indicator. -bildschrelbryhre projection-type television receiver. - fenster projection aperture, p. gate. -spule projection coil. -tubus projection tube (micr.)

Propeller, Verstell- feathered (variable-pitch) propeller or airscrew. proportional, umgekehrt od. verkehrt inversely proportional.

Proportlonalitätskonstante constant of proportionality.

Prozent, Gewlchts- percent by weight. Raum- percent by volume.

Prozentgehalt percent content, percentage.

prozentuale Aussteuerung percentage modulation, m. percentage.

Prozess manufacturing process or method; action, lawsuit, cause, suit, trial, legal case, court c. (law). Anfechtungs - contested action.

prozess-kosten law costs, c. of proceedings, c. of a legal action. -1 adung writ of summons.

prüfen, nach-re-examine, re-check, retest.

Prlifer, Erlchsen- Erichsen cupping machine. Gl attheits- smoothness meter, glossimeter. Härtehardness tester (Brinell, Rockwell sclerometer and scleroscope). Lichtsinn-visual photometer. Linsen- lensometer. Material-, el ektroni scher aniseikon. oberflächenspannungs- (nach Abreissmethode) adhesion-balance type of surface tensiometer. Profil(electronic) protilometer. Rauhelts- smoothness meter (determines degree of polish), glossimeter, profilometer. Ritzhärtesclerometer, scratch hardness tester. Röhren- tube checker, $t$. tester. Stromschluss- continuity tester. Tonhohe- tonvariator. Universal- multiple-purpose tester, multimeter. Windungschluss(inter-)turn short-circuit tester. prifbank (lens) test bench (opt.). prifen test, assay, probe, try, prove, examine, inspect, verify, scrutinize.

Prif-feld test field, proving ground. -feld, stoss- potential impact, flash or impulse testing field. - generator signal generator, test oscillator, all-wave 0 . 
-gerat trouble shooting device, tester, set analyzer (radio). -1 Ing test piece, $t$. specimen, $t$. model, t. sample. -pult test desk. -rohre fur Fernseher phasmajector, monoscope, monotron. -schnarre test buzzer. -sender service test oscillator. -sonde, Klaven- clawtype test probe or prod, search electrode. - spitze penetrat or (of hardness tester); indenter. - stabeinschnurung waist (forming in bar under tensile test). -stand test bed. -strelfen buzz track (on a film). -verfahren prosecution (of a patent application), period during which Fatent of $f$ ice Examiner reviews novelty and merits of a disclosure; test method.

Prufung (s.a. Probe, Prufer, Messer, Messgerät, Messung). exanination, testing, perusal, consideration, inspection, investigation, reviewing, surveying, verification, oheck, search, probe. -, nichtzerstörende, zerstörungsfreie non-destructive test. Abnahmeacceptance test. Daverstandfestigkelts--(long-time) creep test. Eisenpulver- Magnaf lux inspection (using iron dust).

Impuls- s. Prïfung, Stoss-. Mater|al-testing of materials. Rauhheits- testing with smoothness meter or glossimeter. Regenrain test, wet $t$. (of an insulator). Stoss- impact, flash or impulse test (of insulation). Treib- cupping test. Verschleissabrasion test, t. to ascertain wear and tear. Zerrels-, Zlehtensile test, breaking t., rending t.

Pseudo-dämpfung loss of selectivity by parallel internal resistance. -schwund pseudo-fading (due to swing or swaying of antenna).

Puffer grommet, cushion, pad, buffer. shock absorber. -betrleb floating battery operation. -losung buffer solution. -stufe buffer stage, separator s.

Pul sation pulsation, beat, throb. Pulser pulsator (fatigue tester)

Puls-verfahren, Ein- monopulse method. -verstärker, Bildsynchronisierungs- frame synchronizing (or low sync) impulse amplifier.

Pult, Misch-mixing, monitoring or supervising desk. Prüf-test desk. -befeuerung firing on stepped grate bars. -ofen black-f lame hearth.

pulverartig pulverulent, pulverous, powdered; dust-like.

Pulverdiagramm powder pattern. pulverlg powdery, pulverulent, pulverous, dust-like.

Pulverkern, gepresster Eisenconpressed iron-dust core.

pulvern powder, pulverize.

Pumpe, Kreisel- centrifugal pump, turbine p. Lade- charging pump, compression $p$.

Pumpenlamelle vane (of Gaede vacuum pump).

Pumpstengel pumping lead (short piece of tube connecting vessel to be evacuated and vacuum pump), exhaust tube, $\theta$. vent.

Punkt, ausgezelchneter cardinal point (opt.) Beriuhrungspoint of contact, tangency or osculation, touching p. Brennfocal point (opt.) Einschnürungscrossover (in electron-optics). Flucht-vanishing point. Hauptprincipal point, unit point (opt.) Kardinal-cardinal point (opt.) Kehr-cusp. Knotennodal point (opt.) Morsebreiter, spitzer, lengthened dot, clipped dot. Nabel-umbilic. Wende- cusp.

Punktallinse toric lens.

punktförmlg punctif orm; crater shaped. -(e) Indukt Ivität lumped inductance, concentrated $i$. - (e) Quelle point (shaped) source or emitter (of light, electrons, 
etc.), fous lamp.

Punkt(glimm)l ampe point or crater neon lamp, tungsten arc lamp, Fointolite, focus 1.

punktleren, strich-dot-dash.

Punkt-kathode point source cathode, crater c. -lagen point positions. -licht point (crater-shaped)

light source. -losigkeit astigmatist. -marke spot reading (on dial of direction-finder). -raster point (dot or granule) raster, screen, plate or grating. -schreiber, Eln- single-point recorder. -schweissung spot welding. -singularltät point singularity. -strich semicolon.

punktsymmetrisch having point or radial symmetry.

punktuelle Abbildung point-focal vision or imagery.

Punkt-verlagerung, $\mathrm{Bild}$ - spot shift (causing "plastic", telev.) -verteller,Bild- picture scanner. punktwelse Auswertung point-by-point evaluation.

Pupille, erwelterte dilated pupil. Ausgangs-, Austritts- exit pupil. Elngangs-, Elntritts- entrance pupil. Pupillen-grösse pupillary size, p. diameter. -soiel response of pupil -weitemesser korometron.

Pupinspule loading coil, Fupin c. (for reactance balance).

Puppe, Kohle- carbon rod.

Purpur, Seh- visual purple, rhodopsin.

Pustlampe blow-lamp.

putzen clean, cleanse, scour, polish, trim.

Pyramiden-stumpf truncated pyramid, frustum. -wirfel tetrahexahedron.

Pyrometer, Gluhfaden- optical pyrometer, disappearing-filament p., monochromatic $p$. 
Q Faktor $Q$ factor, magnification $f$. $Q$ Gruppen $Q$ code groups. Quadrate, Methode der kleinsten method of least squares.

quadratisch square, quadratic, tetragonal (cryst.), quadrangular. -(e) Abtastung square-law scan. -(er) Gleichrichter square-law rectifier or detector. - (e) Gleichung quadratic equation. -(er) Kondensator square-law condenser or capacitor. -(er) Mittelwert root-mean-square (rms) value, virtual v. Quadrat-well lengener ator generator of square or rectangular waves. -wurzel ziehen extract square root.

quadrieren square; raise to 2nd power.

Quadrupolmoment quadrupole moment. Quant, Licht- photon, light quantum. quanteln quantize.

Quantelung, $R$ aum-, $R$ icht- directional quantization, space $q$.

Quanten-ausbeute quantum yield, q. efficiency. -bedingungen quantum conditions.

quantenhaft pertaining to quanta or the quantum theory.

Quanten-hypothese quantum hypothesis. -sprung quantum transition (abrupt transfer of a quantum system from one of its stationary states into another), quantum jump or leap. -theorie quantum theory.

Quantenzahl, innere inner quantun. number. Oszillations- vibrational quantum number. Rotations- rotational quantum number.

Quantisierung quantizing.

quantitative Messung quantitative measurement, $q$. analysis.

Quantum, $\mathrm{Pl}$ anck's Wirkungs- Planck's constant, quantity of action.

Quantum-ausbeute quantum yield, q. officiency. -berichtigung quantum correction. -gewichte quantum weights, statistical w. mechanik quantum mechanics. -ïbergang quantum transition, q. jump, q. leap. zustand quantum state, energy level.

Quarz, optisch inaktiver racemic quartz, racemate. Linkslevogyrate quartz crystal, lefthanded q.c. Rechts- dextrogyrate quartz crystal, right-handed q.c. stever- (frequency) stabilizing quartz.

quarzähnlich quartzosé, quartzous. -artig quartzic, quartzous, quartzy. Quarzfaden quartz thread, q. filament.

quarzgesteverte Steuerstufe crystalstabilized master or drive (oscillator) stage.

quarzig quartzy, quartzose.

Quarz-inaktivierung, optische racemization. -keil, Doppel- double quartz wedge. -kristall, fertiges piezoid, finished blank (including electrodes). -kristall, optisch inaktives racemate. -mehl quartz powder. -resonator piezo-electric resonator, quartz r. -sender (piezo-electric) crystal or quartzstabilized transmit.ter. zelle, Piezo- supersonic light valve (predicated on Brillouin and SearsDebye effect).

quasi-elastisch quasi-elastic. -unendlich quasi-inf inite.

Quecksil berdampfl ampe mit Heizkathode phanotron, tungar and similar types of tube (with argon filling). -mit steuergitter mercury-vapor tube of the thyratron, ignitron, etc., type.

Quecksilber-faden mercury thread, $m$. column. -falle mercury trap (vacuum pump operation). -halogen mercury halide. quecksilberhal tig mercurial, with $\mathrm{Hg}$. 
Quecksil ber-hochdruck-Entl adungsrohr high-pressure mercury discharge tube or vessel. Kathode mercury (pool) cathode. -leglerung mercury alloy, analgam. -lichtbogen-Kathodenfleck, fixierter anchored cathode spot of mercury arc. -masse mercury pool, m. sump (in bottom of a tube). -mohr black mercuric sulfide. - schal ter mercury switch. - strahlunterbrecher mercury-jet. interrupter, m. break. -tropfelektrode dropping mercury electrode. -vorrat mercury pool (in discharge tube).

Quelle, einfarbige Leuchtstrahlmonochromatic illuminator source, monochromator. -, punktförmige point (-shaped) source or emitter. Elektronen-electron source, $\theta$. enitter. Llcht- source of light, illuminant. Strom- source of current (supply).

Quellenblld,Llcht- light source image.

Quellung swelling, welling, soaking, imbibition.

quer cross, across, transverse, transversal, traverse, diagonal, lateral, oblique, askance, athwart.

quer ab athwart. -Backbord abeam, on the port beam. -steverbord abreast, on the starboad beam. -Pellung taking bearings from athwart or abean stations. Quer-arm cross arm, shunt a. (of network). -aufnahme horizontal picture. -effekt transversal effect (of crystal).

Quer-entzerrer shunt-type attenuation equalizer or compensator. - feld transverse or cross (magnetic) field. - glied, in $1 / 2$-endender Kettenleiter midshunt terminated network. -kondensator bridging capacitor, by-pass c., shunt c. -kontraktionskoeffizlent Foisson's coefficient, rho ratio (lateral contraction: longitudinal extension).
- leltung leakage conductance, leakance; shunt line, cross 1.

-leitungsbrlicke transconductance bridge. -magnetfeld transverse magnetic field. -modulation cross modulation. -pfelfe fif $\theta$. $\rightarrow$ rofil cross-section. -riss transverse crack, cross c., fracture of mineral); crosssection. -schärfe definition in picture direction.

Querschnltt cross-section, transverse s., cross-cut; crosssectional area. Streu- scattering cross-section. Wicklungscross-sectional area of winding. Querschnitts-verm Inderung crosssection reduction (of area), draft, draught. -zusammenzl ehung contraction or reduction of area, formation of a waist (in test piece under tension or tensile stress).

Quer-schrift lateral-wave or Berliner type track (used in gramophone and modern phonograph records). -schwingung transverse vibration, shear v. (sometimes called flexural v.), contour vibration or oscillation (cryst.) -spulenbelastung leak load. -steuerröhre cross-control beam tube, drift t., tube operated on beam deflection. -verbindung, Funkintercommunication channel. -verschiebung und Längsv. cross ad justment and axial adjustment (of electron microscope). -widerstand shunt resistance, cross $r$. (of network) -zusammenzlehung lateral contraction. -zweige cross arms, shunt a. (of network).

Quetsche, Roll en- squeegee.

quetschen pinch, squeeze, crush, mash, squash.

Quetschfuss squash, press or foot (of a tube).

quetschfussfreie Röhre loctal base tube. Quetsch-hahn pinchcock. -kondensator 


$$
\text { Q Faktor }
$$

compression-type condenser.

Q Faktor $Q$ factor, magnification $f$.

$Q$ Gruppen $Q$ code groups. quicken amalgamate.
223 Quotienten

quieken squeak, squeal. Quinte violin E string. quirlen twirl, stir with a whirling motion, turn.

Quotientenmesser quotient meter, ratiometer. 
Rachenlehre gap gage.

Rad, Daumen- sprocket wheel.

Farb- inking wheel, i. roller. Flugel-worm wheel, screw, s. propeller. Hemm- escapement wheel, brake w. Kamm- cog wheel. Kegel- bevel wheel, mitre w., cone w. La Cour'sches phonic wheel. Pfeil-double helical or herringbone gear wheel. Rayleigh-, Schall reaktions- Rayleigh disk (placed in a sound field). Schnecken- worm wheel, worm. Schrittschalt- step-by-step wheel, stepping . Schwungfly wheel, balance w. Sperrratchet wheel, cog $w$. Spiegelmirror wheel, Weiller scanning w. or drun, drum scanner with peripheral mirrors. Steia- escapenent wheel, ratchet $w$. Sternstar wheel. Stift- sprocket wheel. Stirn- spur wheel. stufen- step wheel, cone pulley. Ton- tone wheel. Uml auf- planetary wheel. Vor schub- feed wheel. Zeilenspiegel- line scanning mirror wheel (of Scophony Co.) Rad-bewegung rotary motion, wheel $\mathrm{m}$., rotation. -buchse bushing.

Rudchen, Reiter- jockey wheel, j. roller. Raddrehung rotation; torsion.

Rädern shredded wheat of fect (m.p.)

radförmig rotate, spider-shaped, wheel-s., radial (of a structure). radial symmetrisch radially symmetric, in radial symmetry.

Radier-firnis etching varnish. -grund etching ground.

radioaktiv(e) Emanation radioactive emanation (known as radon, thoron and actinon). -(es) Fol geprodukt metabolon. - (es) Gl eichgewicht radio-active equilibrium (being either transient or secular). $-($ er) Niederschl ag radioactive
R

deposit, film of r. matter, -(e) Produkte metabolons, successive disintegration products of radioactive materials.

Radioaktivität, kUnstliche induced or artificial radioactivity.

Radio-amateur radio fan, $r$. amateur, "ham". -meterflugel radiometer vane. -sonde(fur Wetterforschung) radiometeorograph, radio sonde.

Radium-behälter radiode (glass or metal tube or capsule).

-emanationseinheit curie unit quantity of radon.

Radius, Abrundungs- contour radius. Krümmungs- radius of curvature. -vektor radius vector.

radizieren extract the root, evolve. Rad-kranz wheel rim, tire. -linie cycloid (in stroboscopic aberration), epicycloid. -phänomen wheel illusion. -reifen tire, rim. -satz axle plus two (shrunk-on) wheols. -stand wheel base. -taster wheol-operated means (causing signals, bell ringing, etc.)

Radtkeverfahren sound reproduction method of A.A. Radtke (invented 1917, USP issued 1938); cell impulse amplified, while maintaining and restoring grid potential by high impedance between cell cathode and negative end of source of potential.

Raff-aufnahme, Zeit- time-lapse-motion photography (low-speed shooting, hish-speed projection.) -bild time-lapse picture, fastmotion $p$.

Raffer,Mikro- camera for stop-motion or time- lapse motion micrography. zeit- time-lapse or stop-motion canera.

Raffinieranlage refinery, refining plant.

Rahmen stator, frame, former; loop 
or coil (aerial), frane antenna. Im - der Erfindung inside the scope (or spirit) of the invention. Rahmen, Druck- film trap (in projection). Eisen- iron-cored frame (d.f.) Enttrlibungs- frame to make zero or minimum signal point sharper (free from night effects, etc.), zero clearing or sharpening frane. Entwlckel ungs- developing rack. Kopler-printing frame. Luft-air-cored frame (d.f.) Peilcoil antenna, loop a., frame a. Rahmen-antenna coil antenna, loop a., frame a. -antenne, Kreuz- crossed coil antenna. -entwickelung rack development, tray d. -nachstimmung re-setting or re-check of frame or coil tuning (in automatic radio compass). -negative rack negatives. - sucher wire-frame view-f inder, iconometer.

Rakete, Leucht-, Licht- flare.

Ramankomponente modified frequency of incident light, or Raman spectrum, in a scattering substance.

rammen ram, beat down, tamp, pound. Rampenbeleuchtung footlights.

$r$ ampig shear-scarred (of glass), burred.

Rand edge, border, rim, margin, brim, hem, flange, boundary, periphery, brink. Bördel-

flanged edge, beaded edge. Schirmborder, edge or margin of screen.

$R$ and-anmerkungen marginal notes, $m$. annotations, marginalia. -aussparung (für Tonstreifen) setting aside margin (of film strip) for sound track.

-bedingungen boundary conditions, marginal, fringe, or edge c. -bemerkungen marøinal notes, $\mathbf{m}$. annotations, marninalia. -effekt edge effect, Eberhard e. (of film), fringe or marginal $\theta$.

rändeln edge, border, rim, will, knurl, bead.

$R$ and-kante lateral edge. -kraft force (acting) at edge, marginal force. -präguna (des Films) marginal embossing of film (Pathe'). -strahl marginal ray, peripheral $r$. -winkel angle of contact (between wall and liquid), wetting a. -zons, verstickte nitrided edge or marginal zone.

Rasierstein styptic stick.

raspeln rasp.

rasseln rattle, rustle, clatter.

Rast(e) (bei Fern- und schaltersteuerung) notch, detention point, detent, arrester means (in telemetric, switch, etc., operation). Feder-spring catch, s. detent. rasten, ein- lock in position (as by a detent).

Rasten-arretierung arrester means, locking $\mathbb{m}$., detent. -schalter step switch, multiple-point s. -spreizen bracing members (of piano) -teilscheibe notched index wheel.

Raster scanning field, s. pattern or "raster," unmodulated light-spot scan or scanned area (of a tele$v$ ision pictare); mosaic (in photocathode tube and in iconoscope); grating or screen (of dot, ruled or line type, used in printing). Raster,Druck - printer's screen or meshwork. Farb-color embossing. Fernseh- (cf Raster) television scan surface, scan area or "raster." $\mathrm{Halb}-$, Ineinanderoreifen der interlocking or meshing of fractional scans (in inter laced scanning). Linien- ruled plate, line screen or grating. Punkt- point, dot or granule type of grating, plate, or screen.

Raster, Zwi schenze ilen-, ger adzahl iger even-line interlaced scan.

- -, ungeradzahliger odd-line interlaced scan.

Raster-aufnahme, Farb- color screen photograph. -blende scan hole, scan aperture, raster screen aperture. -element picture unit, p. element or elementary area. - farbverfahren, Linsen- lenticulated or lenticular screen color printing method. feinheit 
resolution, raster detail or definition (of scanning pattern). -fllm,Linsen- lenticular or lenticulated screen film. -frequenz field frequency. -kathode photocathode, mosaic screen. -körner silver globules (of iconoscope mosaic), silver plugs (in barrier -grid m.). -mlkroskop screen scan microscope, raster scan m., electron scan $m$. -platte scan pattern, s. field, s. area, photo-sensitized mosaic plate (of iconoscope); screen (dotted, granule, line or ruled). -punkt elementary scan area, $\theta$. picture point or area. -schirm mosaic screen.

Rasterung definition or resolution of a picture (say 300 lines); embossing or lenticulation (of a film surface). Fein-high-definition scan (of a picture).

Raster-wechselfrequenz field frequency (in interlaced scanning). -zahl number of picture points, p. units or elementary areas (determining definition or resolution of picture).

Rast-klinke detent, latch, paw 1. -nase detent, latch. -polkegel her polhode, space cone. -stern (Im Wellenschalter) notched star (of wave-band switch).

Rastral music ruling pen.

Ratsche rattle. Flihl-micrometer friction thimble.

Rauch-fahne smoke streamer. $-g l$ as tinted glass, smoke g. -klappe vent, stack (of projection booth). rauchschwach smokeless (of powder). rauh rough, raw, roughened, granulated, knurled, burry.

Rauhen granulating, graining, roughing or roughening (superficially).

Rauhheit (Lautsprecher) raucousness, hoarseness (of loudsp.)

Rauhheltsprïfer smoothness meter (determines degree of polish, or lack of polish, on surfaces), glossimeter, profilometer.

Raum space, room; chamber, cavity, enclosure, volume; scope.
-, l.uftleerer vacuum, vacuous space, exhaust s -,überakustischer exceedingly live room or space (with excessive reverberation period). Abtörmixer room, monitoring booth, box. Anfach-, Arbeits- generator space, working or drift s. (in drift or beam tube). Aufnahme- studio, teletorium, telestudio (of audio and video broadcast station, etc.).

Aufschluss- part of electrically prospected volume of soil or earth subject to current or displacement lines. Besprechungsstudio. Blld-image space. Dingobject space. Fall-cathode fall space (in which c. drop occurs). Geschwindlgkelts- velocity space. $\mathrm{Halb}$ - half space. Hall-acoustically live room; reverberation chamber. Nachhall- reverberation room, r. space. Phasen- phase space, extension-in-space. Porenpore space, cell s., cell cavity. Sammel - receiver, receptacle, collector space. Schlag- spark-gap chamber or space. Zuschauerauditorium. Zw/schen- intervening space, intermediate s., interstice, interspace, interstitial s., interval.

Raum-akustik stereo-acoustics, architectural a., space or room a. -beständlgkeit volume stability. -bild stereoscopic picture, plastic p.; space diagram (d.f.). -blldung, Hohl- cavitation (in supersonic wave work, etc.). -bildvorfluhrung stereoscopic picture projection. -chemle stereochemistry. -formel spatial formula. -gebll de space diagram; solid three-dimensional structure. -gefihl space or spatial feeling (in vision). -geometrie solid geometry.

raumger adl inig orthodromic. -(e) Peilstrahlen orthodromes. Raum-geräusch set noise (caused by insufficient sound insulation, in m. p. work), set noise (in radio receiver) comprising Johnson $n$., 
shot n., hum, etc. -gewicht weight by volume. -gitter space lattice, "raumgitter"- -gruppe space group, point g. -halleffekt liveness (ac.) -helligkeit volume brightness. -inhalt volume content, capacity. - - integral space integral. -ladenetz space-chargo grid. -ladungswelle space-charge wave. -ladungswolke concentration of space charges, accumulation of s.c.

råumlich spatial, concerning space, space-geometrical, three-dimensional. - (e)Behinderung steric hindrance (chem.) - (e) Rotationsgruppe space rotation group. -(e) Vertellung spatial distribution, geometric d. - (er) Winkel solid angle (in spheradian units)

Raum-mikrophon non-directional microphone, polydirectional m. -punkt space point. -quantelung space quantization, directional $q$. -resonator, Hohl- cavity resonator, chamber r., rhumbatron (as part of klystron). -sektor solid sector. -sinn space perception. -strahl, Welt- cosmic ray. -strahlenstoss burst or shower of cosmic rays. -strahlung cosmic ray radiation. -tell part by volume, volume. -toneffekt stereophonic sound of fect, binaural e. -verhältnis volume relation, proportion by $v$. -vertellung spatial distribution, geometric d. -welle sky wave, space w., indirect $w_{\text {, ; spherical }}$ พ. -winkel solid angle (in spheradian units). -winkeleinheit steradian, spheradian, unit solid angle. -wirkung (cf Blldplastik) stereoscopic effect (of a picture); spatial e., auditory perspective, stereophonic $\theta$. (ac.)

raumzentrlert body-centered, space-c. (cryst.)

rauschen rush, gurgle, murmur, rustle (in a tube), whistle, roar. Rauschen (back) ground noise (in sound reproduction, radio, film, etc.).
Rohren- tube noise, thermal agitation of tube, hiss. Schrammenscratch noise. staub- dust noise (II.p.).

Rausch-pegel noise level (Schottky effect, thermal noise). -spannung noise potential.

Raute rhombus, diamond.

Rauten-antenne rhombic antenna. - $f$ iach rhombohedron.

rautenformig diamond-shaped, rhombic. R.E. (Richtempfang) directional reception. Reagenzalas test tube.

Reagenzien reagents, reactive means.

Reaktions-glelchung equation (of a reaction). -masse reaction mass, mass resulting from a reaction.

Rebromierung re-bromination.

Rechen-bild nomogram. -fehler error or mistake of calculation or computation. -knecht ready reckoner. -schieber slide rule. -tafel computation table.

rechnen calculate, compute, figure, reckon, count, estimate.

Rechner, Dreleck- triangulator (for trig. calculus). Verzugs-acoustic corrector (sound location).

rechnerisch arithretic, mathematical, by calculation or computation, calculative.

Rechnung, Mischungs- alligation (wath.) störungs- perturbation calculation.

Rechnungs-beamter auditor, accountant. -legung making or submitting an accounting.

recht achteraus right astern. -voraus right ahead.

Recht, Elgentums- ownership right, proprietary r., property right, proprietorship.

recht,flucht- flush, aligned, in true alignment. winkel- rightangled, rectangular, orthogonal.

rechteckige Kurvenform der EMF square emf curve, flat-topped emf c. Welle square wave. rachten winkel, im at right angles, orthogonally, normally, transversely. 
Pecht-fertigung justification, defense, vindication, exculpation. -mässlgkeit lawfulness, legality, rightfulness, legitimacy.

Rechts-anspruch legal claim, title. -beugung miscarriage of justice. -drall right-handed lay or $t w i s t$ (of a cable).

rechtsdrehend dextrogyrate, dextrorotatory, of right-handed or clockwise rotation.

Rechts-drehung dextrorotation, righthanded polarization. -elnwand demurrer, plea, objection. - $f$ all (legal) cause or case, suit, litigation. -gewinde right-handed (screw-) thread. -grund legal ground, 1. reason, argument. -händigkelt right-handedness.

rechtsichtig normal-sighted, emmetropic (of eye).

rechts-kräftig machen make legal or non-appealable, legalize, validate, ratify. -l aufende Welle dextropropagating wave.

Rechts-kurve right-hand curve, starboard $c$. mittel aufheben avoid or remove a renedy of law. - nachfolger legal successor, assign, assignee. -punkt point in or of law. -quarz dextrogyrate quartz, right-handed q. or piezo-electric crystal. -schraube right-handed screw. -spruch judgment, decision, sentence, verdict. -streit(es), Gegenstand des matter in a controversy, case at bar, in issue or in suit. -system right-handed system. -zuständlgkeit jurisdiction, competence.

recht voraus Peilkurs head-on course (with fare-aft axis of vessel or craft directed toward objective). rechtweisender Kurs true (north) course. -WIndkurs true heading. rechtwinklig right-angled, rectangular, orthogonal. -, drelfach trirectangular.

rechtzeltig timely, opportune, punctual.

recken stretch, extend, elongate, pull, rack; subject to traction, strain (metal testing); shingle, tilt (metal).

redigieren edit (film).

Reduktions-getrlebe reduction gear, step-down g. -transformator step-down transformer.

Reduktorlampe adapter-transf ormer lamp.

Referierdlenst abstracting service, reporting s., press clipping $\mathrm{s}$.

reflektierende Brennfläche catacaustic.

Reflexion, ruckweisende retro-directive reflection. -, zerstreute diffuse reflection. splegel-specular reflection, mirror-like $r$. Wellen- wave reflection, พ. echo (with one or more hops). Zlickzackstaggered reflection.

Reflexions-brennflache catacaustic. -ebene plane of reflection. -flecke flare ghosts, flares (phot.) -gonlometer ref lecting goniometer. -höhe level of $r \theta-$ flection (of waves in ionosphere). -l lehthof halation by reflection. -vermögen ref lecting power, reflectivity, reflectance. -winkel angle of reflection. -zahl number of reflections (of waves from ionosphere involving single or multiple hop).

Refraktometer, Eintauch-inmersion refractometer, dipping $r$. -grenzwinkel critical or limitine angle of a refractometer.

Regel, Drelfinger- right-hand rulo, thumb $r$., Fleming's $r$. Faustrule of thumb, rough and ready $r$. Phasen- phase rule.

Regel-gl led ręulating attenuator. -grad degree of dunamic-range or contrast regulation; waximum range of gain variation.

regellos geordnet with random orientation.

Regelloslgkeltsproblem random problem, haphazard $p$.

Regelmässigkeit regularity.

regeln regulate, adjust, arrange, set, order, control, govern, regularize.

Regel schwankungen hunting (in making 
adjustments or in regulation). Regelspannung automatic volune control (avc) potential; dynamic-range or contrast regulation or control p., senerally; control p. Schwundautomatic volume control (avc) potential.

Regel ung, Nach- re-adjustment, resetting. Rückwärts- indirect control, retroactive action, backward regulation.

Regelverstärker variable-gain anplifier, $R$ avc amplifier; noise or silencing a.; anplifier in which regulator or control potential operates.

Regen rain (in fili).

regenbogenfarbig rainbow, colored, iridescent

Regenbogenhaut iris.

Regenerat reclaimed product (rubber).

Regenversuch rain test, wet test (on insulators ).

Regiepult mixing table, n. desk. Regisseur director.

Register stop (organ). -haltigkeit des films registration stability of film. -ibertragung transfer of registry. -zug draw stop (of orsan).

registrieren record (graphically), register, file.

registrierend, sel bst- self-recording, autonatically $r$. - (er) Spannungsteiler potentiograph. - (er)

Strommesser recording ammeter. Registrierung, Daver- long-period recording, time record.

Regler regulator, governor, controller. Druck- barostat. Dynamikvolune- or dynamic-range control means, compandor, contrast regulator. Film- film traction regulator, pull r., stabilizer. Fliehkraftcentrifugal governor. Hauptmain regulator or governor (of a turbine). Hörbarkeits- audibility network; volume control means (avc). $\mathrm{Käl}$ te- cryostat. $\mathrm{Kl}$ angfarbe- tone control, tonalizer. L- L-section attenuator. Lautstärke- audibility network; volume control means (avc).
Licht- light relay, light modulator (Kerr cell, etc.); light control means. Misch-mixing control. Saal- theater fader. Steckplug-in and socket (volume) control means. T- T section attenuator, double-L-s.a. Temperaturtenperature control, thermostat, thermo-regulator, thermautostat (electronic). Tonbandbreitetone band-width control.

egler-dose control aneroid (for engine). -leitung pilot wire. -pult control desk, monitoring $d$. (in sound recording). -stellung fader setting (in film recording). Regulierband,Zeit- timing tape. regulieren, über- overshoot, overrun, excessively regulate.

Regulierschraube adjusting screw, set s.

Regulierung, Bandbreite- band-width regulation, b. switching.

Reibahle reamer.

Reibe grater, rasp.

reiben rub, grind, triturate, rasp, srate, abrade.

Reiboxydation rubbing corrosion, frictional oxidation,

Reibung, innere internal friction, solid viscosity. Schlüpfungssliding friction, slip.

Reibungs-arbeit frictional work; nagnetic hysteresis. -kegel cone of friction. -moment friction torque. -widerstand, schall-acoustic resistance. reich,flächen- with many faces or sides, polyhedral. linien- rich in lines, with abundance of 1 . Reichsgesetzblatt Imperial (German) Law journal or Gazette.

Reichweite (eines Empfängers) (distance) range (of a receiver apparatus), distance-getting ability of a r.a. Nenn- rated range, nominal $r$.

Reichweitenverteilung range distribution. reif ripe, mature, Reif(en) ring, hoop, tire, collar. 
Reifkeim digestion nucleus.

Reifung ripening, digestion of emulsion (of film). Nach-maturing (of filin).

Relfungskörper ripening (acclerator) substance, sensitizer.

Reihe series, row, succession, sequence, train, rank, file, bank (of contacts, keys, etc.)

Reihe, an dle - kommen take (one's) turn. der - nach sequentially, in sequence or succession. gegensinnlg in - series-opposing. glelchslnnig in - series-aiding. unendliche - infinite series.

Relhe,Bild- sequence or series of pictures, frames or pictorial actions. Fourler-, doppelte double Fourier series. Impuls- series, succession or train of impulses. Potenz- exponential series, power s. (math.) Tasten- row or bank of keys.

Reihen-anordnung seriation or series connection (of resistances and condensers, etc.) -bilder sequence of pictorial actions or pictures, motion pictures. -entwickelung expansion in a (power) series, power seriation. fertigung mass production, assembly-line $p$. -folge sequence, consecution, succession, series, order. folge energet Ischer Bevorzugung sequence or order of energy preference. -funkenstrecke multiple spark gap. -glied series elenent (of a network). -impedanzglied series impedance element, $s$. impedor.

-ryhrenmodulation constant-voltage modulation. -verlustwiderstand equivalent series resistance (of a condenser).

rein (es) farbengleiches("einheitliches")Gelb psychologically unique yellow. $-(e)$ skal a true scale. -(e) Sprache. clear voice. -(e) sinusförmige Bewegung plain harmonic or sinuous motion. $-(e r)$ Ton pure note or tone, simple $t$. Reinartzschaltung Reinartz circuit schene (comprising both capacity and inductance or tickler-coil feedback); also; a special shortwave receiver circuit scheme. reinigen purify, refine, clean, cleanse, wash, purge, scavenge, separate, rid.

Relniger, Klang- acoustic clarifier (on ldspk. baffle). stromharnonic eliminator, filter, stopper or excluder, smoothing means.

Relnigungskrels smoothing circuit, filter c., low-pass f. (following a rectifier in a power unit, to remove ripples).

Rein-kathode bright enitter. -kohle solid carbon.

Reinton (cf Heulton) note with one mode of vibration, single-

frequency tone. -blende biasing stop, noise-reducing s., shutter, vane or gate. -schaltung noiseless recording circuit organization. -verfahren noiseless recording (on film).

Reissblei graphite.

reissen tear, pull, drag; pluck (a string or chord).

Reiss-festlgkelt resistance to tearing or breaking, tenacity, tensile strength. -geblet discontinuity, cut-off or break-off region (of oscillations). -kohle crayon, charcoal crayon. -länge breaking length (cable and paper testing). -verschluss slide fastener, zipper.

Reiter slide contact, slider, cursor. Gewichts- rider. -rädchen jockey roller, j. wheel.

Reiz stimulus, excitation, stimulation. Licht- luminous stimulus, light s. Kerven- nervous stimulus. Seh- optical or visual stimulus or excitation. -schwelle threshold of sensation or stimulus, liminal value of stimulation.

Relais, elektro-optisches electrooptical cell, Faraday c., light relay depending on $F$. effect. $-m / t$ mittlerer Ankerruhestellung 
neutral relay, $r$. with differentially moving armature.

Bremzylinder - dashpot relay. Differenzial- differential relay, discriminating $r$. Distanzdistance relay. Elektronenelectron relay, thermionic $r$, Flacker- flashing relay. Fortschalt- stepping or inpulsing switch or relay. Gegenstromreverse-current relay. Halteholding relay, restraining $r$., guard r. Kupfermantel- copperjacketed relay. Licht- light relay (converting current into light variations) (e.s., Kerr cell, Karolus c., modulated neon lamp, etc.). Licht-, elektrooptisches electro-optic cell, Faraday c., light relay depending on $F$. effect. Minimal-no-load relay, under-current $r$. Ruckstromreverse-current relay. Schneideanker- knife-edge relay, schnellhigh-speed relay. Spannungsunder-voltage relay, low-v.r., no-v.r. stever- pilot relay. Stufen- relay with sequence action. Tast- keying relay, $r$. key. Tauchkern- plunger relay.

Verriegel- locking relay.

Verzogerungs- slow-acting relav, time-lag $r$.

Relais-aberregung de-energization of a relay. -anker relay armature. -vorwähler relay preselector.

Relaxations-schwingungen relaxation, sawtooth or ratchet oscillations. -zeit der lonenwolke relaxation time of ionic atmosphere.

Relief (cf Raumwirkung und Plastik) plasticity (of pictures), plastic effect, three-dimensional appearance of telev. pictures. -schreiber embosser. -träger relief support (of embossed film).

Reportage news reporting or gathering work. -film newsreel, news" film, topical $f$.

reproduzierbar, nicht- non-reproduci- ble, non-controllable, non-repeatable.

Requisitenraum property room.

Reserve,Leistungs- margin of power, standby p. -lampe spare lamp.

Reservierungsmittel resist, reserve (in calico printing, wetal etching, etc.)

Resonanz entgegenwirkend antiresonant.

Resonanz, in resonant, resonating or in resonance with, in unison with, in tune with, syntonized. in - bringen resonate, cause to be in resonce with. -, untersynchrone subsynchronous resonance, submultiple $r$.

Resonanz, Dreh- torsional oscillation resonance. Hohlungs- cavity resonance (of rhumbatron).

Nebenschluss- parallel resonance. Schittel-vibration resonance.

Resonanz-anzeiger resonance indicator (e.s., vibrating reed instrument), tuning $i$. -beschleuniger magnetic resonance accelerator (e.g., cyclotron comprising two dees or half hollow cylinders and spiral beam, and other forms of beam tubes using ions, positrons and electrons), induction electron accelerator, also called rheotron and i. accelerator. -boden sounding board; belly (of violin). fluoreszenz resonance radiation (fluorescence).

- frequenz natural frequency, resonance $f$. frequenz, Untersubmultiple (resonance) frequency, subsynchronous $\mathrm{f}$. -hohl raum resonance cavity or chamber (rhumbatron). -korper sound or sounding board (or other resonant structure). -kreis, spannungsseries resonant circuit. -kreis, strom- parallel resonant circuit, tank c.

Resonanzkurve, schiefe unsymmetric or skew resonance characteristic. -, zweihöckrige, zweispitzige, zweiwellige double-hump or double- 
peak resonance curve.

Resonanzkurven-einsat te lung, - senke crevass of resonance curve, dip. Resonanz-röhre resonance or tuning indicator (mostly glow tube), flashograph. - schärfe selectivity, sharpness of resonance or tuning- - schwingung resonant vibration, sympathetic v., co-vibration. -spannung resonance potential, radiation $p_{\text {. }}$ p. resulting in transition of electron from ground state to next orbit and enission of resonance line spectrum.

-spitze resonance peak. -spitze, abgeflachte flat-topped resonance crest. -sprung transition of electron resulting in emission of radiation, -strahlung resonance radiation (of vapor lamp). Resonator, Hohl raum- cavity resonator, chanber r., enclosure r. Quarzpiezo-electric resonator, tuning or resonance indicator.

Resotank micro-wave generator (electron-oscillation tube or magnetron t. conf ined in hollow space or sphere), cavily resonator magnetron. Rest-abweichung residual aberration (of lens). -dampfung net transnission equivalent, net attenuation, - flüssigkeit residual liquid.

- härte residual hardness (of water).

- keim residual nucleus. -pl asma residual electrons, plasua (region practically without resultant charge). -strahl residual ray, $r$. radiation, "reststrahl".

Retortenhelm retort head, $r$. helm. Reusenantenne cage antenna.

Reversionspendel reversible pendulum, Kater $p$.

Revolver revolving nosepiece (in microscope). Spulen- coil switching assembly (for wave-band change). -kopf lens turret (of canera).

Rezension review, criticism.

Reziprok, Widerstands- inverse resistance, resistance reciprocal (= conductance).

Rhodan thioyanogen, sulf oyanogen.
Rhombendodekaeder rhombic dodecahedron.

Rhomboeder rhombohedron.

Rhombus rhomb(us), lozenge.

Rhythmus, im in tune, synchronism, rhythm or unison with; at the rate of.

Richt-antenne directional antenna, unilateral a. -bogen clinometer. - charakteristik directional characteristic, space pattern. -dorn mandrel. -effekt rectification effect; directional action. -empfang directional reception. -empfänger, Einpeilen auf Punksender tune directional receiver to radio station (to take bearings).

richten direct, turn, arrange, adjust, set, straighten (out), dress, align, lay (a gun), train or aim (at). -auf train on, aim at. sich - nach be suided by.

richtende Eigenschaft directional property (of a loud-speaker, screen, antenna, etc.)

Richter, strom- rectifier of thyratron, ignitron or other mercury-arc type. Wechsel- inverter, inverse rectifier, $d-c$ to a-c transverter.

Richt-fähigkeit directivity, directional property. -faktor rectification factor. -funkfeuer directive beacon station, d. transmitter s., radio range s., beall $s$. -gerät bombsight, sight.

richtig right, correct, just, true, or tho. -, farben- orthochromatic (phot.)

Richtigkeit correctness, accuracy, faithfulness, fidelity (of reproduction).

richtigphasig true-phased, in correct phase relationship.

Richt-kanonier gunlayer. -kennlinie rectification characteristic; directional diagram, space pattern. -konstante rectification constant. -kraft directive force, directing f.; versorial f. -l autsprecher dírectional loudspeaker. - linie guide line, guiding 1., directive. 
-mass standard gage. -mikrophon directional microphone (uni-d., bi-d., ultra-d., etc.) -moment torsional rigidity (couple re-quired to twist spring a certain angle). -platte orientation plate (iicr.) -scheit rule, straight edge. -sendeanlage beam station, radio beacon s., radio range $s$. -spannung output voltage furnished by rectifier, $d-c$. potential of r. -strahler directional antenna; d. louds peaker or projector of sound. -strom output current (d.c.) of a detector; rectified (d.c.) component of plate current. Richtung, ausgepeilte bearing. Anflug- direction of approach, homing direction (of airplanes). Aus - $f a l$ sche misalionment (of track or image). Glelt- slip direction (cryst.) Kristallachsencrystallographic axis orientation. Vorzugs- privileged direction. Richtungs-anzeige, eindeutige unidirectional direction-finding (with sense-finding). -anzeiger direction-finder, d. indicator; course indicating radio beacon, -bestimmung direction finding.

Richtungscharakteristik directive or directional characteristic or diagram, space pattern. -DoppelKreis- figure 8 characteristic, tangent-circle pattern.

Richtungseffekt head and tail offect (in developing machine); directional action.

richtungsempfindlich with directional response, dependent upon direction or orientation, non-astatic.

Richtungs-und Entfernungsbestimmung ranging (say, by echo method).

Richtungs-fehler deviation (of pencil or beam), directional distortion (telev.) -funkbake(zum Anfliegen) runway localizing beacon (terminal marker, used in approach procedure). -grter director, directive antenna. -hören aural determination of direction (of airplanes), airplane spotting or locating. -kurve directrix. -navigatlon directional navigation. -quantelung directional quantization. -sender beacon station, beall s., radio range $s$. -sinnbestimmung sense-finding, sensing(determines sense of direction or absolute d.)

richtungsunempfindlich non-directional, astatic.

Richtungs-vermbgen directivity. - verzerrung direction distortion (in sound reproduction). -zerstreuung scatter of direction (beam).

Richt-vermbgen directivity. -verstärker amplifying detector, platecurrent d. -verstärker, schlrmgitter- screen-grid tube with plate-current rectification. - we iser radio beacon, $r$. range, beall station. -widerstand unidirectional resistance (to flow), valve effect, rectifier $\theta$. -wirkung directional ef fect, directivity. -zylinder directional cylinder.

Riefe groove, furrow, channel, flute, slot, milled knurl. Ziehdrawing groove, d. scratch.

riefeln mill, groove, knurl.

Riegel (sliding) bolt, lock, fastening.

Riemen strap, belt, band, thong. -, halbgeschränkter quarter-turn belt. Umhänge- shoulder strap.

Riemen-fett belt dressing. -scheibe belt pulley.

Rieseln rippling, oushing, trickling; swarming (of film); friable, non-caking condition (of materials).

Riesel turm scrubber, wash tower, spray t. or column,

Riffel-fal tenl autsprecher loudspeaker with folded and corrugated horn. -l autsprecher Riffel loudspeaker (with diaphragm corrugations and substantially horizontal radiation.) -membrane pleated 
diaphragm, d. with circular corrusations.

riffeln furrow, channel, groove, ripple (flax).

Rille furrow, groove, track (of phonograph disk).

Rille, Führungs-, Schall-groove or track with undulations (of phonograph record or disk).

Rindenschicht cortical layer.

Ring, geteilter split ring, segnented $r$. Absaug- (cf. Saugspannung) annular or ring-shaped collector electrode. Aufschraub- lens flange. Befestigungs- ring fastener, fastening $r$. DebyeScherrer-D.-S. ring or circle, powder pattern, Hull $r$. Dichtungsgasket ring, gland. Lauf- race, raceway, ball-race (in bearing). Peil-direction-finder loop or frame. Schleif- slip ring, collector $r$. Teil- index ring. Verschluss- locking collar (of magnifier).

Ring-bildung ring formation. -bolzen eyebolt. -elektroden, abbildende focusing rings. -entladung ring discharge. -figur zone plate (of Fresnel), Huygens $z$.

ringförmig ring-shaped, annular, cyclic, toroidal, toric.

Ring-kopf annular recording head. -leitung, Antennen- multiple-receiver connection to antenna. -lochscheibe filn scan disk (with apertures arranged circularly). - übertrager repeating coil.

ringungesättigt cyclically unsaturated, containing an unsaturated ring.

Ringwulst tore, torus, toroid, "doughnut".

Rinne channel, groove, gutter, trough.

rinnen run, flow, trickle, leak. Rippe rib, fin, vane, ripple, flute, corrugation, undulation, section (of a radiator). Kühl-cooling fin, c. flange, radiator fin.
Rippentrichter ribbed funnel. Riss fissure, crack, flaw, rent, tear, sap, opening, elevation (say, front or side).

Riss, Haar- micro-flaw, hairline crack,craze, capillary hair

fissure. Schatten- silhouette. Seiten- lateral elevation, side $\theta$. $\mathrm{Spalt-cleavage}$ crack, c. $\mathrm{f}$ issure.

Riss-bildung cracking, fissuration. -festigkeit crack strength, c. resistance. -härteprobe abrasion test, scratch $t$.

Rissigkeit, Schweiss- chinking or fissuration due to welding.

Ritze rift, slit, fissure, crack, chink, cleft, scratch. stimmglottis, glottic catch or cleft.

Ritzhärte scratch hardness, scleronetric h. -prüfer scleronieter, scratch hardness tester.

Ritzspalt scratched slit (for instance, in silver coat, in sound recorders).

Roentgen.....s. Röntgen...

roh raw, coarse, rough, unref ined, unwrought, unworked, unf inished, in blank form; broad (radio tuning).

Roh-block (raw) ingot. -bramme slab, ingot. -film blank fili, raw stock, -formel empirical formula. -gang abnormally fast drive or run, irregular working (netallurgy). - ling blank, unworked, unf inished or unfashioned piece or part.

Rohmannshaut Rohmann's skin.

Rohr (s.a.Röhre) tube, valve (radio); pipe, duct, conduit. -, hartgeschlossenes rigidly. terminated tube. Abzugs- vent stack (of an arc-lamp) Befehls- pilot or master thyratron (firing first in a trigger circuit). Beobachtungsobservation tube (of electron microscope). Dunst- ventilating pipe. Folge- piloted thyratron (fires second in a trigger circuit). 
Hebe- syphon (tube), Hosen- syphon or $\mathrm{Y}$-pipe. Hill-encasing tube, jacket pipe. Neben-side or branch tube or pipe. Perlbead tube (tilled with glass $b$. to break up a fluid). Skalenscale tube (of spectroscope). Stern-astronomical telescope. ibersetzungs-( in Schwundregelung) converter tube (in ave circuits, between regulator and regulated tubes). Zähl-counter tube (Geiger - Müller).

Rohr-bildung tubing or piping action. -blatt reed (of clarinet). Röhrchen tubelet, capillary tube. Schreib- pen, syphon (of a recorder).

Röhre (cf Rohr) tube, valve, pipe, duct, conduit. -, abgeschmolzene sealed of $f$ tube. -, fremder regte, fremdgesteuerte master-excited tube (with independent drive circuit). -, tusslose 10 tal tube. -, gassgefillte gas-content tube, gaseous t.; gassy t. -, harte hard tube, highly evacuated $t$. -, indirekt geheizte heater-type tube, indirectly heated $t$. negativen Widerstandes dynatron ascillator. - quetschfussfreie loctal base tube. -, selbsterregte selt'-excited or self-ascillatory tube (in a direct drive circuit). -, weiche soft valve, ionic v., gas-filled tube, gassy $t$. - , zer legbare demountable tube. Röhre, Abstimm- tuning indicator §low-tube (e.g., flashograph), $c,-r$. indicator $t$. or magic eye. Achtelektroden- octode. Amplituden(glinm)- glow-tube amplitude indicator, resonance i., tuning i. of neon type. Ballast-absorber valve, a. tube (takes energy during spacing periods): Beelnflussungs-modulator tube. Bildabtaster, mit mechanischer $\mathrm{Bl}$ ende Farnsworth dissector or electron camera tube, Dieckmann Hell magnetic scanner, Campbell-Swint on $c .-r$. scanning or pickup tube. Bildschrelbetelevis or tube, viewing $t_{\text {. }}$, picture-reproduaing tube using cathode rays. Bildspelchersignal- or charge-storage tube, normal iconoscope. Bildspeicherund $\mathrm{Bi}$ ildwand ler-supericonoscope. Bremsfeld-( cf Bremsfeldschaltung) retardation valve, BarkhausenKurz retarding-field or positivegrid t. Doppelgitter-bigrid tube, b. valve, double-grid t. Doppelsteverend- power pentode wherein cathode grid is connected with control g. rather than with cathode. Doppelzweipol- duodiode. voppelzweipol-Dreipol- duodiodetriode tube. DoppelzweipolVierpol- duodiode-tetrode tube. Dreielektroden- triode. Dreifachthree-unit tube, three-purpose $t$. Eichel-acorn tube. Einschlusssealed. tube. Elektronen- thermionic tube, electronic t., electron tube, e. valve. End- power tube, end t. Entladungs-discharge tube, vacuum t. Exponential- exponential tube, variable- $\mu$ t. Fünfpolpentode tube (either of power type or of screen-grid type). Gasentladungs- gas discharge tube or valve. Geräuschunterdrückungssquelch tube, noise-reduction $t$. Glättungs- glow-tube stabilizer. Habann- type of split-anode magnetron, dynatron ascillator (strictly, dynatron uses secondary electrons). Haupt- main ascillator (in independent drive system). lonensteuerungs - thyratron. I solier-butfer tube, isolating $t$. Kathodenstrahl- thermionic tube (obs.); beam t.; Lenard cathoderay t.; Braun and other c. $-r$. tubes working with beam and screen such as oscilloscope, iconoscope, emitron, kinescope, Farnsworth dissector and oscillight, etc. Klppentladungs - time-base discharge tube. Klein-miniature tube. Knopf- shœbutton tube, 
acorn t. Kopplungs- coupling tube (RF input t. associated with antenna). Kraftschwing- power oscillator. Lautzeit-drift tube, beam t., tube involving transittime or phase effects. LenardLenard $c_{.}-r$. tube (used in radiology and experiments on phosphors). Magnetfeld- magnetron type of tube working with constant magnetic field (strictly, a $\mathrm{m}$. operates with variable magnetic field), permatron. Mass-measuring tube, gage t., graduated t., burette. Mehrfach- multi-valve, dual- or multi-purpose tube, multi-unit t. Mehrgitter-multigrid tubo (say, tetrode, pentode, etc.). Misch-mixer tube. Misch selbstschwingende self-heterodyning mixer tube. Modulations- mixer tube, modulator $t$. Mosaik-, doppelseitige image-multiplier iconoscope. Negativwiderstandskallirotron or dynatron types of negative-resistance tube. Niveaulevel or leveling tube. 0xydkathoden-tube with oxide-coated filament, dull emitter t. Plationamplifier tube with filament placed between control grid and plate; (one model has photo-electric g.) Pressboden-loctal base tube. Prüf-,für Fernseher phasmajector, monoscope, monotron. Quecksi l berhochdruck- high-pressure mercury (-vapor) discharge tube. Quersteuer- cross-control beam tube, drift t., t. predicated for its operation on transverse action on beam. Resonanz- resonance indicator, tuning i. (mostly glow-tube), flashograph. Röntgen-, mit Heizkathode hot cathode X-ray tube, Coolidge t. Schirmgitter-, Schutzgitter-shield grid tube, shielded-plate t., screen-g. $t$. Schwing-, fremderregte master-excited oscillator tube (in an independent drive circuit).

Schwing-, selbsterregte self-ex- cited or self-oscillatory tube (connected in direct drive circuit). Sechspol- hexode. Si ebenelektrodenheptode. Sondenbildfang- Farnsworth dissector tube. Speicher(signal) storage or storing tube (telev.) Speicher-und Bildwandersuper-iconoscope. Stab- arcotron. Stever-master, drive or pilot oscillator, exciter tube. Trennisolating tube, buffer $t$.

Trennstrom- spacing valve. Triftdrift tube, beam t. (e.g. Klystron). Ubersetzungs- Umkehr- converter tube (used in avc schemes between regulator and regulated tubes). Vakuum- vacuum tube, discharge $t$. Vervielfältigungs- multiplier, multipactor. Wasserkühl- watercooled tube. Wiederyabe-, Braun'sche Braun cathoce-ray or electronic picture reproducing tube. Zeichenstrom- marking valve. Zweiel ek troden- diode. Zweifachzweipol- duodiode tube. Zweifelddrift tube working with two fields (Heil types of tubes). Zwischenstrom- spacer valve.

Röhren-empfang mit gleichzeitiger HF und AF Verstärkung dual or reflex (valve) reception.

- fassung, federnde cushioned socket or anti-microphonic tube or valve-holder. -fuss pinch, press, squash, stem or foot of a tube or valve. -fuss, umgebördelter re-entrant squash or press. -kapazität interelectrode capacitance. -kappe top cap (to bring in control grid lead). -kolben bulb of a tube, vessel or container of a tube or valve. -konduktanz transconductance. -löscher tube quench. -lot pipe solder. -mehraufwand tube complement. - prüfer tube tester, t checker. -rauschen tube noise, thermal agitation of a tube, t. hiss, valve rustle. -sockel tube base. - spannungsmesser vacuum tube voltmeter. -sperrung cut-off of tube. -stativ 
telescope tripod. -stempel press or squash of a valve or tube. -summer vacuum tube $A F$ oscillator. -träger tube or pipe support. -übersteuerung tube overloading (causing blasting). -vol tmosser vacuum tube voltmeter. -voltmesser, selbstgleichrichtender self. rectifying tube voltmeter. -wippe kipp relay, trigger circuit. -wulst tubular tore, "doughnut". -zerplatzen, nach innen implosion of a vacuum tube. -zwischenstecker tube adapter, valve a.

Rohr-holz cane. -krümmer pipe bend, p. elbow, p. knee.

-leitung, Hohl- co-axial or concentric line, pipe 1.; wave guide (dielectric or conducting). -pfeife reed pipe. -schl ange coil (of pipe), worm, spiral tube. -schlüssel pipe wrench, -sitz cane seat. -streifen skelp or strip (for forming tubes). -stutzen short piece of pipe (serving as an opening, outlet, socket, or connection), nipple, short cylindrical piece, bush or sleeve: -verlegung pipe laying. -verstopfung pipe clogging, stop-up, choke. -welle dielectric wave.

Rohstiick blank.

Rollbahn cycloidal path (of electrons); roller type conveyor; runway or taxiway (of airfield). -kreis cycloidal path (motion of electrons).

Rolle roll, roller, pulley, reel, spool, coil, sprocket; records, file-wrapper, file, docket.

Rolle, Abzieh-pull-down spool or sprocket. Auflauf- take-up reel. Auftrags- application rolzer, inker, inking $r$. Belichtungsprinting drum (of film printing machine); sound recording d., scanning point, translation p. (in s. film reproduction).

\section{Röntgenbeugungsaufnahme}

Druck- impression roller; presser or pad $r$. (in film feed). Farb-inking roller, i. wheel. Film- reel, takeup r. or spool. Filmtransportfilm feed sprocket. Führungssprocket (wheel). Gummi andruckrubber-tired presser or pad roller. Haupt- (part played by) leading star. Kordel-milled or knurled roller. Leit-idler, guide roller. Nachwi ckeltake-up sprocket. Schnurgrooved roller, pulley.

Schwungmassen- rotary stabilizer, roller with fly-wheel action, impedance wheel. Seilpulley. Spann-tension roller (m.p.). Sprossen-sprocket drum or wheel. Titel-part played by leading star. Transport- sprocket wheel (film feed). Umlenk-flanged idling roller, deviating $r$. Vorratsmagazine, m. roll. Vorwickelupper feed sprocket, pull-down s. (m.p.). Zacken-sprocket wheel.

rollen curl (of film).

Rollen-darsteller,Haupt- principal, star. fenster roller film gate. -käfig roller cage. -l aufbahn mit Gummiüberzug rubber-tread of a pad roller. - nummer docket number, register n. -quetsche squeegee. -scheibe chain sheave. -zug block and tackle.

Roll-glasschneider wheel glass cutter, g. cutting w, -kassette film roll darkslide. -kreis cycloidal path (of electrons). - schweller crescendo pedal (organ). -treppe escalator. -walze roller squeeze.

römi sche Kerze Roman candle, magnesium torch, smoke pot.

Röntgen-aufnahme röntgenography, $\mathrm{X}$-ray picture, skiagraph. - beugungsaufnahme X-ray diffraction exposure or picture. 
Röntgenbremsstrahl

-bremsstrahl Röntgen rays caused by collision and checking. - dosismeter X-ray dosimeter. -einheit roentgen (international $X$-ray quantity unit); Xu, X unit, Siegbahn u. (wavelength). -messer roentgenmeter, iontoquantimeter. -photographie röntgenograph, X-ray photograph, skiagraph. -röhre mit

Hei zkatho de hot-cathode X-ray

tube, Coolidge t.-röhre, fensterlose windowless X-ray

tube. -röhre,Hartwerden hardening of X-ray tube (due to cleanup and loss of vacuum).

-strahl enhärtemesser penetrometer, qualimeter. - strahlenmesser röntgenmeter, iontoquantimeter, dose meter, dosimeter, intensimeter, roentgenstrahlen-undurchlässig radiopaque.

Rose,Kompass- card of compass, scale of $c$.

Rosettenbahn rosette-shaped path (of electrons).

Rost rust; grate, gridiron. Etagen- step grate.

Rostanfressung corrosion, honey combing, pitting, tubercular or channel-shaped corrosion.

rösten calcine, roast, sinter. Rost-festigkeit corrosion resistance, non-corrodibility. - grübchen, -narbe pit.

rostsicher non-corrodible, corrosion-resistant, rust-proof, non-rusting.

rot,hell-bright red, of brightred incandescence. kirschcherry red, of cherry-red incandescence.

Rotation, behinderte hindered or inhibited rotation (of molecules).

Rotations-bewegung rotational motion, rotary m. -gruppe, räumliche space rotation group. -körper body or solid of rotation or revolution.

rotationsparabol isch full-parabolic.
Rückführung

Rotations-quantenzahl rotational quantum number. - schwingungsband rotation vibration band.

- schwingungsspektrum rotation vibration spectrum.

rotationssymmetri scher Körper body presenting rotation symmetry.

Rot-auszug red record (color film). -bruch red-shortness (of metal). -bruchversuch hot breaking test. -buchenholz red beech.

Röteltonung russet toning, brownreddish $t$.

rotempfindlich red sensitive.

Rotglihhitze red heat (of incandescence).

rotierend(er) Dämpfer rotating stabilizer (film feed). - (e) Uml aufverschlussblende rotary flicker shutter, $r$. light-cutoff.

Rotorfunkenstrecke spark-gap rotor.

Rotterdammgerät radar.

Rotverschiebung shift of spectral lines toward red or longer waves.

Rouleauverschluss roller blind shutter.

Ruck jerk, jolt, sudden shift or motion, tug.

Ruickbewegung back stroke, return s., retrogression, flyback (of beam, telev.)

rückbilden form again, re-form.

Rück-bildung back (reversible) reaction, regression; re-formation. -dehnung damping capacity (of metals). -dreher,

Phasen- phase lagger, p. shifter. -druck reaction pressure, back p. Rückenwind tail wind.

Rück-flussdämpfung structural return loss. -flusskijhler reflex condenser, return c., returnflow cooler. -führfeder retractile spring, restoring $\mathrm{s}$. - führshnecke return screw conveyor, return worm. - führung 
return lead, r. path, r. circuit; flyback, retrace (telev.) - gang return, retrogression, decline, backstroke; flyback (of pencil).

rückgekoppeltes Audion regenerative grid-current detector, ultraudion.

Rück-gewinnung recovery, recuperation (of current or energy); reclamation, regeneration. -griff(cf Durchgriff) inverse grid transparency. -heizung extra or additional heating (of cathode, from other electrodes). -hörbezugsdämpfung side tone reference equivalent. -hören side tone. -hub back-stroke, return s. -kehrpunkt cusp. -kippgeschwindigkit flyback speed, time-base unlock s. rückkoppeln regenerate, feed back (a tube), put in reactive coupling relation (either positive or negative).

Rückkopplung regeneration, reactive coupling, feedback c., reaction. -mit Hilfsfrequenz super-regeneration (using quench potential).

Rückkopplung, lonen- ion feedback. Mehrfach- multiple regeneration. Pendel - super-regeneration (with quench potential). Reinartzfeedback (of Reinartz) using both capacity and inductance. schall-acoustic feedback, a. regeneration.

Rückkopplungsaudion regenerative grid-current detector, ultraudion.

rückkoppl ungsfrei non-regenerative, free from feedback action.

Rückkoppl ungs-kreis regenerative circuit, reactive c., feedback c. -spannung positive bias potential, p. quench potential (impressed upon gria, in superregenerative circuit organiza-

\section{Rückspiegelung}

tion). -sperre reaction suppressor, "vodas, "feedback s. -spule tickler coil, feedback c. -unterdrückung decoupling, balancing out, tuning out, reduction or suppression of feedback.

Ruicklauf return of pencil, flyback or retrace of cathode spot or beam. -der KIppspannung flyback of sweep potential, return of time-base to zero.

rückl aufend recurrent, retrograde, in return or opposite direction. $-($ er) Blitz return lightning stroke.

Rücklauf-verdunkelung flyback or return trace elimination, blackout of flyback, gating (by blanking pulse). -zelt retrace period, flyback p.

Rück-leiter return wire, r. lead, r. conductor. -leitung, Erdground return (circuit). -meldefeld revertive signal panel. -melder revertive signal or position repeating means, check-back position indicator (in telemetric or remote-control work). - nahmestreit action for withdrawal.

Rückprall rebound, bump, reaction, repercussion, strikeback, back-stroke, recoil, return, back-fire. -elektrode (cf Prallelektrode) rebound electrode, target e., impactor e. (in Weiss mesh multiplier). Rück-projektionsanlage reprojection equipment; back projection e. -reaktion back reaction. -schlag see Rückprall. -schl ag, Bogen- arc-back. -schl agventil check valve.

rückschreitende Welle regressive wave, reflected w.

Rück-seite back side, posterior face, rear surface, reverse. - sicht, mit - auf with due regard for, because of, in the light of. -spiegelung specular 


\section{Rückspielen}

reflection, regular r. -spielen playback, dubbing. - sprung ( $c f$ Rückprall) rebound, ricochet. rijckspul en re-wind.

Rückstand, in - kommen fall behind, be in arrears. Gas- residual gas, remnant of $g$. (or vapor). Kondensator- residual charge of condenser. Trocken-dry residue.

rückständig in arrears, backward, not progressive, retrogressive, reactionary.

Rückstellkraft restoring force, resiliency, retractility, elastic f., restitutive $f$. or pressure.

Riickstoss repulsion, rebound, backward push, recoil, back stroke, reaction, shock. -atome recoil atoms. -bahn recoil track. -elektron recoil electron, Compton e. -potential recoil potential.

Rijck-strahl echo, reflected wave, reflection. - strahlbrennfläche catacaustic. - strahldiagramm back reflection photogram.

- strahler reflector, re-radiator. -strahlung, spiegelnde specular reflection, regular $r$. -strahlungsfaktor reflectance, reflection factor. -strahl ungsmesser reflectometer. -strahlverfahren (Ultramikr.) reflection method (ultra or electron microscope using a thin foil). -strom inverse current, backward c. (in rectifier). -stromrelais reverse-current relay.

rücktreibende $\mathrm{Kr}$ aft repelling force, reflecting f: (acting on electron pencil).

Rijckverwandlung reconversion. rïckwärtige Stromstossgabe revertive pulsing.

Rückwärts-l eitung high-resistance direction. -regelung indirect control, retroactive control action, backward regulation. Rïckweg return path.

\section{Ruhebel I chtung}

ruckweise by jerks, jars or jolts, intermittently, discontinuously, spasmodically.

rickweisende Reflexion retrodirective reflētion.

rückwerfende Brennfläche catacaustic.

Rick-werfer rejector, reflector, throwback means. -wirkung, Anoden- anode or plate feedback or reaction. -wirkung, Blind-reactance (either in the form of inductance or capacitance). -wirkung, Schallacoustic impedance (comprising resistance and reactance, the latter being either in the form of inertance or compliance, corresponding to inductance and capacitance in electricity). -wirkungslertwert reactive admittance, susceptance (either capacitive or inductive). - zugfeder retractile spring. -zugtaste back-spacing key. - zündung backlighting, arcback, backfiring; backlash (imperfect rectification, in valves).

Ruf ring, ringing, call (signal). Not- SOS or distress call, mayday (radio telephone d. call). Rufer developer.

Ruf-horn signal horn. -zeichen, Funkstellen- station call, cude-sign, code signal, "signature".

Ruhe rest, repose, quiescence, silence, $Q$. state, unmodulated, unexcited or neutral condition, stationary equilibrium.

Ruhe.... (in Zusammensetzungen) no-signal..., when in unexcited or unelectrified state, $Q$ or quiescent condition (of a valve).

Ruhe-arbeitspunkt $Q$ point, quiescen condition (of valve). -belichtung unmodulated lighting, nomodulation 1., average or steady illumination, no-sound 7. (in variable-density sound 


\section{Ruheelektrizität}

recording). -elektrizitätslehre electrostatics. -kontakt

spacing contact, rest or inoperating c. (closure occurs upon de-energization of relay), contact of back part of key lever. - lage equilibrium position, balanced p. - lage, Strahlspot zero. - licht unmodulated lighting, no-modulation 1., average illumination, steady i., no-sound i. (in variable-density sound recording). -masse (der Teilchen) rest mass (of particles).

ruhendes Bild still picture, "still", unanimated p., ordinary photograph.

Ruhe-punkt point of rest, fulcrum; quiescent or $Q \mathrm{p}$. (on tube characteristic).

- schwärzung unmodulated density, no-sound d., (in variabledensity sound recording).

- spannung bias potential, steady p.; no-signal p., repose p., $Q$ point p. -stellung, Anker-, Relais mit neutral relay. - stellung, Tonstreifenzero modulation of track.

-streifen unmodulated track. -strom no-signal current, quiescent c.; anode-feed current (d.c. component of anode c.) -strombetrieb closed-circuit operation. -trägerfrequenz center frequency, resting $f$. (in $f-m$ ). - transparenz unmodulated transmission (of film). -wert (cf Ruhezustand) steady, static, siationary, no-signal, neutral or quiescent value, $Q$ point (e.g., when a tube furnishes no oscillations or no potential difference exists between grid and filament). -winkel angle of repose, a. of friction (rarely used).

-zustand state of rest, s. of quiescence, $Q$, neutral or repose condition.

\section{Rutengänger}

Rürapparat stirring apparatus, agitating a., stirrer, agitator. rijhren stir, agitate, puddle, pole, rabble.

Rühr-scheit paddle, rake, stirrer.

- stab stirring rod, s. pole, paddle, rabble.

Rumpf body (of engine, etc.); trunk; hull (of a ship); fusel lage (of airplane); core (of ions), kernel (stable inner electron group); negative unvaried portion of luminous glow (in g. tube). Ionenion core.

Rumpfelektron inner electron (forming part of core, kernel or rumpf e. group).

Rundblick-aufnahme panoramic view, p. photograph, panning shot, pan, pam (m.p. slang). - fernrohr panorama sight, panoramic telescope. -kamera panoramic camera.

runderhaben convex.

Rundfunk, Leitungs- AF wire broadcast, $A F$ rediffusion.

Rundfunk-tonfilm sound telecast, s. telecine (of $\mathrm{film}$ ), broadcast of television program with sound accompaniment (or of video and aural signals simultaneously). -welle (cf viellenabgrenzung) broadcast wave, medium-frequency w., b. band (frequencies between 550 and $1600 \mathrm{kc}$ in USA).

rundhohl concave.

Rund-kerb round notch, Charpy n. (with root radius). - laufen der lonen circulation of ions. -strahl antenne polydirectional antenna, omni-d.a.

runzelig wrinkled, puckered, shriveled; mottled, reticulated (of film gelatin).

Runzeln mottling, wrinkling. Ruissel nose, snout, nozzle. Rute, wiinschel- dousing or divining rod. Rutengänger, Wünschel- douser. 


\section{Rutherford}

242

Rutherford-Bohr Atomnodell R.-B. atom model or conception. Rutil rutile, titanium dioxide. Rutsche chute, shoot, slide. rutschen glide, slide, slip, chute. Rutsch-erscheinung slip phenomenon
Ritteltisch

(cryst.). -kupplung slip clutch. Rüttelformmaschine jolt molding machine, jar raming $m$. riitteln shake, jolt (by jerky motion).

Pitteltisch jarring, jolting or bumping table. 
S. A. (Selbstanschluss-System) automatic telephone system.

Saal, Probe- rehearsal hall.

Saalregler theater fader.

Sache subject(-matter), cause, case, theme, topic, matter, affair, docket, in re.

sachgemäss appropriate, in an expert way.

Sach-kenntnis,-kunde expert knowledge, expertness, competence, knowledge in an art.

sachkundig experienced, expert, skilled, trained in an art or profession.

Sachlage state of affairs, factual situation.

sachlich objective, material, neutral, real, in a matter of fact way.

Sach-verhalt factual situation, facts of a case. -verständiger expert, authority, specialist (in technological art or science, etc.)

-verzeichnis subject index.

- wörterbuch encyclopedia.

Sack bag, sack, pocket, pouch, sac. Wind-wind cone, w. hose (airport).

säge-artig saw-like, serrated. -förmige Spannung, doppel- double sawtooth potential.

Sägemehl saw dust.

Sägezahn-generator sawtooth generator (telev.) -kurve, steilabfall der abrupt drop of sawtooth wave. -spannung sawtooth voltage, ratchet $\mathrm{v}$.

saigern liquate.

Saite, ausgespannte stretched chord, s. string. -, geschlagene percussed chord or string. -, gezupfte plucked string. -,mitschwingende sympathetic string, co-vibrant s. Begleit- accompaniment string (zither).

Saiten-galvanometer string galvanometer, vibration \&. -summer chord Luzzer. -zug string tension.

salbig salvy, unctuous.
Salz,Fixier- fixing salt.

salzgetränkte Kohle mineralized carbon, impregnated c.

Salzkristall, Seignette- Rochelle salt crystal.

sämi sches Leder Chamois leather.

Sammel-anode collector anode, gathering a., output a., ultimate a. -anschluss p.b.x. Line, private branch exchange.

- anschlussteilnehmer subscriber with several lines. -elektrode output electrode, collector e., gathering e., catcher (klystron). - gefäss receiver, reservoir, collecting vessel, receptacle. $-\mathrm{gl}$ as converging lens; preparation tube, specimen t. -1 inse condensor lens, converging 1. , convergent 1., positive 1. - l inse, Haupt- second lens (in electron gun). -linse, Vorfirst focusing lens, cathode 1. (like Wehnelt cylinder, apertured disk, etc., in cathode-ray tube). sammeln concentrate, focus, collect, gather, accumulate, assemble, catch. Sammel-raum receiver, receptacle, collector space, catcher space (in Klystron). -rohr collector pipe, mains. -schiene busbar, omnibus bar. -spule focusing coil. -system collective or condensor system or element (projector). -zylinder focusing cylinder.

Sammler accumulator, storage battery; collector. Wind-air reservoir, compressed-air tank.

Samtdichtung velvet trap.

Sandform sand mold (foundry). sandfuhrend sand-bearing, sandy, areniferous, arenaceous.

Sandguss sand casting.

sandig sandy, areniferous, arenaceous. sanft soft, gentle, mild, smooth. Saphir sapphire. - spat cyanite. satinieren satin, glaze, burnish. 
Satiniermaschine, heisse hot burnishing press.

Sattdampf saturated steam, saturated vapor.

Satte bowl, dish.

Sattel crevass, dip (of resonance curve).

sättigen, über- supersaturate.

Sättigungs-aktivität saturation activity. -apparat saturator.

sättigungsfähig saturable, capable of saturation.

Sättigungs-kapazität saturation capacity. - strom saturation current. -wert valence.

Satz deposit, sediment, settlings; set (of things); sentence, principle, law, rule, theorem, axiom. -vom ausgezeichneten Lichtweg law of extreme path.

Satz, Federn- spring bank, s. assembly. Flächen- theorem of conservation of areas. Glasglass batch, g. composition, g. charge. Gleichverteil ungsprinciple of equipartition of energy (Maxwel1-Boltzmann). Hauptfundamental principle, f. law, axiom (math.) Impulserhaltungstheorem of conservation of momentum. Knall- detonating composition. Kontakt- bank of contacts. Lehr- abstract theorem or doctrine. Leit- guide rule, guiding principle, basic p. Linsenlens combination, system of lenses. Lücken- vacancy principle. Objektiv- set of lenses, system of 1., lens combination. Permanentpermanence principle, sum rule. Rad- axle and two wheels. Summensum rule, permanence principle. Verschiebungs- displacement law. Verteilungs- principle of distribution, d. law. Vorder- antecedent, premise. Wechsel- exchange principle.

sauber clean, neat.

säuern acidify, sour.

Sauerstoff-ion anion, negative ion. -messer eudiometer. -pol oxygen pole, anode. -verbindung oxygen compound.

Saugapparat suction apparatus, aspirator.

saugen suck(up), absorb, imbibe, aspirate.

Sauger suction apparatus, aspirator, exhauster. Well en- wave trap, smoothing choke coil, series reactor.

Saug-fähigkeit absorptive capacity, absorptivity, imbibition power. -feld positive field, suction f., f. which draws away. -festigkeit resistance to suction, s. strength. -filter suction filter. -flasche filter flask. -geschwindigkeit rate of evacuation (of vacuum pump). -gitter space-charge grid. -hahn suction cock. -heber syphon. -höhe suction head, s. height, absorptive height. -kreis acceptor circuit; impedance or absorption wave trap. -leitung suction pipe, s. piping. -luft vacuum. -messer vacuometer. -näpfchen suction cup. - spannung anode, driving or positive potential (of photo-cell), saturation $p$. -spule smoothing coil. -stutzen pump intake, p. nozzle. -transformator booster transformer, sucking t. -ventil suction valve. Säule column, pillar, prism (cryst.); pile (electr.). Gl imm- light column, glow c. (of tuning indicator). Licht- light column. Quecksilber- mercury column. Volta'sche- voltaic pile. Säulenachse prismatic axis. säulenartig columnar, prismatic (cryst.)

Säulen-lampe pillar (lamp), batten. -tischfernsprecher desk (stand) telephone set. Saum seam, hem, edge, fringe. -, farbiger color fringe. säumen hem, edge, border. Säureballon acid carboy. säure-beständig stable to acid action, fast to acid. -bildend 
acid forming. - haltig containing acid, acidiferous.

Säureschwemmverfahren acid flotation method.

säurewiiderstehend resistant to acid, fast to a.

Säurezahl acid number.

sausen rush, whiz, whistle, hum.

Schaar, Kurven- family, group or system of curves.

schaben scrape, shave, grate, rub, abrade.

Schablone pattern, template, stencil, mold, form, model, former.

Schablonenstreifen controlling strip (automatic film printing).

Schabsel scrapings, parings, shavings.

Schacht, Licht- light tunnel (of sensitometer).

Schadenersatz festsetzen assess damages or indemnity. $-k$ lage action for damages.

schädlich spurious, stray (of radiations), noxious, injurious, dangerous, harmful, detrimental. -machen vitiate, contaminate.

Schadloshal tung indemnification, indemnity, compensation.

Schaft shaft, shank, stock, stem, handle, stalk. Niet- rivet shank, $r$. stem.

Schake link (of a chain).

Schäkel shackle.

Schale dish, basin, cup, pan, bowl, scale, tray; husk, shell, rind, skin. Abtropf-drainer, drip, jar, dish. Anoden- anode segment (of magnetron). Dampf- evaporating dish, e. basin. El ektronen-, beinahe geschlossene nearly closed electron shell. Kugel-partial sphere, cup, spherical shell, cupshaped or hemispherical part, calotte. Unter-sub-shell (of electrons). Zweier-duplet rings.

Schal eabschi rmungskoeffizient shell absorption coefficient (shielding function).

Schalen-bau der El ektronenhïlle structure of electron shell. -entwicklung tray development, dish d. -guss chill casting. -gussform chill. -kupplung sleeve coupling. - lack shellac. -windstärkemesser cup-type anemometer,

Schall(s.a. Akustik, Geräusche, KI ang \& Ton) sound, acoustic action, sound a., ring, peal, noise (=disturbing s.)

Schall,Körper- material conduction of sound (say, through solids). Luft- air conduction of sound. Tritt- impact sound (transmission). Schall-abstrahlung sound radiation or projection. -abwehr noise abatement, n. suppression, sound attenuation, s. absorption. - aufnahrie, akusti sche direct mechanical recording (as distinguished from electrical r.) -aufnehmen sound recording; s. pickup, s. collection, s. receiving (by a microphone). -aufnehmen, Gl immröhren- glowtube or g.-lamp sound recording (e.go, by Aeolight). -aufzeichner sound recorder. -ausbreitung, vertikale vertical spread or diffusion of sound. -ausschlag displacement of a particle (ac). -bandaufnahme magnetic steel tape recording. -becher bell (wind instrument). -bestrahlung, hohe high radiation distribution ef ficiency. -brett baffle(board). - bündel pencil of sound. -dämmung sound reduction, s. attenuation, s. deadening, s. absorption, silencing (of noise). -dämmzahl sound transmission characteristic.

schall dämp fend sound absorbing, s. attenuating, damping, deadening (for sound).

scnall-dämpfer silencer, sound $a b-$ sorbing means, muffler. -dämpfungseinheit unit of sound $a b-$ sorption (e.g., Sabin unit). - dämp fungsfaktor sound absorptivity, s. absorption coefficient. -dämpfungsmittel sound absorbing, 
s. attenuating, s. damping, s. deadening means or material, sound absorbent or absorber, $\mathrm{s}$. insulation. - deckel sounding top (of mus. instrument), sounding board, sound b., abat-voix. -druck, effektiver effective sound pressure, rms (root-mean-square) acoustic p. -durchlässigkeit sound transmission, s. transmittance. -einfall, streifender glancing incidence of sound wave. -empfang sound collection, s. reception (of a microphone). - empfänger sound pickup, sound collection device (e.g., microphone), s. receiver. - emp findungsschwelle threshold of audibility, $t$. of acoustic perception or percipience (represented by audiogram). -ereignis sound action, acoustic a. schallen sound, resound, ring.

Schallfärbung timbre, tone color,

tone quality.

schallharte Trichterwand rigid or non-absorbing horn wall.

Schall-härte acoustic stiffness, sound hardness (denoting ratio pressure amplitude to velocity amplitude as a function of frequency). - impedanz acoustic impedance. -ingenieur sound technician, acoustician. -leistung response (of an acoustic system). -mikrophon acoustic microphone. -öffnung louvre (of bell tower). -ortung sound location. -platte für Rundfunk $16^{\prime \prime}$ transcription record for broadcast studios. -platte, Bild- sound and picture on disk. -platte, Mess- frequency record, phonograph disk bearing sliding notes or constant notes; parlophone record (produces warble note). - pl attengeräusch record noise, surface n. -plattensteg wall, land or barrier between record grooves. -quelle sound generator, sound source, source of acoustic energy. -reaktionsrad Rayleigh disk, etc. (placed in a sound field). -reibungswiderstand acoustic resistance (dissipative component of acoustic impedance). -reiz, über schwelliger superthreshold (sound) intensity level. -rille groove or track (on a disk). -rückkopplung acoustic feedback, a. regeneration. -rückwirkung acoustic impedance (comprising resistance and reactance, the latter either in the form of inertance or of compliance, corresponding to electrical inductance and capacitance.). - sammler sound or microphone concentrator. -scheinwerfer sound reflector, acoustic radiator. - schirm baffle. -schlucker sound absorbent, $\mathrm{s}$. deadening material. -schluckung sound absorptivity, acoustic a. - schnelle volume current (ac.). - schnell eempfänger velocity microphone. -schreiber sound recording stylus, s. recorder. - schutz sound proofing, s. insulation. -schwellenstärke threshold sound intensity (of audibility and of feeling).

schall sicher sound-proof.

Schall-stärkemesser phonometer, sound level meter. -strahlbür 'el pencil of sound. -tilgung scund absorption, s. suppression, s. deadening, s. damping, s. attenuation. -tilgungsmittel gobo, nigger, baffle (in form of a wall, etc., in $\mathrm{m} . \mathrm{p}$. work).

schall tot non-resonant, non-oscillable, acoustically inert or inactive.

Schal 1-treibwerk loudspeaker operating mechanism, motor element. - übertragungsanl age (public) address system; electroacoustic transducer (rare). -umfang sound range, s. contrast. - umwandl ungse inrichtung electroacoustic transducer. -verstärkungsmittel sound re-inforcing system (in halls, auditoria, etc.) -verzugs, Ausschal ten des acoustic correction (sound locator). 
- wahrnehmung des menschlichen Ohres human tone or sound perception, auditory percipience. -wand(cf Tonführung) baffle (partition or boaru), loudspeaker screen (to prevent acoustic t'eedbacks), sound panel (for either s. absorption or s. reflection). -weglänge sound path length.

schallweich sound absorbent.

Schall welle, Uber- supersonic wave, ultrasonic w.

Schall well en-aufzeichner phonodeik (records on film). -ausgleich neutralization of sound waves (prevented by baffles). -widerstand acoustic impedance; complex quantity comprising acoustic resistance corresponding to internal friction responsible for energy dissipation, and acoustic reactance either in the form of inertance (=electric inductance) or of compliance (=electric capacitance). Schal I-widerstand ratio pressure amplitude: velocity a. -wiedergabe, Radtkeverfahren s. Radtke verfahren. -wiedergabequal ität realistic or faithful sound reproduction (with "atmosphere" or "room tone"). -wirkungswiderstand acoustic resistance.

Schal t-band controlling strip (of printing machine). -bild circuit diagram, wiring d., hook-up. -buchse jack. -element circuit element, c. means.

schalten, gegeneinander connect in opposition, c. differentially. -, in Brücke bridge (across). -, vielfach multiple. -, vorwärts step forward, notch $f$.

Schal ter, hal bver senkter semi-sunk or semi-recessed switch. -mit 5 Ausgängen switch with five points, five-point s. -, sechsteiliger, mit zwei Stellungen two-position sixpoint switch. Bogenlösch- Deion circuit breaker. Dämmerungs- twilight switch. Dreieck-Sterndelta-star switch. Dreiwege-three- way switch. Druckgas- cross gasblast switch. Druckluft- autopneumatic circuit breaker, air blast switch. Expansions- hydroblast or expansion circuit breaker or switch. Einaus- off-on switch. Hebel-, zweipoliger double-lever switch. Hörsprech- talk-listen switch (of intercommunication system). Impulsöl- oil blast circuit breaker. Kipphebel- toggle switch. Messer- knife (blade) switch. Mithör-listening key. Nadelvibrating reed rectifier.

Peilseiten- direction-finder senseswitch, sensing or sense-finding s. (operates df and sense-finding loops). Pressgas- gas-blast switch. Pressluft- airblast switch. Quecksilber-mercury switch. Rasten- step switch, multi-point s. Schnapp- snap switch, tumbler s. Sprechspeaking key. Stern-Dreieck stardelta switch. S'euer- sequence switch, master s., control s. Stöpsel- plug switch. Stromstossimpulsing switch, stepping s. Stufen- multi-point switch, multicontact s., step s. Thermothermal lag switch. Trenn-isolator, isolating switch, disconnecting link. Uberwachungs- monitoring key. Verstimmungs- wavechange switch, w. changer (in a transmitter). Verzögerungstime-lag switch. Wahl-selector switch. Wasser-hydroblast switch. *echsel-double-throw switch. Wellen(bereich)- wave-band switch. Wende- reversing switch, $r$. key. Zeitschnell- high-speed switch, quick-break s.

Schalter-arbeit switch work. -kamm sequence switch cam. -leistung cf Abschaltleistung.

Schal t-kapazi tät circuit capacitance (radio). - leistung, Ab- rupturing capacity, circuit-breaking c. (of a power switch). -mittel circuit element, c. means; switching 


\section{Schal tnocken}

248

device. -nocken trip cam, trigger c. -periode shutter pariod, shift p., moving $p_{\text {., }}$ feed stroke (m.p.) -rad, Schritt-stepping wheel.

- rolle sprocket wheel (film feed). - schema diagram of connections, circuit d., hookup. -spannung bias reducing potential, unblocking potential (silent tuning). -strom current on contact. -trommel intermittent (motion) sprocket (film feed).

Schaltung (s,a. Stromkreis und Kreis) connection, circuit arrangement, c. organization, c. scheme, hookup; switching.

Schal tung, bildweise intermittent film feed or movemant. -, stete continuous film feed or movement.

Schal tung, Abwäge- comparator circuit organization. Bild-feed or movement of frame or picture (m.p.), frame ratcheling. Blink- blinking arrangement, ratchet, relaxalion or sawtooth generator scheme with RC time-base and neon lamp. Bremsfeld- retarding-field circuit organization (with positive grid and negative plate), Barkhausen-Kurz circuic, oscillatingelectron or electron-oscillation scheme with reflecting electrode. Brücken- H. and T. see Entzerrungskette. CW- capacity-resistance circuit scheme, c. $-r$. network. Doppel röhren- tandem-tube circuit arrangement, push-pull c.a. Doppelweg- full-wave rectifier circuit organization. DreipunktHartley (oscillator) circuil scheme, potentiometer circuit scheme. Entlade- circuit causing fast drop of sawtooth voltage. Film- pulldown film movement, film feed. Filmfort- film feed, f. travel, f. movement. Gl imml ampenkipp- neonlamp time-base circuit scheme. Graetz- four electrolytic rectifier bridge-arrangement. Greiferclaw film feed. Gruppen- series parallel connection. Hintereinanderseries connection or arrangement,

\section{scharf}

seriation, cascading a., tandem a., concatenation (of motors).

Kaskaden- cascading connection, concatenation, tandem arrangement (of motors). Ketten- relay chain circuit. Lade- circuit means causing slow rise of sawtooth voltage. Mischer-keying and mixer tube scheme (for adding synchronizing and video signals). Mittelpunkttast- center-tap key modulator arrangement. Neutral isations- neutrodyne circuit organization. Parallel-parallel connection, p. circuit organization, multiple connection, paralleling. Pendel- (Armstrong) super-regeneration circuit. Reinartz- regeneration circuit (comprising capacity and tickler coil). Reintonnoiseless recording circuit organization. Schläger- dog or plunger movement or mechanism. Schleifen- circuit looped back and forth. Spar-circuit organization designed to economize (plate) current (e.g., Nestel's). Zeilen-vertical stepping-down movement, picture traversing. schaltungstechnischer Aufbau cunstructional details of circuit organization or wiring.

Schalt-vorgang switching operation, s. process. -wähler, Schrittstep-by-step selector.

schaltweise Bewegung intermittent movement or feed, discontinuous $m$.

Schalt-welle wiper shaft. -werk film feed, intermittent or shuttle mechanism (m.p.); control switch m. or gear, trip or stepping $m$. -werk, Fort-, -werk, Schritt- stepby-step mechanism, stepping or switching $\mathrm{m}$, -zeichen symbols of radio circuit elements, legend (list of symbols) letters or numerals).

Schamottestein firebrick. scharf strident, shrill, piercing (of sound); peaked (of a curve). - begrenzt highly selective (of resonance). 
Schärfe resolving power, resolution, definition; sharpness (of selection, distinction, discrimination or tuning), degree or measure of distinctness (e.g.., of optical actions), acuity.

Schärfe, gestochene microscopic sharpness. Abstimm- sharpness of tuning or of resonance, selectivity. Bild-picture definition, $p$. resolution. Fleck- spot focus, sharpness of $f$. Gehör- acuity of hearing. Längs- definition in line direction. Mitten- central definition, sharpness of center. Quer- definition in frame direction. Resonanz- selectivity, sharpness of resonance or of tuning. Seh- visual acuity, sharpness of vision, visual focus. Tiefen- depth of focus, d. of field. Trenn- selectivity, filter discrimination.

Scharfeinstellung sharp focusing (opt.); automatic tuning indication.

Schärfen,Minimum- zero cleaning or clearing, z. sharpening (d.f.)

Schärfenfeld (in Bezug auf öfnung, Entfernung und Brennweite) focal field (in respect of aperture, distance, and focal length).

Schärfenfeld-grenzen limits of sharpness or of definition; camera lines (delineating good focus area). -tiefe depth of field. Schärfen-fläche surface of sharp or distinct vision. -messung, Gehöraudiometry. -tiefe depth of vision, definition in d., depth of focus. -winkel angular resolving power. -zeichnung sharpness of delineation.

Schärfereglung, Trenn- automatic bandwidth selection.

scharfgängig property of a screw cut with triangular thread.

Schärfung, Nullpunkt- zero or minimum point cleaning or clearing, sharpening of minimum (d.f.). scharf-winklig acute-angled. -zeichnende Linse achromatic lens. Scharnier hinge, joint, articulation. Dreh- swivel pivot.

scharren scrape, scratch.

schartig notchy, nicked, jagged, serrate.

Schatten shades, shadows (of picture); umbras. Funk- radio shadow, $r$. pocket, r. dead spot. Halbpenumbra, half-shade or shadow, partial shadow. Kern- umbra.

Schatten-anzeiger shadow tuning indicator, shadowgraph. -apparat, Halb-half-shade apporatus, half-shadow a. -bild silhouette. - grenze, Hal b- penumbra boundary. -kompensation, Halb- penumbral compensation. -mikroskop shadow microscope. -riss silhouette. -veränderung change in shade or shading values. -verhältnis (zwischen Drähten und freier Gitterfläche) cover ratio (say, 1/3), transparency. -wurf sound shadows. -zeiger shadowgraph (tuning indicator).

Schattierung shading, hatching, shade, tint.

Schattierungsverfahren variable-density recurding method.

Schaubild diagram, graph; hookup; perspective view.

Schauer, Höhenstrahlen- bursts or shower of cosmic rays (traced by hodoscope).

Schaverstrahlen shower radiations, Schaufel blade, bucket (of turbine), shovel, scoop, beater, vane. Laufrunner blade, $r$. bucket (turbine.)

Schaufel abstand circular pitch (of turbine).

Schau-gl as display glass; specimen g., sample g. -hörerschaft aural and visual audience, persons listening to and seeing simultaneously audio and video actions.

schaukeln oscillate, rock, reciprocate, move to and fro, swing. Schau-linie graph, curve. -linse viewing lens. -loch peephole, 
inspection h., bezel, observation port (for projectionist, to view the screen).

Schaum, chromatischer chromatic soft focus, c. bleeding. Farb- fringing (of film). Glas- glass gall, sandiver.

Schaum-abheber froth skimmer, drosser. -gold Dutch metal. -gummi sponge rubber. -löffel skimming spoon, skimmer. -ton Fuller's earth.

Schau-versuch lecture experiment, demonstration e. -zeichen indicator signal, visual s., optical s., telltale means.

scheckig dappled, spotted, mottled.

Scheibchen, Beugungs- diffraction disk. Zerstreuungs- circle of confusion, blur circle.

Scheibe disk, slice, pane, dial; pulley, wheel. -, stroboskop ische stroboscope, stroboscopic pattern wheel. Ablese- dial (of directionfinder). Abtast- Nipkow scanning disk, exploring d. Ausgleichschwung- rotary stabilizer, roller with fly-wheel action. Blendenauxiliary rotary shutter disk, stop d. with spiral slot cyclically co-operating with holes of quadruple scanning disk. Daumen- cam (with lobe). Drehturntable (part of m.p. studio equipment). Exzenter - cam. Fall drop shutter, d. annunsiator. Finger-dial switch, finger disk, $f$. wheel. Funkbeschickungs- compensator cam, zero-clearing $c$. Kettenrollen- chain sheave. Kl inken- ratchet disk, notched $d$. Kombinator- combiner disk, c. wheel. Korrektur - compensating cam (of d.f.) Kurbel-crank disk. Leer-idler wheel. Linsen- lens disk (scanner). Loch- Nipkow scanner disk. Matt- ground glass, frosted g. (plate or pane). Mehrfachspiralloch- multi-spiral disk (for scanning). Nipkow- N. scanning disk. Nummern- dial switch, number plate, dial p. Peil- bearing plate. Riemenbelt pulley. Rollen- chain sheave. Sektoren- spinning disk (in flicker test). Sperr-ratchet wheel. 'Spiralloch-Nipkow scanning disk, spiral d. Stufencone pulley, tapered p., stepped p. Teil-index disk, i. plate. Ubersetzer- combiner disk, c. wheel. Unterlag- washer; Supporting disk. Vielkant- polyhydral mirror (of scanner). Wählerdial type selector switch. Wechselblenden- quxiliary rotary disk with spiral slot. Zwischenlagwasher.

Scheibenanode,Loch- apertured disk anode, ring a.

scheibenförmig disk-shaped, discoid. Scheiben-isolator disk insulator, dish i. -l inse, Loch-aperture disk lens. -strahl disk ray.

scheidbar separable, analyzable. Scheide-anstal t refinery. -linie boundary line, separating 1 . scheiden separate, isolate, analyze, decompose; pick, sort; sever, divide.

Scheide-verfahren refining method, separation $\mathrm{m}$. -wand partition, separator (wall), diaphragm, septum; baffle (ldspk.). -wasser nitric acid, aqua regia.

Sche in lustre, light, shine, glow. scheinbares logarithm. Dämpfungsdekrement equivalent logarithmic decrement.

Schein-dämpfung apparent attenuation. -echo pseudo echo.

Scheinergrad Scheiner degree, d. of S. scale.

Schein-leistungszähler apparent power meter (in volt-ampere units). -leitwert admittance. -verbrauchszähler apparent power meter, trivector. -werfer, Linsen- spotlight. -werfer, Facettenspiegel- segmented or facetted searchlight reflector.

Scheinwiderstand apparent resistance, impedance, impedor (part having i.). 
Bl indkomponente d. - (es) reactive component of impedance (either inductive or capacitive). Wirkkomponente d. - (es) active, watt or dissipative component of impedance.

Scheit, Richt- rule, straight edge. Rühr-paddle, rake, stirrer.

Scheitel vertex, summit, crown, top, apex. Brücken- bridge gap. Gl aslens vertex.

Scheitel-brechwert, Grund- primary vertex refraction. -linie vertical line, zenithal 1. -punkt vertex, zenith, apex.

scheitelrecht vertical, perpendicular, plumb.

Scheitel-spannungs-Vol tmesser peak voltmeter, crest v. - tiefe depth of vertex, vertical d. -wert peak value, crest v., maximal v., amplitude. -winkel vertex angle, opposite a., vertical a.

Schelle clip, clamp, shackle. Erdground clamp.

Schellenbaum bell tree, crescent.

Schellhammer riveting snap, s. hammer.

Schema schema, scheme, diagram, blank, form, model, pattern. Schal t- diagram of connections, circuit d., hookup. Term- term diagram. Wikk-actual or practical work diagram.

Schenkel leg, limb, foot, shank, side, side piece, branch. Achs(axle) journal. Aussen- outer leg, o. limb. Magnet- limb or leg of a magnet. Mittel-center leg.

Schenkel-drossel,Drei- three-legged reactor or choke. -rohr bent tube, b. pipe, V-tube, elbow tube, e. pipe.

schenkl ig legged or limbed.

Scherenspreizer lazy tongs.

Scher-festigkeit shearing strength. -schwingungen shear vibrations.

Scherung, Bild- shearing of image. Kurven- correction (of induction curve, etc.)

Scherungs-modulus shear modulus, torsion $m_{.}$, rigidity $m_{\text {. }}$-winkel angle of shear.

Scheue, Licht- photophobia. Schicht layer, stratum, bed, coat, film, skin, lamina. - auf Schicht emulsions placed together so as to face each other.

Schicht, atmosphärische atmospheric layer, sphere (troposphere, tropopause; stratosphere or isothermal region; ionosphere or Heaviside layer or KennedyHeaviside layer; Appleton 1.). -, gerasterte mosaic (photosensitized) coat or screen. Bremsvermögen der photographischen stopping power of photographic emulsion. Feinkorn-fine-grain emulsion. Filmbild- film emulsion, f. coat. Grenz- interface, boundary layer. Hohl- air space. K.-H.- Kennedy-Heaviside layer. Oberflächen-surface layer, s. barrier. Sperr- barrier layer, stopping 1., blocking 1. (of cell).

schichten arrange in layers, stratify, pile (up), stack. Schichtengitter, Schichtgitter stratified lattice, layer 1. Schicht-Kathode cathode bearing, layer or coat (of oxide, lightsensitive substance, etc.)

-körper stratified structure, s. body. -länge path length (of bands). - seite emulsion or sensitized side or face (of a film). -textur stratified texture, sheet intergrowth.

Schichtung stratification, arranging in layers, piling or stacking (up).

Schiebekontakt adjustable slide, slide contact, cursor.

schieben push, shift, thrust, shove; crab (of airplane).

Schieber slide, slider, slide bar; slide valve, damper (regulating draft).

Schieber, Belichtungs- exposure lid, e. shutter. Kontakt- adjustable slider, slide contact, cursor. 
Rechen- slide rule.

Schieber-kassette roller blind darkslide. -körper slide valve or gate.

Schiebe-rohr sliding means to tune an oscillator (by $\mathrm{C}$ or L variation).

Schieber-rahmen lantern slide carrier. -verschluss, Fall - drop shutter. -widerstand slide rheostat.

Schiebkraft thrusting power, thrust, shear.

Schiebungs-fläche slip plane (cryst.) -modulus torsion modulus, shear m., rigidity $m$.

Schieds-richter arbitrator, referee, umpire, arbiter. -spruch arbitration, arbitrament, award. schief oblique, sloped, slanty, askew, skew, crooked, warped, unsymmetric, skew-symmetric (of curves).

schief-achsig oblique axial. - symmetrische negative $P$ aare skew-symmetric "negative" pairs. Schiefe dissymmetric condition; skew. Schiefeinfall grazing incidence, oblique $i$.

Schiefer slate, shale, schist. -bruch slaty, scaly or laminar fracture.

schief-l iegend inclined, sloping, obliquely positioned. -symmetrisch skew-symmetric (of curves). -winklig ob! iqueangled. -winkliges Parallelogram rhomboid, oblique (angled) parallelogram.

Schielen (beim Goniometer) squinting (minimum signal points are displaced an angle other than 180 degrees).

Schielwinkel angle of strabism, a. of squint (eye).

Schiene rail, strip, bar; rim; tire (of a wheel); splint, splice. Andruck- pressure pad or guide. Druck- pressure shoe (film feed). Kontakt- contact bar. Sammel- bus bar, collector b. Strom- third rail.

Schienenstoss rail joint.

schiessen shoot, dart, emit, dash, bombard, set off.

Schiffchen, Porzell an- porcelain boat. Verbrennungs- combustion boat.

Schild label, sign, plate, shell, shield.

Schiller lustre, shine, iridescence, play of colors, opalescence.

schillerfarbig iridescent with metallic color or lustre display. Schirm screen, shield (of dissector tube); shade, umbrella, visor (of a cap); can (of a screened or shielded coil, etc.)

Schirm, kapazitiver electrostatic screen or shield. -magnetischer magnetic shield, m. screen, can (of a coil). -, neutraler neutral screen. -, ununterbrochener, zusammenhängender continuous photosensitized screen or photocathode (in Farnsworth dissector and superemitron).

Schirm, Bild-projection screen. Blenden-gobo (of sound absorbing material). Durchsichts- (der Speicherröhre) transparent screen (of storage type television tube). Leucht- fluorescent screen, phosphor s., luminescent s., Iuminous s., target. Mosaik-, Uberfahren d. sweeping out of mosaic (with beam). Rastermosaic screen. Schall-bafflo. streifen- rod screen. Tonsound screen. Verstärkungsintensifying screen (X-ray work). Schirm-aussteuerung full utilization of screen (up to border, in cathode-ray tube, etc.).-breite screen width.

schirmende Spule, selbst- astatic coil, self-shielded c.

Schi rm-gitter-Richtverstärker screen-grid tube with platecurrent rectification. - gitterröhre screen-grid tube, shieldgrid t., shielded-plate $t$. 
-luminophore phosphors, fluorescent substances (for cathode-ray screens, etc.). -membrane screened diaphram (of microphone). - $r$ and margin of screen, s. border or edge. -substanzen, Leucht- phosphors, fluorescent substances (for cathode-ray screens, etc.) -träger carrier, foundation or support for screen phosphor material (in CR tube).

schlacke slag, cinder, scoria, dross, clinker.

schl acken slag, form slag, scorify. Schl acken-bildung slag or ash formation, scorification. -wolle rock wool, slag w., mineral w.

schl ackig slaggy, drossy, clinkery, scoriaceous.

schlaff slack, loose, flabby, flaccid, soft, exhausted, tired.

schlaffes Trumm slack (or driven) side or end of a belt.

Schlag stroke, blow, percussion, impact, knock, shock, kick, beat, pounding; wobble (of phonograph disk); click, thump or chatter (of a key or contact); lay (of a cable). Kalt-cold heading die. Platten- disk wobble (of phonograph). Tast-key click or thump, chatter.

Schlag-beanspruchung shock, blow or impact load or stress. -biegeprobe shock, blow or impact bending test.

Schlägel mallet, maul, beater, pestle, hammer, drumstick.

Schlägerschal tung dog or plunger movement.

Schlag-feder impact spring. -figur percussion figure. $-f l a ̈ c h e$ impact surface, striking s. -gold leaf gold. -instrument percussion instrument (mus.). -länge lay, length of twist (of cablo). - lot hard solder, copperzinc brazing mixture. -lot, Messing- brass solder. -probe impact, blow or percussion test. - probe, Dauer - continuous impact or shock test (piece). -raum spark-gap chamber or space. -ton strike note (of bell, followed by hum n.) -weite spark, flashover or striking distance (of a gap.) -werk breaking machine, impact toster (of Charpy); signal device, striking mechanism. -werk, Dauer - continuous impact testing machine. -zeug percussion instrument (mus.)

Schl amm mud, sludge, pulp, slime, slush, sediment.

Schlämmkreide precipitated chalk, prepared c.; whiting.

Schl ange, Kühl-cooling coil, spiral condenser pipe or tube. Rohrspiral tube, coil.

Schl angen-hol z snake wood, letter w. -kühler spiral condenser, coil c. -kurve serpentine.

Schlauch hose, tube, tubing, pipe (of flexible material).

Sprech-speaking tube.

schlechte Abstimmung mistuning, off-tuned position.

schleichen sneak, creep.

Schleier veil, haze, slight cloudiness, mist, fog, turbidity (in liquids); reflected, double or ghost image causing veiling (in images or pictures).

Schleier, dichroitischer dichroic fog, silver f., red $f$. -, zwe if arbiger dichromatic fog. Entfernungs- distance fog. Grund- inherent fog, ground $f$. (of emulsion, resulting in background noise). Kalkchalk fog. Luft-air fogging (chemical action of atmosphere). schleierig foggy, fogged, hazy, misty.

Schleier-korn fog grain (of film). - schwärzung fog density. -wert fog value.

Schleife loop, noose, knot; slide, chute. Bahn- orbital loop. Erd- earth circuit, ground c. Film- film loop. Leitungsloop, metallic circuit. Oszillographen- oscillograph 
loop, o. vibrator.

schleifen grind, sharpen, polish, cut; slip, slide; loop (a line).

Schleifen, Kristall- lapping, precision grinding of quartz crystal plates. Plan- surface grinding.

Schleifen-bildner loop setter. -fänger feed and take-up sprocket mechanism. -flug ("U") turn, loop. -messung, Erddraht- loop test. -oszillograph loop, string or vibrator oscillograph.

-schaltung circuit looped back and forth.

Schleifer sliding wiper, copper wire interrupter (sliding over nickel disk to break up c.w. and render them audible).

Scheif-feder wiper, slide spring. -kunst,optische optical grinding artifice or art. -maschine grinding machine, grinder; abrasion or hardne ss tester. -mittel abradant, abrasive. -pulver grinding powder, polishing p. -rille groove, furrow. - spalt slit stop (opt.) -wirkung abrasive action, attrition.

Schleisse splint, splinter. schlenkern swing, sling, fling. Schlepp-antenne trailing (wire) antenna (of airplane). -kontakt trailing contact, make-beforebreak c., shorting c.

Schleuder centrifuge, centrifugal machine; sling. Elektronenelectron gun.

Schleuder-fuss gun press. -guss centrifugal casting.

Schleuse, Licht- Iight valve. objekt- object airlock chamber, air-lock (of electron microscope). Vakuum- airlock (of electron microscope).

schlicht smooth, even, plane. schlichten smooth, plane, dress; adjust, arbitrate or settle (a dispute).

Schlichtgleitsitz plain sliding fit. Schlieren streaks, schlieren, striae (of glass), cords (when heavy). -methode striae method. -zone
Schlucker, Schall-

dead zone, skip z., shadow z., shadow.

schliessend,gut- tight-fitting, tightly or hermetically sealed.

Schliessung und Unterbrechung make and break (of a circuit).

Schl iff grinding, sharpening, polish, cut (of a gem); ground section, g. specimen, o. slide, micro-section surface (as of metals, for etching); ground-in joint.

Schliff, Ätz- ground section (for etching metal). Fett- greased joint. Kern- male part of groundin joint. Kugel-ground spherical ball-and-socket joint. Normalstandard (interchangeable) ground joint. Metall- polished section, micro-s.; metal filings.

Schl iff-kolben, -stopfen ground (-in) stopper. -verbindung, Gl asground glass joint.

Schlinge loop, noose, tie. Bahnorbital loop (of elcctrons).

Schl ingerstativ cardan-suspended tripod.

Schlitten slide, sliding carriage, slide rail, chariot (in Hughes apparatus). Führungs- slide carriage. Kreuz- compound slide.

Schlitz slit, slot, fissure, cleft, incision, split. Aufzeichnungsrecording slit, $r$. aperture (of contact or of optical type). Ausgangs- exit slit (in spectrograph). Ton- reproducer aperture, r. slit.

Schlitz-blende slit stop. -brenner batswing burner. -effekt apertural effect, slit e. -kopiermaschine slit-type printer, contact p. (for film). - lampe slit lamp (of microscope). -magnetron, Zwei- two-split magnetron, two-segment $\mathrm{m}_{\text {. }}$-verschluss focal plane shutter, slittype rotary disk shutter.

Schluckbeiwert absorptivity. schluckend, licht- light absorbent, optically a. or absorptive.

Schlucker, Schall - sound absorbent 
material, s. deadening m., s. insulation. Wellen- wave trap (of absorptive or impedance type). Schluckerzahl, Schal I- sound absorption coefficient, acoustic or sound absorptivity (e.g., in sabin units).

Schluck-grad sound absorptivity, s. coefficient. -stoff absorptive material, absorbent, imbibent.

Schluckung, Schall - sound absorptivity, acoustic a.,s. absorption. Schlupf slip, slippage. schlupffreier Antrieb (s.a. zwangsläufig) non-slip drive, geared d. (free from backlash).

Schlupfkupplung slip clutch, s. coupling.

Schlüpfrigkeit lubricity, slipperiness, oiliness.

Schlïp fung slip, slippage.

Schlipfungs-messer slip meter. -reibung sliding friction, slip.

Schluss short-circuit. -, magnetischer magnetic shunt. Eisenpath closed or passing through iron (for magnetic flux), magnetic or iron path, magnetic shunt, keeper (of a permanent magnet). Elektroden- electrode shortcircuit. Erd- earth connection; (arcing) ground. Wasser- water seal, trap.

Schlussblende final diaphragm (opt.) Schliussel key, switch (for make and break); code. Mithör- listening key. Mutter- nut wrench. Nachmaster key, pass k. Sprechspeaking key. Strom- key switch, make and break. liberwachungsmonitoring key. Verwürfelungsjumble code.

Schlïssel-bolzen clutch key. - gruppen $(Q)$ code groups.

Schliusselung coding, cryptographing. Schluss-fehler, Erd- ground fault. - gebühr final fee. -kondensator terminating condenser or capacitor, short-circuiting c. -löschvorrichtung, Erd- ground fault neutralizer. -prüfer, Strom- con- tinuity tester. -prüfer, Windungs(inter-) turn short-circuit tester, continuity $t$.

Schmal-film substandard film, narrow f. -kante narrow edge, n. side.

Schmelz enamel, glaze; fusion, melting bath, melt.

schmelzbar fusible, meltable, nonrefractory. -, schwer- difficultly meltable.

Schmelze melting, fusion; smelting, smeltery; fused mass, melt; melting heat, blow run; fluid solution (metallography); batch (of glass).

Schmelz-fluss fused mass, melt. - führung smelting practice or schedule, conduct of heat, run. - gl asur enamel. - kirsche igniting pellet. -mittel flux. -schweissung, Gas- gas torch autogenous welding.

Schmelzung heat (run). Durchburnout (causing open circuit), blowout (of fuse). Ubersuperfusion, enameling.

Schmelzungslinie fusion curve. Schmerz-grenze, -schwelle threshold of feeling (of ear).

schmiedbar malleable, forgeable, capable of being wrought.

Schmiede, Gesenk- drop forge. schmiegsam flexible, pliant. Schmiegungsebene osculating plane. Schmier-loch oil hole, oil run. -nute oil groove, oilway.

Schminke make-up.

Schmoren scorching, freezing or melting together (of contacts); mike stew, frying (in radio).

Schmutzgeräusch dirt noise.

schmutzig dirty, soiled, contaminated, unrefined, polluted, filthy, smutty.

Schnabel bill, beak, nozzle, nose.

Schnapp-schalter tumbler switch, quick-action s., snap s. -schuss snapshot. -stativ self-locking tripod.

Schnarre, Prüf- test buzzer.

Schnarrwecker buzzer (alarm).

Schnauze snout, mouth, nose, nozzle, 
spout. Ausguss- pouring lip, p. nozzle, p. mouth.

Schnecke worm, endless screw, volute, spire. Eintrag-feed worm. Rückführ- return screw conveyor, $r$. worm.

Schneckenfeder coil spring, coiled s.

schneckenförmig spiral, helical, worm-shaped.

Schnecken-gangfassung helical lens mount. -gewinde helix. -rad worm wheel.

Schneidbrenner cutting torch.

Schneide knife edge, edge (of a blade). -haltigkeit ability to preserve keenness, cutting power or edge.

Schneiden,Film- editing of film. Gewinde- thread cutting, tapping. - ankerrel ai s knife-edge relay. -aufhängung knife-edge suspension. -lagerung knife-edge suspension or supporting.

Schneider, Roll gl as- wheel-type glass cutter, g. cutting w.

Schneide-stichel cutter, cutting stylus, c. tool, engraver, cutting head (phon. record). -ton edge tone, flue stop (of f. pipe).

Schneid-narbe cutting scar, burry condition. -winkel cutting angle (known as dig-in or drag a., when other than 90 degrees) (in record making).

Schneise radio beacon course, equisignal track, sector or corridor. Anflug-, Einflug- approach track, a. sector, a. corridor.

Schnell-anlauf high-speed start, rapid starting, high acceleration. -binder quick-setting cement.

Schnelle quickness, velocity, speed, fastness, celerity, swiftness. Schall- volume current (ac.). -empfänger, Schall - velocity microphone.

schnellflüssig easily fusible, readily meltable.

Schnell-kopie quick print, hurry p. -kraft elasticity. -methode rapid or high-speed method, quick procedure. -photographie instantaneous photograph, snapshot. -relais high-speed relay. -schalter, Zeit- high-speed circuit breaker. -verfahren ( $\mathrm{Film}$ ) high-speed film processing. schnelltrocknend quick-drying, siccative.

Schnitt cut, cutting, incision, section, slice; cutting and editing (m.p.)

Schnitt, Frequenz- crossover frequency (in woofer-tweeter combination at which equal power is delivered to both ldspks.) Haupt-, erster(1.) primary plane, tangential p., meridional p. (opt.) Haupt-, zweiter (II.) secondary plane, sagittal p. (opt.) Kegel- conic section.

Kristall - see Kristall schnitt. Strahlen- jet contraction, contractio venae.

Schnittbild-entfernungsmesser splitfield range-finder. -versetzung, Ausschalten der halving adjustment (of coincidence range-finder).

Schnitt-brenner slit burner, batswing b. -fänger section lifter (micr.) -fläche sectional plane, section; surface of a cut or section. schnittig, wind- streamlined. Schnitt-kopie editorially cut print, edited p. -punkt crossover (beam), point of intersection. - schraube grub or headless screw. -weite distance between back lens and image, intercept length, -weite, kon jugierte conjugate intercept, c. distance. zeichnung crosssectional drawing, illustration, view, picture or figure (Fig.)

schnitzeln, schnitzen cut, carve, chip, whittle.

Schnitzer knife, whittle, paring tool.

Schnorchel (cf Luftmast) air intake, breathing mast, air funnel, a. mast (on modern undersea craft, for submerged Diesel operation). schnut string, cord, chord, line, 
twine, tape, band. -rolle grooved roller, pulley.

Schnürspur squeeze track, matted $t$. (sound film). -blende mask to make squeeze track.

Schnürung, Ein- squeezing (film), compression, bindup, constriction.

Schoopieren spraying with Schoop gun. Schoop'sche Spritzmethode Schoop

spray (gun) method, aerograph $\mathrm{m}$. schöpfen draw (liquid, etc.), scoop, dip; create.

Schornsteinwirkung stack effect, chimnөy $\theta$,

Schott bulkhead, partition, separation.

Schottky Effekt Schottky effect, shot e., autoelectronic e.

Schottky'sche Gerade Schottky line. schraffieren shade, hatch, line. schräg oblique; sloping, slanting, inclined, beveled, chamfered, skew, askance, canted.

Schräge slope, slant, bevel, obliquity, diagonal, cant.

Schräglage,Kraftlinien- slope of flux lines.

schrägstellbar inclinable, tiltable. Schräg-stellen tilting (of electrode in a magnetron). -strahlen skew rays.

Schramme optical scratch, shadow s. (film); slash, scar.

Schrammenrauschen scratch noise.

Schranke, Licht- light berrier.

Potential-potential barrier.

schränken cross, put across; set (a saw).

Schraubdeckel screw cover, s. cap, s. lid.

Schraube, Achter- octet ring. Einstellset screw, adjusting s., leveling s. Feinstell-micrometer screw, vernier. Flügel - thumb screw, wing nut. Kl enmclamp scr $\in$ w, binding post, terminal. Knebelgriff- tommy screw. Lappenthumb screw. Links- left-handed screw. Luft-, versetzbare aircrew or propeller of variable pitch (feathered). Maden- headless screw, grub s. Mutter- bolt and nut.
Rechts- right-handed screw (thread). Regulier-adjusting screw, set s. Schnitt- headless screw, grub s. Senk- countersunk screw. Spannturnbuckle screw, (magnetic) chuck (in machine tool). Spiegelmirror screw (scanner). Stellset screw, adjusting s. Stifttap bolt.

Schrauben-bohrer screw tap, auger, twist drill. -feder coil spring, helical s. -kopf, ver senkter countersunk screwhead. -lehre micrometric calipers. -linie helix, helical line. -nut helical groove, h. slot, worm. -quetschhahn screw pinchcock. -zahngetriebe helical gear, spiral g., worm.

Schraub-lehre micrometer, micrometric gage. -lehre,Uhr- dial micrometer. -sockel screw base (of lamp). -stöpsel screw plug.

Schrecksekunde time elapsing between shock on molecular system and reaction.

Schreibempfang visual reception, recorder $r$.

schreiben write, record (graphically), type, delineate, trace, reproduce (an image or picture).

schreibende stimmgabel tuning-fork chronoscope.

Schreiber, Bild-picture reproducer, p. delineator, p. recreator, p. receiver, video-signal receiver, televisor, viewing tube. Einpunktsingle-point recorder. Farbinker, printer. Fern- teletype, teletypewriter, telegraph printer; t. operator. Heber- syphon recorder. Hell- Hell type recorder. Kurven- oscillograph, oscilloscope (when no graphic record is made), curve tracer. Mehrpunkt- multipoint recorder. Pegel- level recorder. Relief- embosser. Schallsound recording stylus. Tintenstrahl- ink vapor jet recorder (in picture telegraphy).

Schreib-feder stylus, recording pen, 
style. -fenler clerical error, typographic e. -fläche record sheet or surface. -instrument graphic instrument, recording i. -kopf recorder head. -röhrchen pen, syphon (of a recorder). -röhre, Bildpicture receiver tube, viewing t., electronic reproducing device. -stift cutter, stylus, cutting tool, engraving t. -thermometer thermograph, recording thermometer. Schreien, Uber- overmodulation.

Schreiner, Modell- wood pattern maker. Schrift, Amplituden-variable-area or variable-width recording or track. Beschwerde- plaint, appeal papers. Dichte- variable-density recording or track. Gegentakt- push-pull track or record. Nach-picture recreation; postscript. Normalstandard track. Quer-,Seitenlateral track, Berliner t., horizontal cut (gramophone). Sprossenvariable-density record or track. Tiefen-hill-and-dale (Edison) track or recording, vertical sound groove. Tonfolge- sound track or record (on film, disk, etc.) Verteidigungs- defense, plea. Viel doppel zacken- multiple doubleedged variable-width track. Vielzacken- multilateral sound track. Zacken- variable-area track, v.-width t. Zweizackenbilateral track.

Schrift-boden recording base. -material literature references (e.g., of prior art), bibliography, documentation; file wrapper (of patent case), records. -metall type metal. -mutter type mold, matrix. - satz written pleadings, memorial, brief. -stück bill, brief, papers, document. -tum literature (sources), bibliography, documentation, source material, references (from magazines, patents, etc.) -tumverzeichnis bibliographic list.

Schritt, Erfindungs- object of invention, step of $i$. Hebe-, Höhen- vertical step (in automatic telephony). Wähl erhebevertical stepping of selector. Schrittschal twähler step-by-step selector.

schrittverkürzte Wicklung shortpitch winding.

Schrittweite interval (between spectral lines).

Schrödinger-de Broglie Materiewelle de Broglie elementary particle (electron, proton), electron wave, phase w.

schroten granulate, bruise, roughgrind, crush coarsely.

schrott-effekt (small) shot effect, Schottky e., auto-electronic e. -geräusch shot noise (due to Schottky effect).

schrumpfen shrink, contract, shrivel. Schrumpf-mass amount of shrinkage or contraction. - spannung contraction or shrinkage strain or tension.

Schrumpfungsausgleich shrinkage compensation.

Schub shove, push, thrust, throw, shear. -kurbel crank. -lehre sliding gage or calipers. - spannung shear stress. -stange thrust rod, push(er) r. -vektor thrust vector. -weg finite distance.

Schuh, Gleit- pressure pad, p. guide. Kabel- cable eye, c. socket, connector lug, sweated thimble.

Schuhmacherwachs cobbler's wax.

Schuldigerklärung verdict of guilty, conviction.

Schuppe scale, flake.

schuppig, fein- fine-scaled.

Schurre chute.

Schüttel-herd bumping table. -mikrophon granule microphone.

schütteln shake, agitate, churn, toss.

Schuittel resonanz vibration resonance. Schüttwinkel angle of repose, (sometimes) angle of friction.

Schutz screen, shield, protection, guard, preserving or conserving 
means, safeguard.

Schütz relay, contactor.

Schutz, Berührungs- protection against electric shock hazard. Schallsound proofing, s. insulation. Ubersteuerungs- overload limiter. Schutz-einrichtung, Fehl- fault-clearing device. -funkenstrecke spill gap. -gerät,Knall- crack silencer, pistol shot s., noise killer. -gitter shield (in shield-grid thyratron). -gitterröhre screengrid tube, shield-g.t.; shielded plate t. -hille protective cover, p. coat, p. sheath. -kappe,Lichtlight hood. -korb arcing ring, guard $r$. (of insulator). -marke trade-mark. -masse resist (used in printing, etc.) -mittel, Lichthof- anti-halation means or substance. -netz, Anoden- screen grid, plate shield. -rechte patent rights, trade-mark r., etc. (granted by or registered in Patent office). -wulst, Sprüh- anti-corona collar or tore, guard-ring.

schwach, rauch- sinokeless (of powder). schwach ausgesteuerter Tonstreifen low-modulation track.

schwächen weaken, enfeeble, impair, attenuate, dilute, dim, absorb (light), mute (sound).

Schwächer, Fünfstufen- five-step weakener (phot.) Stufen- step-type weakener (spectr., phot.)

schwachsichtig weak-sighted, amblyopic. Schwächung, Licht- light absorption, 1. dimming, 1. drop, 1. loss, cutting down or reduction of 1 . intensity, 1. diffusion.

Schwächungs-gl ied attenuator. -periode fading period. -widerstand gain controller, g. regulator, potentiometer.

Schwanenhals goose neck.

schwanken fluctuate, oscillate, vary, shake, rock, sway or swing (of an. antenna).

Schwankung, Frequenz- frequency flutter, f. drift, lilt (when slow); swing or deviation of $f$. (above and below assigned resting or center
Schwärzungse inhe it

$f$., in $f-m)$. Lautstärke- fading, fade out, volume or intensity fluctuation. Regel-hunting (in making adjustments or in regulating). Ton-pulsation of reproduced sound intensity, flutter (known-as wowows when up to 6 cycles per second, as flutter when 6-30, as gargles when 3u200 , and as whiskers when over 200 cps.) ' Volumen- volume flutter.

Schwankungs-frequenz ( $c f$ Tonschwankung) frequency of flutter. -messung, Ton- flutter measuring instrument. -schwächer, Frequenzdiscriminator (converts frequency drift into d.c. potential).

-theorem fluctuation theorem.

Schwanz, Bl ind- arrangement making resistance alternately zero and infinite, in dipole feeders; stub. Film-trailer or run-out (piece of blank film attached to film end). Wellen- wave tail (in facsimile). Schwanzbanden tail bands (in after ${ }_{\varepsilon}$ glow).

schwanzloses Flugzeug tailless airplane.

Schwanzstück, Kurven- toe region of film characteristic (or of gamma curve of emulsion).

Schwärme, Mol ekül- swarms of molecules. schwarz(e) Temperatur black-body temperatur, cavity $t$.

Schwarz-druck black printing, $p$. in b. -pegel black level. - sender unlicensed transmitter, illicit t., "underground" t. station. -strahl black (-body) radiation, cavity r., Planckian r. -temperatur black-body temperature, cavity $t$.

Schwärzung blackening, density. Kopier-printing density. Ruheunmodulated density, no-sound d. (in variable-density recording). Schleier- fog density.

Schwär zungs-abstufung density graduation. - bereich density range $(\boldsymbol{r})$ (say, from.3 to 3.0). -dichte density (of film). -einheit unit 
of (film) density. -hof halo. -kurve characteristic film curve, H \& D (Hurter \& Driffield) curve. -messer densitometer. -messer, Kreiskeil- circular wedge densitometer. - umfang density scale, range or latitude of $d$. variations. -verfahren variabledensity method.

Schwarzweissbildsendung black and white facsimile picture or phototelegraphic transmission.

Schwarzwert black level value (telev.)

Schwebefähigkeit buoyancy, floating power, suspension $p$.

schwebende Stimme voix céleste. Schwebe-stoff, -teil chen suspended particles, s. substance, s. matter.

Schwebung beat, interference action (opt., ac.) Helligkeitsflutter of brightness.

Schwebungs-amplitude surging amplitude, beat a. -empfang beat reception, heterodyne $r$. -frequenz,Null- zero beat frequency. -null zero beat. -periode beat cycle. -summer audio oscillator, beat-frequency o. - ton beat note.

schweifen curve, chamfer, tail. Schweige-trichter cone of silence (marker). -zone skip distance, shadow region.

Schweiss, Gl as- glass gall.

Schwe iss-backe welding die. -bl ase arc crater. -druck welding upset. -düse welding tip. -eisen weld iron.

schweissen, stumpf- jump weld, buttw. -, übereinander-, ubberlapptlap-weld, scarf-w.

Schweiss-fuge welding seam, w. line, w. joint, shut. -hitze welding heat. -löcher pores (of wood). - naht shut, weld, weld seam. -raupe, breite spread bead. -rissigkeit "chinkin el" or fissuration due to welding. -stelle welded or welding area, shut. -tropfen welding dribble, w. beads. -umformer welding converter. - umspanner welding transfurmer.

Schweissung, Kehl naht- fillet welding. Naht- seam welding. Punkt- spot welding.

Schwelle, Emp findl ichkeits-(Schall)

threshold of audibility or acoustic perception. Potentialpotential barrier, p. threshold. Potenz- (Kerndurchdringung) nuclear penetration function. Schmerz- threshold of feeling. sichtbarkeits- threshold of vision or visibility.

Schwellen-frequenz threshold frequency, critical f., photoelectric threshold. -reiz threshold or liminal (stimulation) value. -stärke, Schall - threshold sound intensity (of audibility or of feeling). -wert, Farb- liminal value or threshold of intensity of a color. -wert der Platte exposure factor of plate (phot.)

Schweller, Roll- crescendo pedal (of organ).

schwellig liminal, concerning threshold. unter-subliminal.

Schwell-kasten swell box. -pedal swell pedal (organ). -werk swell organ.

Schwel ung low-temperature carbonization, slow burning, smoldering. Dampf-destructive distillation with steam.

Schwemm-stein porous brick (from clay and gravel). -verfahren flotation method.

schwenken swing, turn around, pivot, fulcrum, swivel, rotate, revolve, slew, tilt (telev. camera).

Schwenkungswinkel angle of traverse (gunnery).

Schwerbetrieb heavy-duty operation or service.

schwerdurchlässig difficultly permeable, of low permeability or transmittance, highly opaque.

Schwere heaviness, weight, gravity. -feld gravitational field. -messer 
barometer, gradiometer (measures gradient of earth gravity field). -pendel gravity pendulum.

Schwererde heavy earth, baryta. schwer(es)Elektron heavy electron, barytron, dynatron, mesotron, penetron, x particle. -(er) Wasserstoff deuterium (with mass of 2), tritium (with mass of 3 ). schwerflüchtig difficultly volatile, d. volatilizable.

Schwermetall aktivator heavy-metal activator or phosphorogen (in phosphors).

Schwerpunkt mass center, c. of gravity. Leucht- Iuminous "center of gravity," photometric c., p. centroid.

Schwerpunktsystem center of gravity system, c. of mass system. schwerschmel zbar difficultly fusible, refractory.

Schwimmen lateral flicker (in overspeedy panorama work).

Schwimmer float, pontoon, swimmer. Schwimm-kraft buoyancy. -methode flotation method. -regel, Ampère's Ampere's right-hand rule. -spannung blurring potential, p. causing sway of picture; floating, spurious or stray p. schwinden shrink, contract, fade. Schwing-amplitude der Membran amplitude of diaphragm excursion. - anker oscillating armature. - audion oscillating detector, autodyne, endodyne. -audionwell enmesser autodyne wave-meter or frequency $\mathrm{m}$.

Schwinge link.

schwingen vibrate, oscillate, swing, rock, pivot, reciprocate (to and fro), surge (back and forth). Schwingen, plötzliches spilling over (of an amplifier), sudden undesired oscillating (attended with fringe howl), trigging of a tube. schwingen, durch-( cf Ball aströhre) swing through (operating condition in which wave is not interrupted or cut off). mit- co-vibrate, oscillate in resonance, sympathy or unison, experience sympathetic vibration.

schwingender Draht swinging of filament (in magnetron).

Schwingentl adung oscillatory discharge, oscillating d.

Schwinger oscillator, vibrator. -, offener open oscillator.

Frei- free radiator (loudspeaker). Kipp- time-base oscillator. Sperr-blocking oscillator. schwingfähig oscillatory, vibratory, oscillable, vibrant, vibratile. Schwing-feder vibrator(y) spring, reed (of a chopper). -kennlinie oscillating characteristic, c. of an oscillatory system, resonance characteristic. -kontaktgleichrichter vibrating-reed rectifier. -kristall crystal oscillator. -löcher dead spots (trouble in direct-coupled antenna). - - rel ai s trigger relay.

Schwingröhre, fremderregte masterexcited oscillator valve (connected in an independent drive circuit). -, selbsterregte self-excited or self-oscillatory valve (connected in direct drive circuit). Kraftpower oscillator.

Schwing-spiegel oscillatory mirror, vibrating $\mathrm{m}$. - spiegelabtaster vibrating mirror scanner (of Mihaly's Telehor). -spule moving coil, signal-current c., voice c., speech c. (of loudsp.)

Schwingung vibration, oscillation, undulation, swing, wave, highfrequency a.c., "oscar"(m.p. studio slang).-durch Nebenkopplung stray or spurious oscillation. -, erzwungene forced oscillation, constrained o. -, freie free oscillation. -, gedämp fe damped oscillation, decadent 0 . -, ungedämpfte undamped oscillation, persistent $o_{\text {., }}$ sustained 0 . -, zusammengesetzte complex (harmonic) wave.

Schwingung, Biegungs- flexural 
Schwingung, $B$ ild-

vibration, lateral v. Bildvideo or picture impulse or signal. Dicken- thickness vibration (cryst.) Drehfeld- rotatingfield oscillation. Drehungs-, Drillungs- torsional vibration. Gitter- lattice vibration (cryst.) Grund- fundamental wave, firstharmonic w. Intermittenz- relaxation, sawtooth or ratchet wave or oscillation, time-base o. Ionenionic oscillations, fringing effect, waving in the breeze (across image). Kipp- see Intermittenzschwingung, Längs- longitudinal vibration or oscillation. Laufzeit- electron oscillation (of magnetron). Magnetron- magnetron oscillation (of three kinds: electronic, negative-resistance and rotating-field oscillations). Mit- resonant vibration, sympathetic v., co-vibration (acting in unison). Nebenkopplungs- spurious oscillation. Quer-transverse vibration, shear v. Resonanzresonant vibration, sympathetic v., co-vibration (acting in unison). Relaxations- sawtooth, relaxation or ratchet oscillation. Scher- shear vibration. Ubertragungs- signal oscillation, s. พลve. Umfangs- circumferential oscillation or vibration. Untersubharmonic oscillation, subfrequency 0 . Unterbrechungs,Unterdrückungs- quench oscillation (in super-regeneration). Wärmeheat vibration. Zwangs- forced oscillation, f. vibration.

Schwingungs-abreissen quenching, suppression or discontinuance of oscillations. -abschnitt "bit" of vibration (in sound recording investigation). -amplitude, Membran- excursion amplitude of diaphragm. - anfachung wave generation, wave excitation. -aufzeichner vibrograph (to record mechanical vibrations). - ausschl ag(einer Saite) deflection, excursion or swing (of a chord). -band, Rotationsrotation vibration band. -bauch loop or anti-node of an oscillation or vibration, internode. -bogen amplitude of oscillation. -dämpfung vibration or shock absorption, damping (out) of oscillations or vibrations. -dauer, Kipp- relaxation period. -einsatz, harter hard start of oscillations. -einsatz, weicher gentle or smooth start of oscillations.

schwingungserzeugende Kraft vibromotive force.

Schwingungserzeuger, selbstsperrender self-blocking oscillator. -, Vielfach- multivibrator.

Schwingungser zeugung, sel bständi ge self-oscillation, spontaneous or undesired oscillating, spilling over (attended with fringe howl and whistling in amplifier). schwingungsfähig oscillable, vibratile, oscillatory, vibratory. Schwingungs-festigkiet vibration strength. -form mode of vibration, form of $v$.

schwingungsfrei, eigen- aperiodic, deadbeat.

Schwingungs-knoten oscillation or vibration node or nodal point. -kreis oscillatory circuit, oscillation c., tuned c., tank c. -neigung tendency towards, or on the verge of, oscillating or spilling over (with fringe howl and whistle). -schleife loop, antinode or internode of stationary wave or oscillation. - spektrum vibrational spectrum. -transformator(Tesla) Tesla coil, T. transformer, oscillation transformer. -versuch, preh- oscillation torsion test. -weite amplitude of oscillation or vibration. -zahl, Uml auf- rotation frequency. -zeit time of vibration.

Schwund, Nah- fading or radio fadeout $80-100$ miles around transmitter. Phasen- phase fading. Pseudofading due to swinging or swaying of antenna. 


\section{Schwundausgleich}

Schwund-ausgleich volume control, fading compensation. - automatik automatic volume control means (avc). - regelspannung (automatic) volume control potential, avc p. - verminderungsantenne anti-fading antenna.

Schwung vibration, oscillation, swing. -bahn sound take-off drum (m.p.) -gewicht pendulum. -hebel balance beam, rocker, rocking lever. -kraft centrifugal force, vibratory power, vibrating $p$. -maschine centrifugal whirler. -masse,öl-rotary stabilizer (in film feed). -massenrolle rotary stabilizer, roller with fly-wheel effect (film feed), impedance wheel. -moment moment of rotation, moment of inertia;fly-wheel action. - rad fly-wheel, balance w. -radkreis parallel resonance circuit, fly-wheel c. -scheibe, Ausgleich- rotary stabilizer, roller with fly-wheel action (film feed), impedance wheel.

S.E. (Siemens-Einheit) Siemens (resistance) unit.

S.E. Faktor secondary-emission factor.

sechs-eckig hexagonal. -fach sixfold, sextuple.

Sechs-flach, - flächner hexagon, hexahedron. -polröhre hexode. sechsseitig six-sided, hexagonal. Sechstelkreis sextant.

sechs-wertig hexavalent, sexivalent. -winklig six-angled, hexangular, hexagonal.

seegriun marine green.

Seel icht marine phosphorescence.

Segerkegel Seger cone (for temperature determination), fusion $\mathrm{c}$.

Segment, Wähler-bank of stationary contacts.

segmentförmig segmental.

Sehen, e inäugiges monocular vision, non-stereoscopic v. (eye, opt.). -, zweiäugiges binocular vision, stereoscopic v. Farben- color perception, c. vision, Netzhautzenter-foveal vision.
Seher, Gegen- two-way television or video apparatus (as in video telephone.

Seh-feldblende field diaphragm, $f$. stop. -funktion visual function. -kegel angle of vision, cone of v. or view. -lehre optics. - I inie line of sight, 1. of vision, l. of collimation.

Sehne chord (geometry); fiber (metal), fillet.

Seh-nerv optic nerve. -nervenapparat organ of vision. -purpur visual purple, rhodopsin. -reiz optical stimulus, visual s. or excitation. -schärfe visual acuity, sharpness of vision, resolving power (of eye). - schärfegrenzwinkel angular resolving power, critical angle of visual acuity. -schärfeneinteilung focusing scale. - schwäche, vorgetäuschte simulated amblyopia. -tiefe depth of focus, depth of field. -vermögen resolving power (6f eye), visual faculty, power of sight, p. of vision. -weite distance of vision, visual range; distance between scale and mirror (times 2 in telescopic measurements). -winkel visual angle.

Seiden-faden silk fiber, s. thread - papier tissue paper.

Seifen-bildung formation of soap, saponification. - lamel le plane soap film or membrane. -leim soap glue, s. paste, size (paper). seigern liquate.

seignettesalzkristall Rochelle salt crystal.

Seiher strainer, filter. Einsteckplug-in inlet strainer.

Seil rope, cable, chord, line. Abspann- guy rope, span $r$.

Seilrolle pulley, sheave.

Seite, Emulsions- face bearing emulsion, coated side (of film).

Seiten-abrutschanzeiger side slip indicator. -abweichung lateral deviation. - ansatz side tube, lateral arm, 1. appendage. - ansicht lateral elevation, 1. view, side e. -arm side tube, 
Seitenaufriss

lateral arm, 1. appendage (of a vessel); side branch. -aufriss lateral elevation, side $e$. - bandübertragung, Ein- single side-band transmission.--Zweidouble-side-band transmission. - bandunterdrückung side-band suppression. -bestimmung sensefinding or sensing (determination of absolute direction or sense of direction); determination of side towards which airplane is off course. -eck lateral summit. - elektroden end electrodes, wing e. (of a magnetron). -fehler lateral deviation. -fläche lateral face, flat side, facet. -frequenz side frequency (one of sum or difference frequencies). - führung (von Film) lateral guidance, side guiding. (of film strip in gate). -gleitflug side-slip (of airplane). -kante lateral edge. -navigation, -ortung directional navigation, d. avigation. -riss lateral elevation, side e. -schalter, Peilungs- sense-finding switch, d.f. sense s. -schrift lateral track, horizontal t. or recording, Berliner track (of gramophone). - strahlungen secondary radiations, lateral $r$. , stray or spurious lobes (of space pattern): - veränderungen turns and banks (avigation). -verhältnis, Bildpicture aspect ratio. -wind wind on the beam, side or cross w., w. blowing across course. -windabtrift, Kurs mit Vorhal tewinkel bei course heading into wind, crabbing (due to drift). ...seitig -sided, lateral,... angular.

seitlich lateral. - (es) Abrutschen side-slip (of airplane). -(e) Führung lateral guidance. -(em)Minimum, Antenne mit directive antenna. - (e) sphäri sche Abweichung transverse spherical aberration. selbsterregt

Sektor, Anflug-, Einflug- sector of approach, a. sector, a. track, corridor of a. Raum- solid sector.

Sektoren-membrane sectorial cone (loudspeaker). - scheibe spinning disk (in flicker test). Verschluss sector shutter.

Sektormembran (non-rigid) sector diaphragm or cone.

Sekundär-emissionsfaktor (SE) secondary-emission factor. - emi ssionsvervielfacher electron multiplier tube, e.m. phototube (with plurality of secondaryemission reflecting electrodes, known as dynodes in the e.m. photot.) - struktur secondary structure gridwork (cryst).

Sekunde, Sonnenzeit- solar second.

Sekundemeterkerze meter-candlesecond.

Sekundenuhr time piece with seconds hand.

selbstabgleiche nd self-balanoing. selbständige Entladung spontaneous discharge, self-sustained d., unassistad. d. Funktion selfconsistent function. -mitgenommene Kippschwingmethode self-running conirolled time-base method.

selbstangetrieben self-propelled.

Selbstanschluss-System automatic telephone system.

selbstanzeigender Peiler automatic or direct-reading direction-finder.

Selbstauslösung automatic release, a. tripping action.

selbstdichtend self-sealing, s. tightening, s. packing.

Selbst-einstellung self-adjustment, s. setting. -energie self-energy (of electron). - entzündung spontaneous combustion, self-ignition. -erhitzung self-heating, spontaneous h.

selbsterregender Sender self-oscillatory transmitter.

selbsterregt(e) Schwingröhre selfexcited oscillator tube. - (er) Trägerwell e, Superhetemp fänger mit homodyne receiver apparatus. 
Selbsterregung

Selbsterregung self-oscillation, self-sustained o.; spontaneous (undesired) o., spilling over (with attendant fringe howl) of regenerative receiver.

Selbst-farbe self-color, solid c. -gang automatic feed, a. operation, a. action.

selbstfarbig entirely of the same tint, self-colored.

selbst-gehendes Erz self-fluxing oré. -gleichrichtendes Röhrenvoltmeter. self-rectifying tube voltmeter.

Selbst-kapazität self-capacitance, distributed $c$. of a circuit (due to proximity effects). -konzentration self-focusing, gas. $f .-l$ aut vowel. -lautdeutl ichkeit vowel articulation.

selbstleuchtender Lichtmodul ator light modulator of the glowtube, neon lamp, sodium 1. type, light relay with inherent light.

Selbst-leuchter self-luminous substance, fluorescent s, phosphor. - leuchtverfahren self-luminous or s. -emissive method (using electrons arising on object, in electron microscope). -lötung autogenic soldering.

selbst-rechnender Komparator computing recording comparator. -registrierend self-recording, automatic r. -schirmende Spule self-shielded coil, astatic c. -schwingende Mischröhre selfheterodyning mixer tube, autodyne.

Selbstschwingungsvorrichtung thermionic trigger device.

sel bst-sperrender Schwingungserzeuger self-blocking oscillator. -ständige Entl adung spontaneous discharge, self-sustained d., unassisted d. -ständige Funktion self-consistent function. -ständige mitgenommene Kippschwingmethode self-running controlled time-base method.

Selbststrahl ungsverfahren (Elektronenmikroskop) selfemissive electron microscope or self-illuminating method (based on electrons arising on surface of object).

Selbsttönen ringing or squealing (of a tube).

selbstonendes $\mathrm{Papier}$ self-toning paper.

Selbst-überl agerung autodyne, selfheterodyne. - umkehrung self-reversal (of spectral lines). - unterbrecher trembler, selfinterrupter, buzzer. -zünder pyrophorus lighter, self-igniting 1., automatic 1 .

Selektion, Amplituden- amplitude selection, a. filtering.

Selektivkreis selective circuit, selector c.

Selen-chlorid selenium chloride. -cyanid selenocyanate. -halogen selenium halide.

selenhal tig containing selenium, seleniferous.

Sel en-kupfer copper selenide. -metall metallic selenide. zelle selenium (photo-conductive type of) cell, photronic c.

Sel tenheit rarity, scarcity, infrequency.

Sende-anl age, Richt - beam, beacon or radio range station. -bild outgoing subject copy. -kanal transmission channel, signaling $c$.

Sender, gepeil ter tuned-in beacon

(whence bearings are taken). -, modul ationsgesteuerter transmitter with carrier being varied with AF amplitude; AF modulated transmitter. -, selbsterregender self-oscillatory transmitter. -, tönender musicalspark transmitter, singing-s.t.

Sender und Empfänger, Kombinierter transceiver (radio); microtelephone, handset.

Sender,Ball-re-broadcast station, repeating s., station belonging to a network. Bild-video transmitter; facsimile t. Diapositiv-film transmitter. Einflug- boundary marker beacon. Einstrahlbeam transmitter, unidirectional $t$. Fernseh- television transmitter (comprises strictly both the aural and video signal transmitting means). 
Gemeinschafts- chain broadcast station, network s. Hauptmain oscillator (in independent drive system). Hilfs- auxiliary transmitter, local oscillator (in heterodyne apparatus). Kontroll- monitor transmitter, check-up t.; service test oscillator. Leitfunk-, Leitstrahlradio beacon, beam station, $r$. range. Markierungs- marker beacon, marker. Maschinenalternator transmitter. Mutterkey station, master $\mathrm{s}$. (in chain broadcasting). Prüf- service test oscillator. Quarz- (piezoelectric) crystal or quartz stabilized transmitter. Richtungsbeam station, beacon s. Schwarzunlicensed transmitter, illicit t., "underground" t. Sperrkippblocking oscillator. Steuermaster oscillator, drive 0 . (working on main transmitter in independent drive). Stimmgabeltuning-fork oscillator, vibratingreed transmitter. Stör - interfering station, jamming s.; shadowing signal, spurious irregular shading signal (telev.). Tel egr aphie-, tonmodul i erter $\mathrm{AF}$ or tone-modulated telegraphic signal transmitter. Telephonie-, sprachgeschal teter voice-modulated telephony (signal) transmitter, $\mathrm{v},-\mathrm{m}$. radiophone. Tochter - repeater or affiliated station (related to key station, in chain broadcasting and in b. networks). Ton- modulated c.w. transmitter; audio or aural transmitter (of sound part of a telev. program). Tonfunken- singing or musical (quenched) spark transmitter (telograph ). Zeichen- signal transmitter, s. station. Zwi schenre-transmitter, repeater station. Sender-abstand interference guard band, tolerance frequency. -durchmodul ierung, hohe operation of transmitter station at a high modulation percentage. sendereigner Ton note or pitch peculiar to a beacon station, code note; tuning $n$.

Senderöhre, frequenzgesteuerte frequency-stabilized transmitter tube. Sendung transmission or emission (of radio waves or signals), program. sengen singe, scorch, parch, flame. Senke crevass, dip (of resonance curve), trough, valley, depression; negative source.

senken depress, lower, sag, slope, droop (of curve), de-accentuate (a band).

Senkkörper sinker, bob.

Senkrechte vertical, normal, perpendicular, plumb (line). -fällen let fall a perpendicular. -errichten erect a perpendicular. Mittelmedian perpendicular.

senkrechte Beleuchtung scaffold lighting, overhead 1., top 1.

Senkspindel specific gravity spindle, hydrometer.

Senk-rechtstellung vertical position, perpendicular p., plumb line p. - schraube countersunk screw. Senkungswinkel depression angle. Senkung sag, droop, depression, dip. Sensitometer sensitometer (for comparing and grading film), densitometer.

Separatabdruck reprint, separate impression, special i.

Sequenzillusion sequence illusion.

Serien-apparat series camera. -entwicklung series expansion (math.)

serienmässige Herstellung quantity production, large-scale manufacture.

Serienresonanzkreis series-resonance circuit, acceptor c.

sicher, feuchtigkelts-damp-proof, moisture-p. hochspannungssafe against high-voltage breakdown, possessing high puncture strength, proof against high-potential shock.

Sicherheit,pfeifpunkt- singing margin, whistling $\mathrm{m}_{\bullet}$, stability $\mathrm{m}$. (of radio tube).' Stand- stability or steadiness (to shock or vibration).

Sicherheits-koeffizient safety factor. 
Sicherheitslampe

-l ampe safety lamp, s. light. sicherstellende Vorkehrungen preventive measures, precautions, prevautionary steps.

Sicherung, Grob- spark gap or similar arrester. Lage- locking in position. Luftleerspannungs- vacuum arrester.

Sicht visibility, sight.

sichtbarkeit, ïber schwellige superthreshold visibility.

sichtbarkeits-messer visibility meter (e.g., Lukiesh-Taylor). -schwelle threshold of visibility, t. of vision.

sichtbarmachen render visible or visually perceptible, visualize, indicate visually or optically.

sicht-messer visibility meter. -peiler visual or direct-reading direction-finder. -weite directsight range or distance, line of sight, optical distance. -winkel angle of sight. -wirkung sifter effect, sorting, classification. -zielflug visual homing flight, flight depending on visual indication or reading.

sicke reinforcing seam, crease or pleat, $r$. fin (bent, pressed or otherwise fashioned in metal shoeting).

Sieb-filter, network; sieve, screen, bolter, strainer. Amplitudenamplitude filter, a. discrimination selector.

siebeneckig heptagonal.

Siebenel ektrodenröhre heptode.

siebenwertig heptavalent.

Siebgebilde selective system, filter means, network.

si ebkette ladder-type filter, bandpass $f$. or transmission network. -, mehrgliedrige multi-mesh filter, multi-section f. -von grosser Lochweite broad-band filter.

Sieblochbreite, Frequen z - transmission band, transmitted b., b.-width of filter, spacing between cut-off points.

Siebschale dish with perforated bottom.

\section{Signalpl atte}

Siebsteilheit sharpness of selective network.

Siebung screening, shielding; sifting, straining, filtering, sorting, bolting, classifying.

Siebwiderstand contact resistance (of barrier cell).

Siede-erleichterer boil adjuvant. -kolben boiling flask. -kühlung, Kathode mit vapor-cooled cathoda. -messer, Wasser- hypsometer.

sieke reinforcing seam, pleat, crease or fin (bent, pressed or otherwise fashioned in metal sheet).

Siemenseinheit (S.E.) Siemens unit (of resistance).

Signal, tönendes tonal signal, musical spark s.

Signal, Abschattierungsstör - spurious signal, shading s. (in iconoscope operation, known also as "shading," "black spot," "tilt and bend," etc.) Bild-video signal, picture s. Drehlicht- revolving beacon. Entwarnungs- all-clear signal. Fernseh- video signal, television s. Freigabe- clearance signal. Gleichlauf- synchronizing signal, sync impulse; tripping s. Hauptinner marker signal, main entrance s. (airport). Kenn- tuning note (characteristic audio signal or note for tuning). Misch- signal spectrum (comprising video and sync impulses). Not- distress signal, SOS s., mayday (radio telephone). Nutz- intelligence signal, s. conveying information. Stör- shadowing signal, shadow s., spurious s. (iconoscope operation); jamming signal, interference s. Synchronisier- synchronizing signal, sync impulse, tripping s. Vor-caution signal, warning s., first or outer marker s. (airport). Warn- alarm signal, caution s., alert, raid al arm.

Signal-horn bugle. -l ampe signal lamp, telltale l. - l ampentafel signal lamp or annunciator board or panel. -mittel signaling means, communication $m$. - pfeife siren, whistle. -platte signal plate (of 


\section{Signal überdeckung}

iconoscope). - - überdeckung

blanketing or swamping of signals.

-verlauf signal spectrum, s. pattern. -wand wall of signals,

curtain of $\mathrm{s}$.

Silben-abschneidung clipping, obliteration or mutilation of syllables (in signal art).

- frequenzgerät syllable vodas (of Bell Co.) -umkehrung inversion of syllables (in secret, garbled or scrambled telephony). -verständlichkeit syllable articulation, syllable intelligibility.

silber-belegung silver coating, s. plating, s. film, silvering. -blech silver foil, (thin) silver plate. -glätte litharge. - gl immer common mica, muscovite. -halogen silver halide. -lot silver solder. - papier silver paper, silvered p.; tinfoil, tinfoiled $p$.

sims molding, shelf, cornice. Simultanleitung earthed phantom. singende Bogenl ampe singing arc lamp, Duddell arc.

Singulette singlets.

sinken sink, fall, decline, droop (of a curve).

Sinkstoff deposited substance, settled s., settlings, sediment.

Sinn, im bejahenden in the affirmative (sense). Ladungs- nature of charge, sign.

sinnähnlich substantially synonymous, in an analogous way or sense.

Sinn-bestimmung, Richtungs- sensefinding or sensing (determination of sense of direction or absolute direction). -bild symbol, emblem.

sinn-gemässe Anwendung analogous, rational, logical, corresponding or equivalent application or use. - getreu faithful (e.g., in translation).

Sinnverständ ichkeit intelligibility. sinn-verwandt synonymous. -voll intelligent, clever, ingenious, very suitable, logical. -widrig absurd, senseless, contrary
Soffittenl ampe

to sense or reason, illogical.

sinterkohle sintering coal, noncaking $c$.

sintern sinter, form s., concrete, frit, consolidate, vitrify (cer.)

sinter-quarz siliceous sinter. - schl acke clinker.

sinterung sintering, fritting, fusing, vitrification, baking, caking, agglomeration, consolidation.

sinus, hyperbol i scher hyperbolic sine (sinh).

sinusförmige Bewegung, reinsimple or plain harmonic motion, sinusoidal $\mathrm{m}$.

Sinus-linie sine curve. - schwingung sinusoidal wave, sine w. -winkel sine of angle.

Sirene, Kondensator- condenser chopper or siren (produces pulsating current). Licht- light chopper, c. wheel. Zahn- tooth-wheel synchronizer, tone-w.

Sirutor Westector, rectifier of the copper or oxide type.

sitz seat, fit, lodgment. Presspress-fit, force fit, driving $f$. Schlichtgleit- plain sliding fit. Treib-driving fit.

Skala, reine true scale (mus.) Entfernungs- distance scale. Frequenz- frequency spectrum, $f$. scale. Linear-slide-rule dial (with straight scale).

Skal en-intervall scale division, s. interval. -rohr scale tube (of a spectroscope).

slip side slip (of airplane). smaragdgriun Guignet's green.

Smar agdsp at green feldspar, amazonite.

SMK (Sekunden/Meter/Kerze) metercandle-second.

Snellius'sches Brechungsgesetz Snell's law of refraction.

Sockel base or cap (of lamp or tube); pedestal. Röhren- tube base, valve $b$.

socken crystallize out, contract (of metal).

Soffittenl ampe overhead scoop, scaffold light or lamp. 
Sog, Hohl- cavitation.

soggen crystallize out, contract (of metal).

Sohle sole, bottom, bed, floor, level, base.

solarisationsfreie Platte antihalation dry plate (phot.)

Sole brine, salt water.

Solinglas crown glass.

Soll-kurs charted course, prescribed c. -wert nominal, rated, prescribed, assigned, normal, face or theoretical value.

Sonde probe, search electrode, test prod, sound, sonde (radio).

Abtast- scanning aperture, hole of Farnsworth dissector tube.

Hitzdraht- hot wire anemometer. $\mathrm{Kl}$ auenprüf-claw-type test prod or probe. Prijf- test prod, probe, search electrode. Radio-,für Wetterforschung radio meteorograph, radio sounding, sonde or sondage devices (for weather recording).

Sondenbildf angröhre Farnsworth type of pickup tube, F. dissector t. (with a scanning aperture).

Sorider- special, separate, exclusive. -abdruck reprint, separate $p$.

sondern separate, sever, segregate, sort (out).

Sonne (Klieg) sun, sun arc, Klieg lights. Gegen-parhelion, mock sun.

Sonnen-ausbruch solar eruption. -bahn ecliptic (apparent path of sun). - bestrahlung insolation, irradiation by solar rays, exposure to sunlight. -blende sun shield, black screen. -ferne aphelion. -fleckperiode sunspot period, s. cycle. -nähe perihelion. - schutz sunshade. -strahl ungsmesser solarimeter, pyroheliometer, actinometer (for solar flux density). - tätigkeit solar activity. -wärmemesser solarimeter, pyroheliometer. -zeitsekunde solar second.

sorbierender Stoff sorbent, sorptive. sorbierter Stoff sorbed substance, sorbate.

sortieren sort, assort, size.

Sortierung, Phasenaus- phase focusing

(in beam or drift tube such as

Klystron).

sossio spotty, bleary (of a picture). Sourdine mute.

Spähne, Ei sen- iron turnings, borịngs, filings or chips.

Spalt crack, gap, split, rent, fissure, slit, intei stice, aperture, lacuna.

Spalt, Aufzeichnungs- recording slit, $r$. aperture. 'Stufen- step slit (phot.) Ton- sound recording aperture or slit.

Spalt-ausleuchtung slit illumination. -bild,optisches optical slit image. -bildgerät photographic soundhead. - blende slit, apertured stop. -breite width of slit (of spectroscope, etc.), apertural width. -brüchigkeit cleavage brittleness. - ebene cleavage plane, c. face. -effekt aperture effect, apertural e. -fläche cleavage face, c. plane. - flächenzeichnung cleavage plane or crystal marking.

spaltfreie Beleuchtung apertureless or slitless illumination.

Spalt-körper cleavage substance, c. product. -öffnung chink (in clarinet). -optik microscopic apertureimaging optical means or "optic". -prozess fission process. -rohr collimator. -riss cleavage crack, c. fissure. -streuung slot leakage, s. stray. -ton slit tone, jet t.

Spaltung, Phasen- phase split. Strahlbeam splitting, split beam.

Spaltungs-kristall cleavage crystal. - produkt fission product, cleavage p. - untergrund fission background. Sp an chip, splinter; shaving, boring, turning (mostly used in plural form).

spanabhebende Bearbeitbarkeit freecutting machinability. - Formgebung shaping or fashioning by machine tool, with incidental removal of chips. 
Spange

270

Spange clasp, buckle, clip, staybolt.

sp annbar tensile, extensible, ductile, capable of being tensed or tensioned.

spannen stretch, strain, set, wind, tense, tension, tighten, span, clutch, grip.

Spann-kraft tensional or elastic force, tension, load, extensibility, expandibility, elasticity. -rolle tension roller, spring-loaded idler (m.p.).

- schraube turnbuckle, turn screw; chuck (magnetic).

Spannung (cf Potential) potential, voltage, tension, pressure (in electricity); strained state or condition; strain (of molecules).

Spannung, abgeflach te flat-topped potential (wave). anlaufen gegen eine- buck against a potential. an - liegender Draht negative wire. elastische- elastic strain. magnetische- magnetic potential (line integral of field intensity). mitlaufende- follower potential. sägeförmige- sawtooth potential, ratchet p. -verriegelnde- cutoff biasing potential.

Spannung, Abl enk-, deflection potential, sweep p. Abreiss- stopping potential, cut-off p. Anregungsexciting potential, stimulating p., pre-ionizing p., p. causing electron to be raised to a higher level out of its atomic bond. Ausgleich- transient potential; compensating p. Beschleunigungsgun potential, accelerator p. Bild-, Untergehen der swamping of video or picture signal potential (in noise level). Bildwellenvideo (signal) wave potential. Bogenzünd-ignition potential, arc-strikins $\mathrm{p}$. Brems- negative anode potential (in electronoscillation tube). Brenn-constant glow potential (is slightly lower than striking p.), normal. running p. (of photo-glow tube, etc.) Bruch- ultimate stress, breaking s. Brumm- hum potential, ripple p.

\section{Spannung, Resonanz-}

D- Dee voltage (of cyclotron). Druckeigen- residual compressive stress. Durchschlag- breakdown, rupture, disruptive, puncture or flash-over potential ( $\theta l$. engg.). Eigen-residual stress, inherent or internal tension or strain. Einsatz-(cf Zündspg.) sparkingover or flash-over potential; breakdown p. (in grid glow tube), starting p. Empfangs- signal potential, incoming $p$. Federspring tension, elasticity. Filmfilm tension, f. pull. Gipfelpeak potential, crest p. Glasstrain or internal tension of glass. Gleich-, wellige ripple potential, pulsating p. Glimmglow potential, breakdown p. (indicates incipient glow discharge in grid-glow tube); anode p. (at which glow discharge begins in photo-cell), stopping potential (lies slightly below glow p. in photo-glow or grid-glow tube, and is the value to which p. must be dropped to stop glow discharge once it has started). Grenzflächeninterfacial tension. Haft- adhesive stress. Ionisierungs- ionizing potential, ionization p., p. sufficient to detach electron from its atomic bond. Kipp-, Ruickl auf der flyback of sweep potential. Kreis"circle" voltage. Lösch- stopping potential (at which glow discharge is stopped; s. p. is lower than glow p. in photo-cell and glow tube); extinction p., critical p. (at which t. goes out); cut-off p. (of thyratron). Nutz- useful potential, signal p. oberflächensurface tension. Peil-directional signal potential (picked up by directional antenna; to be distinguished from auxiliary a.p.) Regelautomatic volume-control (ave) potential; dynamic-range or contrast regulating p.; generally: control p. or regulator p. Resonanz- resonance potential, radiation p., p. resulting in transition of electron from ground state to next orbit, with 
Spannung, Richt-

incidental emission of resonance spectrum. Richt- output voltage of rectifier, d.c. v. of rectifier. Ruhe- bias or steady potential; no-signal p., repose or quiescent $p_{.}, Q$ point $p_{\text {. }}$

Sägezahn- sawtooth potential, ratchet p. Saug- anode potential, driving p., positive (d.c.) p. (of photo-cell), saturation p. (if all electrons are drawn to anode). Schalt- variable bias potential (in silent tuning and automatic volume control).

Schrumpf-contraction or shrinkage strain or tension. Schubshear stress. Schwimm- floating, spurious or stray potential, blurring p., p. causing sway of picture, in telev. Schwundregel- automatic volume control (sve) potential. Sperrcut-off potential, quench p., stopping $p_{.}$, biásing $p_{\text {. Stoss- }}$ impulse potential, shock p. streck- yield stress. Teilpartial potential, componental p. Tonwell en- audio(signal) wave potential. Trübungspotential causing quadrantal error ( $Q E E)$. iber- overvoltage, excess-voltage, overvolting. Uml auf- magnetomotive force (mmf) (being the line integral of magnetizing force). Verkettungs-interlinked voltage. Versorgungs- supply (source) potential, voltages. Verzögerungs- delay voltage, delayedaction v. (in ave). Vorbiasing potential (impressed on a grid, magnet, etc. ); prestress (of metals); inherent bias tension (of a spring) to restore, say, to normally tensed or tensioned condition; anode, drive or positive p. (of photo-cell); normal or initial tension (in materials). Vor-, verriegelnde cut-off biasing poential. Vorgl imm- sustaining potential (in pre-glow range, slightly below critical discharge p.)

\section{Spannungsresonanz}

wiederkehr- recovery voltage. Zeichen- signal potential. Zünd-firing potential, striking p. (of ignitron, thyratron, etc.); $\mathrm{p}$. at which in glow-tube discharge changes from assisted to selfsustained form. Zusatz- booster potential, additional p.

spannungführender Draht live wire, charged w., hot w.

Spannungs-änderung change of strain (in metal). - aussteuerung, Anodenplate voltage excursion. -Dehnungsdi acramm stress-strain diagram, stress-deformation d. -differenz, Zündlbsch- striking-extinction potential difference. - -isen iron in voltage circuit (of a meter). -entlastung stress relief, anti-fatigue or anti-vibration means (in transmission lines). - erhöher voltage step-up means (e.g., transformer); booster. -festigkeit puncture strength, dielectric s.

spannungsfrei oliihen strain relief anneal or thermal treatment. -(es) $\mathrm{Gl}$ as strain-free glass.

Spannungs-glättung smoothing device (filtering out ripples), potential stabilizer. -knoten voltage node, potential n. -kreis potential circuit (of a meter). -kurve, Herz- electro-cardiogram.

spannungslos free from tension or strain, untensed; dead (absence of electrical potential).

Spannungs-messer, oberflächen- surf'ace tensiometer. -messer, Röhrenvacuum tube voltmeter. -modulation velocity modulation. -netz spacecharge grid.

spannungsoptisch photo-elastic. Sp annungs-prüfer, oberflächen-, nach Abreissmethode adhesion-balance type of surface tensiometer. - punkt terminal, tap. -reihe contact potential series, electromotive s., electro-chemical s. -reihe, Thermo- thermo-electric series. -relais undervol tage relay, low-v.r., no-v.r. -resonanzkreis series-resonance circuit, voltage 
r.c. -rücklauf,kipp- flyback of sweep potential, return of timebase p. to zero. -schutz, Berührungs- protection against electric shock hazard. -sicherheit breakdown strength, puncture proofness. -sicherung, Luftleervacuum arrester. -spitzenanzeiger crest-voltmeter, peak-voltage indicator. -spitzennivellierer vol lage peak limiter. -sprung voltage step, v. leap, abrupt change of v., discontinuity. -spule voltage coil (in two-waltmeter method). -stoss impulse vollage, polential i. or pulse. - strom curreni floriing in potential circuit (of a meter). -teiler voltage or potential divider, potentiometer. -teiler,Glimm- glow-tube potentiometer, g.-t. voltage regulator. -tensor stress tensor. -verlust drop of potential. -wechsel stress alternation, s. variation, s. cycle. -zeiger voltmeter; voltage vector.

Spannvorrichtung jig, chuck, holding, gripping or clamping device. sparren rafter, spar (of airplane). Sparschaltung (Nestel) circuit organization designed to economize plate current.

sparstoffarme Legierung alloy low in scarce or critical materials. Spartransformator auto transformer. Speckstein soapstone, steatite, talc. Speichelkammer moisture chamber (of soldering iron).

Speicher accumulator, means to store up electrons (or energy in general). Stromstoss-impulse storer, digit storing register. Wärmeheat accumulator, regenerator. speicher-platte storage pickup. -röhre signal or charge storing or storage tube (telev.) -röhre, Bild- signal or charge storage tube, normal iconoscope.

-wandl erröhre, Bild- super iconoscope. -wirkung signal storage, charge accumulation.

Speise-apparat feed apparatus. -hahn feed cock. -leitung (aerial) feeder lead, downlead, energyfeeder cable or wire, transmission line. -rohr feed pipe, supply pipe or tube.

Speisung supply, s. source, feeding, excitation, energization.

spektral zerlegtes Licht spectroscopically dispersed or separated light.

spektral analytisch spectroscopic, spectrometric.

Spektral-apparat spectroscopic apparatus, spectroscope. -bereich spectral range, s. region. - darstellung representation by spectra. - linie spectral line, spectrum 1. - linienfal tung "raltung" of spectral lines. - photometer spectrophotometer. - tafel spectral chart.

Spektrograph, Geschwindigkeitsvelocity spectrograph, v.

analyzer. Massen- mass spectrograph, m. spectrometer.

Vakuumgitter- vacuum grating spectrograph. Zweikristalldouble-crystal spectrograph.

Spektroskop, Luftplatten - FabryPérot interferometer, étalon (when not variable).

Spektrum, El ektronensprung- electron transition (or jump) spectrum. Feinstruktur- fine structure specirum, micro-s. s.

Fl ammen-flame spectrum.

Funk - see Wellenabgrenzung. Grosstrukturmacro-structure spectrum. Magnetorotations- magnetic rotation spectrum. Streifen-band spectrum. Übersichts- general spactrum. wellen-(cf wellenabgrenzung) spectrum of electromagnetic waves; radio wave $\mathrm{s}$.

Sperr-bereich suppression band or range, attenuation $b$. exclusion $b$. -blende blocking light stop, limiting s. -dämpfung stop band attenuation.

Sperre (cf Sperrer) wave trap, stopper, rejector; lock, seal, baffle. Band- 
Sperre, Rückkopplungs-

band rejection filter, elimination f., exclusion $f$. Rückkopplungs- reaction suppressor, feedback s., "Vodas". Spiegel-

frequenz- image-frequency stopper. Stör- noise gate.

sperren shut, shut off, block, stop, bar, space (apart), guard (a position), stopper, exclude, preclude, lock, cut-off or choke ? a tube).

sperrend, selbst- self-locking, automatically 1 .

Sperrer (cf. Sperre) trap, suppressor, excluder; lock, block. Echoecho killer, e. suppressor.

Sperr-feder click spring. -filter rejection filter, $r$. circuit, stopper c., suppressor f. - flüssigkeit sealing liquid. - glelchrichter barrier-film rectifier, blocking r., electronic r., metal r., cuprox $r$. -glled suppressor. -greifer pilot pin. - hahn stopcock. -haken pawl, latch, catch, trip, dog, click, detent, ratchet. -hebel pawl, latch, arresting lever, lock 1. -holz plywood. -kegel pawl, detent, click. -kette low-pass filter. -kipposzillator, -kippsender blocking oscillator. -klinke pawl, latch, catch, trip, dog, click, detent, ratchet.

-kondensator stopping condenser, insulating c., blocking c. -kreis rejector circuit, parallelresonance c.. wave trap c. -kreis,HF- low-pass filter or selective circuit. -kreis, NFhigh-pass filter or selective circuit. -kreiskopplung parallel resonance coupling. -membrane stop diaphragm. - rad ratchet wheel, $\operatorname{cog}$ w. -relais locking relay, guard $r$. -richtung high-resistance direction, backward d. (of a rectifier). -scheibe ratchet wheel. - schicht barrier layer, blocking 1., stopping 1. -schichtzelle barrier-type cell, photo-voltaic c., photronic c.,
Spiegelbild

rectifier c. (operating with a blocking layer). - schwinger blocking oscillator. - spannung biasing potential (impressed upon grid), cut-off p., quench p. (in super-regeneration). -strom backward current, inverse c. (of rectifier). -topf wave trap.

Sperrung, Röhren- cut-off of a tube, quenching of discharge. Strahlbeam cut-off, b. eclipsing, b. gating (telev. tube, by blanking pulse).

Sperr-ventil stop valve, check v. -wirkung (cf, sperren) brake action, retarding a., delay a., lagging, valve a. -zeit, Teil-partial restoring time (in echo suppression). -zelle barrier-type cell (working with blocking layer).

Spezialfilm hoher Steilheit highcontrast emulsion film.

spezifisch(e)Ladung specific charge, charge-mass ratio. - (er)widerstand resistivity.

spezifizieren specify, itemize, particularize.

sphärisch überkorrigiert spherically overcorrected. -unterkorrigiert spherically undercorrected.

spheroidaler Zustand spheroidal state. Sphärokristall spherical crystal, sphero-crystal.

Spiegel mirror, reflector, speculum, polished or reflecting surface, any deposited layer or coat (inside a tube, etc.), silvering; surface or level (of a liquid, etc.)

Spiegel, Augen- ophthalmoscope, skiascope. Fang-collecting mirror. Geräusch- noise level. Gettergetter patch, g. film. Hohl- concave mirror, c. reflector. Kipposcillating mirror. Kugelspherical mirror, s. reflector. Lamell en- strip-like mirror (in screw scanner). Parabol- parabolic reflector. Schwing-vibratory mirror, oscillating $\mathrm{m}$. stör- noise level.

Spiegel-belag mirror coating, reflecting film, silvering (of a mirror). -bild speculum image, mirror i.; 


\section{spiegelbildich}

flare ghosts or spots (camera), double or reflected (ghost) image (in telev.).

spiegel-bildlich homologous, mirror-picture condition, specular. -bildlich(es) Verhältnis inverse relationship. -blank highly polished.

Spiegel-bogenl ampe reflector arclamp. -feld revertive-signal panel, check-back position indicator (giving readings of remote instruments, in telemetering work). -frequenz image-frequency (in radio). - frequenzsperre image-frequency stopper or rejector. $-\mathrm{gl}$ as plate glass. -gleichheit mirror symmetry.

spiegelig specular, mirror-like. Splegel-kranz row, array, rim or set of mirrors (in scanner), drum scanner. -kreuzlibelle reflectortype cross-level (panoramic telescope). -metall speculum metal. spiegeln reflect, shine, glitter, be reflected, glare (from glass surfaces).

spiegelnde 0 berfläche specular surface. -Reflektion specular reflection, mirror-like $r$.

Spiegel nei gungsmesser Abney level (of reflecting type, to measure vertical angles).

Spiegel-rad mirror drum (e.g. of Weiller). -rad,Zellen-line scanning mirror wheel (of Scophony). -reflexion specular reflection, regular $r$. -schraube mirror screw (scanner). -symmetrie mirror symmetry, specular s.

Spiegelung reflection, specular effect, picture mirroring; replica (of curves), homologous condition or relation. Dreh-combined rotation and reflection. Rückspecular reflection, regular $r$. viel-multiple of image.

Spiegel-weil e reflected wave. -wirkung cut-off, reflecting or mirror effect (of electron lens when electrons are unable to cross barrier).

\section{Spinvertellung}

Spiegler reflector.

Spiel play, playing; working, operation, action (of mechanical parts), cycle of action; backlash, shake, play or lost motion (of wheels, gears, etc.); deflection, throw or excursion (of meter or instrument needle); set (of things).

Spiel, Flanken- backlash (of screwthread). Glocken- carillon (set of bells).

Spiel-art touch (mus. instrument); variety. - aufbau, Ioni si erungsTownsend structure or buildup.

Spieler,Platten- disk-type phonograph or gramophone. Rück- playback, dubbing device.

Spiel-haus, Licht- motion-picture theater or playhouse, movie. - leiter director of play. -raum play, latitude, margin, range, scope, freedom of action, elbow room, clearance, backlash. -raum, Bell chtungs- latitude of exposure. -saiten fingerboard strings (zither). -zeugkino home movie.

Spiesskant diamond.

Spin, Einheits- unit spin. Kernnucleus spin.

Spinbahn-aufspaltung spin orbit splitting. -kupplung spin orbit coupling.

Spindel spindle, pivot, axle, arbor, shaft, axis of revolution.

sp indel förmi g spindle-shaped, fusiform.

Spindelführung screw control (of recording cutter).

Spindeln hydrometer test.

Spindel trieb worm drive, "rear.

Spin-drehimpuls spin angular momentum. Spinelle spinel (cryst.)

Spin-glleder spin terms. -impul skopplung spin orbit coupling. -momentdichte spin momentum density. -multipletten spin multiplets.

Spinne,Lautsprecher- spider of loudspeaker (inside or outside, to keep voice or signal coil centered).

Spin-vektor spinor (two-dimensional vector). -verdopplung spin doubling. -verteilung spin distribution (math.) 
Spiral antenne

Spiral antenne (flat) spiral aerial or antenna, extensible s. antenna, s. loop.

Spirale spiral, helix, coil, twisted structure.

Spirallochscheibe Nipkow scanning disk with helical array of apertures, spiral d. scanner.

Mehrfach-multi-spiral scanning disk. Vierfach- quadruple scanning disk.

Spiral-steigung pitch or lead of a spire or spiral. -täuschung spiral illusion.

Spitze point, peak, crest, tip, top, apex, vertex, summit. Prüfpenetrator (of hardness tester). Resonanz- resonance peak or crest. Stecker- tip of telephone plug (fitted into tip jack or pup $j_{\bullet}$ )

Spitzen-anzeiger, Spannungs- crest or peak voltage indicator, $c$. voltmeter.- - aufhängung point suspension, pivot s. -elektrode needle electrode, point $e$. - entladung point discharge, needle (-gap)d.

spitzengelagert journaled on points. Spitzen-glas reticulated glass. -lagerung jeweled bearing, pivot jewel, point suspension, p. support. -leistung record (achievement, performance, or accomplishment), peak output. -nivellierer, Spannungs- voltage peak limiter. -zähl er needle counter. -zirkel compasses, dividers.

spitzer Mor sepunkt clipped dot.

spitzig pointed, acute, sharp, tapering.

Spitzlichter bright lights, highlights (in a picture); tangential lighting.

spitzwinklig acute-angled.

Splint sap, sapwood; pin, peg, key, splint, cotter, split pin. -holz sapwood, sap.

Splitter,Kristall- crystal chip, c. grain.

Sprach-aufnahme speech, voice, vocal or dialog recording. -beeinflussung voice control, modulation by v. -deutlichkeit articulation,
Spreize

intelligibility (of vowels and consonants, etc.)

Sprache speech, voice, articulate sound, language; code.

sprachgeschalteter Tel ephoniesender voice-modulated telephony (signal) transmitter, v. $-m$. radiophone.

Sprachlaute speech sounds, articulate s.

sprachmoduliert speech-modulated, voice-m.

Sprach-untersuchung speech study, s. analysis. -verständlichkeit articulation, intelligibility of speech or articulate sound (ascertained, e.g., by logatoms). -verstärkungsanlage (public) address system. -wi edergabe voice reproduction; demodulation of speech.

Spratzen flicker, sputter or spitting (of a cathode).

Sprechaufnahme speech, voice, vocal or dialog recording.

sprechende Bogenlampe speaking arc, Simon arc, Duddell a.

Se recher, syntheti scher voder. Fernseh- television telephone, video $t$.

Sprechfaktor, Fern- telephone influence factor. -frequenz voice frequency, speech $f$. - hörer microtelephone, telephone handset, transceiver. -kapsel condenser microphone. -kopf soundbox, recording soundhead. -kopf, Abnehmer- sound pickup, reproducing head. -l eistung response of an electroacoustic system. - schalter speaking key. - schl auch speaking tuhe. - schliussel speaking key. -strom voice, speech, sound, telephone or signal current. -stromkreis circuit carrying voice or $\mathrm{AF}$ signal currents (in loudspealer). -transformator speech transformer, AF t. -verkehr, Fernseh- video telephone traffic, television telephone communication.

Spreize spreader, spacer, strut, outrigger. 


\section{Spreizenkamera}

Spreizenkamera, Spreizkamera strut camera, extension c.

sprengen explode, blow up, blast, burst; sprinkle (with water). sprenkeln sprinkle, spəckle, mottlə, spot.

Spriegel hoop.

springen burst, break, crack, fracture; spring, jump, leap.

Spring-faderwage spring balance. -kraft springiness, elastic force, elasticity, power of recoil or rebound, resiliency. -punkt critical point (where a break or discontinuity occurs). Spritze, El ektronen- electron gun. Licht- recorder lamp with constricted neon arc (e.g., Ewest "light gun," pointolite, etc.) spritzen squirt, spurt, spout, spulter, spray, exude, inject (syringe).

Spritz-entladung initial discharge caused by rapid surge of ions; needle-gap d. -guss (pressure) die-cast. -pistole spray gun, s. pistol (of Schoop), aerograph. spröde brittle, short. -machen embrittle.

Sprödigkeit,Kalt- cold brittleness. Spross, Sprosse shoot, sprout, germ; rung (of a ladder).

Sprossen-rolle sprocket drum. - schnürspur, Gl ei ch takt- variabledensity single squeeze track. - spur,Gleichtakt- single variabledensity track. -trommel sprocket drum or wheel.

Sprossung germination.

sprühen spray, spit, scintillate, scatter, produce corona (on wires). Sprïh-festigkeit spray proofness.

- ionen spray ions. - schutzwulst anti-corona collar, guard ring. - verlust corona form of discharge, brush d. loss.

Sprung (cf Sprungentfernung) crack, chink, fissure, flaw, fault; jump, bounce, leap; break, discontinuity (of a curve), transition (in spectra and quanta); hop (of waves). Absorptions- absorption discontinuity. Barkhausen- Barkhausen
Spule, Achter-

effect (abrupt changes of magnetization). Bild-picture repetition frequency, p. cycle; break or shift of vision. Phasen- phase angle shift. Quantum- quantum transition, q. leap, q. jump. Spannungs- voltage step, v. leap, abrupt change or discontinuity of $\mathrm{v}$. Sprung-entfernung skip zone, s. distance (between beginning and end of wavehop). -faktor, Zeileninterlace factor or ratio. sprunghaft irregular, non-sequential, sudden, by leaps and bounds. Sprung-höhe pedestal level or height, amplitude of return or flyback (telev.). -methode, Zeilen-interlaced, interleaved or intermeshed scanning method (with odd or even line interlace). -punkt (cf. Springpunkt) transition point. - spektrum, El ektronen- electron transition or jump spectrum. -stelle point of sudden irregularity, unsteadiness, change, or discontinuity. -wahrscheinlichkeit transition probability, leap p. sprungweise (cf ruckweise) by steps or stages, by leaps and bounds, intermittently, discontinuously, non-sequentially.

Sprung-winkel, Aus- angle of reflection. zeit transit time, transtion t. (of electrons).

Spule, angezapfte tapped coil.

-, angezapfte, in der Mitte centertapped coil, mid-tap c.

-,flächenhafte laminar or areal coil, non-filamentary $c$. -, gepanzerte, wenig streuende iron-core deflection yoke unit (with low spot and pattern distortion). -,gleichstromvormagnetisierte d.c. controlled, saturable coil (of loudspeaker). -,kleine bobbin. -,körperlose structure with entire mass of vibratory system contained in oil. -, selbstschirmende self-shielded coil, astatic c.

Spule, Ablenk- deflection coil, deflector c, d. yoke. Abwickelfeed spool, f. reel. Achter- 
figure- $\varepsilon$ coil. Auflauf- take-up reel. Aufsteck- plug-in coil. Auslöse- trip coil. Beruhigungsfilter choke, snoothing coil. Betätigungs- working coil, tripping coil, differential $c$. Binokular-binocular coil (form of closed-field c.) Dämpfungsdamping coil, amortisseur $c$. Dreh- moving coil, rotating c., rotor: c. Dreh-, mit einem Lager unipivotal moving coil.

Ei senblätterkern- laminated ironcore coil. Eisenstaubkern- irondust-core coil, Ferrocart core c. Feld-field coil, nagnetizing $c$. Felderreger-field exciting coil (in electro-dynamic loudspeaker). Filmvorrats- film wagazine roll, magazine. Hon i gwaben- honeyconb coil. Induktanz-, ei sengeschlossene closed-core coil. Induktions-, variable varioneter. Käfigcanned coil, shielded c. Kondensor- condensor coil (electron microscope). Kopplungscoupler coil. Kopplungs-, veränderliche vario-coupler. Korbbodenspider-web coil, basket-type c. Kreis-tuning coil. Litzenlitzwire coil. Luft-air-cored coil. Luftdrahtverlängerungsaerial loading coil, lengthening c. Magnet- solenoid, field coil. Massekern- compressed iron-core coil, dust-c.c., Ferrocart c.c. Mehrlagen- multilayer coil, banked c., pile c., honey-comb c. with banked winding. objektivobjective coil. Projektionsprojection coil. Pupin- loading coil, Fupin c. (for reactance balance). Quer- leak coil. Ring- toroidal coil. Rückkopplungs- tickler coil, retroactive c. Sammel- focusing coil. Saugsmoothing coil. Schwing- moving, speech, voice or signal-current coil. Spannungs- voltage coil (in wattmeter). Steck- plug-in coil, detachable c.; demountable reel (film). Striktions-focusing coil. Strom- current coil (in two-wattmeter method). Such- search coil, probe c., rotating c., exploring c. (of d.f.); flip c. (in magnetic field tests). Tauch- moving coil (of loudspeaker), telescoping c. Teil-fractional coil, component or subdivision of a coil. Variometer-,drehbare rotor of variometer. Varlometer-,feste stator of varioneter. Verlängerungs- serial loading coil, lengthening c. Vorratsmagazine, m. roll (m.p.) Wabennoneycomb coil, lattice-wound c., duolateral c. Zeilen- line scan coil, 1. sweep c.

spulen wind, reel, coil up.

Spulen-abzweig coil tap. -achse bob of spool, core of s. (m.p.) -anzapfung coil tap. -arm nasazine or spool arm or support. -durchflutung nagnetic potential of coil. -fahrstuhl band-width regulator with reciprocating auxiliary coupler coil parallel to oscillating c. halter, Filmfild roll holder. -kern bob of spool, core of s.(m.p.) -kette low-pass filter. -körper coil forn, c. forner, spool. -mark nolded coil core. -revolver coil switch mechanisu (for waveband change). - topf can, canned coil. -träger niagazine or spool arm or support. -windungsschlussprüfer inter-turn short-circuit or continuity tester.

Spund stopper, plug, bung; tongue (of dovetailed board).

Spur track, trace, trail; gutter, channel. -, vorionisierte preionized track. Brumm- buzz track. Ton-, eingeprägte track enbossed on film strip. Ton-, eingeschnittene track engraved in film strip. spurenweise in traces. Spur-faktor tracking factor (phonograph recording). -l ager thrust bearing. -methode, Nebel- Wilson cloud trask method (using a streak of droplets and an ionizing particle). -verzerrung tracking error, t. distortion (phonogr.) -zapfenlager (end) thrust bearing. 
-zeit,Bild-Watkins factor, development $f$.

Stab rod, bar, stick. -antenne rod antenna, whip a.

Stäbchen rod (of nerve endings of eye), rodlet.

Stabfeder, Dreh- torsion bar spring. stabilisieren stabilize, steady, wake constant, unif orm or normal, regularize.

Stab-kopf rod eye. -mikrophon carbon stick microphone. -röhre arcotron.

Stachel sting, thorn, spine, prickle, prong.

Staffeln, Gl asplatten- (Michel son) echelon spectroscope.

Stahl, naturharter self-hardening steel. Edel-refined or superior alloy steel.

stahlband-armiert,-bewehrt steel-tape arnored, ribbon wrapped.

Stahl-bandaufnahme ragnetic stee 1 tape recording. -drahtaufnahme sound on wire recording system. -kiesstrahlen,-kugelblasen steel shot blasting. -stechen steel engraving.

Stamm-baum flow sheet (of working process). -farbe prinary color. -holz sten, heart or trunk nood. -körper parent substance, p. body. - Datent parent patent.

Stampfe stanp, staniper, pestle, punch, ramier, tamper.

stampfen ran, tamp, pave, pound.

Stampfwerk stanp nill.

Stand, helliger, der Technik present state of the art (or technology).

Stand, Achs- wheel base. Führercontrol cabin, pilot's cockpit, p. compartment (airpl.), tank connander's position. Prüftest bed.

Stand-anzeige dead beat indication or reading (of an instrument). -anzeiger bearing indicator means, d.f. dial.

Standarte lens carrier, base board: stand-bild still film picture, in- aniuate p. -entwicklung slow developnent, stand $d$.

Ständer stand, standard, pillar, post, pole, pedestal, uprisht, colunin, housing (of rolls); stator, field systen (of dynamo). Abtropf- arainer. Lupen- lens stand.

Standfestigkeit stability, rigidity, steadiness, solidity, resistance to deformation, creeping strength (of materials). Dauer- long-tine creep strength.

Standfestigkeitsprüfung (long-time) creep test.

standhaft steady, constant, firn, stable.

Stand-linie base line, position Iine. - linienpeilung position-line bearins, great circle b. -linienverfahren base-line method of measurenent.

Standort station, stand; position (d.f.), fix. -bestimmung obtaining a $f$ ix, position or location finding (by two or more d.f. stations), fix, position-line bearing, great circle b. -daten positional infortation. - peilung see Standortbestimmung.

Standsicherheit stability or steadiness (to shock and vibration).

Stange stick, rod, bar, pole, post. Abspann- stay, terninal or strain pole or mast. Kreuzungs- transposition pole. Schub- thrust rod, push rod, pusher $r$. Zahn- rack rod.

Stanniol tinfoil.

stanzen punch, perforate, stanp or blank out (from a solid sheet).

Stanz-matrize cutting dieplate, punching die. -presse starping press, punch. -verfahren stalping nethod, punching $\mathrm{m}$.

Stapel staple, pile, neap, stack. stark belastet heavily loaded, highly 1., carrying a heavy load.

stärkegrad intensity, degree of strength or of concentration. Starkstromstöruns interference from power systens; power (supply) failure. 
starr stiff, rigid, non-yielding, Starrheit rigidity, stiffness.

Startbahn runway.

starten, durch- "open up" and repeat landing procedure (in $\mathrm{ZZ}$ landing method).

Startweite (der Elektronen) distance from origin (of electrons), distance from object to lens, object distance.

stationär(e)Messung deadbeat neasurement. $-($ er $)$ Zustand steady, stationary or stable state.

Stations-melder station indicator, tuning i. -wähler station selector.

Stativ stand, support, tripod. Kurs- class microscope. Röhrentelescopic tripod. Schlingercardan-suspended tripod. Verfolgungs- running tripod, followshooting $t$.

Stativlupe stand magnifier.

staubdicht dustproof, dust tight.

Staub-figuren powder pattern, dust

figures (of Lichtenber $\xi$, Bitter,

Debye, Scherrer, Hull, etc.).

-kern dust core, molded $c$.

-rauschen dust noise (of film).

-welle acoustic dust pattern

(showing nodes and antinodes).

-zähler dust counter.

Stauchdruck compression.

stauchen compress (by blow), knock, beat, upset, clinch (a rivet).

Stauchmatrize,Kal t- cold upsetting die.

stauen stow, dan up, choke, baffle.

Stau-gerät baffle type fluid flow gage. -gitter suppressor grid, baffle grid: -körper baffle, barrier.

Stauung, El ektronen- electron accumulation or cloud (in virtual cathode).

Stauwirkung baffle or accunulator effect, damiing e.; resistance.

stechen stick, prick, pierce, sting, tap; engrave.

Stechkolben pipette.

Stecker, berïhrungssicherer shockproof plug. -, dreiteiliger three- point plug, three-way p. -, unverwechsel barer non-interchangeable plug.

Stecker, Blind- dumny plug. Dreifachthree-pin plug, triplug. Klinkenjack, plug switch. Netz- power supply plug, light socket p.

Zwi schen-socket adapter, a. plug.

Stecker-buchse,-hül se plug socket, connector s. - säule multiple socket and plug device, multi-way s. and p.d. -spitze tip (of telephone plug).

Steck-regler plug-in and socket

(volume) control. -spule demountable magazine or reel (m.p.); plug-in coil, detachable c. -umschalter plug-in switch.

steg path, small bridge (of string instrument), cross piece, strap, bar; material or wire portions of a grid (between meshes); any narrow strip of solid material between holes or recesses, as in w.p. film between perforations, or between tracks or grooves in phonograph disks, called barrier or land. Druck- pressure bar, harmonic b. Gitter- grid stay; solid portions of g. mesh, §. strips used for wires. Steg-abstand distance between grid wires (or g. supports or stays). - gitter grid in which all wires are parallel to axis and surround cathode cage-fashion. -zahl number of grid wires, stays or supports.

Stehbild still picture, non-animated p. -verfahren lantern slide projection method.

Stehen des Bildes steadiness of inage. --, mangel haftes jumping or unsteadiness of imase.

stehende Figur stationary pattern, s. figure. welle standing wave, stationary $w$.

Steh-kolben flat-bottomed flask. -lager pillow block, pedestal bearing.

steif stiff, rigid, firm, non-yielding.

Steife prop, strut, brace; rigidity, stiffness. 
steigen rise, ascend, mount, increase.

Steigerer, wucht- dynanic expander.

Steigerung increase, increment, raise, boost. Dynamik- dynamicrange expansion. KontrastCallier effect, print contrast (phot.)

Steig-höhe elevation (in capillary tube), height of rise or of ascent, pitch (of a screw).

- leistungsmesser rate of clinb neter ("varioneter" neasuring both $r$ ise and descent). -rad ratchet wheel, escapement $w$.

Steigung, Gewinde- pitch (of screw thread). Spiral-pitch of a spire, lead of a $\mathrm{s}$.

Steigungsmesser, Luftschr aubenpropeller pilch indicalor.

Steigwinkel rise angle. steil,zu too high a gamna (in film printing).

Steilabfall der Sägezahnkurve abrupt drop of sawtooth wave.

Steilheit sradient, nutual conductance (nutual characteristic), slope (of tubes); contrast ( $\mathrm{f} i \perp \mathrm{n}$ ).

Steilheit, Emulsions- steepness of gradation. Entwicklungs- slope (of $\mathrm{D}$ and $\mathrm{H}$ curve). Film-, hohe high contrast (of film). Filtersharpness of selective network. Flanken- width of transition interval between transmission and attenuation bands; steepness of sides or slopes of a curve or of upstroke and downstroke of an ilpulse. Gradationskurven- ganna. value or slope of response line. Sieb- sharpness of selective network. Transponierungs- slope or Iutual conductance (of a mixer tube). Umwandlungs- mutual conductance, slope (of mixer tube, etc.)

Steilheitsmesser derivator, tangent neter (for curves).

Stein-bildung, Kessel- boiler scale formation, incrustation. -gut white ware (with white absorbent body and soft glaze). -kohlenschlacké coal cinders. -lager jeweled bearing. -öl petroleum. -porzellan hard porcelain.

Stelle spot, site, locale, location, place. an - und Stelle in situ. Stelle, abgeschirmte dead spot, radio shadow, r. pocket. Dezimaldecinal place. Locker- Loose place, 1. spot (cryst.) Nullzero place. Peil-control station (in sroup of $d$. f. stations). stellen place, put, set, regulate, adjust.

Stellenzahl position number, atonic n.; index (nath.), number of digits. Stellhahn regulating cock. stellig,drei- three-place, t.-figure (value). fünf-(e) Cenauigkeit five-figure accuracy. sechswith six decimal places.

Stell-kraft, Rück- restoring, retractile, elastic or restitution force or pressure. -schraube set screw, adjusting $s$.

Stellung, Arbeits- working position, operative $p$.

stellvertretender Patentkommissar deputy or acting patent commissioner. Stempel stanp, stanper, die, punch, pestle, piston, matrix. Lamoenpress or squash of a lallp. Presspress rall.

Stempel rohr plunger tube, piston $t$. (used in acoustic inpedance tests). Stengel,Pump- pumping lead, punp tube (short piece of tubing connecting with vacuun punp), exhaust vent. Stereogrammetrie stereophotogrammetry. Stern-Dreieckschal ter star-delta switch.

sternförmig star-shaped, stellate. Stern-gliedkette T (-nesh) network. -rad star wheel. -rohr astrononical telescope. -tag sidereal day. stet, stetig continuous, constant, stable, steady.

stetiger bildwechsel continuous, steady, non-internittent feed or motion (of picture strip). steuerbar nodulable, capable of being modulated or controlled; steerable (of antenna charactistic and polarization); nanageable, nanoeuvrable, controllable. 


\section{Steverblende}

Steverblende modulating electrode, modulation shield, $\mathrm{m}_{0}$ grid (in cathode-ray tube).

Steuerbord achteraus starboard af $t$. - voraus starboard bow. -kurve starboard (right-hand) curve (airplane approach).

Stever-daumen sequence switch cal. -drossel masnetic modulator, $\mathbb{m}$. choke, Heising modulator. - frequenz pilot frequency, synchronizing f. -generator drive oscillator, naster o. -gitter control grid; shield (of a c.-r. tube). -kurs steered course, conpass c. -mann, Ton- operator controlling sound volume (in theaters).

steuern steer, regulate, control, modulate, guide, direct.

herunter- regulate down, decrease carrier amplitude.

Steuer-nocken sequence switch cam. - quarz (frequency) stabilizing quartz crystal. -relais pilot relay. -röhre naster oscillator tube, drive t., drive o. -röhre, Quer- cross-control tube, beall tube (depending for its operation on b. deflection). -schal ter master switch, control s., sequence $\mathrm{s}$. - sender naster oscillator tube, drive t., d.o. (working upon main transmitter, in independent drive). -stelle sound gate $(\mathrm{f} i \perp \mathrm{n})$; impulse receiving place or point (in remote-control, telemetering, etc.) -stelle, Ton- tone-control point, exciter p.y sound gate. -strich dot or pointer to observe or read bearings. -stufe, fremdgesteuerte, quarzgesteuerte crystal-stabilized oscillator stage, master or driver stage stabilized by quartz crystal.

Steuerung auf Dunkel modulation to dark condition, negative $\mathrm{m}_{\text {. }}$-auf Hell modulation to light condition, positive 1 . Ausblend- obturator modulcation, cut-of $f$. Dichtigkeits- charge-density modulation. Dunkelwert-adjustment to value of darkness. Fern- remote-control

\section{Stift}

action, telemetric c. (with selsyn motor and synchro system). Frequenzvon Senderschwingungen frequency stabilization of transuittęr oscillations. Geschwindigkeits- velocity modulation. Gitter-grid sweep, g. swing, g. excursion, g. excitation; s. control (in ignitron, etc.) Helligkeits-, Intensjtäts- brilliancy modulation, intensity m., brightness I. or control. Kristall- crystal stabilization, quartz control. Ladungs(dichte)- charge density modulation. Leitungsstrom- conduction-current modulation. Lichtiight scanning, 1. modulation, 1. control. Linien- velocity modulation. Zeilen-velocity modulation, variable-speed $n$.

Steuerungs-bereich drive range, swing of anplifier grid, operation inside straight part of characteristic. -einrichtung, Licht- light valve, light relay.

Steuer-verstärker amplifier between power and input stage (operating on pure voltage amplification). -welle control wave (in chain broadcasting), pilot w. -zylinder fousing grid, Wehnelt g. or cylinder (in cathode-ray tubes).

Stich prick, puncture, stab, sting, stitch; engraving; tap, tapping; pass (rolling nill). Form- shaping pass (rolling).

Stichel, Schneide- cutter. cutting stylus, c. tool, c. head, engraver (phonograph recording), scriber. stich-flamme fine pointed flame, dart of $f$. -leitung tie line, tap 1. -loch tap-hole. -pfropf taphole plug. -probe sample taken by tapping, random sampling, assay of tapped metal. -wortverzeichnis (key-word) index.

Stickstoffhalogen nitrogen halide. stiel handle, shaft, stem, stalk. stift pin, peg, tack, stud, pencil. Gewinde- headless screw, grub s. Justier-pilot pin, registration p. Mittlungs- centering pin. Schreib- stylus, style, cutting or engraving tool. Tast- tracer 
point, ieeler.

Stift-lagerung pin suspension, $p$. supporting. -rad sprocket wheel. -schlüssel pin wrench. -schraube tap bolt.

stilb stilb (unit of luminosity in

Hef ner candles per square cr. stille Entladung corona, silent discharge, effluve. -Zone

skip zone, dead spol.

Still-abstimmung silent tuning, quiet t. -einstellung silent tuning, g.a.v.c. tuning. -l eben still life.

still-schweigend tacit, inplicit, implied; silent, quiet, caln. -stehen stand still, stop, arrest, be stationary.

Stimme,gemischte nutation stop

(organ). -, schwebende voix

céleste. Grund- foundation stop

(organ). -, Lautstärkenbereich der rienschilichen human voice intensicy range. -, Modulation der inflection of voice.

Stimmflöte tuning pipe, pitch pipe.

Stimmgabel, schreibende tuning fork chronoscope. - sender tuningfork buzzer oscillator, vibratingreed transuitter. -steuerung tuning fork control (in phonic whee1). -unterbrecher tuningfork interrupter. -zinken prong or tine of a tuning fork. stimm-hafte Konsonanten sonant,

voiced or vocal consonants.

-lose Konsonanten surd. non-vocal or breathed consonants.

Stirim-pfeife tuning pipe, pitch p. - platte reed board, $r$. plate (of accordion). -ritze slottis, slottic catch or cleft. -schlüssel tuning key, wrench (of kettle drun) -stock wrest plank (piano). -ton tuning pitch. -wirbel wrest pin. stimmung, hohe high pitch. -, Flöte mit flute with tuning slide.

Tremolo- tremolo.

stimmungsbild key picture, sertimer.t

p. -, dunkles lok-key picture. -, überhelles high-key picture. Stirn, wellen- wave face, w. front, w. head.
Stirn-ansicht end view. -bügel headrest (of magnifier). - fläche end face; face, front. -holz cross-cut wood, end-grain w. -rad spur wheel. -streuung overhang leakage flux. -verbindung face connector. -wand front wall, plate or panel; end w. -welle onde de choc, inpact w., bow w. -widerstand (front) end resistance, leading-end $r$. -zapfen trunion, end journal.

Stocheisen poker (bar or iron), fire p., stirrer, stoker, rake.

stock stick, staff, stock. stocken stop, slacken, hold up, arrest; mold, decay.

Stockflöte stick flute.

Stockung stoppage, stagnation, obstruction, standstill, jan, "bottle neck".

Stoff substance, matter, material, stuff, fabric, cloth. Trockensiccative, drying substance.

Stoff-aufwand expenditure or reouirement of material. -bahn web of fabric or cloth (textile). -bedeckung, wandbekleidung dreping, gobo, tormentor (when in the form of a portable wall with sounci-absorbent material, in m.p. studio). - mangel shof tage of material, underfill

(in rolling). -patent patent covering substance or material, product p., substance p. -teilchen particle of matter, corpuscle. -verbindung composition of matter.

Stöpfel, Stopfen stopper, plug, cork. Stopfen, Griffel- stopper with thumb piece. Schliff-ground-in stopper (of glass).

Stopf-ton (der gedackten Trompete) stopped tone (of $\mathrm{s}$. trumpet). - werg oakum.

Stoppbad short-stop treatment. Stöpsel, geschlitzter split plus. Blind-duminy plug. Glas- glass stopper.

Stöpsel-glas stoppered glass. -hahn stopper cock, c. stopper. -körper plug body.

stöpseln plug in, insert a p. falschnisplug. 
Stöpsel-rheostat resistance box (plug operated). -schalter plug switch.

Stör-ampl itude noise level. -anfälligkeit susceptibility to trouble, "X", interference or noise, trouble incidence. - befreiung elimination of interference, jaming, fading, strays, static or atmospherics. -befreiung, Einrichtung zur static eliminator, $X$ stopper. -breite detuning "width" (detuning required to reduce resonance amplitude to 1 percent).

Storchschnabel pantograph. stören interfere, jan, disturb, trouble, perturb, derange. Störer,durch - verdeckte Zeichen swanped signals.

Störernullung connection of case of interfering device with neutral wire (in d.c. mains). Stör-faktor, Fernsprech- telephone interference factor. -feld stray field, interference $f$. -festigkeit imunity from noise, disturbance or distortion. - gebiet mush area (in chain broadcasting), disturbed area. - geräuschatmen fluctuation of noise, waxing and waning of $n$. -gries low noise in picture background due to thernal effects, Brownian movement, mechanical properties of tube, and lowfrequency fluctuations of local enission density, i.e., shot effect. -lautstärke noise level. -muster spurious pattern, false p. (telev.) -nebel chief interference $z$ one, maximum noise z. -niveau noise level. -pegel,"ntergehen der Bildspannungen im swamping of picture signals in noise level. -schutz interference elininator, trouble elinination, anti-parasitic means. -schutzpackung noise suppression, shielding harness (for airplane engines), interference eliminator kit. - sender shadowing signal (telev.); interfering or jamming station. -signal, Abschattierungsspurious or shadowing signal (in iconoscope operation; known also as "shading," "black spot," "tilt and bend" etc.). -signalKompensation conpensation of spurious sienal in iconoscope operation by shading circuit. -sperre noise gate. -spiegel noise level. -stelle center of disturbance (cryst.); point of disturbance or perturbation; discontinuity. -suchgerät interference locator, signal tracer (used stage by stage in receiver). Störung dísturbance, perturbation, noise, distortion, interference, statics, atmospherics, jamming (in signal transnission); disarrangement, derangenent, trouble. -, atmospärische atmospherics, static, stray, "X's", QRN. -, ausserirdische extra-terrestrial or interstellar noises or disturbances. -, luftel ektrische statics, atmospherics, "X". Eigen- internal trouble. Gitterlattice distortion, lattice dislocation. Laufzeit- delay distortion. Lichthof- halo disturbance. Nah- nearby interference, local i. (from sources close to receiver site). Starkstrom- interference from power systems; power failure. störungs-rechnung perturbation calculation. -suche trouble "shooting", interference location. - theorie perturbance theory. -welle parasitic or interfering wave, jaming w., garbling w. (in secret telephony). -well enweg path of interference wave.

Stoss impact, shock, collision, pulse, count or burst (e.g., of cosmic rays), inpulse; push, stroke, jolt, recoil, thrust; joint, junction (of rails). Durchbruch- burst puise. Höhenstrahl- cosuic ray burst or shower. Rück- back stroke, recoil. Schienen- rail joint. Spannungs- potential inpulse, p. pulse, impulse voltage. Treppen- 


\section{Stossdämp fung}

lap joint.

Stoss-dämp fung damping or attenuation of pulsations or vibrations, shock absorption. -dauer duration of collision or shock. -durchschlagfestigkeit inpulsevoltage breakdown strength.

Stösse, Dreier- triple collision, three-fold c. Dunkel- backǵround counts (in Geiger-Müller tube).

Stössel, Stösser pestle, stamper, ranimer, tamper, sten (of valve), inpulsing, pushing or inpacting rod (in loudspeaker), driver rod.

stossen push, thrust, hit, knock, impact, ram, pound.

Stoss-erregung shock, inpact or collision excitation.

-feder,Strom- impulse spring (in dial). -festigkeit shock, impact or inpulse (breakdown) strength. -fuge joint, junction. -galvanometer ballistic galvanometer. -generator inpulse or test wave generator. $-k r a f t$ percussive power, notive p. -kreis impulsing circuit. -linie boundary line, joining 1. -messer, Kraft- ballistic pendulum. -prüffeld (potential) inpact or inpulse testing field. -sender inpulse exciter, i. transuitter. - spannung inpact potential, shock p. -stelle joint area or point, junction $p$. contact point. -ton beat note; throb. -verbreiterung inpact broadening. -wage ballistic pendulum. -welle percussion inpact or inpulse wave; w. impacting upon a joint. -winkel butting angle, a. of joint. -zahl number of callisions, impacts, or bursts.

Strafe,bei - der Nichtigkeit on pain of nullity.

Straf-gesetz penal code. -recht criminal law. -summe penalty, fine.

Strahl (cf Strahlung) ray, radiation, bean (in d.f., telev., etc.), pencil, jet (of fluid), flash (of lightning); straight line, radius (geonetry).

Strahl, achsenparalleler paraxial ray.

-, ausfall ender energent ray.

-, ausserachsial er Extra-axial ray,

abaxial r. -, ausserordentlicher extraordinary ray. -, durchfall ender transcident ray, transuitted $r$. -, einfall ender incident ray. -, ordentlicher ordinary ray. -, schräger skew ray, oblique r. -, streifender glancing ray. -, ultraroter infrared ray, ultrared $r$. - ultravioletter uitraviolet ray, vita-r. (between 2900 and $3200 \mathrm{~A}$.)

Strahl,Ab- reflected ray. Anodenanode ray, canal $r_{\text {. }}$ positive $r$. Brems- (Röntgen) X-rays due to collision or checking. Dunkelobscure radiation, heat $r$. (from a light source), invisible (actinic) $r$. beyond the violet. El ektronen-, weiss er heterogeneous bean of electrons. Faden- pencil ray (electrons); beal or pencil of nonuniform cross-section, in gasfocused c. r. tube, due to positive ions and secondary electrons, thread beam. Fahr- radius vector (wath.). Gegen- ref lected ray, reflection. Gesichts- visual ray Grenz- border-line ray, infraRiöntgen r. H- H-ray (consisting of positive hydrogen ions and protons). Kanal- canal ray. Knoten- cf Strahl, Faden-. Leit- guide ray, g. beam, radio beacon; radius vector (wath.) Licht-, maximale Aussteuerung des - (es) clash point of light valve (in sound recording). Luft- air jet. Molekular - molecular ray, m. bean: Peil-,raumgeradliniger orthodrowe. Rand- marginal (zone) ray, peripheral r., extraaxial r. (opt.) Rest- residual ray, $r$ radiation, reststrahl. Röntgenbrems- X-rays due to collision or checking. Schräg- skew ray. Schwarz- black (body) radiation, cavity r., Flanckian r. Streuscattered radiation, s. signal, stray radiation, stray light. 
Weltraum- cosnic ray. Zentralcentral ray, axial r. (opt.)

Strahl-abblender means to mask, stop down, occult, eclipse, gate or diaphragm rays, beam douser, blanking or blanketing means, dimmer. -ablenkung ray deflection, pencil d., beam d. or deviation; ray refraction.

Strahlabtaster (mit unsichtbaren ultraroten Strahlen) noctovision scanner (using nicro- or infrared rays). El ektronenelectron scanning pencil, beak, brush or lever. Kathoden- electron bean scanner. Licht- spotlight scanner, brush or pencil.

Strahl-abtastung scanning or sweeping with a light or electron pencil. -apparat jøt apparatus, injector (of steam).

strahlartige Entladung needle-point corona or streamer discharge, leader-stroke d.

Strahl-ausfall emergence of beam. -brechung refraction or splitting of rays. -bündel beam, pencil, bundle, bunch or brush of rays. -bündel, Kathoden- cathoce-ray beam or pencil; (loosely) gun. strahlen emit rays, radiate, be radiant or radiating.

Strahlen-brechungsmesser refractometer. - bündel(s.a.strahlb.) bundle of rays or radiations, cone of r. -bündel, Schallpencil of sound. -einfall, streifender glancing incidence of rays. - gang path of rays, trajectory of r., ray tracing, geometric configuration of rays. - härtemesser penetrometer, qualimeter (for X-rays), -kegel cone of rays. -messer radiometer (heat rays), actinometer (actinic r.), solarimeter (sun r.). -optik geometric optics. -reichweite range of rays or corpuscular emission. - schnitt jet contraction, contractio venae. -stoss, - Höhen-, Raum- burst or shower of cosmic rays, cosmic ray track (traced by hodoscope). - teiler beam splitter.

Strahlentladung, Faden- thread ray discharge.

Strahlentstehungsort source or origin of electron pencil or gun.

Strahlenzieher protractor.

Strahler radiator (of radio waves, loudspeaker, etc.); heater (of a cathode). Massen- mass radiator. Mehrfach- bank or pillar of lamps, battens. Richt- direciive antenna, directional a.; directional loudspeaker or projector, radiator. Rück- reflector, re-radiator. Temperatur - incandescent light source, luminous radiator.

Strahl-erweiterung spread of beam (telev. tube). -erzeugerfuss gun press. -erzeugungssystem gun (comprising electron source, first and second lenses); ray radiator system. -fläche radiant area, radiator surface, radiation-emissive s. -hebel arm pencil leverage, deflectibility of electron beam.

strahl ig, l ang- elongated, needlelike (cryst.)

Strahl-intensitätskontrolle modulation of beam intensity (by grid, shield or Wehnelt cylinder, telev.) -methode, Atom- atom or molecule ray method. -neigung inclination of ray. -quelle, Leucht-, einfarbige monochromatic illuminator, $\mathrm{m}$. source, monochromator. -röhre, El ektronen-( $c f$. Kathodenstrahl röhre) beam tube; cathode-ray tuning indicator t. or magic eye. -ruhel age spot zero. -schauer, Höhen- cosmic ray shower or burst. -schreiber, Tinten- ink vapor jet recorder (facsimile telegraphy). - sender, Leit- beacon; radio range or beam station. - spaltung split-beam, b. splitting. -sperrung beam cut-off or eclipse, gating of the $b$. -stösse, Höhen- cosmic ray bursts.

Strahlung, aktinisch undurchlässige adiactinic radiation.,$- \mathrm{fal}$ sche stray radiation. Grau- grey radiation. Hohl raum- cavity radiation, black-body r. Raum- cosmic 
ray radiation. Resonanz- resonance radiation (of vapor lamp). Rück-, spiegelnde specular reflection, regular $r$. Schwarzkörperblack-body radiation, cavity $r$. Seiten- secondary radiation, lateral r., spurious lobe (of space pattern), Temperatur - thermactinic radiation. Über- irradiation. UItra- cosmic rays. Vernichtungsannihilation radiation.

Strahlungs-bel astung radiation load. - dämpfung radiation damping, $r$. resistance. -diagramm radiation diagram or characteristic, space pattern, radiation p. -druck radiation pressure. -empfänger radiation-sensitive or responsive pickup, r. receiver, record sheet or surface (on which radiations impinge for measuring color temperature, etc., consisting of photographic plate, selenium cell, bolometer, etc.) -fläche (electron) emitting surface, emitting area, radiator s. -formel, $\mathrm{Pl}$ anck'sche Planck's equation (of spectral energy distribution). - gegendruck radiation reaction. - gl eichgewicht radiation equilibrium. -messer actinometer (for actinic rays and solar flux density), radiometer (thermal rays), solarimeter (sunrays). -messer, Sonnen- pyroheliometer, pyrheliometer, solarimeter, actinometer (for solar flux density). - potential radiation potential, resonance $\mathrm{p}$. (to excite atom or molecule). - temperatur radiation temperature, effective t. -verfahren, Selbstself-emissive or s.-illuminating method (used in electron microscope) based on electrons arising on object surface. -verluste corona losses, radiational 1 . -widerstand effective resistance (loudsp., antenna, etc.), radia-

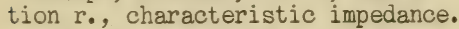
-werfer beacon or beam station. Strahl-unterbrecher, Quecksil bermercury (jet) interrupter.

\section{Streckgrenze}

-vergenz vergency of rays (either convergence or divergence).

-verlauf path of rays, course of $r_{.}$, trajectory of $r_{0}$, ray tracing. - wegablenkung, Peil- distortion of bearing, lateral deviation (due to diurnal or seasonal factors, terrain, local conditions, etc.)

Strang rope, chord, cord; web (of paper, cloth, etc.), strand; line (of drive shafts).

Strasse, Feinblech- sheet rolling mill. wirbel- vortex avenue.

Strebe,Gitter-grid stay, g. support. strebenlos unsupported, unstayed, unpropped.

streckbar extensible, extensile, ductile, malleable.

Strecke distance, space, stretch, extent; path (e.g., grid-filament). Strecke(z.B.a-b) interval a-b, element of length $a-b$.

Strecke abschneiden lay off a distance.

Strecke, Gas- gaseous path or gap (in

a tube). Gitter-Kathoden- grid-

filament path or circuit.

strecken stretch, extend, spread, draw, elongate, pull.

Strecken-befeuerung, Nacht- route beacon for night flying service. -dienst, Lang- long-range or longdistance avigation (navigation) service. - element linear element (math.) -feuer, Haupt- main or trunk route beacon, airway b. - funkfeuer range beacon. -funkfeuer, Haupt- main route radio beacon. -karte route airway map. - leitstrahl long-distance or route avigation (navigation) beam. -messung end-to-end measurement, straight-away m. -navigation long-distance avigation, route a. -netz air routes. -spektrum continuous spectrum. -teilchen linear element (math.)

Streckfestigkeit resistance to stretching, $r$. to elongation. -figur flow lines, lines of stress, surface bands. -grenze yield 
Streckspannung

point, limit of elongation.

-spannung yield stress. -stahi rolled steel. -werk rolls, rolling mill.

streichen stroke, rub; paint, coat, varnish; erase, cancel; bow or stroke (a string).

Streich-instrument bowed or string instrument. - schale oilstone, whetstone.

Streifen strip, stripe, ribbon, tape, fringe (in light interference), band, streak, stria, vein.

streifen graze (as in a shock or collision), glance, skirt, brush against; stripe, streak, striate. Streifen, Abdeck- squeeze unit, mask, mat (m. p.); barrier (between frames). Absorptionsabsorption bands, a. spectrum. Bitter'sche Bitter (magnetic powder) pattern or bands.

Brumm- buzz track; hum (broad transverse bands appearing on c. $-r$. tube screen). Endtrailer (blank end of a film). Interferenz- interference fringe (opt.). Kontroll- monitor slip. Längs- longitudinal stripes, striae or streaks. Leucht(in Entladung) luminous streamers (in discharge). Löt- tag strip, terminal s., connection s. Mach'sche Mach bands. Rohrskelp, strip (for forming pipes). Ruhe- unmodulated track. Tonsound track.

streifender Einfall grazing, oblique or glancinǵ incidence (of rays or sound waves).

Strelfen-filter, dreifacher tricolor banded filter. -gefüge laminated structure, banded s. -keil photometric wedge. -lichtverfahren variable-density 'recording method. -schirm rod screen. - spektrum band spectrum. -verschmälerung, Ton- squeezing of sound track, matting.

Streifungen striations, striae (metal coatings, etc.)

Streifungen, Translations- transla-
Strich, Teil-

tion gliding striae.

Streit, Patent- patent litigation, p. action, p. suit. Rücknahmeaction for withdrawal.

streitanmerkungen annotations of litigation.

Streiter contestant to a suit, litigant, disputant.

Streit-fall,-frage,-punkt,-sache issue, question at issue, contested case, dispute, controversy, matter at bar, $m$. in issue, $m$. in suit.

strengflüssig viscous, semi-fluid, difficultly fusible; refractory, streuen scatter, strew; fluctuate, disperse, "spread" or scatter (of values).

Streu-faktor scattering factor (of $x$-rays), s value. -feld stray field, leakage f., extraneous $f$. - impedanz leakage impedance. -induktivität stray inductance. -kapazität stray capacitance, spurious c. -kopplung stray coupling, capricious c., spurious c. -licht stray light. -querschnitt scattering cross-section. -richtung scattering direction. -strahlen scattered signals, s. radiations, stray radiations, stray light.

streuung scattering effect, diffusion e., dispersion, stra.y leakage, spread (d.f.) $\mathrm{Fl}$ anken- side leakage, s. stray. Spalt- slot leakage, s. stray. Sti rn- overhang leakage flux. Streu-winkel angle of scattering. -zelle scattering cell.

Strich stroke, streak, stria, line, dash, mark, grain. Ab- downstroke or trailing edge of a synchronizing impulse (telev.). Auf- upstroke or leading edge of a synchronizing impulse (telev.)

Dauer- long dash, permanent note (N-A interlock in d.f.). Gittergrating line. Kompass- point of compass. ober- maximum or peak carrier current, m. picture signal. Stever- dot or pointer to observe and read bearings. Teil- graduation line, division 1., gage mark (of a scale). 
Strichdiapositiv

Strichdiapositiv line transparency, 1. diapositive.

Stricheinstellung, Bild- framing (by observing frame lines). Bild-, fal sche misframing, out-of-frame condition.

stricheln shade, hatch, streak. Strich-fokus line focus (of x-rays). -formullerung chemical formula. using dashes or lines (to represent bonds). - geräusch frameline noise (form of motor boating). - gitter ruled grating, simple line g. -kreuzplatte cross wire plate (of telescope), reticle, graticule.

strichliert broken (of a line), dash-lined.

Strich-mass line standard. -methode stroke method, line $\mathrm{m}$. (paper test). -punkt semicolon. strichpunktiert broken (dot-dash) (line).

striktionsspule focussing coil. stroboskopische Scheibe stroboscopic disk, s. pattern wheel. Strohgeige Stroh violin (with diaphragm or contact microphone attachment).

Strom, abgehender outgoing current. -, ankommender incoming current, input c. -,eingeschwungener steady-state current. -, phasenverschobener out-of-phase current, phase-displaced c., dephased c., quadrature c. (when shift is $\left.90^{\circ}\right) 0^{-2}$, vagabundierender leakage current, stray $c$. -, verzweigter branched current, branch c. -, welliger ripple current, undulated c., pulsating c.

Strom, Ableitungs- leakage current, stray c. Arbeits- watt current, working c. Ausbreitungs- dispersion current. Ausgleichbalancing current (of a bridge), compensating $c$. Ausschwingdecay current, decaying $c$. Bild-video current, picture c. $B l i n d-$ reactive current, reactance c., wattless c. Brücken-

\section{Strom, Wechsel-}

ausgleich- bridge balancing current. Dauer- persistence current. Dunkel- dark current (flowing in photocell, in absence of light). Elnschwing- building-up current. Erst- primary current. Gespensterghost current. Halte- holding current, retaining c. Kreiscircular current; c. flowing in a circuit. Kriech-creep current, sneak c., surface-leakage c. Lade- charging current (anomalous and normal, of condenser); charging c. (of battery). Leckleakage current, leakance c. Leit- conduction current; pilot c. (in wired radio). Leitungswechselconduction alternating current. Licht- light flux,1. current (in lumens); lighting c. Nachwirkungsabsorption current, residualcharge c. (of condenser). Nutzuseful current, signal c. Pegelpilot current. Photo- photo-cell current. Quer- leak current. Richt- output current (d.c.) of detector, rectified d.c. component of plate c. Rück- reverse current, backward c. (in rectifier). Ruheanode-feed current (d.c. component of plate or anode c.); no-signal c., quiescent c. Sättigungs- saturztion current. Schalt- current on contact. Spannungs- current flowing in potential circuit (of a meter). Sperr- backward current, reverse c. (of rectifier). Tonvoice signal current, sound $c$. Träger-,plötzlich veränderter wobbled carrier. Verdrängungscapacitance current, displacement current, dielectric c. Verlustwatt current, active c., energy c. Verschiebungs- capacitance current, displacement c. dielectric c. Vor-, dunkler dark pre-sparking current. Vormagnetisierungsbiasing current (for magnetic polarization). Wand- wall current (of amplifier). Wechsel- alternating current (a.c.) (mostly singlephase current is meant specifically), 
indirect c., any periodic c. wellen- pulsating current, ripple c. Zeichen-marking current (telegr.) Zwischen- spacing current (telegr.)

Strom-abnehmer tap, collector of current. -arbeit Joule heat. - aufnahme, Blind- drawing or taking wattless, idle, or reactive current. - aufnahme, Gifferdrawing of current by grid.

- aussteuerung, Anoden- plate current excursion. -bahn,flächenartige laminar or areal path of current, sheet c. -belag current coverage, c. distribution (in terms of amps. per centimeter of periphery). - dämpfung, Echoecho current attenuation, active c. loss. - dichte current density (in amps. per unit of crosssection); luminous flux (in lumens).

stromdurchflossen alive (of a wire), traversed by current.

Strom-einsatz, Gitter- point where grid draws current, incipient $g$. c. flow. -faden streamer (in discharge), current path; stream line. - flächenformel current sheet formula.

stromführend current-carrying, c.conducting or conductive, live, charged, "hot".

Strom-gattung kind of current, system of c. -gebiet, Anlaufrange or region of incipient current flow (e.g., at grid). - gitter atomic lattice.

Stromkreis (s.a.Kreis), an Ende kurzgeschlossener short-circuited circuit or line, end-shorted c. -, am Ende offener open-ended circuit. - unbel asteter unloaded circuit. -,verzweigter branched circuit, divided c. Abstimmtuned circuit, tuning c., tank. Bezugs- reference circuit. Geräu schunter drückungs- noise reduction circuit, squelching c., silencing c. Grundgeräusch(back) ground noise reduction circuit. Impedanz- impedor, circuit with impedance. Nutzsignal circuit, utilization, working or useful c., active c. Reinigungssmoothing circuit, filter c., lowpass filter (following rectifier in power unit). Sprech-circuit carrying voice or signal current (in loudspeaker). Still abstimmungssilent tuning circuit, q.a.v.c. circuit.

Strom-leistung electric power (wattage). - IInienaufzelchner device (often electrolytic tank) to map or plot potential, fields, lines, tubes of force, etc., on a model.

stromlos currentless, de-energized, dead.

Strommesser, registri erender recording ammeter. Hitzdraht- thermal ammeter, hot-wire a.

Strom-modulation, El ektronen- electron quantity modulation. -reiniger harmonic eliminator, $h$. excluder (filter), smoothing means, stopper. -resonanzkreis parallel resonance circuit, tank c. -richter, gittergesteuerter rectifier of thyratron type, mercury $r$. or ignitron with control grid. - schiene third rail, busbar. -schlüssel key switch, make and break. - schlusspriffer continuity tester. -spule current coil (of a wattmeter).

Stromstoss current pulsation, pulse or impulse. Anlass- starting rush or impulse of current. -feder impulse spring (of dial switch) - gabe impulsing, pulsing. -gabe, rückwärtige revertive pulsing. - schalter impulsing switch, stepping relay. -übertrager impulse repeater. -verhältnis impulse ratio.

Strom-tor thyratron. -tor-I gnitron Kombination mutator. -ibernahme transfer of current, tapping of $c$. strömung current, stream, flow, flux, circulation, fluid movement; tendency, trend. -, wirbellose flow free from vortices, eddies or turbulence, irrotational flow. 


\section{Strömungspotential}

Stromungs-potential flow potential, streaming p. -widerstand flow resistance (of a test piece); aerodynamic $r_{\text {., d drag. }}$

Strom-verdrängung skin effect, current displacement, Heaviside e. - verzweigung network of conductors, dividing or branching of currents. -wage ampere balance. - wandler, Durchführungs- bushingtype current transformer.

- wechsler commutator, reverser. - weiche current divider (network or filter). -wender commutator, reverser. -wert current value, amperage. -zeiger ammeter; current vector. -zuführung current supply lead.

Strudel eddy, vortex, whirl (pool), maelstrom.

strudell os irrotational, non-eddying. Struktur, feinschuppige magnetische fine-scaled magnetic structure. -, hyperfeine-, ubberfeine hyperfine structure. -, zell enartlge honeycombed structure, pitted s., cellular s. Fein-fine structure, micro-s. (cryst.)

Sekundär - secondary structure gridwork (cryst.). Übersuperlattice; superstructure. Uberfein- hyperfine structure. Verzweigungs- lineage structure.

Struktur-anal yse, Grob- gross or macro structure analysis.

-chemie structural chemistry.

strukturlos structureless, amorphous.

Stück, Flächen- area element, areal e., unit area. Leiter- conductor element, portion, part or (short) length of conductor. stückfärbung tissue. staining, staining in toto (microscopy). stückweise piecemeal, piece by piece.

Stufe step, stage, degree, grade, gradation; digit (automatic telephony), point (in multipoint switch). -, nachgeschal tete gesteuerte main oscillator stage (in independent drive system). stufe, Beschleunigungs- target (of

\section{Stumpf, Kegel-}

multiplier), acceleration stage. Elner- units digit. Farb-,Farbencolor gradation, tint, shade. Graukell- intermediate shading value. Helligkeits- (intermediate) brightness stage, i. grey value (between black and white), shading or tonal v. Lautstärkenloudness unit, sensation $u$. Licht- printer step (copying machine). Misch-mixer stage, m. first detector s. Nachlauf(cathode) follower stage. Niveauterm, energy level (of electron). oxydations- stage or degree of oxidation. Puffer-separator stage, buffer s. Überlagerungsmixer stage, first detector (in superheterodyne receiver).

Zehner - tens digit.

stufenartig graduated, step-like, graded, gradual, echeloned, stepped.

Stufenfolge succession or sequence of steps or stages, graduation. stufenförmige Echos echelon formation of multiple echoes.

Stufen-gitter echelon grating. -kell step tablet, stepped photometric absorption wedge (in x-ray, etc., work). -l inse echelon lens, Fresnel 1. -messer, Farb- tintometer. -methode gradient method (of cooling, etc.). - platte sensitometer tablet. -prisma echelon prism. -rad step wheel, cone pulley. -rel-ais relay with sequence action. - schalter multi-point switch, m.contact s. - scheibe cone pulley, taper p., stepped p. - schwächer step-type weakener (spectroscopy, photography). -spalt step slit (phot.).

stufenweise in stages or steps, stepwise, by degrees, successively, sequentially, gradually.

stulpe cuff.

stiilpen turn inside out, t. upside down, put over or upon in inverted position.

Stummfilm silent film.

Stump f stump, butt-end. Kegeltruncated cone, frustum-cone, 
Stumpf, Pyraniden-

frustum. Pyramiden- truncated pyramid frustum. Wellen- butt end of a shaft.

stumpfe Verbindung butt joint. stumpfeckig blunt cornered.

stumpfkegel truncated cone, frustum. stumpf schwei ssen butt-weld, jump-w. Stumpfungtläche, Ab- truncating face (oryst.)

stump fwinklig obtuse-angled.

stürmisch stormy, turbulent, violent, vivid, agitated.

Sturz plunge, fall, sudden drop or decline, chute.

Stürzung, Bild-toppling of plcture or image (pictures turned an angle in relation to one another).

stütze support, stay, prop, bearing.

Trag- supporting stay or prop.

stuitzen support, prop, stay, rest or bear on.

Stutzen, Ausblick- objective lens socket (opt.) Rohr- short piece of pipe or tubing serving as an opening, socket, or connection; nipple, short cylinder, bush or sleeve. Saug- pump intake, $p$. nozzle.

Stütz-i sol ator pin insulator, rod $i$. -pfeiler supporting pillar, s. column, bedding pile. -punkt point of support, bearing surface, fulcrum; airbase, base airport.

Substanz, Mutter- parent substance. subtraktive Modulation downward modulation, positive m.

Suche, Störungs- trouble "shooting", $t$. locating, interference location.

Such-el ektrode probe electrode, exploring e., collector e. (in vacuum t. work). - empfang straight-ahead reception, singlecircuit r., direct r., nonheterodyne $r$.

Sucher searcher, locator, detector, finder, probe, view-finder (opt.) Durchsichts- direct-view finder, eye-level f. Fehler-aniseikon (for detecting cracks electronically), electromasnetic crack detector (by flash magnetization), materiologic devices (with X-rays). objekt- object-finder (opt.)

Prismen- prismatic seeker or

finder. Rahmen- wire-frame viewfinder, iconometer. Ziel(auf)radar (radio detection and ranging apparatus), sonar (underwater sound navigation, detection and ranging).

Sucher-bild seeker picture, monitoring p. -korn backsight of viewfinder. okular focusing lens. - parall ax seeker parallax, finder p. Such-gerät, Stör- interference locator, signal tracer (traces progress of signal by stages). -licht searchlight. -spule search coil, rotating c., exploring c. (of d.f.); flip c. (used in magnetic field tests). -tonanalysator heterodyne analyzer. -tonverfahren beatnote method (using heterodyning search frequency).

südliche Breite south latitude. Südlicht aurora australis. Summand term of a sum (math.). Summar (Leitz) Summar objective. Summe,Licht- light sum, total 1. emission. Linien- line integral. summen hum, buzz; sum up, add (up). Summen-frequenz summation frequency, sum $f$. -geber,Fernmess- telemetric integrator. -gleichung summation equation. - satz sum rule, permanence principle. -ton summation tone-wirkung combined or compound action or effect.

Summer buzzer, small AF oscillator. Dynatron- dynatron oscillator. Gl imm- glow-tube type of buzzer oscillator. Magnet- magnetic interrupter or buzzer-type of AF oscillator. Röhren- vacuum tube AF oscillator. Schwebungs- audiofrequancy ( $A F$ ) oscillator, heterodyne buzzer o.s beat.f.o. Stimmgabel tuning-fork $\mathrm{AF}$ buzzer oscillatior. Überlagerungs- heterodyne $A F$ warbler oscillator.

Summer-erregung buzzer excitation. -well enmesser buzzer wave meter. Summlerungsgerät integrator. Summ-spur,-streifen buzz track (on film). 
Sumpf swamp, marsh, bog; pit, sump, pool (of mercury in a vessel), pouring basin.

Super-łeitfähigkeit supra-conductivity, super-c. -het(erodyne)emp fang mit selbsterregter Trägerwelle homodyne reception. Supra-leitelektronen super-conduction electrons. -leiter super conductor.

Surrerscheinung motor-boating (in radio receivers).

Süsswasser fresh water.

Symmetrie symmetry, balanced condition. -ebene plane of symmetry, crystallographic plane.

symmetrieren, symmetrisieren balance, symmetricize, symmetrize, align, make symmetric, neutralize. Symmetriezentrum inversion center. symmetrisch, achsen- axially symmetric, in axial symmetry. el ektrisch-homopolar. höhensymmetric about vertical axis (cryst.) kugel- spherically symmetric, spherosymmetrical. mitten- centro-symmetrical. plan-planisymmetric. punkthaving point or radial symmetry. radial-radially symmetric, in radial symmetry. rotationsin rotation or revolution symmetry. schief- skew-symmetric. spiegel- mirror symmetric, with specular symmetry. zentralcentro-symmetric.

Synchron i sator, Zahnrad-, magn. cogged or tooth-wheel synchronizer. Zeiger-dial scoring machine (m.p.)

Synchronisieratelier scoring stage. Synchronisieren s, a, Gleichlauf. synchronisieren, lokal geregeltes local synchronization (by tuning fork, etc., in telev.)

-, sellbständiges self-running synchronization (telev.) -, unselbständiges distance- or signal-controlled synchronization (telev.) Nach-post-scoring (of silent film), dubbing, re-recording (first picture, then sound). Nach-(von Trickfilm, mit springendem Ball) bouncing-ball synchronization (m.p.). Vorpre-scoring (first sound, then picture). Zwangs- locked symchronization.

Synchronislerimpuls, Bild- frame synchronizing impulse or signal, low sync i. Zellen-line synchronizing impulse or signal, high sync i. - Abtrennung oder -Aussiebung synchronizing separation (separating control or sync impulses from video signals), amplitude separator, clipper.

Synchronisier-klappe clappers to mark sound and picture track for synchronization. - l ïcke, Zeil enline synchronizing period. -zeitmarke marking of time, synchronizing $m_{.}$, time scale. -zwang locked synchronism, 1. synchronization.

Synchroni si erverfahren, Lücken- gap, underlap, interstice or interval synchronization method (sync signal introduced between end of line traversal and beginning of next line).

synthetischer sprecher voder. Systematik systematology. Szenen, Ateller- regular stage scenery or sets. Effekt-scenics, light effects, etc. 
T Regler T type section attenuator. tabellarisch tabular, in tabulated form.

Tabelle,Belichtungs- exposure time table. Peil- bearing chart. Umwandlungs- conversion table.

tabellisieren tabulate, tabularize, Tatletten, Färbe- varitone tablets. Tafel table, tablet, plate, slab, sheet (of metal), cake (of chocolate), pane (of glass); slate, blackboard, panel.

Tafel,Einstell-focusing board, test chart. Farten- color chart, c. scale. Fluchtlinien- alignment chart. Misch- mixing panel, monitoring p. Rechen- computation table. Signallampensignal panel, annuciator board or p. Spektral- spectrum chart. Umrechnungs- conversion table, reduction t., conversion scale (say, meters to kilocycles). Zahlen-numerical table, $\mathrm{n}$. tabulation, table of figures.

Tag,Stern- sidereal day.

Tagl indheit hemeralopia.

Tages-gans,örtlicher local diurnal variation. -lichtfaktor day-lisht factor (occasionally called window efficiency ratio, phot.), -lichtkassette daylight loading magazine ( $u \cdot p$.)

täglich daily, diurnal.

Takt time, cadence, measure (nus.), tempo, rate, rhythm, stroke (of engine).

Takt-funkensystem timed-spark system. - oeber impulsing means, impulser, time-valve, time tapper, t. beater, metronome.

-geber,Kristall-crystal monitor, c. stabilizer. -impuls synchronizing impulse, timing $i$.

taktmässige Ablenking timed deflection, t. sweep.

Tal, Wellen- trough, valley or dip of a wave.
Talg tallow, (solid) fat, grease.

Talwegkurve thal weg.

Tambourinbecken tambourine jingles.

Tangens, hypertolischer hyperbolic tangent, tanh (math.)

Tangente, Wende- tangent to reversing point.

Tannenbaumantenne christmas tree antenna.

Tannenbaumkristall dendrite (cryst.)

Tanz, Antreten zum formation of electron groups, bunching (in beam tube), phase focusing. El ektronenoscillatory (erratic) movements of electrons (caused by electric or magnetic actions).

Tanzen, Bild- unsteadiness or jumping of picture.

Tarnung camouflage.

Tasche pocket, pouch, bursa, trap. Ausfrier-liquid-air trap (vacuum punp).

Tastarm wiper (arm).

Tastatur, Universal- universal keyboard.

Tast-blende, Ab- scanning aperture, $\mathrm{S}$. hole (Farnsworth tube). -bolzen gage finger, tactile part. -drossel magnetic modulator.

Taste, Abstands- blank key, spacer k. Lösch- cancel key, clearing k., release k. Ober- sharp (mus.), black key. Rückzug- back-spacing key. Unter- long key, white k. Tasten keying, k. modulation; feeling, exploring, scanning, probing. Tasten, Hinunter- carrier caused to drop to low value (zero) by synchronizing signal.

Tasten-feder,Brumm- spring for bass keys (accordion). -feld keyboard. -reihe bank of keys, row of $k$.

Taster calipers, feeler, keying device (radio), tactile part or device; scanner, explorer (telev. picture).

Taster, Aussen- outside calipers. Fadendicke- calipers with jaws measuring thread gage capacitively. Gewinde- thread calipers. Innen- 


\section{Taster, Rad-}

inside calipers. Rad- wheel operated means causing signals, bell ringing, etc. Tiefendepth gage.

Taster-skala feeler scale. -zirkel gaging calipers.

Tastgerät stabilizer; keyer and limiter leveling amplitude variations in incoming video signals (telev。) Vibrationsvibro-tactile device.

Tast-geräusch key chirp (occurring after key has been opened), key thump, k. click (caused on closing key). -klick key thump, $k$. click. -prellen see-geräusch. -relais keying relay, r. key. - schal tung, Mittelpunkt- centertap key modulator circuit. -schlag key click, k. thump, chatter. -stift tracer point, feeler.

Tastung (cf Abtastung) control, keying, modulation by key action; impulsing, pulsing. Einkanal- single-channel pulsing (telev. synchronization). Hoch- working impulse transmitted on voltage substantially above normal. Spiral- spiral scanning.

Tatbestand factual findings.

Tätigkeit, Sonnen- solar activity. Tatsachen, Erfahrungs- empirical experience, practical facts or data.

Tatze paw, claw, cam.

Taubheit, Leitungs-(des äusseren ohres) conduction deafness or impairment of hearing (outer ear). Nerven- (d. inneren Ohres) nerve deafness, perception d., nervous perceptive impairment of hearing (inner ear).

Tauch-bahn (der Elektronen) dip orbit (of electrons). -batterie plunge battery.

tauchen immerse, dip, plunge, steep.

Taucher-aufnahme submarine shooting. -kol ben plunger.

Tauch-kernrelals plunger relay. -lack dipping varnish. -spule moving coil, signal coil (of loudspeaker); telescoping $c$.
Teiler, Strahlen-

- spul enmikrophon moving-coil microphone. -zylinder plunge cylinder, plunger.

tauglich fit, proper, appropriate, useful, serviceable, usable, good, suited.

Taumel bewegung tumbler movement, $t$. action.

Taupunkt dew point, thaw $\mathrm{p}$.

Täuschung, Augen- optical illusion. Spiral-spiral illusion.

Technik engineering art, technology, technics, technique. Funkradio technology, r. engineering art. Nachrichten- signal art, communication art. Stand derstate of the art.

Techniker, Aufnahme- camera technician, c. man.

technisch commercial (grade, etc.), technical, technological, practical, industrial. gl as- glass technological, vitrological.

technische Frequenz commercial frequency. -Wechselströme commercial alternating currents, industrial a.c. -Werkstoffe industrial, commercial or technical materials.

teiglg doughy, pasty, mellow, plastic, kneadable.

Teil...sub-...,fractional, divisional, partial, componental.

Teil-abtastung fractional scan, partial s., coarse s. -bild compound image (of color record).

Teilchen particle, small part, corpuscle, element (math.) -, harte primaries. Grund- smallest particles, fundamental p., atoms. Schwebe- suspended particles. Stoff-particle of matter, corpuscle. Strecken- linear element (math.)

Tellchen-dichte particle density. - feld particle field. -geschwindigkeit particle velocity.

Teildruck partial pressure.

Teiler divider (of potential, etc.), divisor (math.), submultiple. Frequenz- frequency divider, demultipler or submultipler of $f$. Phasen- phase splitter. Spannungs- voltage divider, potentiometer. Strahlen- beam 
splitter (m.p.)

Teilerdkapazität direct earth capacitance.

tellerfremd aliquant.

tellfremd prime to each other.

Tell-frequenz component frequency (say, of a spectrum). -kanal sub-channel. -kopf index head. -kraft component force, componental f. -kreis pilch circle, p. line, divided or graduated circle, dial, graduated circular plate. -maschine dividing engine, d. machine, ruling e. - nehmer, Sammel anschluss- subscriber with several lines. -raster fractional scan, f. sweep (in interlaced scanning). -ring index ring. -scheibe index circle, i. plate. - scheibe, Rasten- notched index wheel. - spannung partial voltage, component v., submultiple v. -sperrzeit partial restoring time (in echo suppression). -spule fractional coil, component or subdivision of a $\mathrm{c}$. - strich graduation line, division 1., d. mark (on a scale). - ton partial, p. note (either a higher harmonic or a sub-h.) -trommel divided drum, graduated d.

Teilung division, graduation, partition, pitch, scale, submultiplication. Gleich- equipartition. Kern- nuclear division. Niet- rivet spacing. Vierquadripartition. Zeilen- Iine pitch (telev.) Zwei- bipartition, bisection.

Teilungs-ebene plane of division. - fehler faulty pitch (telev.), line p. defect (e.g., underlap of lines). -gesetz law of partition. -koeffizient distribution coefficient.

Teilvierpol section of recurrent structure.

Tel eaufnahme telephotographic work.

Telegraphie, Bild- picture telegraphy, radio or wire picture transmission, phototelegraphy, facsimile, wire-photo. Drahtwell enwired wave telegraphy, wire radio t., "wired wireless" . Unterlagerungs- sub-audio frequency telegraphy system, composite operation (tèlegraphic and telephonic signals passed over one wire, often attended by thump noise).

Tel egraphier-pause space (in telegraphic work). - sender, tonmodulierter $\mathrm{AF}$ or tone-modulated telegraphic transmitter.

Telegraphon télegraphone (Poulsen, Blat tnerphone, etc.)

Telephon, Fernseh- television telephone, video t. Funk- radiophone. Zungen- reed or Brown-type telephone receiver.

Telephonie, Geheim- scrambled, garbled or secret telephony (based upon band transposition or inversion, use of scrambling circuits, speech inverters, etc.) Mehrfach- multiple telephony.

Telephonie-drossel magnetic modulator. - sender, sprachgeschal teter voicemodulated telephony (signal) transmitter or radiophonic transmitter.

Teller, Auflage-, Platten- turntable (of phonograph).

Teller-fuss plate- or dish-shaped foot, press or base. -gerät, Zweidouble turntable type of player. - isolator dish or disk insulator for antenna supporting.

Temperatur, charakteristische (Debye's) characteristic temperature.

-, schwarze black-body temperature, cavity t. Anlass- tempering temperature, annealing t. Farbcolor temperature.

temperaturabhängig temperature responsive, $t$. dependent, be a.function of temperatur $\theta$.

Temperatur-beständigkeit temperature stability, t. constancy, condition of being unaffected by temperature (changes). - feld temperature gradient, t. "field" pattern. -gang variation of temperature, function or effect of $t$. - leitfähigkeit temperature diffusivity, thermal conductivity. 
Temperaturregler

-regler temperature control, thermostat, thermo regulator, (electronic) thermautostat. -strahler incandescent light source, i. Iuminous radiator. -strahlung thermactinic radiation.

Tempoknopf speed regulator (in shape of a knob).

Tensor, Spannungs- stress tensor.

Tensoral gebra tensor algebra.

Terme, anharmonische anharmonic terms or constants.

Termin zur Verhandlung einer Sache anberaumen sel a date for a hearing or a case, appoint a $h$.

Term-schema term diagram, $\imath$. scheme. -verschiebunq term shift.

Tertiärempfang three-circuil receplion.

Terzflöte chird flute.

Tetraeder tetrahedron.

Teufe depih.

Textur, Schicht- sheet intergrowth, stratified texture.

$\operatorname{tg} \alpha \tan \alpha$.

T-Glied T-type section or filter mesh, T-type network $\mathrm{m}$.

Thallium-sulfidzelle,-zelle thalofide (photo-conductive) cell.

Theaterkopie release print.

Thema theme, subject, subject-matter, topic.

thermische Fehlordnungserscheinung formation of holes (cryst.)

thermoel ektrischer Effekt thermoelectric effect, Seebeck e. - wärmemesser thermel.

Thermo-el ement, Vakuum- vacuum thermocouple. -galvanometer Duddell's thermo-galvanometer. -kraft thermo-electric force. -kreuzbrücke thermo-couple or junction, thermal cross.

Thermometer, thermo-el ektrisches thermel. Registrier-, Schreibthermograph.

Thermoschalter thermal-lag switch. Thorerde thoria (thorium oxide). thorieren thoriate.

thoriertenkathode, wi ederbel ebung der re-activation or rejuvenation of thoriated cathode, flashing. thorisch toric.

Thoriumemanation thoron.

thor lumhal tiger Wol framf aden

thoriated tungsten filament.

Tief-ätzen intaglio etching (whole metal coated with resist, pattern cut out with scriber). -decker low-wlng airplane. -druck intaglio printing process.

Tiefe deplh of focus (of eye). Eindring- depth of penetration. Modulations - s. Modulationsgrad. Schärfen- depth of vision, $d$. of focus, definition in $\mathrm{d}$. Schärfenfeld- depth of field. Seh- depth of focus, $d$. of field.

Tiefen bass-notes, low-frequency n., low-pitch n.; dark picture portions.

Tiefen-bestimmung, Fehler- stereoscopic radiograph for locating defects, depth determination of flaws. hervorhebung bassy condition (low AF overemphasized). -kompensation reed armature arrangement to accentuate or emphasize low frequencies (in loudspeaker). -konus low-frequency cone loudspeaker, woofer. -messer fathometer, ocean sounding meter (e.g., supersonic), sonic depth finder. -schärfe depth of focus, d. of field. -schrift hill and dale track, Edison t. or sound groove, vertical recording (of phonograph disks). -taster depth gage. -unschärfe insufficient, or lack of depth of, focus. -unterschei dungsvermögen power of differentiating depths. -winkel angle of elevation below horizontal, a. of depression. -wirkung plasticity of image, plastic or stereoscopic effect (of picture).

tiefer Ton low-pitch sound, bass s. or note.

Tiefpassfilter low-pass filter. tief-rund concave. -schmelzend low-melting. -schwarz jet black.

Tieftemperaturvergasung low- 
Tieftonkonus

temperature distillation or carbonization, partial c.

Tieftonkonus low-frequency cone loudspeaker, woofer.

Tiefung cupping (or drawing in drop press to make concave or dished articles).

Tiefungswert cupping value, ductility $v$.

tiefziehen deep draw, cup, dish.

Tiefzieh-fähigkeit capability of being cupped or drawn into article with deep concavity in drop press, etc., cuppability. -probe cuppability test (specimen), Erichsen test. -verfahren technological method of drawing sheet articles in drop press, cupping method.

Tiegel, Porzellan-porcelain crucible, p. pot.

Tiegel-brennofen crucible oven. -flusstahl crucible cast steel. -form crucible mold.

Tierleim animal glue, a. size.

Tikker ticker, tikker; radio chopper, c. พ. train interrupter.

tilgen eradicate, destroy, cancel, erase, blot out, delete, efface, obliterate.

Tilgung (und Ausleuchtung) extinction or quenching (of fluorescence), evanescence.

Tilgung, Schall- sound absorption, suppression, damping, deadening or attenuation, abatement of noise.

Ton, dumpfer all-bot tom sound. -, gehal tener sustained sound. -gleitender glissando, sliding note. -, hoher h.-frequency note or sound. -, hohler boomy sound, dull s. -,krächzender,

kreischender all-top sound or voice. -, reiner pure note, p. tone, simple t. -, sendereigner note or pitch peculiar to a beacon station, code note. -, tiefer low tone, low-pitch tone, bass note. -, unreiner impure tone, ragged note or $t$.

Ton, Anbl as- Aeolian tone. Ausfluss- jet tone, slit t. Daver- permanent note, tone, sound or signal. Differenz-
Tonanalysator

difference tone. Dinas- Dinas clay. Farb- color tone, tint. Fever-fireclay. Fremd- alien frequency or tone (in sound reproduction). Ganz-whole tone. Halb- semi-tone, sharp (mus.) Heul- sound with large number of modes of vibration. Hieb- Aeolian tone or note. Interferenzbeat note, interference n. Kammer- "A" of tuning fork. Kapsel- sagger clay, capsule c. Keller- tunnel effect, boom $e$. Kombinations- combination tone (either difference or summation $\mathrm{L}_{0}$ ) Licht- photographic sound recording. Magnet- magnetic sound recording. Mis- distorted note, off-pitch sound. Nadel- stylusor needle-recorded and reproduced sound. Netzbrumm- motor boating (of a.c.), a.c. pickup, mains hum. ober- overtone (often a harmonic), upper partial. Reln- note with one mode of vibration, singlefrequency tone. Schlag- strike note (in a bell, followed by hum n.) Schneide- edge tone, flue stop tone (of flue or organ pipe). Schwebungs- beat tone, interference t., b. note. Spaltslit tone, jet t. Stopf-(einer gedackten Trompete) stopped tone. Stoss- beat note, throb. Summensummation tone. Teil-partial note, partial (being either a subharmonic or a higher harmonic or overtone). Triller-trill, quaver, warble. Übergangstransient. Wechsel- warble note, w. sound (air alarm). Zwischen-medium or intermediate tone or shade (of color).

Ton-abnahmestelle sound aperture, s. ¿ate. -abnehmer pickup (of phonograph), soundhead (m.p.). -abnehmertrommel sound take-off drum, scanning $d_{\text {. }}$-abstufung grading of tones, graduation of tonal intensities. -abtastspalte sound scanning slit. -abtaststelle sound gate, s. pickup point, s. scanning slit. -analysator, such- 
heterodyne analyzer of sound. - angeber, chromatischer chromatic pitch pipe. - ansatz, Lichtphotographic sound film head. - ansatz, Madel- sound-on-disk attachment or head. -arm tone arm. -art tonality, mode (major or minor), key. -ateller sound stage, studio, teletorium. - aufnahme-Kofferappar at box equipment, trunk unit. -aufnahme,Lichtsound film recording. -aufnehmer sound recorder. -aufzeichner, magnetischer magnetic sound recorder, magnetophone, Poulsen telegraphone, Blattnerphone, etc. - bandbreiteregler ( cone) band width control. -belichtungsstelle sound gate, point where sound track is scanned, scanning light. -beseitigungsdrossel hum-bucking coil, hum eliminator choke. - bild tonal pattern, tone spectrum. -bildwand transoral screen (m.p.). -blende tone-control means, tonalizer; fader (continuously variable or otherwise). -blende, Reinbiasing or noise-reduction stop, gate, vane or shutter. -darbietung sound entertainment, acoustic e. - effekt, Raum- stereophonic sound effect, binaural e. -empfang modulated reception, tonal $r$. - empfänger, Bi.ld- audio-video receiver combination. - empfindung acoustical perception, sound sensation, auditory percipience. tönend(es) Signal tone s., tonal s., musical s. gleich- unisonant, consonant. selbst- ringing or squealing (of a tube, in radio apparatus).

-Tonerde alumina, argillaceous earth.

tonerdehaltig containing alumina, aluminiferous.

Ton-erdesalz, aluminum salt. - $f$ arbe timbre, tone quality. - färbemittel tone shading means, tonalizer. -fenster sound gate, s. slit.

Tonfilm, Rundfunk- broadcast of video and aural signals, sound telecine or s. telecast, television program with S. accompaniment. Trick- sound cartoon. -optik soundhead lens or optic (m.p.), sound "optic". -rundfunk audio and video film broadcast, sound telecine. -wagen sound truck, location t., S. van. -zusatzgerät sound film head or attachment. Ton-fixierbad toning or fixing bath. -folgeschrift sound track, s. record. -formstück, mehrzügiges muliple tile or clay conduit. - frequenz audio frequency, audible f., voice f., a-f. -frequenzen, höhere treble, high a-f or audio frequencies (handled by tweeter loudspeaker). - frequenzen, niedere bass frequencies, low a-f (handled by woofer loudspeaker). -führung labyrinth (of loudspeaker). - funkensender singing or musical (quenched) spark iransmiller. - funkenstrecke quenched spark gap. tongebend sound generative, acoustic, sonant.

Ton-gehör, absolutes absolute (genuine) pitch. -generator a-f generator, tonal or musical g. -gerät,Lichtsound film head or at tachment. -haltungspedal tone sustaining pedal. -helligkeit pitch (of sound sensation). -höhe pitch. -höhevergleicher tonvariator, (sometimes) pitch pipe. -kamera sound recording camera. -kameramann recordist. -klebestelle blooping patch or splice (of sound film). -kodaskop sound kodascope. -kontrolle,Kopfhörer-monitoring with headset. -kopf sourá film head or attachment. -kopie sound film used for reproduction. - l ampe exciter lamp(mostly incandescent light-source) operating photocell. - l aufwerk sound film feed mechanism. -leiter musical scale (natural, diatonic or just s., and tempered s.) -1 ichtl ampe exciter lamp (mostly incandescent) operating photo-cell. -masse paste (cer.) -meister monitoring operator (working at control or 
mixer desk), recordist, sound engineer, director of sound. -messer tonvariator. -mischer tone fader, t. mixer.

tonmodulierter Telegraphiersender tone- or AF-modulated telegraphic transmitter.

Tonmuffel clay retort.

Tonnenfehler barrel distortion, positive d.

Ton-optik soundhead lens, s. "optic" (m.p.) -papier tinted paper. -probe sound test; tonsil t. (for recording voice of a singer). - punkt, Licht- elementary shading value or density v. -rad tonewheel.

tonrichtiger Filter orthochromatic, true-color or correct-tone filter (opt.)

Ton-röhre singing valve. -rolle recording roll (of sound film). - schiefer clay slate or schist, argillaceous slate, argillite. - schirm sound screen. - schlitz reproducer slit, $r$. aperture, sound $\mathrm{g}$. -schwankung pulsation of reproduced sound intensity (known as wowows up to 6 cycles per second, as flutter between 6 and 30 cps, gargles 30-200 and whiskers over $200 \mathrm{cps})$, generally called flutter ragardless of cps (sound film). -schwankungsmesser flut ter reter, f. measuring instrument (sound film). -sender modulated c.w. transmitter, tonic t.; audio, aural or sound t. (accompanying video program). - spalt sound slit, s. aperture. -spektrum sound spectrum. -spur, eingeprägte track embossed on film strip. -spur, eingeschnittene track engraved in film strip. -spurbreite, Zackenwidth of variable-area sound track. -steuermann operator controlling sound volume in a theater. -steverstelle control or exciter point (sound film).

Tonstrelfen sound track, s. recording. -, doppel spuríger doublo-edged variable-width sound track. -, e inspuriger single-edged variable-width sound track. -, schwach ausgesteuerter lowmodulation track.

Tonstreifen, Licht-, veränder $1 \mathrm{i}$ cher Breite squeeze track (bearing photographic sound actions).

-verschmäl erung sound track squeezing, mat ing.

Ton-strom sound current, signal c., volce-signal c. -träger soundtrack support. - transportrolle sound-film feed roller or sprocket wheel. -trickfilm sound animated cartoon. -trommel scanning drum, sound take-off $d$. -überbl endung sound fading, s. fade. -überlagerung AF modulation. - umfang tonal range, bandwidth, compass. -umschal tungseinrichtung fading or shading device.

Tönung density value, shading v. Beiz-mordant toning. Wärmeheat tone (thermochemistry), h. quantity, h. effect.

tönungsrichtiges Bild picture of proper shading and contrast.

Tönungswert tonal value, gradation (of image).

Tonveredler high-frequency cutoff, h.f. filter, h.f. de-accentuator, sound clarifier, tone control comprising blocking condenser shunted to loudspeaker, tonalizer.

Tonverfahren, $\mathrm{Kl}$ ar- noiseless film method. Magnet- magnetic sound recording, telegraphone $r$. method, Blatinerphone m., etc. Kadelsound on disk method. Rein- noiseless film method.

Ton-vol umen, mi ttl erer power level (read in volume indicator), average 1. -wagen sound truck, location t. or unit, lorry set, s. van. -wahrnehmung acoustical perception, sound or auditory sensation. -welle recording drum shaft. - well enspannung audio wave potential (of sound accompaniment, in telev.) -wender tone converter. -wledergabe sound 
Tonwieder gabeopt ik

reproduction. -wiedergabeoptik soundhead lens or "optic".

-zeitdehnung acoustic slow motion (Silka method), -zelle sound cell (used in talking film). -zentrum tonal or acoustic center (of "gravity") of frequencies. -zerlegung,-zersetzung analysis of sound. -zusatz, Licht- photographic sound on film head or attachment. -zusatz, Nadel- sound on disk attachment or head. -zuschlag aluminous flux.

Topf can, shield; pot. Sperr-wave trap.

Topf-kreis tank circuit. -magnet shielded magnet, ironclad m. - photometer photometric integrator. Tor unit of millimeter mercury column.

Tor, Strom- thyratron.

Torfkohle peat charcoal.

torkeln wobble, tumble, stagger.

Torsions-kop' torsion head.

-modulus rigidity modulus, tor-

sion $m_{0}$, shear $m_{\text {. }}$-wage torsion balance.

tot(es) Feld dead zone (of a ribbon microphone), plane of ribbon. -(er)Gang lost motion, backlash. -(es)Material inert material, stable $\mathrm{m}_{\bullet}$; neutral m., inaotive $\mathrm{m}_{0}$ -(e)Wiedergabe dead reproduction. - (e)windung dummy turn, d. spire, idle turn, dead end.

-(e) Zone skip zone, dead z.

tot, schall- non-resonant, acoustically inactive or inert, non-oscillable.

Totale medium-long shot, full shot, full-length figure (m.p.)

Halb-(close) medium shot.

totbrennen overburn.

Töter, Krach- automatic silent tuning means, noise suppressor; $\mathrm{n}$. gate (film recording).

Touren-Bel astungskennlinle speedload characteristic.

Trabanten satellites (spectr.). träge inert, inactive, neutral, sluggish, slow-responding, lagging.

Träger carrier, bearer, supporter, foundation, bed, supporting means,

\section{Träghe i tsmoment}

base (of something), vehicle, backing; beam, girder.

Träger,positiver positive carrier, ion. Aufzeichnungs- recording or sound track or support (sound on film, on disk, etc.). Bl enden- diaphragm carrier. Film- film base, f. support, f. foundation. Hllfs-auxiliary carrier, sub-carrier. Ladungscharge carrier. Lichttonphotographic sound track support. Mikroskop-microscope stage. objekt- object support, O. carrier (plate), specimen holder (e.g., celluloid skin or film), slide, mount, stage, stand. Schirm- carrier, foundation or support of screen (coated with phosphor substance, in cathoderay tubes). Wicklungs-former, skeleton form, winding frame, coil form. Zusatz-missing or suppressed carrier re-introduced in receiver (in side-band signal transmission). Zwischensub-carrier.

Träger-follen supporting foils. - frequenzfernmessung carrier telemetering (e.g., metameter of GE Co.). -plättchen, 0 bjektobject support, o. lamina, 0 . slide. -seite film base side or face. -strom, zeltlich veränderlicher wobbled carrier current, warbled c.c. -underdrückung carrier suppression (in quiescent c. telephony).

-wegtastung carrier gating, c. suppression, c. interruption (in synchronizing work). -zusatz re-insertion of carrier.

Trag-fähigkeit buoyancy, lift, bearing, carrying or supporting strength, carrying capacity; yield, productiveness. - flächenholm wing spar. -flügelprofil aerofoil section.

Trägheit inertia, sluggishness, inertness, slowness, persistence (of eye or retina), time-lag. Netzhaut- retinal persistence. Trägheitsmoment moment of inertia. 


\section{Tragkörper}

Trag-körper(des Magnetsystem) chassis (of magnet system). -stütze supporting stay, s. prop. tränken steep, soak, saturate, impregnate.

Transformator, Abwärts- step-down transformer. Anpassungsmatching transformer. Anzapftapped transformer (especially center-tapped t.), split t. Aufwärts- step-up transformer. Ausgleich-hybrid- transformer, three-winding t., balanced t., differential to Dreiwicklungshybrid transformer, threewinding t. Käfig- shielded transformer. Hetz- mains transformer, power t. Phasen- phase shifting transformer. Reduktionsstep-down transformer. Saugbooster transformer, suction t., sucking t. Spar- auto transformer. Treiberröhre- driver tube transformer. Zwi schen-inter-stage transformer.

Transformator-gehäuse transformer shell, t. container, to tank. -kurve amplification curve of a transformer.

Translations-fläche translation plane, slip p. (cryst.) -linie slip band (cryst.) -streifung translation gliding striae, crystal ridges.

Transpar enz transparency, transmittancy, transmissivity (for light). -schwankungen modulus of transmission (film).

Tr ansponi erungs-empfang heterodyme reception. -steilheit slope or mutual conductance (of mixer tube).

Transport,Film- film feed, f. movement, film travel. Moment- momentum transfer.

Transporteur vernier, protractor.

Transport-kette conveyor chain, transfer c. -mechanismus feed mechanism, conveyor $m_{\text {. }}$-querschnitt transport cross-section. -rolle, Film-picture film feed sprocket. r rolle, Ton- sound film feed sprocket. - theorie, Impulsmomentum transport theory (of

\section{Trennungsilinie}

turbulent flow). -theorle, wirbel- vorticity transport theory. -trommel intermittent sprocket.

Transversalmethode variable-area. method (of sound recording).

Trapezfehler keystone, trapezium or trapezoidal distortion (due to dissymmetry to earth of deflection plates, to electron beam angle, etc.)

Trefferzahl number of impacts or shocks.

Treffweite (=Brennwelte in Optik) focal length (of electrons $=f$. distance in opt, )

T Regler T-type section attenuator.

Treiber driver, punch; propelling means. -röhre-Transformator driver tube transformer. -system driver system.

Treib-mittel driver, driving, motor or motive fluid or substance, fuel, propellant. -probe cupping test. -sitz driving fit. -werk, Schallloudspeaker operating or driving mechanism or means, motor m.

Tremolostimmung, Tremulant tremolo, tremulant effect. trennen separate, sever, sunder, divide, resolve, decompose.

Trenn-faktor separating factor. -festigkeit separating strength, static crack s. -filter dividing filter, separating $f$. - frequenz cut-off frequency. -netzwerk dividing network. -röhre isolating tube, buffer t. - schalter isolating switch, disconnecting link, isolator. - schärfe selectivity, filter discriminator. -schărfereglung automatic bandwidth selection. -stromröhre spacing valve (in telegraphy).

Trennung selection, filtering, separation (by filtering network, etc.); sorting (of ions, in mass spectrometer).

Trennungs-fläche parting plane, cleavage p., surface of separation. -linle dividing line, 
Trennungswinkel

boundary 1., partition 1., demarcation 1., 1. of separation. -winkel angle of separation (of solid moving through fluid.)

Treppe, Roll- escalator.

treppenförmig (s.a.stufen) in the

form of stairs, stepped, echeloned, terraced.

Treppen-rost siep grate. -stoss lap joint.

treppenweise echeloned, scalariform, stepwise, by stages, by degrees, gradually.

Tretkurbel foot pedal, treadle.

Treue, Farb-color fidelity (of color film), e. match, orthochromaticity.

treue wiedergabe faithful reproduction, fidelity of $r$. , orthophonic r. (of sound).

Trichter horn (of loudspeaker, with throat at narrow end and mouth at end with largest flare); funnel, hopper, cone, gate (in foundry); electromagnetic horn (of wave guide).

Trichter, Exponential-, abgestufter multi-flare exponential horn. -, aufgewundener twisted or curled exponential (loudspeaker) horn. -,gefal teter folded exponential horn.

Trichter, Riffel- corrugated horn. Rippen- ribbed funnel. Schweige- cone of silence marker (for airplane landing).

Trichter-einlage filter cone. -hals throat of (loudpeaker) horn. trichterloser Lautsprecher hornless loudspeaker, direct radiator 1 .

Trichter-mundöffnung mouth (opening) of horn, largest flare.

-mundstück throat of horn, narrow inlet of $h$. -mündung mouth opening of horn, largest flare.

-rohr funnel tube, tube funnel. -vorhof air chamber of loudspeaker (between cone and horn). -wand, schallharte rigid or non-absorbing horn wall.

Trlck trick, artifice, slight of hand, stratagem; trick effect or "stuff" (m.p.). -bilder trick

\section{Trockenmittel}

shots, t. pictures. -film, Tonsound animated cartoon. -film, Zeichen- animated cartoon. -filmzeichner animator. -kamera trick camera. -tonfilm sound cartoon.

Trieb, Spindel-worm drive. Zahnrad- rack and pinion drive, toothwheel drive mechanism.

Trieb-element motor element (of loudspeaker) -gehäuse gear housing, g. casing, g. box. -knopf milled head, pinion head (in microscope). -kraft moving force, motive power, propelling f. -mittel driving or motor means; fuel, propellant. - schraube coarse adjustment (in microscope). -werk machine, engine, drive mechanism, motor element (Ioudspeaker, etc.); gearing, transmission gear.

Triftröhre (cf Laufzeitröhre) drift tube, beam t. (e.g., Klystron).

Trillerklappe shake key (wind instrument).

trillern trill, quaver, warble.

Trillernote (cf Wechselnote, Heulton) trill note, warble note.

Trlograph electrocardiograph with CR tube.

Tripelpunkt triple point (in metallography).

Tritt kommen, in (cf Phasenaussortierung) come in step or phase, bunching, p. focussing. (of electrons).

Trittschall impact sound (transmission). -dämpfung foot $\mathrm{fall}$ sound attenuation.

Trocken-apparat drying apparatus, drier, desiccator, dehydrator. - entgasung dry distillation. - gerüst drying frame, d. rack, - gleichrichter copper rectifier, cuprous-oxide r., seleniumoxide r., metal r., dry r. -kompass dry compass. -kondensator dry electrolyuic condenser. -linse dry objective, non-immersion o. -mittel drying agent, 
drier, siccative, desiccative. -rückstand dry residue. -stoff siccative, drier, drying substance. Trog,elektrolytischer electrolytic tank (for field pattern mapping).

Trogbatterie trough battery.

Trommel drum, cylinder; tympanum. -, grosse bass drum. -,kleine snare drum.

Trommel, Belichtungs- exposure drum, recording $d$. (on which film is exposed to modulated light beam). Linsen- lens drum (scanner). Loch- scanning drum (of Jenkins). Mess- graduated drum. Nachwickellower take-up reel. Schaltintermittent sprocket ( $\left.m_{\bullet} p_{\bullet}\right)$. Sprossen- sprocket drum. Teildivided drum, graduated d. Tonscanning drum. Transport- intermittent sprocket. Vorwickelupper pull-down sprocket. Wirbelhigh side drum. Zacken- sprocket wheel.

Trommel-blende scanner drum, $d$. with scanning apertures. -fell drumhead; tympanic membrane, tympanum (of ear). -flöte fife. -schlägel, -stock drum stick. -welle, Antriebs- drive sprocket shaft. Trompete trumpet.

tropfbar capable of forming drops or of dropping or dripping, liquid. Tröpfchen globule, spherule, droplet, small bead. -methode,01oil drop method.

Tropf-düse dripping nozzle. - el ek trode, Quecksil ber- dropping mercury electrode.

tropfen drop, drip, trickle. Tropfen, Schweiss- welding dribble, w. bead.

Tropfenmesser stalagmometer, stactometer.

Tropf-gewichtmethode drop weight method.- -hahn dropping cock. -trichter dropping funnel.

Tropfungszeit dropping time. Trübe dross, pulp, slush, slurry, slime; turbidity, cloudiness. trübe turbid, cloudy, dull, thick, veiled, foggy, frosted, dim, opaque, muddy.

Trübgl as frosted glass, opal g., glass diffusing light.

Trübung. (cf Enttrübung) tarnishing (on metal surface); clouding (of glass), opacity; night and other effects tending to impair sharpness of zero in d.f., wavering of signal beam, blurring (of minimum). Funk- blurring of minimum signal or of zero point in radio d.f. or radio bearings, uncleared zero.

Trübungs-grad degree of turbidity . -koeffizient coefficient of turbidity, Angstrom c., degree of t. -messer (cf Bellichtungsmesser) turbidimeter, opacimeter, nephelometer (type of photometer). - spannung potential causing $\mathrm{QE}$ (quadrantal errors).

Trug-bild illusion, phantom, fata morgana. -schluss erroneous conclusion, fallacy, fallacious argument.

Trumm film strip. -, schlaffes slack end (or driven portion) of a belt, rope, etc. -,straffes driving or taut end (of a belt).

Trümmer fission products, f. fragments, remains.

Tubus, Ansatz- extension tube. Auszieh-draw tube (of microscope). Einstell- micrometer adjusting means, adjuster tube (in electron microscope). optik-optical. barrel. Projektions- projection tube (micr.)

Tubus-schlitten tube slide. -träger tube support.

Tunnel effekt tunneling effect. Tüpfel analyse spot analysis, drop a.

tüpfeln dot, spot, speckle, stipple, mottle.

Turbine, Anzapf- bleeder turbine. Elektronen- cyclotron and inverted (or Hollmann) forms thereof, magnetic resonance accelerator, induction electron accelerator (also called rheotron).

Turbinen-dichtung turbine (labyrinth) 
Turbinen

seal. -dichtung, Kämmen der rubbing of turbine seals.

- unterbrecher turbine interrupter,

i. break.
Turm, wickel - mandrel.

Turmalinkristall tourmaline crystal.

Türöffner, lichtelektrischer photo-electric door opener. 
überakustischer Raum(mit übertriebener Nachhall dauer) abnormally live space (having undue reverberation period).

Überanpassung, widerstands- overmatching of impedance (load resistance greater than internal resistance). über anstrengen over-exert, overstrain. Uber-anzeige overswing (of volume indicator). -belichtung overexposure. -besserung over-correction, o.-compensation.

überbl asen over-blow (of a flute). Uberbleibsel residue, residuum, remainder, remnant.

überblenden dissolve, fade-over, mix, lap.

Uber-blender fader, means to cause change-over from one projection to the next (m.p.); sound fader, means for fading in and out, blending and mixing (sometimes with central tap); fading mixer (telev.) -blendung, Ton- sound fading, s. fade. -blendungsbl ende dissolving shutter, lap-dissolving s. (for gradual transition from one scene into another). überbrïcken shunt, bridge, by-pass. überbrückungskondensator by-pass condenser, bridging capacitor. über-dauern outlive, outlast, survive. -decken swamp, blanket, mask, cover up, eclipse, conceal. überdeckung, Zeil en- line overlap. über-drehen super-speed operation (in film work). -drehzahl excessive speed. -druck, Atmosphären(Atii) atmospheres excess pressure. Ubereinandergreifen overlap (in diffraction pattern, scanned lines in telev., etc.); engage over one another.

Übere inander lagerung superposition, superimposition.

Ubereinanderliegend superjacent, superposed.
Über-einanderschwe issung 1 ap weld. -einkommen agreement, arrangement, convention, contract, compact. uber-einstimmen agree, coincide, harmonize, acquiesce in, correspond, register (one part with another), be in unison or synchronism (of phases). -endlich transfinite. -entwickelt overdeveloped, overdone, "cooksd" (m.p. slang). - exponiert overexposed.

Überfahren des Mosaikschirmes durch Strahl sweep out mosaic with beam.

überfällig overdue.

Über-fall rohr overflow pipe. - fang covering. - fanggläs er flashed opal glasses. - fangschicht glass liner, g. lining.

über-feine Struktur hyperfine structure. -flüssig superfluous, overabundant, in excess, excessive; overflowing. -führen convert, transport, transfer, convey, reduce (to practice or to a practical form); convict.

Überführung, weg- overpass, overhead crossing.

Überführungs-geräte(Kabelkasten) terminal equipment (cable box). -kasten test box; cross-connecting terminals. -zahl transport number (of ions), transference $n$. Übergang transition, passage, crossing, (gradual) change (from one place or condition to another), blending, shading (of colors). -von...zu transition or change from....to; replacement of ....by, substitution of.... for. Übergang, erl aubter permitted transition (in accordance with selection principle). -,erzwungener forced transition, non-spontaneous t. -, unerl aubter, -, verbotener forbidden transition. -, verfl auter 


\section{Übergang, Bewagungs-}

softly shaded transition (lacking definition) (telev.).

Bewegungs- motional transient.

Phasen- phase transition.

Quantum- quantum transition, q. leap.

Uibergangs-einrichtung change-over (from one projector to another). - gl leder transition types, t. members. -leltwert transconductance, transfer characteristic. -punkt transition point, place of t. or transfer. -schicht transition layer (opt.) -stelle transition point, place of $t$. or transfer. - töne transients. -verlust contact loss, transition $1 .$, junction 1. -wahrscheinl ichkeit (der El ektronen von einem Niveau zum andern) transition probability (of electrons to leap from one level to another). -widerstand contact resistance. -zahlen transference numbers (of solution). -zustand intermediate stage, intermediary s., transition s., transiency. Übergewicht overweight, extraw., unbalance, desequilibrium, preponderance, predominance. über-glasen glaze, overglaze. -greifen overlap, engage over, encroach, transgress, cross a boundary. -helles stimmungsbild high-key picture. -hitzen superheat (beyond saturation), overheat.

Überhöhung bank or cant (in a curve), hump, super-elevation (of rails), lobe (as on a cam).

ibberhol en overreach, outstrip, outrun, overtake, catch up with. Über-hol ungsgebiet der El ektronen catchup, overtake or bunching range of electrons (in phase focusing, in beam-tube drif t-space). -hörfrequenz supersonic, ultrasonic, ultra audible, superaudible or ultra-audion frequency.

iber-individuell super-individual. -kal ten supercool, undercool. Über-korrektur overcorrection, overcompensation. -kreuzungspunkt, Elektronen- electron crossover

\section{Übernahme}

(point where principal rays from first lens cross axis = exit pupil in optics; in electron optics, crossover lies between cathode and first anode). -krümmung excessive curvature. -kuhl ung supercooling, overcooling, underc. -l ader supercharger (of engine). -lagerer heterodyne, local oscillator (based on beat principle) e.g., of endodyne, autodyne or selfheterodyne type. -lagerer, Selbstautodyne, self-heterodyne. Überlagerung heterodyning, production of difference frequency or beat; superposition, impression of one wave or $f$. upon another. Ton- AF modulation, tonal $\mathrm{m}$. Überlagerungs-emp fang heterodyne reception, beat $r_{0}$-kreis circuit in which heterodyne or beat action is produced, heterodyne c. -stufe mixer stage, first detector (in superheterodyne receiver). -summer heterodyne warbler oscillator.

überl appt schwelssen lap-weld, scarf-weld.

Ujberlappung over-lap (of lines, in telev. image).

überl astet overloaded; overrun (by working a lamp in overvolted condition).

Überlastung overload, overcharge, overtax; overrunning (of a lamp by operating it at voltage far above rated), overvolting. über-legung consideration, reflection, deliberation. -leltfähigkeit superconductivity, suprac. -l i chtung over-exposure. -mass allowance (in press fits). -mikrometer ultra-micrometer. -mikroskop, Durchstrahl ungstransmission type ultramicroscope, super $-m_{\bullet}$, electron $m_{0}$ -mikroskop, Selbststrahl ungsself-emissive electron microscope, self-illuminating $\mathrm{m}$. (based on electrons arising on object surface). -nahme,strom- transfer of current, tapping of $c_{.}, c_{.}$tap. 


\section{Überregul i erung}

-regul ierung overshooting, excessive regulation, e. compensation, over c.

über-rot infrared, ultra-red. -sättigen supersaturate.

ijberschall-lichtzelle supersonic light relay or valve. -wellen supersonic, ultra-sonic or $u_{0}-$ audio waves. - well enanhäufung (zone of) accumulation of ultrasonic waves. zelle supersonic light relay or valve.

überschi ebung transvection. -schlag nose over (of an airplane).

- schlag, Funken- sparkover, flashover, breakdown (say, of a sparkgap). - schmelzung superfusion, enameling. - schneidung overlapping, intersection (of lines), intercept; overcutting (when adjaccent record grooves touch).

- schreien overmodulation. -schreitung exceeding (a certain limit, mark, level or value); transgression. -schrift heading, caption, title. überschwellige Schallreizt superthreshold (sound) intensity levels. - sichtbarkeit super-threshold visibility.

Übersee-Emp fänger transocean receiver, transatlantic $r$.

übersehen oversee, overlook, survey, sweep (with eye).

Übersetzung, Frequenz- frequency transformation.

Übersetzungs-rohr converter tube (used in avc schemes between regulating and regulated tubes). -verhältnis transformation ratio, gear $r$.

ijbersicht survey, review, summary, extract, synopsis, abstract, digest. über-sichtig long-sighted, hypermetropic. - sichtlich easily followed up, easily understandable, visible, clear and simple, not complicated or intricate.

ijbersichtsspektrum general spectrum. überspannt(er) Betrieb overvol tage operation, overvolted o., overrunning. - (e) Entl adung overvolted discharge.

Überspannun: overvoltage, excess v., overrunning, overvolting.

Uber-spannungs funkenstrecke

surge arrester, s. gap, s. absorber. - spielen re-recording. -sprecherscheinung cross-talk, babble (when from several channels), "monkey chatter"; distortion, lack of sensitivity.

über-stehend projecting, protruding, salient, standing over, surmounting; surnatant, supernatant (of liquids). -stevern overshoot, overload, overmodulate. -stevert over-"shooted" (m.p.)

Übersteverung overloading (of tubes, causing blasting); over-modulation (in film recording), sound overshooting.

Überstec erungs-abschneider overload chopper. -messer overload indicator. -punkt overload point (of a recorder). -schutz overload limiter (for film).

Über-strahlen der Ramanlinien

swamping or outshining of Raman spectral lines. -strahlung irradiation. -streichen des

Leuchtschirmes sweep out of television screen. -streichung eines Frequenzbereichs durch einen Kondensator sweeping of a frequency range by a condenser, coverage of f. band, scanning a range. -struktur superlattice; superstructure.

übertragen transfer, transmit, transport, convey; translate, transcribe; assign, vest in, delegate, confer, grant, cede.

Übertragender assignor, grantor, transferor.

übertrager, Brïcken- differential transformer. -stromstossimpulse repeater.

Übertrager-kondensator grid condenser (in audion or gridcurrent detector). -kreis intermediate circuit, transformer c.

Übertragung, mehrwegige multipath transmission, multichannel t. Bild-fade-over $\left(m_{0} p_{0}\right)$. Einseitenband- single-side- 
Übertragung, Leitungs-

band or vestigial s.-be cransmission. Leitungs- wired radio, w. wireless, carrier telegraphy or telephony, wire broadcast. Übertragungs-band signal band. -einrichtung, Schali- (public) address system, electroacoustic transducer. -erklärung assignment (declaration), deed of transfer. -faktor transfer factor, image t. constant. -frequenzlinie transmission-frequency characteristic. -gegenstand teleview object, televised $0_{0}, 0_{\text {. }}$ to be televised or transmitted by video signals. -güte transmission performance (in rating). -kanal transmission channel. -kurve transmission-frequency characteristic. -mass image transfer, attenuation constant. -mittel transmission medium, to means. -schwingungen signal oscillations, s. waves. -urkunde deed of assignment, assignment. -weg transmission channel, t. path, ¿. medium.

ibber-tretung contravention, of fense, violation, transgression. -verdichtung supercharging. - vergrösserung extra or supplementary magnification, over $-m$. überviolett ultraviolet, of vitaray nature (when of wave-length 2900-3200A).

Über-wachsungen overgrowths.

-wachung tell-tale device, monitoring, supervision, watching. -wachungsschal ter od.-schlüssel monitoring key.

Uberwi egend preponderant, predominant, prevalent.

Uberwucht unbalance, imbalance. uberziehen cover, coat, line, plate, overlay, put on or over. Überzug flashing, coat, cover, skin, plating, lining, envelop; incrustation, crust. Gummi-, auf Rollenlaufbahn rubber-tread of roller.

üblich conventional, customary, usual, common, ordinary. u.f.,uff. and the following, et $\operatorname{seg}(q)$.
Uhr,Film- film footage counter. Haupt-,Mutter-master clock. Neben- secondary clock.

Uhrschraubenlehre dial micrometer.

U K W (UI trakurzwell en) (cf well enabgrenzung) ultra-short waves, micro w., midget w., uhf w., quasi-optical w.

Ulbricht'sche Kugel Ulbricht sphere type photometer.

ultrakurze Wellen ( of Wellenabgrenzung) ultra-short waves, uhf w., quasi-optical w., micro w., midget w.

ultrarot infrared, ultrared.

UItrarot-abtastung noctovisor scan. -durchlässigkeit diathermancy, ultrared or infrared transmittancy.

ul trarotundurchl ässig athermanous, opaque 10 infrared.

UItra-schallzelle supersonic light valve. -schwärzung blacker-thanblack condition, infra-b.c.

UI trastrahlung, kosmi sche cosmic rays or radiations.

ultraviolette Strahlen ultraviolet rays, vila r. (between 2900 and $3200 \AA)$.

Ultra-wasser optically empty water. -zentrifuge ultra-centrifuge, high-speed c.

umändern alter, change, modify, amend (an application).

Umänderungsprozess, Kern- nuclear disintegration.

um-biegen bend over, round or back, double-back, crimp.

-bilden re-model, re-form.

-bördeln bead over, flange (all around). -drehen turn around, twirl, rotate, revolve.

Umdruck reprint, circular. -papier transfer paper.

um-fällen dissolve and re-precipitate. -falzen crimp over, bead. Umfang circumference, periphery, circuit, circle, contour; scope, extent, compass, range, dimension, latitude, size, volume, confines. Aufzeichnungsrecording range. Helli gkeltsbrilliance, brightness or contrast range, key (of a picture), brightness relationship. 
Umfang, Lautstärke-

Lautstärke- volume range. Lichtrange of light oscillations.

Negativ- intensity range of negative. objekt- range of brightness (in subject). Schall- sound intensity range, s. contrast.

Schwärzungs-range or latitude of densities, density scale. Tontonal range, compass.

Umfang-kopieen, Gross- "hi-range" or wide-range prints. -Kopien, Klein"lo-range" prints. -kraft tangential force, circumferential f., peripheral $f$.

Umfangs-geschwindigkeit circumferential or peripheral speed. - geschwindigkeitsmesser tachometer (e.g., stroboscopic, with strobotron). -schwingungen circumferential oscillations or vibrations.

umfassen embrace, comprise, span, encompass.

umfassend comprehensive, extensive, embracing.

Umfläche circumferential, contour or ambient surface.

umfliessen flow around or in contact with, circumcirculate, skirt in flowing.

IImformbarkeit re-formability, deformability.

Umformer converter, inverter (from d.c. to a.c.), thyratron; transducer. Mess- measuring transducer. Pendel-vibrating rectifier. Schweiss- welding converter.

Um-gangsklappe by-pass (clack) valve. -gebung surroundings, environment, ambient, ambiency. umgehen by-pass, circumvent, obviate, dodge, avoid, evade; go around, circumnavigate.

Umgehungsschal tung by-pass circuit, b. - p. connection.

umgekehrt proportional inversely proportional.

umgekehrter oder umgestülpter Fuss re-entrant squash or press (of a (ube).

umgiessen re-cast, cast around (something).
Umgrenzungslinie contour, boundary, peripheral or circumferential line.

Umgrösserung enlargement or reduction, change in size or dimension. Umgrösserungsverhältnis ratio of enlargement, r. of magnification. Umguss transfer (by pouring), decantation; recasting, recast. Umhängerlemen shoulder strap. Umhüllung covering, wrapping, casing, envelope, jacket, sheathing, shrouding.

IJmhïllung, Papier- paper wrapping. Umhüllungsl.inie envelope, contour line.

Umkehr,Selbst- self-reversal (of spectral lines)

Umkehr-absorptionskanten reverse absorption edges or limits. -bad reversing bath.

umkehrbar reversible, revertible, capable of being turned over or out.

Umkehrentwicklung reversal processing, $r$. developing.

Umkehrer, Phasen- phase inverter, p. reverter.

Umkehr-erscheinung(bei Beleuchtung von Schichten) photographic reversal or solarization (in layer exposure). -film film resulting from developing by reversal. -prisma(cf. IImkehrungspr.) inverting prism. -punkt reversal point. -röhre converter tube. Umkehrung, Band- speech band inversion or transposition (in garbled or scrambled secret telephony). Bild- image inversion, $i$. reversion (when two axes, i.e., right and left, top and bottom simultaneously interchanged); solarization (reversal of gradation sequence, due to overexposure) silben-syllable inversion (in garbled telephony). Umk ehrungspri sma, Doppel- inversion prism (inverts in both axes of image).

umkippen unlock time base. Umkl appen reversal (say, of motion). 
umkl eiden

um-kleiden line, sheath, cloak, surround. -klöppeln braid, pur braiding around.

Umkopie optical reduction print, o. p. of altered dimensions. Umkopieren optical printing. Unkreis periphery, circumference;

perimeter; surroundings.

umkreisen circle, encircle, revolve, spin, rotate, gyrate (around).

Umkristallisieren re-crystallizing (resulting in offsetting in tungsten wires).

Umladung change in charge, c. in sign (of molecules and acoms). um-l agern re-arrange, re-group. - laufen revolve, rotale, circulate, gyrate (spirally).

- l aufend circulating, circular, gyrating, revolving.

Umlauf-frequenz rotalional frequency (of electrons). - getriebe planetary gear, sun and planet $g$. -integral contour integral. - räder planetary wheels. -schwingungszahl rotation frequency. -spannung magnetomotive force, mmf (line integral of magnetizing force). - verschlus's rotary shutter. - verschlussblende, rotierende rotary (flicker) shutter, light cut-off.

uml egbarer $\mathrm{Kl}$ appsucher reversible or folding finder.

umlegen reverse, tilt, tip, throw (a switch); lay over or around, Nrap.

I!ml enk-prisma deviating prism. -rolle flanged idler roller, deviating $r$.

um-lernen learn anew, unlearn and re-learn. -manteln jacket, sheath, case, encase.

Um-modulierung re-modulation. -polung reversal of polarity. umpressen press (-fit) around, put or apply (around something) by pressure.

Umrandungskurve envelope. Umrechnung re-calculation, recomputation, conversion. Umrechnungs-faktor conversion fac- tor, reduction f., change ratio. -tafel conversion scale (say, meters to kilocycles).

Um-richter (cf nechselrichter und

Gleichr.) (ransverter (Lo change a-c lo d-c or vice versa). -risszeichnung linear drawing, sketch, outline illustration, -roller re-winder. -satz, Leistungs- energy exchange, iransduction.

umschaltioar adapted to circuit

changes or changes in connection, switchable, reversible (by switch or similar action).

Umschalter(cf Schalter) reverser, commutator switch, switch, reversing gear; swilchboard or panel. Gabel-hook switch. steck-plug-in switch.

Umschaltung, Lauf- reversal of motion.

Umschal tungseinrichtung, Tonfading or shading device, fader. UImschlag cover, covering, wrapper, envelope; sudden change; hem, collar. Anker- armature travel, a. excursion, a. transit.

Umschlagspunkt transition point. umschl ingen wind around, wrap a., clasp, loop, cling to, embrace. Umschl ingungswinkel looping angle. umschmelzen re-cast, re-melt, refound; melt around (something). !!mschnürung, Faden- serving of thread (on a cable).

IImschreiben re-writing, transcription, circumscription, description (malh.); re-recording, electric transfer of sound track onto film or disk.

umschreibender Kreis circumscribing circle.

Umsetzung transposition, double decomposition, conversion, change, transformation, transduction.

Imsetzungsgerät transducer, transductor.

Umspanner, Schweiss- welding transformer.

um-spielen re-record, play back. - spinnen cover, braid or spin around.

Um-springen jumping, abrupt 
reversing, arising of sudden irregularity or unsteadiness.

-spulen re-winding (of film). umspïlen flow around or in contact with, skirt, circumcirculate. Umstände,mildernde extenuating circumstances.

umstel l en re-adjust, re-adapt; reverse, transpose, invert.

Umstellungspunkt transposition point.

umströmen flow around, $f$. in contact with.

umstülpen overturn, invert, put upside down or inside out.

Umwandler mutator, converter, transformer, transducer, sink. Energietransducer, sink.

Umwandlung transmutation, conversion, transformation, change. Kernnuclear transmutation.

Umwandl ungs-einrichtung, Schall(electro-) acoustic transducer. -punkt transformation point, iransition p. -steilheit mutual conductance or slope (of tube). - tabelle conversion table.

-vorrichtung transducer, sink. -wärme heat of transformation. Um-wegl eitung by-pass (lead). -welteinfluss environmental influence.

urn-wendèn turn, turn over, about, sideways or upside-down, invert, revert. -wickeln wrap round, cover, wind on or over; re-wind.

Imwindung convolution.

unabgestimmt untuned, aperiodic, unresonated, non-resonant. unabhängig, frequenz- free from frequency effect, independen of $f .$, not a function of $f$.

Unabhängi gkeit, Frequenz- frequency independence, condition of a quantity of being independent of, or unaffected by, frequency.

Unabhängigkeitssatz independence theorem.

un-auffällị inconspicuous, concealed, attracting no altention. - aufgelöste Linienbilder unresolved line patterns. - ausdehnbar inexpansible, non-ductile, inextensible. - bearbeitet raw, crude, unwrought, unfinished, in blank form or b. condition.

Inbefugter intruder, unauthorized person.

un-bel astet unloaded. -bemannter

Ballon unmanned balloon.

- benannt unnamed, anonymsus;

indefinite (math.) -beschadet

without prejudice to.

- beschichteter Blankfilm uncoaled plain film base. -besetztes Eand empty band. -bestimmt undetermined, indeterminate, indefinice.

Unbestimm theitsrel ation indeterminacy relation, uncertainty $r$.

un-bewaffnetes Auge unaided eye, naked e. -bezogene Farben unrelated colors. -bunte Farben hueless colors, achromatic c., c. devoid of hue. - dehnbar inextensible; non-ductile. - dicht untight, pervious, leaky. -durchdringlich impermeable, impervious, impenetrable. -durchlässig impermeable, impervious, opaque.

undurchlässig für aktinische

Strahlen adiaclinic. - für Ultrarot. athermanous (co infra-red rays). - für Roentgenstrahlen radiopaque. licht- light-lighl, opaque or impervious to light, non-diaphanous. wärme- athermanous or impervious to heat, heat insulating.

un-durchsichtis opaque, impervious, non-transparent, non-diaphanous. - echt pseudo...., non-genuine, imilated. -echte Längenmessung indirect measurement of length. -edel base, not noble or rare. -el astisch gestreut unelastically scat tered. -empfindlich insensitive, non-reactive, nonresponsive, immune from, neutral, inert, unsusceptible. -empfindlich, richtungs- non-directional, astatic.

Unendlicheinstellung infinity adjustment (opt.)

un-endliche Reihe infinite series. 


\section{unentfl ammbar}

-entflammbar flame-proof, noninflammable, non-ignitable, noncombustible. -entflammbarer Film safety film. -entgeltlich gratuitous, gratis, free (of charge). -entwickelt implicit (math.) -erlaubter Übergang forbidden transition. - erwïnscht undesirable, objectionable. Unfähigkeit disability, inability, incapacity, incapability. unfrel constrained, engaged, tied, bound.

Ungänze,kleine minor discontinuity or defect.

un-gebundene Ladung free charge. - gedämpfte Anzeige ballistic or non-aperiodic reading or indication (of instrument). -gedämpfte Schwingung undamped oscillation, sustained o., continuous wave (c.w.), persistent w. -gefasstes $\mathrm{Gl}$ as rimless lens. - geordnete Geschwindigkeit velocity of agitation. -geordneter Zustand disordered state, d. arrangement. - gepufferte Lösung unbuffered solution. -gerade uneven, out of true, not straight, non-linear, crooked, odd (harmonic, multiple). - geradwertig of odd valence. - gerichtet(e) Antenne non-directional antenna. -(es) Mikrophon astatic microphone, non-directiona. m. -geschaltet unwired.

-gesehnte wicklung full-pitch winding. -geteilter Ring unsplit ring, solid r.

Ungewissheitsprinzip uncertainty principle, indetermination p., indeterminacy $p$.

ungleich unequal, unlike, uneven, dissimilar, odd, not uniform, different, unmatched.

Ungleichgewicht desequilibrium, unbalance, imbalance.

un-gleichnamig unlike, opposite (of poles). -gleichseitig scalene (triangle).

Un-gleichung inequality, non-equality. -giltigkeit invalidity, nullity, state of being void, annuled or not in force.

\section{Unselektivität}

unhaltbar untenable. -hämmerbar non-malleable.

Universal-gelenk universal joint, Cardan $j_{*}$, ball and socket $j$. - gl imml ampengerät universal neon tube tester, -nebenschluss Ayrton shunt, universal s. - prijfer multiple-purpose tester, multi-meter. -tastatur universal keyboard.

unkennbar undiscernible, unrecognizable.

Unkosten, General - general expense, overhead e.

un-lauterer Wettbewerb unfair competition. -lösbar undissolvable, indissoluble; undetachable, locked. - messbar immeasurable, incommensurable (math.) -mischbar imiscible. -mittelbare Betrachtung direct viewing. -okular monocular. -paarwertig of odd valence. -periodisch aperiodical, nonperiodic, deadbeat, non-recurrent. Unruhe fluctuation, irregularity, instability (of physical quantities); disquiet, unsteadiness; balance (of time piece). Anoden- fluctuation of anode feed current, rippled condition in plate c. Heiz-fluctuation in heating or filament current, $f$. c. ripples. Netz-mains or supply line fluctuations (of potential or load); mains ripples.

un-rund non-circular, cornered, out of round. - scharf unfocused, poorly defined, out of focus, blurred, fuzzy or ghosty (of image or picture).

Unschärfe insufficient focus, lack of $f .$, out-of-focus condition, blur or fuzziness, lack of definition (of picture); flat tuning condition. Tiefen-insufficient depth of focus, lack of $d$. of $f$. unselbständige Entladung assisted discharge, non-self-sustaining d., non-spontaneous d. -fremdgesteverte KI ppschwingungen distant-controlled or signal-c., non-selfrunning time-base (telev.)

Unselektivltät lack of selectivity. 
unsichtbar

unsichtbaren Strahlen, Abtastung mit noctovisor scan with invisible (or infra-red) rays.

Unsinn, offenbarer manifest absurdity or nonsense.

un-starre Moleküle non-rigid molecules. -stät,stetig unsteady, unstable, variable.

Unstetigkeit im Filmzug flutter in film pull.

Unstetigkeitsstelle point of unsteadiness, instability or discontinuity.

Unstimmigkeit discrepancy, disparity, lack of harmony.

unstreckbar non-extensible, nonductile, non-malleable.

Unsymmetrie asymmetry, dissymmetry; (condition of) unbalance, unilateralness.

Unter...sub-..., fractional, partial. unterabteilen subdivide, make subcompartments or partitions.

Unter-anpassung under-matching (of impedance). - anspruch sub-claim. - anzeige underswing (of volume indicator). - art subvariety, subspecies. -bel astung underload. -belichtungsberelch toe range (of under-exposure).

unterbrechen interrupt, break, stop, arrest, make discontinuous.

Unterbrecher, Motor- motor-driven make-and-break. Quecksilberstrahl-mercury (jet) interrupter. Selbst- trembler, self-interrupter, buzzer. Stimmgabel- tuning-fork interrupter. Turbinen-turbine interrupter or break.

Unterbrechung und Schllessung break and make.

Unterbrechungs-bad short-stop bath, interruption b., short-s. trough. - funken break spark, circuitopening s., wipe s. -schwingungen quench oscillations (in superregeneration). -vermögen

circuit-breaking, rupturing or opening capacity or power (of a switch).

unterdrehen low-speed shooting. Unterdruck reduced pressure (below
Unter lage, Filz-

atmosphere), partial vacuum.

Unterdrlickung, Fluoreszen zquenching or suppression of fluorescence (by killer, poison, etc.) Seitenbandside-band suppression. Tatsachenconcealment or suppression of facts. Träger-suppression of carrier (in quiescent telephony).

Unterdrückungs-schwingungen quench oscillations (in super-regeneration). -stromkreis, Geräuschnoise reduction, quelching, suppression or silencing circuit. Unter-fläche under surface, under

face, under side, base. -form sub-variety, subspecies.

- formanten sub-formants.

- führung, Weg- underpass.

Untergehen swamped state, swamping (of a station). - der Bildspannung im störspiegel swamping or blanketing of picture signal by noise level.

untergeordnet subordinate, subsidiary, secondary (in rank, quality or order), immaterial.

Untergericht inferior court, lower c. (of law).

untergeschoben spurious, suppositious.

Unter-glasur underglaze. -grund background, subsoil, subterrarean soil, underground. -grund, Spaltungs- fission background. - gruppe subsidiary group, subordinate g. -haltungswert entertainment value. -hörfrequenz sub-audio frequency, infrasonic f. -hülle subshell.

unterirdisch subterraneous, subterranean, underground.

Unter-korrektur undercorrection, undercompensation. -kuihlung supercooling, undercooling, overcooling.

Unterlage support, base, substratum, backing, foundation, lining, bed, carrier. Fllzfelt base, f. support, $f$. under-layer, $f$. foundation. 
Unterlage, Vergleichs-

Vergleichs- basis of comparison. unterlagert subjacent, infraposed, underlying.

Unter-l agerungstel egraphie subaudiofrequency telegraphy system, composite operation ? telegraph and telephone signals passed over one line wire; of ien attended with thump noise). -lagscheibe washer, supporter disk.

unter-lassen omit, abstain or refrain from, fail, default, neglect. ,-legen lay under, underlay, put under, infrapose.

Unter-legescheibe washer, supporting disk. -licht horizonial light. unter-liegend subjacent, underlying, placed below or underneath, infraposed, by sub,ject.to. - ordnen subordinate, make secondary or of minor rank.

Unter-ordnung subordination, submission. -patent sub-patent.

-resonanzfrequenz submultiple resonance (of a subsynchronous frequency). -sagung inhibition, prohibition, forbidding, interdict, interdiction, restraint, injunction. -schale sub-shell (of elecirons). - schallwelle infrasonic wave, sub-audio w.

unter-schätzen underestimate, undervalue, underrate. -scheidbar distinguishable, discernible, discriminable, differentiable.

Unterschei dungs-markierung distinctive marking, telltale is -vermögen, Farb-color discrimination faculty of power. -vermögen, Tiefen- power of differentiating depths.

Unter-schiebungskl age suit for passing off goods as though of another person. - schied, Gang- phase difference, path d. (of rays, waves, etc.) unter-schneiden undercut (groove too shallow or lateral movement of stylus too small). -schreiten fall below or short of (a certain value). -schwellig subliminal, below a threshold.

Unterschwingung subharmonic oscillation, subfrequency 0 . unter-setzender Verstärker scaling down vacuum tube. -setzt geared down. -spannen subtend; sub-volt. - spannter Betrieb undervolted operation.

Interspannung under-running voltage, undervolting.

unterstellen presuppose, assume, allege, impute, charge.

Unter-steuerung under-modulation, insufficient m. -stützung eines Einspruches argued support of an opposition.

untersuchen analyze, examine, inspect, check up, investigate, search, research, inquire, scrutinize.

Untersuchung investigation, research, examination, probing, inspection, test.

Unter suchungsobjekt test specimen, t. sample, t. piece.

untersynchrone Resonanz subsynchronous resonance, submultiple resonance.

Unter-taste long key, white.k. - tauchung immersion, submersion, deep dip. -teilung subdivision, submultiplication; partition, partitioning. -teilungspunkt tap (of a battery, etc.).

-wagenantenne under-car antenna. -wasserschall empfänger hydrophone, submarine or subaqueous microphone, pickup or sound detector, asdic (submarine detector).

unterwegs en route, on the way. Unterwind forced draft (introduced from below).

un-tilgbar indelible. - trennbar inseparable, indivisible, unseverable, undetachable. - übersehb ar uncontrollable, hard to follow up or inspect, complex, intricate. -ubertragbar nontransferrable, unassignable, inalienable. - ibertrefflich unexcelled, unsurpassable, unrivaled. -verbrennbarer Film slow-burning film, safety f. -verfaulbar unputrefiable, impuurescible.

Unvergängl i chkeit imperishableness, ever-last ingness. 
unver-gängl iches Lichtbild imperishable picture. -hältnismässig disproportionate.

Unverständlichkeit unintelligibility. un-verträglich inconsistent, incompatible. -verwechselbarer Stecker non-interchangeable plug. -verwïstlich indestructible. -verzeichnetes Bild,-verzerrtes Bild orthoscopic image, undistorted picture.

Unvollkommenheit imperfection, imperfectness, faultiness, flaw, defect.

un-vollkommener I solator imperfect insulation, leaky dielectric. - wandelbar immutable, invariable, unchangeable, imperishable. -widerlegl ich irrefutable, unrebut table. -wirksam inactive, ineffective, inefficient, inert. - wirksam machen render inoperative or inactive, disable, deactivate, inactivate.

Unwucht unbalance, imbalance, desequilibrium. -erreger,-masse unbalance weight.

un-zeitgemäss untimely, unpropitious. -zeitlich untimely, immature. -zerlegtes Licht undispersed light. -zerstörbar indestructible, non-corrodible. -ziehbar non-ductile. -zugänglich inac- cessible. -zweideutig unequivocall, unambiguous, clear.

Uran-tonbad uranium toning bath.

- verstärker uranium intensifier.

Ur-atom primordial atom.

-ei chkreis master reference sys-

tem. -gewicht standard weight, primary $s . w$.

Urheber author, originator, founder. - recht copyright, rights of an author or inventor.

Urkunde ausstellen execute a document.

Urkunde,Besitz- tille deed.

ibertragungs- deed of assignment.

Urmass primary standard.

ursächlich causal, causative. Ursprung origin, source, provenience. ursprünglich original, primitive, parent.., primary, first.

Ursprungs-bild original subject copy. -type prototype, original or primordial type or specimen. Urstoff primary matter, element. Urteil judgment, decision, verdict, opinion. - fällen render a.decision, judgment, an ard, decree, sentence or veraict. Urteil, Versäumn is- judgment by default.

Urteilsvollstreckung execution of a sentence, carrying out a judgment. 
vagabondierende ströme leakage currents, stray c., vagabond c. Vakuole pinhole, vacuole. vakuundicht vacuum-tight, sealed (airtight) to insure a vacuous state.

Vakuum-dichtung vacuum plumbing, v. sealing, v. packing. -fett vacuum grease. -gitterspektro graph vacuum grating spectrograph. - hahn vacuum tap. -mantelgefäss thermos or Dewar vessel with exhaust jacket. -messer,-meter vacuometer, vacuum gage, ionization g. -pumpe, Vorfore-pump, rough-vacuum $p$. -schleuse airlock (of electron microscope). - thermoelement vacuum thermo-couple. -zelle vacuum photo-electric cell.

Valenzkette, Haupt- primary valence chain.

Variationsmethode variational method.

Variometer, Ballon- balloon statoscope. KI app-hinged-coil variometer.

Vater metal master (in disk manufacture).

Vektor, absoluter tensor. Viererfour-component vector.

vektorielle Darstellung vectorial representation.

Vektorindikation vector response index.

Ventil, Ablass- discharge valve, drain v. Etagen- multi-seat valve. Licht- light valve, 1 . relay. Rückschlag- check valve.

Ventil-kliken plug of a valve. -stopfen valve stopper, s. within $\mathrm{v}$.

Venturidise constricted or nozzlelike reducer, throat piece of Venturi meter.

ver-all gemeinern generalize.

-änderlich variable, changeable, fluctuating, unstable, unsteady, non-constant. -änderlich, laufend running variable.

Veränderliche variable, variable quantity (math.)

veränderlich(er)Brennweite und Grösse,Linse von zooming lens for variable focus and variable magnification, "vario-objective." - (e) Kopplung vario-coupler.

Veränderung, Farben- change of color, disculoration.

Veränderungen, Seiten- turns and

banks (in avigation).

verankern anchor, stay or guy (a pole).

Veranlassung von, auf at the request or suggestion of, on behalf of.

ver-antwortlich responsible, answerable, liăble, chargeable. -arbeiten process, work.

Verarbeitung, Film- film processing. veraschen incinerate.

Verband union, association; binding, bandage, fastening, tie, bond (in bricklaying).

Verbesserungs-mittel corrective. -patent patent of improvement. verbiegen bend, buckle, warp. Verbindlichkeiten eingehen incur liabilities.

Verbindung mit angeschärften Enden scarfed joint. -, stumpfe butt joint. Dreiweg- three-way connection. Einlagerungsintercal ation compound. Glasschllff- ground-glass joint. Muffen- sleeve joint. Sauerstoffoxygen compound.

Verbindungs-fähigke it affinity, combining power. -glied connecting link. -klammer brace, clip connector. -klemme connecting means, binding post, terminal. -kraft bonding strength, affinity, combining power. -punkt joining, 
connecting or union point, junction, juncture.

Verbleiung leading, lead lining. Verblitzen des Films dendriform exposure of film (due to static charges).

Verbot prohibition, inhibition, interdict, interdiction, injunction. verboten(e) Linie forbidden line (spectr.) -(er)Platz..forbidden place (cryst.) - (er)übergang forbidden transition.

Verbrauch, Energie- energy consumption, e. dissipation. Energie-, ohne non-dissipative, wattless.

Verbraucher load, consuming device, consumer.

Verbrauchs-messer supply meter. -zähler, Blind- reactive volt-amp. hour meter, sine m., wattless component m., var m. -zähler, Schein- apparent power meter. verbraucht spent, exhausted, used up, worn out, consumed, stale (of air), dissipated.

verbreitern widen, broaden, spread (out).

Verbreiterung spread (of image). Druck- pressure broadening (of gas-spectrum). Linien- line broadening. (telev.) Spektrallinien broadening of spectral lines. Stoss- impact broadening. Zeichen- signal spread (due to echo effect).

verbrennlich inflammable, combustible, burnable.

Verbrennungsschiffchen combustion boat.

Verbund-gl ass compound glass, multilayer \&. -röhre compound tube.

verdampfbar evaporable, vaporizable, volatile.

Verdamp fer evaporator, drier, dehydrator, carburetter (water gas).

verdamp fungsfähig evaporable, vaporizable, vol atile.

Verdampfungsquelle source of evaporation, s. of volatilization.

verdecken cover (up), mask, conceal, hide, eclipse, occutt. verdeckte Zeichen, durch Störer

signals swamped by interference. Verdeckung masking(ac.), overriding (of noise).

Verdeckungserscheinung masking effect.

ver-derben spoil, damage, ruin, decay, rot. -dichtbar condensable, compressible. - dichten condense, condensate, concentrate, compress, compact, press, together, concrete, make denser, inspissate, consolidate. Verdichter, Kreisel- rotary compressor, centrifugal c.

Verdichtungswelle compression wave.

ver-dicken thicken, concentrate, inspissate; coagulate, jell, become viscous. -drahteter Empfänger fully wired receiver set.

Verdrahtung, $8 \mathrm{l}$ ank- bare wiring. ver-drallen twist, strand (together). -drängen di splace, drive out, remove, expel, supplant, supersede.

Verdrängung, Strom- skin effect, current displacement.

Verdrängungsstrom di splacement current, dielectric c., capacitance $c$.

verdrehen distort, twist, wrench, subject to torsional stress, stagger (rotationally), shift (a phase).

Verdrehung (cf. Zerdrehen) rotation, turn (of a picture); twist, warping, torsion.

Verdrehungs-dauerhal tbarke it endurance under torsion stress.

- fähigkeit torsibility.

-moment torsional starting torque, moment of rotation. -wechselfestigkeit strength under alternating torsion stress. -winkel angle of torsion, twist a. Verdreifachung, Frequenz- frequency tripling. ver-drosseln provide with choke coil. -dübeln dowel. Verdunkel ung, Rlickl auf- flyback 


\section{Verdunkel ung}

elimination, return-trace or return-path e., blackout or blanking of flyback trace (by gating and blanking pulse.

Verdunkel ungs-kapazi tät shading condenser. -phase dark interval. ver-dünnbar diluable, rarefiable, capable of being diluted. -dünnen dilute (liquid), rarefy (gas), altenuate, thin, de-concentrate, reduce or dim (light). -dünntes Hel i um low-pressure helium. Verdïnnungs-grad, Helligkeitsloss or reduction of brightness. -mittel diluent, attenuant. - welle rarefaction wave. verdunstbar evaporable, vaporizable, capable of being evaporated.

Verdunstungsgefäss evaporimeter. veredeln (cf vergüten) improve, elevate, refine, ennoble, purify, perfect; plate with a higher grade of metal.

Veredler,Ton- high-frequency filter, hf cutoff, sound clarifier, tone control, tonalizer.

Vereinfachung simplification, reduction (math.)

Vereinheltlichung standardization, normalization, make for uniformiiy, regularization, co-ordination.

Vereinigungs-koeffizient combination coefficient. - stelle junction, joining, meeting or union point. Vereisung ice formation, icing (on wings).

ver-engen narrow, contract, constrict, squeeze. -erzen mineralize.

Verfahren process, method, procedure, proceeding, mode (of action), technique, processing (of film). Beschwerde- appeal action or procedure. Billigkeits- equity suit.

Verfärbung discoloration, shading. verfestigen make firm or strong, solidify, consolidate, concrete, set, harden.

Verfestigungs-kurve plastic stressstrain curve (cryst.) -zeit time of set (of gels). verfilzen felt, mat. verflachen level off, flatten, be- come even, smooth (down), lose selectivity, become flatter or less steep, slope down, broaden or make broad-topped (as a resonance curve).

verflauter Übergang shaded-off

transition (definition lacking). ver-flechten inter-weave, involve. -flüchtigen volatilize, evaporate. -flüssigen liquefy.

Verfolgung, Ziel- tracking a target (by detecting and ranging means using a slewing motor).

Verfolgungs-aufnahme running shot. -stativ follow-shooting tripod, running $t$.

verformbar deformable, plastic, fictile, kneadable, moldable. Verformung deformation, working, warping, strain.

Verformung unter Fllessen, wissenschaft der rheology. -, plastische plastic deformation, p. working.

Verformungsvermögen degree of deformation, deformability.

Verfügung action (of Patent Office), order, decree, decision.

Vergangenhelt, Wärme- thermal antecedent, prior heat treatment. vergänglich perishable, transient. vergasbar gasifiable, vaporizable. Vergaser gasifier, vaporizər, carburetor.

Vergasung, Ti eftemperatur- lowtemperature distillation or carbonization, partial c.

Vergenz vergency (either convergence or divergencz).

Vergiften poisoning, contamination, killing (of phosphor).

Vergitterung grating, gridding, lattice, grille.

vergl asbar vitrifiable.

Vergl asung vitrification, glazing, glaze.

Vergleich comparison, contrast, matching. Farben- color matching.

Vergl eicher, Tonhöhe- tonvariator, pitch pipe.

Verglelchs-feld matching field (in photometry). -masstab standard 


\section{Vergleichsprisma}

of comparison, s. of reference, yardstick. -prisma comparison prism.

Vergleichung comparison, contrasting, matching.

Vergleichunterlage basis of comparison.

vergrössern enlarge, magnify, increase (in size).

Vergrösserung, 1 ineare magnification in diameter. Fernrohr- telescopic magnification. Über- supplementary or extra magnification, overm.

Verguss-k ammer-Einführungsi sol ator pothead (lead-in) insulator. -masse filler or sealing compound.

vergïten refine, improve (quality), temper, coat (a lens).

vergütete $P$ tobe quenched or tempered test piece or specimen.

Vergütung, Glas- refining or treating of optical glass (e.g., by producing low-reflectance coating, dimming, etc., as by Bausch \& Lomb Balcote magnesiumfluoride anti-reflection film on glass).

Verhältnis proportion, ratio, rate, relation, connection, circumstance. Aufteilungs- potentiometer ratio. Molen-mole ratio. öffnungs- aperture ratio, relative a. Stromstoss- impulse ratio. winkel- angular ratio.

Verhältnis-anzeiger exponent (math.) -arme ratio arms (of a bridge).

verhältni smässig proportional, proportionate, commensurable, commensurate.

Verhandlung pleading, proceeding, hearing, argument, discussion. zur mündlichen - gel angen

to be called for oral hearing or argument. einer Sache, Termin zur - anberaumen set a date for hearing a case.

verhärten harden, indurate, set (cement).

verharzen resinify, resining.

\section{Yerkleisterung}

verheddern tangle (of antenna wire, etc.)

Verhinderung, Korrosions- corrosion inhibition.

verhol zen lignify, turn woody.

Verhör hearing, interrogation, examination, trial, questioning.

Ver jährungsgesetz law of limitations or superannuation.

ver-jüngen point, taper, thin, diminish, reduce; constrict, narrow, contract (a tensor). -kabelte Leitung cable line, stranded-wire 1.

Verkabelung, Amts - central wiring, office $w$.

verkanten twist, lilt (of camera). Verkehr, Fernsehsprech- video telephone traffic.

Verkehrsbezirk, Funk - radio controlled aerial navigation (or avigation) district or zone. Nah- approach or local control zone.

Verkehrs-flughafen commercial airport, civil a. -kontrolle,Luftairways traffic control, avigation regulation. - luftfahrt commercial aviation, c. avigation.

verkehrt proportional inversely proportional.

Verkettung linking, linkage, concatenation, bonding.

Verkettung, Fluss- flux linkage.

Verkettungsspannung interlinked voltage.

ver-kitten cement, lute, seal. -kittete Linse cemented lens. -kleben cement, lute, paste, glue, cover, seal or close with adhesive or agglutinant. -kleiden face, case, line; disguise, mask. -kleidet faired (of airplane).

Verkleinerungskopie reduction print.

verkleistern paste (up), turn into p., agglutinate.

Verkleisterung gelatinization (of sterch), agglutination, glutinization. 
verklingen decay, die out (of waves, etc.).

Verkohlung carbonization, coking, dry distillation, destructive d., charring.

Verkokungszeit coking time.

Verkopplung spurious, stray or undesired coupling.

verkürzen shorten, abbreviate, condense, abridge, contract, curtail. Verkürzungskondensator shortening condenser, padding c., aerial series c., antenna c.

Verlagerung displacement, shift, dislocation, misalignment. Bildpunkt- spot shift (causing plastic effect, in telev.). Nullinien-zero setting, electric biasing (in film recording). Peilbearing shift, error or distortion. Zeilen-migration or shift of line position.

verlängern lengthen, extend, prolong; produce (math.).

Verlängerung, Frist- extension of time, e. of term, respite. Patent- renewal of a patent.

Verlängerungsspule, Antennen- aerial load coil.

Verlauf pattern, plot, path, shape, trend, course, slope. Feld-field map, $f$. pattern, $f$. configuration. Kopplungs- coupling coefficient, c. factor.

Verlauf-filter sky filter.

Verlegung,Kabel- cable laying, burying of c. Rohr - pipe laying.

Verleihkopie release print, distributing p. (of film).

verletzen infringe, violate, transgress, contravene, prejudice, injure.

Verletzten, dem - eine Geldsumme zusprechen adjudicate a sum of money to aggrieved or, injured party. Verletzung offense, violation, infringement (upon patent rights).

Verletzungsklage, Patent- action or suit for infringement on patent rights.

Verlorener Kopf deadhead, wastehead, crop end.

Verlust loss, dissipation, drop (of potential), absorption (light). Verlust, Belag- surf ace leakage loss (of condenser). Einführungs- insertion loss. Erwärmungs-Joule loss, joulean heat (dissipation). Luftreibungs- windage loss. Nachwirkungs- residual loss. Spannungs- voltage drop. Sprüh-corona di scharge or brush d. lọss. strahlungs- corona loss. Ubergangs- contact loss, transition l., junction 1 .

verlustarmes Kabel low-loss cable. Verlust-dämpfung damping (loss) resulting in dissipation of energy (by ohmic resistance). -faktor phase angle difference (of a condenser). -kapazität imperfect capacity, leaky c. -konstante attenuation constant. -leistung, Anoden- anode dissipation, effective power d. -leistung, Auffangplatten-collector dissipation (in multiplier).

verlustlos non-dissipative free from loss, lossless.

Verlust-strom watt current, active. c., energy c. -widerstand nonreactive, effective, dissipative or ohmic resistance. -winkel phase angle difference, loss angle (of a condenser). -ziffer phase angle difference.

Verminderer, Machleucht- killer, poison (of phosphors).

Vermittelung agency, intermediary, means, mediation, interposition, intervention, adjustment.

vermöge by or in virtue of, according to.

Vermögen, Abbil dungs- resolving power. Brems- stopping power, retarding p., braking p. Emissions- emissivity, emitting power. Reflexionsreflectance, reflectivity, reflecting power.

Vermutung assumption, supposition, surmise, conjecture.

vernachlässigen disregard, neglect.

Vernachlässigung neglect, di sregard, omission.

vernebeln atomize (under high 
pressure).

verneinenden Falles in the negative case.

Verneinung negation, denial, disavowel, contradiction.

Vernetzung network, reticulation.

Vernichtungsstrahlung annihilation radiation.

ver-pflichtet obligated, bound, under obligation. -puffen puff off, deflagrate, explode, detonate; vaporize, atomize. - regneter Film rainy film.

-riegeln block, lock, inter-lock, cut off, gang (condensers), bolt, latch. -riegelnde Spannung cutoff biasing potential.

Verriegler suppressor. -relais locking relay.

Verrückung displacement, disturbance, derangement, shift, dislocation, dislodgment. Atom- atom displacement.

verrühren mix by stirring, stir up. Versäumnisurteil judgment by default. verschachteln nest, insert into one another.

verschlebbar displaceable, shiftable, movable, sliding, slidable. -,posaunenartig shifiable trombone-fashion, telescoping.

Verschiebe-fehler lack of synchronism; out of frame condition. -zackenspur bilateral variablearea sound track.

Verschiebung displacement, dislocation, drift, slip (in geology), shift (of phases), dephasing. -, dielektrische dielectric displacement.

Verschiebung, B/Id-slow drift of picture, hunting of p. Bild-, seitliche lateral shift of image. Druck- pressure shift (of spectral lines). I sotopie- isotope effect,i.shift. Längs- axial adjustment (of electron microscops). Parallel-translatory motion, t. shift. Phasen- phase displacement, p. shift, dephased or out-of-phase condition.

Zeilen- interlacing, interlaced scan, line-offset s. Zeit- time lag, t. shift.

Verschiebungs-konstante (Dielekzitätskonstante) displacemeni consiani, specific inductive capacily (for vacuum). -I Inie line of displacement. - satz displacement law. -strom displacement current, dielectric d.c., capacitance c.

verschiedenfarbig varicolored. verschieden gegliederte Bildteile picture portions of dissimilar make-up, nature or organization. Verschl ackung scorification. Verschlag partition, compartment. Verschlechterung impairment, deterioration, spoiling, debasement, vitiation.

verschlelern veil, make turbid, fogged, or cloudy, befog.

Verschleierung veiling. glare (of projected image), foggy or fogged condition (of film).

Verschleiss wear and tear, abrasion, attrition. -priffung abrasion test.

verschlungene Blitzbahn tortuous path of lightning flash.

Verschluss closing, closure, shutting, shutter, stopping, stopper, fastening, lock, clasp, trap, seal, zipper, seal-off (of ignition mercury pool, etc.).

Verschluss, el ek tro-optischer electro-optical shutter. -,luftdichter airtight seal, hermetic s. End-cable box, termination b. Fall schieber-, drop shutter. Gas- gas seal, ball and hopper, cup and cone arrangement (of furnace). Rei ssslide fastener, zipper. Rouleauroller blind shutter. Schlitzfocal plane shutter. Sektoronsector shutter. Uml auf- rotary shutter. Hasser- water seal. Verschlussblende, flimmerfreie non- 
Verschlussblende, Umlauf-

non-flicker shutter. Uml auf-, rotierende rotary flicker shutter or light cutoff.

Verschlüsselung coding, cryptography.

Verschluss-kopf cable terminal. -ring locking collar (of magnifier). -stück plug, stopper, seal.

Verschmäl erung des Tonstreifens squeezing of sound track, matting. verschmelzen melt, smelt, fuse, melt together, blend, merge, alloy.

Verschmel zung merging (of pictures). Ver schmel zungsfrequenz critical flicker frequency, fusion or noflicker $f$.

verschmieren smear, daub, lute. verschmoren scorch, freeze together (of contacts).

ver-schobene Phase displaced phase, shified p., dephased or out-of -p. condition. - schränken toggle, cross, interlace. -schrumpfen shrink, contract.

Verschweigung concealment.

ver-schwimmen blur, become indistinct; blend. -schwinden vanish, disappear, evanesce, fade (away). -schwommen indistinct, foggy, indefinite, blurred, bleary, woolly (of zero signal point in df apparatus); fuzzy (lack of sharpness of sound track). -schwommenes Aussehen bleary, blurred or foggy appearance (of image).

Versegelung distance sailed or covered (between $t$ wo bearings); deviation from right course, yaw.

verseilen strand, cable, twist together.

Verseilung cable lay up, stranding (of wires or leads).

ver-sengen singe, scorch. -senken sink, submerge. -senkter Schalter, halb- semi-sunk switch, semirecessed s. - setzbar capable of being mixed or treated. - setzen mix, treat, coinpound, alloy, add (one substance to another); stagger, displace, shift. - setzt staggered, of set, displaced, dislocated, shifted (of phases, in quadrature $=90^{\circ}$, in opposition

\section{Verstärker}

$\left.=180^{\circ}\right)$, dephased, out of phase. Versetzung dislocation (cryst.), permutation, alligation (math.); alloy (metal). Flugzeug- drift, lateral displacemient or shift of airplane, deviation from course. Metall - alloying, alloy. Perforations- slipping of perforation, dislocation.

verseuchen vitiate, poison, infect. Versicherung, eidesstattli che affirmation in lieu of oath, statutory declaration. -,eidliche affidavit, sworn statement, testimony or declaration. versickern ooze or seep away. versinnbildlichen symbolize, express or represent by symbols. Versorgungs-gebiet service area, supply district. -spannung supply (source) potential. verspannen stay, guy, span. versperren obstruct, block, lock. Verspiegl ung, Hochheim 'sche Hochheim special mirror plating or "silvering" substance (of I.G. Farben).

verspleissen splice.

Versprödung embrittling.

Verständigung intelligence transmission, signal t. -im Flugzeug inter-phone. -zwischen Flugzeugen inter-aircraft communication or signaling. Eigen- interphone (on board airplane).

Verständl ichkeit intelligibility (teleph.), transmission audibility, articulation (consonant, vowel, with the use of logatoms). silben- syllable articulation, s. intelligibility.

Verstärkbarkeitsgrenze limiting amplifiableness, maximum (practical) amplification or gain.

verstärken amplify, multiply, intensify; re-inforce, strengthen, increase, fortify, concentrate, magnify, boost; truss (poles, etc.)

Verstärker amplifier, multipler, intensifier ( $R F$ stage between antenna and receiver input), repeater (in a telephone line). 
Verstärker, A- class A amplifier, pre-amplifier (with operating angle of 360 degrees). B- class $B$ amplifier (operating angle 180-360 degrees). C- class C amplifier (operating angle less than 180 degress). - , ausgegl i chener balanced amplifier. -, detonierender heterodyne amplifier. -mit negativem widerstand kallirotron, dynatron type of tube (with negative resistance). -mit zwei gegeneinandergeschal teten Röhren push-pull amplifier. -, untersetzender scaling down vacuum tube. Aufnahme-recording amplifier. Bildfrequenz-,Bildpuls- amplifier for frame synchronizing impulses, low-sync a. Direkt- straightahead amplifier, non-heterodyne a. Druck-Zug- push-pull amplifier. End-power amplifier, final a. Geradeaus- straight-ahead amplifier (in non-heterodyne receiver). Gleichtakt- in-phase amplifier. Kaskaden- cascade amplifier, repeating a. Kathodencathode follower (scheme). Kraft-, Leistungs- power amplifier.

Leitungs- telephone (circuit) amplifier or repeater. Mehrfachmulti-stage amplifier Mikrophonmicrophone pre-amplier, m. amplifier. Nach-amplifier above input stage, (mosily) power a. Nadeltonphonograph-type amplifier. Netzmesh amplifier, m. multiplier (with electron-permeable targets of fine mesh or gauze). Photozellen-electron multiplier phototube; photo-cell amplifier.

Regel-variable-gain amplifier, ave (automatic volume-control) a., noise-reduction or silencing a.; any amplifier in which regulator or control potentials are operative. Richt- amplifying detector, plate-current detector.

Schirmgitter-Richt- screen-grid tube with plate-current rectification. Steuer - amplifier between power and input stage. (operates on pure voltage amplification).

\section{Versteinung}

Uranium- uranium-intensifier (phot.) Vor- input amplifier, pre-amplifier-(class A RF amplifier in superheterodyne receiver). Zeilenimpul s- amplifier to increase line synchronizing impulses, high sync a, Zusatzbooster amplifier. Zwi schen- (inter-) stage amplifier.

Verstärker-brett amplifier rack, a. panel. -bucht,-bunker amplifier or repeater rack or bay. - geräusch amplifier noise, tube n. - gestell amplifier or repeater rack or bay. -gleichrichter amplifying detector. -kette amplifier cascade. -netz amplifier channel. -stufe (zwischen Antenne und Verstärkereingang) intensifier (tuned RF) stage, intermediate circuil. -zug amplifier channel.

Verstärkung, wirk- transducer gain. Verstärkungs-anlage, Sprach- (public) address system. -begrenzer output limiter, o. suppressor, gain spoiler. - faktor amplification factor, voltage f. -mass unit of gain, repeater gain, equivalent r. g. -mittel, Schall- sound reinforcing system (for halls, etc.). -schirm intensifying soreen ( $x$-ray work). -unterdrücker gain spoiler, output suppressor. -zahl amplification constant, a. coefficient, a. factor,

verstecken hide, conceal.

Versteifung stiffening, propping, bracing, strutting.

Versteilerung (Vergrösserung des Amplituden- verhältnisses zwi schen Zeilen oder Signalen) exaggeration of amplitude differences (emphasizing the stronger and supressing the weaker one of two or more signals), expansion. - der Konturen insuring higher definition (telev. pictures), improvement in resolution and black-white contrast (of an image), expansion.

Versteinung devitrification (glass); petrifaction. 
verstellen shift, move, transpose, adjust.

Verstellpropeller feathersd (variable-pitch) propeller.

Verstellung, Höhen- adjustment in elevation.

Verstellungs-knopf,Bild-framing knob (m:p.) - welle, Bild-framing shaft (m.p.)

verstimmt detuned, out of tune, dissonant, untuned, disharmonious, dissyntonized, tuned off resonance. Verstimmungsschalter wave (length) change switch.

verstopfen stop, choke, clog, obstruct, stopper (up).

verstopfte Tromoete stopped irumpet. Verstopfung, Rohr - pipe clogging, stop-up, choke or obstruction in a pipe or piping.

verstreichen fill or stop up, caulk, spread or coat over; elapse, expire.

verstümelt mutilated, obliterated, maimed, clipped (teleph., ac.). Verstümmelung, wörter- clipping of words, obliteration of $\mathrm{w}$.

verstürzen fall to pieces, scatter. Versuch, Beregnungs- rain test, wet t. (of an insulator).

Drehschwingungs- oscillation torsion test. Rotbruch- hot breaking test. Verwindungs- corsion test. Vorführungs- demonstration experiment. Vorlesungs- classroom experiment, lecture demonstration. Versuchs-fehler experimental error. -feld proving ground, field for making tests and experiments. - laboratorium experimental or research laboratory. -stab test rod, t. bar.

Vertagung adjournment, postponement. vertäubtes $0 \mathrm{hr}$ deafened ear. vertauschen exchange, interchange, play reverse rôles, transpose.

Vertauschung exchange, interchange, permutation (math.), transposition (of wires).

Verteidungsschrift argued statement of defense, ploa.

vertellen distribute, divide, dis- perse, diffuse, scatter, spread, space apart.

Verteiler, Bildpunkt- picture scanner (at roceiving end) Licht- beam splitter (m.p.). verteilte Induktion oder

Induktivität distributed inductance, continuous loading (opposite of lumped or concentrated i.) - Kapazität distributed capacitance, self-c. (as distinguished from lumped c.) -Wicklung distributed winding.

Verteilung, räumliche spatial disuribution, geometric d. Empfindlichkeits- sensitivity distribution. Farbenempfindlichkeits-, des Auges spectral response of eye, color sensilivity of eye. Geschwindigkeitsvelocity distribution, $d_{\text {. }}$ in energy. Geschwindigkeits-, Apparat zur Bestimmung der velocity selector. Gleichequipartition. Lichtstärkelight intensity distribution (on photo-cathode). Maxwell'sche Maxwellian distribution (law). Raum- spatial distribution, geometric d. Reichweitenrange distribution.

Verteilungs-anlage relay equipment, distribution gear. - fläche, Licht- isophotic curve. - gesetz distribution law, partition 1. -konstante distribution or partition constant or coefficient. -kurve, Fehler-error distribution curve. -satz principle of distribution, d. law.

Vertikalausbreitung von Schall vertical diffusion of sound.

Vertikal-hinlauf vertical scansion: -navigation vertical guidance, v. avigation. -wechsel vertical or frame synchronizing pulse or cycle.

vertonte 0 berfläche surface bearing sound track, s.-impressed surface or area.

Vertonung, $\mathrm{Nach}$ - dubbing. vertragl ich contractual, (bound) 


\section{verträglich}

by contract.

verträglich compatible.

vertraglich verpflichtet contractual-

ly bound or obligated.

Vertragspartei party to contract, contractant.

Vertrauensbruch breach of confidence.

Vertrieb eines $\mathrm{Filmes}$ releasing or distribution of a film.

Vertrocknung drying (up), desiccation.

verunreinigen vitiate, soil, contaminate, pollute, adulterate, make impure.

Verunreinigungskerne impurity nuclei.

ver-ursachen cause, produce, occasion, result in. -urteilen pass judgment against, sentence, fine, condemn, convict, adjudicate. - vielfachen multiply.

Vervielfacher, Farnsworth- multipactor, F. multiplier. Netz- mesh multiplier, m. amplifier (works with secondary emission, cascaded electron-permeable mesh or gauze targets). Pendel-reciprocating and acceleration electron multiplier predicated on secondary emission. Sekundäremissionselectron multiplier (with plurality of secondary-emission targets or reflecting electrodes).

Vervielfacherzel le, photo-elektrische,mit Sekundäremission electron multiplier photo tube, photo-electric electron-m. tube (with reflecting electrodes or dynodes).

Vervielfältiger, Gitter- mesh-type multiplier.

Vervielfältigung copying, printing (of film).

Vervielfälti gungs-faktor multiplicaiion factor. -kondensator condensor-type d.c. multiplier. -oberfläche dynode (in Farnsworth multiplier). -röhre multiplier tube, multipactor.

Vervierfacher quadrupler.

Verwachsungen inter-growths, intercrescences.
Verwackeln des Bildes unsteadinass of frame.

verwandelbar transformable, convertible, transmutable.

verwandeln transform, convert, change, turn.

Verwandlung, Paschen-Back- P.B. magneto-optic effect.

Verwandschaft relation, affinity. verwaschen obliterated, blurred, vitiated, bleary.

Verwaschungszone blurred zone, bleary z., z. lacking definition, confusion z. (telev.).

Verwehung draft of air, wind. verweilen dwell, sojourn, stay. verweisen, Berufung an den Gerichtshof refer appeal to the court. verwerfen warp, distort, twist; repudiate, reject.

Verwerfung rejection, repudiation; warping, cieformation; slow frequency drift (in radio apparatus, after tuning).

Verwertungsperiode evaluation period. verwickelt complex, complicated, involved, intricate.

verwinden twist, subject to torsional stress.

Verwindungsversuch torsion test. Verwirklichungsform practical embodiment (of an invention). ver-wischen blot out, efface, obliterate, blur, make bleary. -wittern disintegrate, effloresce. -würfeln jumble, garble. -zahnen tooth, cog, indent, provide with (gear) teeth, serrate.

Verzahnung, Evolventen- involute tooth gear.

Verzahnungsgetriebe, winkel- double helical gear.

verzapfen joint, mortise; dispense by tap.

verzehren consume, spend, dissipate, abate (smoke).

Verzeichnung faulty delineation or re-creation of a picture, distortion of a p. ( $e_{.} g_{\bullet}$, pincushion, barrel, etc., distortion, in television).

Verzeichnung, an i sotrope anisotropic 
Verzeichnung, kissenförmige

shear distortion, distortion of orientation (telev.) -, kissenförmige pincushion distortion, pillow d., negalive d.

-, nichtlineare non-linear distortion. -, tonnenförmige barrel

distortion, positive d. verzeichnungsfreie Linse orthoscopic lens, distortion-free 1.

Verzerrer, Dynamik- dynamic compressort.

Verzerrung durch Ein- und Ausschwingung transient (non-linear) distortion, build-up and decay d. -durch Feldkrümmung distortion due to field curvature, curvilinear distortion.

Verzerrung, Ausschwing- decay distortion, non-linear d., overthrow or underthrow d. (in facsimile transienc). Bild-(cf Verzeichnung) picture distortion (in facsimile: jiggers, f. transients known also as hangover, tailing, overthrow and underthrow distortion). Dämofungs- frequency discortion; tone d. Einschwingbuild-up or transient (non-linear) distortion. Frequenz atcenuation-frequency distortion, amplitude f.d., attenuation d., frequency d. Laufzeit-transittime distortion, phase d. Lichtfleck-spot distortion. Phasen- phase distortion. verzerrungsfrei distortionless, d.free, non-distorting. -(es) Okular orthoscopic eyepiece, discortion-free e.

Verzerrungs-leistung distortion power (in cerms of d. voltamperes). - toleranz tolerance of distortion.

Verzicht disclaimer, renunciation, waiver.

verzichten(auf Anmeldung, Patent, etc.) abandon, drop or relinquish (an application, patent, rights, etc.), disclaim, renounce, waive rights.

Verzichtleistung disclaimer, renunciation, waiver, abandonment. verziehen warp, buckle.
Verziehung warpage, buckling.

Verzinkung, Feuer- hot galvanization, pot g.

Verzögerer,Vielfach- BarkhausenKurz type of tube (retardingfield t., magnetron, etc.)

Verzögerung retardation, deceleration, braking, delay, lag, checking. Funken- spark lag. Phasenphase delay, phase lag, (phase) angle of lag.

Verzögerungsbad restraining bath. verzögerungsfrei inertialess, free

from lag, delay or sluggishness.

Verzögerungs-kette delay network.

-1 inse cutoff or stopping. (electron-optic) lens. -relais slow-acting relay, time-lag $r$. - schal ter time-lag switch. - spannung delay voliage, delayed-action v. (in avc circuits). Verzug time delay, t. lag.

Ausschwingungs- hangover, tailings (in facsimile). Entl adungsdischarge delay, d. lag.

Fliess-yield or flow distortion or deformation.

Verzugsrechner acoustic corrector (sound location).

verzweigte Ströme branched currents, branch c. -Stromkreise divided circuils, branched c.

Verzweigung by-pass, shunt, branching (of transmutation products), ramification.

Verzweigungs-struktur lineage structure (cryst.). - verhältnis branching ratio.

Verzwillingen twinning.

Vibrations-gal vanometer, kompen-

siertes Abraham's rheograph

(inertia and damping compensated). - schwelle vibrational threshold. - tastgerät vibrato-tactile device. viel deutig ambiguous, non-equivocal, capable of various interpretations.

Viel-doppel zackenschrift multiple double-edged variable-width track. - ebene multiplane. -eck polygon. vieleckig polygonal, multiangular. vielerlei various, multifarious, variegated.

vielfach manifold, multiple, poly..., 


$$
\text { vielfach, gleich- }
$$

multi..., various, frequent. gleich- equimultiple.

Vielfach-abtastung multiple scanning. -beschleuniger cyclotron (type of) tube (with two dees and spiral beam), betatron (of G.E.Co.) - echo mulitiple echo, flutier e, Vielfaches, ganzes integral multiple, whole m. -,gerades even multiple. - unger ades odd multiple.

Vielfach-konturen multiple outlines along edges of objects or of lines (in pictures or images), general masking of details. - schaltung connection in multiple. -schwingungserzeuger mulcivibracor. -streuung multiple scattering. -telegraphie multiplex telegraphy. -verzögerer Barkhausen-Kurz type of tube (retarding-field, magnetron, etc. tubes). -zackenblende shutter or light stop for making multilateral sound track. -zackenschrift multilateral sound track or $\mathrm{s}$. recording.

Vielfall multiplicity. vielfarbig many-colored, multi-c., variegated, polychromatic.

Vielflach polyhedron.

viel-flächig polyhedral.

- gestaltig multiform, manifold, diverse. -gliedrig polynomial (math.). -gliedriger Kettenleiter multi-mesh network.

Viel-kanal aufzeichnung multi-channel recording. -kantscheibe polyhedral mirror (of scanner).

vielkernig multinuclear, polynuclear, polynucleate; polycyclic.

Viellinien-spektrum discrete band spectrum, manyline spectrum. -sy'stem many-lined system.

vielseitig many-sided, polyhedral, multilateral; having diversity, versatile.

Vielspiegelung multiple of image. vielstimmig polyphonous, polyphonic (of organ).

Vielstufenwärmeaustauscher multipass heat-exchanger.

\section{Vignettierwirkung}

vielteilig multiparite, of many parts; polynomial (math.)

viel wegehahn multiple-way stopcock.

vielwertig multivalent, polyvalent. Vier-bandlichtschleuse four-band

light valve, four-ribbon $1 . v$. -eck, Gelenk- linked or articulated quadrilateral or quadrangle. viereckig four-cornered, square, quadrangular, oblong (of reading slass).

Vierervektor four-component vector. Vierfachspiral lochscheibe quadruplespiral scanning disk.

Vierflach tetrahedron.

vier-fl ăchig ieurahedral,4-f'aced.

-gliedriy four-membered, tetragonal (cryst.); quadrinomial (math.). -gliedrige Farbgleichung four-color equation, four-stimulus e.

vierpol quadripole, four-terminal network, transducer, ideal artificial line (with two input and two output terminals). Teil- section of recurrent struccure.

Vierpol-dämpfung image attenuation constant. -1 autsprecher balanced armature loudspeaker (with permanent magnet). -winkelmass image phase constant. Vierseit quadrilateral.

vierstellige Zahl four-figure number.

Vier-stoffsystem quaterrary system. -teilung quadripartition.

-telkreis quadrant.

viertelkreisige D-Werte quadrantal error (Q.E.) values (due to airplane fuselage, etc., in $\left.d_{.} f.\right)$

vier-telperiode quarter period. -weghahn four-way cock.

Vignettierapparat vignetter. Vignettieren des Lichtbündels vignetting of cone of light.

Vignettier-maske vignetting mask. -wirkung durch Linsenfassung trimming of pencil incensity caused by lens mount, vignelting effecl. 
Viol a

Viola viola, tenor violin.

Viol in $\mathrm{E}$ violin $\mathrm{E}$ string.

Visier sight (of gun, etc.), visor. Abdrift-drift meter. Bombenbomb sight.

visieren sight, aim, gage.

Visier-einrichtung view-finder.

-linie line of sight. -mattscheibe

focusing screen. -richtung line

of sight, direction of vision.

-scheibe focusing screen.

-vorrichtung (visual) sight for

taking direct bearings. -zirkel

gaging calipers.

Vogel-augenahorn bird's eye maple.

-orgel bird's organ. - persoektive

bird's eye view. - pfeife bird

whistle.

Vokale, halb- liquid consonants, semi-vowels, liquidae.

Vokaleinsatz beginning of vowel sound.

Voltanode unsplit anode (of magne(ron). -bogen semi-circular rail (of microscope).

vollfarbig of full (saturated) color.

Voll-flächner holohedron. -l eiter compact, unstranded or solid conductor. -macht ausstellen execute a power of attorney. -machtgeber principal, mandator. -netzanschluss all-electric supply, mains s. -niete solid rivet. -opalscheibe pot opal diffuser disk.

vollstreckbar enforceable, executable, executory.

Voll-weggleichrichter full-wave rectifier. -ziehungsbefehl writ of execution warrant.

Vol tampere(Scheinlei stung) var (unit of reactive power).

Voltapotential Volta potential, V. emf, V. effect.

Vol tmesser, Gleichrichter- rectifying voltmeter. Ionenwind- ionic wind voltmeter. Röhren-, selbstgleichrichtendes self-rectifying tube voltmeter. Scheitel spannungspeak voltmeter, crэst v.

Voltsekunde weber (magnet ic flux unit).
Vorbescheid

Vol umen, Mol- molar volume.

Volumen-gl eichrichtung volúme reclification. -schwankung volume flutter. -verlust loss in volume.

Vol um-gewi cht volume weight, weight of unit v. -prozent percent by volume. -verhältnis volume relation, proportion by $\mathrm{v}$.

Vomhundertsatz percentage. Vor amt, HIlfs- sub-control station. vor angehend preceding, leading, antecedent.

Voranode first anode (c.-r. tube); screen grid connected with cathode and acting as "fore-anode" in power pentode (Pierce circuit). voraus, Backbord port bow. -, recht right ahead. -, Steuerbord starboard bow.

voraus-gehend preceding, leading, previous, prior, antecedent. -nehmend anticipatory (patent law).

Voraussetzung postulate, premise, assumption, supposition, presumption, hypothesis.

Vorbedingung prerequisite, precondition, conditio sine qua non. vorbei-führen (cf vorbeistreifen) skirt, conduct or direct past (something), lead past at a glancing angle. - sehen not to look straight at each other (in (wo-way television telephone). -streichen,-streifen skirt, glance, graze, pass by, brush against (in more or less close contact with something).

Vor-bel adung pre-charge, preliminary c. (of sorptive in sorbent). -belichtung priming exposure or illumination, pre-exposure.-Zellen-primary illumination of a cell. - benutzung, of fenkundige oder öffentliche prior use, public use. -bereitung preparation, line-up, readying. -bereitung für Bildaufnahme lining up for shooting pictures. -bescheld preliminary action (of 


\section{Vorbild}

Patent Office Examiner), interim decision. -bild pattern, model, copy, prototype, type, exemplar. vorbringen argue, plea, reason. Vorder-ansicht front elevation, $f$. view. - blende anterior stop, field s.

vordere 8 rennebene front focal plane. $-(r)$ Brennpunkt first focal point.

Vorder-glied predecessor, antecedent. -licht front light (studio). -1 inse front lens, field 1. -mann stehen, auf lie in the shadow of. -satz premise, antecedent. -seite front side, front, obverse, face, panel.

Vordruck first impression, proof. voreilen lead.

Vor-eilung,Phasen-phase lead. -eilungswinkel angle of lead (of phases). -einflugzeichen fore marker, outer m. (beacon) (of airfield). -entladung pre-discharge. -entladungskanal pre-discharge track. -form gathering mold (in glass manufacture).

vorführen demonstrate, display, bring out, produce.

Vorführer projectionist, motionpicture operator.

Vorführung,Film- film projection; exposure of $f$. (in gate).

Vorführungs-film display film. -kabine,-raum projection room or booth (m.p.) -versuch demonstration experiment.

Vorgang process, action, event, phenomenon, reaction, proceeding, procedure, occurrence, happening.

Vorgang, einmal iger non-recurrent action, unique a., singular a., event or phenomenon. -, unperiodischer non-recurrent action, non-periodic a. or phenomenon. -, zeitllich veränderlicher action subject to time variation, a. variable with time. Ausgleichtransient. Bewegungs- motional action, cinematographic a. Einzel-single or separate process, action, phenomenon or event. Schalt- switching operation, process or action.

\section{Vorlegierung}

Vor-gänger predecessor, antecessor, antecedent, progenitor. -gelege transmission gearing, connecting gear. -geschichte previous history, antecedents.

vorgespannt pre-stressed (mech.), initially tensioned; biased (electrical potential).

vorgetäuschte Sehschwäche simulated amblyopia.

Vorgl imml i cht-gebiet sustaining voltage range, pre-phologlow region (arises at a voltage slightly below striking v.). -zelle photo-emissive gasfilled cell operating at sustaining voltage (in pre-glow region).

vorhall en over-emphasize; preverberate, pre-echo (ac.)

Vor-hal tewinkel drift angle, a. of lead. -hangblende curtain fading shutter. -hängeaufbl endung curtain fade-in. -hof;Trichterair chamber (adjacent horn of loudspeaker).

vorionisierte Spur pre-ionized track. Vor-kammer pre-chamber, ante-chamber; forehearth (oven). -kehrung precaution, provision, precautionary measure or step. -kehrungen, sicherstellende preventive measures.

vorkommen occur, happen. Vorkonzentration (cf Vorsammellinse) preliminary focusing, pre-focus, first focus action (in c. $-r$. tube).

vorladen cite, serve, subpoena, summon,

Vor-ladung appointment, summons, subpoena, writ, citation, notice, notification. -lage original copy, copy, pattern; receiver, condenser (zinc smelting). -1 auf lead or precession of sound (in relation to picture $=$ 19 frames $=361 \mathrm{~mm}$ ). -läufer forerunner, precursor; sign, indication, clue.

vorläufig provisional, preliminary, advance (e. g., notice).

Vor-legierung key alloy. 


\section{Vorlesung}

- lesungsversuch classroom experiment or study, lecture demonstration.

vorliche Kurslinie ahead course or direction, "go-to" indication. Vorlicht priming or biasing illumination.

vormagnetisierte Spule,gleichstromd.c. controlled saturable coil, three-legged reactor.

Vormagnetisierung bias magnetization, magnetic polarization. Vormagnetisierungsstrom biasing current.

vorn in front, anteriorly. vornherein, von from the outsel, from the first, to begin with. Vor-prüfer examiner (in Patent office). -prüfungsverfahren preliminary examination, preliminary search or action. -pumpe fore-pump, backing p. (for vacuum). -rang prioricy, precedence, superiority, first rank. -rat, Metall-, Quecksilbermercury pool (in rectifier).

Vorrats-flasche "Winchester", stock bottle. -leitung spare circuit, standby c. -lösung stock solution. -röhre input tubo, -rolle,-spule magazine, $\mathrm{m}$. roll.

Vorraum ante-chanber, fore-c., outer c.

Vorröhre input tube.

vorrüicken feed (forward), advance, progress, step forward, notch forward (e.g., a switch or controller). Vor-sammel inse first focusing lens, cathode 1. (like Wehnelt cylinder, apertured disk, etc., in c.-r. tube). -satz,Peil- directionfinder means added to standard receiver apparatus. -satzgerät aıtached or accessory device, head (m.p.) - satzlinse anteriorly attached lens, front 1 . attachment, magnascope (to enlarge projector image).

vorschalten cut in circuit, connect in series.

Vorschal twiderstand series resistance; multiplier (of a voltmeter). vorschlagbar capable of being moved, dropped, let or folded down on hinges.

Vorschrift, Bedi enungs- service direction, working instructions, directions for use. Dienst- service or working rules and regulations.

Gebrauchs- directions for use. Vorschub feed, conveyance. Papierpaper feed.

Vorschub-bewegung, zeilen- line sweep, 1. traversing motion. -rad feed wheel.

Vor-selektion pre-selection. -sichtsmassregel precaution, precautionary step or measure. -signal first or outer marking signal, caution or warning s. -spann label (of a print); leader (of a film). -spannorogramm prologue.

Vorspannung biasing potential (of a grid, magnet, etc.), bias, polarizing $p_{.}$; inherent bias tension (as in a spring) to restore normal position, normally tensed or tensioned condition, initial tension, pre-stress (in metal, etc.); anode, drive or positive p. (of a photo-cell). -verriegelnde cut-off biasing potential. Gitter-, automatische automatic bias or self-biasing of grid. Vor-spektrum preliminary spectrum (of Goldstein).

vorspringen project, protrude, be salient or prominent.

Vor-sprung projection, salient, lug, shoulder, protrusion, prominence. -stellung mental picture, conception, ideas, notion; representation, demonstration, performance, display. -stoss adapter, lap (of a tile), edging. -strom dark pre-sparking current. -stufe first or preliminary step; input or first stage (of an amplifier); primer. -synchronisieren pre-scoring (first sound, then picture).

vortäuschen simulate, mislead, 
delude, deceive, cause an illusion. Vortexring vortex (ring).

Vortransport supply sprocket.

-trieb positive drive, forward d., corque. -vakuumpumpe fore-pump, rough-vacuum $p_{\text {. , backing }}$.

-verfahren preliminary procedure or proceedings. -veröffentlichung anticipatory reference, anticipation (in patent or magazine literature), earlier publication. - verstärker input amplifier, pre-a. (class A RF amplifier, in a superheterodyne). -verstärkung gain amplification.

vorwalzen rough down (rolling mill work).

Vor-wärmung pre-heating, fore-warming. -warnung alert signal.

Vorwärts-bewegung active stroke (in film feed), forward motion, translatory movement. -gang

direct action (camera). -regelung

direct control, forward-acting regulation.

vorwegnehmend anticipatory (in the form of a disclosure in prior art). Vorwickel pull-down sprocket. -rolle,-trommel supply reel, (upper) feed sprocker, pull-down sprocket.

Vorwickler upper feed sprocket. Vorwiderstand series resistance. vorwiegend preponderant, predominani, especially, chiefly.

Vor-wölbung prutrusion, anterior

curvalure. -wurf subject (phot.) -zeichen indication, symptor, sign (math.). -zerleger filter (for ultraviolet) mounted anteriorly of monochromator entrance slit). -zugsrichtung privileged direction. 
wabenartig honeycombed, pitted (of structure):

Wabenspule honeycomb coil, latticewound $c_{.}$, duo-lateral c.

Wachfrequenz watch frequency, f. of international automatic alarm signal, distress f., mayday (in radiophony).

Wachs-abdruck impression in wax. -paste cerate or encauslic paste. -platte ( sof $\mathrm{i}$ ) wax disk, w. master (in disk manufacture). -tuch oilcloth, cerecloth (obs.). -tum, Kristallisations- germination, crystal growth. -tumkurve growth curve. -tumzentrum nucleus, center of growth, kernel. Wackelkontakt loose contact, variable or defective c.

wackeln shake, totter, rock, sway, quake.

Wackelung, Ver- frame unsteadiness. Wage,Dreh(feder)-torsion balance: Feder- spring balance, s. scales. Fein- precision balance, micro-b. Hebel- beam scale, lever s.

Mikra-micro balance, precision b. Stoss- ballistic pendulum.

Strom- -ampere balance.

Wagebalken beam of balance, scale b. Wagen, Aufnahme- pickup camera iruck, dolly. Fernsehau fnahmetelevision pickup camera truck, video bus. Lautsprecher- loudspeaker truck, sound t. Ton(film)- sound truck, location t., lorry set, sound van.

Wagenfett axle grease.

Wägeschal tung, $A b$ - comparator circuit organization.

Wagschale weighing-dish, scale or balance pan.

Wahl, Nummern- impulse action, stepping (automatic telephony).

Wähler, Schrittschal t- step-by-step selector.

wähler-bucht bay of selector. -hebeschritt vertical step of selector. -scheibe dial type selector switch. -segment bank of (stationary) contacts. wahllos at random, haphazardly, wi thout choice or selection, nonselective.

Wahl schalter selector switch. wahlweise selective, directional (in signal transmission.

wahre Peilung true bearing. Wahr-heitsbekräftigung affirmation. - nehmbarkeit perceptibility, noticeability, observability, visibility, discernibility, audibility.

wahrnehmen perceive, sense, notice, observe, be sentient.

Wahrnehmung perception, sensation, observation, percipience, sentience. Farben-color vision, $c$. perception. Schall-, des menschlichen Ohres human sound or tone perception. Ton- acoustical perception, auditory or sound sensation.

Wahrscheinl ichkeit, Ansprech-,eines Zählrohres efficiency of counter tube (probability to respond or register).

Wahrscheinlichkeits-gesetz probability law. - rechnung calculus of probability.

Waldhorn French horn, concert $h$. Walkerde fuller's earth.

Walze roller, roll, cylinder, drum, wheel, barrel. Bild-picture cylinder (in Bakewell's p. transmitter).

walzen roll, mill, laminate. vor- rough down (by rolling mill). wal zenförmig cylindrical, barrel shaped. Walzen-mühle roller mill. -strecke roll train.

Wal zwerk, Draht- wire rod mill. Wand wall, partition, side, cheek, shell, panel, screen, baffle. 
Wand, Bild-

wand, Bild-picture (projection) screen. Durchprojektions- translucent screen. Ganzmetall-allmetal (projection) screen. Schallbaffle (board) of a loudspeaker; sound panel (for s. absorption or reflection). Signal - wall of signals, curtain of $s$. Tonbildtransoral screen (m.p.).

Wand-bekl ei dung, schal l schl uckende wall draping, w. lining, baffle, blankets, sound absorbing material put on walls or panels, of ten portable, called "gobos" or "tormentors" (m.p. and sound stage). -durchführungsi sol ator wall lead-in insulator, bushing $i$.

Wander-licht (Personenabtaster) splotlighi scanner for persons. -marke wander mark, measuring $\mathrm{m}$. (in range finder).

wandern migrate, shift, crawl, creep, wander (of beam), travel (of waves or surges, over electric lines). wandernder Lichtstrahl flying spot, scanning spotlight.

Wanderung, Material- creep or flow of material (or metal).

wanderungs-geschwindigkeit rate of migration, crawl or creep (in telev., ions), m. velocity. -sinn direction of migration (of ions). nander-welle traveling wave, transient w., surge. -wellengenerator surge generator, impulse g., "lightning" g. -well enmesser klydonograph, surge indicator, s. recorder.

Wandler transformer, converter (of picture or image), transducer. - lichtelektrischer photo-cell. Bild-image converter, picture transf ormer, transducer (changes optical into electric images or patterns, etc.) Durchführungsbush-type transformer. Fernsehphoto-cells, light relays and similar devices(changing light into currents and back), television transducer. Frequenzfrequency iransformer, $f$. changer. Kurzwellen- short-wave converter. Mess- measuring
Wärmel eitzahl

transformer, instrument $t$. Wandlerspeicher röhre, Bild- supericonoscope.

Wand-stoffbekl eidung draping, baffle, blanket, sound-absorbing material, called gobo or tormentor when in form of portable wall (m.p. studio). -strom wall current (of a tube).

Wange cheek, side piece, end p. Wanne trough, tub, casing (of a muliiple condenser and its plates). - - pneumatische pneumatic trough (in gas drying). warmblasen hot blasting (water gas manufacture).

warmbrüchig hot-short, brittle when hot.

Wärme, gebundene latent heat.

Arbeitswert der mechanical equivalent of heat. Bildungsenthalpy, heat of formation. Eigen- specific heat, body heat, animal $h$.

Wärme-abführung, - abgabe, -ableitung, heat dissipation, h. evacuation, loss of h., h. "abduction", the carrying or conducting away of $h$. - arbeitswert mechanical equivalent of heat. - austauscher, Vielstufen- mulil-pass heat exchanger. - bewegung thermal agitation, heat motion.

wärmedurchlässig diathermic, thermanous.

Wärme-durchschlag breakdown due to thermal instability. - einheit (w.E.) thermal unit, heat u. -el ektrizität thermo-electricity. - entbindung, - entwicklung development, disengagement or evolution of heat, loss of $h$. -fluss heat flow, thermal $f$. or flux. - geräusch thermal noise (sort of Brownian motion of electrons in input circuit).

wärmegleich isothermal.

wärme-ionisation thermal ionization, temperacure i: -kapazität thermal capacity. -kraftlehre, -mechanik thermodynamics.

- leitvermögen,-leitzahl thermal conductivity. 


\section{wärmemesser}

Wärmemesser thermometer, calorimeter. -, thermoelektrischer thermel.

Sonnen- solarimeter, pyroheliometer. Wärme-rauschen thermal noise (thermal agitation or motion of eləctrons).

-schwingung heat vibration.

- speicher heat accumulator, regenerator. -stoff caloric, thermogen. -strahlung thermal radiation, iemperature $r_{\text {. }}$, heat $r .$, radian heal. -tod heal death. -tönung heat cone (in thermochemistry), heat quantity, h. effect. wärmeundurchlässig athermanous, impervious to heal, heat insulating. Wärme-vergangenheit thermal antecedents, prior heal ireatment. -verlust heat loss, thermal 1. -wirkung cemperature effecl, heat action, thermal a. warm-fest heat resistant. -gepresst hot-pressed, hot-press-fitted, subjected to thermo-plastic treatment.

Warnsignal (cf Entwarnen) air-raid alarm, caution signal.

Warnung, Vor- alert signal. Warze wart, nipple, pin, knob, boss. Wasser,Ultra- optically empty water. Wasser-abguss, Fagott- bassoon syphon. -ablass,-ableitung water drain, w. drainage, w. outlel.

wasser-abstossend repelling wacer, w. repellent, non-hygroscopic.

- anziehend water or moisture attracting, hygroscopic. -beständig stable in water or towards action of w. -bindend hydrophylic, waterabsorbent, w. imbibent.

Wasser-blase bubble of water; vessel or container for water heating. -druck hydraulic pressure. -entziehung dehydration, removal of water, desiccation. wasser-fest water-tight, w.-proof, resistant to w. -frei dehydrated, desiccated, anhydrous.

Wasserhahn water cock, w. tap, faucet.

wasserhaltig hydrous, hydrated, aqueous, containing water, w. bearing.

wässerig walery, hýdrous, aqueous.

\section{Wechsel bl ende}

Wasser-kraftl ehre hydrodynamics. -kühlröhre water-cooled tube. - luftpumpe water vacuum pump. -mantel water jacket.

wassern airplane traveling in contact with water. ab-rise or start from water. an- "land", alight or descend on water.

Wasser-schalter hydroblast switch. -schluss water seal, trap. - siedemesser hypsomeler.

Wasserstoff, schwerer deuterium (mass of 2), iritium (mass of 3 ). - ionenkonzentration pH value, hydrogen-ion concentration. -kern hydrogen or $\mathrm{H}$ particle or nucleus. -knallgas detonating gas (explosive mixture of hydrogen and oxygen). -strahl H ray (consisting of $\mathrm{H}$ particle or posi(ive hydrogen ion). -zahl hydrogen-ion concentration, $\mathrm{pH}$ value.

Wässerung washing (of film,etc.) Wässerungsgestell washing rack (phol.)

wasserunlöslich insoluble in water. Wasser-verschluss waler seal. -wert water equivalent (of a calorimeter).

watte absorbent cotton, wadding (of collon, glass wool, etc.), pad. Watt-leistung,-zahl wallage, power in terms of wall.

W.E. (wärmeeinheit) heal unil, thermal u.

Wechsel change, shift, alternation, cycle. Bild- (cf Vertikal-) picture cycle, moving period, feed stroke (m.p.) Horizontalhorizontal or line sync impulse or cycle. Last- cyclic stress, stress application cycle. Lichtvarialion of exposure. Polreversal of polarity, change of $p$. Vertikal- vertical or frame synchronizing pulse of cycle. Zeilen-s. Horizontal Wechsel-bezi ehung inter-relation, correlation, mutual or reciprocal relationship. -blende allernating shutter (in stereoscopy); masking disk (with spiral slou 


\section{Wechsel druck}

cyclically cooperating with quadruple scanner disk or drum apertures), auxiliary rotary shuiter disk with spiral slot. - druck alternating pressure. -festigkeit, Biege- alternating bending strength. -festigkeit, Verdrehungs- strength under alternating torsion stress. -frequenz,Bild-picture or frame frequency, repetilion f'. - frequenz, Zeilen- line frequency. - getriebe, Geschwindigkeitschange-speed gear. -hahn change cock. - induktion mutual inductance. -kardioide swilched cardioid (d.f.) -kontakt makeand-break contact. - licht light intensity variations (striking photo-cell). -note warble note, warble sound (in airplane alarms); appogiatura note (mus.) -periode, Zeilenzug- f'ield frequenry ( $=2$ x frame $\left.f_{0}\right)$-richter inverter, inverse or inverted (d.c. to a.c.) rectifier, d.c. to a.c. transverter (mostly combined with transformer).

-richter, Pendel- vibratory inverter, vibrator. -satz exchange principle. - schalter double-throw swilch.

wechselseitig reciprocal, mutual, interchangeable, alternate.

Wechsel sprechen, Funk - alternate twoway radiophone communication. Wechselstrom alternating current, indirect c., a.c. (specifically, single-phase current is mostly meanc), any periodic c. Leitungsconduction alternating current. Wechsel-stromempfänger a.c. electric radio set or receiver apparacus. -stromton hum in a lelev. picture (causing wavy edge or sides, pairing of lines, elc.) - tastverfahren reversing switch method (in d. f'. work); make-andbreak switch or keying method. -ton warble note or sound (used in airplane alarms); appoggiatura note (mus.) -verhältnis reciprocal relalion, $r$. proporition. -winkel alternate angle.

-wirkung inter-action, mutual or

cooperalive a., reciprocal effect. Wechselwirkungs-energie mucual potential energy. -glieder interaction terms. -potential interaction potential.

Wechsel-zahl, Bild- frame frequency, picture f., repetition f. -zahl, Last-alternating stress number. -zeit,Bild-frame frequency, picture f., repetition $f$.

wechsler, ob jektiv- revolving nosepiece (in microscope). plattenrecord changer (in phonograph). Strom- commutator, reverser. Wechslung, Fall system- drop system record changer.

Wecker, Schnarr- buzzer alarm. Weg (in Mechanik) displacement (in centimeters, in mechanics, equivalent lo charge, in coulombs, in electricity, and to volume displacement, in cubic centimeters, in ac.)

Weg, Eisen- iron path, magnetic or ferro-m. circuit. Gleis- rail track. Gleit- glide path, landing curve (of an airplane), landing beam. Neben-, by-path, by-pass. Übertragungs- transmission channel, t. path, l. medium.

Weg-ablenkung,Peilstrahl- distoriion of bearing, devialion (due lo diurnal or seasonal factors, weather, terrain, local conditions, etc.); lateral deviation. -abschnitt element of path, length or distance, portion, fraction or subdivision of length. weg-diffundieren dif't'use away. -heben, sich cancel oul (math.) wegig, ein- single-channel, one-way, simplex. mehr- of multi-channel or m.-path nature.

Weglänge,mittle freie mean free path. Schall- sound path length.

Weg-leitung directions for tracking trouble. -strecke distance, suretch, (element of) length. -tasten des Trägers, Synchronierung durch carrier interruption, suppression or gating of $c$. , 
synchronizing impulse by c. gating. -ïberführung overpass, overhead crossing. -unterführung underpass. -winkel transic angle (of klystron and other beam tubes). -zeitkurve trajectory.

Wehneltzyl inder Wehnelt cylinder, W. grid, W. shield (Lelev.)

weich gentle, smooth, yielding, soft; weak or of low contrast (of picture or image).

weich, schall- sound absorbent. weiche Röhre soft valve, ionic tube, gas-filled t., gassy $t$.

Weiche dividing network, d. filter, separator (for sync and video signals) comprising limiter tube and network.

weiche,Strom- current divider (filter).

weichen soak, steep; yield, give way; soften, plasticize.

weichlöten sof l-solder.

Weichzeichner sof $\mathrm{t}$-focus lens.

weigern refuse, decline.

Weihnachtsbaumantenne christmas tree antenna.

Weiser pointer, indicator, hand, guide. Kurs-, Richt- radio beacon, $r$ range.

weissbrüchig white fracture, pale $f$. Weissbuche white beech.

weisser El ektronenstrahl heterogeneous beam (of electrons).

weissglühend while-hot, incandescent. Weiss-guss white metal (cast).

- lot soft solder. -metallfutter,

Lager mit babbilled bearing. -wert bright level value (celev.)

Weisung direction-finding, guiding (by radio compass, etc.). Fehldirectional or d.f. error, quadrantal e. (Q.E.), compass deviation.

Weite, lichte inside diameter, $i$. width, lumen. Bild-distance between screen and lens, image intercept, i. distance. Brennfocal distance, f. length, convergence d. Einstell-focal range, focusing $r$. Gegenstandsdistance from object to lens, 0 . distance, d. from electron source (or from stop or crossover) to main 1. Schnitt- distance from back lens to image, intercept length. Schwingungs- amplitude of vibration, a. of oscillation. Seh-distance of vision, visual range. Sicht-optical distance, line of sight, direct-sight range or distance. Start- (der El ektronen) distance from origin, $d$. from objecl to lens, object d. Weiter-bildung furcher development (of an invention), evolutionary development or progress. -drehen des Schaltarmes stepping around or notching forward of the wiper, controller handle, elc. -führung einer Anmeldung prosecution of an application (for letters patent). weit-gehend far-reaching, substantial, thorough, appreciable; broad (of a patent specification, its disclosure or claim).

- geöffneter Konus wide-open cone or pencil. -maschiges Gitter open or wide-meshed grid, coarse g. -sichtig far-sighted, long-s., hyperopic.

Weitwinkelbild wide-angle picture, "Wide-scope" p.

weitwinklig wide-angled (phot., etc.) - (e) Aufnahme magnascopic picture. - (en) Kohlenstiften, bogenlampe mit scissors-type arc lamp.

Welle (cf. Wellenabgrenzung) wave; shafl, arbor, axle, spindle, roll, roller.

Welle, direkte ground wave, direcl w. ("hugging" curvature of earth). -, ebene plane wave. -, extremkurze ultrashor wave. -, fortschreitende progressing or progressive wave. -,gedämpfte discontinuous, damped or decadent wave. -,glei chstromüberlagerte wave superposed on or with d.c. -, impulsmodulierte pulsemodulated wave (used in radar). - , l inksl aufende sinistropropagaling wave or surge. 
Welle, rechteckige

-, rechteckige square wave.

-, rechtsl aufende dextro-propagating wave or surge. -, reflektierte reflected wave. -, rückl aufende retrogressive wave, reflected w. -, stehende standing wave, stationary w. -,ultrakurze ulirashort wave, quasi-optic w. -, ungedämpfte undamped wave, persistent w., continuous w. (c. w.). Welle, Anregungs- exciton (strictly an electron in a crystal moving in a field of a positive hole). Antriebszahntrommel- drive sprocket shaft. Boden- direct or ground wave ("hugging" curvature of earth). Bug- (eines Geschosses) nose wave (of a projectile). de Brogliede Broglie wave, electron w., phase w. Dehnungs- dilational wave. Dezimeter-micro-wave, decimeter w. Eigen-natural wave. Elektronende Broglie electron wave, phase w. Elementar - wavelet (of Huygens). Feld-field wave. Front-onde de choc, impact wave, bow w. (preceding a projectile's nose). Hohl (metali) rohr- metal-sheath conducting wave (in tubular or hollow pipe wave guidar). Huygens'sche Elementar- Huygen wavelet. Knallshock wave. Kopf-onde de choc, impact wave, bow w., shell w., ballistic w. (ballistics). Kreiscircular (electric) wave. Kugelsphericál wave. Leitbahn- transittime or electron-path oscillation. Luft- sky wave, space w., indirect w. Mantel- wave on outer surface of co-axial cabłe, "shell" wave. Materie- de Broglie elementary particle (electron or proton) wave, elementary w., phase w. Mikromicro wave, hyper-frequency w. (1-100 centimeters). Nuten-slot ripple. Phasen- de Broglie wave, phase w., electron w. Raum- space wave, sky w., indirect w.; spherical w. Rauml adungs- space-charge wave. Rohr-dielectric or conducting wave (solid or hollow pipe พave guide). Rundfunk-(cf

\section{Wellenberg}

Well enabgrenzung) broadcast wave, medium-frequency w. Schalt- wiper shaft, switch s. Spiegel- reflected wave. Staub-,Kundt'sche acoustic dust pattern (showing nodes and antinodes). Stevercontrol wave (in chain broadcast). Stirn- onde de choc, impact w., bow w. Störungs- garbling wave (in secret or coded telephony); parasitic w., interfering w., jamming w. Stoss- percussion wave, impact w., impulse w.; wave impacting upon a joint (say, in a pipe). Uberschall- supersonic wave, ultra-audio w., ultrasonic w. Ultrakurz-(cf. Well enabgrenzung) ultra short wave, uhf w., quasi-optical w. Unterschall- subaudio wave, infrasonic w. Verdichtungs- compression wave. Verdünnungs- rarefaction wave. Wander-traveling wave, transient (wave), surge. Zeichen- marking wave, signal w., keying w. Zwerg(cf Well lenabgrenzung) micro wave, midget w., dwarf w. Zwischenzeichen- spacing wave, back w.

Well enabgrenzung und - Einteilung frequency and wave-band designalion suggested by Fed. Communic. Comm. (FCC): very low-frequency (vIf) 10-30 kc; 10w-f. (If) 30-300 kc; medium-f. (mf) 300$3000 \mathrm{kc}$; high-f. (hf) $3-30 \mathrm{mc}$; very-high f. (vhf) 30-300 mc; ultra-high f. (uhf) 300-3000 mc; super-high f. (shf) 3,000-30,000 mc.

Wellen-antenne wave antenna,

Beverage a. - anzeiger cymoscope (ubs.), wave detector, oscillation detector, coherer. -aufzeichner, Schall-phonodeik (record on film), phonautograph. - ausbreitung in Hohlmetall rohr wave propagation in hollow metal pipe, metal-sheath conducting wave p. in wave guide. - bereichmelder wave band indicator. - berel chschalter wave band switch. - berg 
Wellenberglochwe ite

peak, crest or hump of a wave. -berglochweite distance separating peaks, intercrest d. -betrieb, Gleich-operation of stations on one wave, chain operation (from key station), network operation. - bündel wave packets, beam of electrons and ions. - bündelung beaming, directional effect of waves. -dämpfungskonstante wave al lenuation constant. -detektor cymoscope, wave detector, oscillation d., coherer. - echo radio (signal or wave) echo (involving sinsle-, double-or muliiple-hop). -feld wave field. $-f$ läche,

Fresnel'sche Fresnel zone (of half-period elements).

wellenförmic undulatory, wavy, corrugated, rippled.

Wellen-front, geneigte tilied wave froni, l. wave head. -frontwinkel wave fron angle, w. tilt. - führung wave guide (either dielectric or conducting). -funktion wave function.

well lengerader Kondensator straightline wave-length condenser.

wellen-geschwindigkeit wave velocity, phase v. -gipfel peak, crest, or hump of a wave. -gleichung, d'Alembert'sche d'Alembertian wave equation. -konstante wave constant (meters per millimeter of piezo-electric resonator). -länge, Grenz- limiting wavelength. -leitung wave-guide (for dielectric or conducting waves). -linie wavy line, undulatory l., sinuous 1., rippled 1. -mechanik, Geometrisierung der geometric derivation of wave equation. well enmechanisch wave mechanical. Wellenmesser (cf Frequenzmesser) wave meter, Fleming's cymometer, frequency meter, ondometer.

Schwingaudion- autodyne wave meter or frequency $m$. Summer- buzzer wave meter.

Well en-rückstrahlung wave reflection (by hop to ionosphere and back). - sauger smoothing choke, series reactor, wave trap.

\section{Wender, Strom-}

- schalter wave-band switch. -schlucker(cf-sauger) wave trap (of absorptive or of impedance type). -schwanz wave tail (in facsimile). -spannung, bildvideo wave potential (telev.) -spannung, Ton- audio wave potential (telev.) -spektrum (cf - abgrenzung) spectrum of electromagnetic waves, radio wave specirum. -stirn wave face, w. front, w. head. -strom pulsating current, ripple c. -stromfilter ripple filter. -stumpf but end of a shaft. -tal trough or valley of a wave. -telegraphie,Drahtwired-wave telegraphy, wiredradio $t$. -widerstand characterislic impedance, surge i. (distinguished by some authors). -zahl wave number. -zugfrequenz group or wave-train frequency, spark f. -zugkohärenzlänge coherence length of wave trains. wellig wavy, rippled, undulated, pulsating. -, l ang- long-wave, of greal w.-length. -(e) Gleichspannung ripple potential, pulsating $p$.

Welligkeit ripple, rippled condiiion, humpiness (of resonance curve), pulsation factor; lack of linearity. Mehr-multi-wave property (of a crystal).

well rohr corrugated tube or tubing. Wellung rippling, corrugation, undulation.

Welt, Aussen- external world. welt-beschreibung cosmography. -raumstrahlen cosmic rays. weltzeitlicher Anteil des Potentialgefälles universal diurnal variation component of potential gradient.

Wendekurve turn in landing (in $\mathrm{ZZ}$ method), U turn.

wendel spiral, helix, coil. Kehrdoppel- reversed double loop. Hendel system, Doppel- biplane filament system.

Wendepunkt inflection point, reversing p., cusp, turning p. wender, Strom- current reverser, 
Wender, Ton-

commutator. Ton-tone converter. Wende-schalter reversing switch, $r$. key. -tangente langent to reversing point. -zeiger turn meter, $\boldsymbol{\imath}$. indicator (of airplane).

Wendigkeit maneuvrability, manageableness, ease of handling or operaling.

Werbung, Licht- light-advertising, propaganda using illumination effects.

werfen, sich warp, distort, deform. werfer,bild-picture projector. Strahlungs- beacon or beam station.

Werg oakum, tow.

Werk, Lauf- feed mechanism, drive m. Schalt- film feed mechanism, intermittent $\mathrm{m}$. (m.p.)

Werk-blei raw lead. -photo studio still. -stoff, technischer industrial, technical or commercial material. -stoff, Austauschsubstitute material. -stück work, piece of material or metal (to work on), blank to be machined or tooled, etc.

Wert, quadratischer Mittel- rootmean-square ( $\mathrm{rms}$ ) value, virtual v. Behaglichkeits- comfort value. Bei- constant, coefficient, factor, parameter, co-ordinate. Effektiv- effective value, rootmean-square $v_{0}$ (rms), virtual $v_{\text {. }}$ Nenn- nominal, normal, assessed, face or rated value. Schwarzblack-level value. Unterhaltungsentertainment value. weissbright-level value (telev.)

wertanzeiger, Maximum- peak indicator, crest i. Mininum- minimum indicator (for instance, of voltage).

Wertbestimnung valuation, evaluation, appraisal, estimate.

Werte, auseinandergehende divergent or discrepant values.

wertig,gerad- of even value.

Wertigkeitsformel valence formula, linkage $f$.

Wertung valuation, evaluation, appraisal, estimate.

Wesen (einer Erfindung) essence or essential feature of an invention, wi derbekl agter

(main) object of invention. wettbewerb, unl auterer unfair competition.

Wetter-forschung, Radiosonde für radiomeleorographic sonde, radiosonde. -messinstrumente mit funkentelegraphischer Fernübertragung radio-telemeteorologic or telemetric instruments.

Wetzstein whetstone.

wichte specific gravity (sp.gr.), unit of weight.

wickel, Kondensator- tubular condenser, paper capacitor, rolled or wrapped fixed condenser (of melal foils and paper or mica insulation), Nansbridge c. Vorpull-down sprocket.

wi ckel-blockkondensator tubular condenser, paper capacitor, wound or wrapped c. of fixed value (with metal foils and paper or mica insulation), Mansbridge c. -drähte, Gittergrid coil wires. -körper coil form, former.

wickuln wind, wrap, roll, twist. wi ckel turm mandrel.

Wi ckler, Nach- lower take - up sprocket. Vor-pull-down sprocket.

wicklung, angezapfte, in der Mitte center-lapped winding. -,gesehnte short-pitch winding, chord w. -,kleine bobbin. -, schrittverkürzte short-pitch winding. -, verteilte distributed winding. - Bifilarbifalar winding, Ayrton-Perry $w$. Dämper- amortisseur winding, damping w. Dritt- tertiary winding (of a transformer). Durchmesser full-pitch winding. Erstprimary winding. Korb- basket winding, low-capacitance w. Stufen-banked winding, pile w. Zweidraht- bifilar winding, Ayrton-Perry w. Zweit- secondary winding.

wi cklungs-faktor space factor, copper f. -halter,-träger winding form, coil form, c. support, skeleton form, former.

wider-beklagter cross-clain 


\section{wi derdruck}

defendani, counteraction d.

-druck counterpressure, reaction.

- haken barb. -hall repeated echo,

e. reflection, reverberation,

resonance. -hall, Flatter-

flutter echo, muliiple e. (be-

tween two parallel surfaces).

-hallzeit reverberation period,

r. time. -kläger cross-claim

plaintiff, counteraction p.

- lager abutment (immovable point

or surface sustaining pressure or

reaction). -legung rebuctal,

refutation, disproof.

wider-rechtlich illegal, unlawful,

contrary to law. -sinnig in or

of opposite or contrary sense or

direction (say, of rolation);

devoid of sense or logic, non-

sensical, illogical, irrational,

absurd. - spiegeln reflect.

- sprechend contrary, contradic-

tory, inconsistent, opposite.

wider spruch opposition, protest, objection, inconsistency, in opposition or conflict with.

widerstand ein- und ausschalten

cut in and out resistance.

-, induktionsfreier non-inductive

resistance, onm r. - , innerer(im

Innern) volume resistance, in-

sulation $r$. - magnetischer re-

luctance, magnetic resistance.

-, ohm'scher ohmic resistance, d.

c. resistance. -, spezifischer

resistivity (mass or volume), specific resistance. -, winkel-

freier resistance with zero

phase angle.

widerstand, Ableitungs- leak re-

sistance, leakance, resistance

leak (of a tube). Abschluss-

cermination or terminal re-

sistance or impedance.

Abzweig- leak coil, 1. resistance

(of a lelegraph repeater).

Ausbreitungs- diffusion resistance.

Ausgleich-compensating resistance, balancing $r_{0}$, build-out $r$.

Ball ast-ballasting resistance, ballast resistor. Beruhigungssteadying resistance, ballasting
Widerstand, Schallwellen-

r. Bewegungs- motional impedance. $B l$ ind; induktiver inductive reactance, positive r., magnelic r. Blind-,kapazitiver capacitive reactance, negalive r.; condensance. Dämpfungs- nonreactive, active, dissipacive or ohmic resistance. Dunkel-dark resistance (of a cell). Durchgangs-volume resistance (exclusive of surface $r_{.}$), insulation $r$. Durchlass- forward resistance (of a rectifier). Ei senwasserstoff- iron-hydrogen resistance. Entknurrungs- antigrowl resistance, $r$. to suppress growling noise due to $R F$ or tickler coil in regenerative path. Erder-grounder resistance. Fahr- aerodynamic drag (of an airplane). Falz-folding resistance. Federungs- compliance (ac.). Fusspunkt- terminating impedance, base-loading $i$. (of antenna). Haut- dermal or skin resistance; s. effect $r$. Isolations- insulation resistance, insulance (reciprocal of leakance). Kenn-characteristic impedance, image i., indicial i. Kenndämpfungs-, Ketten- ilerative impedance. $k$ lemmen- Lerminal impedance, $t$. resistance. Kopplungs- coupling resistance, Längs- line resistance, series $r$. (of a filter or network). Leerlauf-opencircuit impedance. Luft- air resistance, aerodynamic drag (of airplane). Massen- inertance. Nutz- (cf wirkwiderstand) useful resistance, signal r. oberflä chen- surface resistance; skin effect $r$. Quer-cross resistance, shunt r. (of a network).

Reibungs- (Schall) acoustic resistance (sound). Reihenverlustequivalent series resistance (of condenser). Richt- unidirectional resistance, valve effect. Schall- ratio pressure amplitude: velocity a. Schall relbungsacoustic resistance. Schallwellen- 
Widerstand, Schal l wi rk

acoustic impedance (comprising a. resistance and reactance).

Schallwirk- acoustic resistance. Schein- impedance, apparent resistance; impedor (part having i.) Schein-, Blindkomponente reactive component of impedance. Wirkkomponente active or dissipalive component of impedance.

Schieber- slide rheostat. Schwächungs- gain controller, g. reg lator, polentiometer. Si ebcontact resistance (in barrier cell). Stirn-(front) end resistance, leading end $r$.

Strahlungs- effective resistance (of loudspeaker, antenna, clc.), radiation $r_{\bullet}$, characteristic impedance. Strömungs- aerodynamic resistance, drag (of air).

Ubergangs- contact resistance. Ubergangs-, einer Probe flow resistance of test specimen. Verlust- non-reactive, effeclive, ohmic or dissipative resistance. Vor- series resistance. Vorschalt- series resistance; multiplier (of voltmeter).

Wellen-characteristic impedance, surge i. Wirk- non-reactive,.active, effective, dissipative or onm resistance. Zersetzungselectrolytic resistance.

Wi derstand-Reakt anzverhäl tn is

$Q$ factor, magnificalion $f$.

widerstands-ball ast resistance ballast, ballasting r. -brücke resistance bridge, Wheatstone b. - bijchse resistance box.

- fähigkeit,knick- folding endurance (of film). - fal schanpassung mismaiching of impedances. -kette vollage divider, potentiometer. -körper baffle, damper.

widerstandsloser $\mathrm{Krei}$ sesistanceless circuit (devoid of resistance or impedance).

Widerstands-messer ohm meter, megger, -moment section modulus (metal testing). -rauschen circuit noise (due to Brownian movement). -rezlprok inverse resistanne, $r$. reciprocal. -iberanpassung overmatching of impedance (Ioad resistance exceeds internal tube $r_{\text {. }}$ ) - unteranpassung under-matching of impedance. -verteilung (Im Potentiometer) laper (called linear $t$. if distribution of resistance is uniform per unit of length). widerstrahlen reflect, re-radiate. widrigenfalls in default of, failing which.

wi eder-abtretung re-assignment. - aufbau re-construction (of a facsimile picture). - aufnahme re opening (of a legal case); re-take (m.p.). -bel eb ig re-activation, rejuvenation (of a thoriated filament, etc.). - einfuhrung re-insertion, restoration.

wiedereinschal ten re-close (a switch), cut or connect in circuit again.

Wieder-erl angung recovery. -eröffnung re-opening (of a law suil).

wiedererstatten return, re-imburse, restitute.

Wiedergabe, $f l$ ache flat reproduction. -,flimmerfreie flicker-free reproduction. -, getreue good definition (of image by a lens, by telev, or facsimile, etc., receiver); orthophonic reproduction (of loudspeaker). - , hohle boomy reproduction ( $\mathrm{hf}$ cul off.) -,kontinuierliche flicker-free reproduction. Farben- color reproduction. Frequenz- frequency response (characteristic).

wiedergabe-brillianz brilliance of sound reproduction, "bounce." -dose sound pickup (head). -natürlichkeit faithfulness, fidelity or realism of reproduciion, brilliance. -optik, Tonsoundhead lens, s. optic. -qualität quality or faithfulness of reproduction (with "atmosphere" or "room tone"). - röhre,8raun'sche Braun c. $r$. tube or electronic picture reproducing tube.

wiedergewinnen recover, recuperate, re-claim. 
Wiederhall (repeated) echo,r. reflec. lion, reverberation, resonance. Flatter-flutcer echo, multiple e. -formel Ejring formula. wi ederholbar reproducible, repeatable, reiterable. wi eder-holungsaufnahme re-take. -holungsfrequenz repetition frequency; $f$. of recurrence. -instandsetzung re-conditioning, restoralion, renovation. wi eder-kehrend recurrent (periodically or not).

wieder-kehrspannung recovery voltage. -schein reflection, reflex. wieder-soi egeln,-strahlen reflect. wieder-vereinigunc re-combination (of ions in gas); focusing (of rajs, electrons, etc.). -zündung re-striking, re-ignition.

wilde Kopplung stray or spurious coupling, undesired c.

willkürlich arbitrary, haphazard, (at) random. -verteilt randomly distributed, scallered at random.

wil son-kammer cloud chamber, expansion c., fog c. -nebel spurmethode Vilson cloud track melhod (using streak of droplets and ionizing particle).

wind, dem - entgegendrehen head into the wind, crab. geçen den fliegen fly up wind. mit dem fliesen fly with tail wind. wind, Gesen- head wind, contrary w. kiicken-tail wind. Schall-fllow or draft of matter particles. Seiten- side or cross wind, wind on the beam, wind blowing across course.

winddreiecksrechnung airdrift

triangulation.

winden wind, coil, wrap.

wind-geschwindigkeit air velocity. - yeschwindigkeitsmesser anemomeler, wind meler. -kurs course heading in wind (coincides with map or headc. in absence of wind). -kurs, rechtweisender true heading. -1 ade wind chest, sound box (of organ). -maschine fan, blower. -sack wind cone, w. hose (airporı). -sammler air reservoir, compressedair lank.

wind-schief warped, deformed or twisted (out of shape). schnittig streamlined.

Windstärkemesser, Schalen- cup anemomeler.

Windungen, Ampere- ampere turns (als).

wintungs-fluss curn-filux, f'luxliens, magnelic linkage. - ganghöhe pilch (of curns). -kapazität internal capacitance, inter-turn c., self-capacitance. -schlussprüfer inter-turn shortcircuil tester or continuily tester.

Windwinkel angle of the wind, angle between head-on course and direction of $w_{\bullet}$, yaw angle.

Winkel, einspringender re-entrant angle. -, räumlicher solid angle (measured in steradian uniıs). Winkel, Abgangs- angle of deparcure. Ablenkungs- angle of deflection, a. of refraction. Abprallangle of ricochet. Achsenaxial angle. Anlauf- anste of approach. Anstell- angle of attack; a. of pitch, blade a. Aufnahme- shooting angle. Ausfallangle of emergence, $a_{\text {. }}$ of reflection. Aussprung- angle of reflection. beugungs- diffraction angle. sild-angle of image, a. of view. Blick- angle of view. brechungsangle of refraction. Dämpfungsphase angle difference, loss a. Drall- angle of (wist. Einfallangle of incidence. Einspringre-entrant angle. Ergänzungscomplementary angle. Erhebungsangle of elevation. Fall- angle of inclination. Fehl- phase displacement angle, shift a. Flächenplane angle. Gesichts- visual angle, optic a., facial a., camera or viewing angle. Glanz-glancing angle. Gleit- glide ansle. Grenz- crilical angle, limiling a. (of refractometer). Grenzflächen- interfacial angle 
(between two adjacent crystal faces). Grundrichtungs- base angle. Halb- half angle. ral böffnungs- semi-apertural angle. Haupteinfall-angle of principal incidence. Höhenangle of elevation, azimuth a., angular height, vertical visual a. Inklinations- magnetic inclination, m. dip. Komplementcomplementary angle. Konvergenzangle of convergence. Kursazimuth of target (gunnery); magnelic a. Lauf- transit angle. Leitstrahl-angle of beam. Luftangle of the wind. Luv-drift angle, a. of lead. Macheilangle of lag. sieben- adjacent angle, adjoining a. Peigungsinclination (angle), a. of slope, a. of dip. öffnuncs- aperture ansle, apertural a. Phasen-impedance angle, phase a.;6 facior, quality $f$. Polarisationspolarizing angle, 3rewster a. Prall-angle of reflection. Rand- angle of contact (between wall and liquid), wetting angle. kaum- solid angle. keflexionsangle of reflection. Ruhe- angle of repose, angle of friction (rarely used). Schärfenangular resolving power, focal angle. scheitel- opposite angle, vertex a., vertical a. Scherungs-, schiebungs- angle of shear. schiel-angle of strabism, a. of squint. Schneid-cutting angle (called dig-in a. or drag angle when other than 90 degrees, in record making). Schuitt- angle of repose, a. of friction (rare). Schwenkungsangle of traverse (in gunnery). Seh- visual angle. Sehschärfencrenz- angular resolving power, critical angle of visual acuity. Senkungs- depression angle. Sicht- angle of sight. sinus- sine of angle. Steigrise angle. Stoss- butting angle, a. of joint. Streu- angle of scattering. Tiefenangle of elevation below horizontal, a. of depression. Trennungs- angle of separation (in solid moving through fluid). lmschl inguncs- looping angle $\left(m_{*} p_{.}\right)$. Verdrehunys- angle of torsion, twist a. Verlustphase angle difference (of condenser), loss a. Voreilungsangle of lead (of airplane). Vorhalte-drift angle, angle of lead. wechsel- alternate angle. weg- transit angle (of klystron). hellenfront- wave front angls. wind-angle of the wind, angle between head-on course and wind direction. Zentri-sector angle, center a.

vinkel-abhängi gkeit angle dependence, as function of an angle. -abweichung angular deviation. -aufblendung angle fade-in. -bild, weit- wideangle picture, "Wide-scope" p. - eikonal angle iconal (opt.). winkelfreier widerstand resistance with zero phase angle.

winkel-frequenz angular frequency, radian f., pulsatance. -funktion trigonometric function, angular $f$. -geschwindigkeit pulsatance $(\omega)$, angular velocity, frequency in radians. -grad degree of angle, radian (unit of circular measure of angle). -halbierende bisector, bisłctrix (of angle). -hebel bell crank lever, angle 1. -konvergenz half-convergence error. -linie diagonal. -mass phase constant, wave-length c. (denotes phase shifi between input and output potential, and is the imaginary component of image transfer constant); set square. -mass, Kenn-iterative phase constant. -mass, Vierpolimage phase constant.

winkelmesser protractor, goniometer, angle gage (for eyes), theodolite (for surveys), angleometer (for measuring external angles). Karten- map protractor. Neigungs- 
Winkelmesser, 0 ffnungs-

clinometer. öffnungs- apertometer.

Winkel-messung, Kristall- crystallographic goniometry. -minute onesixtieth of one angular degree. -punkt infection point (of a curve).

winksl-randig with beveled edge. -recht right-angled, rectangular, orthogonal.

Winkelstellung angular position winkeltreu of true angle or bearing.

Winkel-unterschied, Konvergenzbinocular parallax difference. - verhältnis angular ratio. -verteilung angular distribution, d. in angle. - verzahnungsgetriebe double helical gear.

winklig,gleich- equi-angular, equiangled, isogonal. scharf- acuteangled. schief- oblique-angled. spitz- acute-angled. stump fobtuse-angled. weit- wide-angled (phot.)

winzige Gewebegebilde ultramicrons (of eye tissue).

Wippe rocker, balance, counterpoise b. Pohl'sche - Pohl commutator (double-pole, d.-throw switch). Robren- Kipp relay, trigger circuit.

Wirbel vortex, whorl, eddy, turbulence; spigot, button, collar, swivel, peg (in string instrument). Stimm- wrest pin.

Wirbel bewegung vortex motion, eddy m., eddying, whirl.

wirbel-frei irrotational. -lose strömung flow free from vortices, eddies or turbulence, irrotational $\mathrm{f}$.

wirbeln whirl, spin, warble.

Wirbel-strasse vortex avenue. -transporttheorie vorticity transport theory. -trommel high side drum.

Wirkdämpfung transducer loss. wirken act, work, operate, effectuate, effect, result in.

Wirk-komponente energy, active, watt, dissipative or wattful component, in-phase c. -leistung active wöl bungsfrei

power, actual p., true p. (in watts). -leitwert active admittance, conductance, -samkeit activity, effectiveness, efficacy, efficiency. in - setzen render operative, throw in gear, start. - schema actual or practical operating or working diagram. -strom active current, energy c., watt or wattful component.

Wirkung, Fern- telemetric action, distance a., remote effect.

Wirkungs-grad, Gesamt- overall efficiency, total e., commercial e. - grösse action magnitude, a. quantity. -kreis sphere of aotion, s. of influence, action radius.

wirkungslos inactive, ineffectual, inefficient, ineffective.

Wirkungs-quantum, $\mathrm{Pl}$ anck's $\mathrm{Planck}$ 's constant, quantity of action. - querschnitt effective crosssection or c.-sectional area.

Wirk-verbrauchsmesser active power meter. -verstärkung transducer gain. -widerstand non-reactive, active, effective, dissipative or ohm resistance. -widerstand$B l$ indwiderstandverhältnis $Q$ factor, quality $f$.

Wischkontakt self-cleaning or -wiping contact.

wissentlich deliberately, wilfully, knowingly.

wobbein wobble (intentional frequency variation, as by wobbulator, used in $f_{\text {. }}$-response test of radio apparatus); warble (as in secret telephony).

Wohnsitz legal or postal address, residence, place of living, domicile, office (in case of a company). wol ben vault, arch, make arcuate, curve, camber.

wolbung buckling, curvature (of image); arching, vaulting. Bildfeld-curvature of image field, lack of flatness. Filmfilm buckling.

wöl bungsfrei flat, uncurved, nonbuckled, flattened (of image or field). 
Wol fram-bogenlampe tungsten arclamp, pointolite. - faden, thoriumhaltiger thoriated tungsten wire or filament. - faden, wi ederbel ebter rejuvenated or re-activated tungsten filament, (subjected to flashing).

Wol fraumsaures Calzium calcium tungstate.

Wolke, El ektronen- electron cloud; $e$. shell. Ionen- ionic atmosphere. Rauml adungs- concentration or accumulation of space-charges.

Wolkenbildung formation of clouds, clouding.

Woll astonprisma Wollaston prism, double-image $p$.

Wollfilz wool felt.

Wortabschneidung clipping, obliteration or mulilation of words (in telephony).

Wörter-buch, Sach- encyclopedia. -verstümelung clipping, obliceration (of words).

wörtlich verbal, verbatim, literal, literatim.

wubbeln wobble, warble (by wobbulator, in coded (elephony).

Wucht, El ektronen- collision or bombarding force of electrons, impact of e. Mündungs- kinetic energy ac the muzzle. Ûber- unbalance, imbalance.

wuchtsteigerer dynamic expander. Wulst, Ring- tore, torus, toroid,

$X$-Einheit unit of wave-length for Röntgen rays, etc., Siegbahn unit.

y-geschnittete $\mathrm{Pl}$ atte $\mathrm{Y}$-cut plate or crystal.

Yucon (cf. schweres Elektron) "doughnut." Röhren- tubular tore. Sprühschutz- guard ring (against corona).

wul stförmig toroidal, doughnut-shaped. wunder Punkt snag, trouble, difficulty, moot point.

winschel rute divining rod, dousing $r$. wünschel rutengänger douser.

Wurf throw, cast, projection (of pictures). Schatten- sound shadows.

würfel, Lummer-Brodhun'scher L.-B. contrast photometer, cube p. (with cubical cavity). Pyrami den-tetrahexahedron. würfelchen, Abbe'sches Abbe drawing cube.

Würfeleck corner of cube, cubic summit (cryst.)

Wurfel ektrode, Bild- target elecirode.

würfelförmig cubic, cubical. Wurf-kraft projectile force. -lehre ballistics. - linie line of projection, curve of $p_{.}$, irajectory. -parabel irajectory parabola.

würmerkriechen swarming (indicative of excessive graininess, m.p.) wurstförmig toric, tire-shaped. Wurzel, Kubik- cube rool. Quadratsquare root.

Wurzel-zeichen radical, root sign. -ziehen extracting a root, process of evolution.

$\mathrm{x}$-geschnittene $\mathrm{Pl}$ atte $\mathrm{X}$-cut plate or crystal.

Yukawa particle or heavy electron (barytron, now known as mesotron). 
Zacke prong, tine, tooth, jag, serration.

Zackenblende vane with serrated or triangular edge, t. aperture (used in sound recording). Vielfach- light stop for making multi-lateral sound track.

Zackenrolle sprocket wheel.

Zackenschrift variable-area sound track, v.-width s. recording. Mehrfach- multilateral sound track. Zweifach- bilateral track.

Zackenspur, Abdeckdoppel- duplex variable-area track. Abdeckeinfach-, Einfach- unilateral variable-area sound track. Gegentakt- push-pull variable-area track. Verschiebebilateral variable-area track.

Zacken-tonspurbreite breadth of variable-width or variable-area sound track. -trommel sprocket drum, s. wheel.

zackig jagged, toothed, indented, serrated.

zäh tough, tenacious, viscous, viscid (liquids).

Zähflussigkeit viscosity; refractoriness.

Zähigkeits-einheit (poise) unit of viscosity. -kehrwert fluidity (inverse of viscosity). -messer mit Fallkörper viscosimeter, viscometer of balldrop type.

Zahl, dreistellige three-figure number. -, ganze integer, integral number.

-, ger ade even number. -, naturliche natural number. -, unbenannte indefinite number. -, ungerade odd number. -,vierstellige four-figure number, four-place n.

Zahl,Bild- picture frequency, frame f., repetition f. Dehnungs- coefficient of expansion, c. of extension. Gute- figure of merit; guality of $Q$ factor (ratio reactance-resistance of coil).

Hall raum- chamber coefficient (ac).
Haupt- cardinal number. Kenncharacteristic factor; office code. Kerbwirkungs- fatigue stress concentration figure. Kernladungsnuclear charge number. Koordinations- co-ordination number. Massen- mass number. Ordnungsordinal number, atomic n., serial n. Stellen- position number, atomic n.; index (math.). Überfuhrungstransport number (of ions), transference $n$., Hittorf $n$. Übergangstransfer number (of solutions).

Zählader meter wire, pilot w., marked $w$.

Zahlen-tl ank figure space, $f$. blank. -lehre arithmology, arithmetic, numerology.

zahlenmässig numerically, as far as numbers are concerned. -erfassbar numerically evaluable.

Zahlentafel table of figures, numerical table of tabulation.

Zähler,Bild- frame indicator, $f$. counter. Bl indverbrauchs- reactive volt-amp.-hour meter, sine m., wattless component $\mathrm{m}$., var-hour $\mathrm{m}$. Bl utkörper- haemacytometer, blood counter. Faden- thread counter, linen tester. Filin- footage counter (m.p.) Funken- spark counter. Meter- footage counter (m.p.). Scheinverbrauch- apparent power meter, trivector. Spitzenneedle counter (e. g., of Geiger). Staub- dust counter.

..zählig ...nnary (binary, ternary, etc. ) ...fold.

Zahn und Trieb rack and pinion. Zahn,Sperr- ratchet tooth, pawl, detent.

zähneln, zahnen tooth, indent, denticulate, notch, mill, knurl.

Zahn-getriebe, Schrauben- helical gear, spiral g., worm. -kranz tooth-wheel, gear rim, toothed $r$. -motor mit stimmgabelsteuerung 
phonic drum motor, p. wheel (of La Cour). -radfeinbewegung slow-motion gear. - sirene tooth-wheel synchronizer, tone-wheel. -stange rack (rod), tooth rack. -trieb rack and pinion drive, tooth-wheel d. -tronnelwelle, Antriebs-drive sprocket shaft. -werk gearing.

Zain ingol, bar, rod, pig.

Zängchen small forceps or tweezers. Zance (pair of') tongs, pincers, nippers, pliers, forceps, tweezers. Zance, Feder - spring pliers.

Zäpfchen, farbenemp findliche color distinguishing cones (of eye).

Zapfen peg, lenon, pin, plug, pivot; lap, spigol, bung; axle, journal, irunnion.

Zaofen,oreh-pivot, trunnion. Lager-journal. Lauf-journal, neck (of rolling mill). stirntrunnion, end journal.

Zaofen-kurve spectral response curve of eye (cones). -lager plain bearing, socket, bush, collar.

Zapfstelle tap, tapping point, tap connection.

Zapon varnish.

Zaser fiber, filament.

Zeemanstruktur, aufgelöste resolved Ze enen pattern.

Zehneck decason.

Zeliner-logarithmus logarithm to the base 10. -system decimal base, $d$. system.

Zehntellösung tenth-normal solution. zehntelnormale Lösung decinormal solucion.

Zeichen signal, sign, symbol, mark, stamp, imprint.

Zeichen, durch Störer verdeckte swamped signals.

Zeichen, Akten- serial number, file n., docket $n$. betrachtungsreference letter, r. numeral, symbol. Bezugs- reference symbols (numerals or letters, used in drawings). Bodenfunkstel lenrufground station call signal or sign. Einflug-boundary marker signal. Funkruf- radio call signal or sign, code signal,

\section{Zeiger}

"signature". Gleichheits- equality sign. Gleichlauf- synchronizing pulse, sync signai. Haupteinflug- inner marker signal, main entrance s. Kenn- characteristic feature, mark, sign, indication, clue, symptom; object (of an invention or palent). Kurz- symbol, symbolic denotation. Mehrfachmultiple (or multiplied echo) signal. rot-distress signal, SOS s. or call. Peil-call sign, code signal (e.g., of ground station). kuf-call or code signal or sign, "signature" (of a station). Schalt- symbols of circuit elements. Schau- optical signal, visual s., indicalor s., telltale means. Sender - transmission signal, transmitter s., station s. Voreinflug-fore or outer marker (of beacon). wurzel- radical sign, root s., radical. Zeittime signal.

Zeichen-frequenz signal frequency. -schiefer pencil slate. -spannung signal potential. -stromröhre marking valve. -trickfilm animated cartoon. -verbreiterung signal spread (due to echo effect). zeichnen, eine Kurve plot a curve, map a graph.

zeichnende Linse, scharf- achromatic lens.

Zeichner, Schall- sound recorder. Trickfilm- animator (of animated cartoons). Heich-sof $t$-focus lens.

Zeichnung drawing, sketch, cut, view, illustration, delineation or recreation (of an image, etc.), tracing, graphic representation.

zeichnung, Akten- drawing in docket, in case-records or file wrapper (of a patent application or legal case). - Block- block diagram, schematic drawing, diagrammatic illustration. Fern-perspective drawing. Schnitt- cross-sectional drawing, illustration, view or picture. Umriss- linear drawing, sketch or outline.

Zeiger pointer, hand, index hand, 
needle, indicator; measuring instrument, meter ( e.g. $_{0}$, wattmeter, etc); index, vector (math.).

Zeiger,kurs- navigator's compass. Leucht- dial light resonance or tuning indicator. Licht- spot lighting, point 1. Maximumwertpeak indicator, crest i. Messerknif $\epsilon$-edge pointer. Peil-pointer of d.f. apparatus. Schattenshadowgraph (tuning indicator). Sekunden- seconds hand. Spannungsvoltmeter; voltage vector. Stromammeter; current vector. Wendeturn indicator, t. meter (of airplane).

Zeiger-diagramm vector diagram. -instrument pointer-type instrument. -länge length of brush or pencil (in cathode-ray tube). -synchronisator dial scoring machine $\left(m_{0} p_{.}\right)$

Zeile,Dipol-dipole array.

Zeilen-ablenkung line scan, 1. scansion, 1. sweep. -abstand separation between lines, pitch, inter-line distance (telev.) - bewegung line scan (telev.) -breite line width, strip w., spot diameter (telev.). -durchlauf, Bild- line traversal (telev.) - fl immer, Zwi schen- inter-line flicker, shimmer, weave (telev.). -fol geimpulse fractional scan or partial s. impulses, linesequence or $I_{\text {. }}$ - set $i$. (in interlaced scanning). -frequenz line frequency, strip f., horizontal (scan) fo -hinlauf (cf Rücklauf) active line trace, scansion (of pencil or spot). -kippeinsatz incipient flyback. -kipper line time base. -kippschwingung line time base impulse. -raster, Zwi schen-interlaced scan. -raster, Zwi schen-, unger ader odd-line interlaced scanning. -rückl auf flyback, return, retrace (of pencil or spot). - schaltung picture traversing, vertical stepping down movement. -spiegelrad line scanning mirror drum (of scophony) -sprungfaktor interlace factor or ratio. -sprungmethode interlaced, intermeshed or interleaved scanning method. -spulen line scan coils, line sweep c. -steuerung velocity modulation, variable speed $\mathrm{m}$. Zeil ensynchroni si erungs-impuls line synchronizing impulse, high sync i. -lücke (cf Lückensynchronis.) line gap or interval (for in(roduction of line syne pulse). Zeilen-teilung line pitch (Lelev.). -überdeckung line overlap, spot o. -verlagerung migration or shift of line position. -verschiebung interlacing; line offset scan. -verstärker line or high sync impulse amplifier (telev.). -vor schubbewegung line sweep, line traversing motion. -wechselfrequenz line frequency. -zug line sequence, 1. series. -zugwechselperiode field frequency $(=2 \times$ frame $f$., (elev.)

Zeit, Abkling- decay period, dieout p., quench p. (in superregeneration). Anfach-buildingup period (iime required for oscillating to start). Anheiz(cathode) heating time (till stable temperature is reached), warm-up period, thermal time constant. Ankling- starting time, onset t. (of a tone). Anschwingbuild-up period ( $t$ ime required for oscillating to start). Aufbau- formation time (in spark discharge). Aufschaukel - time constant of resonant amplification. Ausregel- decline period (of control potential). BildspurWatkins factor, development $f$. (film). Dunkel- dark interval, d. period. Einregelungs- building-up or waxing period (of control potential). Einschwingbuild-up period (required for starting of oscillations). Eintauch-immersion period. Nachhal 1- reverberation period. Nachwi rk- hang-over period, decay p. Zerfall- decay time. 


\section{Zei tabl enkung}

Zeit-ablenkung time base. -abschnitt element of time, interval of $t$. - aufnahme time exposure. -blinker chronograph, time recording camera. - daver, Abstoppen von stopwatch measurement of time values or intervals of time. - dehnaufnahme high-speed camera shooting or picture (for slow-motion projection). - dehner slow-motion device. - dehnung high-speed camera shooting; time scale. -dehnung, Tonacoustic-slow motion. -festigkeit time strength, endurance s. (found by time test). -folge time sequence, consecution. - funk time topics program, timely t. -härtung time hardening. -kreiskondensator time-base condenser.

zeitlich temporal, concerning time, temporary. -veränderlicher Trägerstrom wobbled carrier current. -veränderlicher Vorgang action variable with time, a. subject to time variation. - (er) Verlauf shape of curve as function of time, t. slope.

Zeit-l upenverfahren high-speed take and low-speed projection method, slow-motion or retarded-action m. -marke, -mass time scale, marking of time (for synchronization). -masstab time scale. -inesser chronometer, time piece, watch, clock. -messer,registrierender chronoscope, chronograph. -messgerät chronographoscope. -messgerät, Mikro- microchronograph. -modulation velocity modulation, variable-speed m., variable-speed scanning. -raffaufnahmeverfahren time-lapse photography, low-speed shooting for high-speed projection. -raffer time-lapse-motion camera, stop-motion device.

zeitraubend time-consuming, tedious. Zeit-rechnung chronology. -regulierband timing tape. -relais, Invert- inverse-time relay. -schnell schal ter high-speed circuit breaker or switch. -schriftenliteratur journal literature, magazine $1 . \quad-$ si gnal time
Zelle, Vervielfacher-

signal. -skal eninstrument timescale instrument. - spirale time spiral. -verschiebung time lag, t. shift. zeichen time signal. zellähnlich cell-like, celloid, cellular, pitted, honeycomb-like. Zelle,lichtelektrische od. photoelektrische photo-electric cell, electric eye (either photoconductive, p.-voltaic or p.emissive, the latter preferably called photo-tube). -, photoel ektrische, mit Sekundärel ektronen - Vervielfachung photo-electric electron-multiplier tube, $e_{0}-m$. with photo-cathode.

Zelle, Alkali- alkali-metal (photoemissive) cell. Einheitsunit cell, elementary c., unit crystal, lattice u. El ek trophoreseelectrophoresis cell. El ementarunit cell, elementary c., u. crystal, lattice u. Gas- gasfilled (photo-emissive) cell. Gleichrichter-rectifier cell, barrier-layer c. Gl imm- photoemissive gas-filled cell (operated at potential slightly below critical discharge p.), photoglow tube. Haar-hair cell, capillary c. Hinterwand- photovoltaic barrier-layer cell with posterior metallic 1 . Lichtphoto (-electric) cell.

\section{Lichtsteuer- (Debye-Sears)} supersonic light valve, D.-S. lighi modulator. Mehrfachplatten-, von Karolus-Kerr Karolus multiple-plate Kerr cell. Piezoquarzsupersonic light valve (predicated on Brillouin and Debye-Sears effect). Selenium- selenium (photoconductive) cell. Sperr(schicht)barrier-layer photo-voltaic or photronic cell, rectifier c. loperating with a blocking or stopping layer). Streu- scattering cell. Thallium (sulfid)thalofide cell. Über schallsupersonic light valve. Vakuumvacuum photo (-electric) cell, photo-emissive (alkali) c. Vervielfacher-, mit Sekundäremission 
electron multiplier photo-tube (working with dynodes).

Vorderwand- photo voltaic barrierlayer cell with anterior metallic layer. Vorgl imml icht- photoemissive gas-filled cell operated at a sustaining potential in preglow region (slightly below striking or critical discharge p.).

zellenartige struktur honeycomb, pitted or cellular structure.

Zell en-kabel, Photo- low-capacitance shielded cable (connecting photocell and amplifier), video cable, concentric c., co-axial c. -verstärker,Photo- electron multiplier phototube; photocell amplifier. -vorbelichtung priming illumination of a cell.

zellig, zwischen- intercellular.

Zellstoff cellulose, pulp (paper).

-pappe pulp board, carton, millboard.

Zentralstrahl central ray.

zentral symmetrisch centro-symmetrical.

Zentren,Farb- F centers. Keimgrain centers.

zentriert, flächen-face-centered (cryst.). raum- body-centered, space-c. (cryst.)

Zentrierungsfeder spider (of loudspeaker coil).

Zentrifuge,Ultra- high-speed centrifuge.

zentrifugieren centrifuge, centrifugalize.

zentrisch centric, central, cocentric.

Zentriwinkel center angle, sector a. Zentrum,Kurbel- crank center. Massen- center of mass, c. of inertia, centroid. Ton- tonal center, acoustic c. (of "gravity") of frequencies. Wachstum- center of growth, nucleus kernel. zerbrechlich breakable, fragile, brittle.

Zerbrechungsfestigkeit resistance to breaking strain, b. strength. zerbröckelnd crumbling, crumbly, friable.

Zerdehnung, Bild- radial distortion of picture or image (pincushion effect).

Zerdrehung, Bild- rotational or tangential distortion, twist (barrel effect; telev. pictures). Zerdrückfestigkeit resistance to crushing or compression strain, crush strength.

Zerfall decomposition, dissociation, disintegration, decay. Atom- disintegration of atom, splitting or fission of $a$. Korn- grain disintegration. Mesonen- meson decay.

Zerfall barkeit, Nicht- nondisintegrability.

Zerfall elektronen electrons resulting from disintegration (say, mesotrons), decay e. zerfallen decompose, dissociate, disintegrate, undergo fission, break up, divide, crumble, fall apart, decay, breakup.

Zerfall-kurve decay curve. -produkte, radioaktive metabolons, successive disintegration products of radioactivity, -prozess decomposition process.

Zerfalls-elektronen decay electrons (resulting from disintegration, say, mesotrons). -konstante disintegration constant, transformation c., radio-active c., decay coefficient.

Zerfallzelt decay time, disintegration $t$.

zerfliessen deliquesce, melt, dissolve, run.

Zerhacker chopper. Licht- light chopper, episcotister.

zerhackt flicked of $f$, chopped. zerlegen decompose or split up (light beam), refract, diffract; take apart, demount, cut up, dissect, analyze, resolve, separate, knock down.

Zerleger scanner, dissector (of Farnsworth), exploring means. Bildfeld-picture scanner, image dissector. Vor-filter mounted anteriorly of mono-. chromator (for ul traviolet rays). -blende dissector aperture, 
zerlegtes

scanning hole.

zerlegtes Licht, spektral- spectroscopically dispersed or separated light.

Zerlegung (cf zerlegen) analysis, decomposition, dissection, dispersion, resolution, definition. Bildfeld-image (field) definition. Licht- light dispersion, 1. decomposition, 1. refraction. Ton- sound analysis.

Zerlegungsvorrichtung scanner, exploring means.

Zerplatzen, Kern- nuclear fission, n. disintegration. Vakuumröhren-, nach innen implosion, inward collapse or burst of a tube.

Zerrbild distorted picture, p. made with an anamorphotic lens. zerreiben pulverize, powder, triturate, disintegrate or comminute by grinding or abrasive action.

zerreiblich friable, crumbly, triturable.

Zerreibung pulverization, trituraiion, atrition, comminution (by abrasion).

zerreissen tear, lacerate, break, rend, rupture, wear out, shred.

Zerreissprobe breaking test, tensile $\iota .$, rending $t$.

Zerrlinse anamorphosing lens, distorting lens.

Zerrungskreis annulating network; suppressing $n$.

zerschlagen break in pieces, smash, shatter, crush, batter.

zersetzen dissociate, decompose, disintegrate, analyze.

Zersetzung, Ton- analysis of sound. Zersetzungs-kunst analysis, art of analyzing. -widerstand electrolytic resistance.

Zerspanbarkeit free cutting and machining property.

Zerspanung removal of metal by cutting tool (chipping, turning, shaving, etc.).

zerstäuben spatter, sputter, disintegrate, vaporize, atomize, reduce to dust or mist, spray. Zerstäubung disintegration, sputter,

\section{zeugen}

splutter or spatter (of cathode); spraying, atomization.

zerstörend, holz- Iignicidal.

Zerstörung, lochfrassähnl iche

pitting, destruction in form of

pits, honeycombing.

zerstörungsfreie Prüfung non-

destructive test.

zerstossen pound (to pieces), bray, bruise, powder, pulverize.

Zerstrahlung annihilation radiation (due to collision and mutual annihilation of atoms, electrons and positrons).

zerstreuen disperse, scatter, dissipate, diffuse.

zerstreuend, licht- light dispersive, 1. diffusive, 1. scattering.

Zerstreuung dispersion, diffusion. Energie- scatter of energy.

Farben- chromatic aberration, $c$. dispersion, chromatism.

Richtungs- scatter of direction (of beam).

Zerstreuungs-bild image formed by divergent lens. - bilder des Auges blur circles of эye. -kreis circle of diffusion, blur c. -linse dispersive lens, divergent 1., negative 1. - scheibchen circle of (least) diffusion, blur c. -vermögen dispersive power (opt.) -wirkung scattering (of elecirons, etc.)

zerteilt,fein- finely divided.

zertrümmerbares Material fissionable or disintegrable substance.

Zertrümmerbarkeit der Materie disintegrability of matter.

Zertrümmerung fragmentation, breakifig up into fragments, shattering, smashing, disintegration, fission (of atom).

Zertrümmerungswahrscheinl ichkeit probability of transmutation or disintegration.

Zessionar assign, assignee, transferee.

Zettel label, slip of paper, card, ticket, check, note.

Zeugelinie generatrix, Eenerating 1 ine. zeugen testify, give or bear testimony; procuce, create, generate. 


\section{Zeugengebühr}

Zeugengebühren witness fees.

Zeugnis testimony, testimonial, evidence, witness. - ablegen bear testimony or witness.

Zickzack-Reflexion staggered reflection.

zi egel rot brick-red.

Ziegenleder kid. leather.

zi ehbar ductile.

Zieh-diamant diamond die, -eisen drawing die, dieblock, draw plate, die p. (for drawing wire).

ziehen (cf Zi ehvorgänge) draw, pull, tug, drag; warp; describing a phenomenon in which oscillating suddenly ceases on loosening feedback coupling.

Zi ehen eines Bildes photographic travel ghost.

Ziehen,Kubikwurzel- extraction of cuoic root. Quadratwurzelextraction of square root. wurzelroot extraction, process of evolution.

Zieh-fähigkeit,Tief- cuppability, capability of being drawn or cupped in drop press. -grad ductility. -kraft drawing or tractive power, traction. - presse drop (forge) press (for drawing and forming sheet metal articles, etc.). -probe tensile test, t. t. specimen. -riefen fissures, drawing grooves or scratches. -vorgänge pulling, oscillation hysteresis, resonance discontinuily, protraclion or instability phenomena.

Ziel goal, aim, object, end, destination, mark, target, objective, boundary, limit.

Ziel-abflug flight away from object. -anflug flight towards object. - aufsuchen detection of target or object (by radar or sonar, etc.). zielen auf aim at, strive for, direct or tend to, adjust (sight).

Zielender, Geschütz- gunlayer.

Zielfernrohr sight telescope, bombsight, rifle s., telescopic s. Doppelblick- double direct sighting telescope.

Zielflug, Gehör- aural homing flight. sicht- visual homing flight.

\section{Zinnpfeife}

-empfänger homing receiver apparatus. -verfahren point to point avigation method, homing me thod.

Ziel-gerät course indicator. -kontroll apparat radio timing apparatus.

Ziel-kurs, ablaufender course away from transmitler or beacon. -, anl aufender course towards transmitter or beacon. -, mi sswei sender magnetic course to sweer. -, rechtwei sender true course to steer.

Ziel-linie finish line (in racing); line of sight, 1. of collimation (opt.). -marke sight graticule or reticle.

Zielpeilung, rechtweise nde Richtung vom Flugzeug zur bodenpeilstelle true reciprocal course (from airplane to ground station). -,missweisende Richtung vom Flugzeug zur Bodenpeilstelle magnetic reciprocal course (from airplane to ground station).

Zielpunktes, Anzlelen des sighting of target.

zielstrebig purposive.

Ziel-sucher radar (radio detection and ranging apparatus), sonar (under-water sound navigation and ranging). - verfol gung tracking a target (with detecting and ranging equipment and with directors, using a slewing motor).

Zierschiene grille bar.

Ziffer figure, digit, number, cipher. Anteil-percentage share. Formshape factor, theoretical stress concentration $f$. Kenn- index (of a log.), characteristic, code number. Verlust- phase angle difference $(\tan \delta)$.

Zifferblatt, Leucht- luminous dial.

Zinkbecher zinc case or cylinder (of dry cell).

Zinken (cf Zacke) prong, tine (of tuning fork); spike, tooth, jag, lug.

Zink-pfeife zinc pipe. - - ool zinc pole, cathode.

Zinnpfeife tin pipe. 
Zipfel lobes, ears, secondary beams, stray or side radiations (of a beacon station).

Zipfel, Yaupt-major lobe (of space pattern). Neben-minor lobe or ear (of space pattern).

Zirkel circle; pair of compasses. Spitzen-compasses, dividers. Taster-, Visier-gaging calipers. Zirkonbrenner zirconium burner, $z$. filament.

zischen hiss, sizz, fizz, sizzle. Zi schl aute sibilants, friccatives. Zi ther, Akkord- auto harp.

Zitter-bewegung circular fluctuation movement (of spin). -elektrode vibrating electrode, vibratory e.

zittern tremble, shake, quiver, vibrate, quake.

Zittern, Bild- unsteadiness of picture, jumping of $\mathrm{p}$.

Zone, stille oder tote skip zone, dead z. (radio reception).

Zone, Anhäufungs- zone of accumulation (of ultrasonic waves).

Auslösch-dead spot, skip distance, skip region (of silence). Leitequisignal zone, glide z. or path. Schlieren- dead zone, skip z., shadow z., shadow. Schweigeskip distance, shadow region, zone of silence (airplane landing). Verwaschungs- confusion zone, $z$. lacking definition, blurred or bleary z. (celev. pictures). Zonen-blende limiting stop (diaphragining out a given zone or area). -fehler zonal aberration. zu dicht overdense. - $\mathrm{fl}$ ach too low a gamma.

Zubehörteile accessories, spare parts.

zubereiten prepare, dress, finish, make ready.

züchten grow, raise, produce, cultivate.

Züchtung growth or growing (of crystals).

Züchtungsgefäss growing vessel.

Zuckanzeige ballistic, kick or flash (instrument) reading or indication (d.f., etc.)

Zuckerbestimmung saccharimetry, de-

\section{Zugkraft}

termination or analysis of sugar (with polarimeter).

zuerkennen adjudge, adjudicate, award. -, eine Entschädigung award damages.

zuerteillen award, allot.

zufällig accidental, incidental, haphazard, casual, random, by chance, fortuitous.

Zufälligkeit accidentalness, casualness, chance, probability, vagary, fortuity.

Zufalls-ergebnisse fortuitous results, f. findings. -gesetz law of chance, 1. of probability.

Zufluss flow, flux, afflux, influx, inlet, admission.

Zufuhr admission, inlet (for instance, in water-cooled tube), feed, supply, afferrence.

Zug drawing, pull, traction, draft, train, stroke, flue, drawtube (of microscope).

Zug(e), im - des Verstärkers in the channel or cascade of an amplifier. im - des Tonspaltes along the slit, in line with s.

Zug,künstlicher forced draft. Banden- band progression. Haupt- main, principal or outstanding feature or trait, main object (of an invention or a patent). Kurven-,abklingender decay train. Verstärkeramplifier channel. Zeilenline sequence, 1 . series.

zugänglich accessible, amenable. Zügen, in rohen in rough outline, roughly, approximately, practically, substantially, closely.

Zugentlastung traction relief. zugestandenermassen admittedly, concededly.

Zug-feder tension spring, traction s., retractiles. -festigkeit tensile strength, tenacity. - gitter positive grid; space charge g.

zügiger Gang intimate or positive threading (without play or backlash).

Zug-kraft traction, tractive force or effort, tension, attraction. 
-linie tractrix. -loch vent hole, air h. -luft (air) draft.

-posaune slide trombone. -rohr air pipe, vent p. or tube, stack. -wechselperiode, Zeilen- field frequency ( $=2 \mathrm{x}$ frame $\mathrm{f}$. )

Zuhörer auditor, audience (plurality of listeners).

Zuleitung, Antennen- antenna lead, downlead, feeder line, transmission 1.

Zuleitungsinduktivität lead inductance.

zumischen admix, mix with, add. Zündel ektrode ignitor (in ignitron). zünden ignite, start (an arc), set afire, kindle, light.

Zünder lighter, igniter, fuse, tinder. Zünder, Dampfdruck-vapor-pressure igriter. Doppel- lime and percussion fuse. Druck- pressure igniter. selbst- (pyrophorous) self-igniter, automatic lighter.

zunderfest tinder-proof.

Zünd-flämmchen pilot flame. -kirsche ignition pellet. -löschspannungsdifferenz striking-extinction potential difference. -papier ignition paper (phol.). -spannung firing potential, striking p. (of arc-lamp, rectifier, thyratron, ignitron, etc.), break-down p. (of insulator), flashover (of sparkgap), glow p. (at which initial glow-discharge begins, in photocell), p. at which, in glow-tube, discharge changes from assisted to self-sustained form. -stab ignitor, ignition rod, striking $r$. (in ignitron).

Zündung ignition, priming. Fehlfailure to fire or ignite (in rectifier, ignitron, etc.); misfiring (of engine). Frühpre-ignition, premature i. Rückbacklighting, arc-back, back-firing, backlash (in imperfect valve rectification).

Zündungseinsetzen initiation or incipience of striking or firing.

Zunge blade, reed, ditton, congue, vibrator, keeper (of a magnet). Kontakt- spring contact, reed, blade, ditzon.

Zungen-freouenzmesser vibrating reed frequency meter. -öffner latch opener.

Zungenfeife reed pipe or flute. Doppel- double-tongued flute. orgel- reed organ pipe.

Zungentelephom reed telephone receiver, Brown-type t.r.

Zuordnung correlation, co-ordination, assignment, allotment. zupassen fit, adjust. zupfen pick, pluck, tug, pull. zurechtmachen get ready, make ready, ready, prepare, adjusi, line-up.

Zurichten prepare, make ready, dress, finish, straighten, adjust.

zurückdrehen turn off, turn down (as in a lathe).

Zurückfallen in Grundzustand return or re-transition to ground state or lowermost energy level (of an atom).

zurück-führen trace (back), ascribe, attribute, fly or lead back, re-convene, reduce, return, restore. -halten retain, hold back, retard, stop. -prallen rebound, recoil, be reflected. -weisen dismiss, reject, refuse, disallow.

Zurückweisung overruling, rebuttal, dismissal, disallowance, rejection (of an application, petition, etc.)

zurückwerfen throw back, reflect (light waves), reverberate (sound waves), echo back, bend back (a ray, by ionosphere).

zusammenbacken cake, stick together, conglomerate, agglomerate.

Zusammenballen bunching of electrons in groups (by phase focusing, in drif $\mathrm{l}$ space of beam tubes); agglomeralion, conglomeralion, coagulation (of colloids and smoke particles).

Zusammen-ballungen bunching ( of charges or electrons). -bau erection, assembly or mounting work.

zusammenbrechen collapse, break down, implode (as an inwardly 
collapsing vacuum vessel).

Zusammendrückbarkeit compressibility. zusammen-drücken compress, squeeze, crush, compact, consolidate. - fallen collapse (as a field), implode (as a vacuum tube ); coincide, register, come together. -fallend coincident, be in congruity or in registration. -fassender Bericht survey, summarizing report or article.

Zusammen-fassung summary, résumé, compilation, abstraction, recapitulation. -gehörigkeit correlation (of things).

zusammengesetzt(er) $\mathrm{Kl}$ ang complex musical sound. -(e) Leitung compound circuit. -(e) Schwingung complex harmonic wave.

zusanmengesprengte $\mathrm{Pr}$ ismen brokencontact prisms (closely adjoined without cement, as in Cornu p.)

Zusammenhalt cohesion, unity. zusammen-hängende Photokathode plain mirror or continuous (nonmosaic) photo-cathode (as in Farnsworth dissector and super-emitron). -häufen heap or pile up, accumulate, aggregate, gather together. -kl appbar collapsible, foldable, knockdown. -laufen run together, converge, blend, coagulate, concur.

Zusammen-pressung der Luftteilchen compression or condensation of air particles (in sound wave). -schmoren von Kontakten scorching, freezing or melting logethar of contacts. - setzblende scanning hole, picture recreator aperture (to focus beam, in Farnsworth oscillight receiver).

zus ammen setzen compose, compound, assemble, fit together.

Zusammen setzungsvorrichtung, Bildpicture scanning disk (of telev. receiver), picture reproducer, recreator or delineator.

zusammensprengen join or fit together, without cement, as prims or objectives, in a broken-contact assembly.

Zusammen-stellung,Linsen- lens combination. -zi ehung, Quer- lateral contraction. -zi ehung, Querschnittscontraction or reduction of crosssectional area, necking, formation of a waist (in piece under tensile teet).

Zusatz addition, addendum, appendix. Lichtton- sound-on-film head or at tachment. Nadel ton- sound-ondisk attachment.

Zusatz-einrichtuns accessory, ancillary or auxiliary means or equipment. -gerät, Tonfilmsound film head or attachment. zusätzlich additional, supplemental, accessory, auxiliary, boosting.

Zusatz-l inse supplementary lens. -nachbildung building-out section (of transmission line). -patent patent of addition (to parent p.) -spannung booster potential, addition p. -träger suppressed carrier re-inserted in receiver (in side-band transmission of signals). -verluste stray load losses. -verstärker booster amplifier.

Zuschauer audience, (movie) patrons, spectators, viewers and/or listeners (sound film). -raum audi torium.

Zuschlag flux.

Zuschlagsgebühr extra tax or fee, supplementary fees, dues or charges.

zuschmelzen seal, melt together. zusetzen alloy, admix, add. zuspitzen point, sharpen, taper, tip.

zusprechen, dem Verletzten eine Geldsumme adjudicate or award a sum of money to injured or aggrieved party.

Zustand state, condition, situation, circumstance. -, eingeschwungener steady state oscillation. - geordneter ordered state or arrangement (of a structure), preferred configuration (of units in crystal lattice). Augenadaptations(state of) retinal adaptation. Dauer-steady state. Elektronen-, entarteter degenerate electron 
Zustand, Entstehungs-

state. Entstehungs- nascent

state, status nascendi.

Gleichgewichts - state of equilibrium, state of balance, stable s. Grund- ground state (of nucleus). Grund-,Zurückfallen in return or re-transition into ground state or lowermost energy level. Ruhestate of resi, repose or quiescence, steady, stationary or neutral s. or condition, no-signal or $Q$ s. (of a lube). (For additional terms of Ruhe...)

zuständic sein als Berufungsinstanz to have appellace jurisdiction. zuständiger Gerichtshof court of competent jurisdiction or venue.

Zustands-diagramm phase diagram. -formel state formula. -gleichung equation of state. -grössen variables of state. -summe pariial function. -verschiebung change in state, displacèment of s. Zustimmung assent, accession, acquiescence, consent, agreement, permission.

zuteilen allot, assign, delegate, give over, allocale; adjudicale, award.

Zutritt,Luft- access or admission of air, in the presence of $a$.

Zuweisung der wellenlängen frequency allocation or assignment.

Zuwiderhandl ung act in contravention of.

Zwang(es), Prinzip des geringsten principle of least constraint or resistance.

Zwang, Ausführungs- compulsory working (of a patent). Synchronisierlocked synchronism.

zwangläuflg (cf kraftschlüssig) positive, by positively acting means, by constraint, in a locked manner, nor-slip (in drive).

Zwangs-l izenz compulsory license. -schwingung forced oscillation. - vollstreckungsbefehl writ of (compulsory) execution.

zwangsweise by mandamus, by compulsion, compulsorily.

Zweckbel euchtung, Zwei - dual-purpose illumination.
Zwecke tack, pin, peg. Kamm- tack. zweckmässig suitable, appropriate, expedient, adapted, fit.

zwei-achsig biaxial. -adrig bifilar. -atomig diatomic. -äugiges Sehen binocular vision, stereoscopic v.

Zweibandspieler two-unit dubber. zwei-chöriges $\mathrm{Pi}$ ano bichord piano, เwo-string p. -deutig ambiguous, equivocal, double meaning, double entendre.

Zwei-drahtwicklung bifilar winding, Ayrton-Perry w. -elementkristall bimorph crystal (either of bender or (wister type).

Zweier-schal en duplet rings. -verfahren two-line-series or two fractional scans per frame (interlaced scanning method).

zweifach dual, double, two-fold.

Zwei fach-diodengl ei chrichter, -zweipolröhre duodiode rectifier, d. valve.

zwei-fädig bifilar. - farblger Schleier dichroic fog, dichromatic f., silver or red $f$.

Zweifeldröhre drift or beam lube working with two fields (of Heil, elc.)

zweiflächis dihedral, two-faced. Zweig branch, leg, arm (of a bridge or filter). Längs- line arm, series arm (of a filter or network). Mess- measuring arm.- P- P branch (spectrum lines). Quer- cross arm, shunt a. (of network).

zweigl iedrig binary, two-membered, binomial (math.) -(er) Kettenleiter two-section network.

Zweig-linien, Null-zero branch lines. - stromkreis divided circuit, branch c.

Zweiheit duality, dyade, couple. zweihöckrige Resonanzkurve doublehump resonance curve.

Zwei-koffergerät outfit in two carrying cases. -krei sempfänger two-circuic receiver. -kristallspektrometer double-crystal spectrometer.

zweiohriges Hören stereophonic or binaural hearing, plastic audition 
Zwe ip ack

or hearing, two-channel listening

(with auditory perspective):

Zweipackverfahren bipack məthod.

zweipoliger Hebel schal ter doublelever switch.

Zweipol-röhre,Zwei fach- duodiode (tube). -strecke dipola tube, dipole path.

zweischenk lig having two limbs, legs or branches, two-legged.

Zwei-schleifenoszillograph twostring oscillograph. - schlitzmagnetron two-split magnetron, (wo-segment $\mathrm{m}$.

zwei schneidig double-edged, two-e.

Zwei sei tenbandüberträger side-band transmitter with suppressed carrier.

zweiseitig two-sided, bilateral. -(es) Arbeiten two-way operation, double action.

zwelspitzige Kurve double-peak curve, double-hump c.

Zwei-stärkenbrille bifocal spectacles. -stoffsystem binary system.

zweistufiger Nocken double-lift cam (with two lobes).

zweiteilig bipartite, consisting of two parts.

Zwei-teilung bipartition, bisection. -tellergerät two-turntable type of player.

Zweitwicklung secondary winding.

zweiwellige Resonanzkurve two-hump resonance curve, double-hump r.c.

Zweiwelllgkeit two-wave property (of a tube, crystal, etc.).

Zwel-zackenschrift bilateral track (sound film). -zenterproblem twocenter problem. -zweckbel euchtung dual-purpose illumination.

Zwergwelle (cf Hell enabgrenzung) micro wave, midget w., dwarf พ.

Zwickbohrer twist drill.

Zwi ckel wedge, trycock. Bal ggusset.

Zwiebellinse biconvex lens.

Zwillings-bildung twinning, twin formation. -gleitung twin slipping. -kerne twin nuclei. -kristall twin crystal. -prisma biprism.

Zwinge clamp, cramp, vise, ferrule, hoop.
Zwischenraum

Zwischen-bild intermediate image, first i. (in super-microscopy). - bürstenmaschine metadyne, metadynamo. -fehler zonal aberration. -filmmethode intermediatefilm method (telev.) -flügel intermediary blade, anti-flicker b., balancing b. (m.p.). - fluoreszenzschirm intermediate fluorescence screen (supermicroscopy). -flüssigkeit intermediate liquid. -form passage type. - frequenz intermediate frequency, $i$. beat or transfer $f$. (in superhet. receiver). -frequen zemp fänger mit Kristallsteuerung crystal stabilized superheterodyne receiver, stenode radiostat. - gefäss intermediate vessel, 1. receptacle.

zwi schengeschichtet inter-stratified.

Zwischengitter-ion interstitial ion. -platzmechanismus interstitial mechanism (of alloys).

Zwi schen-glled intermediate member, connecting m., link, intermediate. -hörbetrieb duplex operation, transmitter and receiver alternately connected with aerial, alternate two-way communication. -kern compound core, intermediate. -kopie lavender copy. -körper intermediate medium or substance, interposed m. or means. -lagscheibe washer.

zwi schenlegen interpose, interlay, intercalate, insert.

Zwischen-l inienfl Immer interline flicker, shimmer, weave. -masse interstitial matter, cement (geology). -mittel intermediate medium or substance, interposed $\mathrm{m}$. or means. -modulation intermodulalion.

zwischenmolekulare Kräfte intermolecular forces.

Zwi schen-optik intermediate optic, interposed 0 . or optical means. -phase inter-phase (liquid dielectric). - positiv master positive. -raum intermediate space, intervening s., interstice, 
interspace, interstitial s., interval (of space). -raum, Polpole clearance, interpolar space or gap. -ring adapter (of microscope).

zwi schenschal ten interpose, interpolate, cut in between, cut in circuit,insert, include, intercalate.

Zwi schen-sender re-transmitler, repeater station. -stecker adapter plug, socket a. -stecker, Röhren- tube adapter, valve a. -stromröhre spacing valve. - ton medium cone, incer-

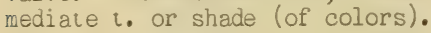
-träger sub-carrier. -transformator inter-stage transformer. - tubus intermediate tube (supermicroscope). -verstärker stage amplifier. -wand partition, separating wall. -zeichenwelle spacing wave, back w.

Zwischenzeil en-flimmer inter-line flicker, shimmer, weave (telev.). -raster interlaced scanning. -raster, ungerader odd-line scanning.

Zwi schenzeit time incerval, interim, meanwhile, intervening $t$. zwi schenzellis intercellular. Zwitschern birdies, canaries (extraneous high-frequency noise).

Zwitscherofeife bird warbIer.

Zwitter-apertur split focus (Walton scanning arrangement). -ion dual ion, amphoteric i., hybrid i.

Zwölf-eck dodecagon. -flach dodecahedron.

zwölfstufiges system duodecimal scale (tonal system).

Zyklotron (cf Resonanzbeschleuniger) cyclotron comprising two dees or half hollow cylinders and spiral beam. (Dther models of resonance accelerators using a bent beam are known as induction electron accelerators, rheotron, synchrotron, etc., all of them to be distinguished from a late 1946 model of accelerator which is of the linear, non-spiral tspe of accelerator gun).

Zylinder,Sammel- focusing cylinder. Steuer- Wehnelt grid or cylinder, focusing g., control electrode, shield (c.-r. tube).

Zymbal dulcimer, cymbal.

ZZ Verfahren ZZ (airplane) landing method. 


